\title{
Liquid Effluent Study: Ground Water Characterization Data
}

\section{Environmental Division}

Westinghouse Hanford Company

Date Published

August 1990

Prepared for the U.S. Department of Energy

Office of Environmental Restoration and

Waste Ni agement

(2) Westinghouse

Hanford Company Richland, Washington 99352

Hanford Operations and Engineering Contractor for the

U.S. Department of Energy under Contract DE-AC06-87RL10930 
WHC-EP-0366

\section{CONTENTS}

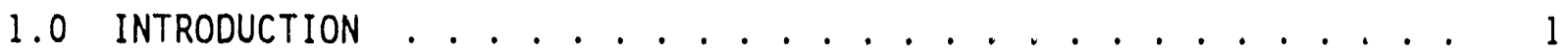

2.0 SAMPLING AND ANALYSIS RATIONALE .............. 1

3.0 QUALITY ASSURANCE/QUALITY CONTROL . . . . . . . ....?

3.1 DOCUMENT HIERARCHY .................. . . . 2

3.2 QUALITY ASSURANCE PROGRAM ............... 5

3.3 QUALITY CONTROL PROGRAM ................. . . . 5

4.0 MONITORING WELL AND SAMPLING CONDITIONS .........

4.1 FACTORS POTENTIALLY AFFECTING ANALYTICAL RESULTS ...... 9

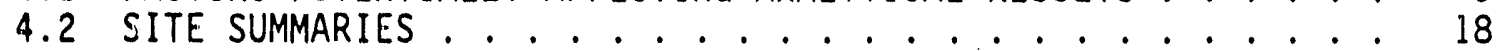

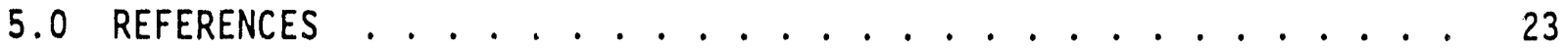

APPENDIX A - GROUNDWATER DATA .............. A-1

APPENDIX B - DATA VALIDATION SUMMARY SHEETS ........... B-1

APPENDIX $C$ - WELL LISTING AND SAMPLING DATES .......... $c-1$

APPENDIX D - INORGANIC SAMPLE REANALYSIS DATA ......... D-I

FIGURES:

1. Document Hierarchy ....................44 4

2. Comparison of Analytical Results for Selected Metals in
Groundwater Samples Analyzed by Two Different Methods .... 8

3. Time Series Concentrations of Selected Metals During Purgewater Removal at Well 299-W18-27 .......... 15

4. Plot of Constituent Concentrations in Non-RCRA Well 3-1-3 Versus Constituent Concentrations in RCRA Well 3-1-7.... 14

5. Plot of Constituent Concentrations in Non-RCRA Well 2-E34-1 Versus Constituent Concentrations in RCRA Well 2-E34-2 ... 17

TABLES:

1. Constituents Above Detection Level in Wells 3-1-3 and 3-1-7...13

2. Constituents Above Detection Level in Wells 2-E34-1 and $2-E 34-2$ 


\subsection{INTRODUCTION}

This report is a support document to the Liquid Effluent Study Final Project Report (WHC 1990c). The focus is on sampling and analysis rationale, quality assurance (QA), data validation, and sampling conditions for the groundwater quality assessment. Interpretation of the groundwater data is provided in the final project report.

\subsection{SAMPLING AND ANALYSIS RATIONALE}

As stated in the Liquid Effluent Study Project Plan (WHC 1990a), groundwater characterization is primarily a reconnaissance effort to assess the occurrence of contaminants in the immediate vicinity of liquid waste disposal sites. The approach was to: (1) use existing data from Resource Conservation and Recovery Act of 1976 (RCRA) monitoring projects and other operational programs, and (2) supplemental sampling from non-RCRA wells. Supplemental sampling from non-RCRA wells was necessary to provide sufficient spatial and temporal coverage to detect any contaminants that might have been associated with recent operational discharges. Relevant Hanford Site groundwater data acquired from January 1, 1989 through May 15, 1990, including the supplemental sampling results for this study, are included in Appendix A.

Because the project plan (WHC 1990a) constrained the time period for supplemental groundwater sampling to less than 1 yr and did not include the installation of new monitoring wells, the degree to which analytical results represent operational conditions is an important consideration. Groundwater data used in this report are representative of a variable portion of past operating conditions. The controlling variables for the occurrence of effluent constituents in groundwater beneath a disposal site are (1) the time required for the effluent to travel through the soil column, and (2) the mobility of the individual constituents. Constituents that have an affinity for sediments will migrate slower than those that remain with the liquid. Thus, the operating history of the waste stream/disposal facility and constituent sorptive properties must be considered in assessing the relationship between groundwater sampling results and contributing waste stream characteristics.

As specified in the project plan (WHC 1990a), all samples collected for this study were analyzed for 40 CFR 264 Appendix IX constituents and sitespecific radionuclides. The Appendix IX list of analytes is a broad spectrum of organic and inorganic hazardous constituents. Site-specific radionuclides not on the Appendix IX 1 ist were selected from relevant effluent stream characterization reports (WHC 1989a).

Analytical data summary tables (Appendix A) for each disposal site list every analyte fior which results were reported by the analytical laboratory (U.S. Testing Company, Inc. [U.S. Testing]). The tables present statistical summary information (average result, minimum result, maximum result) for samples collected and analyzed during the time period specified for this report (January 1, 1989 to May 15, 1990). Only a few results were 
not available in time for this report. Promethium-147, for example, a site-specific constituent in some PUREX waste streams was not analyzed due to contractual problems involving U.S. Testing.

Additional analytical background information and sampling conditions are provided in the following sections. A compelete well-by-well listing of results can be obtained through electronic access of the Hanford Ground Water Data Base. A hard copy listing by well is also available in the Administrative Record files.

\subsection{QUALITY ASSURANCE/QUALITY CONTROL}

This section presents quality controls used to ensure the reliability of groundwater analytical results. The document hierarchy which defined the quality controis is discussed first followed by general and projectspecific controls and interpretations.

\subsection{DOCUMENT HIERARCHY}

Figure 1 presents the document hierarchy. The pertinent sections defining $Q A / Q C$ of each document are summarized below.

- Liquid Effluent Study Project Plan (WHC 1990a). This plan described al? activities to be accomplished during the characterization and assessment effort. The plan stated that groundwater sampling and analysis will be conducted in accordance with established protocol for RCRA compliance monitoring already in place.

- Waste Stream Characterization Report (WHC 1989a). Volume I of this report incorporated the following elements related to $Q A / Q C$ :

- Proposed sampling schedule

- Proposed listing of analytical project parameters and procedures.

Volume IV of this report described $Q A / Q C$ in Section 2.2 (Ground Watelr Sample Collection Procedures). All sampling activities were performed by Pacific Northwest Laboratory (PNL). Section 2.2.11 (Quality Assurance/Quality Control) described the PNL contract $Q A / Q C$ program.

- Groundwater Sampling and Analys is Statement of Work (WHC 1989C and 1990a). Statements of work (SOW) were transmitted for FY 1989 and 1990 from Westinghouse Hanford to PNL to establish the requirements and sampling schedules for groundwater sampling and analysis activities. QA/QC sections were included in each SOW which (1) required a RCRA QA project plan (QAPP) that met the requirements of QAMS-005/80, Interim Guidel ines and Specificationss for Preparing Quality Assurance Project Plans (EPA 1983) and pertinent U.S. Department of Energy (DOE) Orders, and (2) required that all activities would be consistent with the irotocols and recommendations provided in U.S. Environmental 
Protection Agency's (EPA) RCRA Ground Water Monitoring Technical Enforcement Guidance Document (EPA 1986a) and Test Methods for Evaluating Solid Waste (EPA 1986b). Additionally, QC records were to be maintained with which to verify accuracy and precision of analytical data.

Analytical data used in this report for January 1989 to August 1989 were covered by the FY 1989 SOW. The wells sampled during the period of September 1989 until April 1990 (specifically for the Liquid Effluent Study; see Appendix C for complete well listing and sample dates) fell under the FY 1990 SOW.

- Groundwater Sampling and Analys is Program Management Plan (PNL $1989 a$ and 1990a). These plans were prepared by PNL to satisfy a deliverable required in the SOW. Each program management plan describes the administration and technical management of activities contained in the related sow.

- Quality Ass'irance Project Plan (PNL 1989b). A PNL-prepared document which addresses the QA controls for the various RCRA groundwater monitoring project activities. The QAPP is reviewed and approved by Westinghouse Hanford. Organizational structure, data quality objectives, procedural controls, QC checks, audits, corrective actions, reports, and records are defined.

- Analytical Laboratory Contract. $Q A$ and $Q C$ requirements in this document reflect the requirements in the SOW (WHC 1989a and 1990) and the QAPP (PNL 1989). The contract for analytical services during the time period covered in this report was given to U.S. Testing. The contract contained the following QA clauses: the laboratory was to assure the integrity and validity of test results through an internal QC program which included written $Q C$ procedures, QA samples, and corrective actions, (2) the laboratory would maintain EPA Safe Drinking Water Certification, (3) the laboratory would meet QC requirments of EPA's Test Methods for Evaluating Solid Waste - Physical/Chemical Methods (EPA 1986a), (4) the laboratory would participate in interlaboratory comparisons and audits (i.e., DOE/Environmental Monitoring Laboratory Quality Assessment Program, EPA Environmental Radioactivity Laboratory intercomparisons, and EPA Water Pollution and Water Supply Performance Evaluation studies), and (5) the laboratory would produce a quarterly report of interlaboratory comparisons and $Q C$ results.

- Procedures (Laboratory). Procedures regarding internal QC requirements were contained in the U.S. Testing QC manual.

- Procedures (Sampling and Data Management). Procedures regarding PNL activities to collect representative samples, ensure the chain-of-custody of those samples, and manage analytical data received from the laboratory are contained in PNL's Procedures for Ground-Water Investigations (PNL 1389c). 
Figure 1. Document Hierarchy.

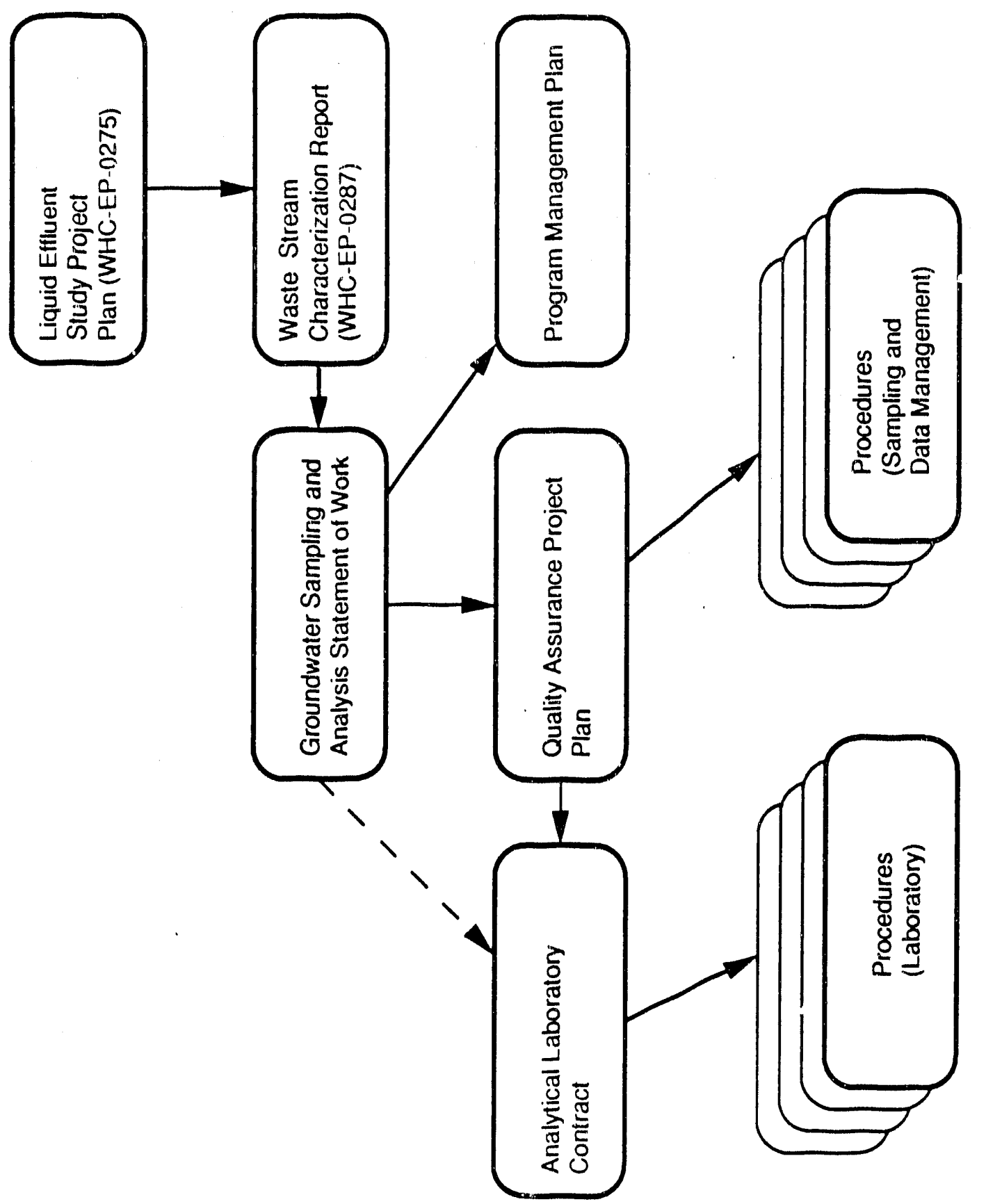




\subsection{QUALITY ASSURANCE PROGRAM}

The PNL RCRA Quality Assurance Project Plan (PNL 1989b) defined the QA requirements for analytical work. Content of this document is summarized above. Audits and surveillances were conducted by PNL, Westinghouse Hanford, DOE, and the EPA on various aspects of this work.

\subsection{QUALITY CONTROL PROGRAM}

\subsubsection{Groundwater Sampling and Analysis Quality Control Program}

Pacific Northwest Laboratory conducts a QC program to assess the quality of data reported for groundwater samples collected under the Westinghouse Hanford SOW. The QC program is based on guidance from EPA, Region 10 and the EPA RCRA Groundwater Monitoring Technical Enforcement Guidance Document (EPA 1986a). Analytical procedures were in place at U.S. Testing for the performance of analytical work in accordance with the current edition of EPA's Test Methods for Evaluating Solid Waste (EPA 1986b). Key elements of the QC program include: (1) submission of blind standards, blanks, and duplicate samples to U.S. Testing; (2) submission of replicate samples to alternate laboratories; and (3) participation of U.S. Testing in the interlaboratory comparison programs.

Evaluation of $Q C$ samples is as follows: For blind standard samples where the concentration is known, 2.0 standard deviations (95\% confidence interval) about the expected value is used as an acceptable target range. If results fall outside the $95 \%$ confidence interval, a request for data verification is warranted. Values from interlaboratory comparisons of field samples must fall within 2.8 standard deviations based on EPA Water Supply or Water Pollution Laboratory Performance Evaluation Studies to de considered equivalent analyses. This calculation takes into account the uncertainties associated with two sources of analyses.

The PNL reports the results from the QC program in monthly reports to Westinghouse Hanford and in quarterly summaries that are included in the RCRA quarterly data report which is submitied by DOE, Richland Operations Office (DOE-RL) to Washington Department of Ecology (Ecology). The quarterly summaries are discussed here (PNL 1989d,e,f; WHC 1990b,c). Prior to October 1989, the quarterly reports noted analytical anomalies. For the three quarters beginning January 1989, and ending in September 1989, the anomalies reported were: (1) sporadic occurrences of methylene chloride in volatile organic hydrocarbons and total organic halogens, (2) chromium in unfiltered samples from new wells, (3) acetone in field b? anks (thought to be due to the reuse of sampling bottles), and (4) matrix interference effects for fluoride analyses. Beginning in October 1989, summaries of the QC blind standards and interlaboratory replicates were added to the quarterly reports. For the three quarters following October 1989, the only analytical anomaly reported was a continuation of the fluoride matrix interference (October through December 1989). The blind standard and replicate results are tabulated below. Reasons for unacceptable (out-oflimit) results are contained in the quarterly reports. 


\begin{tabular}{|c|c|c|}
\hline Quarter & $\begin{array}{l}\text { Bl ind Standards } \\
{[\%, \text { no. analyses) }]}\end{array}$ & $\begin{array}{l}\text { Replicates } \\
[\%, \text { no. analyses })]\end{array}$ \\
\hline Oct-Dec, 1989 & $\begin{array}{l}92 \% \text { acceptable, (113) } \\
8 \% \text { unacceptable }\end{array}$ & $\begin{array}{l}98.3 \% \text { acceptable, (933) } \\
1.7 \% \text { unacceptable }\end{array}$ \\
\hline Jari-Mar, 1990 & $\begin{array}{l}88.7 \% \text { acceptable, } \\
11.3 \% \text { unacceptabie }\end{array}$ & $\begin{array}{l}98.3 \% \text { acceptable, }(514) \\
1.7 \% \text { unacceptable }\end{array}$ \\
\hline Apr-Jun, 1990 & $100 \%$ acceptable, (71) & $\begin{array}{l}98.4 \% \text { acceptable, }(1,150) \\
1.6 \% \text { unacceptabie }\end{array}$ \\
\hline
\end{tabular}

\subsubsection{Liquid Effluent Study Quality Control Activities}

Two activities have been undertaken by Westinghouse Hanford to verify and validate the analytical results for the wells sampled for this report. These checks are in addition to the QC program conducted by PNL. The two activities are (1) a validation of select analytical results and (2) a reanalysis of inorganic samples by another laboratory.

3.3.2.1 Analytical Data Validation. The Westinghouse Hanford Office of Sample Management has conducted a data validation review of select analytical data collected for this report. The procedural steps included in the review are described in Appendix $B$. The validation level chosen for this review is Level B (see Appendix B for a detailed description).

In general, a Level B validation includes a systematic review of analytical data from field samples and their associated laboratory QC samples. The purpose of the review is to make determinations concerning data quality and data limitations based on a review of laboratory performance and implementation of applicable protocols. This is accomplished through documentation and the use of data qualifiers. Each step of the validation procedure is documented. A data validation coversheet is attached to each package which summarizes the entire review and gives an overall assessment of the sampie. Major and minor problems are noted and data qualifiers explained. Data validation coversheets are included in Appendix B.

The sample sets from 10 of the 90 well samples collected specifically for this study were reviewed $(11 \%)$. No major problems were noted. Minor problems noted on the summary sheets are as follows:

- Direct Aqueous Injection

- Holding times - Results for eight wells were flagged as estimated due to missed holding times. This analys is was performed using a U.S. Testing procedure. The Office of Sample Management used a 28-day holding time ir the evaluation. The U.S. Testing procedure specif ed 40 days. Only one of the samples exceeded the 40-day linit $(2-E 25-34)$. 
- Herbicides

- Holding times - One well exceeded the extraction holding sime ( 8 days out).

- Low surrogate recovery - Results for one well flagged.

- High matrix spike duplicate \% recovery - Results for one well flagged.

- High matrix spike \% recovery - Results for eight wells flagged.

- Hydrazine

- Low matrix spike/matrix spike duplicate \% recoveries Results for six wells flagged.

- Dioxin

- Low surrogate recovery - Results for one well flagged.

Based on this survey, it appears that no potentially serious or major violations of procedural controls occurred which would invalidate the data. The most serious problems noted were the instances in which holding times were exceeded. As explained above, in this survey it affected the results from two wells (Direct Aqueous Injection for one, Herbicides for the other).

3.4.2.2 Inorganic Sample Reanalysis. Ten archived groundwater sample sets (nitric acid preserved, including filtered and unfiltered samples) from the sampling effort conducted for the Liquid Effluent Study were reanalyzed by the PNL analytical laboratories as an independent check. The primary objective was to validate the occurrence of a large number of less-thandetection-limit metal results reported by U.S. Testing.

Reanalysis was performed by direct aqueous injection of the sample media into an inductively coupled plasma emission mass spectrometer (ICP. MS). Detection limits with ICP-MS are generally more than a factor of 10 lower than the conventional methods used for the present study. Thus, it was possible to verify the validity of the less-than-detection-limit results as well as to obtain an intercomparison for all positive occurrences.

Results of this colparison are included in Appendix D. Of the 383 metal results, $53 \%$ were reported by U.S. Testing as below detection. The ICP-MS results (Appendix D) confirm that all of the less-than-detectionlimit results reported by U.S. Testing are in fact at or below the stated U.S. Testing detection limit.

A comparison of the positive results, which consist primarily of unfiltered samples (the filtered samples yield primarily less-thandetection-limit result.s from U. S. Testing), is shown in Figure 2.

Considering that most of the results for the comparison were for unfiltered water samples, the agreement is reason-ably good. For example, unfiltered samples may result in some variability due to the inhomogeneity of the sample matrix (i.e., due to nonuniform entrainment of particulates in the injected sample volume). 
Figure 2. Comparison of Analytical Results for Selected Metals in Groundwater Samples Analyzed by Two Different Methods.

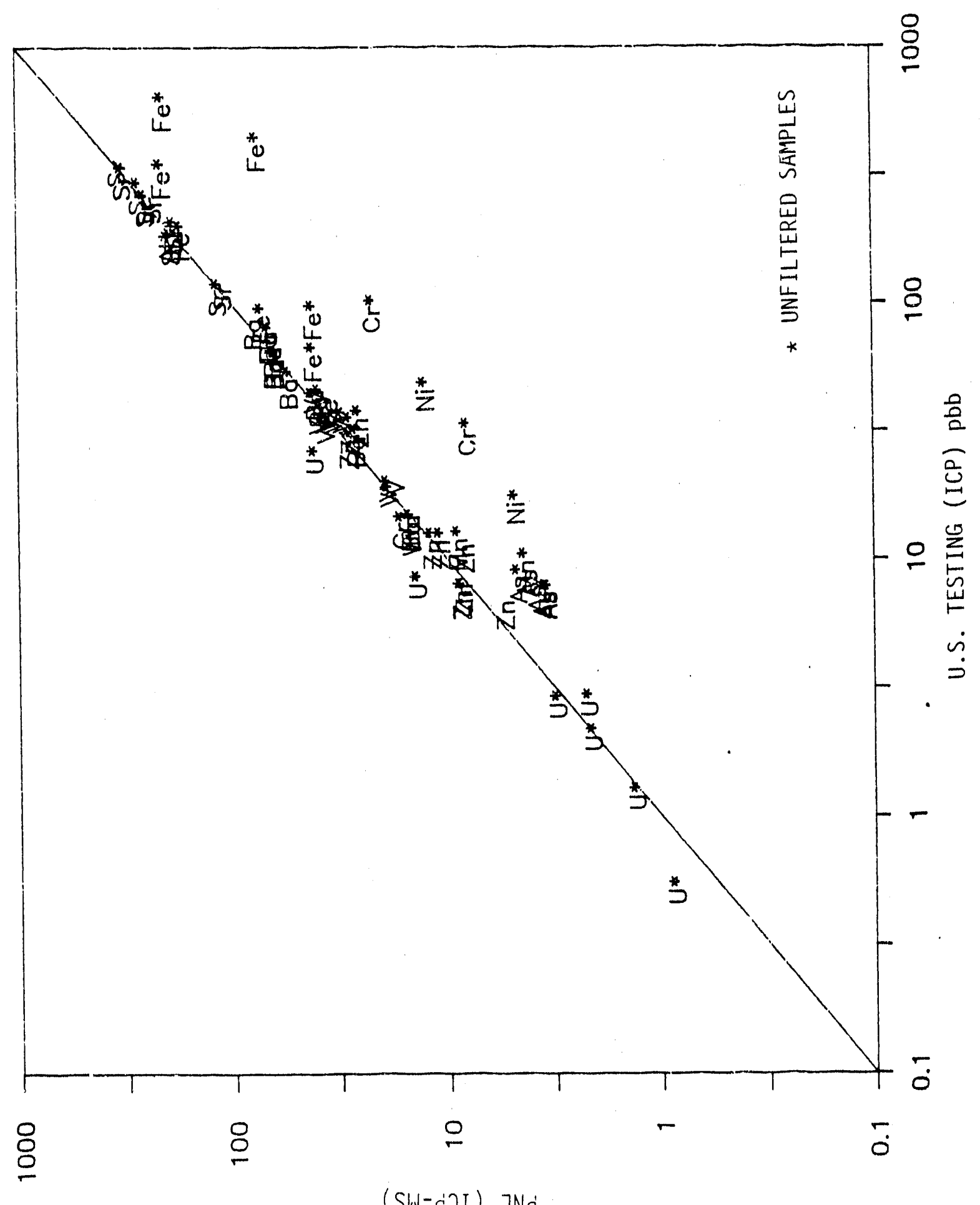

(SW-CJI) $7 \mathrm{No}$ 
WHC-EP-0366

\subsection{SAMPLING AND MONITORING HELL CONDITIONS}

As noted in the project plan (WHC 1990a), a large number of older, nonRCRA wells were included in the monitoring network. These wells were used to provide the broadest possible coverage for assessing groundwater quality in the licinity of active liquid disposal sites. However, well construction, sampling systems, and other factors may bias analytical results for certain analytes in samples from these wells.

These ractors are discussed below followed by site-specific summaries of sampling conditions.

\subsection{FACTORS POTENTIALLY AFFECTING ANALYTICAL RESULTS}

\subsubsection{We11 Construction Effects}

Well construction effects on analytical results are possible in both old and new wells. The problems are more prevalent in the older Hanford wells and can be attributed to a greater number of contributing factors.

Westinghouse Hanford recognized the potential inadequacies of site wells for use in regulatory compliance monitoring systems and contracted Golder Associates Inc. in May, 1989 to conduct a "fitness-for-use" evaiuation of selected groundwater monitoring wells. The final report for that study was delivered in December 1989 (Golder Associates 1989). Reference to this study, however, is made throughout this section to highlight potential problems with wells associated with this study.

0lder wells on the Hanford Site were constructed in accordance with acceptable standards and practices that were in force at the time of construction. Many of the wells do not meet current requirements due to (1) outdated or inappropriate construction materials (e.g., carbon-steel or galvanized casing), (2) inadequate surface and annular seals, (3) inappropriate screen location, or (4) excessive screen lengths (Golder Associates 1989). The most common analytical problam in older wells noted in this study appears to be caused by use of wells with outdated or inappropriate construction materials. For example, the occurrence of elevated metals (iron, manganese, zinc, and cadmium) particularly in unfiltered samples (see discussion in Section 4.1 .2 below). Addition of an acid preservative in the field immediately upon collection of a metals sample may result in partial dissolution of particulate phases (well casing debris or corrosion products) present in the well water. The probler: is exacerbated in bailed samples.

Data anomalies associated with the other three construction problems noted above are not as easily discerned in the analytical results. In the - case of inadequate surface and annular seals, constituents could be more rapidly transported to the groundwater near the well which would not otherwise be present. Inappropriate screen location and excessive screen lengths could result in nonrepresentative samples. 
In some new wells (RCRA compliant, stainless steel), anomalous metal results in unfiltered samples (e.g., chromium, nickel) may be due to cuttings and welding slag being introduced into the wells during casing installation. Recent installation procedures take every precaution to avoid introduction of foreign material during well installation. Filtered results can be checked against the unfiltered data to assess whether particulate metal is a likely problem.

\subsubsection{Sampling Method Effects}

Three sampling methods were used in collecting groundwater samples for this study. The techniques employed were bailer, electric submersible pump, and positive displacement pump. Restrictions in the use of each technique are discussed below.

The least desirable sampling method employed is the use of a bailer. Fifteen wells sampled for this study were collected with bailers. All 15 wells were older, non-stainless steel wells. The bailer collection procedure does not adequately purge the well prior to sample collection and does not allow for filtration of an aliquot of water for comparison; only unfiltered metal results are available. As a result, particulate phase materials (well casing corrosion debris, installation residues, and sedimentary material) may be entrained in the sample. A study to demonstrate the effects of this sampling technique on analytical results was conducted on well 2-W18-17 at the 216-Z-20 Crib.

Wells at this facility had carbon steel casings and were not equipped with dedicated submersible sample pumps (i.e., only unfiltered samples were initially obtained with a bailer). A followup resampling effort for this study was conducted on April 20, 1990, at one of the two wells (2-W18-17) and involved installation of a temporary submersible pump and time series sampling for selected constituents (trace metals and plutonium) during purgewater removal. A time series plot of unfiltered sample results is shown in Figure 3. The typical result for bailed samples would be represented by the time zero concentrations. Iron and aluminum appear to be correlated with the turbidity time-response plot while manganese, zinc, and nickel appear to decline in a regular manner with increasing purgewater removal. Plutonium was below detection in all samples. At the end of the pumping period (after removal of approximately three borehole volumes of purgewater), filtered samples were also collected in which all of the metals were at or below detection limits.

The results summarized in Figure 3 and discussed above, demonstrate the type of false positive results that can occur when sampling from carbon steel casing wells, and the bias introduced by routine bailer sampling from Hanford wells. In the latter case, only unfiltered samples are obtained. When particulate debris in the unfiltered samples is acidified (i.e., the prescribed preservative added), various metals are released to solution. Particulates can include both corrosion products from the casing as well as sedimentary material dislodged during sample recovery. The latter mechanism could account for the elevated aluminum (i.e., disolution of aluminosilicate mineral phases). 
Figure 3. Time Series Concentrations of Selected Metals During Purgewater Removal at Well 299-W18-27.
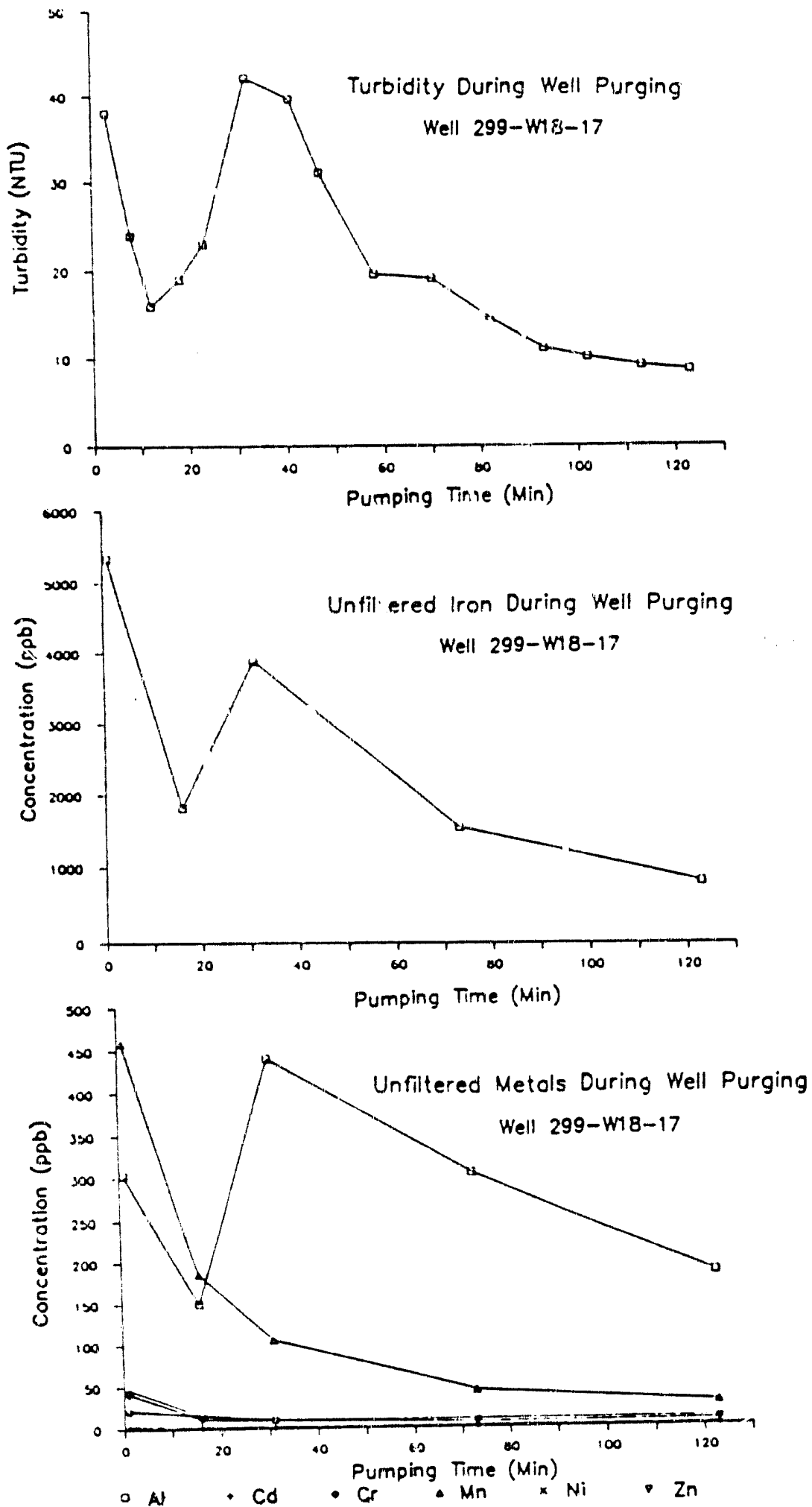
Electric submersiole pumps are the standard sample collection method in older wells. The problem with the collection of volatile organic compounds using this method has been well discussed in the literature. A study reported by Liikala et al. (1988) using two onsite wells and different sampling techniques indicates no significant statistical difference in concentrations for four chlorinated hydrocarbons between the three techniques. Based on the results of this study, volatile organic compound results reported in this study are not expected to be affected by the use of a submersible pump in sample collection.

Positive displacement pumps are the preferred method of sarnple collection. No analytical anomalies are expected in sampls:s c'sllected using this method.

\subsubsection{Studies Related to Data Quality}

Two comparison studies have reen conducted in conjunction with the liquid effluent study to try to discern the effects on analytical results based on (1) different well types, and (2) different sampling methods. The problem of data quality has been examined by the direct comparison of groundwater chemical data for RCRA and non-RCRA wells that are in reasonabe proximity to one ariother and that are screened at approximately the same interval. The results of these studies and the comparisons are presented below.

The first well pair consist of 3-1-3 and 3-1-7, located approximately $650 \mathrm{ft}$ southeast of the 300 Area process trench. Well $3-1-3$ is a carbon steel well completed in 1950 to a depth of $102 \mathrm{ft}$. It is plugged at a depth of $75 \mathrm{ft}$ and the casing is perforated between 25 and $70 \mathrm{ft}$. Well $3-1$ 7 was drilled approximately $20 \mathrm{ft}$ from well 3-1-3 for the purpose of direct comparison of concentrations of volatile organics in different well types and with different sampling techniques (Liikala et al. 1988). Well 3-1-7 was completed in 1985 to a depth of $75 \mathrm{ft}$ with stainless steel casing and screen. The screened interval is between 25 and $70 \mathrm{ft}$.

In the study reported by Liikala et al. (1988), the two wells were sampled over a 4-wk interval using three different sampling techniques. The sampling techniques used during the test included the use of a submersible pump, a bladder pump, and a bailer. Four chlorinated hydrocarbons, perchloroethylene (PCE), 1,1,1-trichloroethane (111-TCA), trichloroethylene (TCE), and chloroform were detected in both wells during the test. Statistical analyses of the test results indicate no significant differences in chlorinated hydrocarbon concentration between samples collected with different techniques or between wells with different. construction materials.

Wells 3-1-3 and 3-1-7 were resampled as part of the effluent study on November 21, 1989. For this study, the stainless steel well was equipped with a Hydrostar positive displacement piston sample pump while a submersible pump was used in the carbon steel well. The concentrations of constituents above detection limits in the two wells are listed in Table 1. The concentrations for the two wells are plotted in Figure 4 , indicating good constituent agieement between the two wells. The only significant deviation indicating a difference between the wells is for unfiltared iron 
which is higher in the non-RCRA well with a carbon steel casing. Filtered iron was below the contractual detection limit $(30 \mathrm{ppb})$ in both wells. It is interesting to note that concentrations for chloroform and TOX are almost identical in the two sets of analyses, confirming the results of Liikala et a). (1988).

Table 1. Constituents Above Detection Level in Wel1s 3-1-3 and 3-1-7.

\begin{tabular}{|c|c|c|c|}
\hline Constituent & $3-1-3$ & $3-1-7$ & Units \\
\hline Alkal inity & 56,000 & 56,000 & (a) \\
\hline Alpha, HDL & 82.5 & 63.9 & $\mathrm{pCi} / \mathrm{L}$ \\
\hline Barium & 22 & 22 & $\mathrm{ppb}$ \\
\hline Barium, filtered & 21 & 21 & $\mathrm{ppb}$ \\
\hline Boron & 17 & 19 & $\mathrm{ppb}$ \\
\hline Boron, filtered & 18 & 17 & ppb \\
\hline Calcium & 23,400 & 23,600 & $\mathrm{ppb}$ \\
\hline Calcium, filtered & 22,200 & 22,000 & $\mathrm{ppb}$ \\
\hline Chloride & 24,600 & 22,500 & $\mathrm{ppb}$ \\
\hline Chloroform & 17 & 17 & $\mathrm{ppb}$ \\
\hline Gross beta & 22 & 18.4 & $\mathrm{pCi} / \mathrm{L}$ \\
\hline Iron & 135 & 53 & $p p b$ \\
\hline Lithium & 15 & 14 & $\mathrm{ppb}$ \\
\hline Lithium, filtered & 16 & 14 & $\mathrm{ppb}$ \\
\hline Magnesium & 4,430 & 4,420 & $p p b$ \\
\hline Magnesium, filtered & 4,220 & 4,250 & $\mathrm{ppb}$ \\
\hline Nitrate & 1,100 & 1,200 & $\mathrm{ppb}$ \\
\hline Potassium filtonod & $\begin{array}{l}2,680 \\
2,520\end{array}$ & 2,740 & $\mathrm{ppb}$ \\
\hline $\begin{array}{l}\text { Potassium, filtered } \\
\text { Silicon }\end{array}$ & $\begin{array}{l}2,520 \\
6,380\end{array}$ & $\begin{array}{l}2,660 \\
6,710\end{array}$ & $\begin{array}{l}\mathrm{ppb} \\
\mathrm{pob}\end{array}$ \\
\hline Silicon, flltered & 6,020 & 6,320 & $\mathrm{ppb}$ \\
\hline Sodium & 11,700 & 12,300 & $\mathrm{ppb}$ \\
\hline Sodium, filtered & 11,100 & 12,000 & $\mathrm{ppb}$ \\
\hline Strontium & 109 & 108 & $\mathrm{ppb}$ \\
\hline $\begin{array}{l}\text { Strontium, filtered } \\
\text { Sulfate }\end{array}$ & $17 \begin{array}{l}104 \\
000\end{array}$ & $17 \begin{array}{l}103 \\
17000\end{array}$ & $\begin{array}{l}\mathrm{ppb} \\
\mathrm{ppb}\end{array}$ \\
\hline $\begin{array}{l}\text { Sultate } \\
\text { TDS }\end{array}$ & 138,000 & $\begin{array}{r}17,000 \\
115,000\end{array}$ & $\begin{array}{l}\mathrm{ppb} \\
\mathrm{ppb}\end{array}$ \\
\hline TOX, LDL & 36 & 37 & $p p b$ \\
\hline Uranium & 66.4 & 56.1 & $\mathrm{ppb}$ \\
\hline
\end{tabular}

aAlkalinity is reported in terms of milligrams/liter of $\mathrm{CaCO}_{3} \cdot--$ 
Figure 4. Plot of Constituent Concentrations in Non-RCRA Well 3-1-3 Versus Constituent Concentrations in RCRA Well 3-1-7.

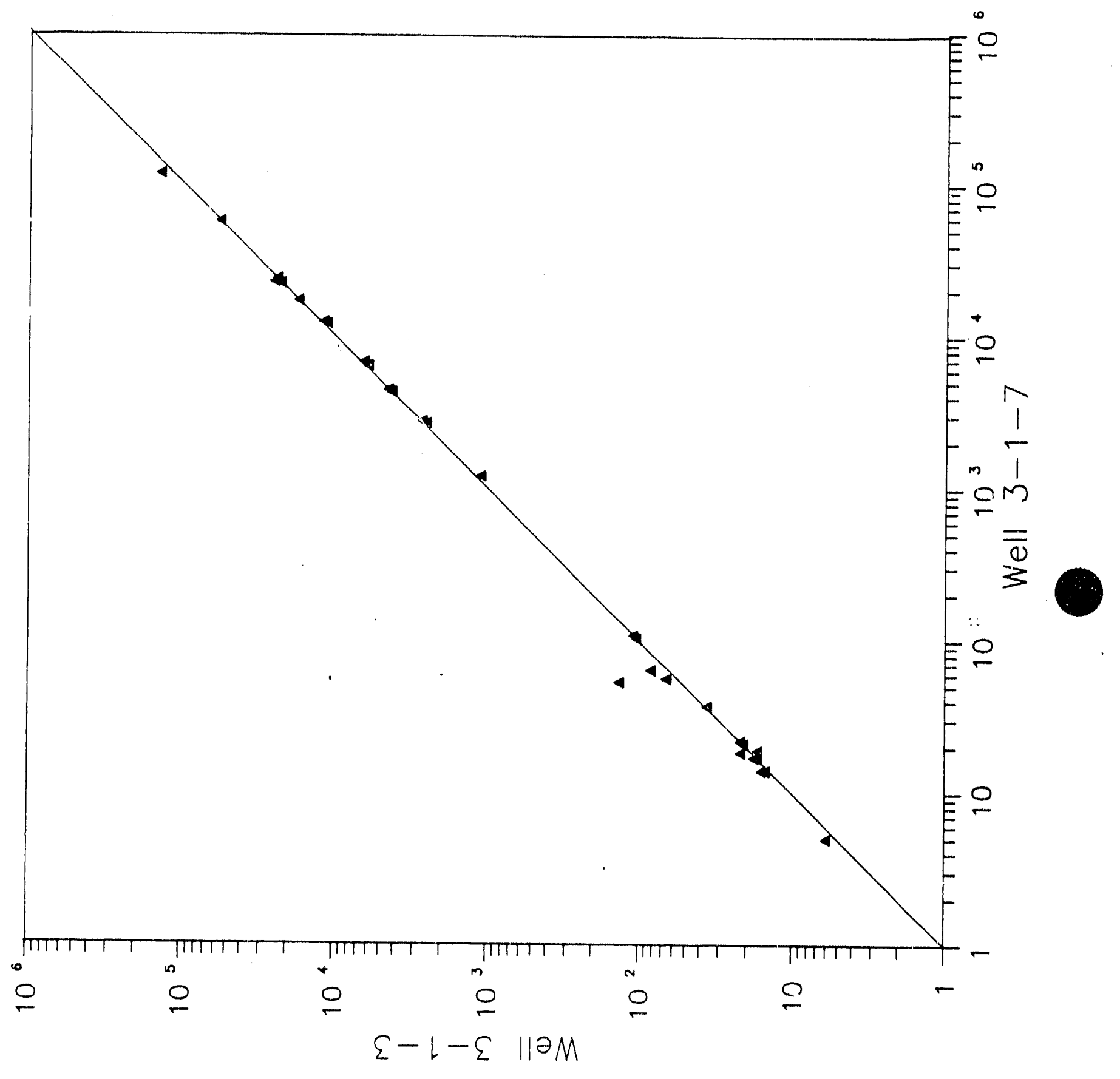


The second we1l pair sampled is made up of wells 2-E34-1 and 2-E34-2 located at the low level waste burial ground in the 200 East Area. We11 2E34-1 was completed in June of 1961 to a depth of 245 it. It has a carbon steel casing that is perforated between 215 and $230 \mathrm{ft}$. Well 2-E34-2 was completed in September of 1987 to a depth of $242 \mathrm{ft}$. It was completed with 4 -in. stainless steel casing with stainless steel screen between depths of 220 and 240 tt.

Well 2-E34-1 was sampled on December 4, 1989, and well 2-E34-2 was sampled on November 27,1989 . The concentrations of constituents above detection limits in the two wells are listed in Table 2 . The concentrations for the two wells are plotted in Figure 5. Analytical values for filtered metals were not reported for we11 2-E34-1, and thus, are not included in the comparison. The data indicate good agreement between the two wells, the major differences being higher iron, chromium, and nickel concentrations in the RCRA compliant well (2-E34-2). These three elements are the principal components of stainless steel and may be a result of stainless steel particulates left in the well during completion. Alternately, ti:oy ilay be a result of stainless steel corrosion brought about by the relatively high chloride content of the local groundwater.

The data available to date, with the exception of higher iron, chromium, and nickel in RCRA compliant well 2-E34-2, indicate that there is little or no differece between the chemistry of groundwater samples collected from older non-RCRA wells and of groundwater samples from more recent RCRA compliant groundwater monitoring wells. The lack of differences in chemical compositions of groundwater samples from the two types of wells is probably attributable to the thorough purging of the wells prior to sampling. The groundwater sampled is in contact with the well materials for too short a time for appreciable reaction to occur at the low temperatures of the groundwater. 
Table 2. Constituents Above Detection Level in Wells 2-E34-1 and 2-E34-2.

\begin{tabular}{|c|c|c|c|}
\hline Constituent & $2-E 34-1$ & $2-E 34-2$ & Units \\
\hline $\begin{array}{l}\text { Alkalinity } \\
\text { Alpha, HDL } \\
\text { Aluminum } \\
\text { Arsenic } \\
\text { Barium } \\
\text { Boron } \\
\text { Calcium } \\
\text { Chloride } \\
\text { Chloroform } \\
\text { Conductivity, Lab. } \\
\text { Gross Beta } \\
\text { Chromium } \\
\text { Iron } \\
\text { Magnesium } \\
\text { Manganese } \\
\text { Nickle } \\
\text { Nitrate } \\
\text { Potassium } \\
\text { Silicon } \\
\text { Sodium } \\
\text { Specific Conductivity } \\
\text { Strontium } \\
\text { gOSr } \\
\text { TOX, LDL } \\
\text { Sulfate } \\
\text { TDS } \\
\text { Tritium } \\
\text { Turbidity } \\
\text { Uranium } \\
\text { Vanadium } \\
\text { Zinc } \\
\text { pH, field } \\
\text { pH, laboratory }\end{array}$ & $\begin{array}{c}94,000 \\
1.1 \\
500 \\
5 \\
24 \\
15 \\
42,400 \\
12,000 \\
5 \\
447 \\
8.7 \\
10 \\
345 \\
12,300 \\
26 \\
10 \\
10,000 \\
8,070 \\
19,700 \\
23,900 \\
438 \\
219 \\
-0.2 \\
3 \\
96,700 \\
289,000 \\
3,480 \\
1.6 \\
1.3 \\
19 \\
5 \\
7.4 \\
7.7\end{array}$ & $\begin{array}{r}97,000 \\
1.2 \\
329 \\
7 \\
35 \\
22 \\
44,400 \\
11,500 \\
5 \\
458 \\
9.7 \\
46 \\
948 \\
12,500 \\
24 \\
46 \\
12,000 \\
7,130 \\
19,400 \\
24,100 \\
487 \\
216 \\
0.3 \\
5 \\
100,000 \\
286,000 \\
3,340 \\
5 \\
1.7 \\
21 \\
8 \\
7.6 \\
7.8\end{array}$ & $\begin{array}{l}(\mathrm{a}) \\
\mathrm{pC} \mathrm{j} / \mathrm{L} \\
\mathrm{ppb} \\
\mathrm{ppb} \\
\mathrm{ppb} \\
\mathrm{ppb} \\
\mathrm{ppb} \\
\mathrm{ppb} \\
\mathrm{ppb} \\
\mu \mathrm{mho} \\
\mathrm{pC} \mathrm{i} / \mathrm{L} \\
\mathrm{ppb} \\
\mathrm{ppb} \\
\mathrm{ppb} \\
\mathrm{ppb} \\
\mathrm{ppb} \\
\mathrm{ppb} \\
\mathrm{ppb} \\
\mathrm{ppb} \\
\mathrm{ppb} \\
\mu \mathrm{mho} \\
\mathrm{ppb} \\
\mathrm{pC} \mathrm{i} / \mathrm{L} \\
\mathrm{ppb} \\
\mathrm{ppb} \\
\mathrm{ppb} \\
\mathrm{ppb} \\
\mathrm{NTU} \\
\mathrm{pC} \mathrm{i} / \mathrm{L} \\
\mathrm{ppb} \\
\mathrm{ppb}\end{array}$ \\
\hline
\end{tabular}


WHC-EP-0366

Figure 5. Plot of Constituent Concentrations in Non-RCRA We11 2-E34-1 Versus Constituent Concentrations in RCRA We11 2E34-2.

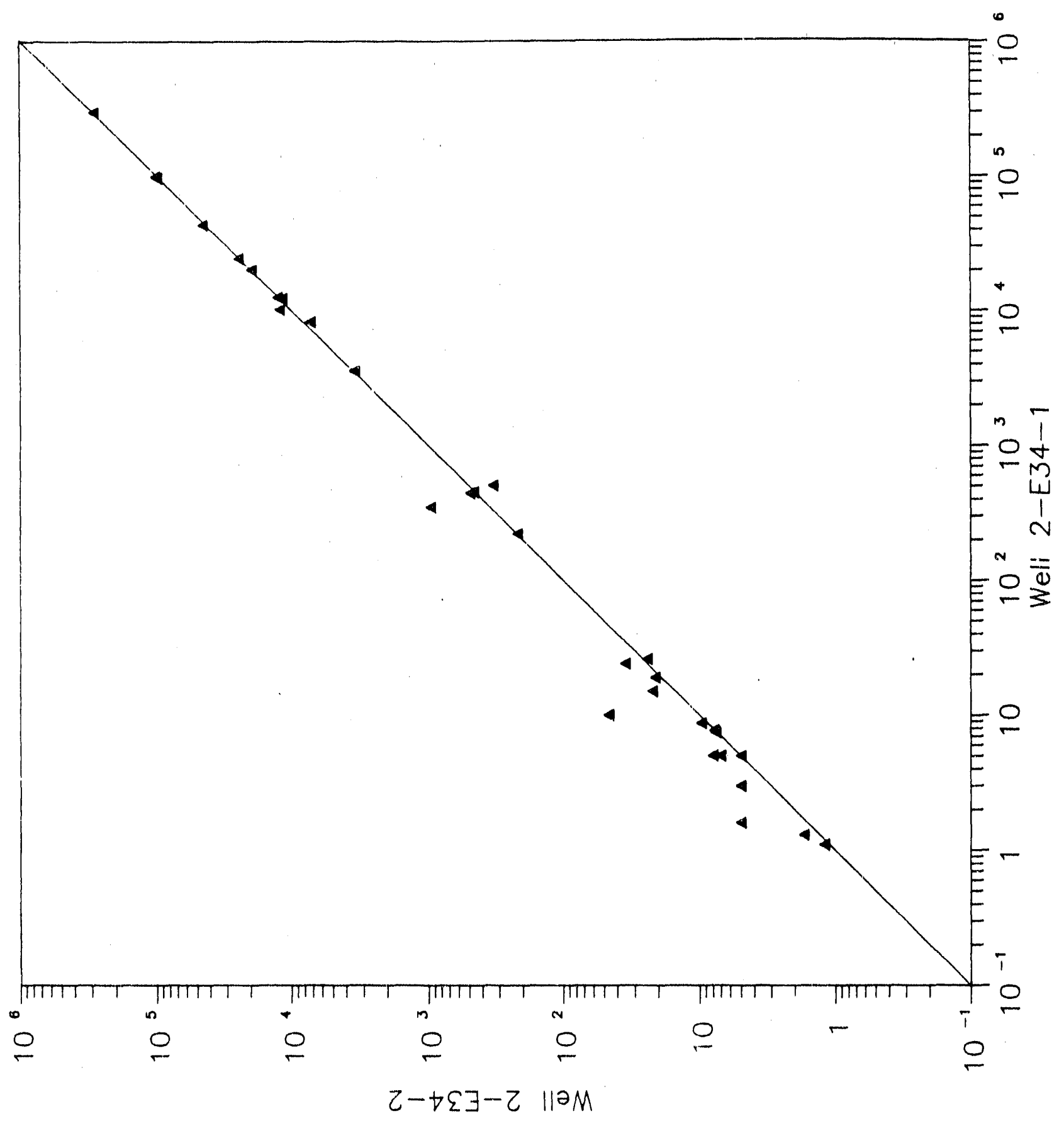




\subsection{SITE SUMMARIES}

Available information on well and sampling conditions that may have a bearing on sample representativeness is summarized in the following subsections.

- 100-D Ponds - Because there are no groundwater monitoring wells in close proximity to the 100-D Ponds, groundwater quality cannot be evaluated with respect to the effluent stream constituents at this time. Future monitoring wells for the facility are proposed for the RCRA program (Tri-Party Agreement milestone M24-16; Change No. $M 24-89-1)$.

- 1324N/NA Pond - Eighteen wells were sampled during the period of interest for the effluent study, of which eight were RCRAcompliant wells. Appendix IX (or equivalent) constituents as well as additional indicator parameters were measured during the period for these wells. One additional non-RCRA-compliant well was sampled only for indicator parameters. It is important to note that six of the ion-RCRA-compliant wells used in this study were sampled by bailer, for which anomalous or elevated metal concentrations are expected. A fitness-for-use evalution (Golder Associates 1989) indicated that six of the non-RCRA well ( $1-\mathrm{N}-16$, $1-N-17,1-N-21$, and $1-N-23$ through $1-N-25$ ) require remediation.

- 1325N Crib - Eleven non-RCRA wells and one RCRA well were sampled in March, November, and December 1989, for the 1ist of Appendix IX constituents and radionuclides. Abbreviated sampling was performed on one additional non-RCRA-compliant well $(1-N-39)$. The 11 non-RCRA wells sampled are older wells constructed with carbon steel casing, which are subject to corrosion. The fitness-for-use evaluation (Golder Associates 1989) recommends that well $1-\mathrm{N}-41$ be remediated. Available documentation for the RCRA-compliant well indicates that water level in the well is approximately $40 \mathrm{ft}$ above the screened interval, providing a substantial column of stagnant water in the casing.

- 216-A-8 Crib - Two non-RCRA-compliant wells were sampled on January 9, 1990, for the list of Appendix IX constituents and radionuclides. Only bailed, unfiltered samples were obtained; thus, elevated metal concentrations are expected due to partial dissolution of particulate phases (i.e., sediment and well casing corrosion products). Indicator parameters were al so available from operational monitoring and related sampling programs. The fitness-for-use evaluation (Golder Associates 1989) recommends that one of the wells $(2-E 25-6)$ be remediated.

- 216-A-29 Ditch - A RCRA interim-status groundwater monitoring program was in place for this facility at the time the effluent study was initiated (Smith and Gorst 1990). This ongoing program was augmented with one sampling event for Appendix IX and sitespecific constituents. Six RCRA-compliant wells were sampled, four of which were equipped with Hydrostar sample pumps and two with submersible pumps. 
- 216-A-30 Crib - The 216-A-30 Crib is monitored by two non-RCRAcumpliant groundwater monitoring wells. Well 2-E25-11 is at the head end of the crib and well 2-E16-2 is at the lower end. Samples from these two wells were collected with a bailer, and anomalously high results for metals are expected. The fitnessfor-use evaliation (Golder Associates 1989) recommends that one of the wells $(c-E 16 \cdot 2)$ be remediated and contends that the other wel1 (2-E25-11) may meet RCRA standards but requires agency confirmation.

- 216-A-36B Crib - A total of seven wells were sampled for Appendix IX and site-specific radioactive constituents. Two non-RCRAcompliant wells (2-E17-5 and -9) equipped with submersible pumps were sampled on May 16, 1989. Five RCRA-compliant wells equipped with Hydrostars were sampled on May 12, 1989 (2-E17-17), on May $15,1989(2-E 17-14$ and 2-E17-16), on May 17, $1989(2-E 17-15)$, and or May 19, $1989(2-E 17-18)$. Other indicator constituents were available from the operational monitoring program.

- 216-A-37-1 Crib - Four non-RCRA-compliant wells (which included one bailer sampled well) were sampled for this study between January 3 and January 9, 1990, for Appendix IX and site-specific constituents. Other results from operational and RCRA programs during the time period of interest were also included in the analytical summary. According to the fitness-for-use evaluation (Golder Associates 1989), three of the four wells (2-E25-17, 2E25-19, and 2-E25-20) may meet RCRA standards but require agency confirmation. One of these wells (2-E25-17) has approximately 2 ft 0 ; water above the screen.

- 216-A-37-2 Crib - Four non-RCRA-compliant wells equipped with submersible pumps were sampled, on December 14, 1989 (2-E25-2.2), on December 20, 1989 (2-E25-24), on January 29, 1990 (2-E25-23), and on Apri? 16, 1990 (2-E25-21), for Appendix IX and sitespecific radivactive constituents. Other indicator constituents were available from the operational monitoring program. According to a recent well-fitness evaluation (Golder Associates 1989), one of the wells (2-E25-22) requires remediation, and the remaining three may meet RCRA standards but require agency confirmation.

- 216-A-45 Crib - Two RCRA-compliant wells equipped with submersible pumps were sampled on April 17, 1990 (2-E17-12) and November 30, 1989 (2-E17-13) for Appendix IX and site-specific radioactive constituents. Other indicator constituents were available from the operational monitoring program. Both of the wells have between a 2- and 5-ft column of water above the screened interval (Golder Associates 1989).

- 216-B-3 Pond System - Thirteen wells were included in the groundwater sampling network, of which, all but two (6-42-40A and 6-4240B) were considered RCRA-compliant wells. The two non-RCRAcompliant wells included one bailed well (6-42-40A) for which anomalously high metal concentrations are to be expected. Two of the wells connsidered RCRA compliant (6-43-43 and 2-E32-4) require a variance from the State, but meet present criteria established 
in the Washingtun Administrative Codes (Golder Associates 198'). The augmented sampling for the effluent study was conducted between December 6, 1989 and January 26, 1990 for whic!. Appendix IX and site-specific radionuclides were analyzed.

- 216-B-55 Crib - Groundwater in the vicinity of the 216-B-55 Crib has been monitored as a part of the Hanford Groundwater Monitoring Program and the Operations Area Monitoring Program for a number of years. Two wells, 299-E28-12 and 299-E28-13, have been sampled over the operational history of the facility. Neither of these wells is constructed to current RCRA standards. One of the wells (2-E28-12) was sampled by bailer, for which elevated metal results are expected. The fitness-for-use evaluation (Golder Associates 1989) recommends that both of the wells be remediated. As a oart of this study, the wells wore sampled and analyzed for an extended list of analytes.

- 216-B-62 Crib - Two non-RCRA-compliant wells (2-E28-18 and 2-52821) equipped with submersible pumps were sampled on lanuary 11 , 1990 for Appendix IX and site-specific radioactive constituents. Other indicator constituents were available from the operational monitoring program. According to the evaluation performed by Golder Associates (1989), both of the wells meet the criteria for RCRA wells discribed in the Washington Administrative Codes, but require agency approval.

- 216-B-63 Ditch - Because there are no groundwater monitoring wells in close proximity to the 216-8-63 Ditch, groundwater quality cannot be evaluated at this time. Future monitoring wells for the facility will be proposed as part of the Westinghouse Hanford RCR.A program.

- 216-C-7 Crib - Because there are no groundwater monitoring wells in close proximity to the 216-C-7 Crib, groundwater quality cannot be evaluated with respect to effluent stream constituents at this time. Future monitoring wells for the facility will be proposed as part of the Westinghouse Hanford RCRA program.

- 216-S-10 Ditch - Because there are no groundwater monitoring wells in close proximity to the 216-S-10 Ditch, groundwater quality cannot be evaluated. Six RCRA-compliant monitoring wells were proposed for the facility, but not completed in time to be incorporated in this assessment.

- 216-S-26 Crib - One non-RCRA compliant well equipped with a submersible pump was sampled January 11,1990 for Appendix IX and site-specific radioactive constituents. Evaluation of the well fitness-for-use (Golder Associates 1989) recommends that the well undergo remediation. Other indicator constituents were available from the operational monitoring program.

- 216-T-1 Ditch - Because there are no groundwater monitoring wells in close proximity to the 216-T-1 Ditch, groundwater quality cannot be evaluated with respect to effluent stream constituents at this time. Given the average volume of effluent discharge and 
the presence of a caliche layer, the potential presence and composition of a perched water zone should be considered in plans for future site monitoring of this facility.

- 216-T-4-2 Ditch - Because there are no groundwater monicoring wells in close proximity to the 216-T-4-2 Ditch, groundwater quality cannot be evaluated with respect to effluent stream constituents at this time. Future monitoring wells for the facility wiil be proposed as part of the Westinghouse Hanford RCRA program.

- 216-U-14 Ditch - Two RCRA-compliant wells equipped with submersible pumps were sampled on November 2, 1989 for Appendix IX and site-specific radioactive constituents. In addition, one nonRCRA well was sampled by bailer on January 2, 1990 for Appendix IX and site-specific radioactive constituents.

- 216-U-17 Crib - The monitoring network for this study consisted of six RCRA-type monitoring wells equipped with submersible sample pumps. Site-specific and Appendix IX constituents were sampled between October 27 and October 31, 1989. Other indicator type parameters available from the operational monitoring program are included in the summary for this study.

- 216-W-LC Crib - One non-RCRA-compliant well (299-W14-10) was bailed and sampled on January 30, 1990 for Appendix IX and sitespecific radioactive constituents. The fitness-for-use evaluation (Golder Associates 1989) recommends that this well be remediated. other indicator constituents were available from the operational monitoring program.

- 216-Z-20 Crib - Two non-RCRA compliant wells (2-W18-17 and 2W18-20) were sampled in January 1990, for Appendix IX and sitespecific radioactive constituents. The fitness-for-use evaluation (Golder Associates 1989) indicates that approximately a $10-\mathrm{ft}$ column of water exists in well 2-W18-17 above the screened interval. Wells at this facility have carbon steel casings and were not equipped with dedicated submersible sample pumps (i.e., only unfiltered samples were initially obtained with a bailer).

- 200-W Powerhouse Pond - Because there are no groundwater monicoring wells in close proximity to the 200-W Powerhouse Pond, groundwater quality cannot be evaluated at this time. Future monitoring wells for the facility will be proposed as part of the Westinghouse Hanford RCRA program.

- 2101-M Pond - Four RCRA-compliant wells (2-E18-1 through 2-E18-4) equipped with Hydrostar positive displacement pumps have been sampled on a quarterly basis from July 1988 to present: The upgradient well (2-E18-1) and one down gradient well (2-E18-3) were sampled for Appendix IX and site-specific radioactive constituents on October 31,1989 and November 27, 1989, respectively. Other indicator constituents are available for the other two wells from the RCRA monitoring program. 
- 300 Area Process Trenches - Groundwater in the vicinity of the 300 Area Process Trenches has been monitored extensively since 1985 in connection with a RCRA interim-status grouridwater quality assessment for this facility (Smith and Gorst 1990). Thirty-one wells were sampled during the time period of interest for the liquid effluent study of which over half were RCRA-compliant wells. Appendix IX (or equivalent) constituents and site-specific radionuclide and chemical constituents were analyzed as well. The fitness-for-use evaluation (Golder Associates 1989) recommends that eight of the non-RCRA compliant wells be remediated. Five of the RCRA compliant wells (3-1-12,3-1-16B, 3-1-16C, 3-1-17B, and $3-1-17 \mathrm{C}$ ) have water columns of $5-, 60-, 130-, 70-$, and $130-\mathrm{ft}$, respectively, above their screened intervals. One of the nonRCRA compliant wells (3-1-7) has approximately $65 \mathrm{ft}$ of water standing in the casing above the screened interval.

- 400 Area Ponds - One nori-RCRA compliant well (6-2-7) with a submersible pump was sampled on April 13, 1990 for Appendix IX and site-specific radioactive constituents. Other indicator constituents were available from the operational monitoring program. 


\section{5. . REFERENCES}

DOE-RL, 1990, Quarterly Report of RCRA Groundwater Monitoring Data for Period April 1, 1990 through June 30, 1990, DOE/RL-90-36, U.S. Department of Energy, Richland Operations Office, Richland, Washington.

EPA, 1986A, RCRA Groundwater Monitoring Technical Enforcement Guidance Document, Office of Waste Programs Enforcement and Office of Solid Waste and Einergency Response, U.S. Environmental Protection Agency, Washington, D.C.

EPA, 1986B, Test Methods for Evaluating Solid Waste: Physical/Chemical Methods, SW-846, 3rd edition, Office of Solid wa: te and Emergency Response, U.S. Environmental Protection Agency, Washington, D.C.

Golder Associates, 1989, Final Report for the Borehole Data Evaluation Checklist and Determinations for Well Fitness, prepared for Westinghouse Hanford Operations by Golder Associated, Corisulting Geotechnical and Mining Engineers, Richland, Washington.

Lijkala, T. L., D. S. Daly, and A. P. Toste, An Evaluation of the Effects of Well Construction Materials and Ground-Water Sampling Equipment on Concentrations of Volatile Organic Compounds, PNL-6585, Pacific Northwest Laboratory, Richland, Washington.

PNL, 1989a, Program Management Plan (PMP) Ground-Water Sampling and Analys is Program, Ltr $8901460 \mathrm{~B}$, dtd 4/6/89, P. J. Mitche11, Pacific Northwest Laboratory, to K. R. Fecht, Westinghouse Hanford Company, Richland, Washington.

PNL, 1989b, RCRA Groundwater Monitoring Projects Quality Assurance Project Plan, OHE-18, Rev. 0, Pacific Northwest Laboratory, Richland, Washington.

PNL, 1989c, Procedures for Groundwater Investigations, PNL-MA-567, Pacific Northwest Laboratory, Richland, Washington.

PNL, 1989d, RCRA Ground-Water Monitoring Projects for Hanford Facilities: Progress Report for the Period January 1 to March 31,1989 , unnumbered, June 1989, Pacific Northwest Laboratory, Richland, Washington.

PNL, 1989e, RCRA Ground-Water Monitoring Projects for Hanford Facilities: Progress Report for the Period April 1 to June 30, 1989, unnumbered, September 1989, Pacific Northwest Laboratory, Richland, Washington.

PNL, 1989f, RCRA Ground-Water Monitoring Projects for Hanford Facilities: Progress Report for the Period July 1 to September 30,1989 , unnumbered, December 1989, Pacific Northwest Laboratory, Richland, Washington. 
PNL, 1990a, Program Management Plan (PMP) Ground-Water Sampling and Analysis Program, Ltr 9000223B dtd 1/16/90, R. H. Gray for M. S. Hanson, Pacific Northwest Laboratory, to K. R. Fecht, Westinghouse Hanford Company, Richland, Washington.

P'.L, 1990b, RCRA Ground-Water Monitoring Projects for Hanford Facil ities: Progress Report for the Period October 1 to December 31, 1980, PNL-7306, Vo1. I, March 1990, Pacific Northwest Laboratory, Richland, Washington.

Smith, R. M., and W. R. Gorst, 1990, RCRA Ground-Water Monitoring Projects for Hanford Facilities: Annual Progress Report for 1989, PNL-7305, Pacific Northwest Laboratory, Richland, Washington.

WHC, 1988, Fiscal Year 1989 Statement of Work for Groundwater Sampling and Analysis, Ltr 8957072 dtd 12/30/88, L. C. Brown, Westinghouse Hanford Company, to P. J. Mitche11, Pacific Northwest Laboratory, Richland, Washington.

WHC, 1989a, Waste Stream Characterization Report, WHC-EP-0287, Vols. I through IV, Westinghouse Hanford Company, Richland, Washington.

WHC, 1989b, Fiscal Year1990 Sampling and Analysis Statement of Work, Ltr 8957072 dtd 12/11/89, L. C. Brown for K. R. Fecht, West inghouse Hanford Company, to M. S. Hanson, Pacific Northwest Laboratory, Richland, Washington.

WHC, 1990a, Liquid Effluent Study Project Plan, WHC-EP-275, Rev. 2, Westinghouse Hanford Company, Richland, Washington.

WHC, 1990b, Quarterly Report of Resource Conservation and Recovery Act Groundwater Monitoring Data for Period January 1, 1990 through . March 31, 1990, Ltr 9053781 dtd 5/25/90, $\pi$. E. Lerch, Westinghuuse Hanford Company, to R. D. Izatt, U.S. Department of Energy, Richland Operations Office, Richland, Washington.

WHC, 1990c, Liquid Effluent Study Final Project Report, WHC-EP-0367, Westinghouse Hanford Company, Ricnland, Washington. 


\section{APPERDIX A}

\section{GROUNDWATER DATA}

This appendix contains the groundwater analytical results arranged by disfosal site in 27 subappendices. A site map with well locations precedes each data table; where there are no monitoring wells, only a site map is included (i.e., the absence of data tables should not be construed as missing data). All analyses performed are listed for every sample analyzed. A "Detected" and "Above Limit" column is provided for which a "yes" entry indicates a detection linit is exceeded or a standard is exceeded (see final project report for definition of standards used as well as additional statistical summary data such as the number of times an analytical result exceeds a detection limit or standard). Listing of the analyte name in the first column from the left signifies that the constituent was analyzed.

Analytes are arranged in four general categories:

- Indicator Parameters

- Drinking Water Parameters

- Groundwater Quality Parameters

- Site-Specific and Other.

The "other" parameters are primarily the Appendix IX organic analytes. Constituents within each of the above groups are arranged alphabetically.

The designation "filtered" following the analyte name indicates the sample was filtered in the field. Analyte/naine entries without ihis designation mean the sample was not filtered.

\section{Hois}

All analytical results listed in data tables are 1 imited to no more than three significant figures; additional places are computer generated (additional places occur only in "average" column; max and min columns indicate correct number of significant figures). 
100-D Ponds 
WHC-EP-0366

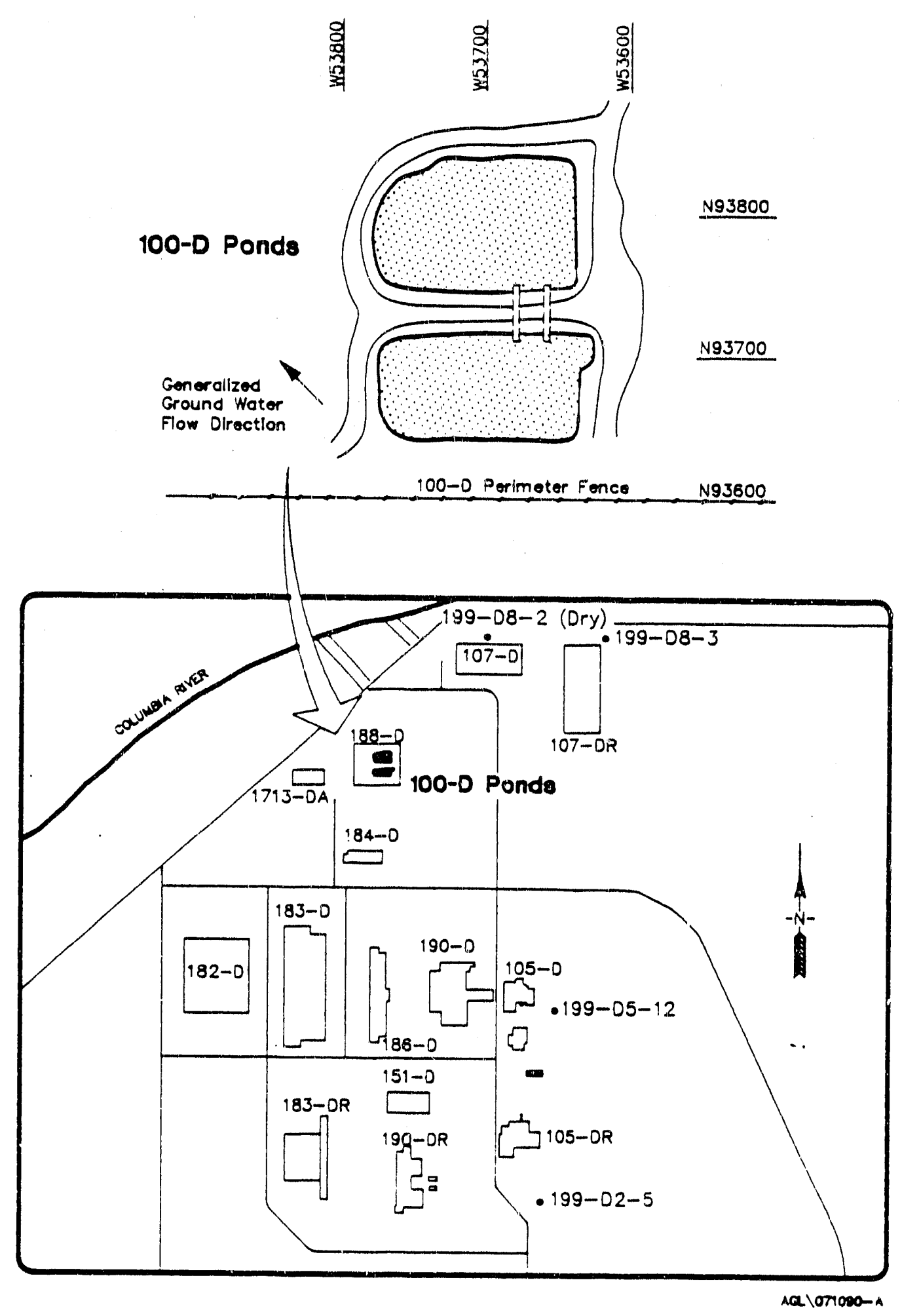

Well Location and Site Map for 100-D Ponds 


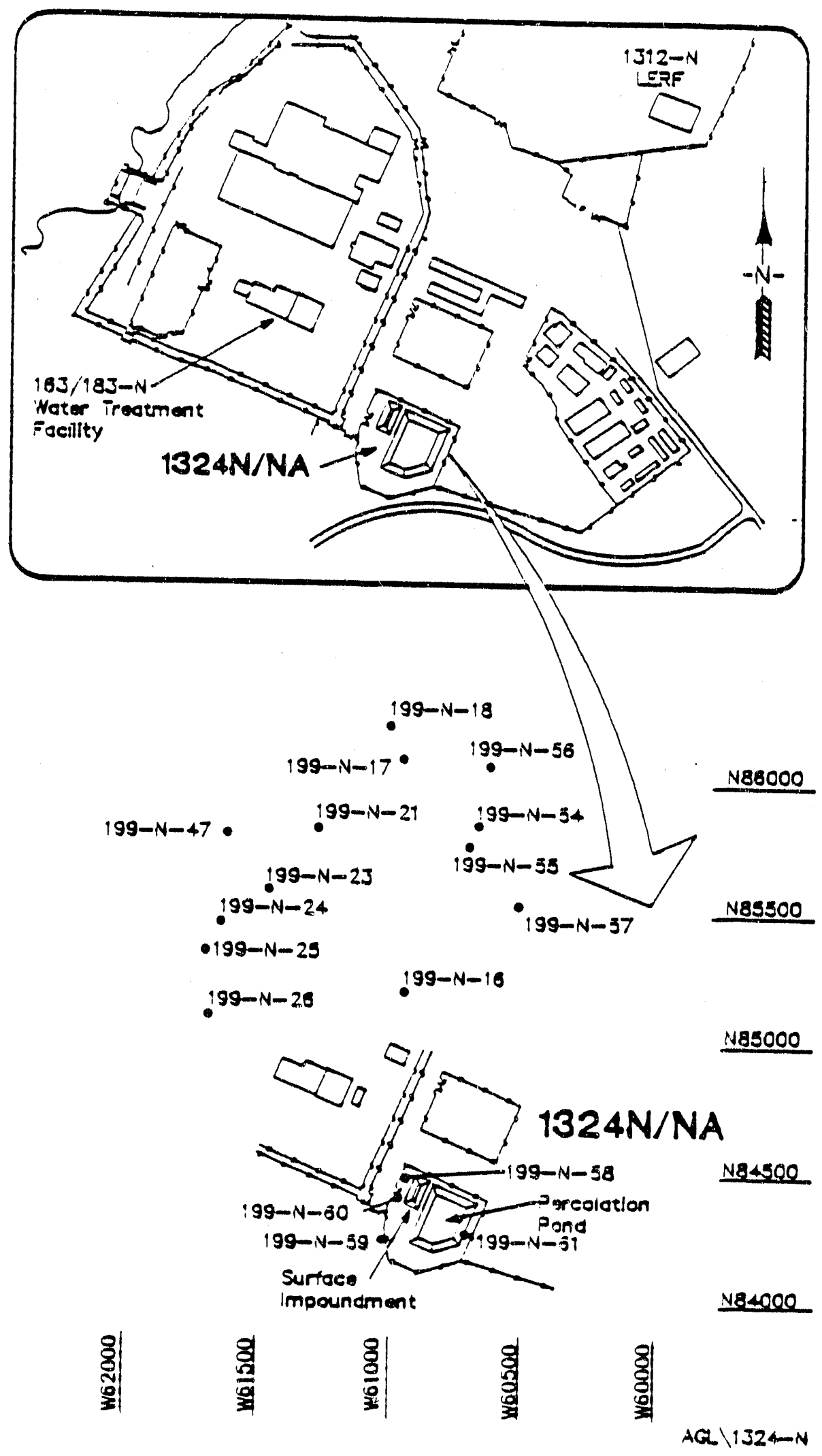

Well Location and Site Map for 1324N/NA Pond 
WHC-EP-0366

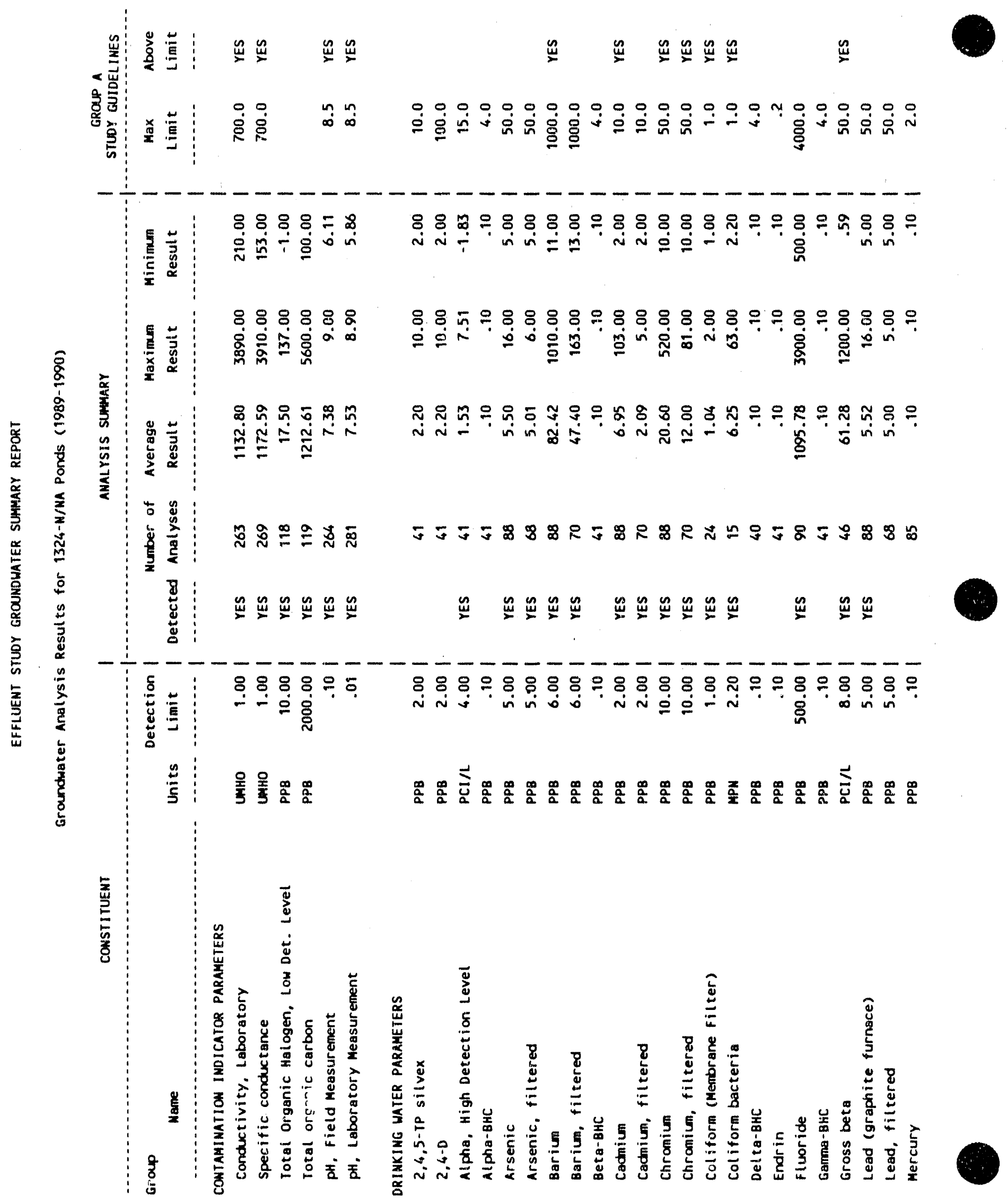




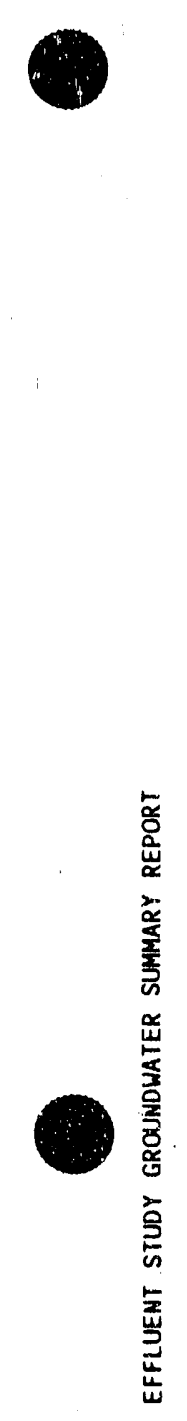

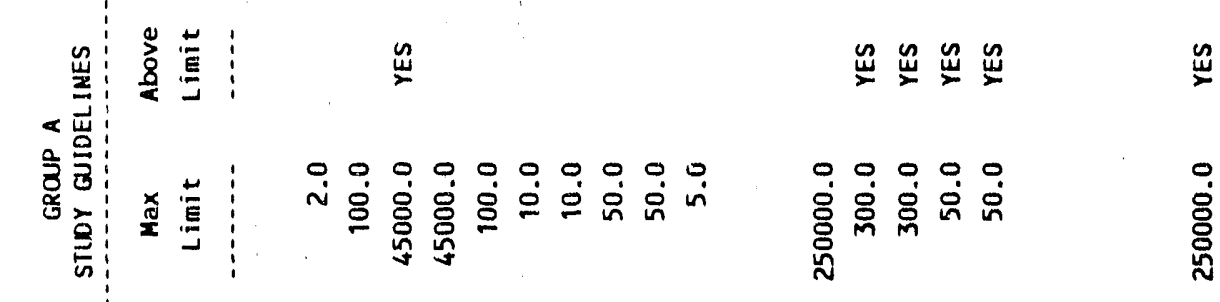

$\stackrel{m}{\mathscr{x}}$

$\stackrel{\circ}{\stackrel{0}{\circ}} \stackrel{\circ}{\circ}$

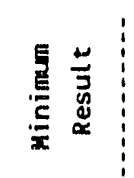

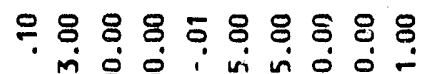

웅 8 8 8 8 888

88888888

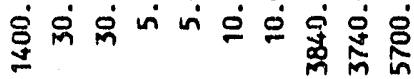

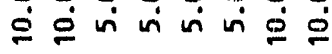

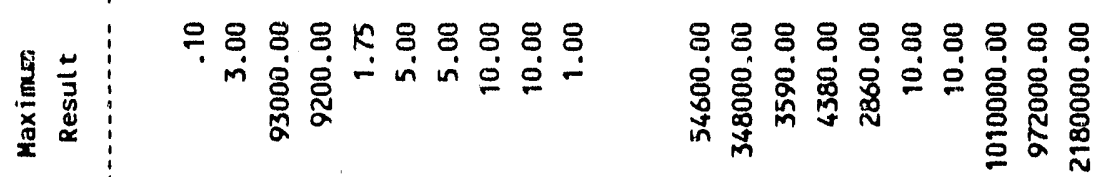

88080888

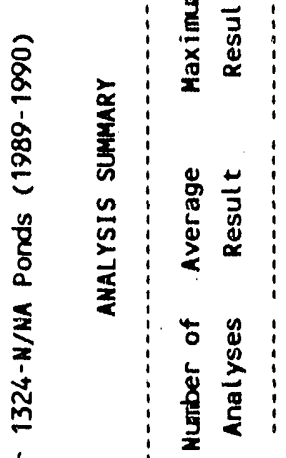

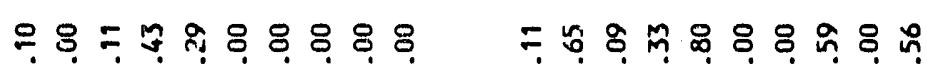

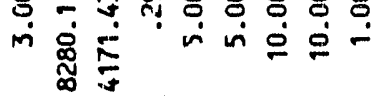

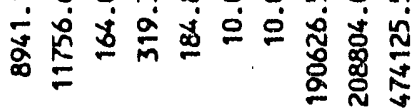

8888888

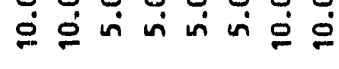

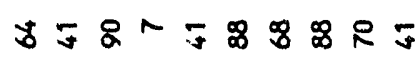

\&

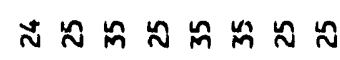

\begin{tabular}{l:l}
$\mathbb{d}$ \\
\hdashline
\end{tabular}

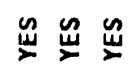

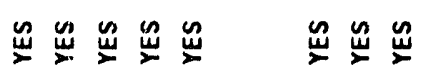

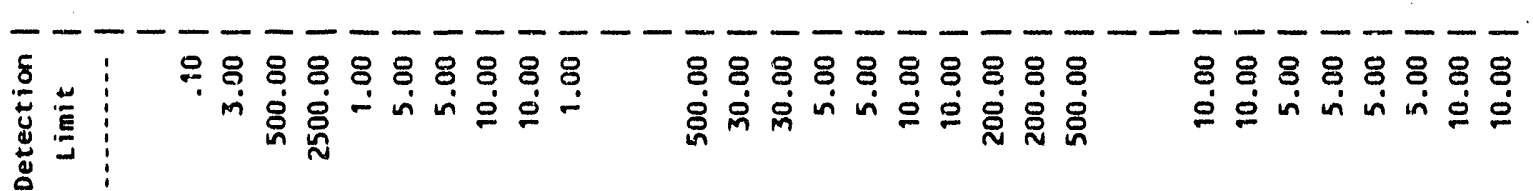

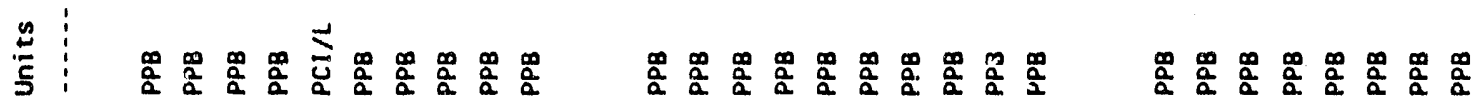

\section{tos}

.

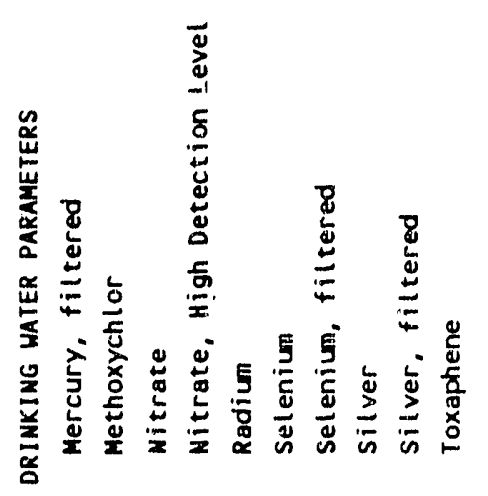
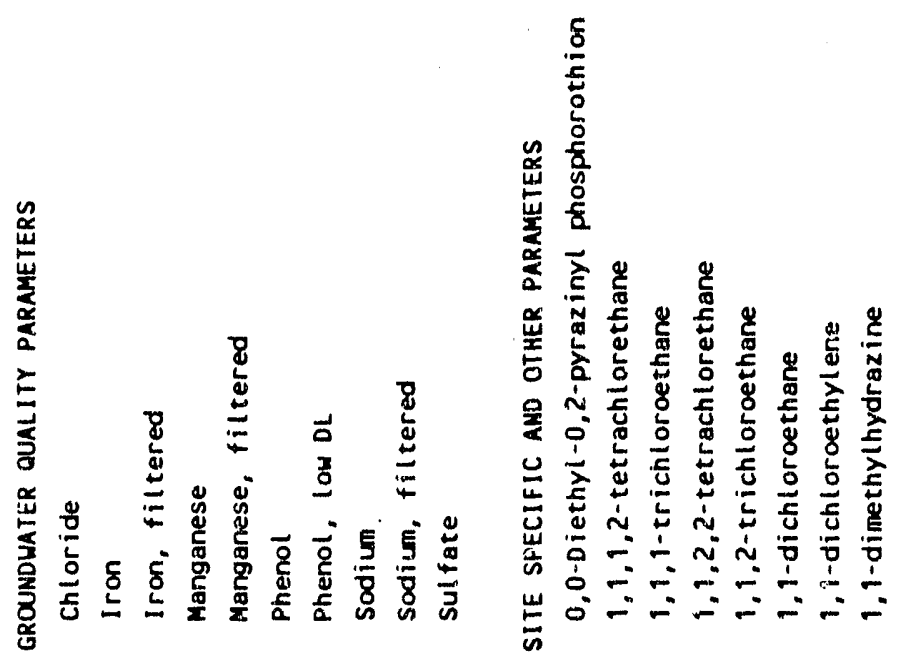
WHC-EP-0366

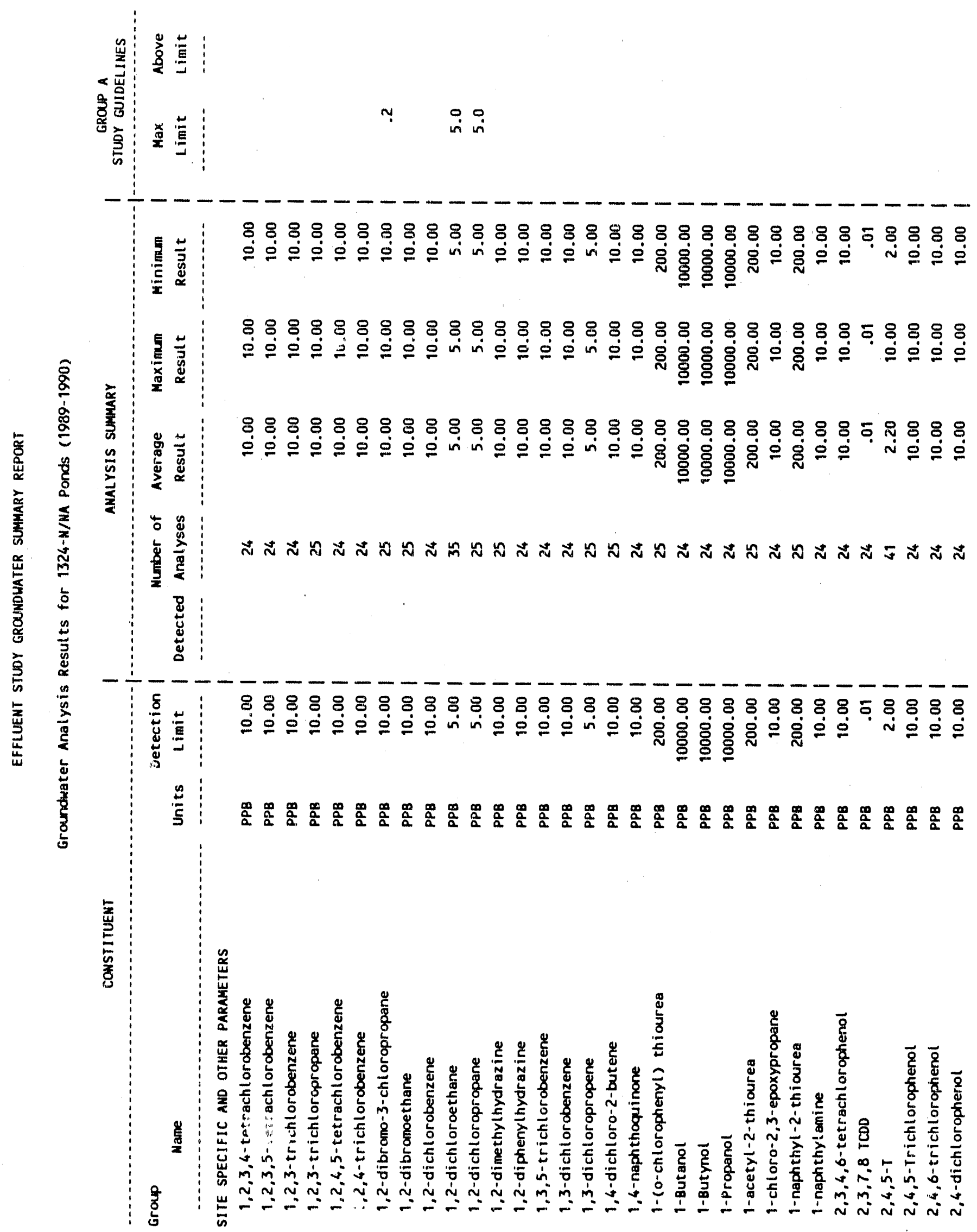




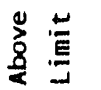

監

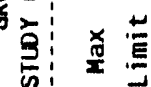

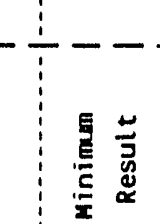

88888888888888888088888888888

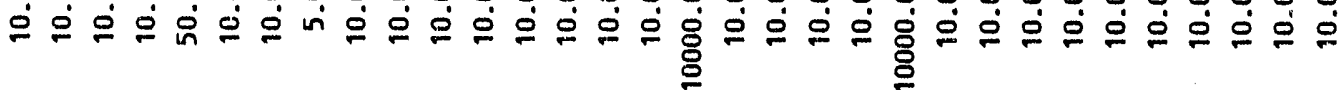

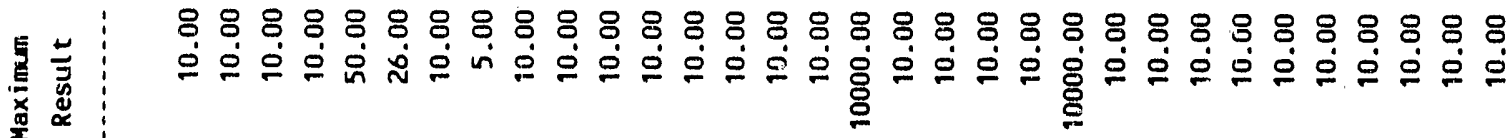

8888 8

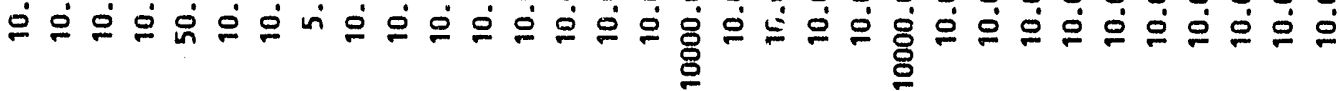

要泀

$\div$

要要

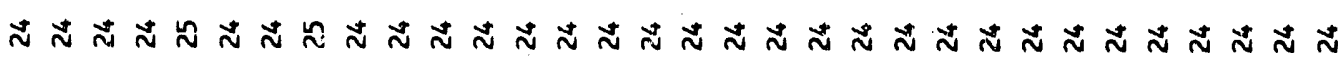
$\stackrel{3}{2}$

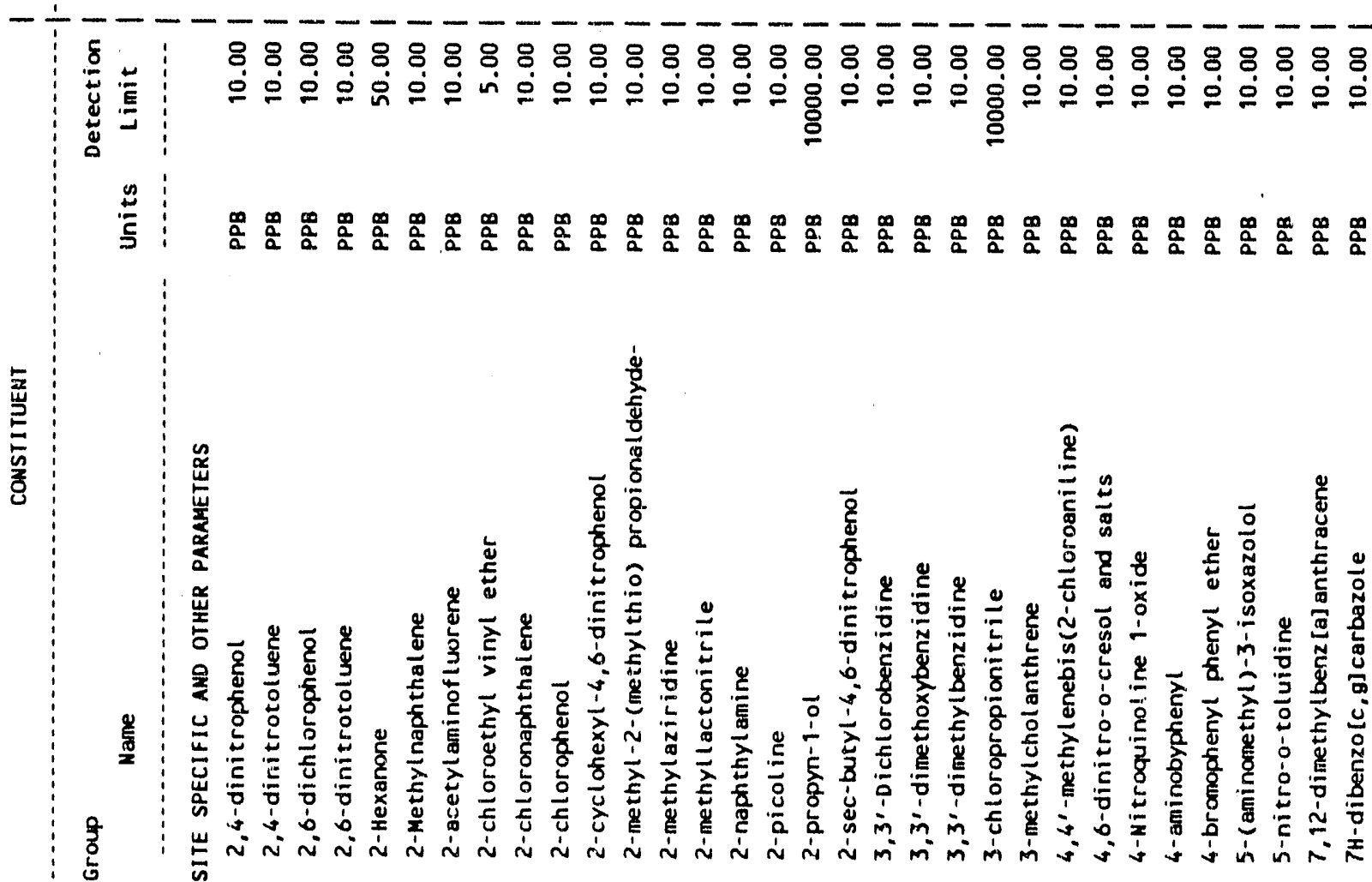




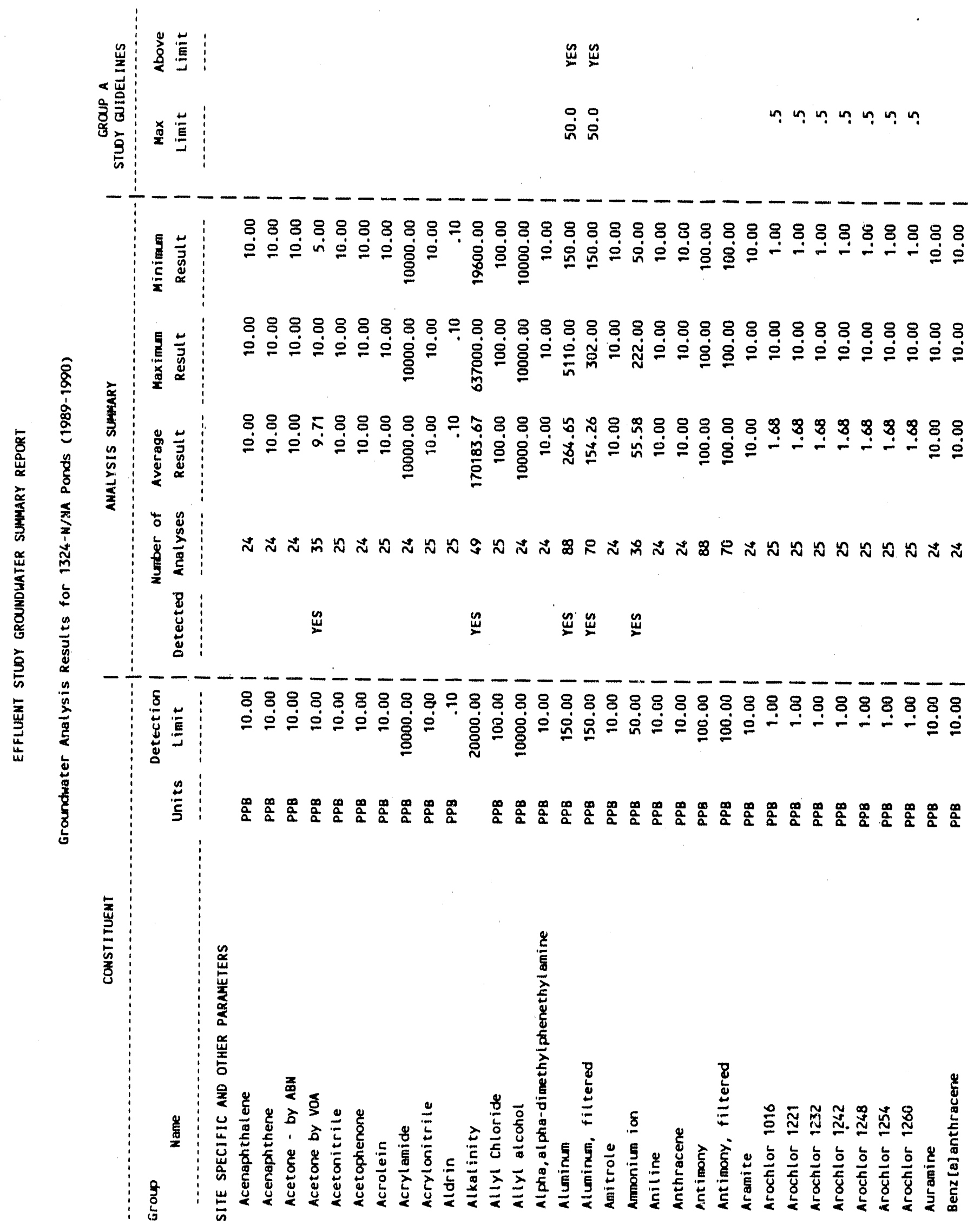




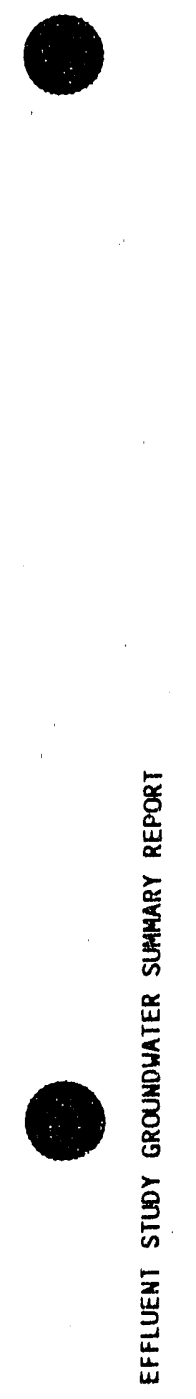

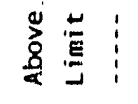

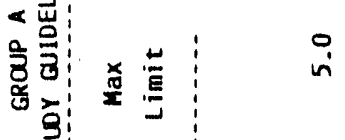

요용

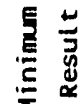

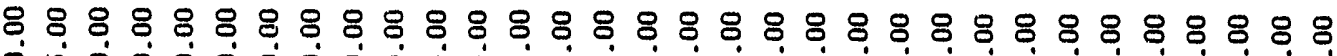

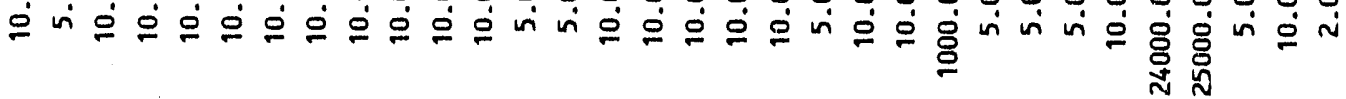

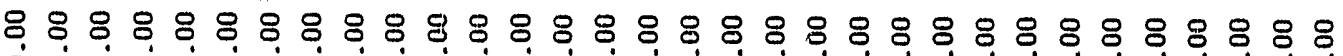

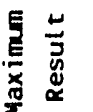
ํ்

岗

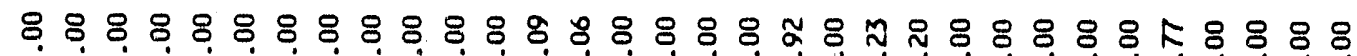

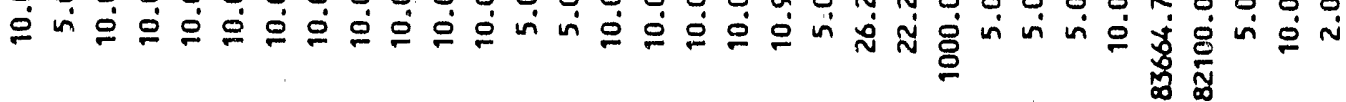

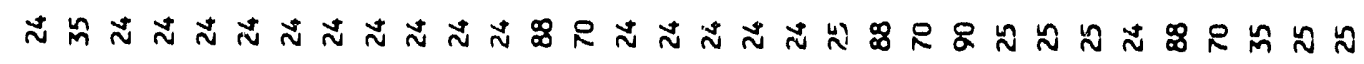

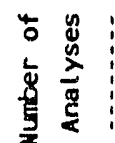

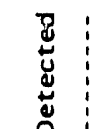
$\stackrel{\mathscr{m}}{\sim}$

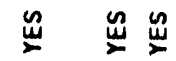

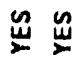

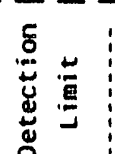

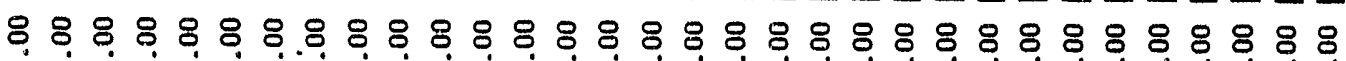

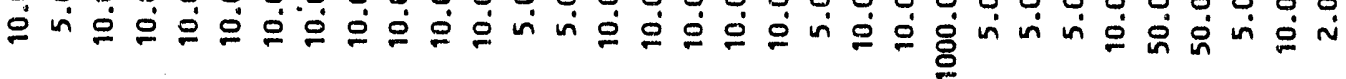

:

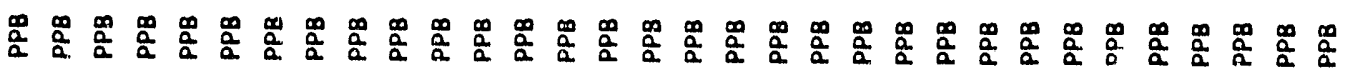




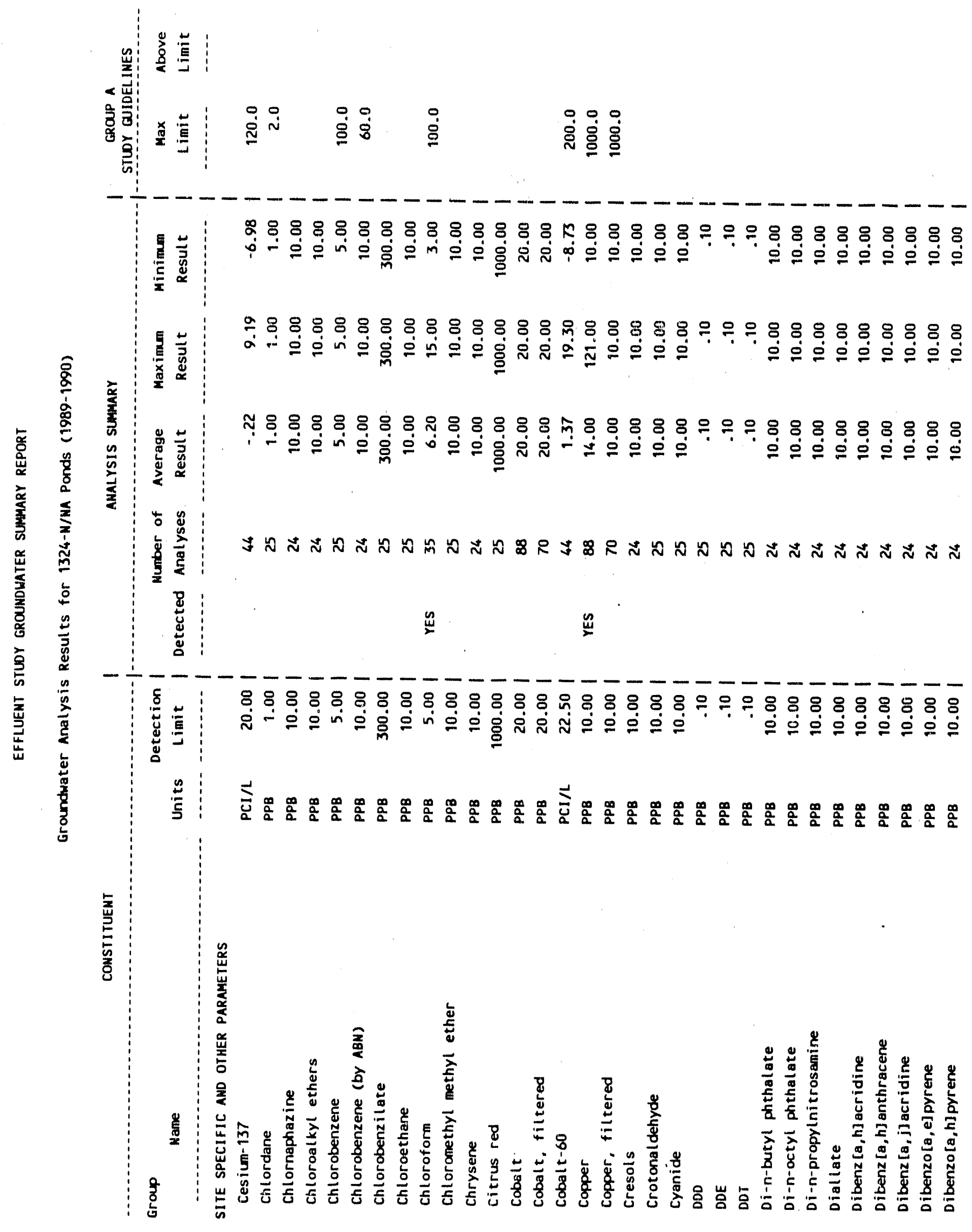


WHC-EP-0366

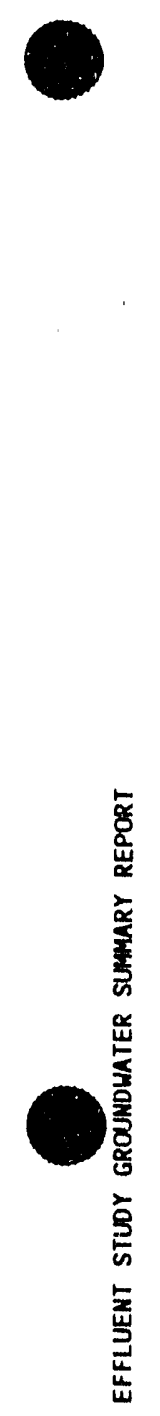

空萣

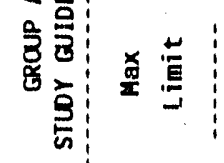

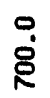

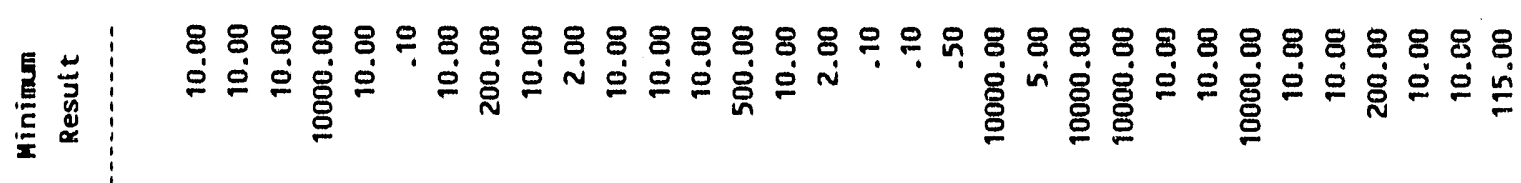

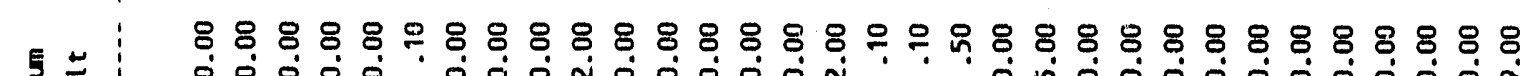
造苛

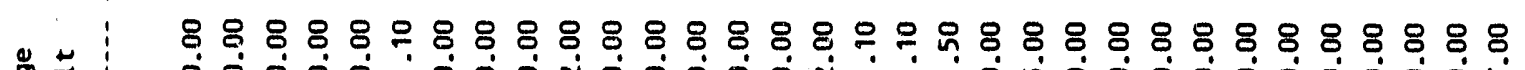
若 声焉 焉焉

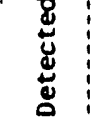

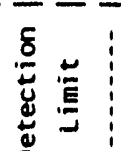

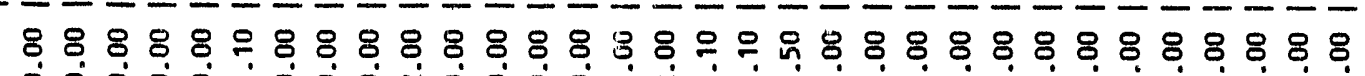

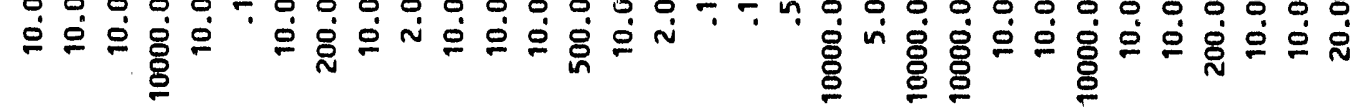

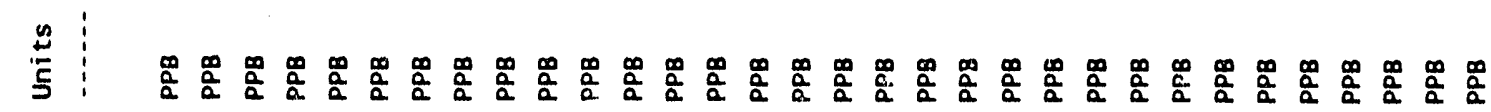

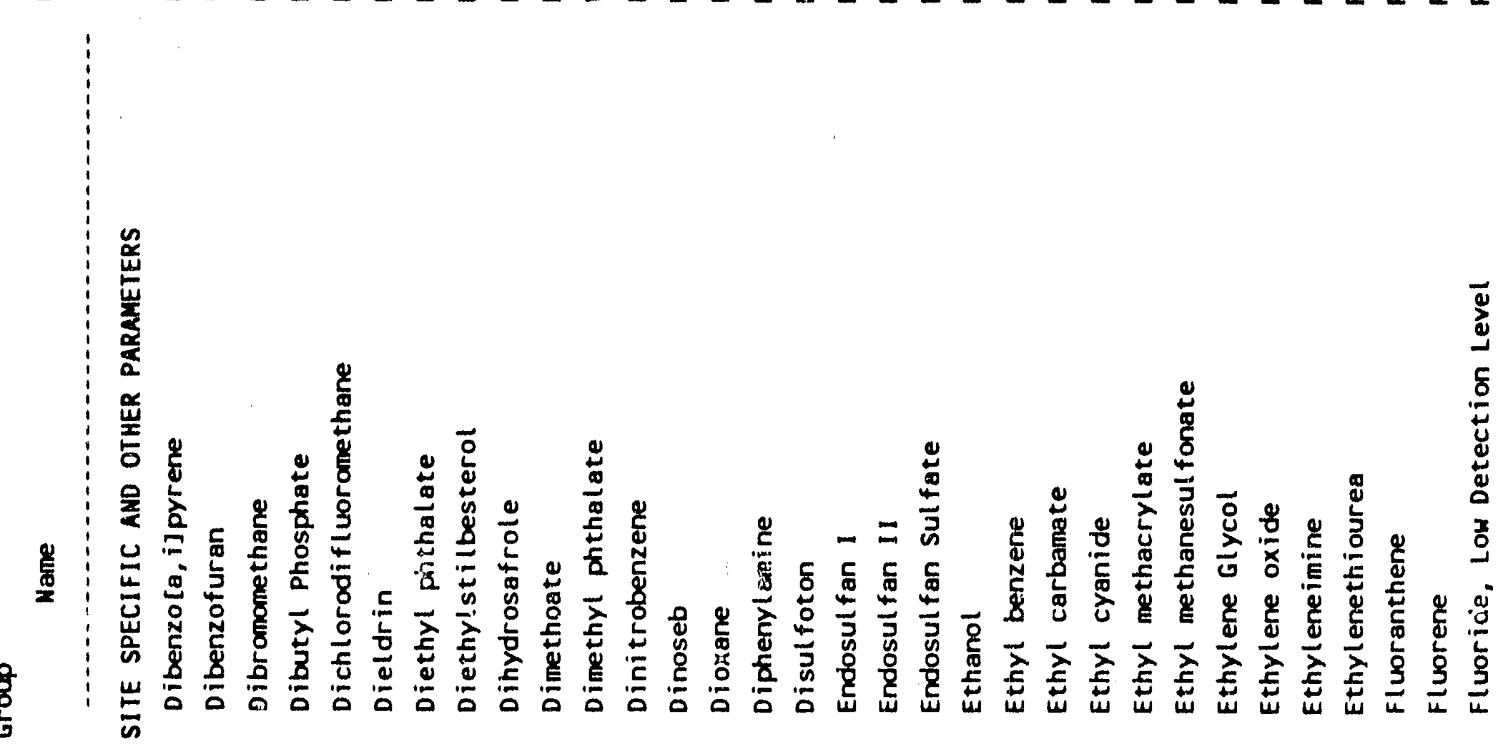




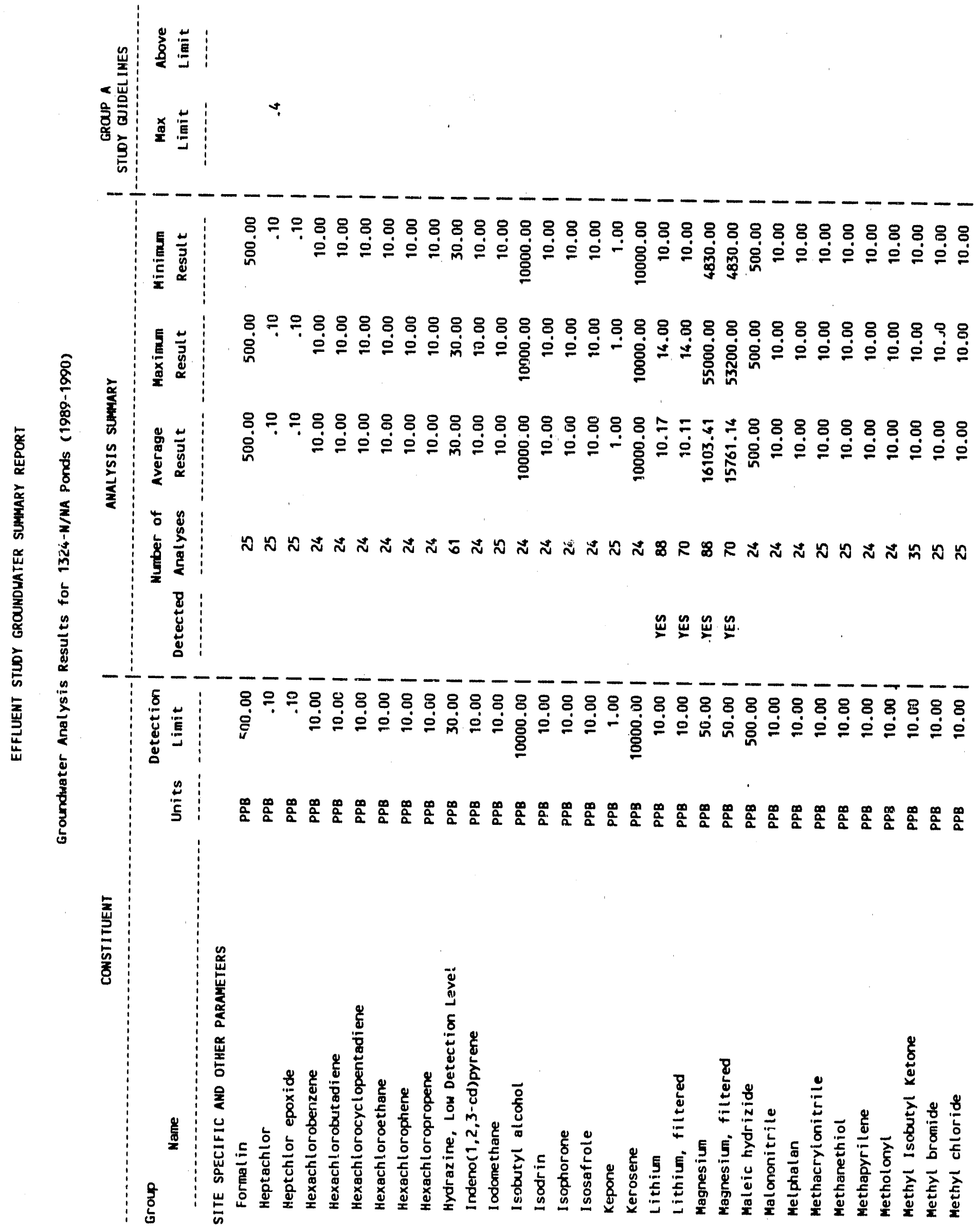




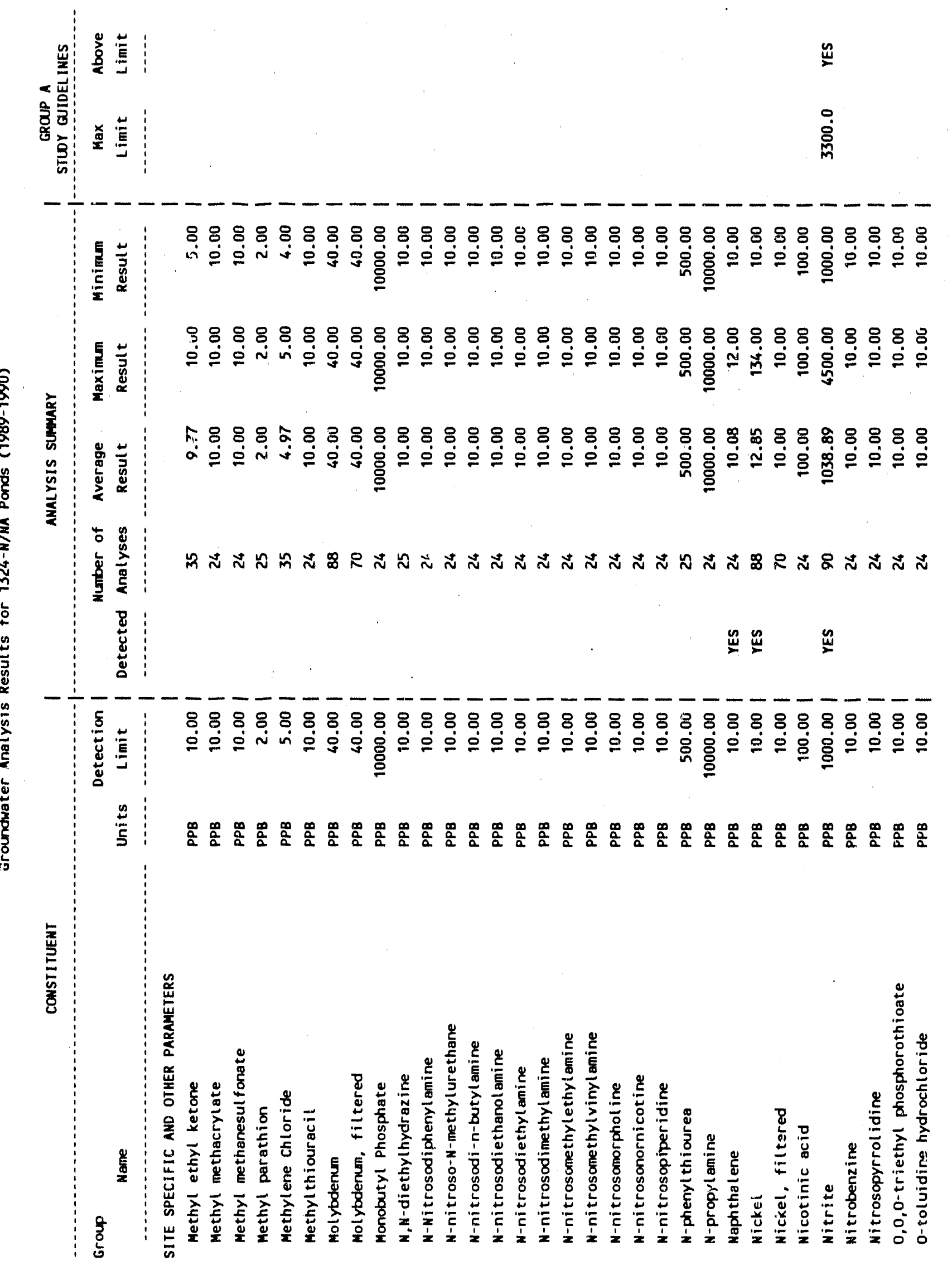


WHC-EP-0366
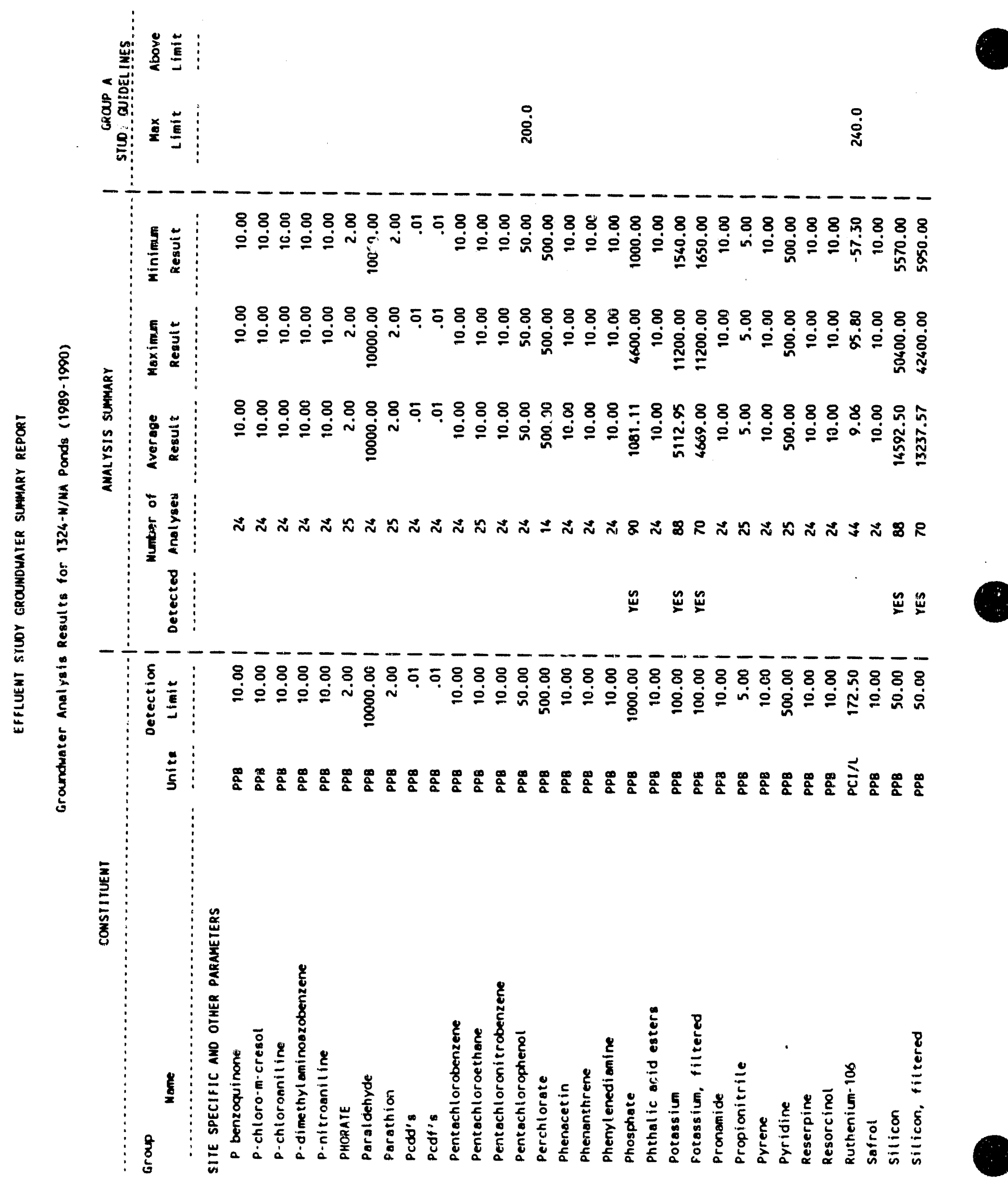


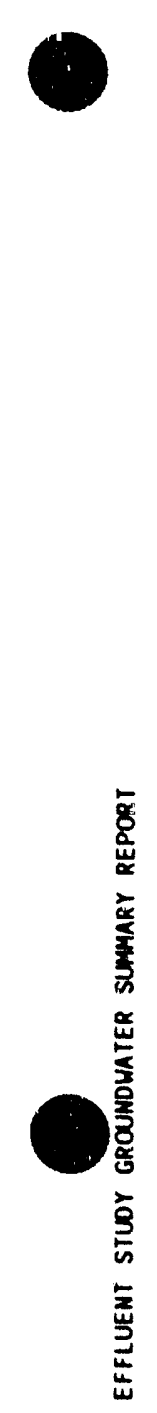

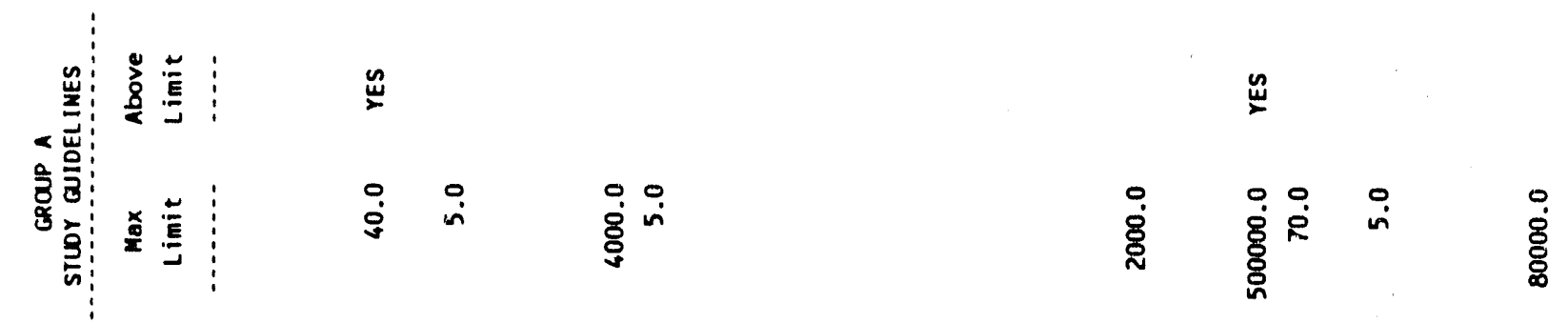

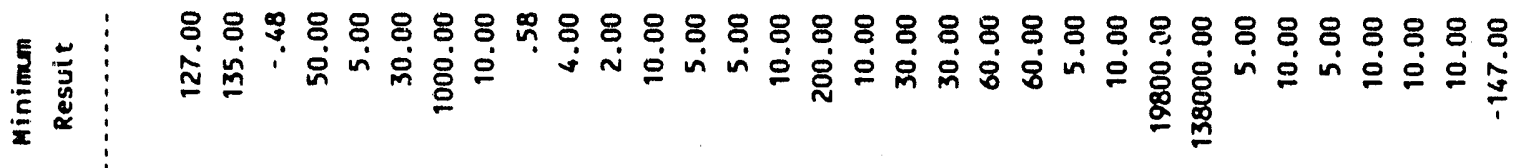

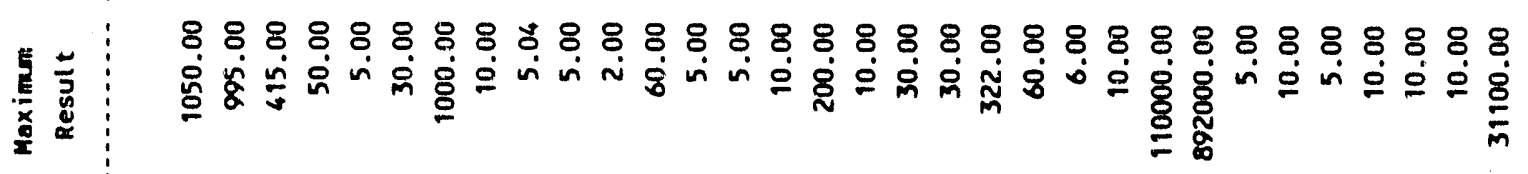

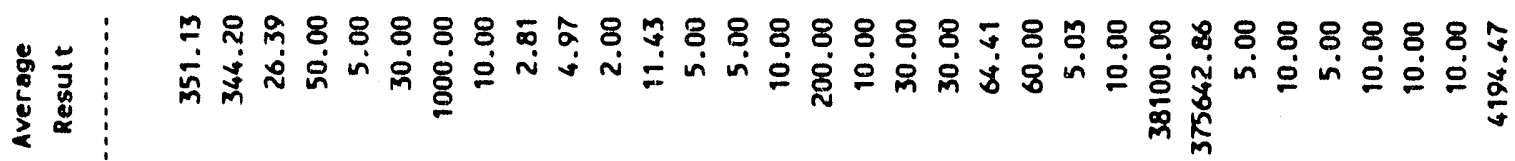

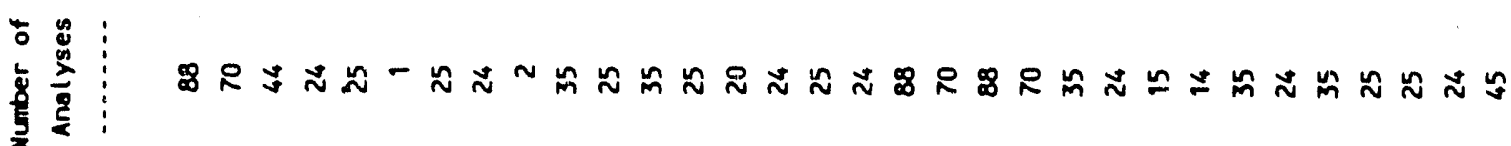

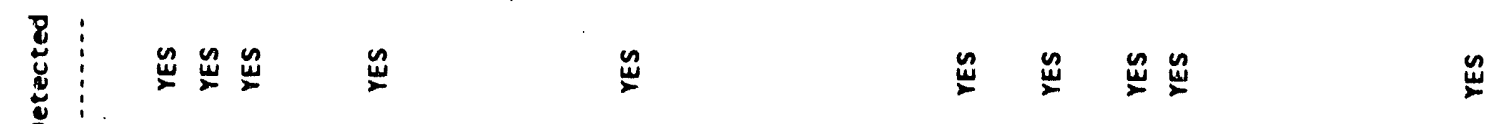

$$
\begin{aligned}
& \text { - } \\
& \text { 过 } \\
& \text { 穿 } \\
& \text { 窟 }
\end{aligned}
$$

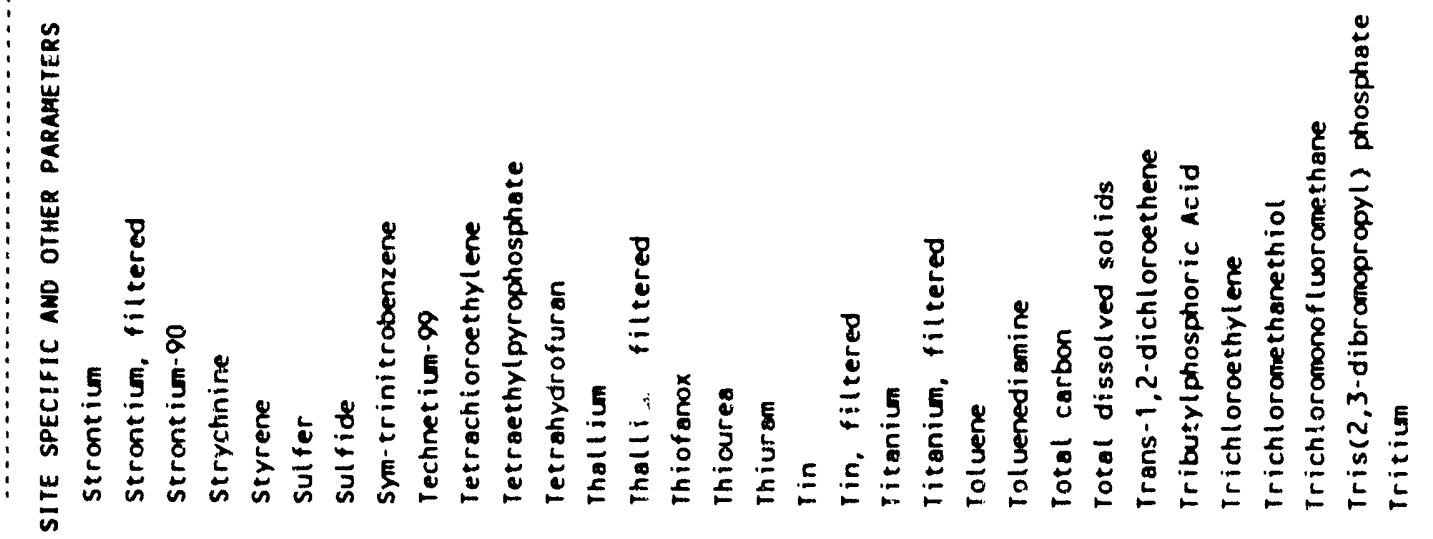


WHC-EP-0366

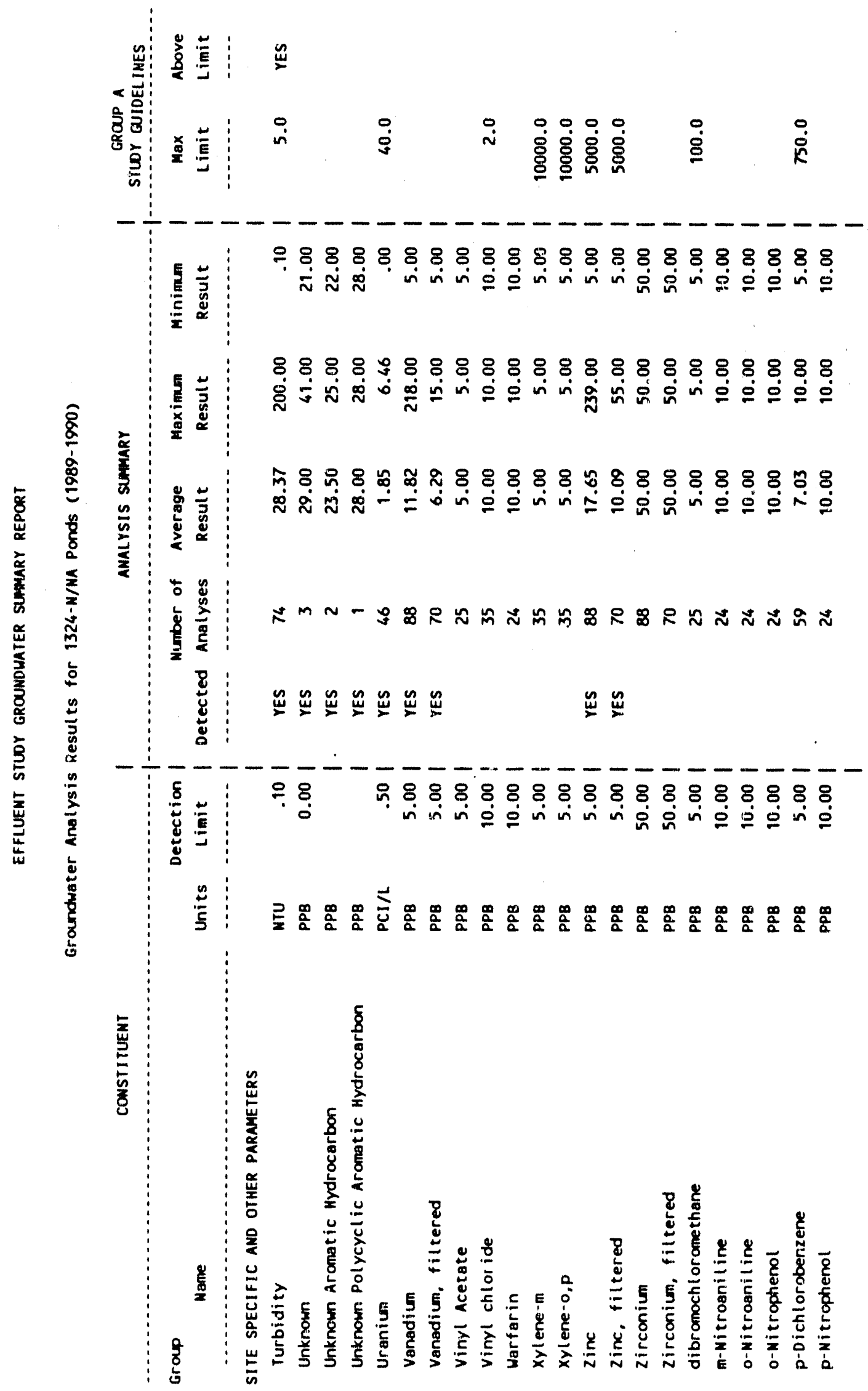


WHC-EP-0366

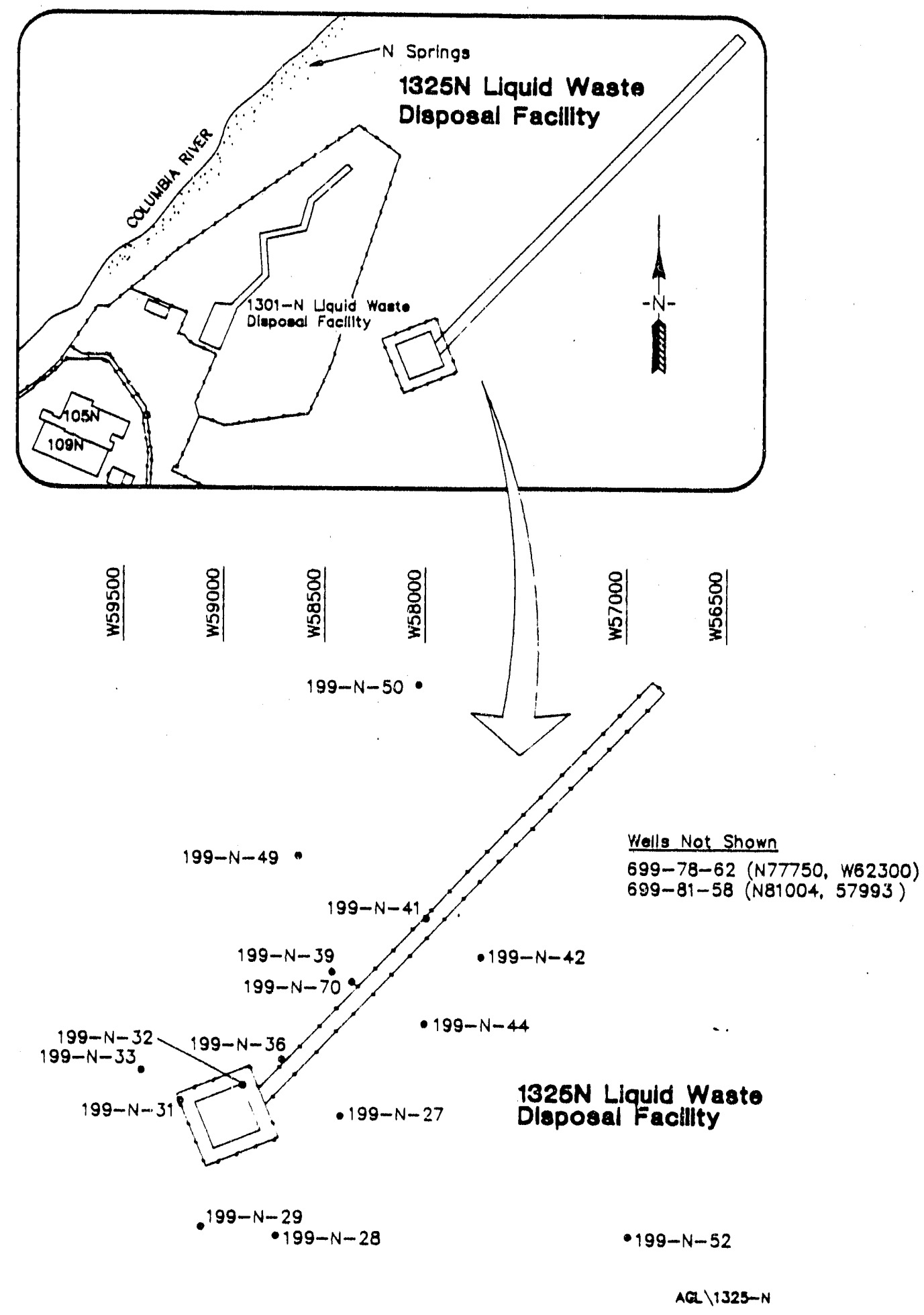

We11 Location and Site Map for $1325 \mathrm{~N}$ Crib 


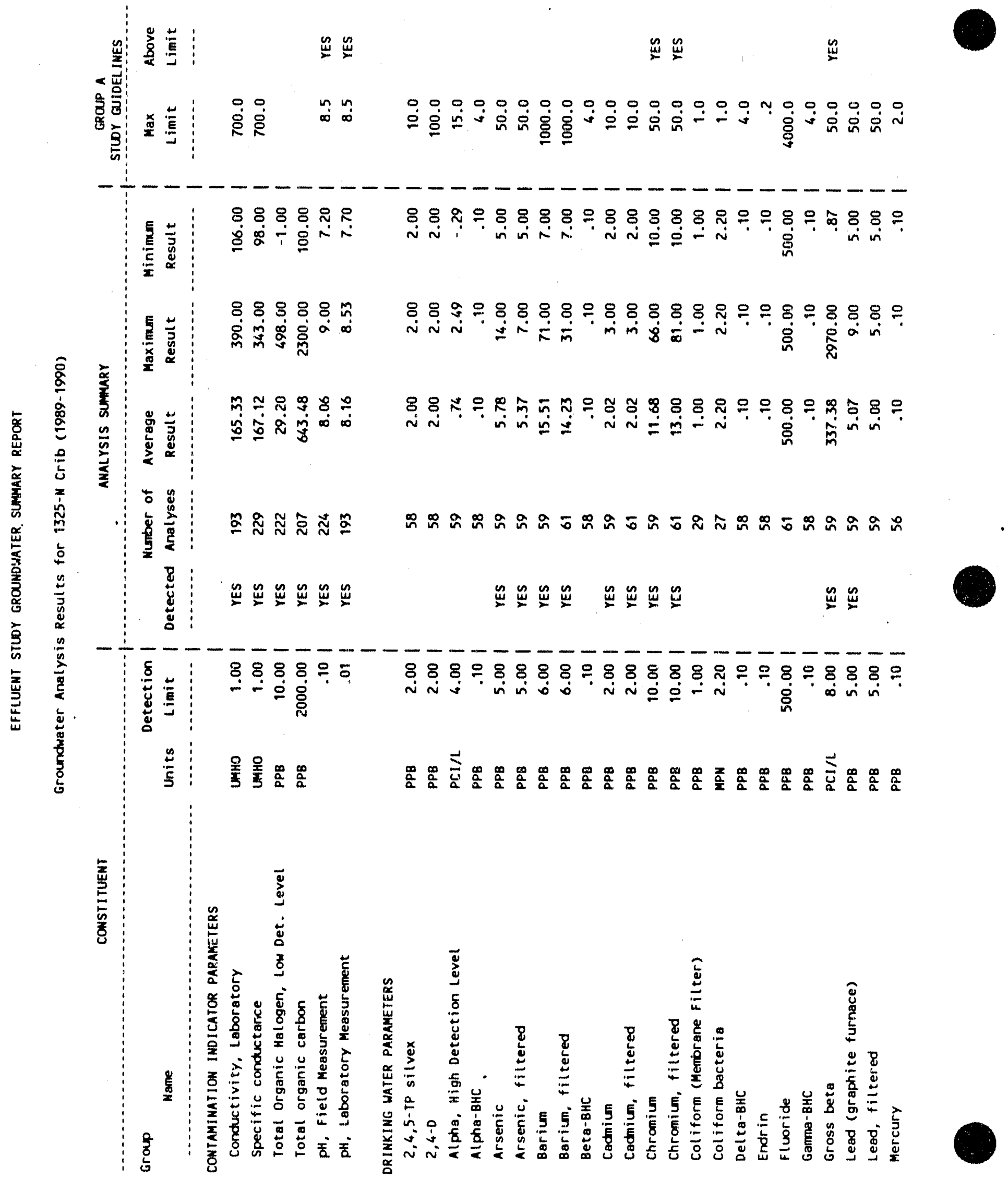


WHC-EP-0366

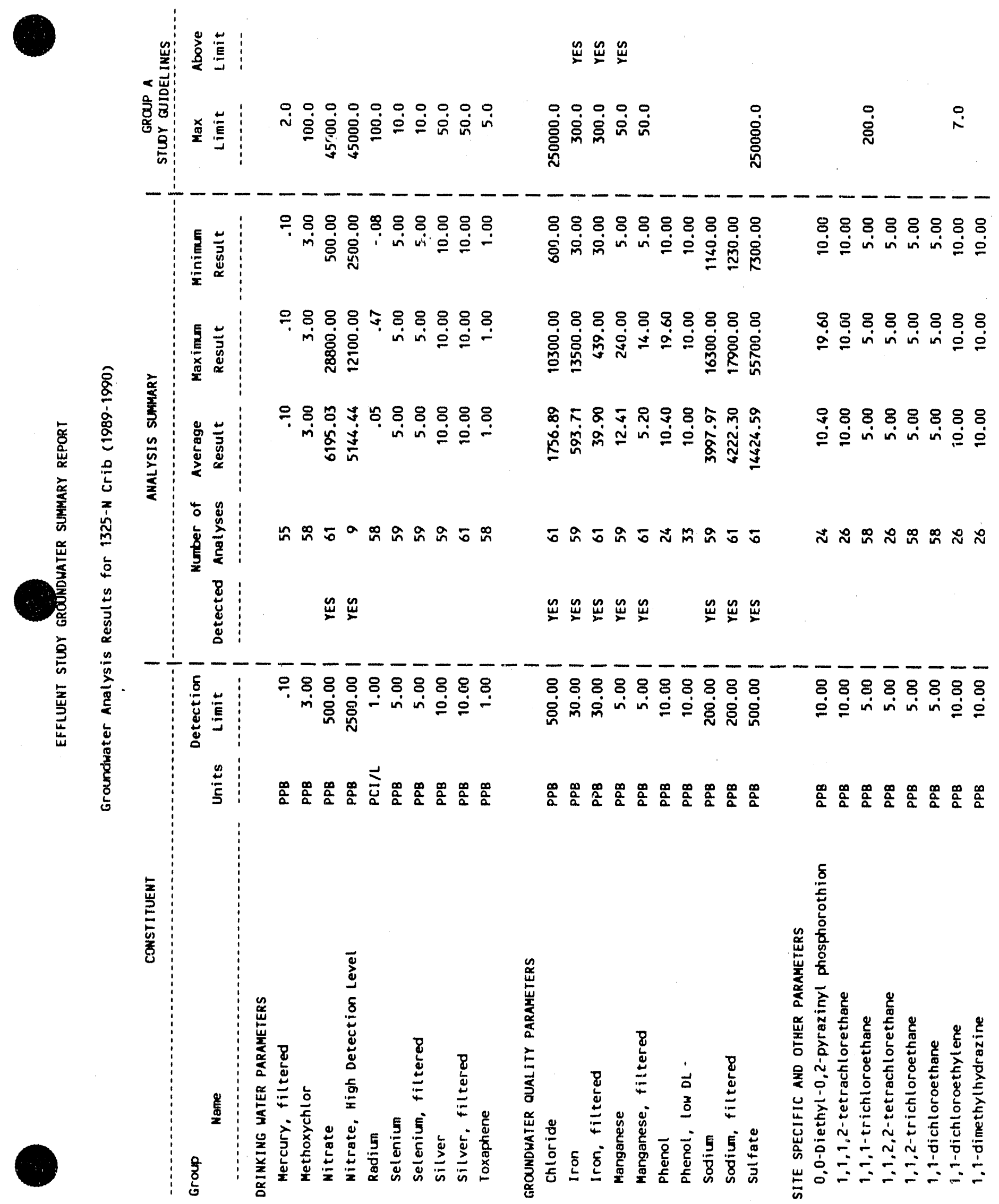




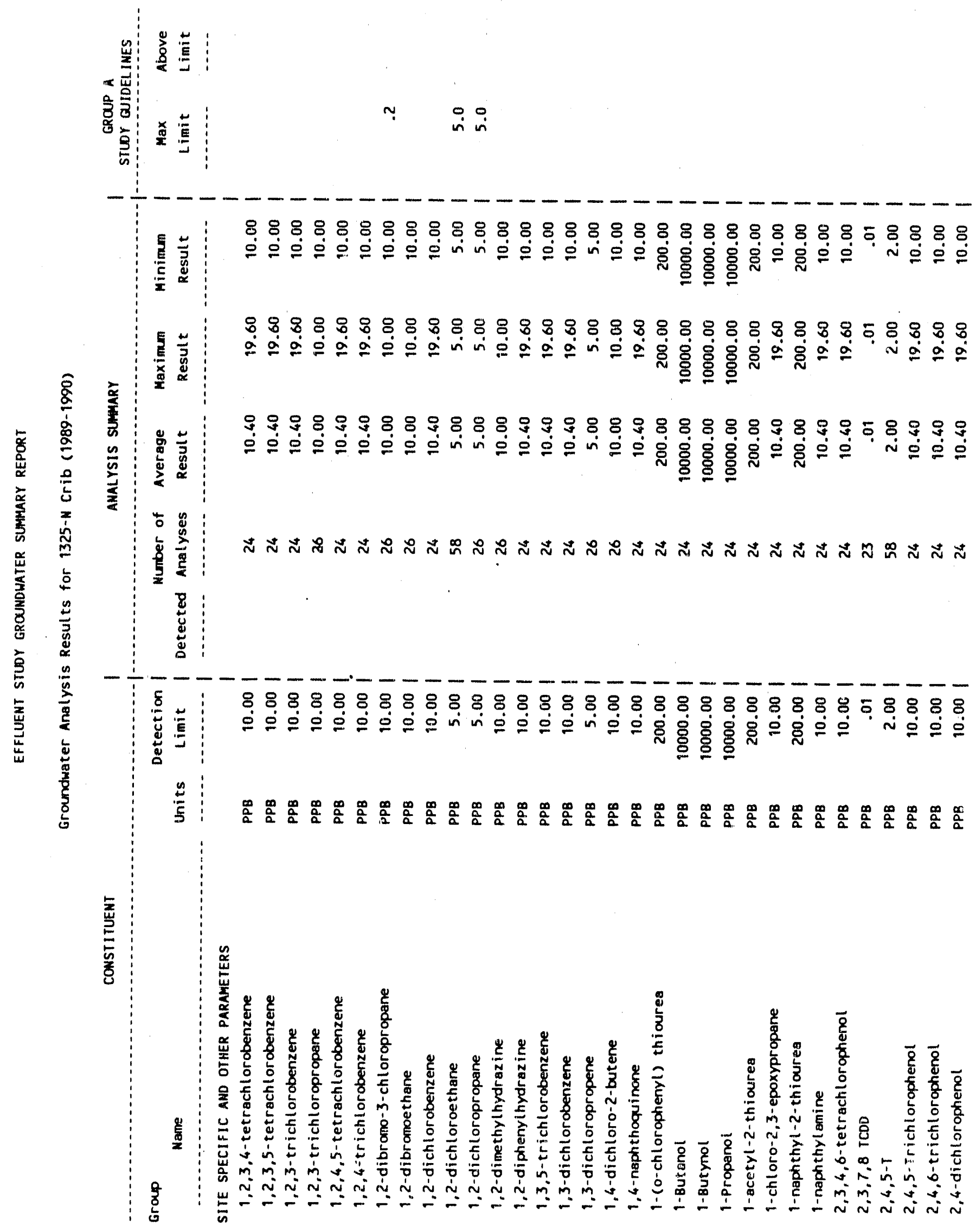


咅:

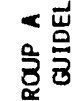

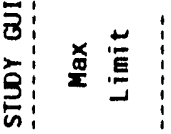

$-\frac{1}{1}$

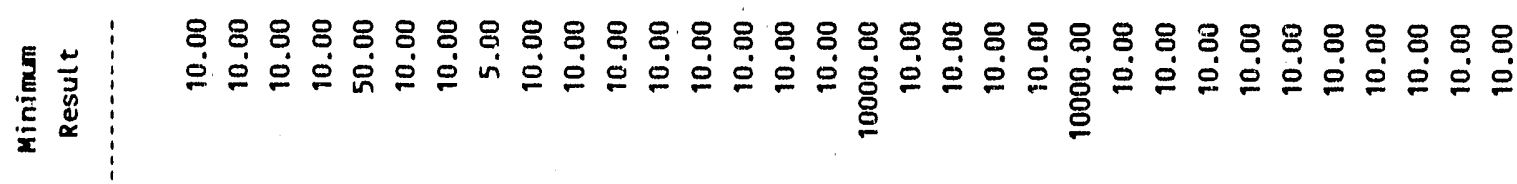

ـ

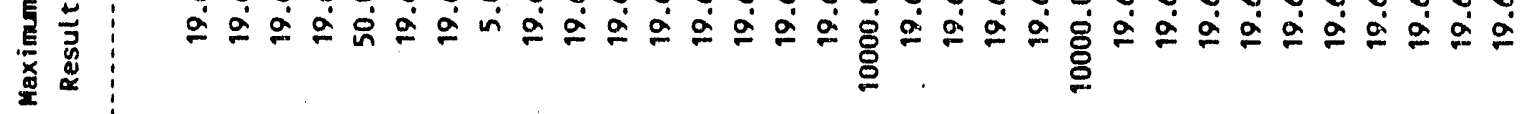

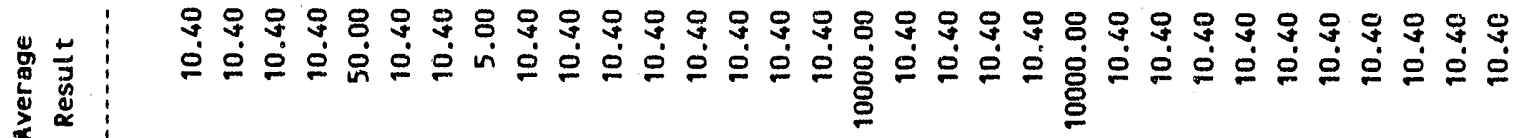
$\stackrel{0}{\gtrless}$ ¿ 莫

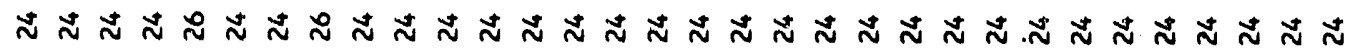
(:

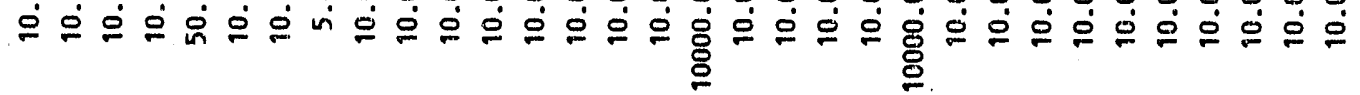

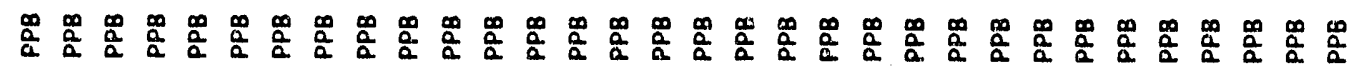

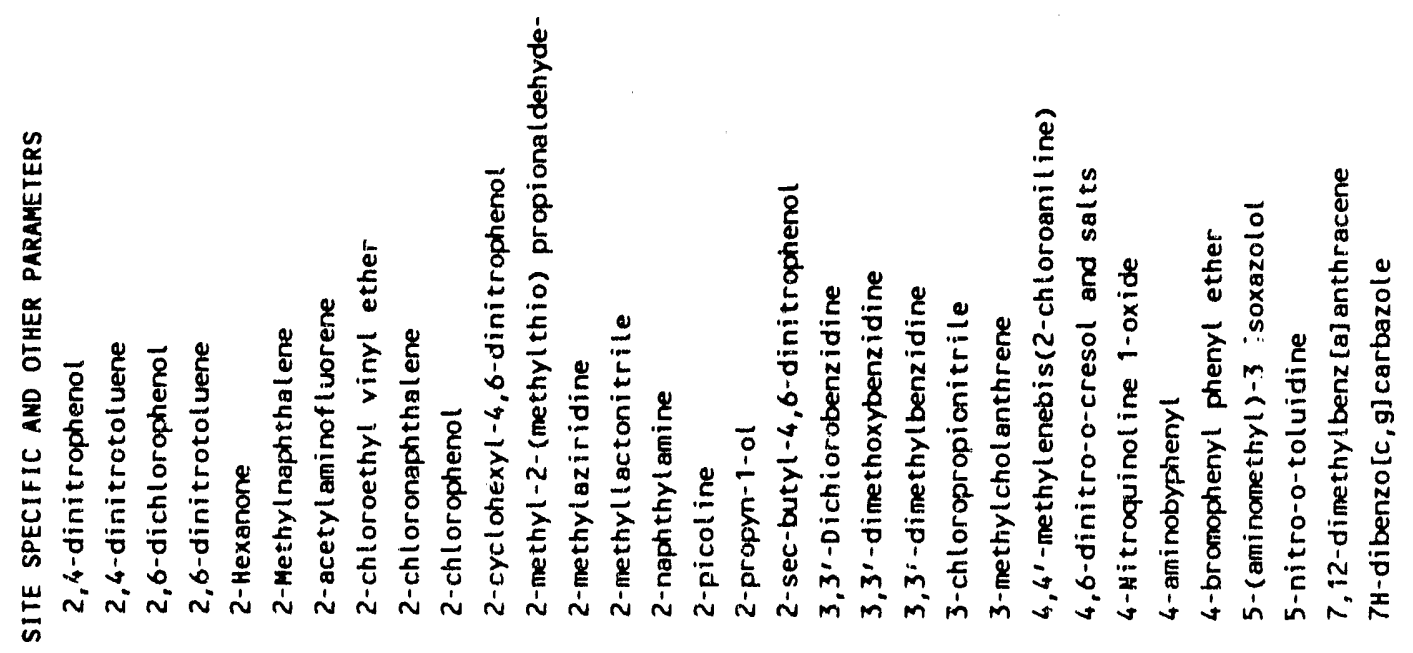



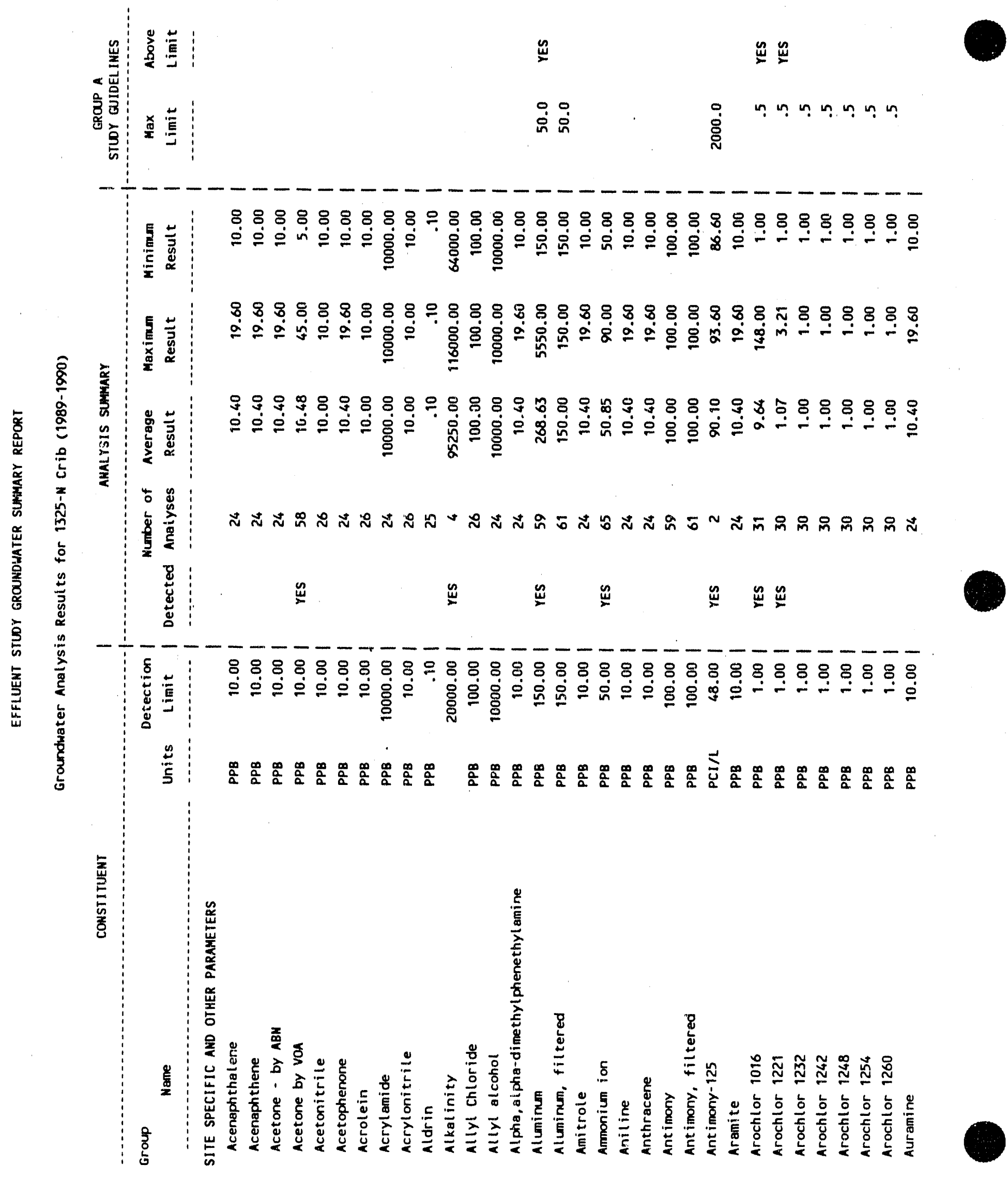
WHC-EP-0366

\section{亘}

$<$ 运

敢:

遂富

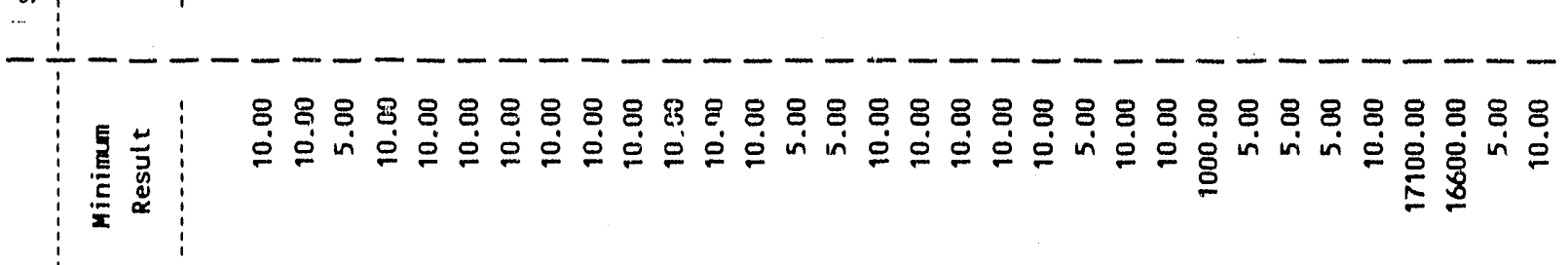

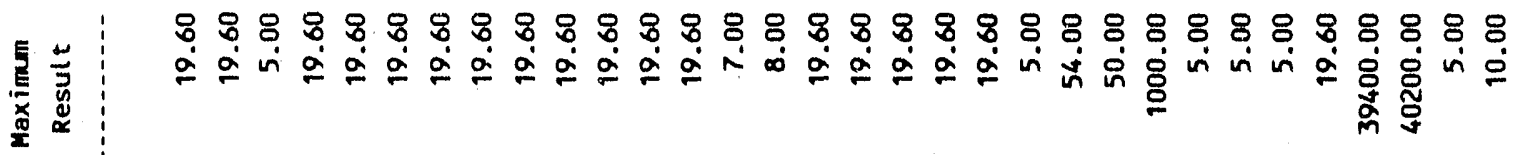

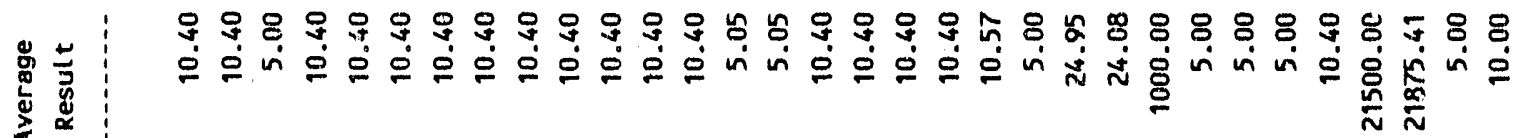

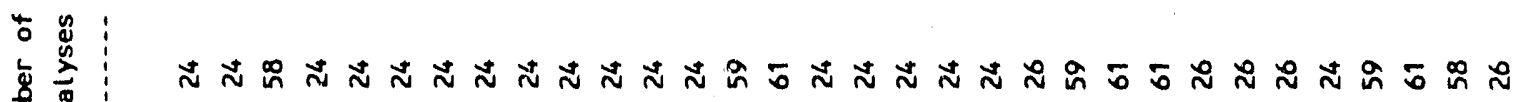

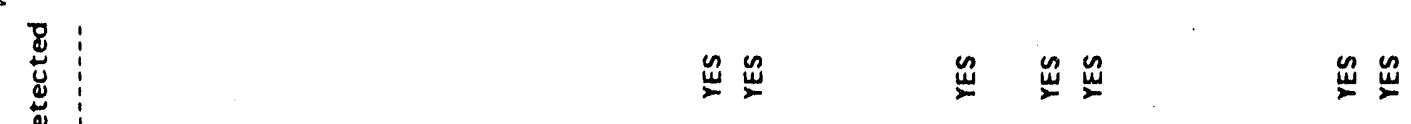

8

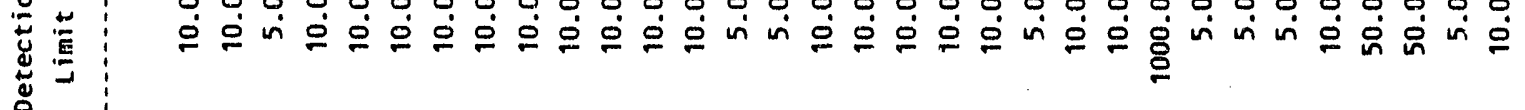

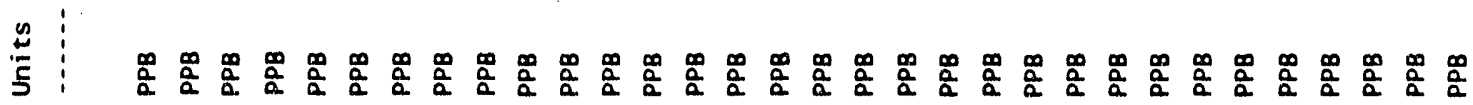

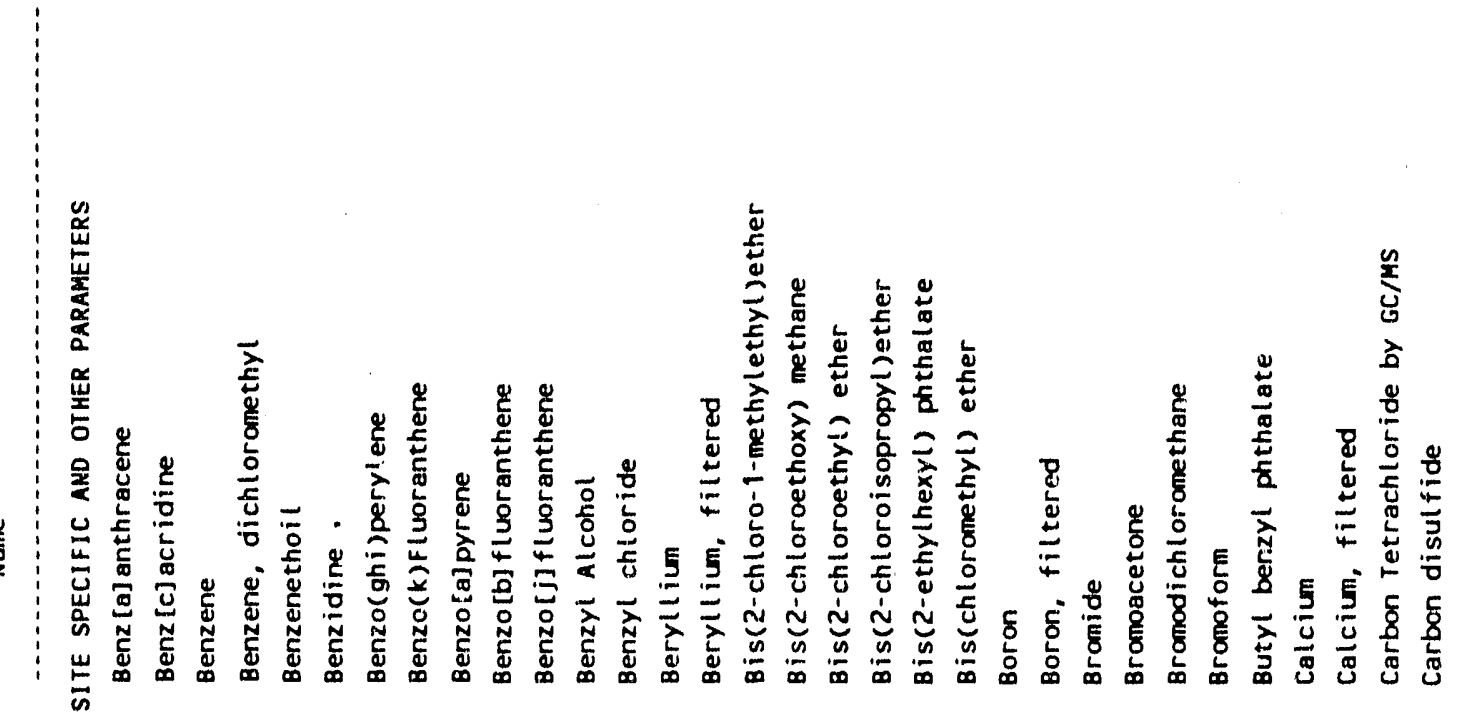




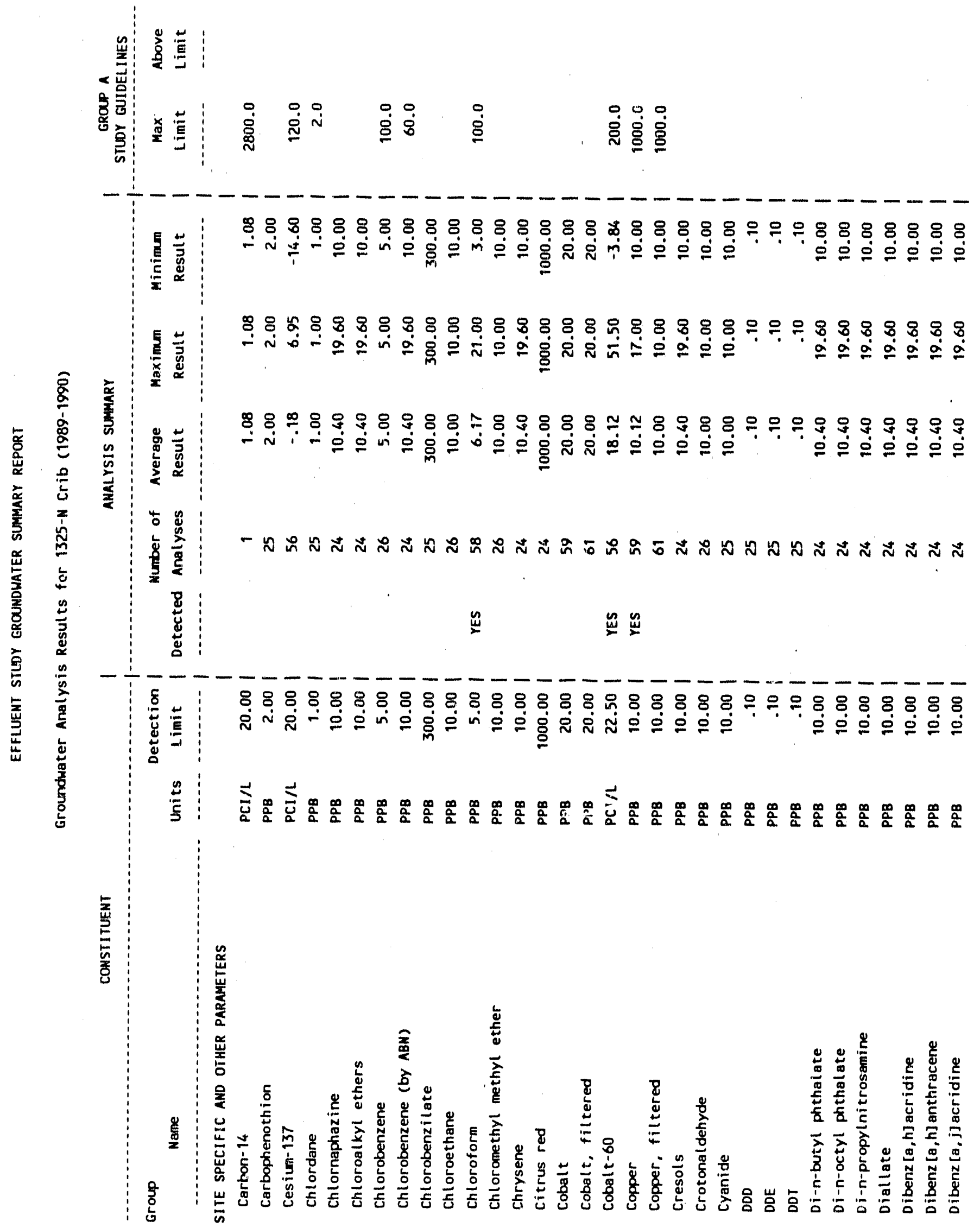


葛

密

空

$\stackrel{\circ}{\check{g}}$

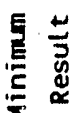

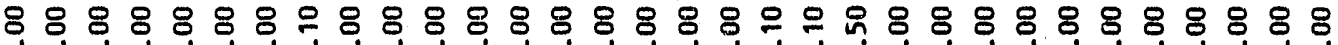

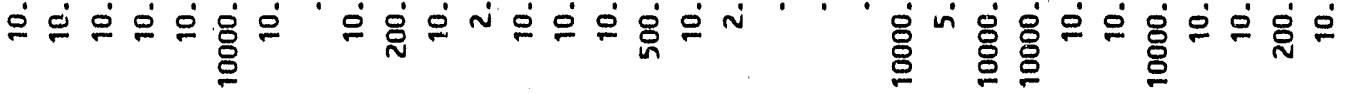

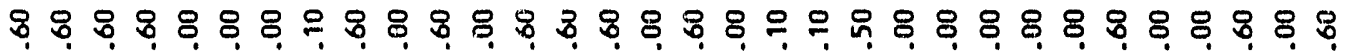

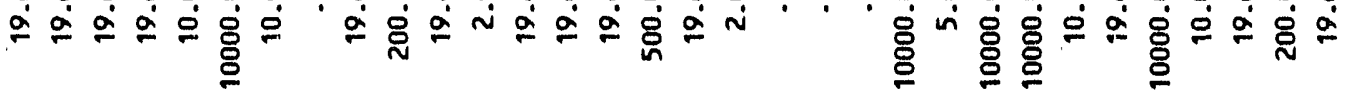

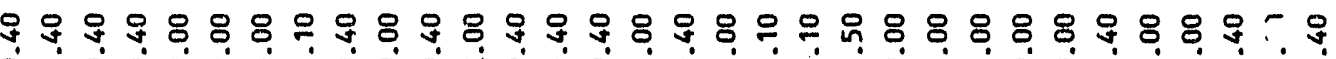

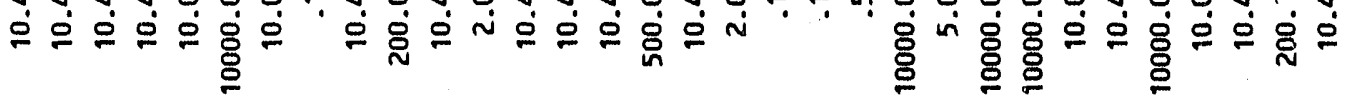

㟔出

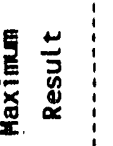

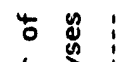

กัง 焉是

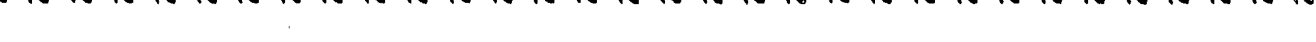

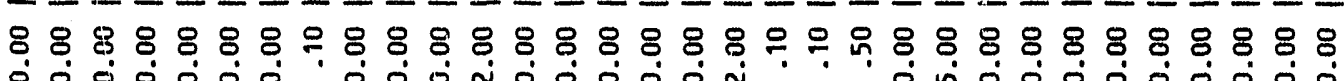

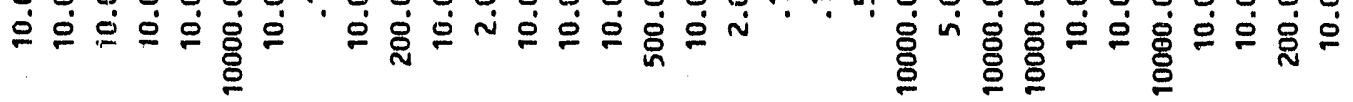

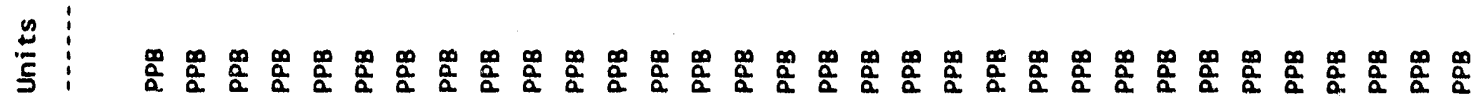

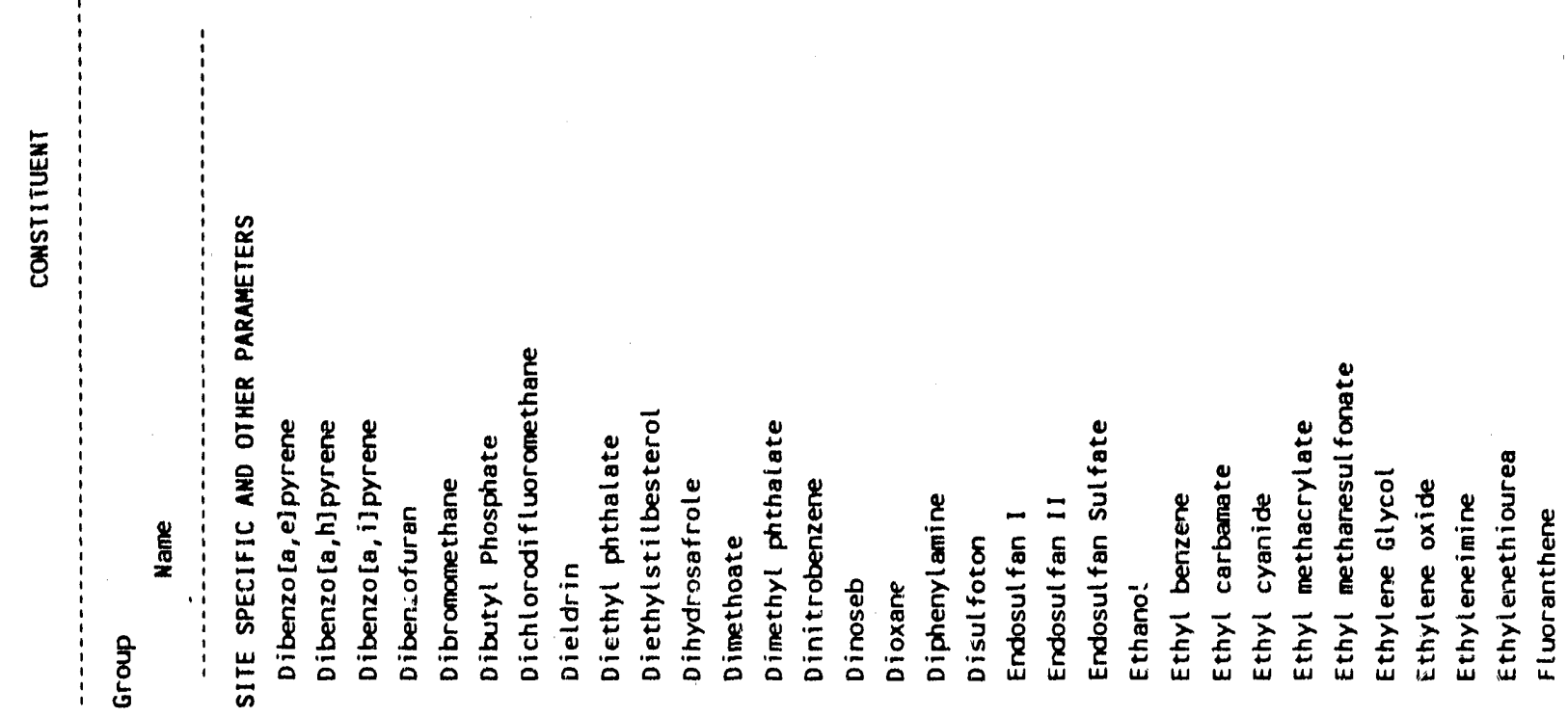


WHC-EP-0366

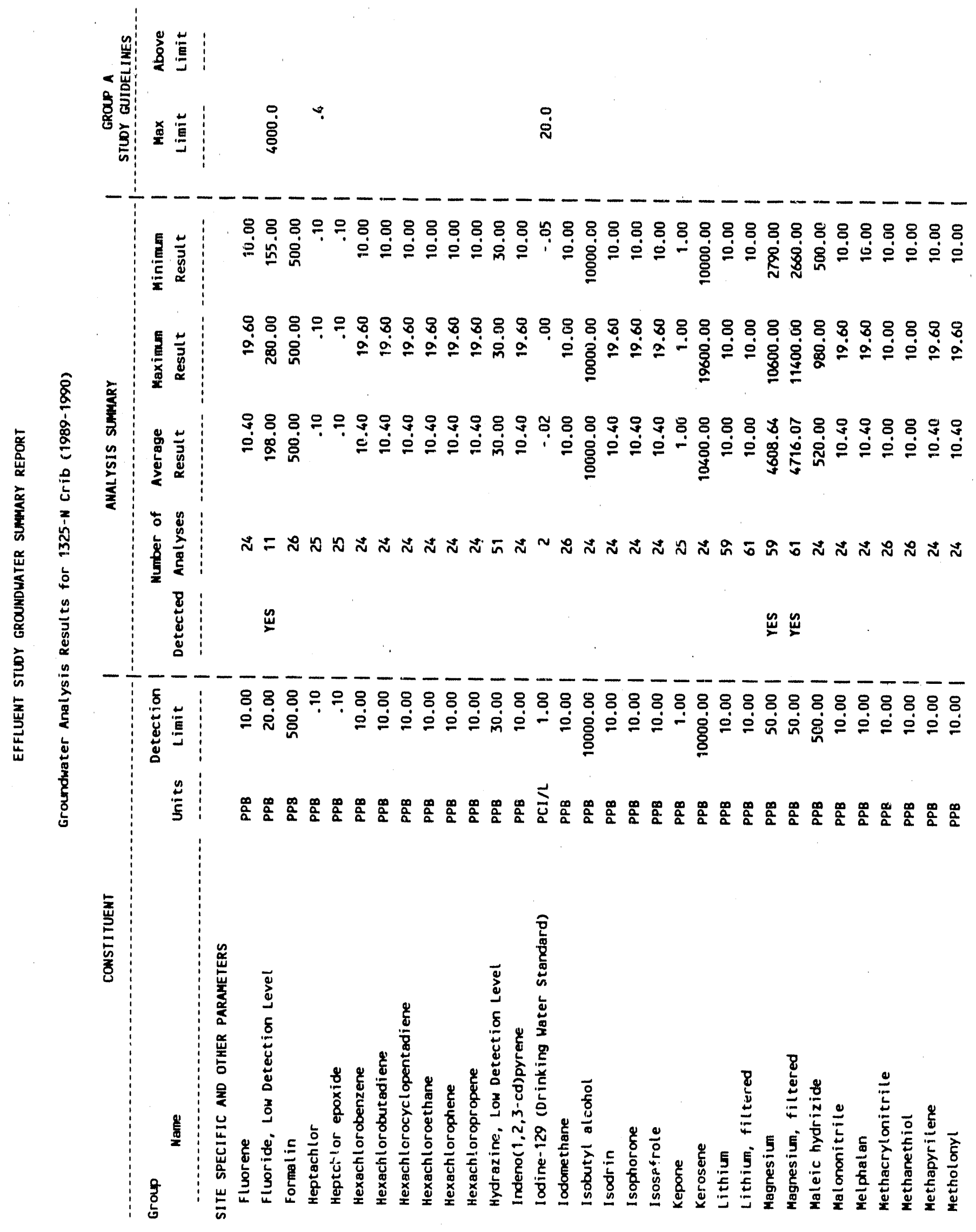


WHC-EP-0366

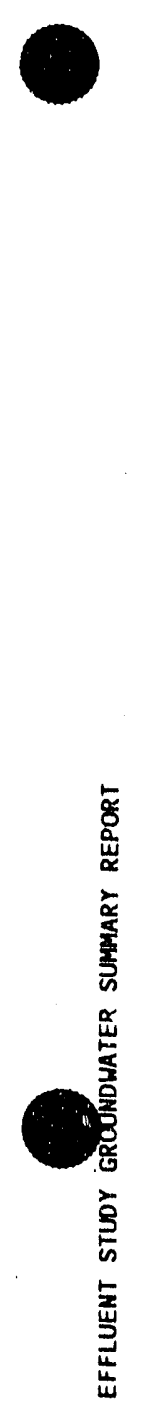

染苜

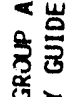

啚荎

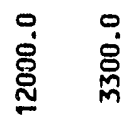

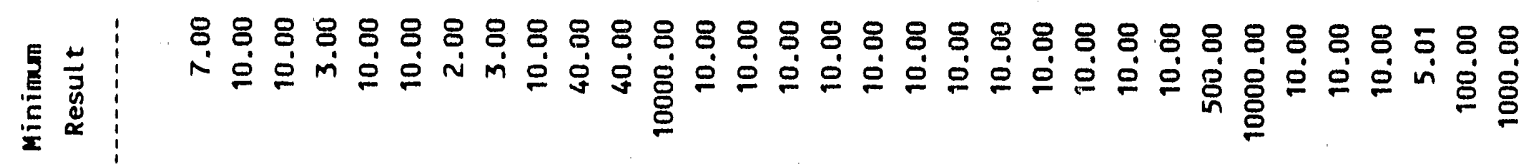

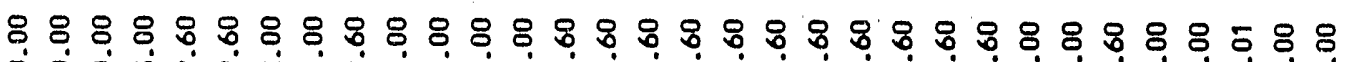

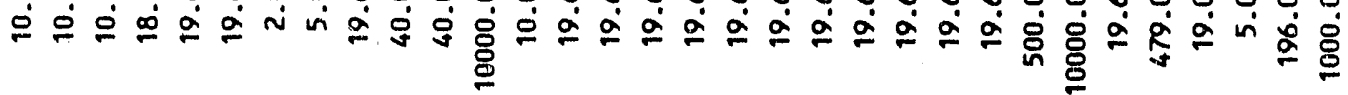

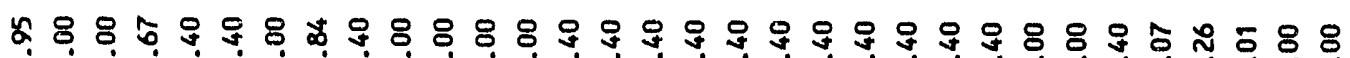

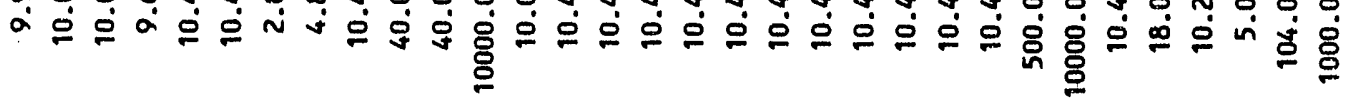

焉

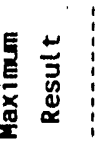

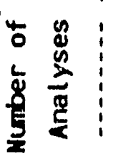

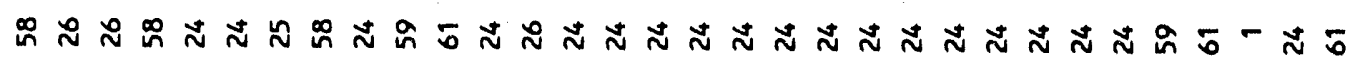

造:

$\stackrel{\mathscr{M}}{2}$

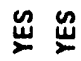

वे

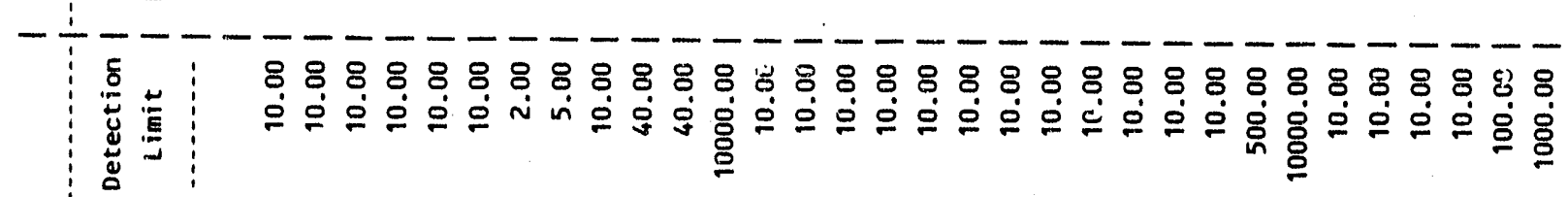

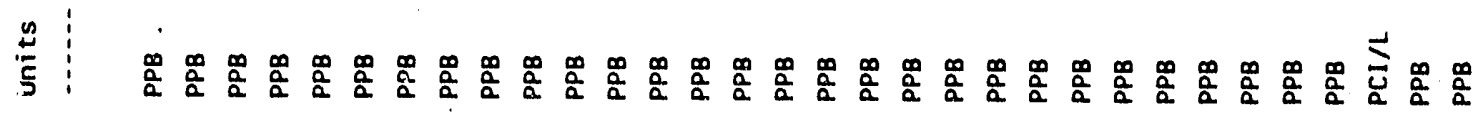

氙

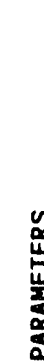

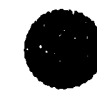

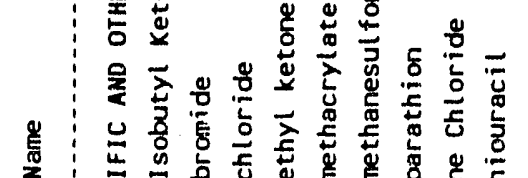

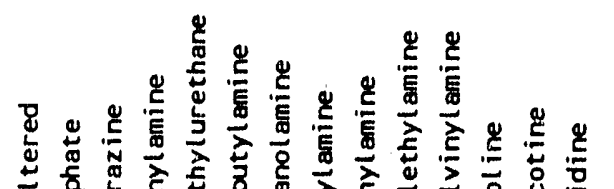

胥尌

:

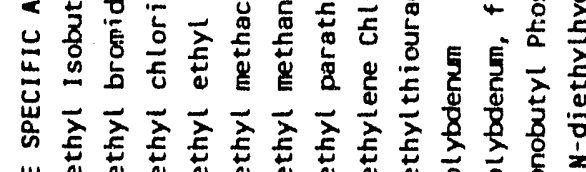

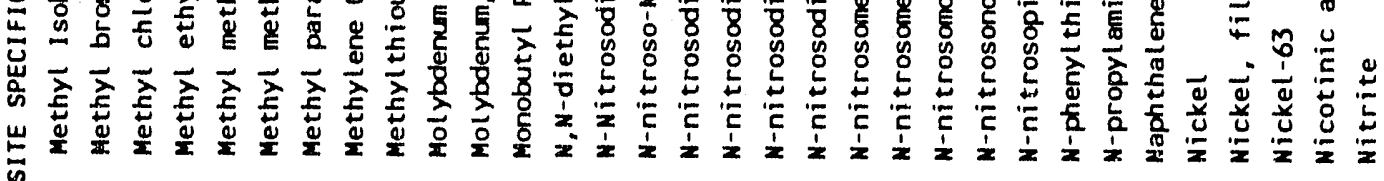




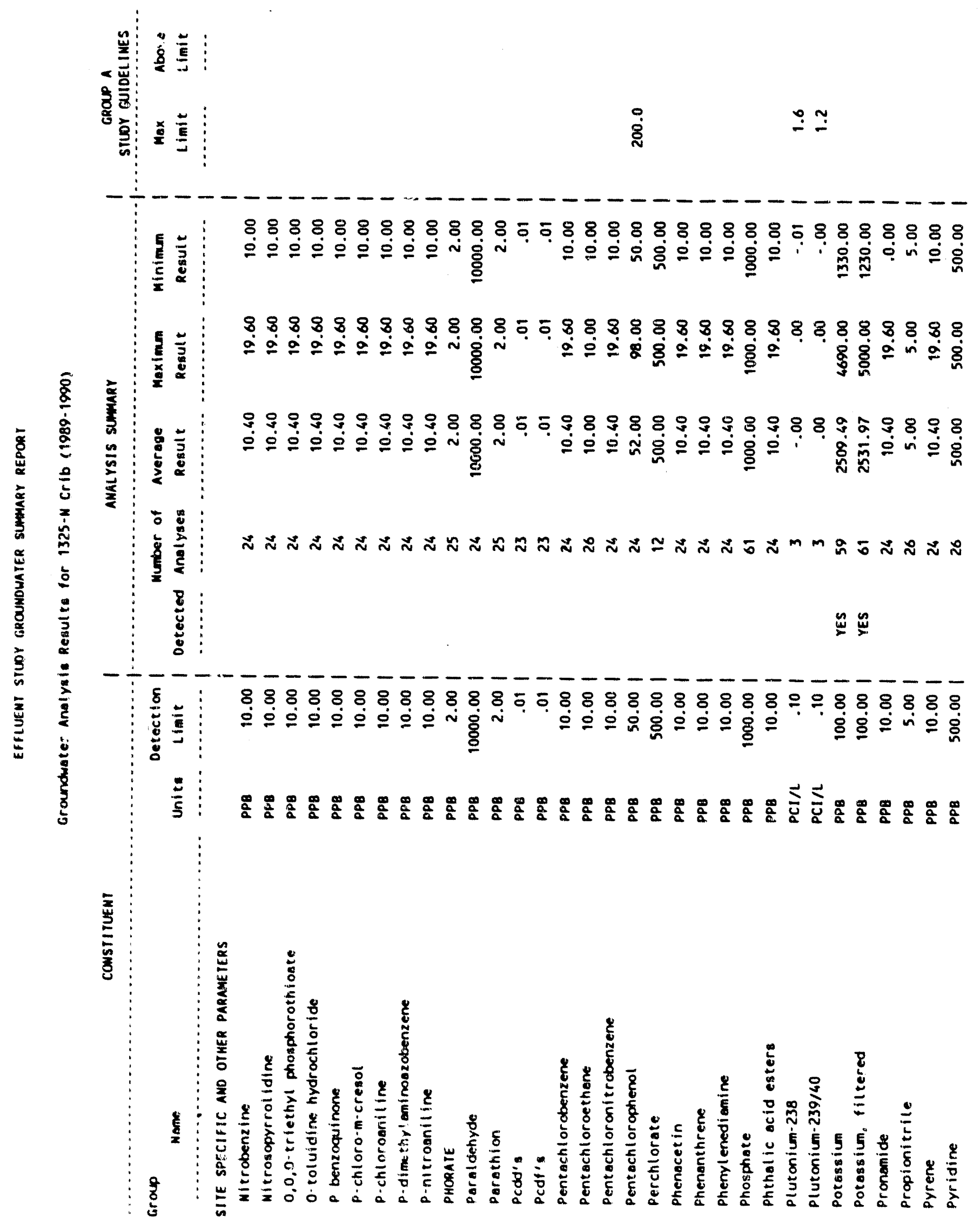




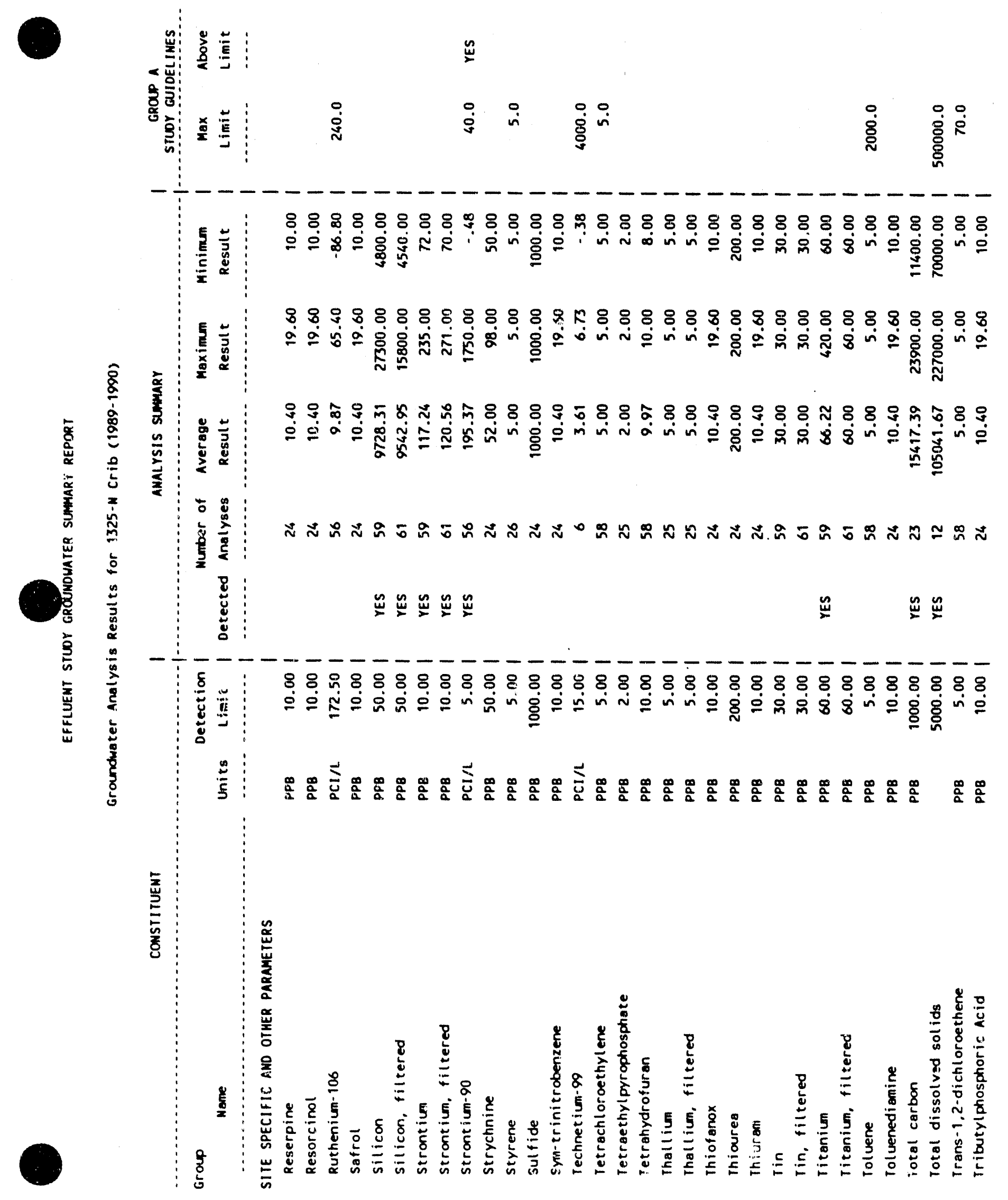




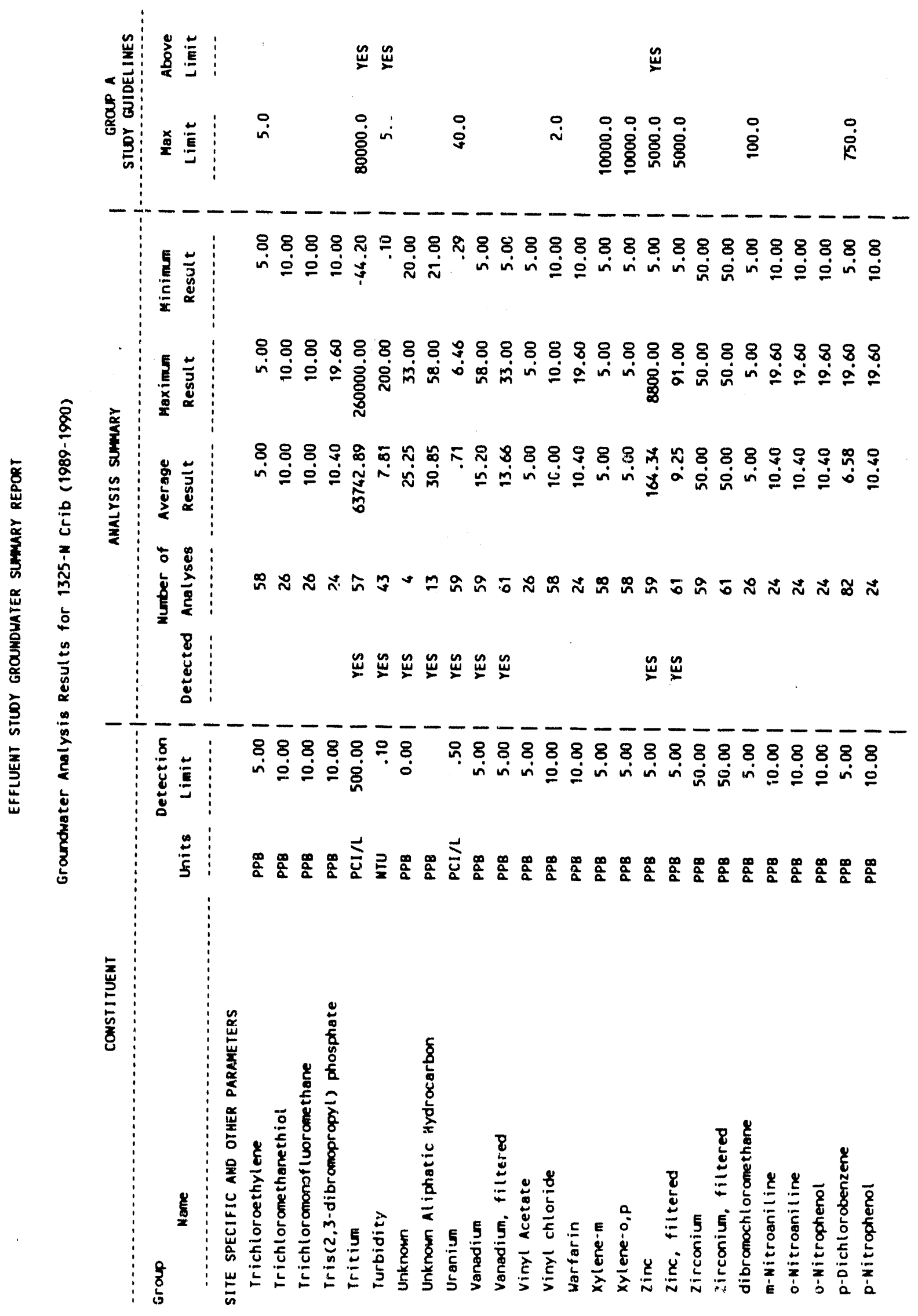


WHC-EP-0366

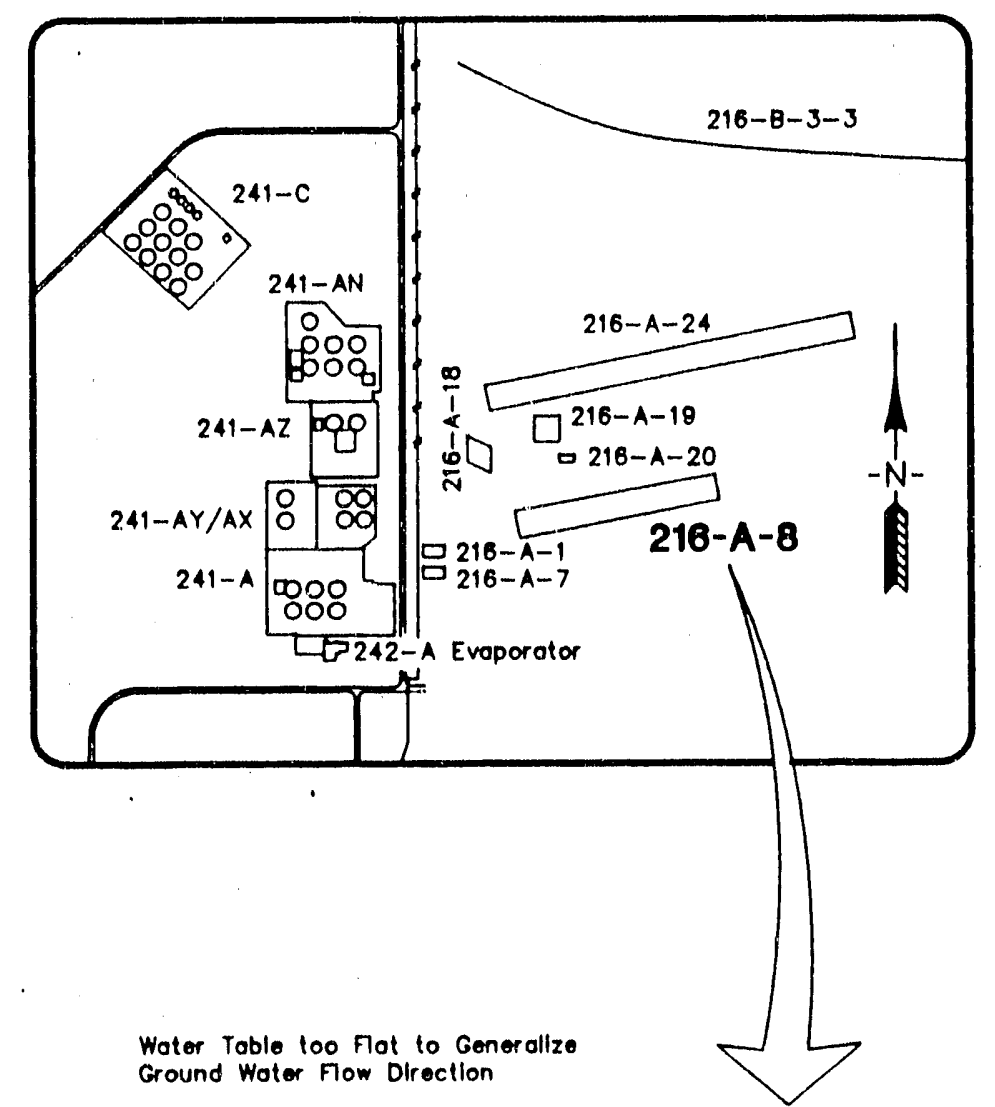

$N 42000$

Table too flat to Genera Ground Woter Flow Direcllon

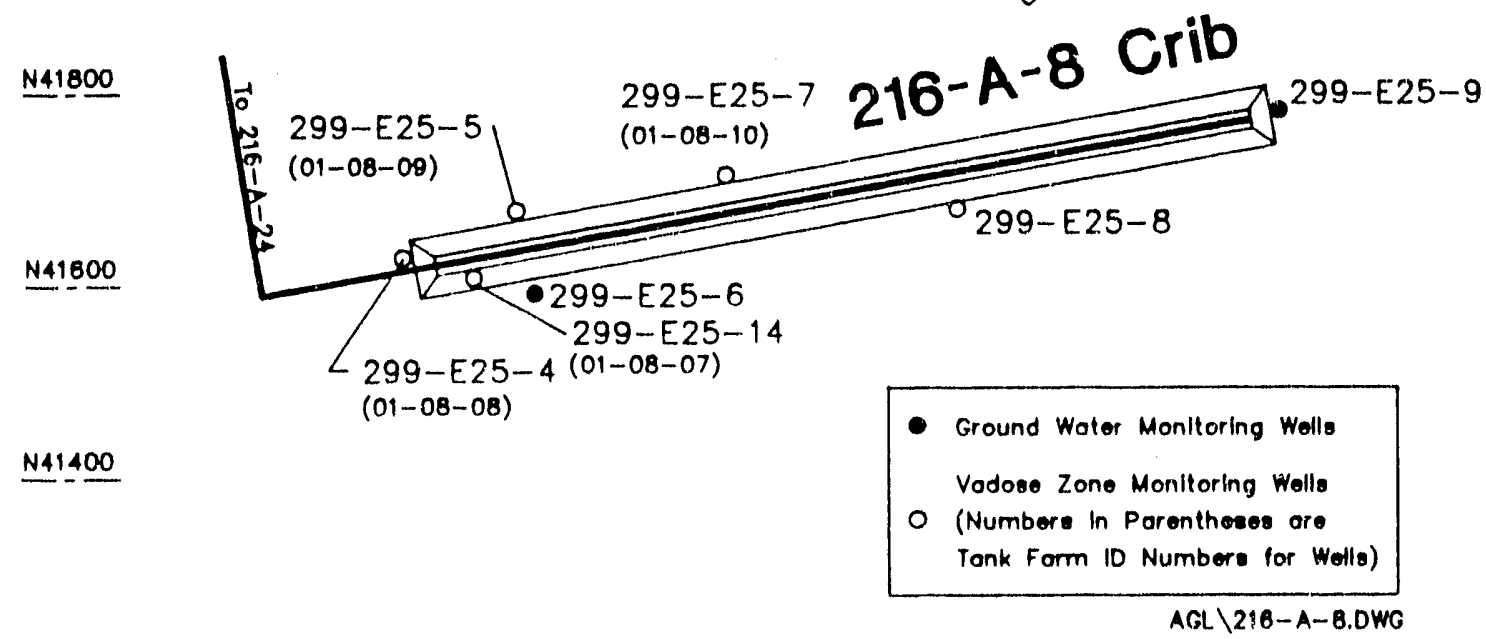

N41200

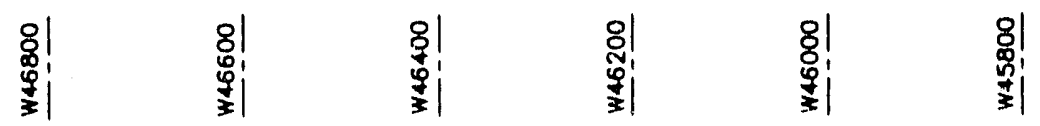

Well Location and Site Map for 216-A-8 Crib 
WHC -EP-0366

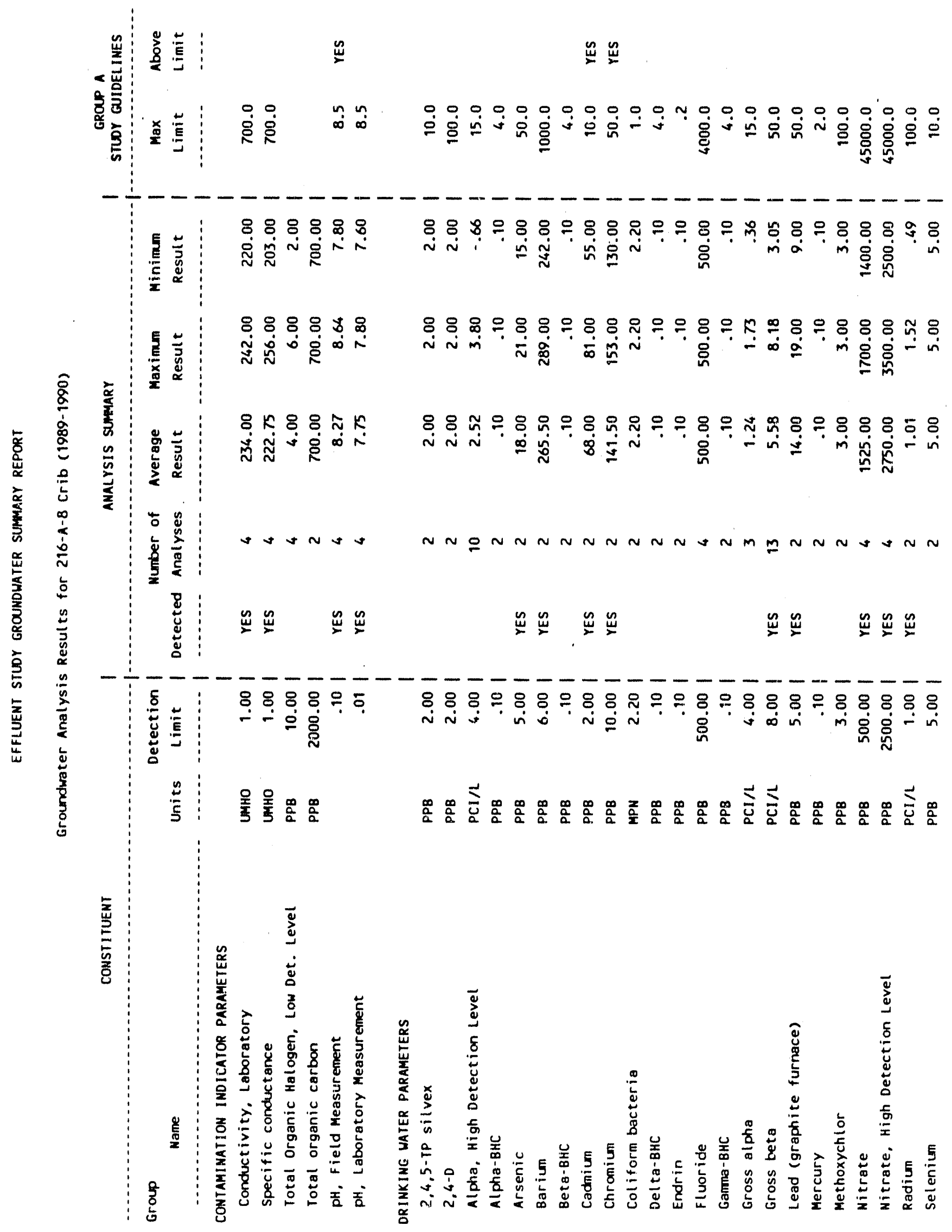


WHC-EP-0366

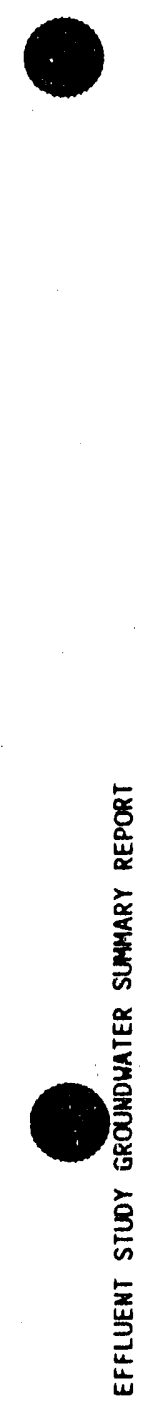

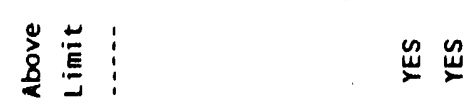

空

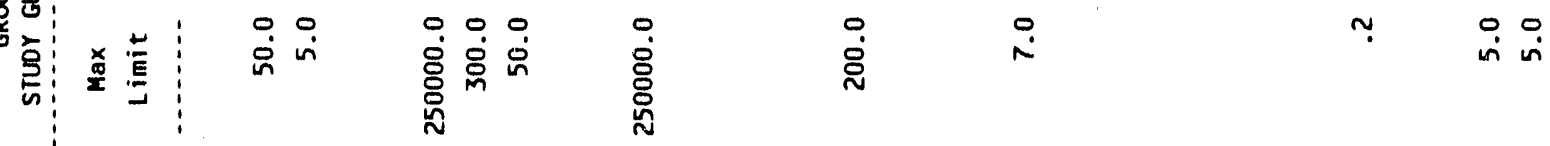

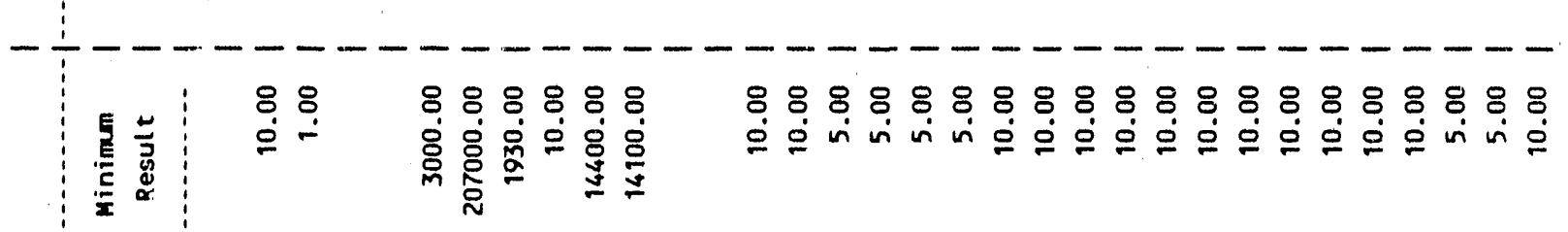
Е

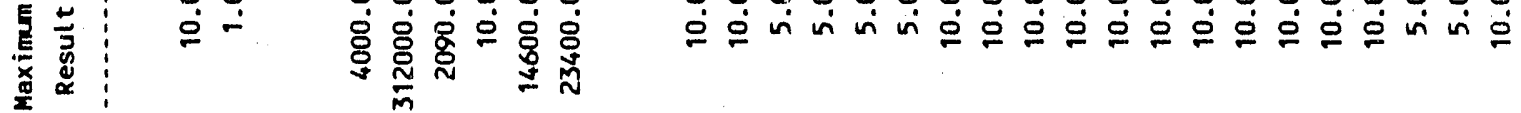

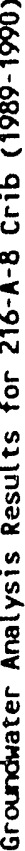

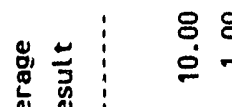

888888

88888888888880888888 $\sum_{2}$

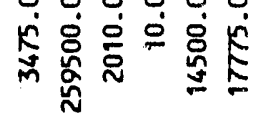

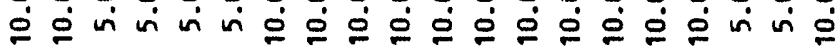
\begin{tabular}{c:c}
0 \\
0 \\
\hline
\end{tabular} 焉要

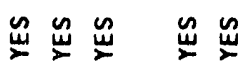

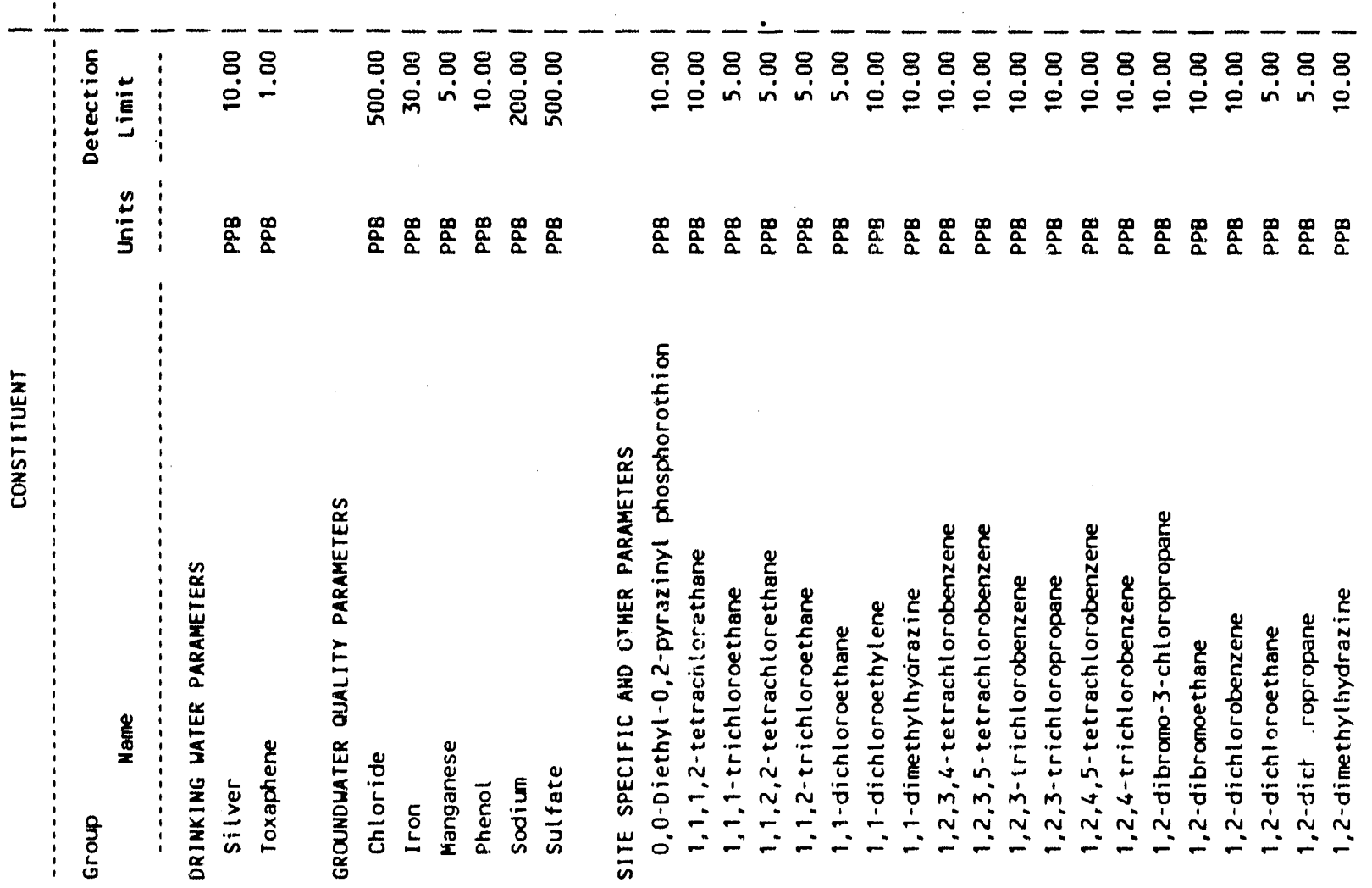


WHC-EP-0366
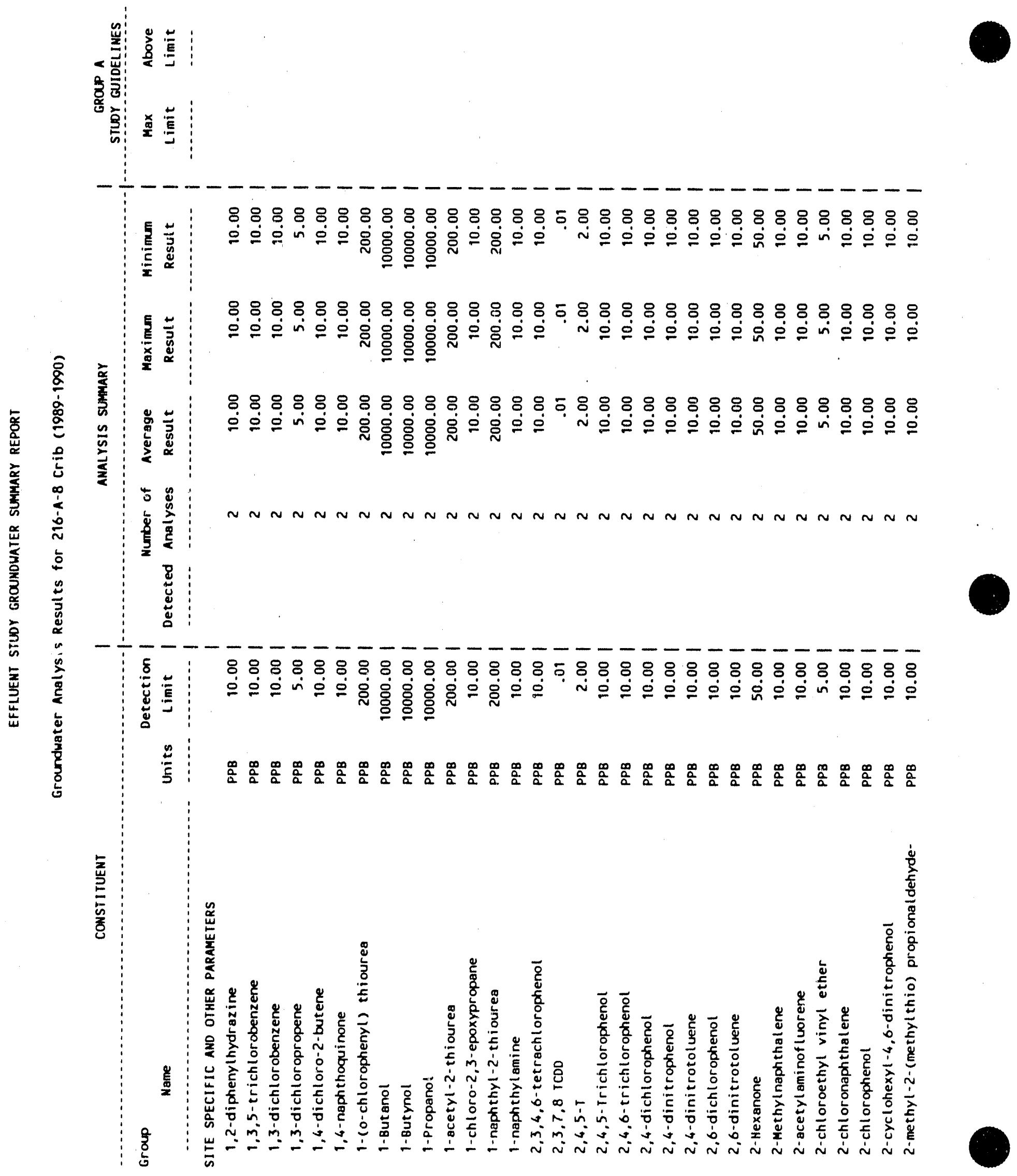
WHC -EP-0366

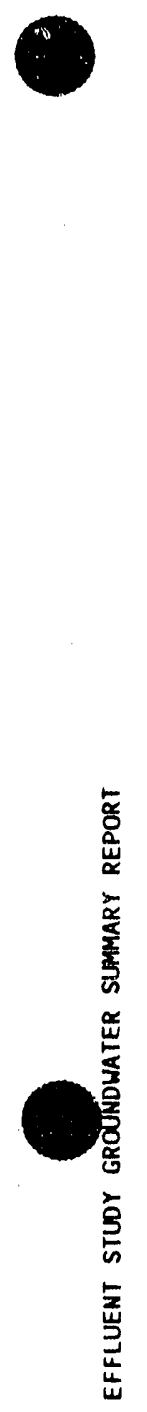

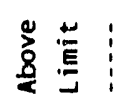

$<$

家

煎

$-\frac{1}{1}$

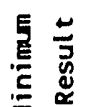

888888888888888888888888888088

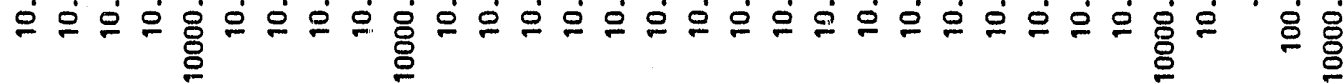

88888888088808888088088888888088 莀

888888888888888888888888888988

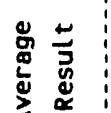
த்த்த் 总: 要要

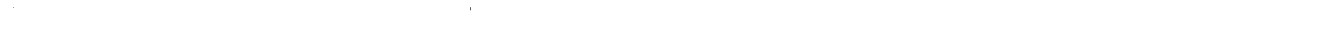
:

喜 8888888888888888888888888888088

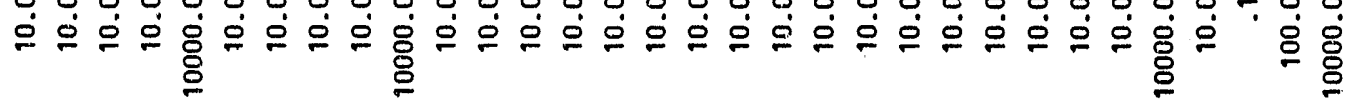

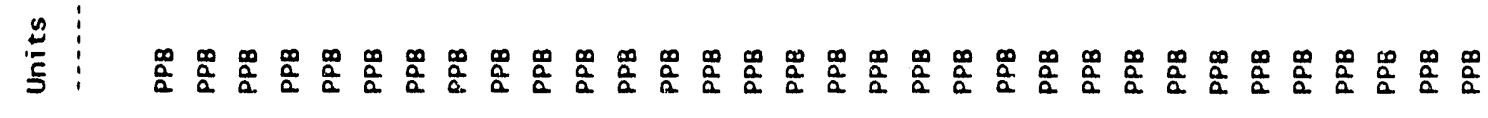
点

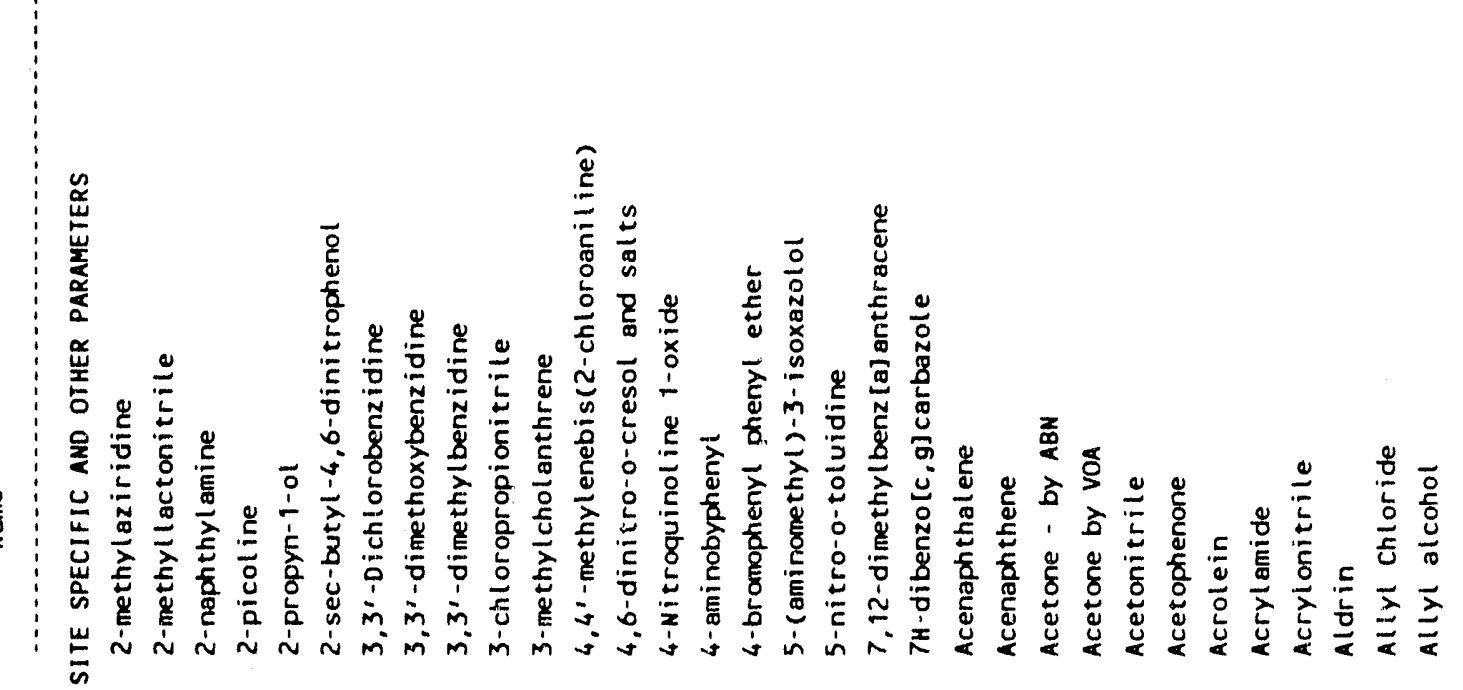


WHC-EP-0366

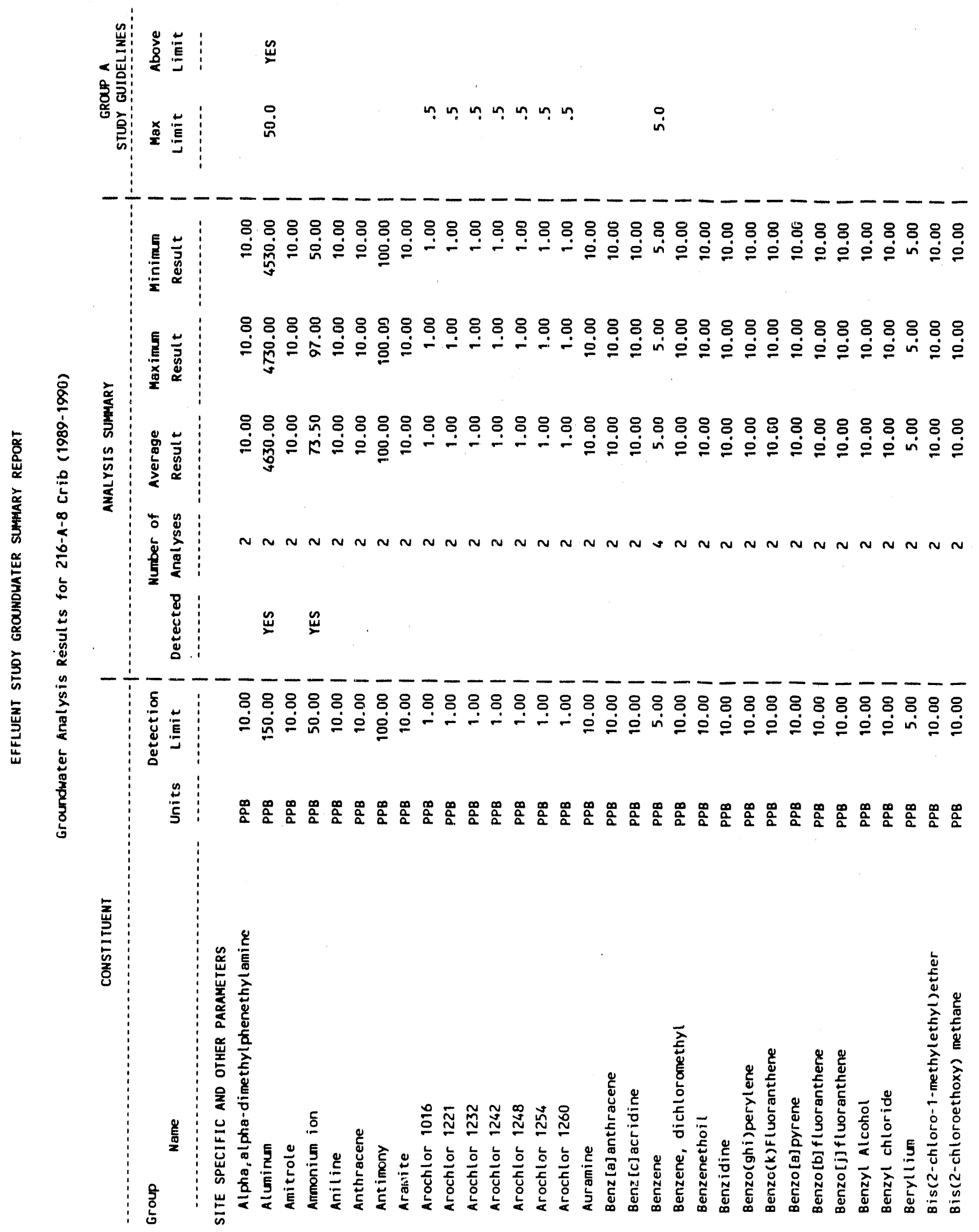


WHC-EP-0366

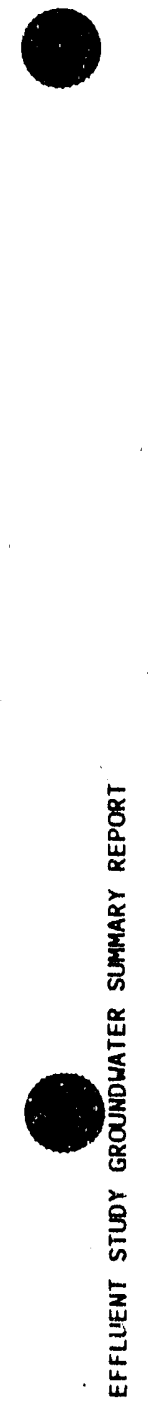

葛

密:

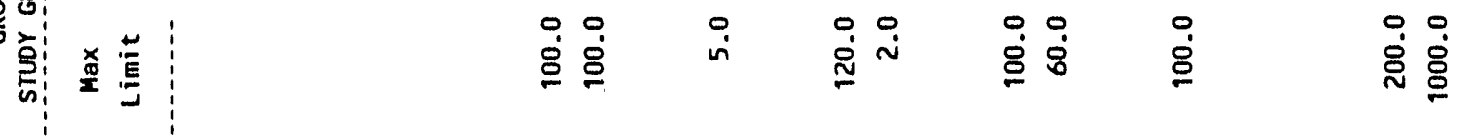

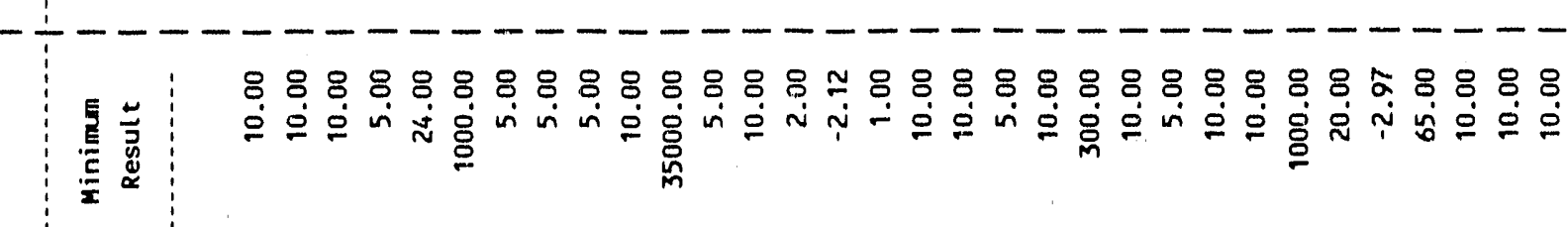

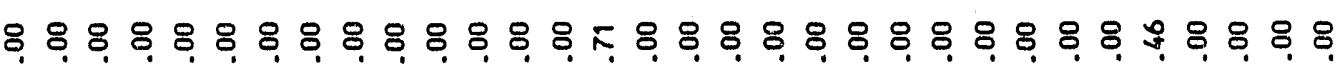

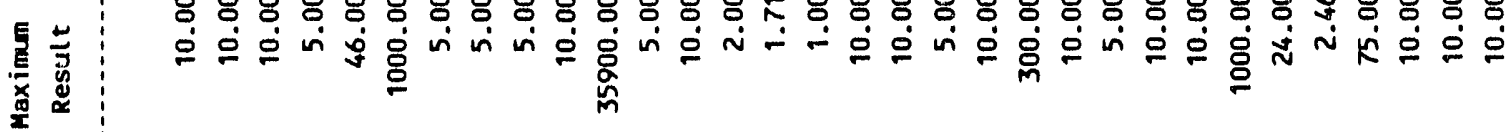

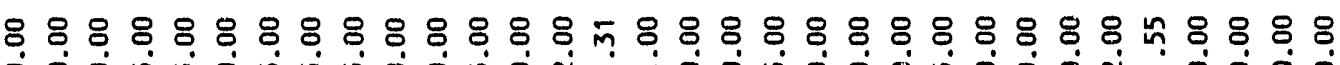

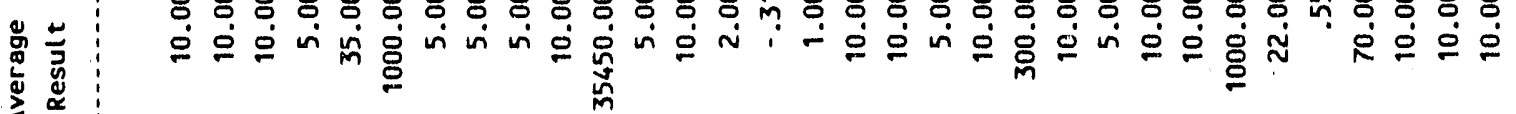

t:

焉寒

\&

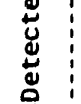

$\stackrel{\mathscr{w}}{\underset{w}{2}}$

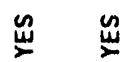

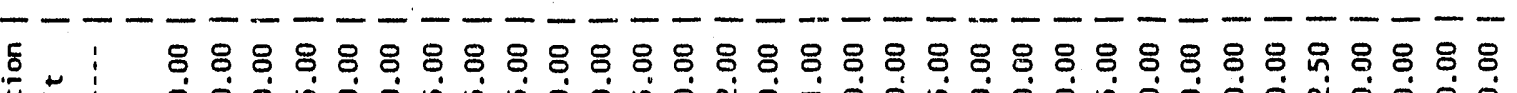

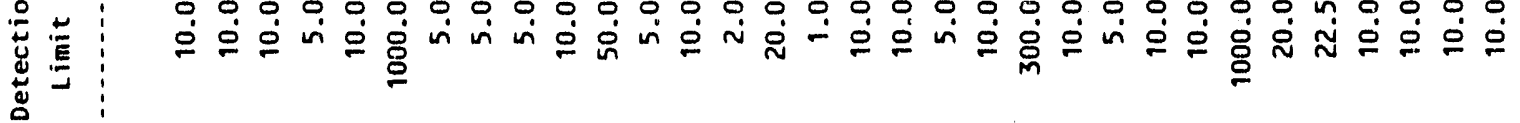

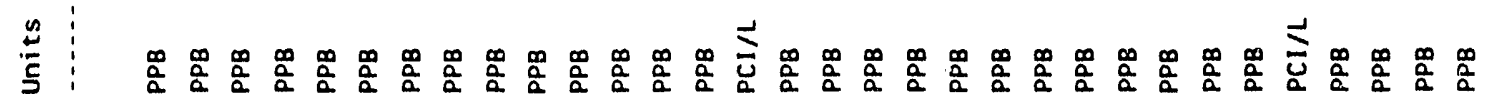

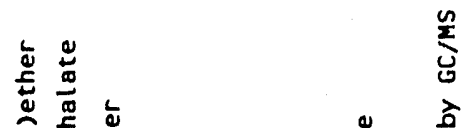

焉焉

웅 है

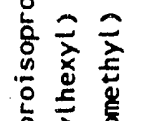

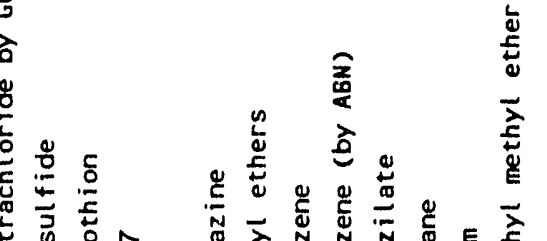

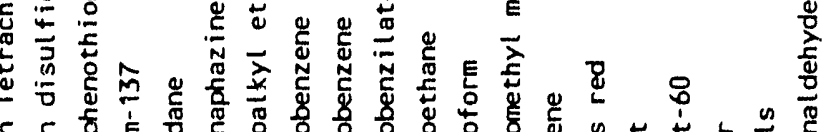


WHC-EP-0366

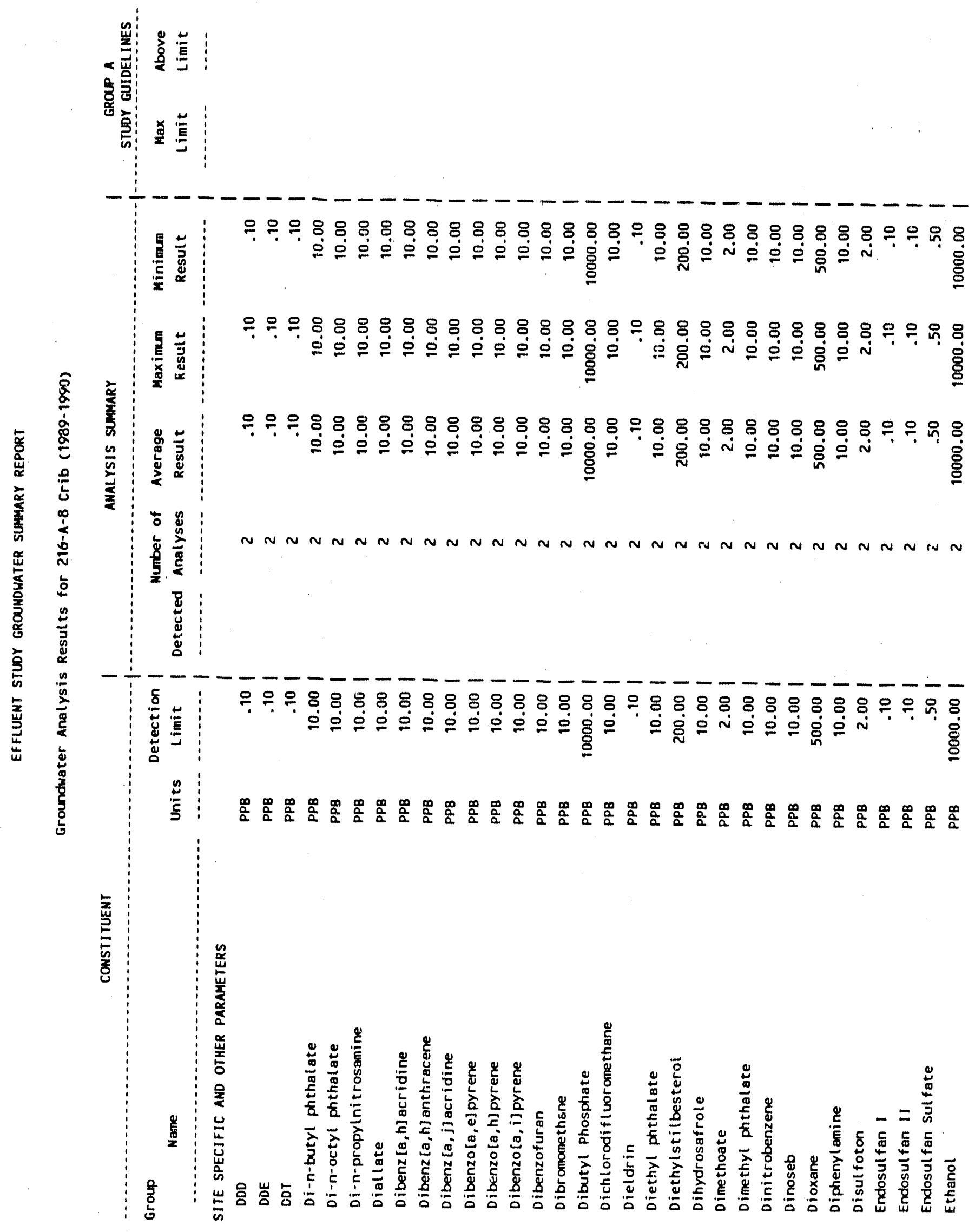


WHC - EP-0366

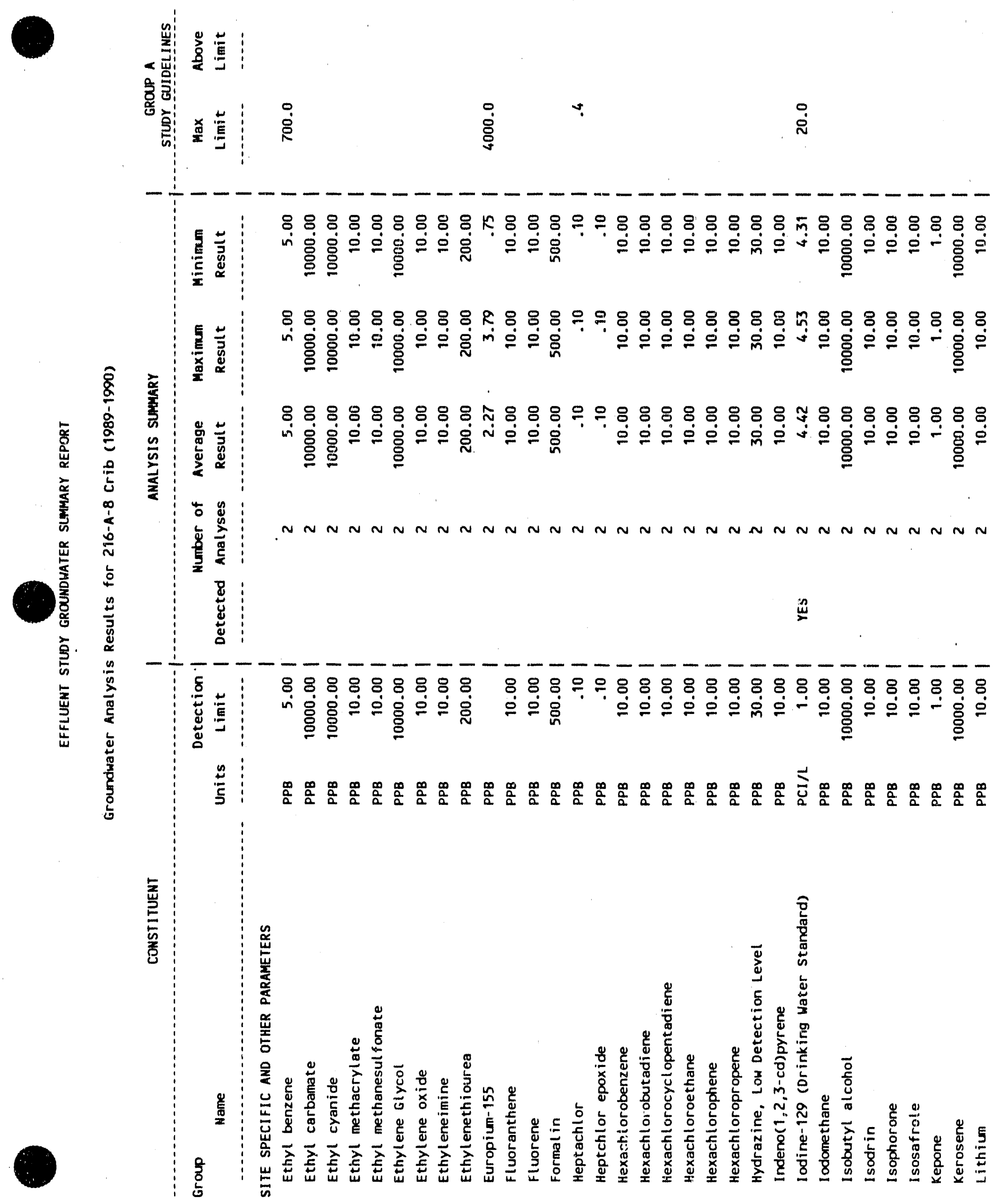


WHC-EP-0366

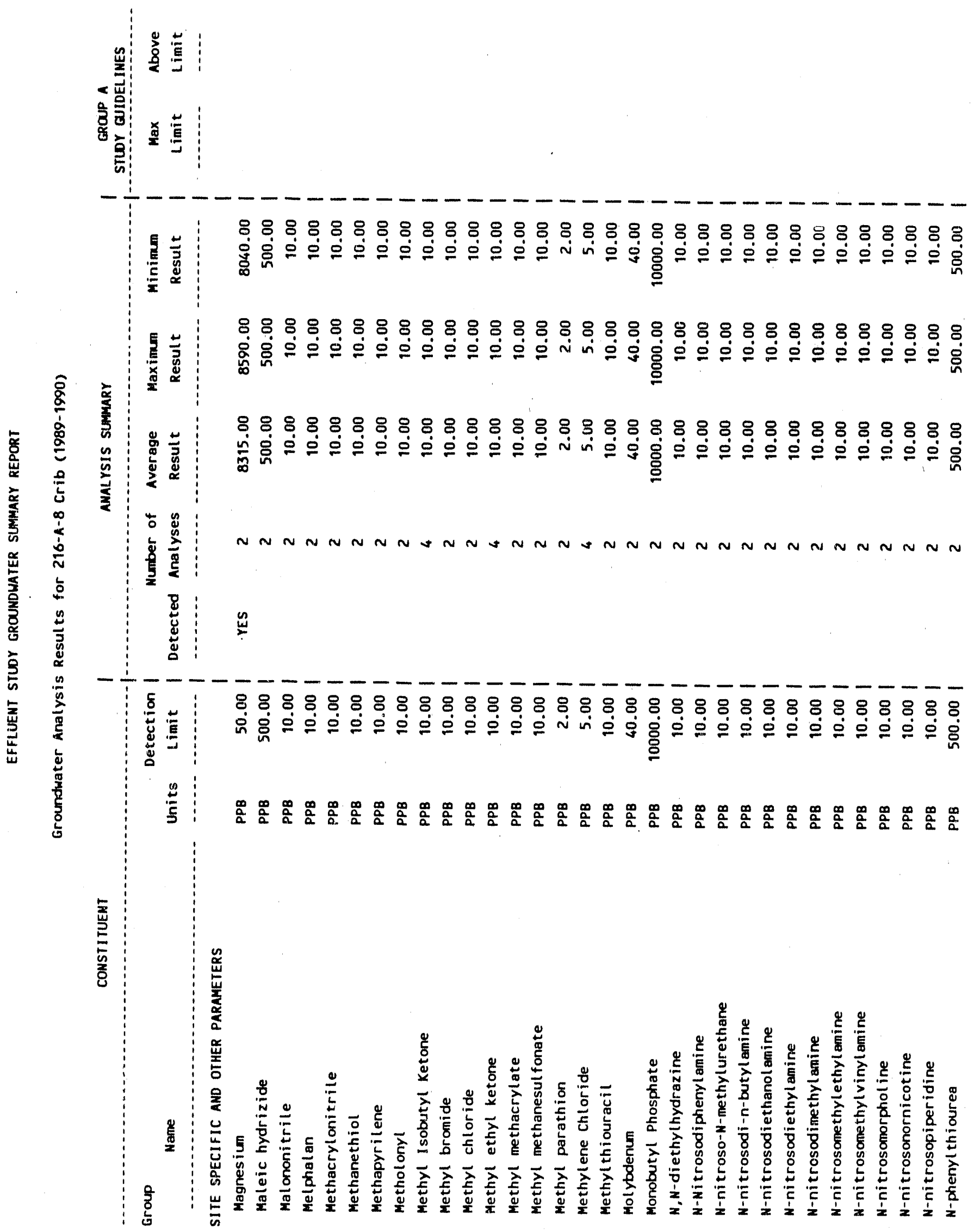


WHC-EP-0366

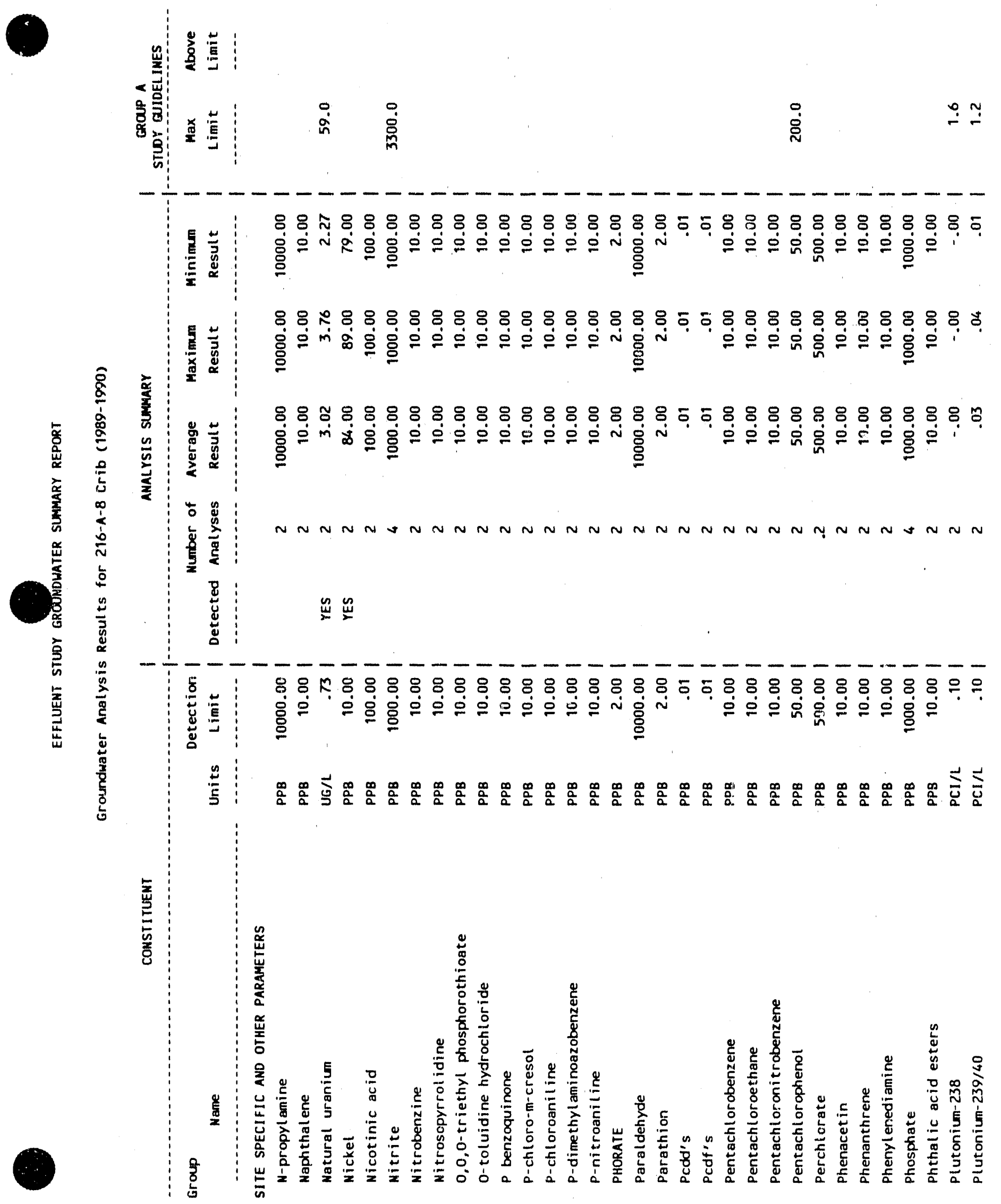


WHC-EP-0366

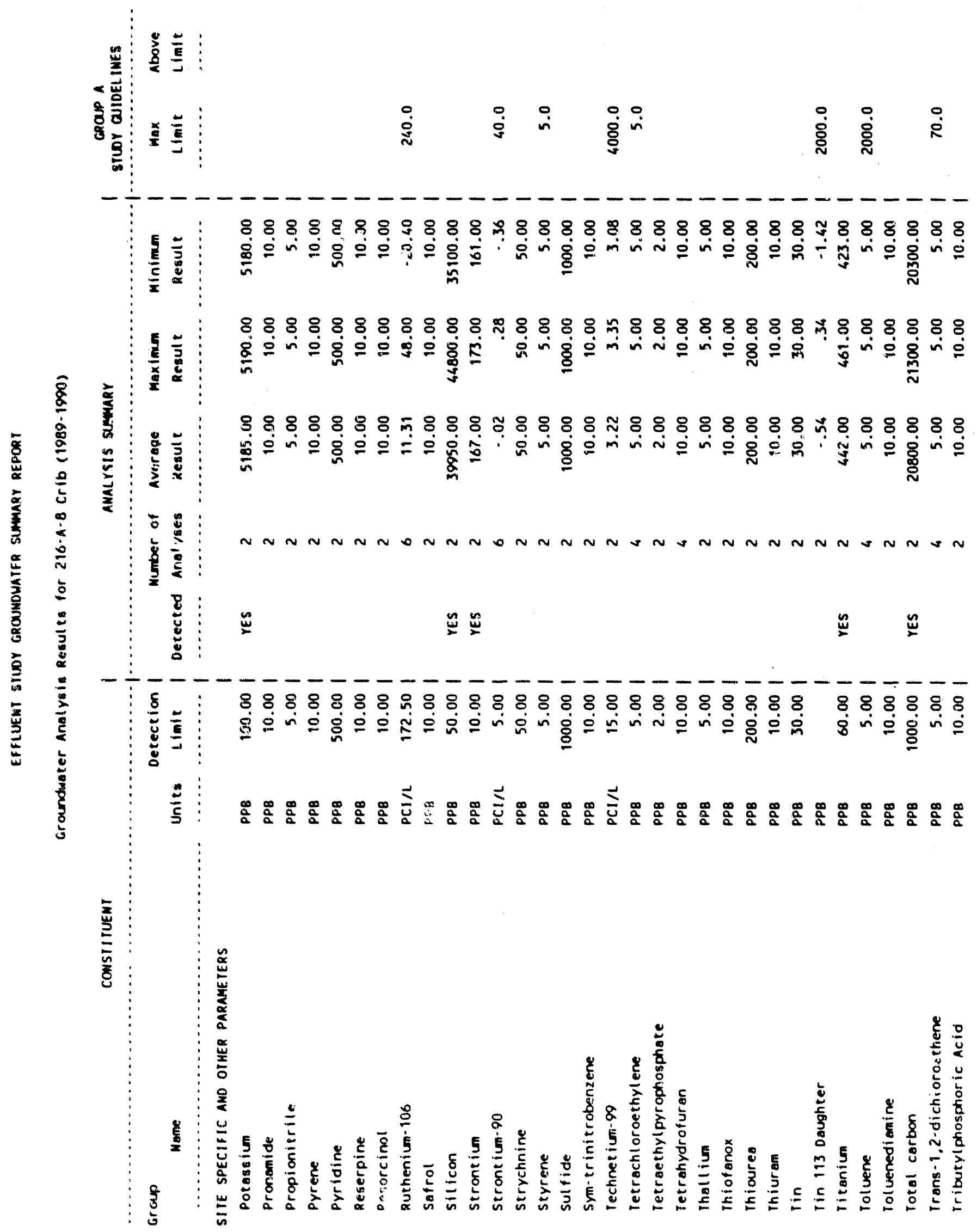


WHC - EP-0366

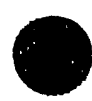

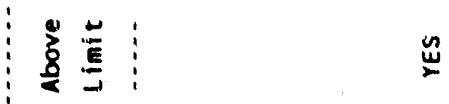

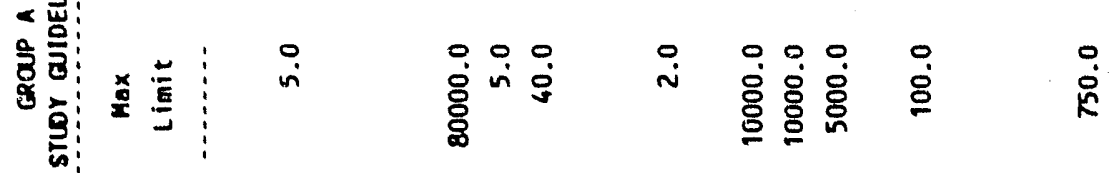

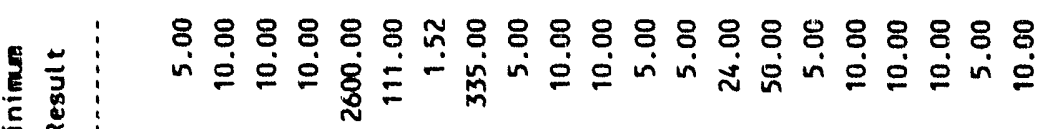

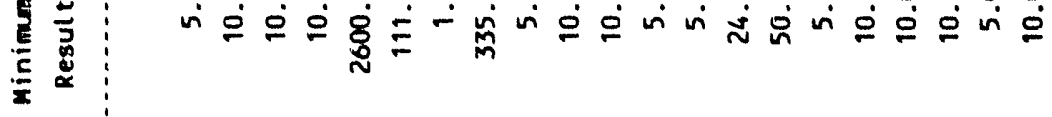

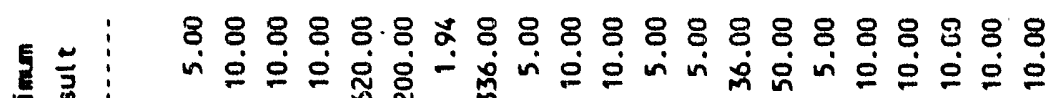

들

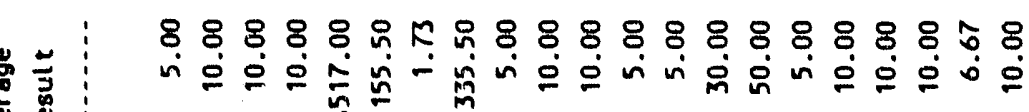

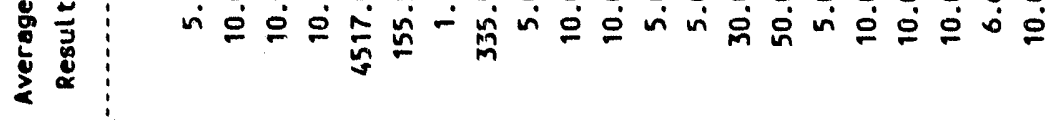

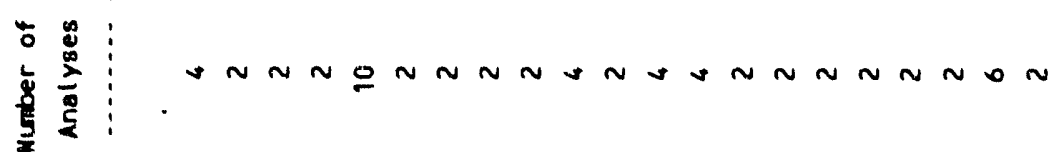

递:

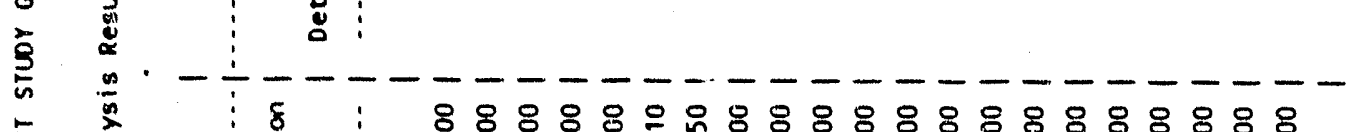

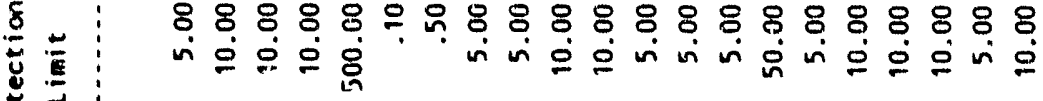

:

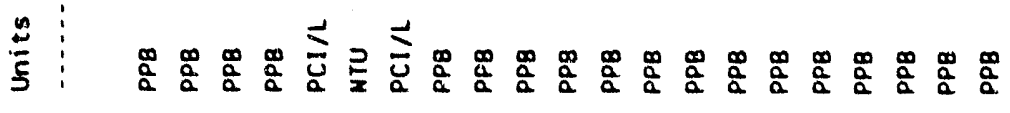

密

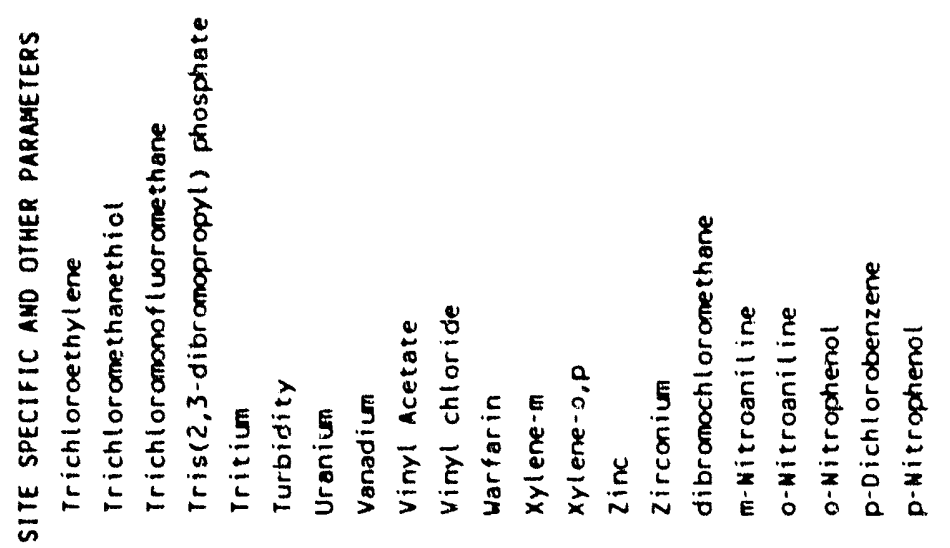




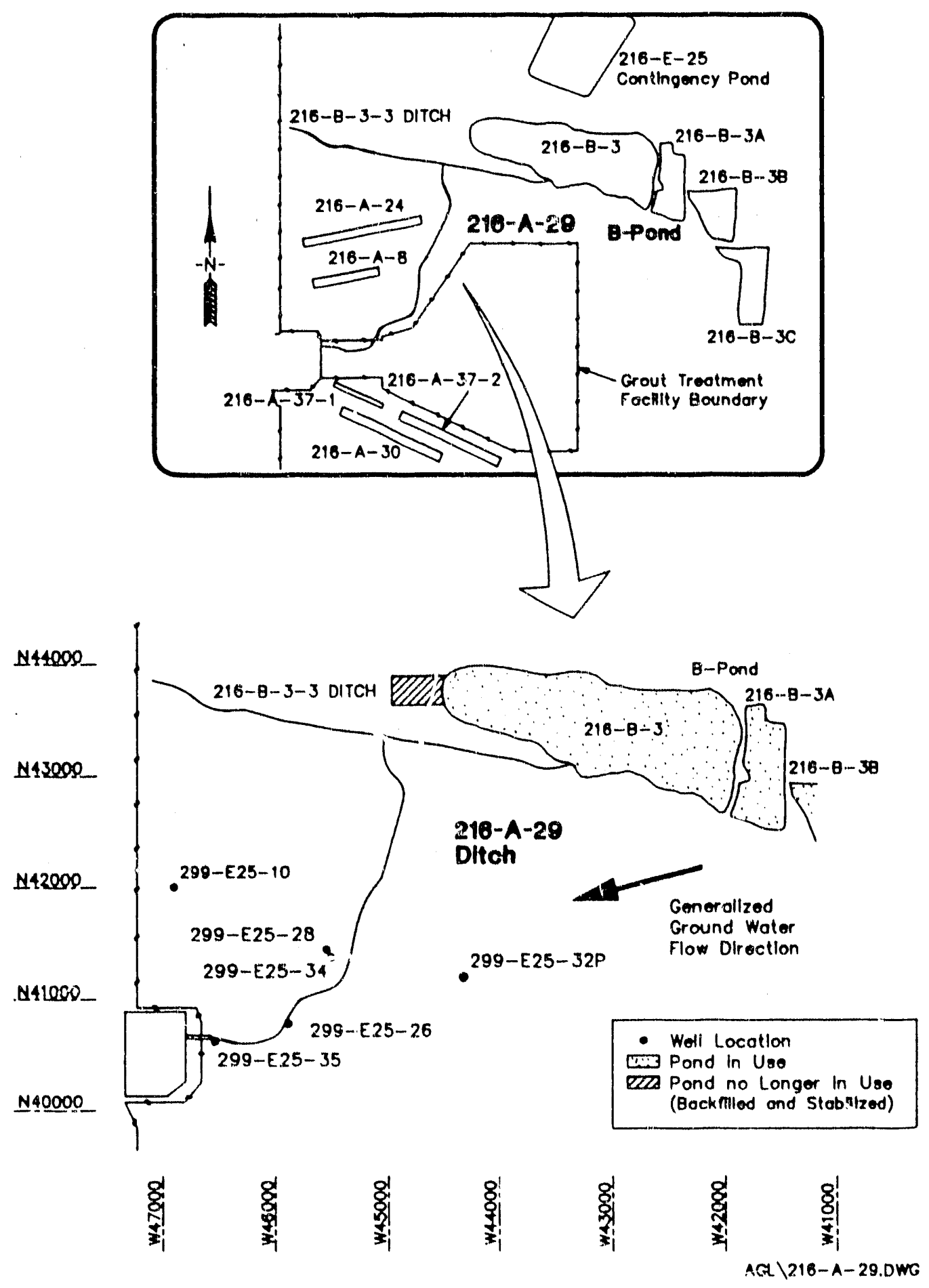

Well Location and Site Map for 216-A-29 Ditch 


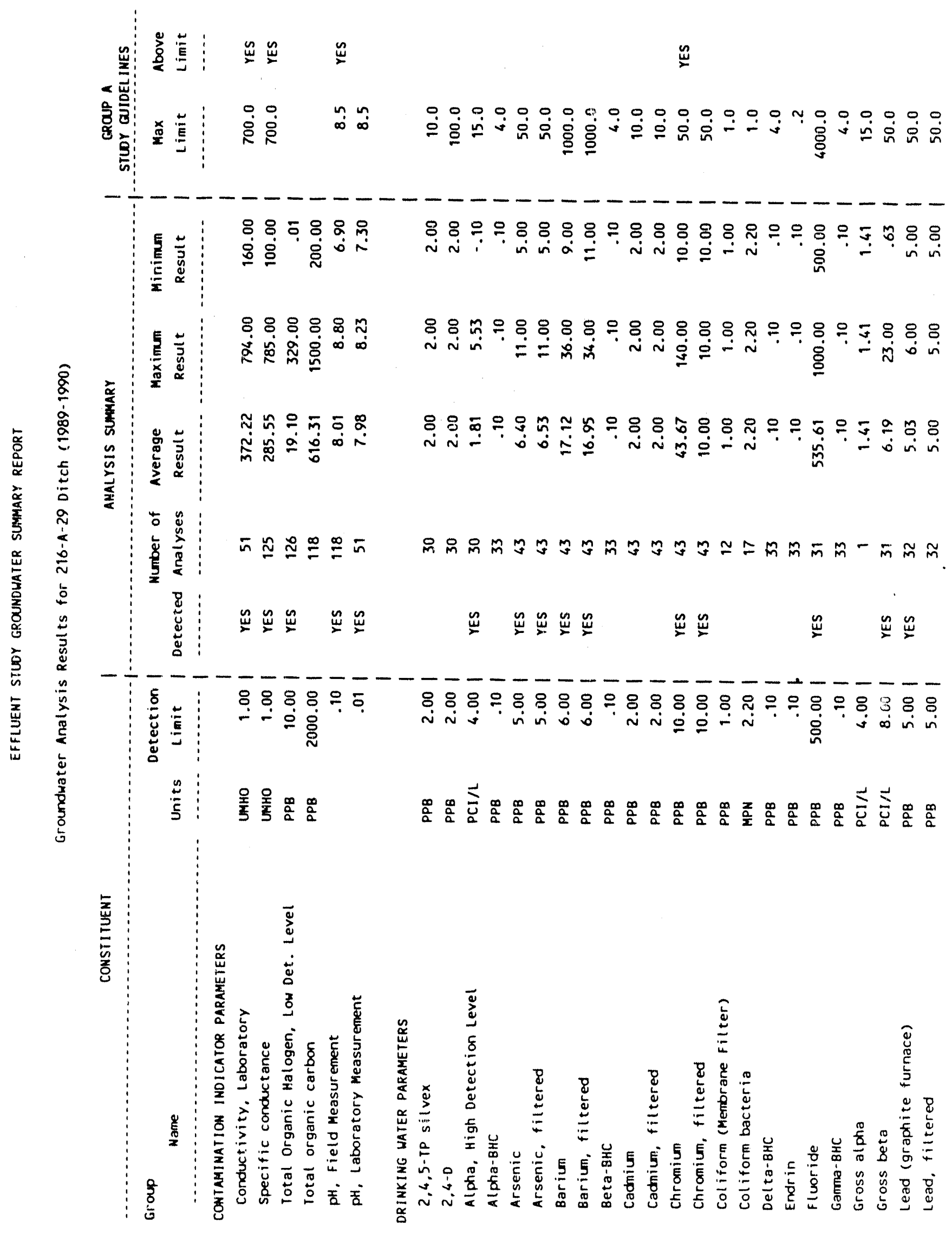




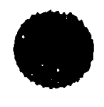

等言

$\stackrel{\dddot{w}}{\check{W}}$

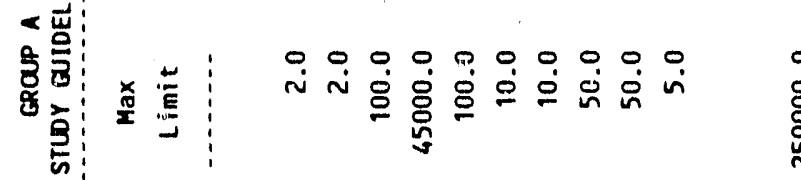

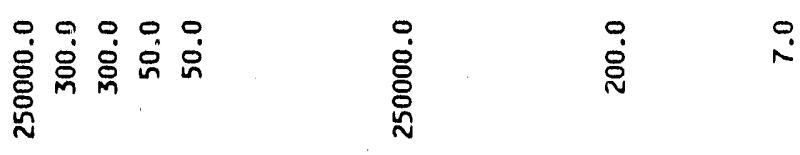

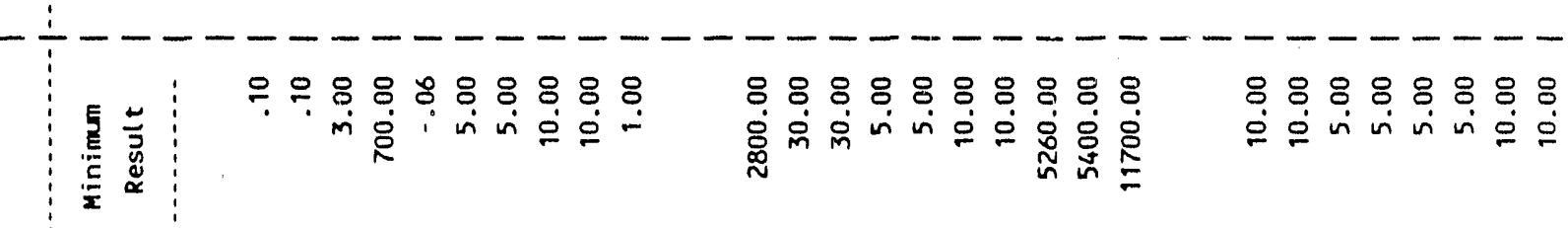
F $\quad$ 웅유 兽

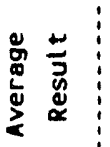

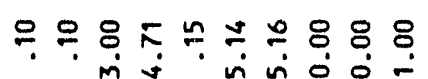

ธำ

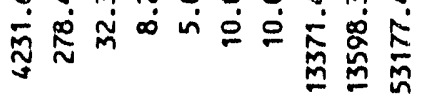

8.88 .88 .8

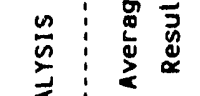

峦 的的过

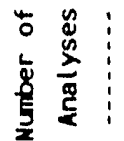

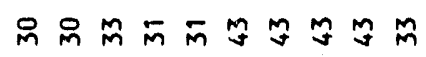

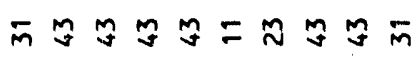

ก

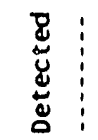

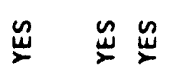

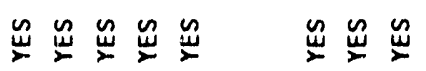

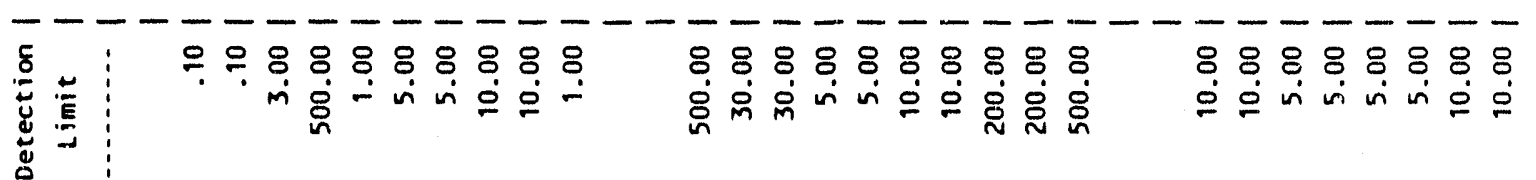

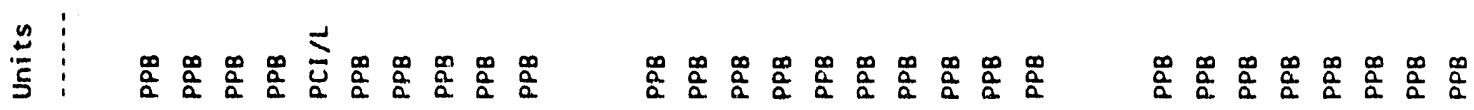
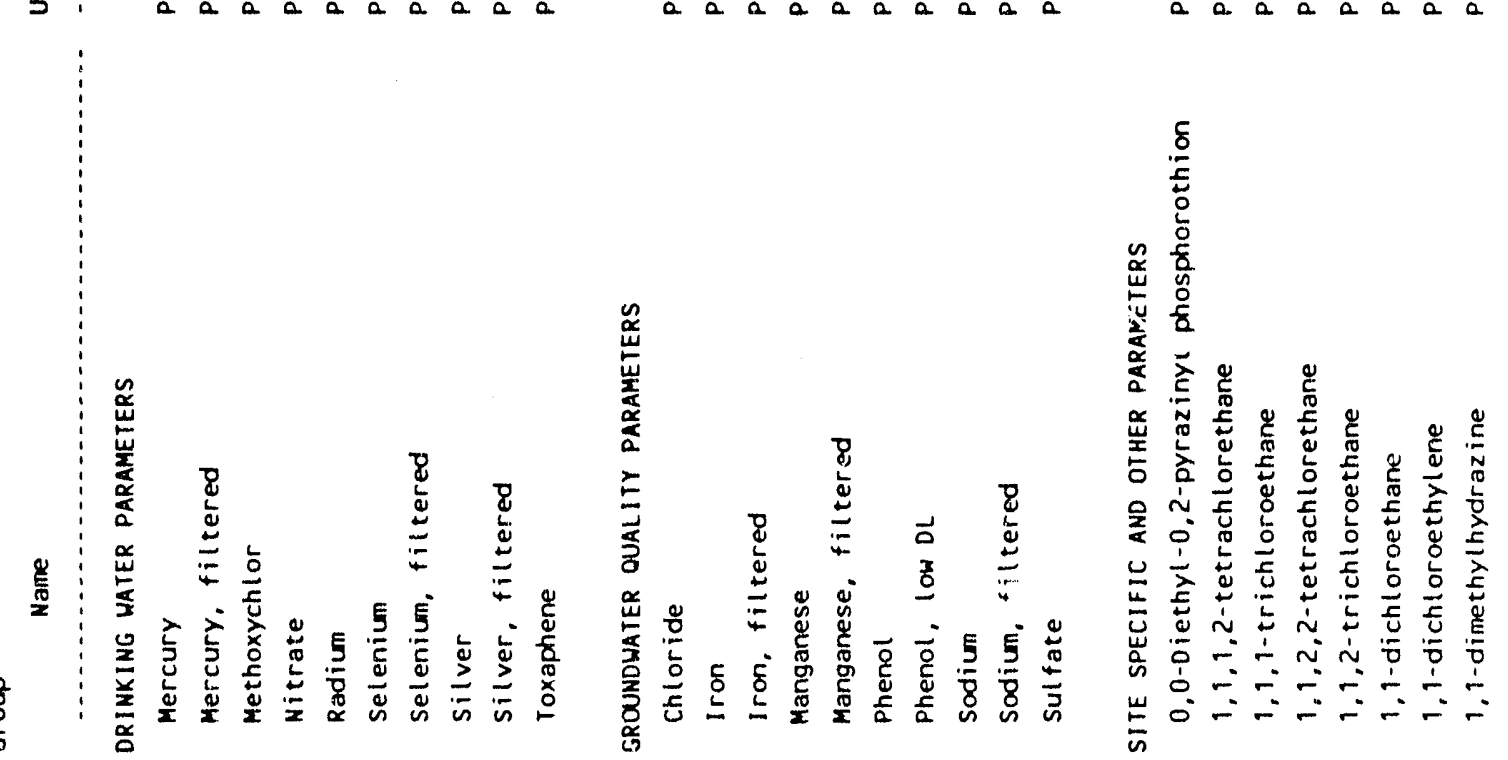
WHC -EP-0366

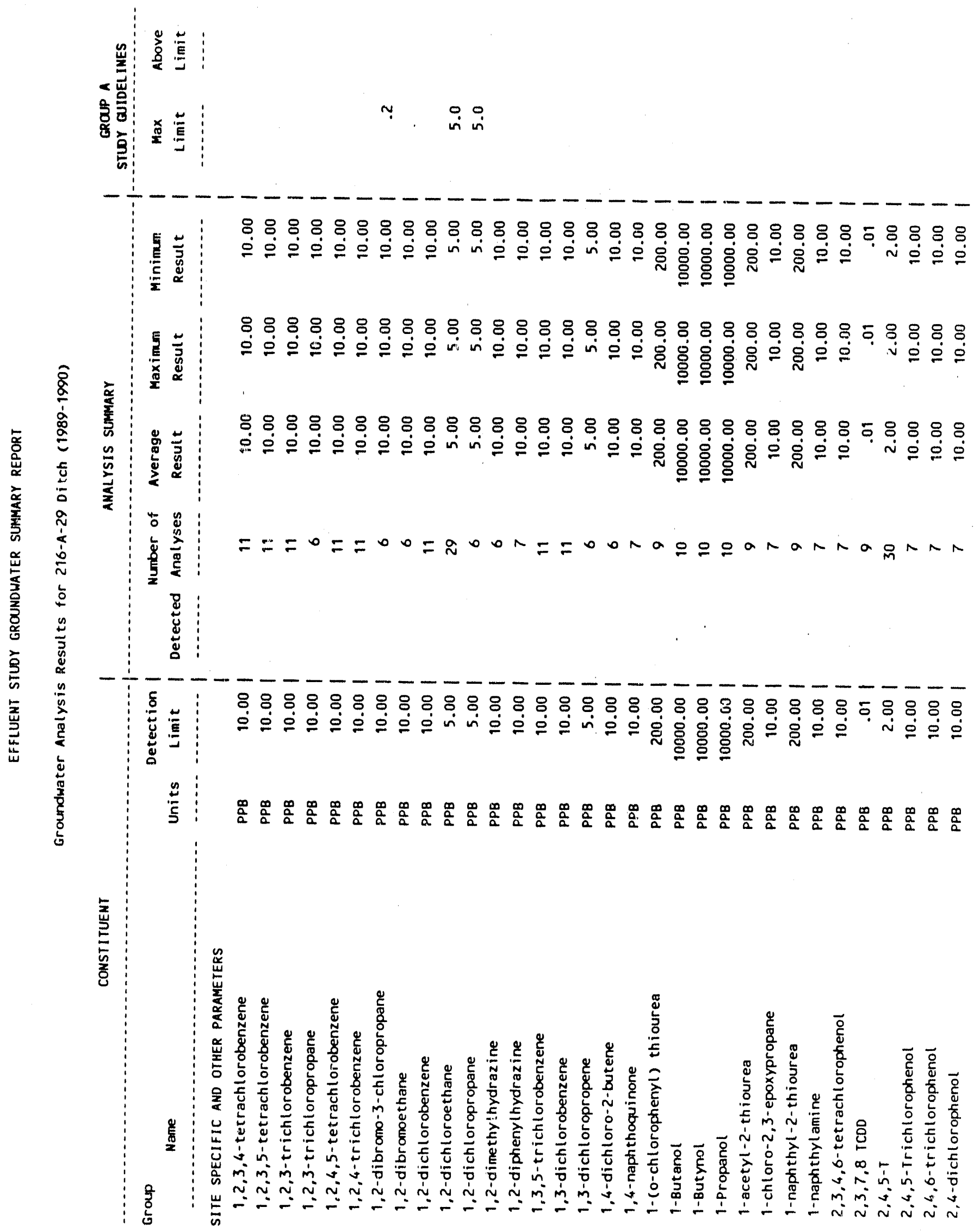




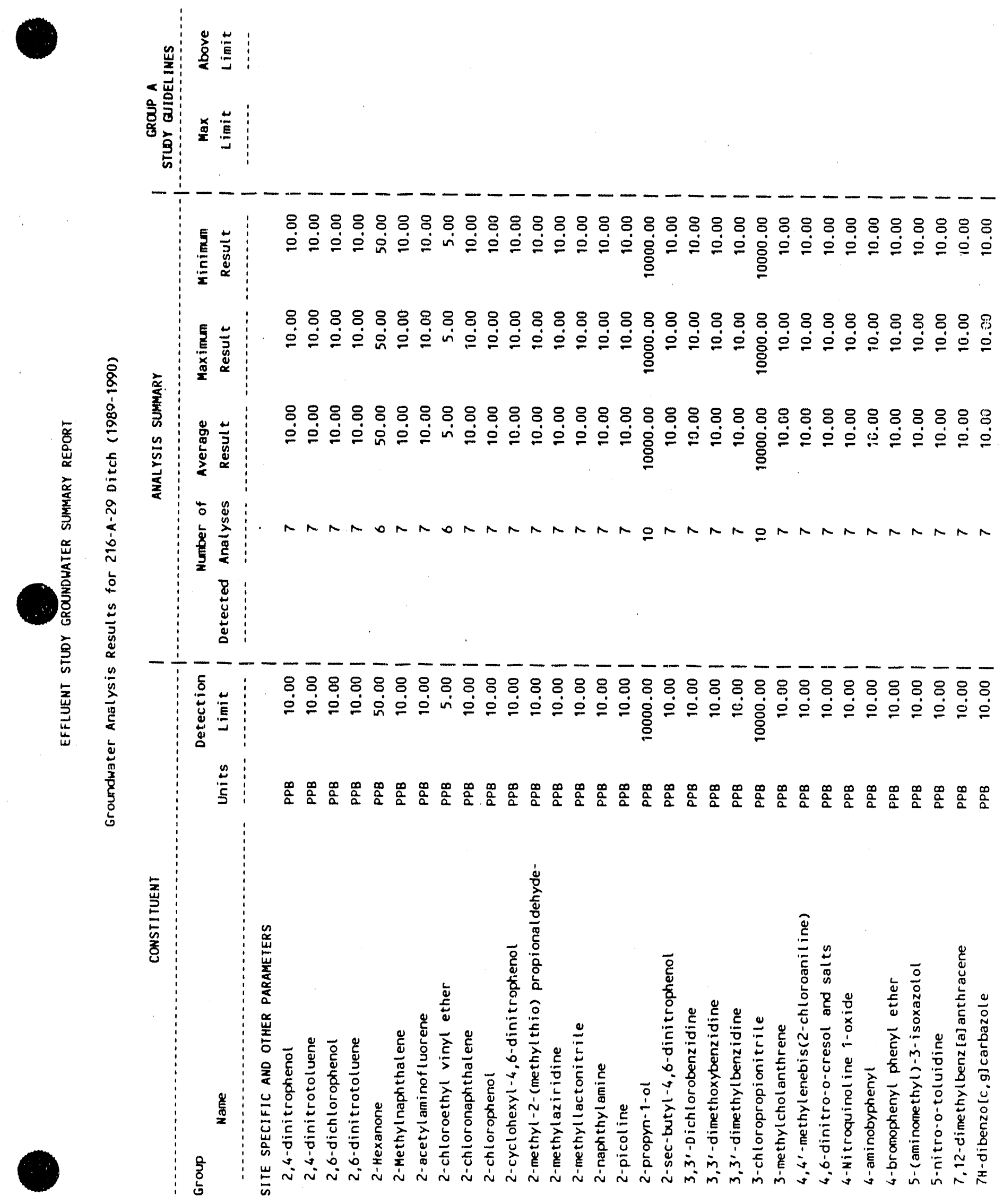


WHC-EP-0366
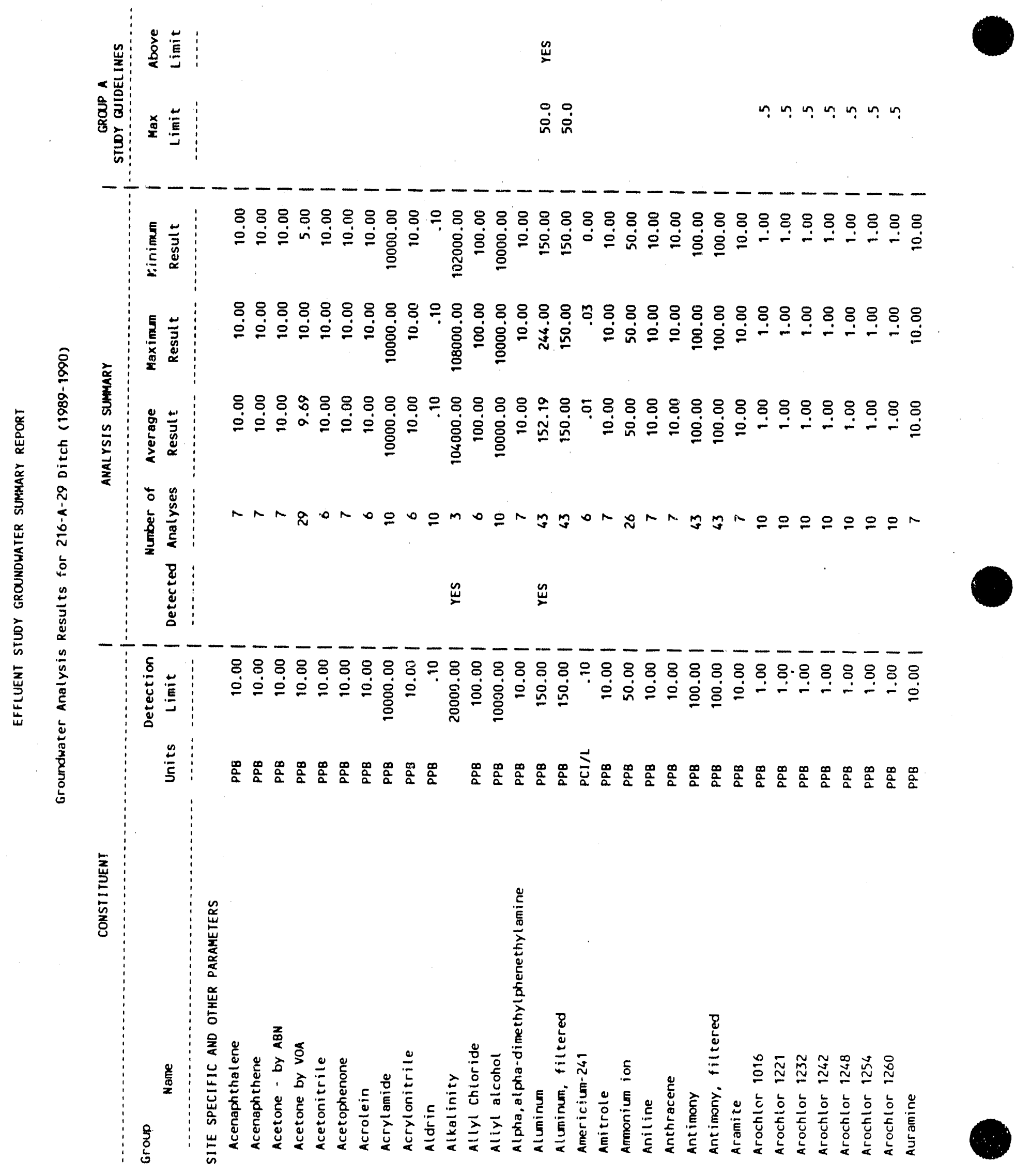
WHC -EP-0366

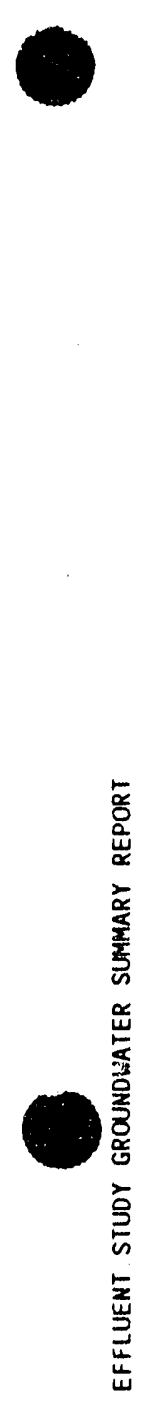

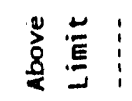

:

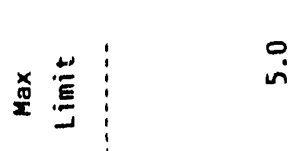

$\ddot{\dot{g}} \dot{\dot{g}}$

$\stackrel{\circ}{n}$

骨喜

8888888888888888888888888888888

○்

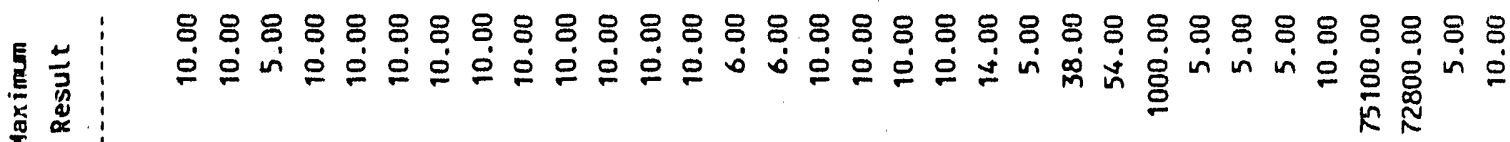

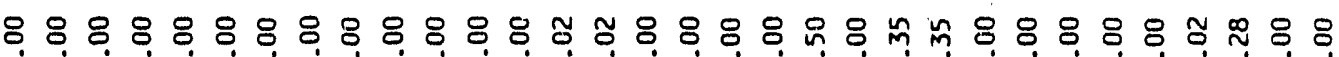

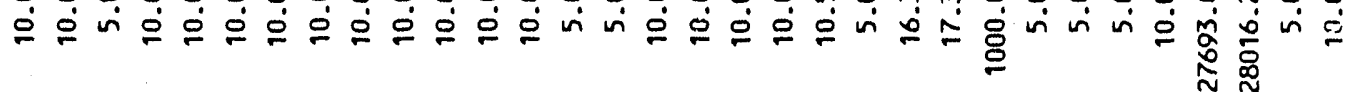

离泀

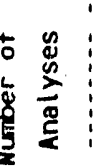

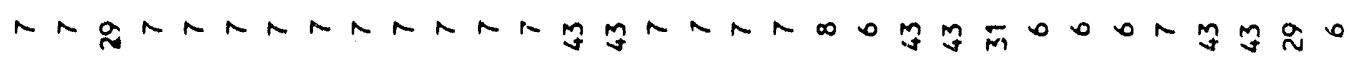

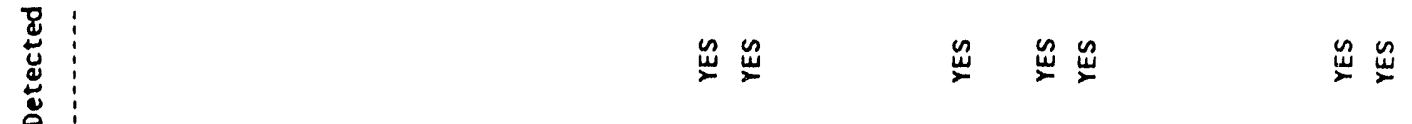

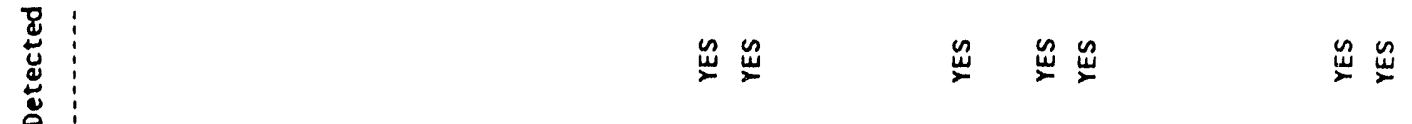

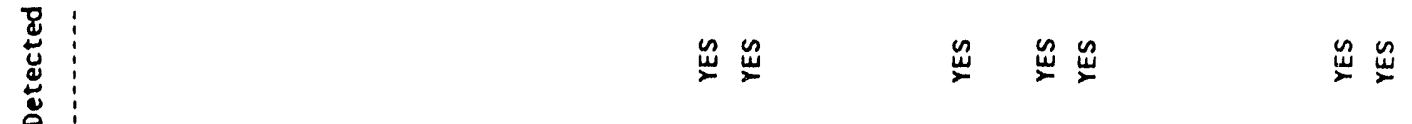
б 苞

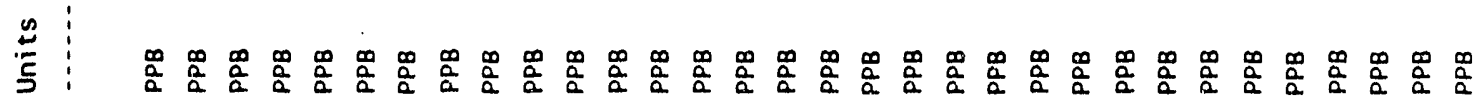
釆

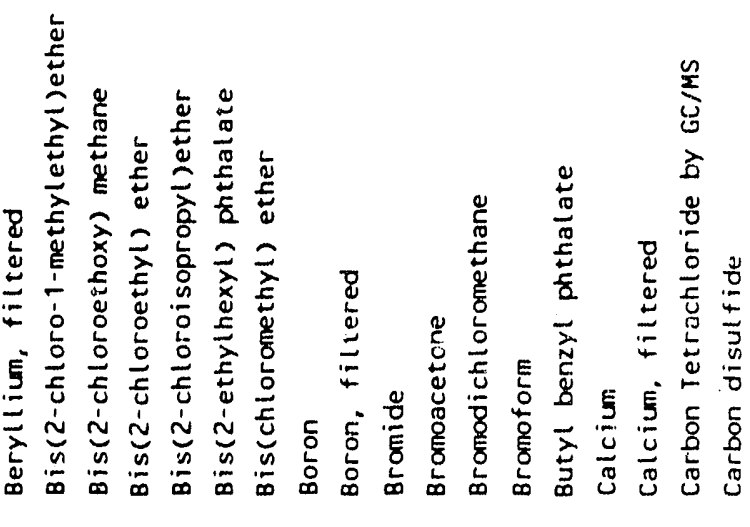


WHC-EP-0366
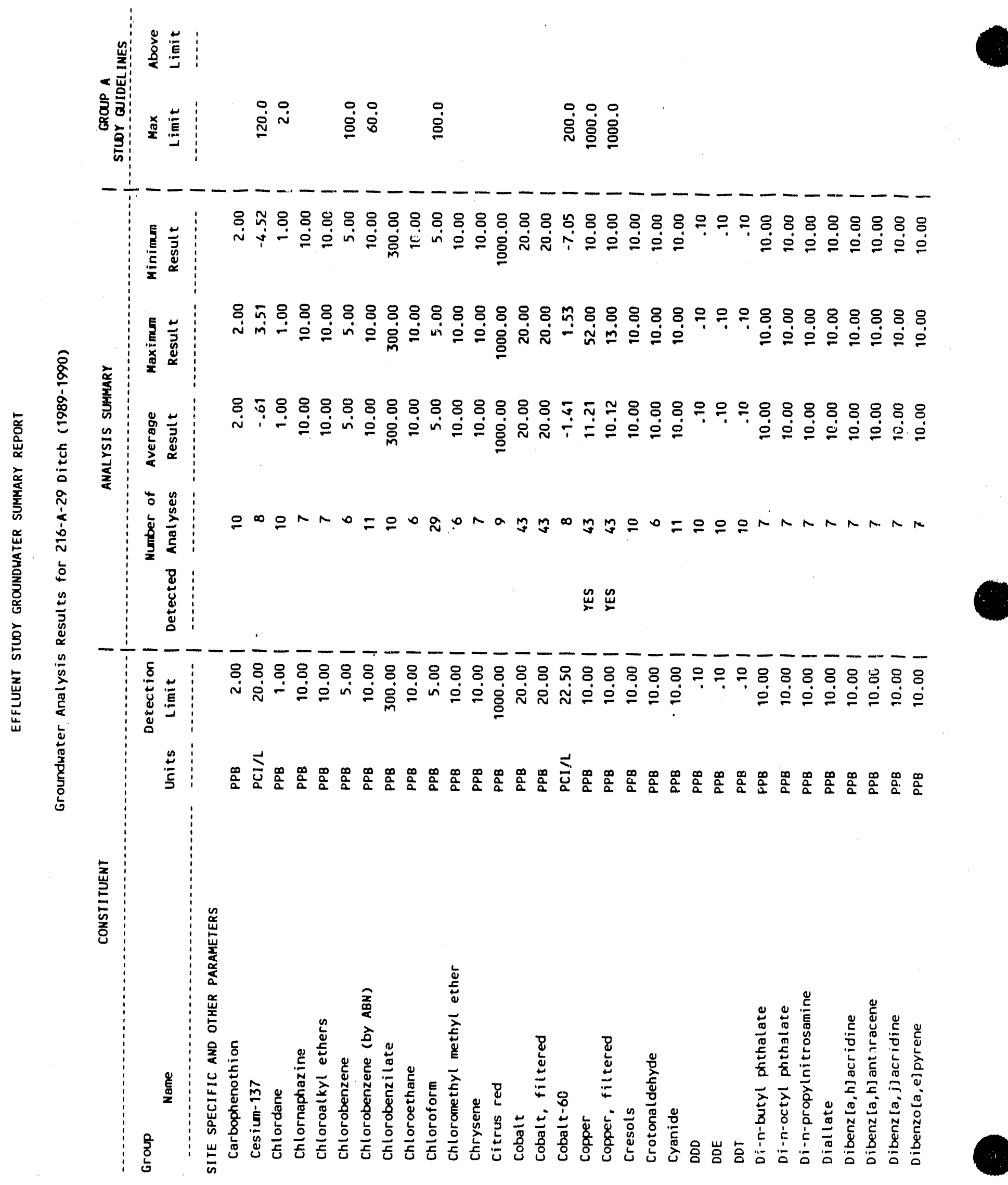
WHC-EP-0366

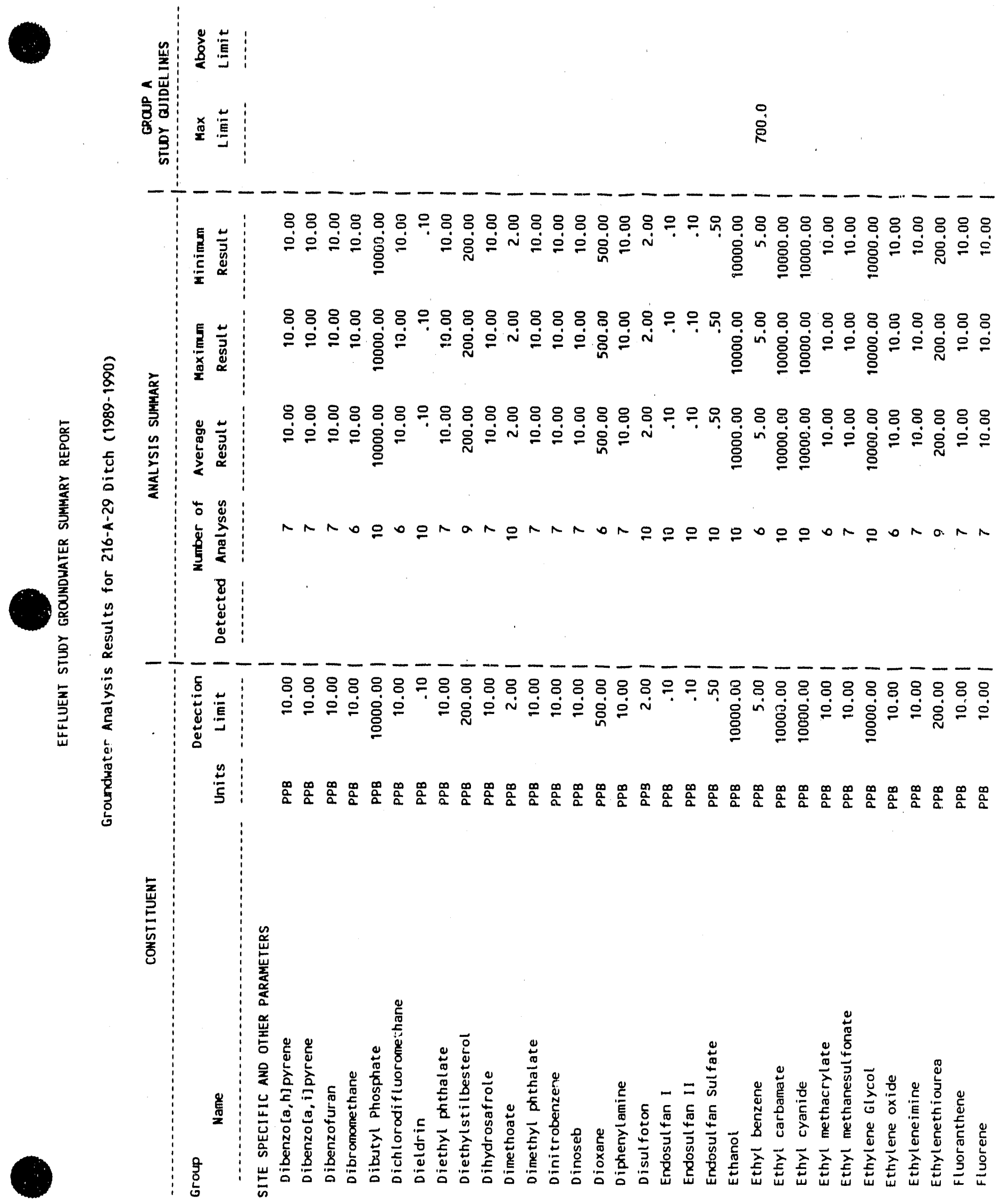


WHC-EP-0366

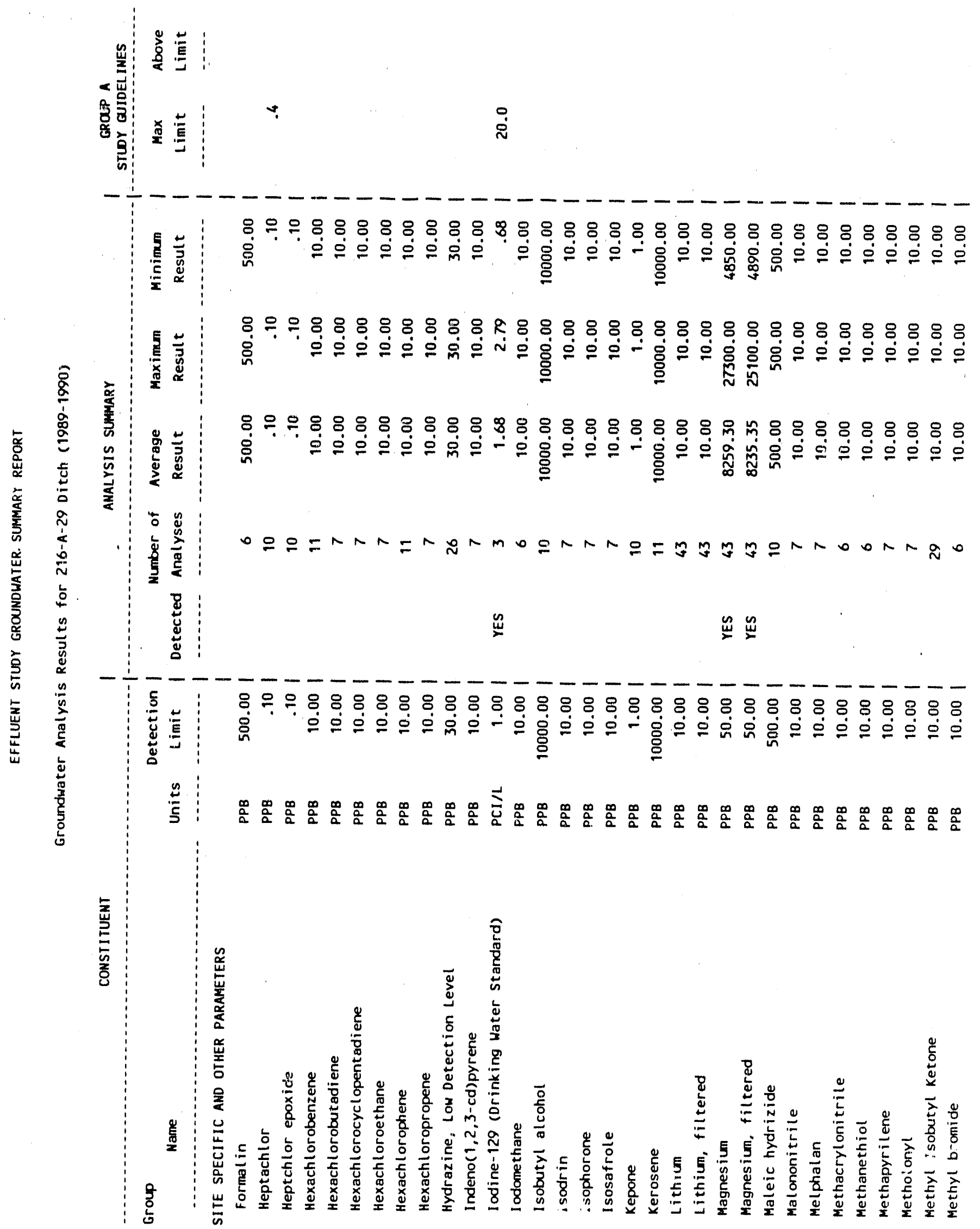



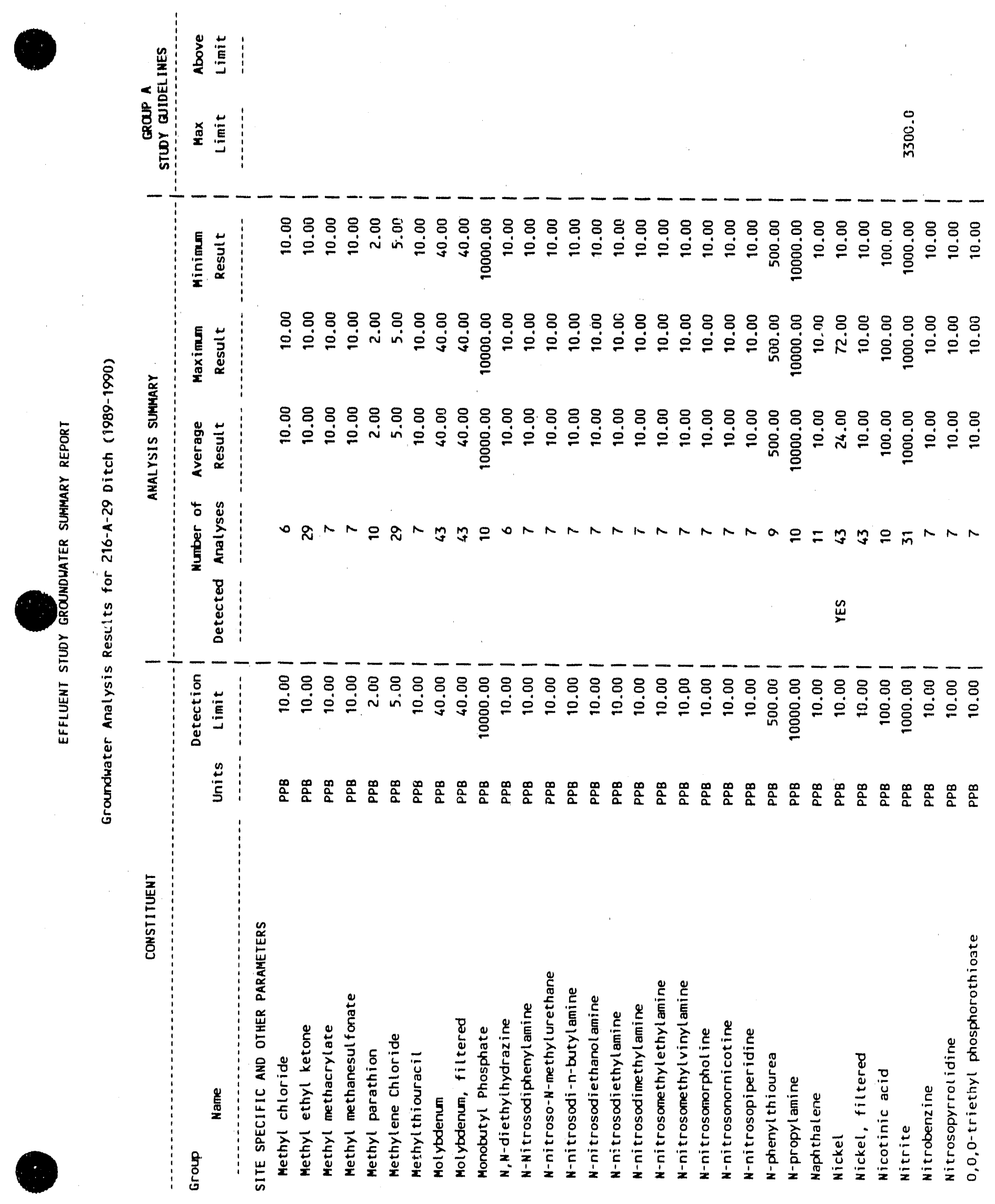
WHC -EP-0366

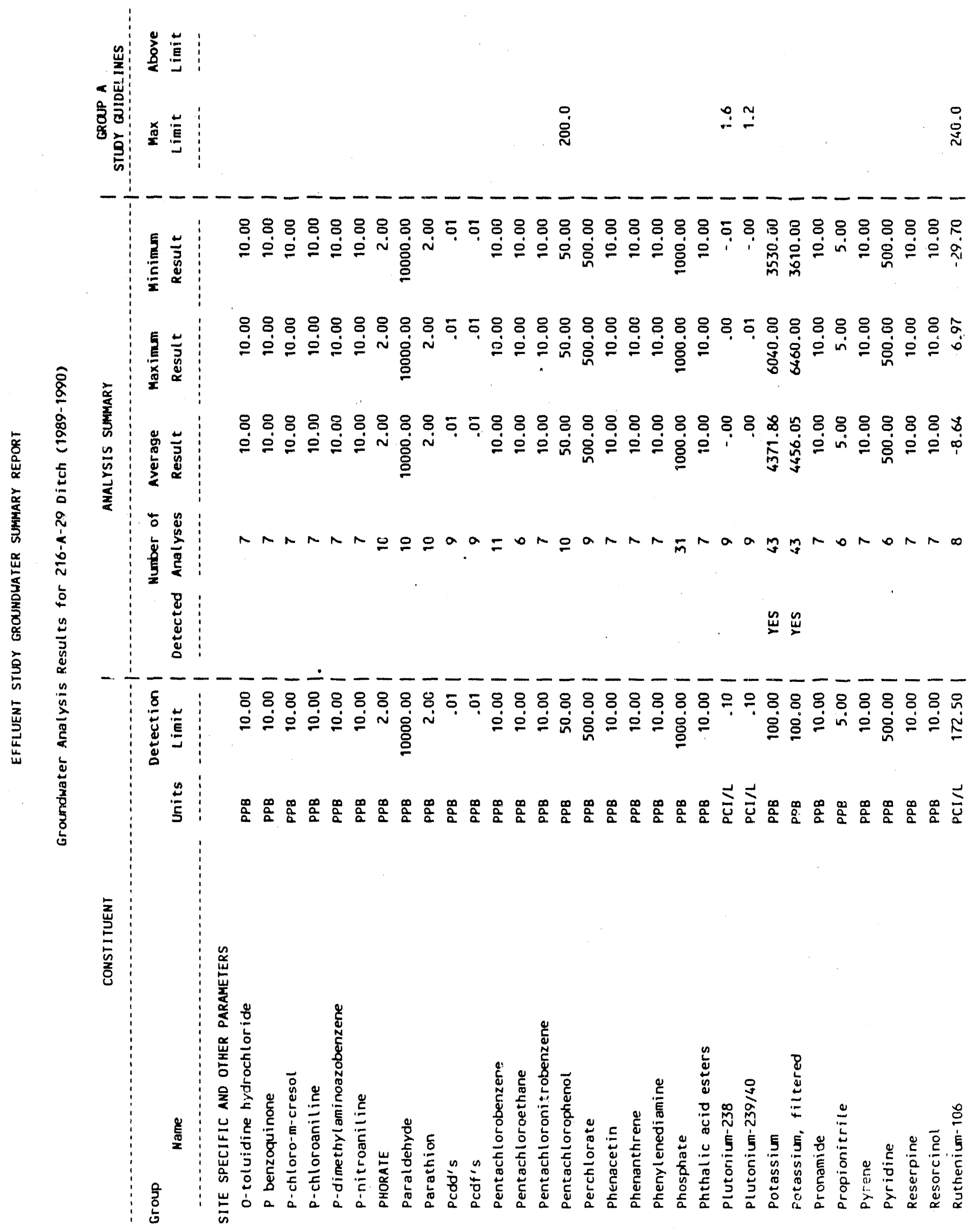




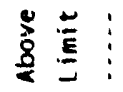

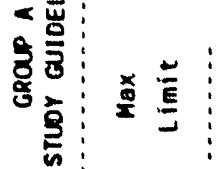

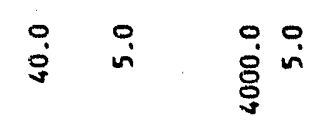

号

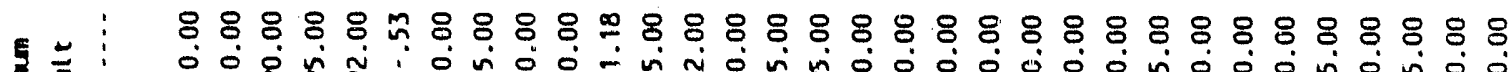

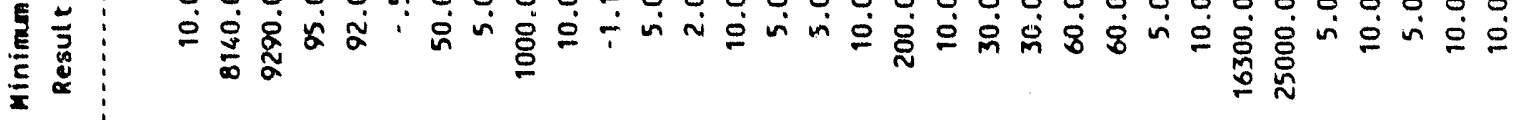

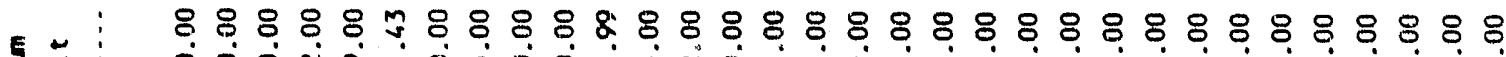

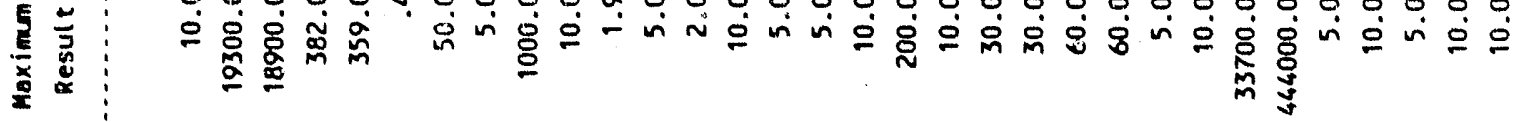

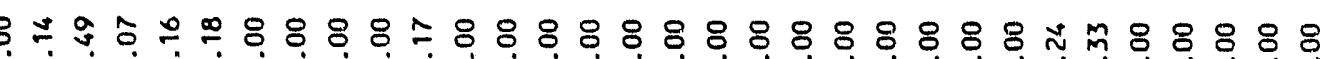

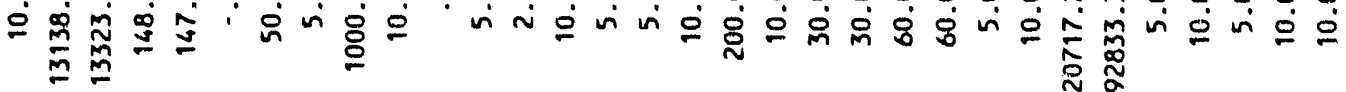

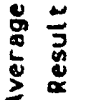

is:

急

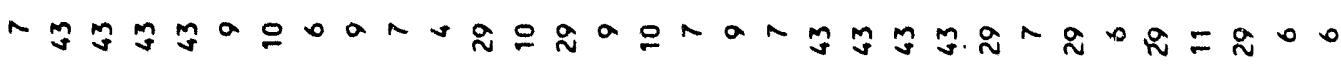

宽:

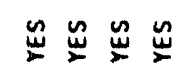

$\stackrel{n}{2} \underset{2}{2}$

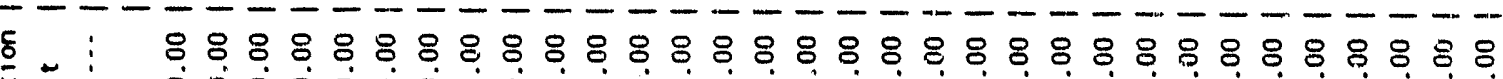

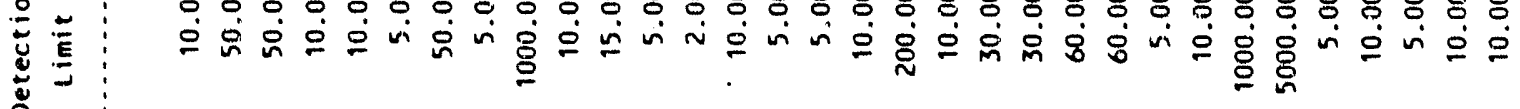

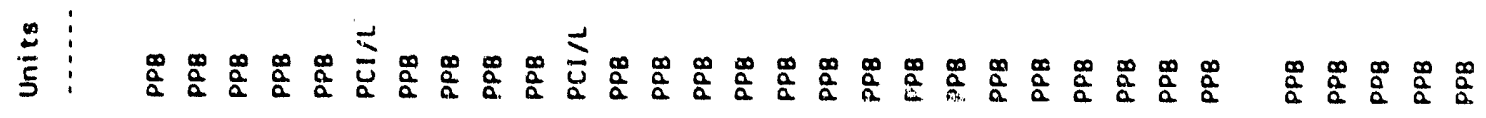

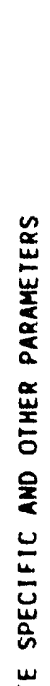

:
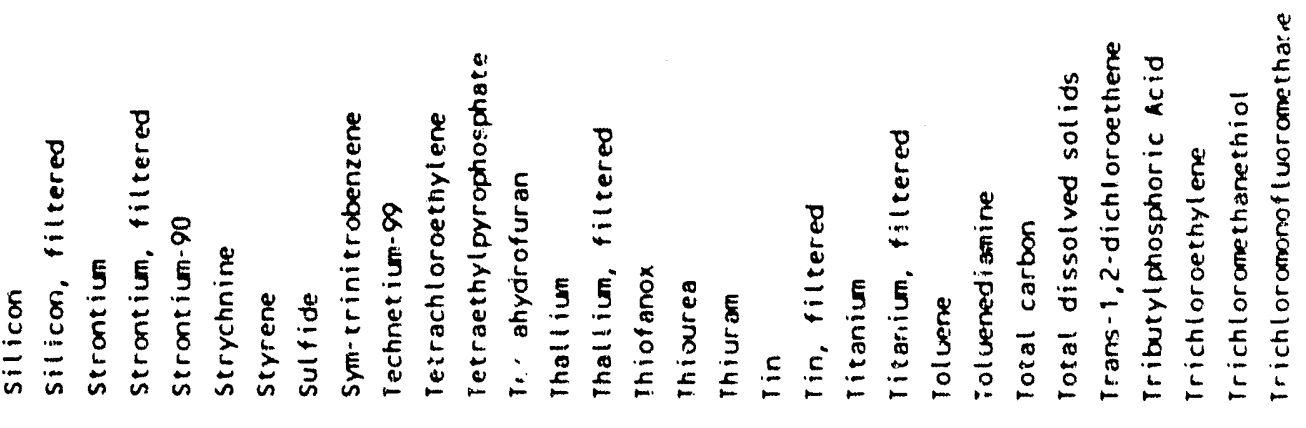
WHC-EP-0366

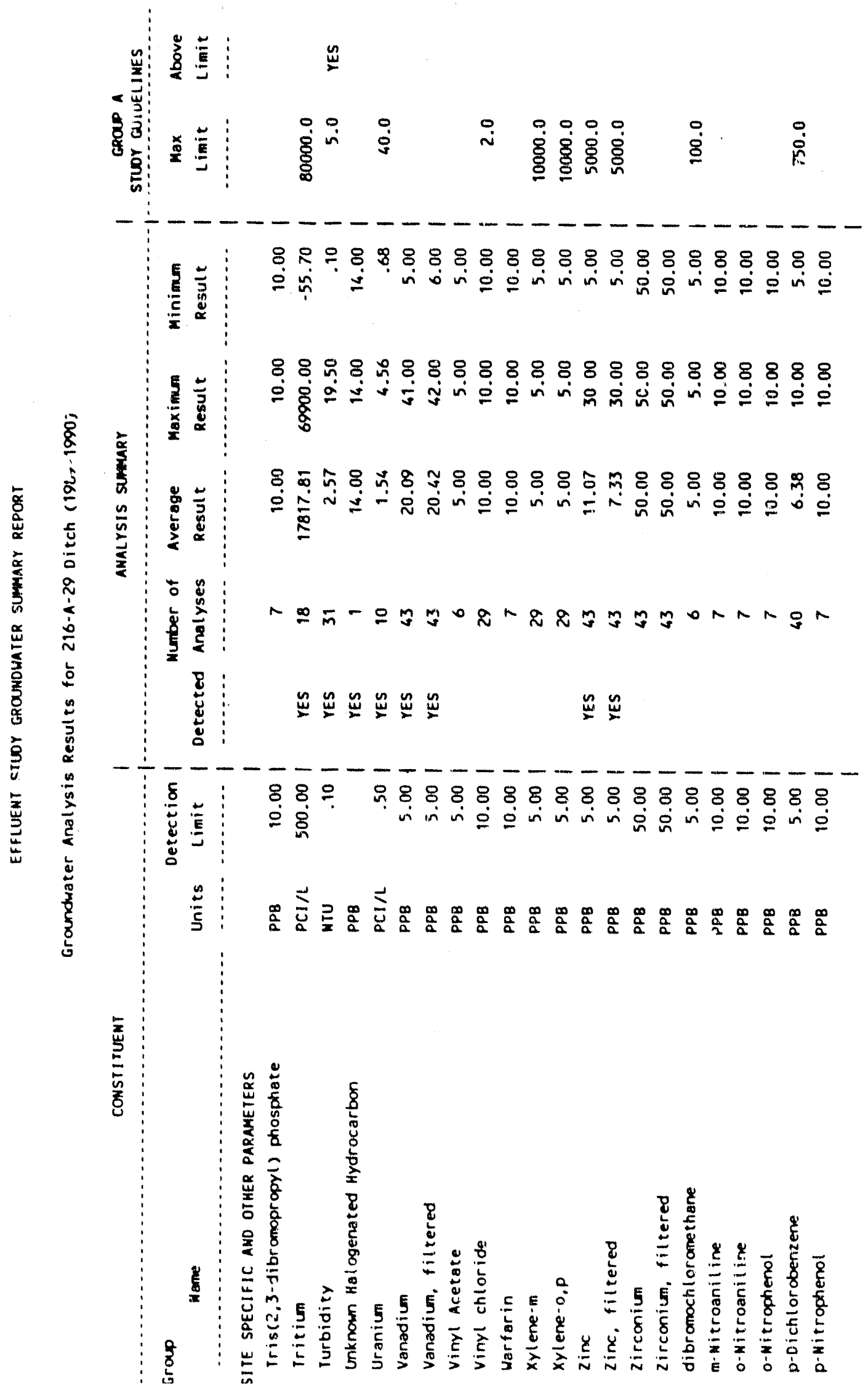




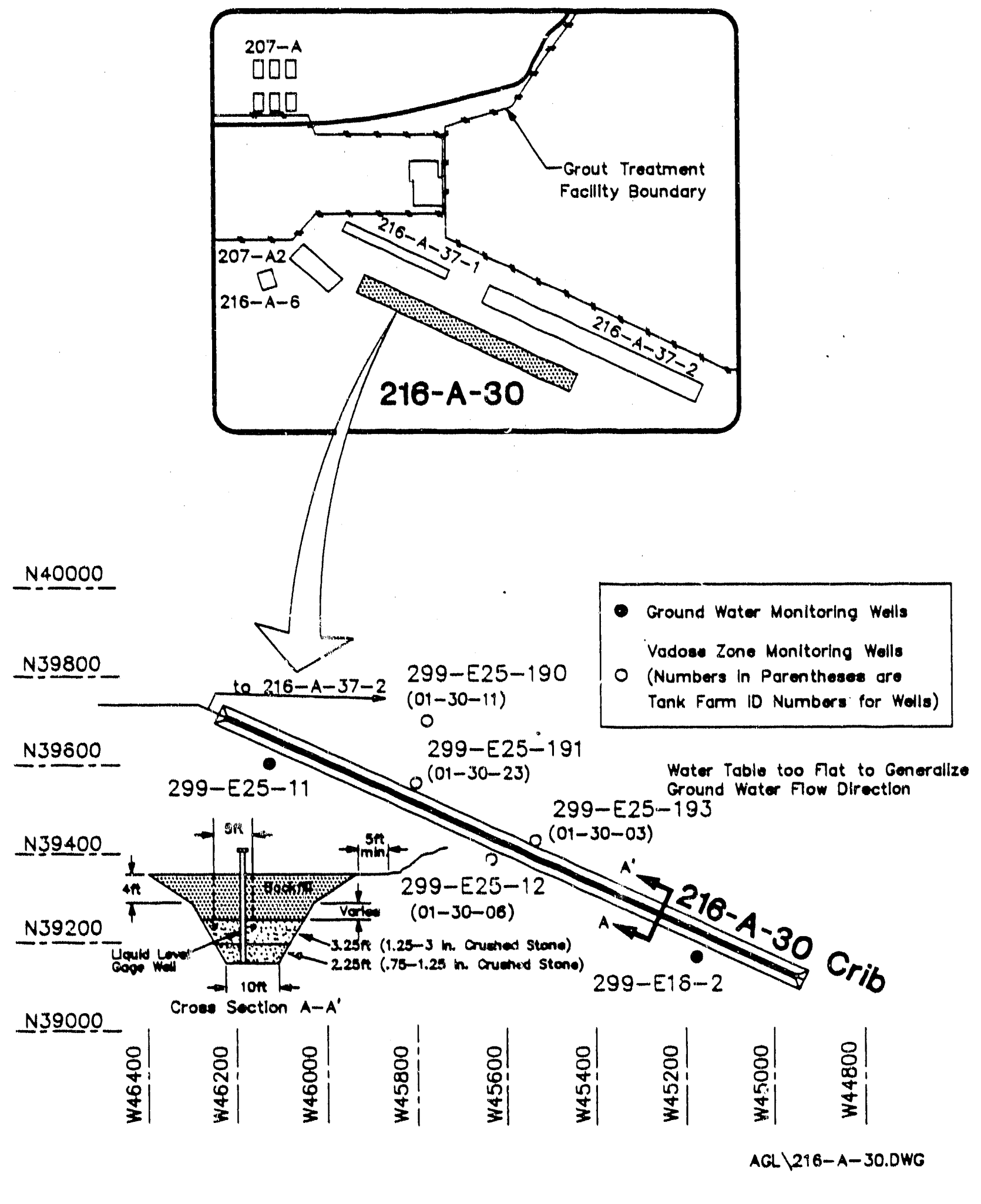

Well Location and Site Map for 216-A-30 Crib 
WHC -EP-0366

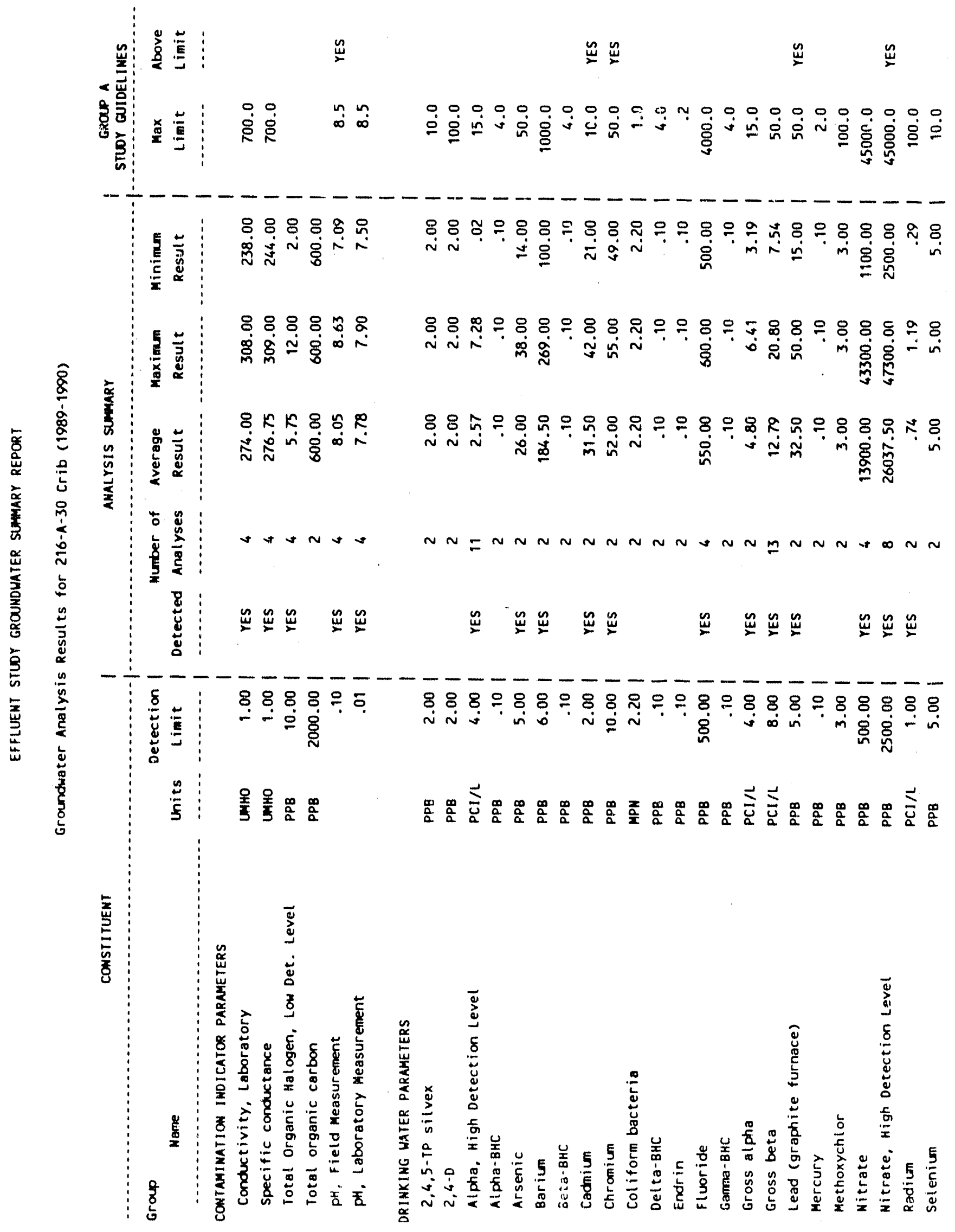




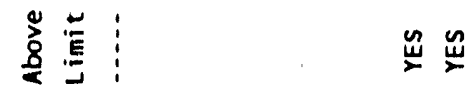

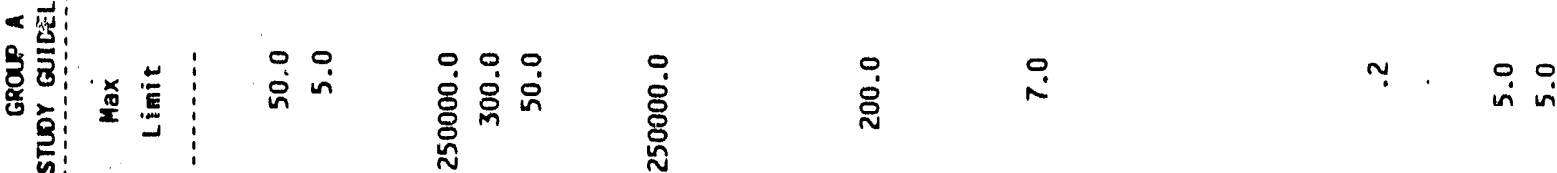

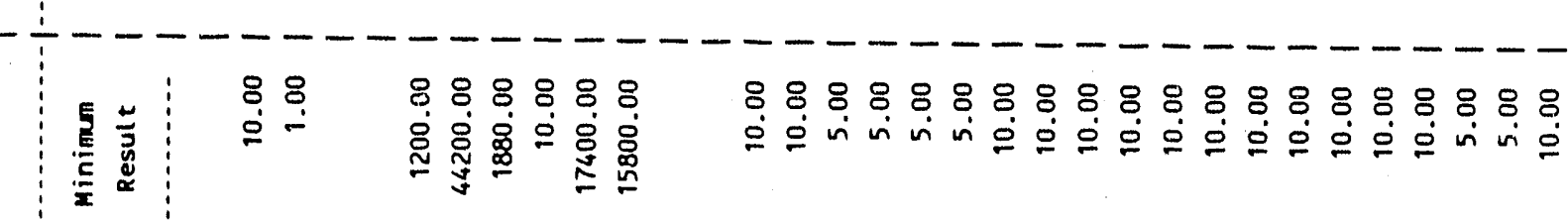

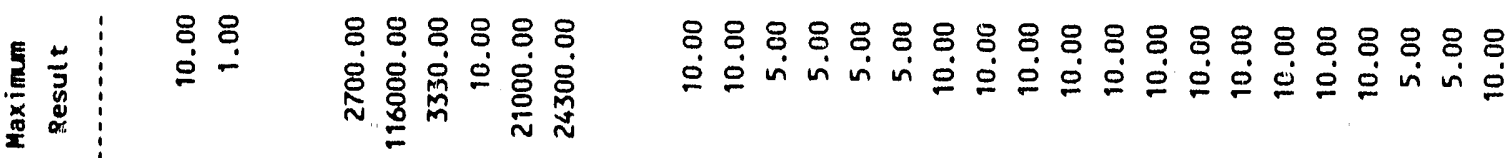

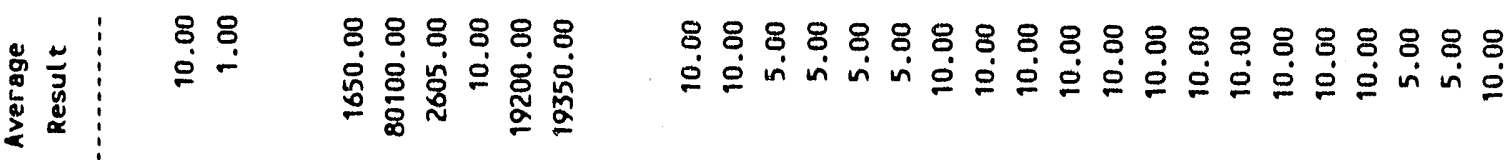

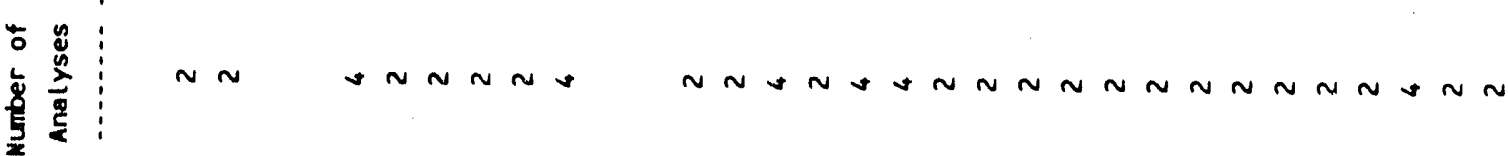

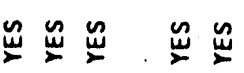

눈
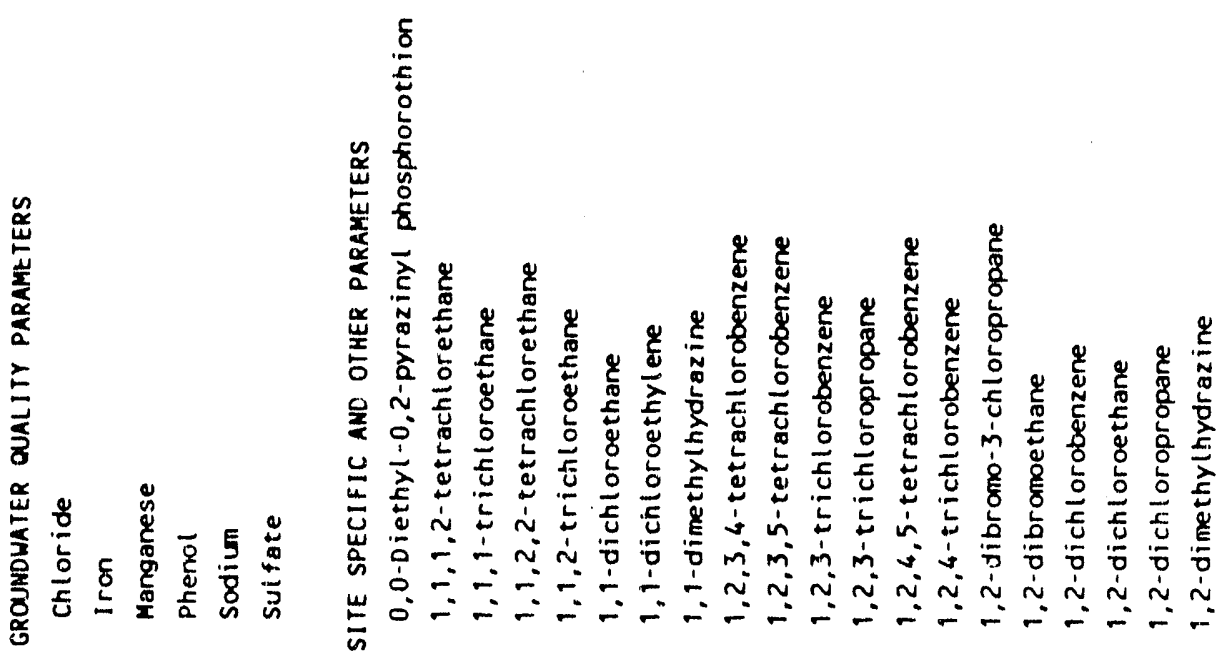


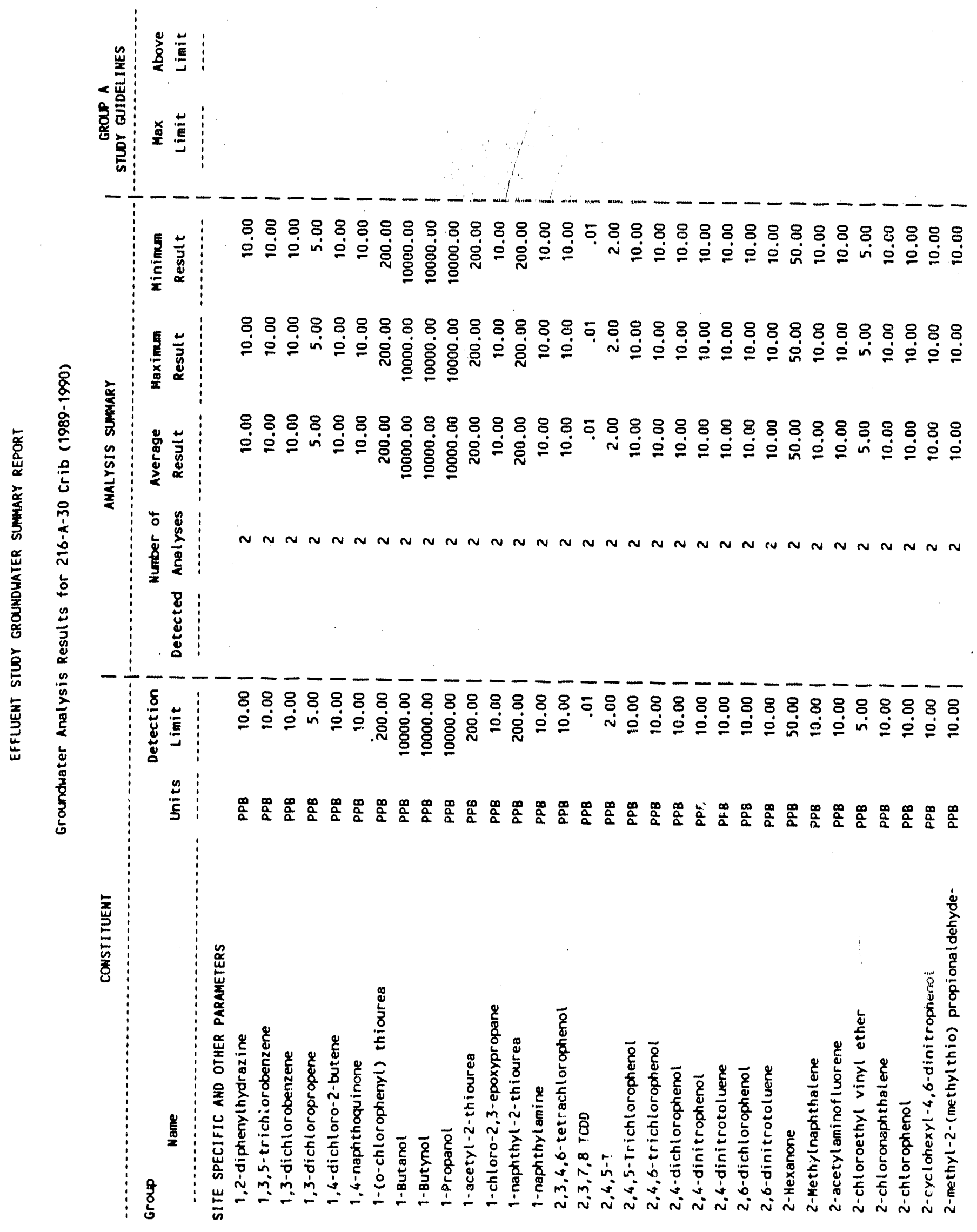


WHC -EP-0366

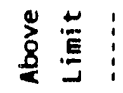

崖:

$<$ 岁:

营

के

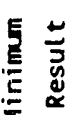

88888888888898888888088888888988

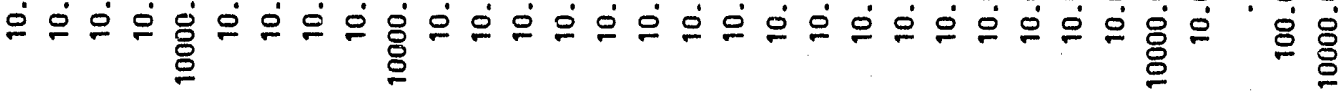

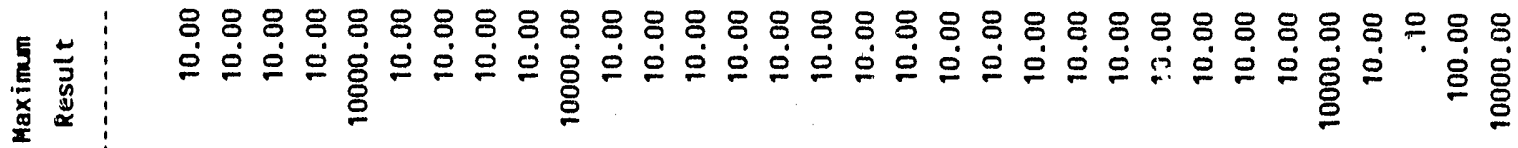

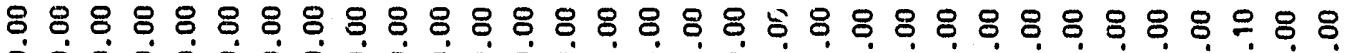

崖苛

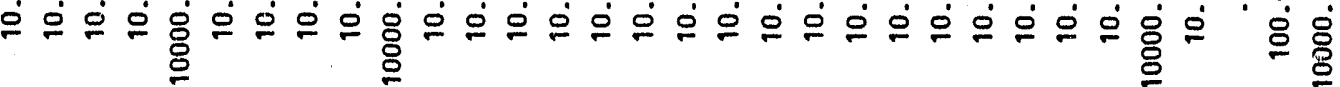

50 and

焉要

:

$\delta$

若

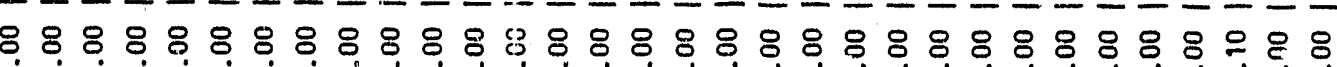

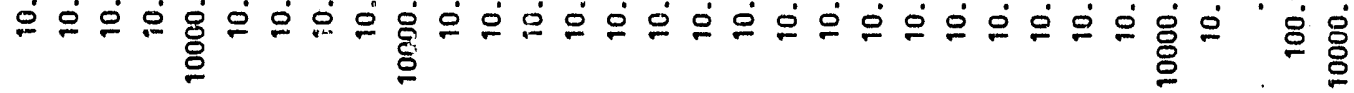

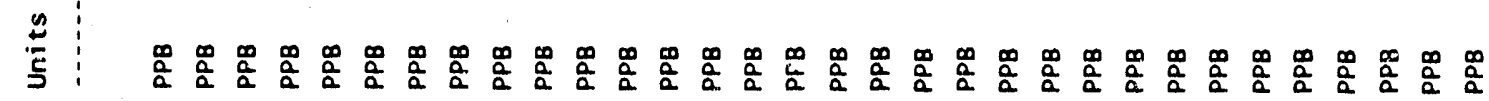

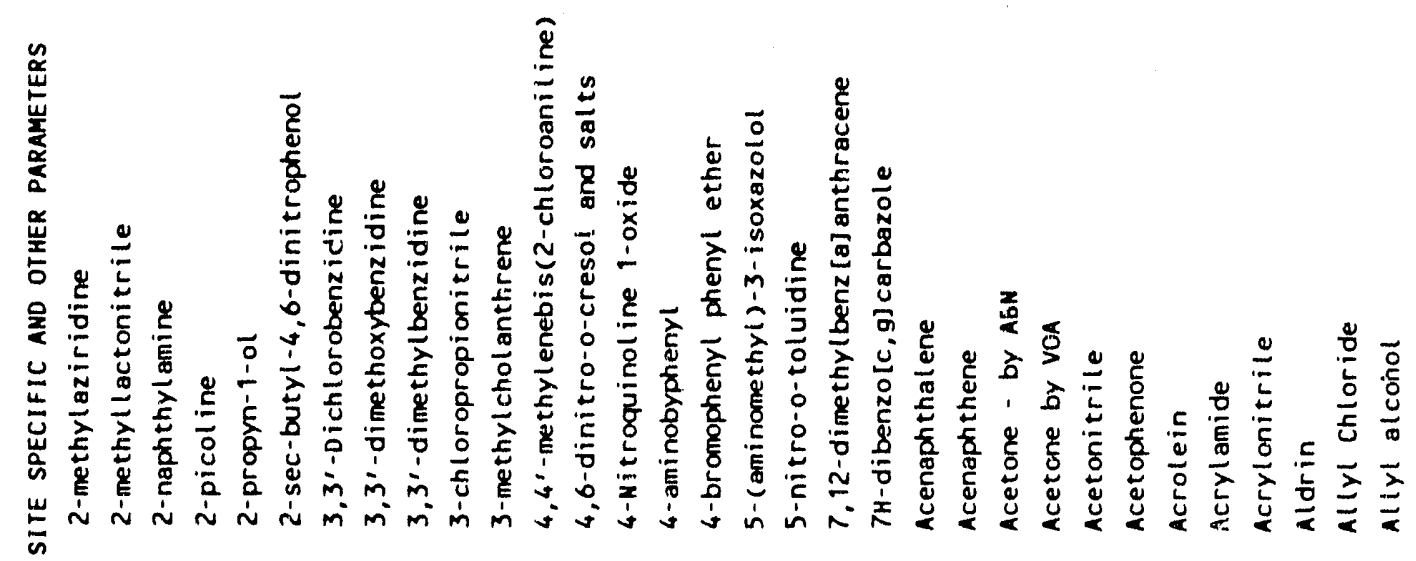


WHC-EP-0366

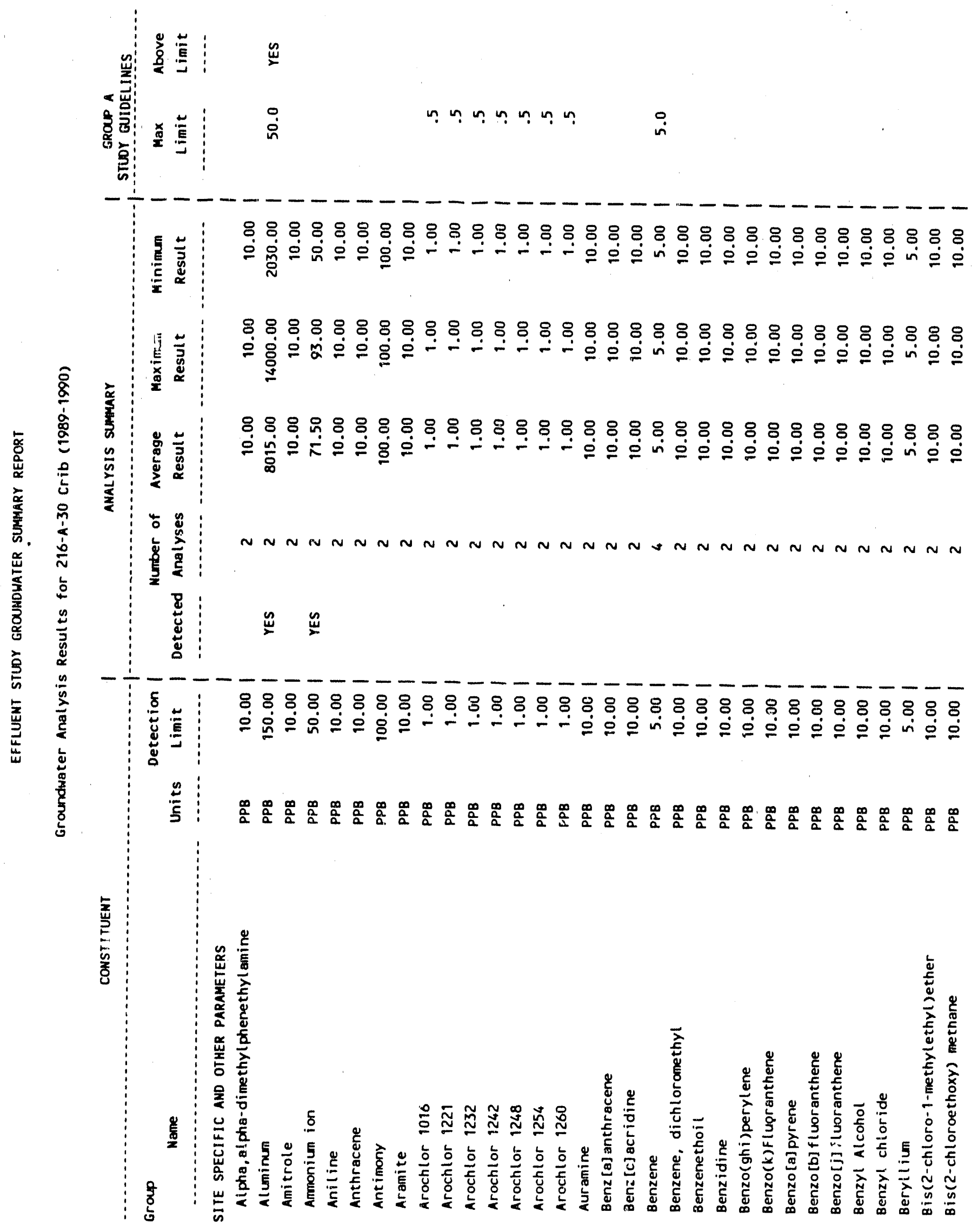




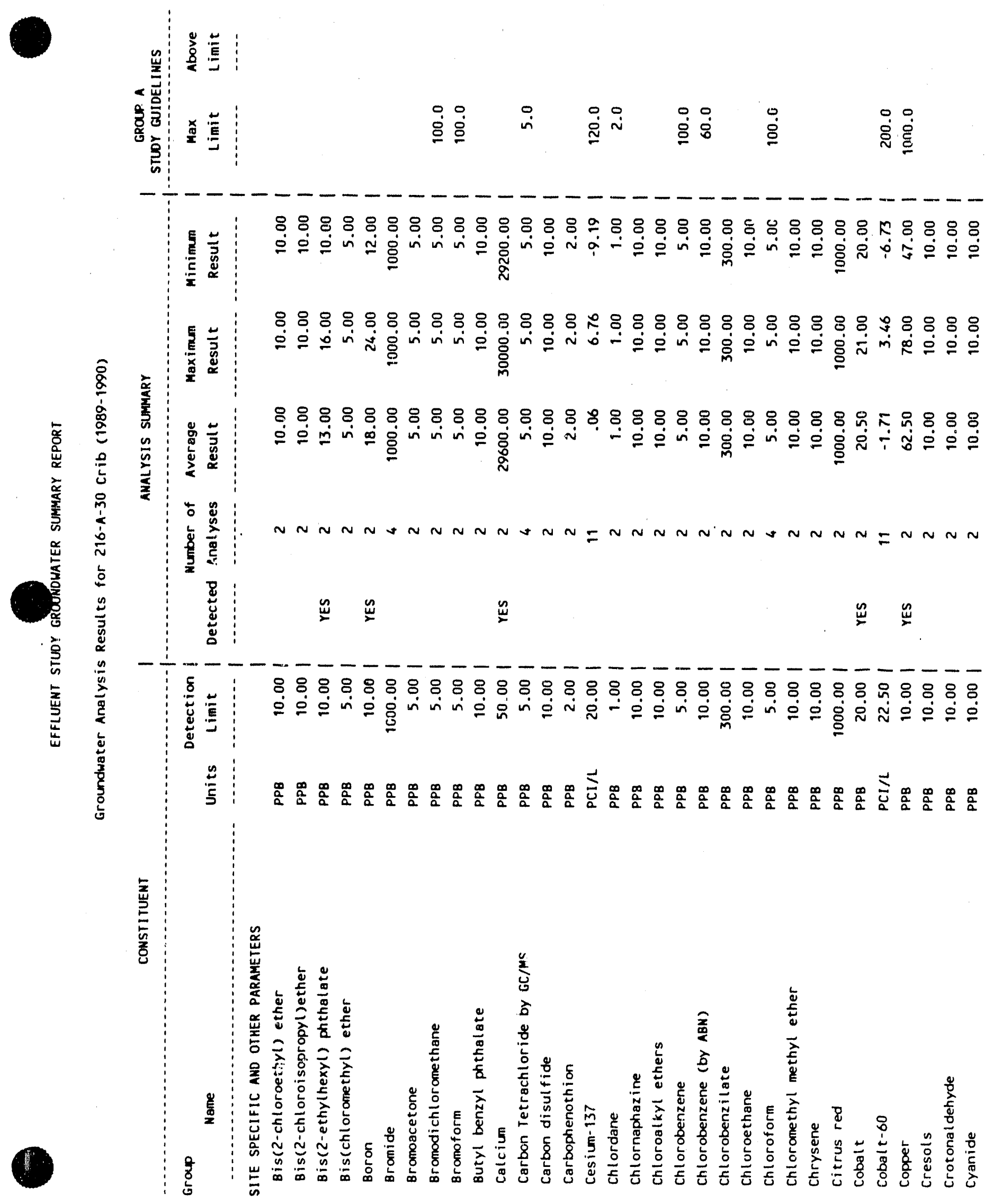


WHC-EP-0366
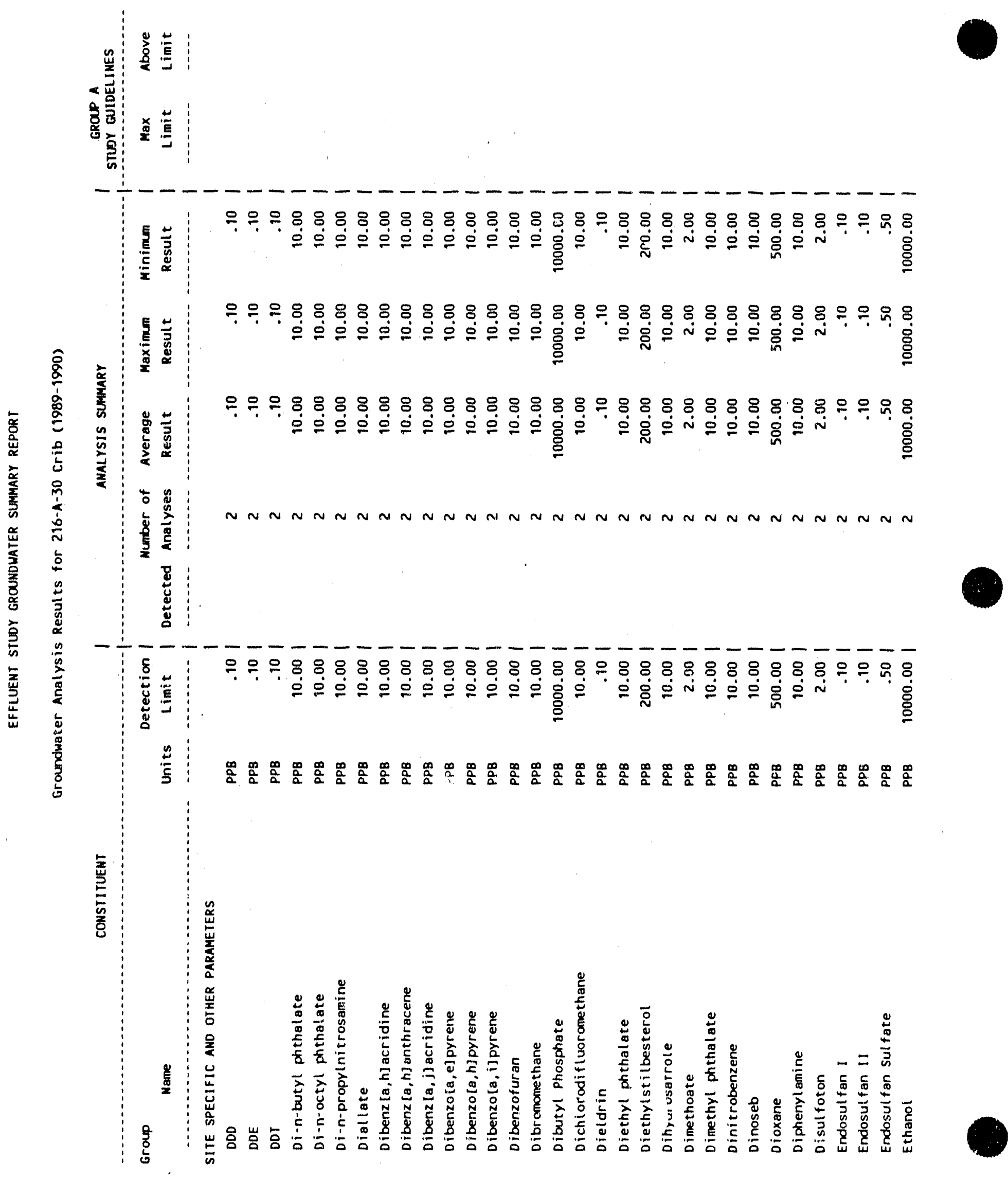


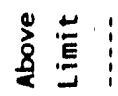

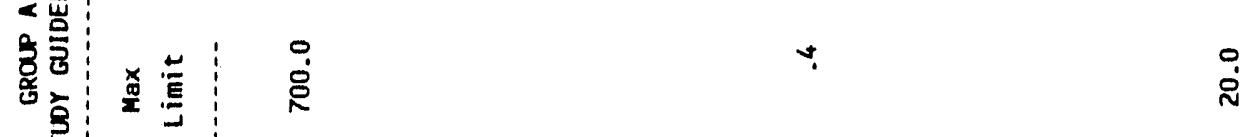

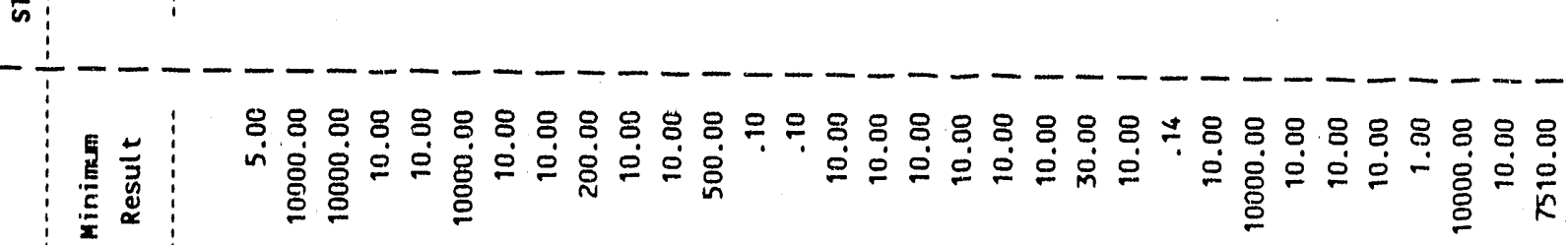

重言

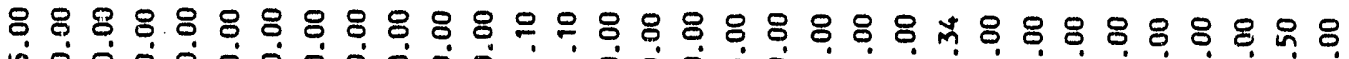

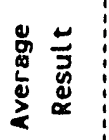

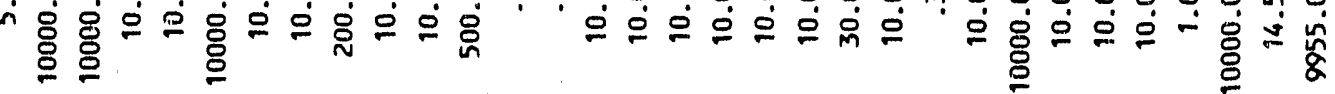

to 告

:

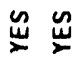

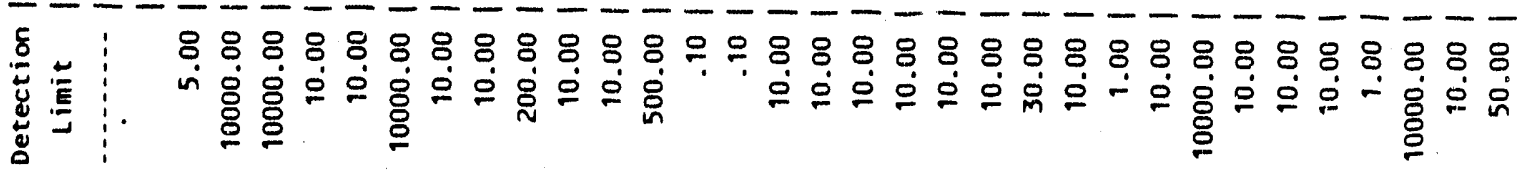

:

䇋

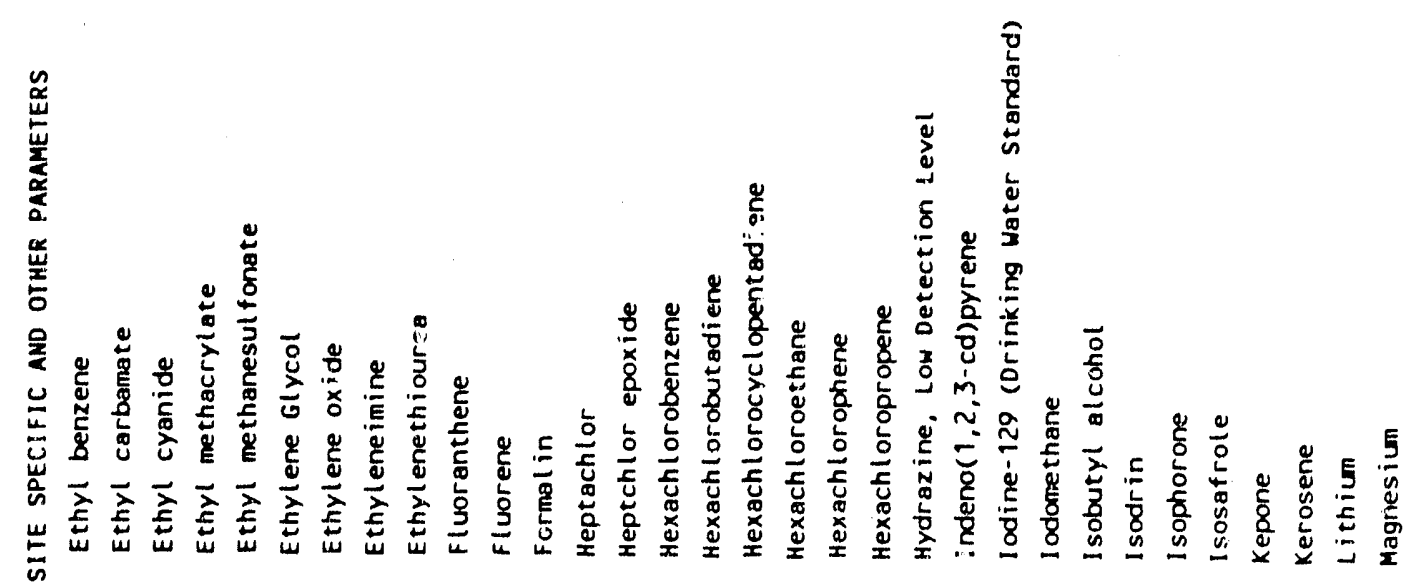




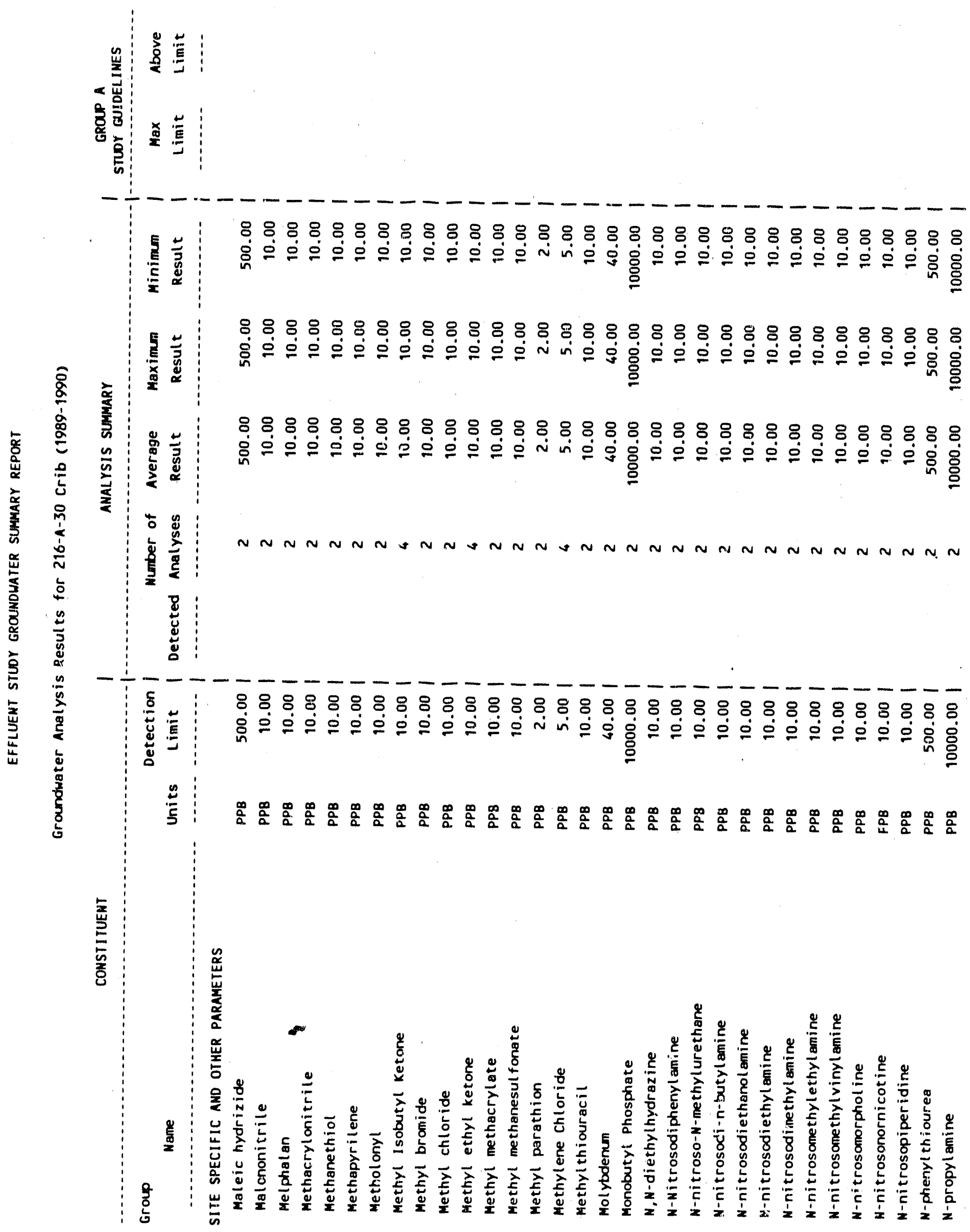


莫

<砣

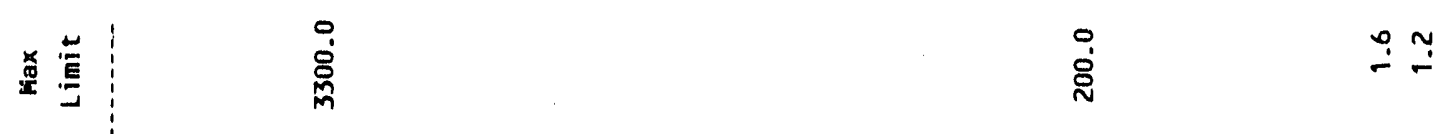

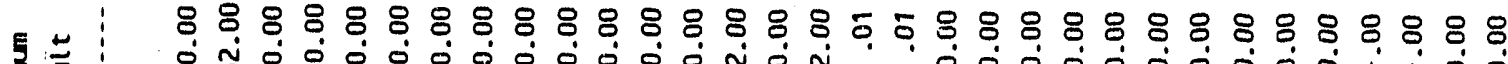

节

5

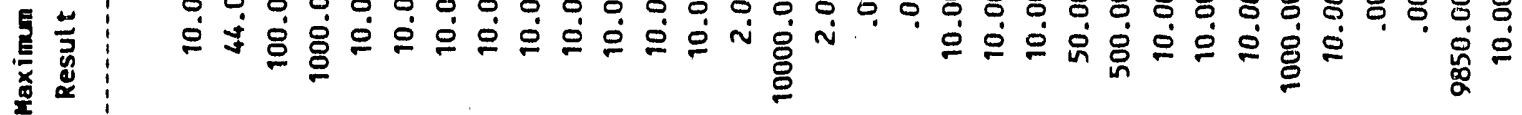

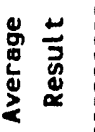

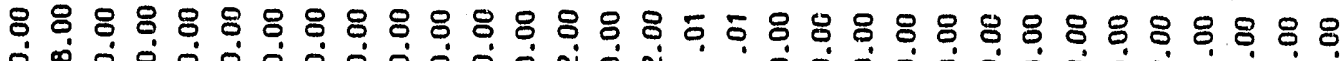

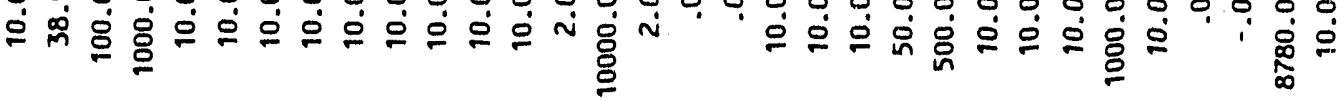

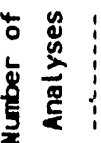

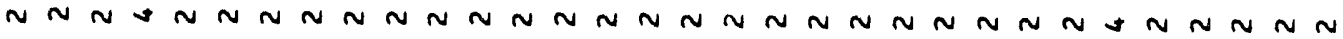

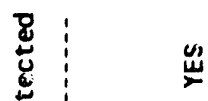

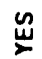

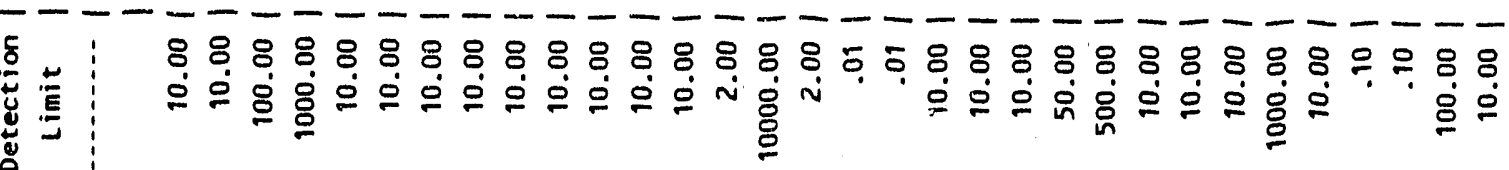

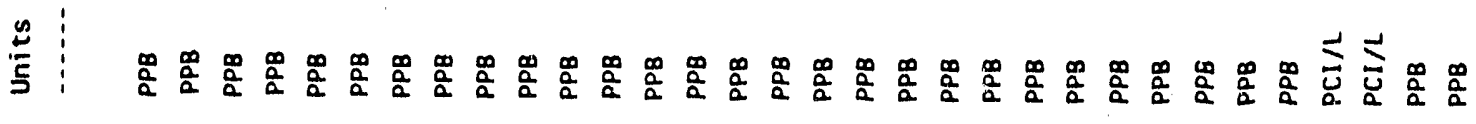

产

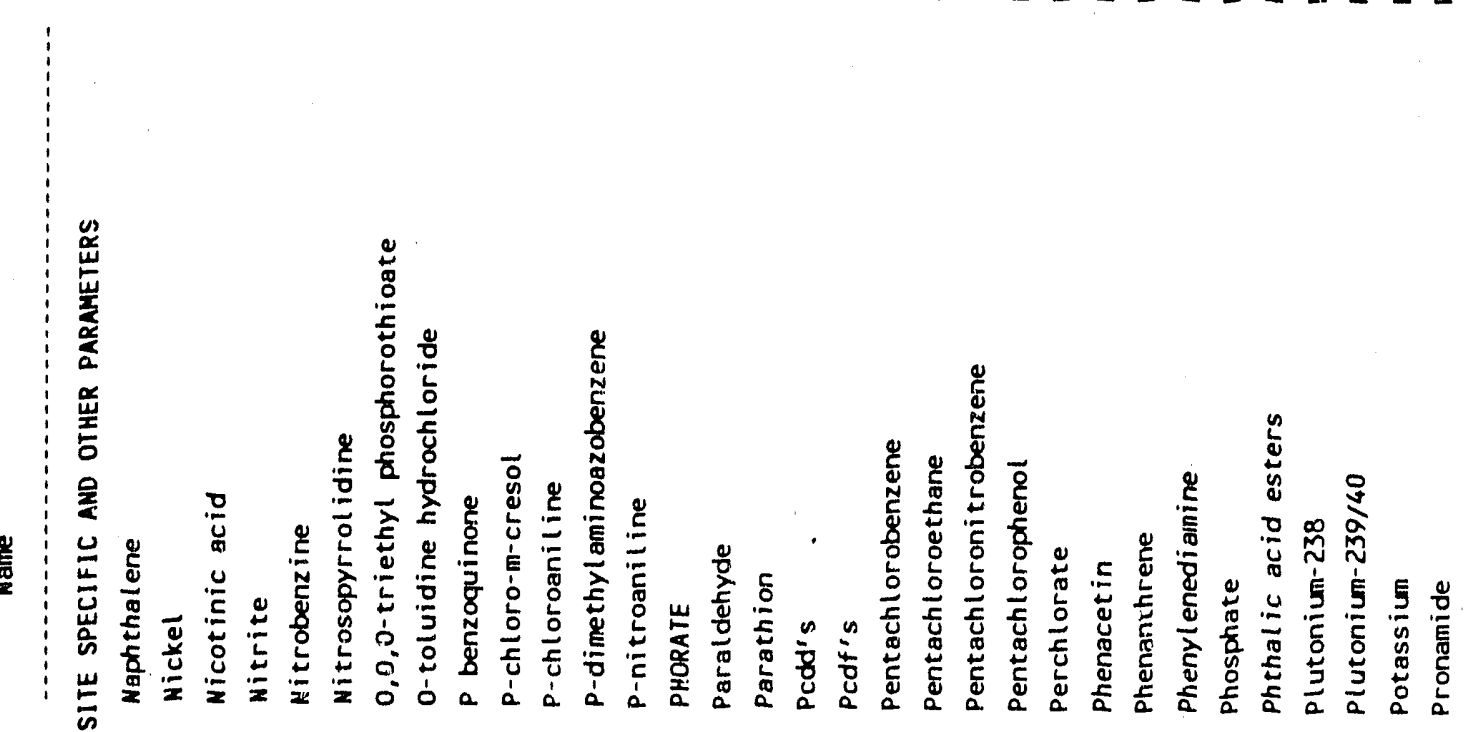


WHC-EP- 3366

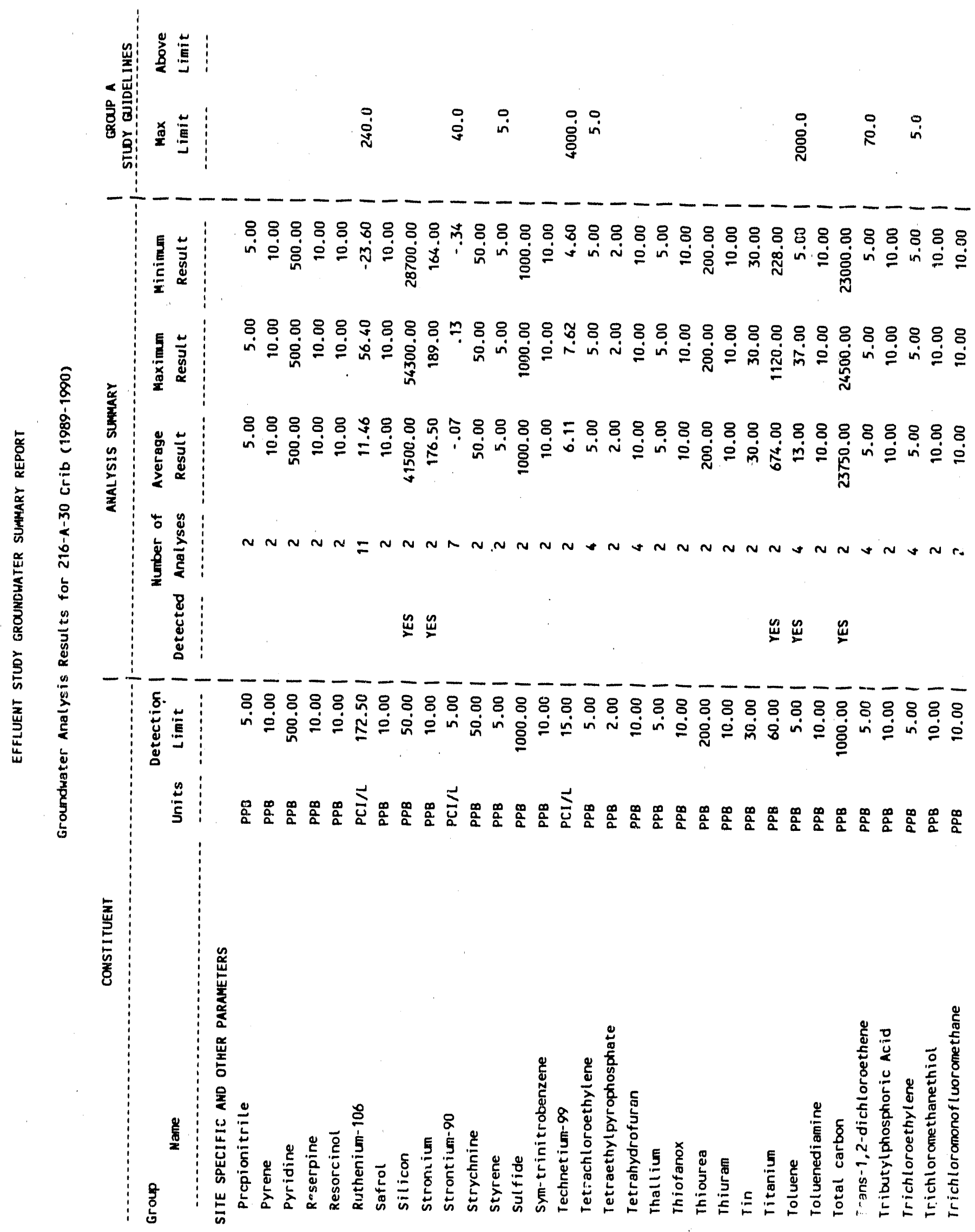




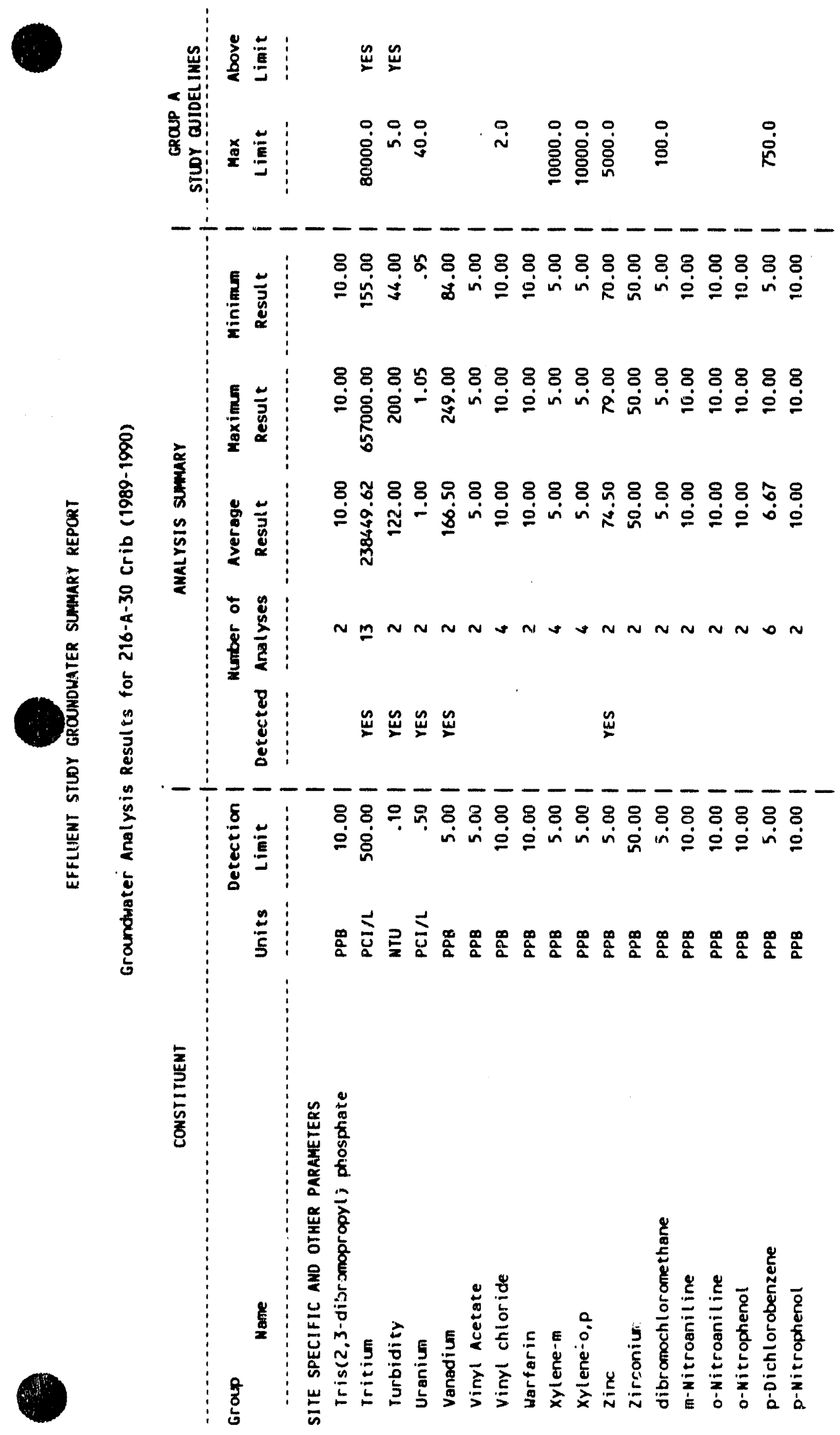




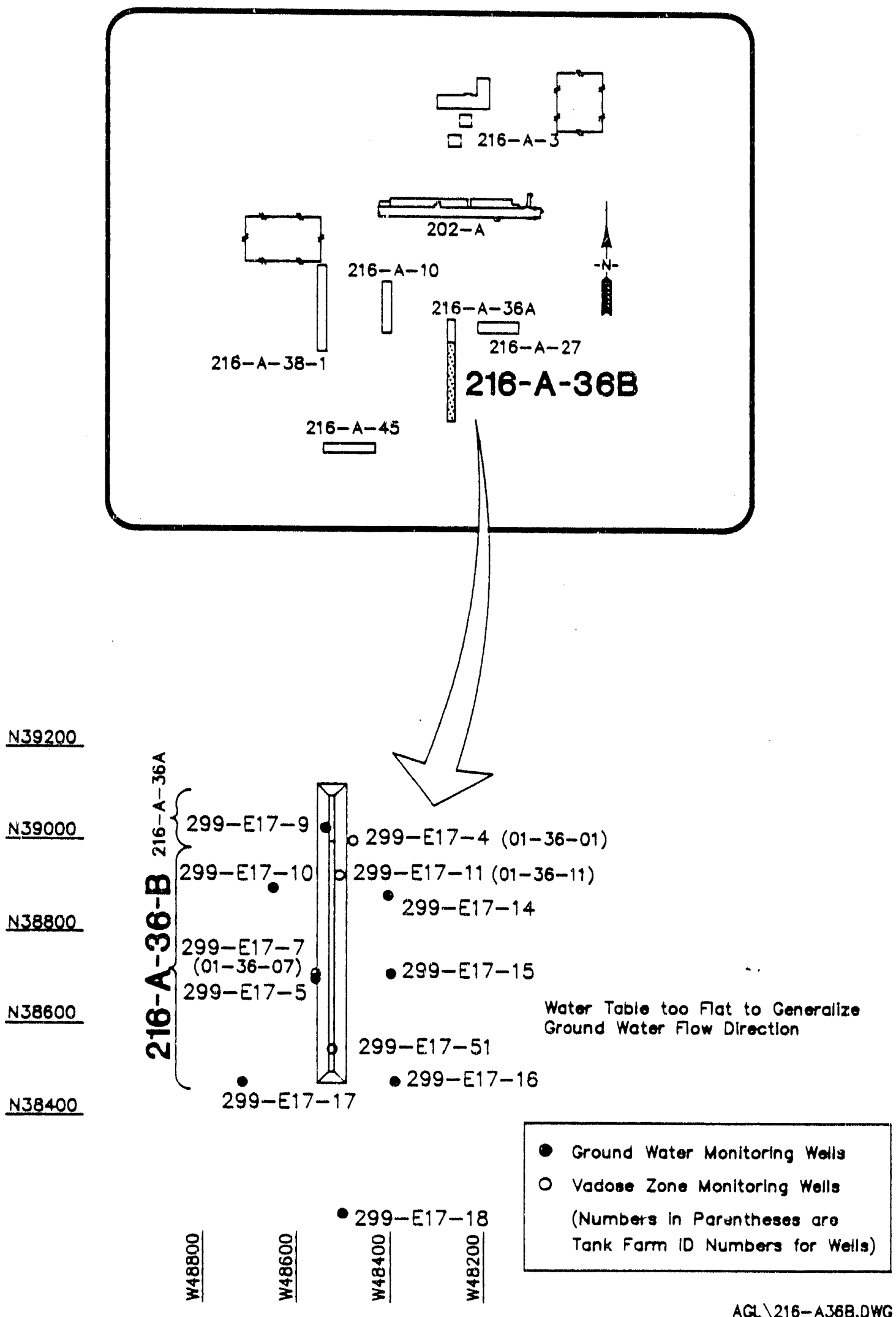

We11 Location and Site Map for 216-B-36B Crib 
WHC -EP-0366

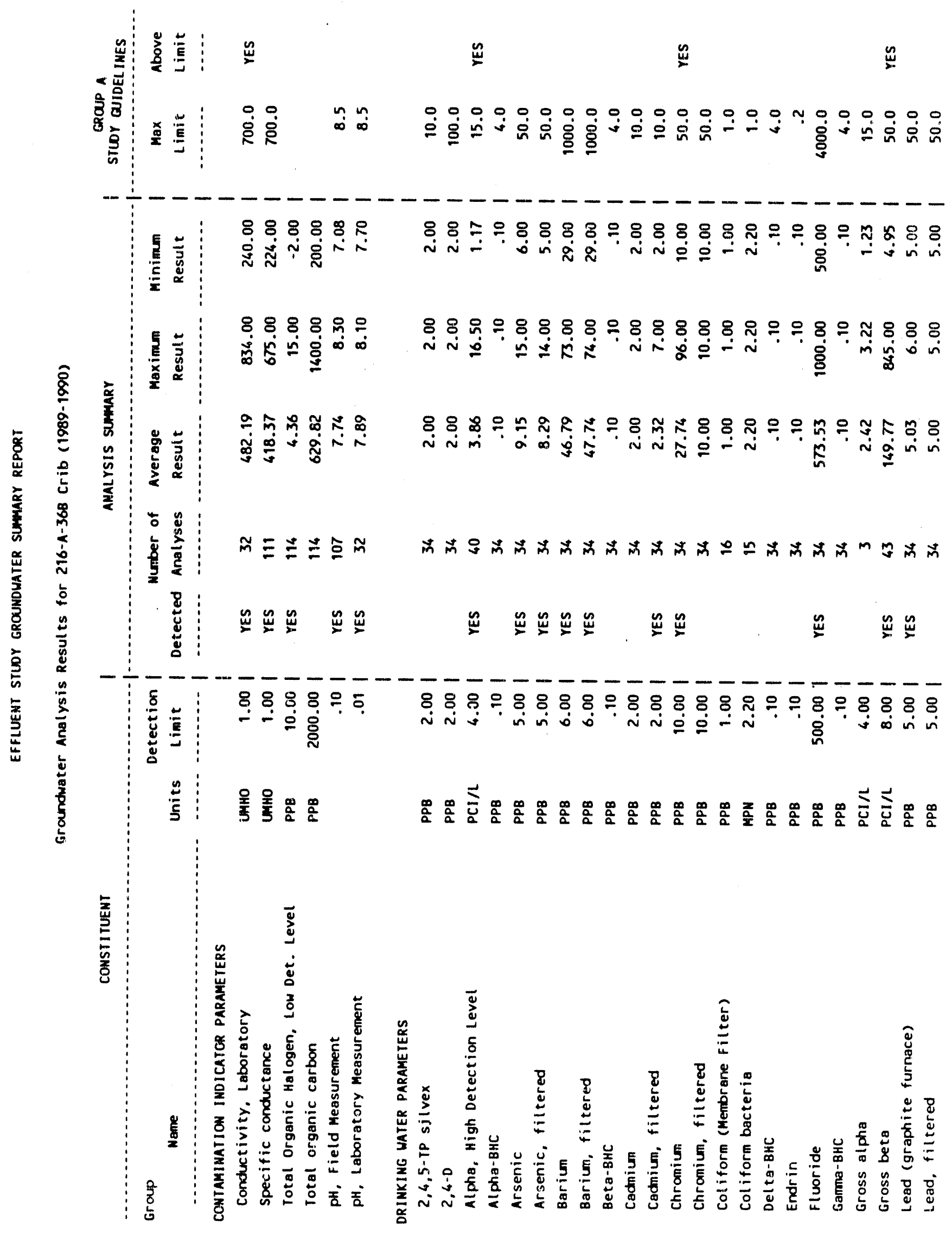


WHC-EP-0366

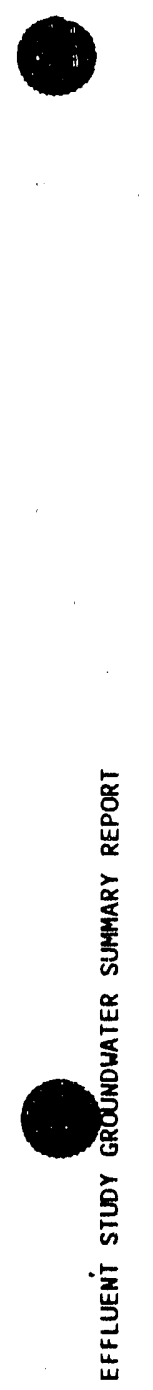

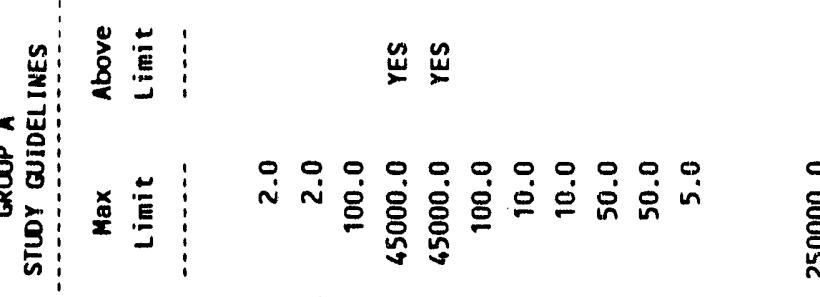

$\stackrel{\sim}{2}$

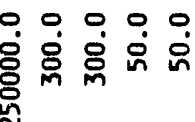

:

官

$\stackrel{0}{1}$

$88.8888 \% 8 \%$

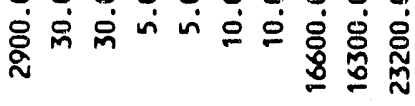

8888888

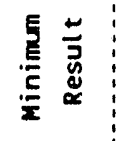

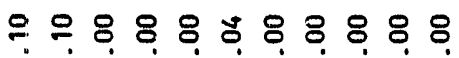

mं 这客

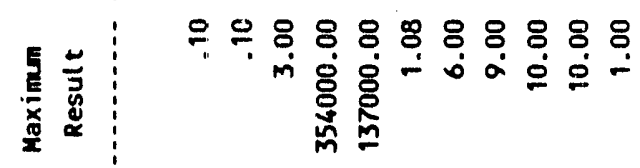

용ㅇㅇㅇㅇㅇㅇㅇ

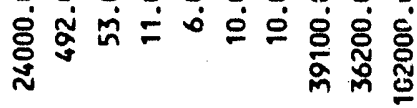

888888.

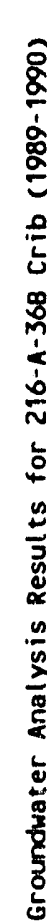

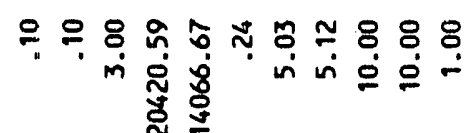

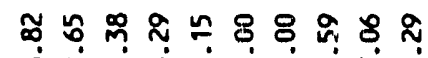

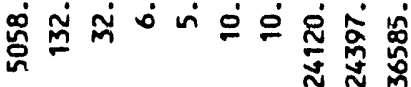

8888888

岁泀

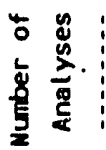

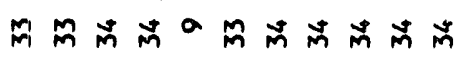

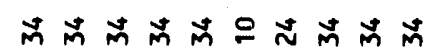

으는

总

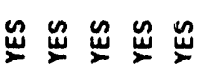

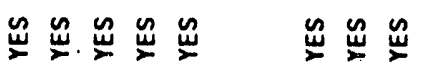

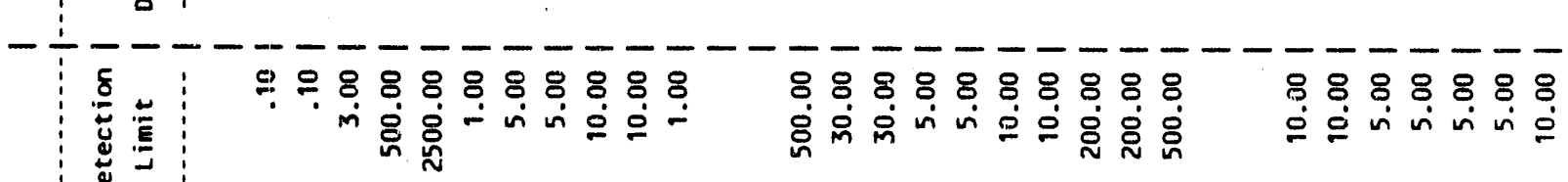

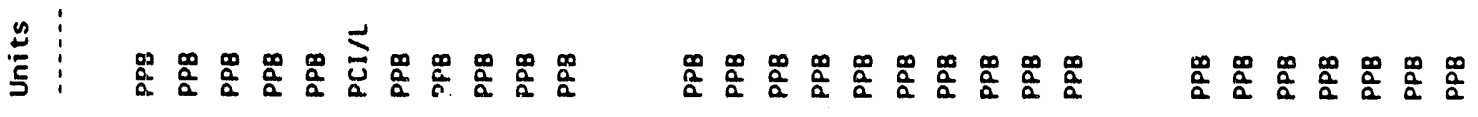
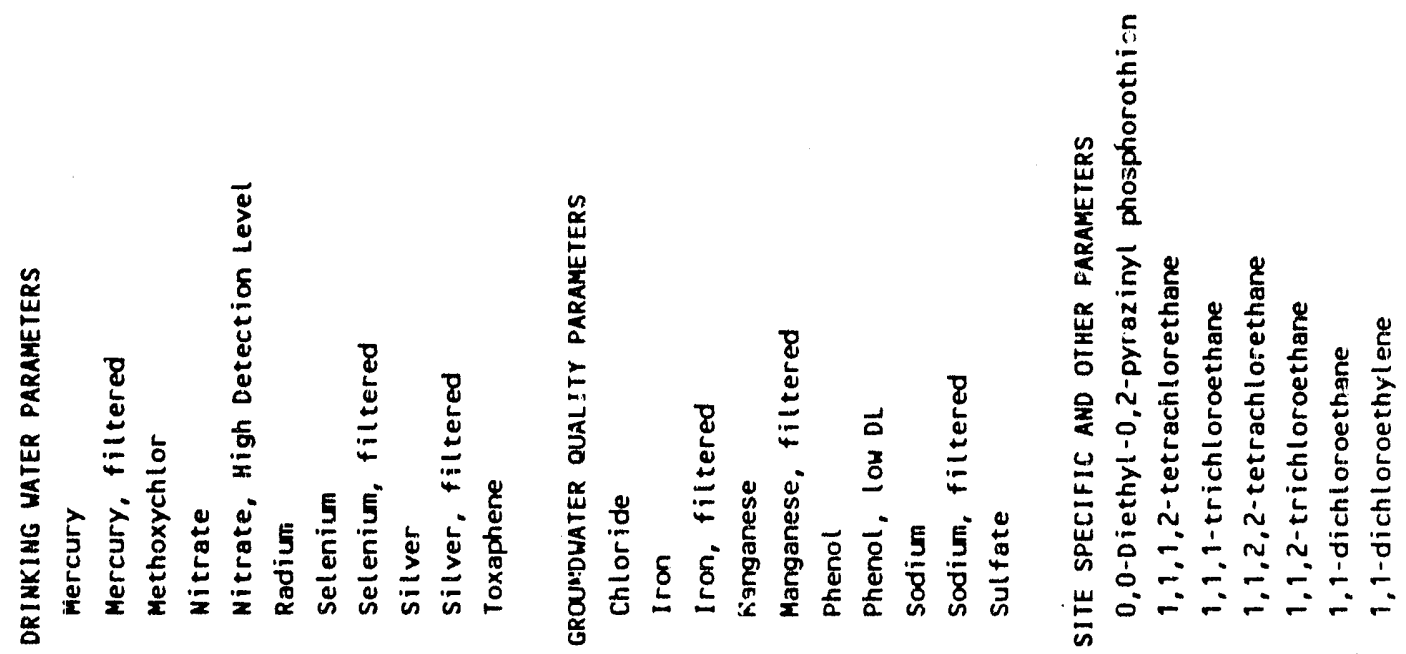
WHC-EP-0366
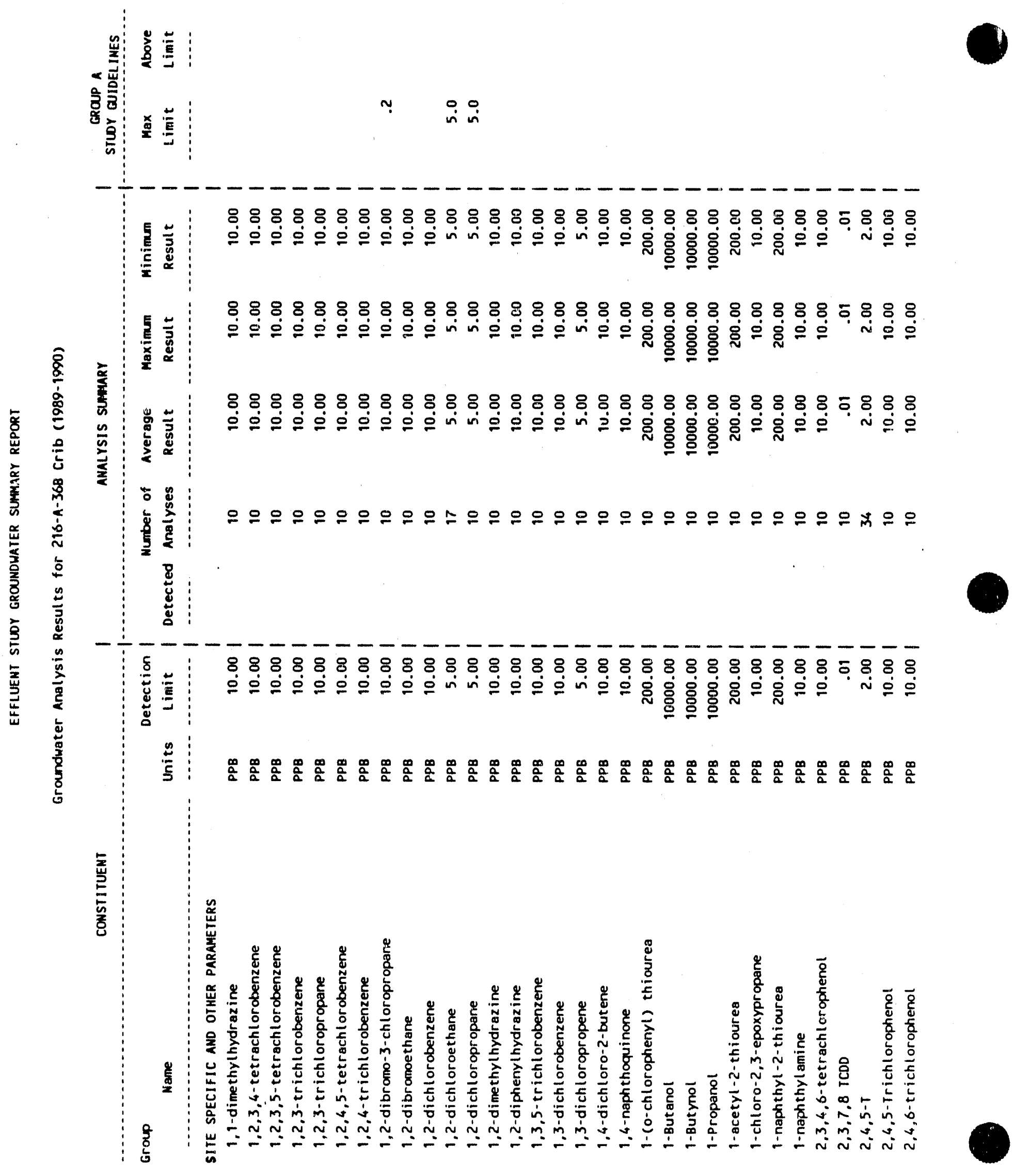
WHC - EP- 0366

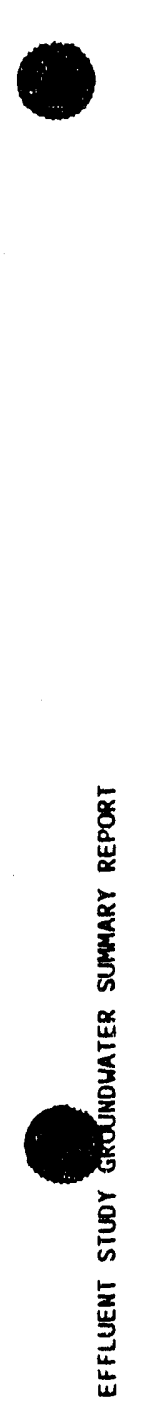

(1)

88888888888888888888888888888888

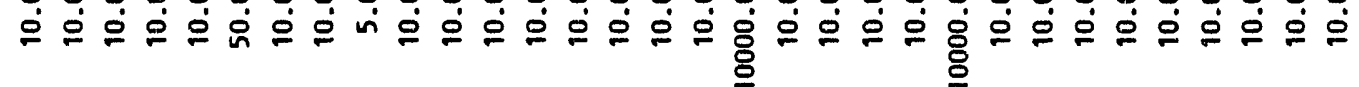

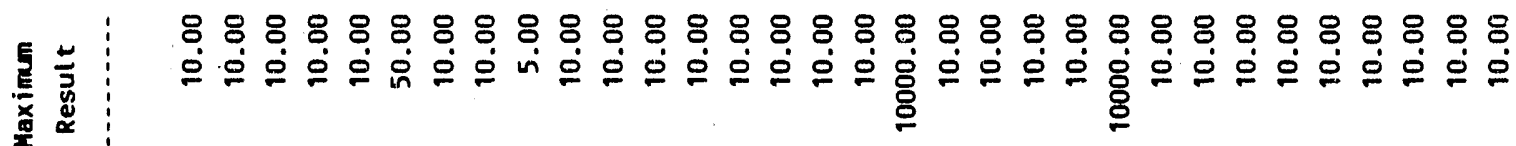

88888888888888888888898888888888

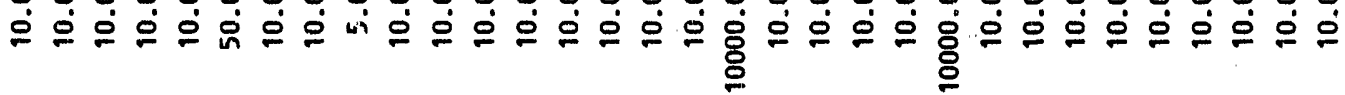

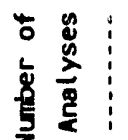

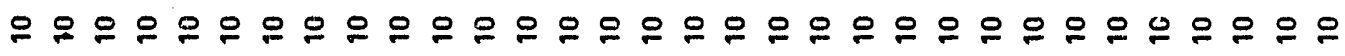
8

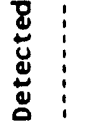

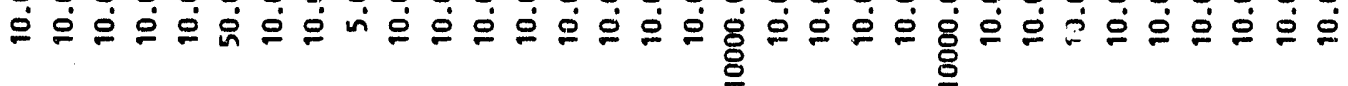

$\stackrel{2}{5}$

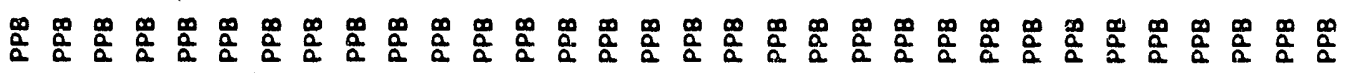

$$
\text { : }
$$

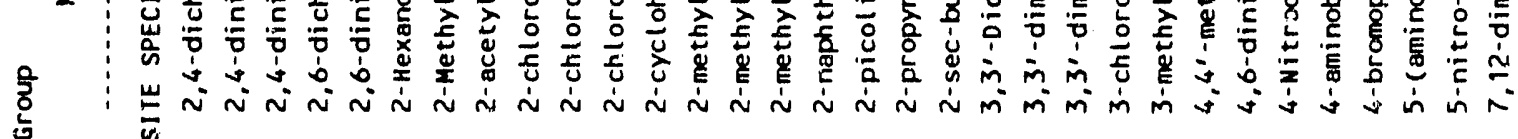




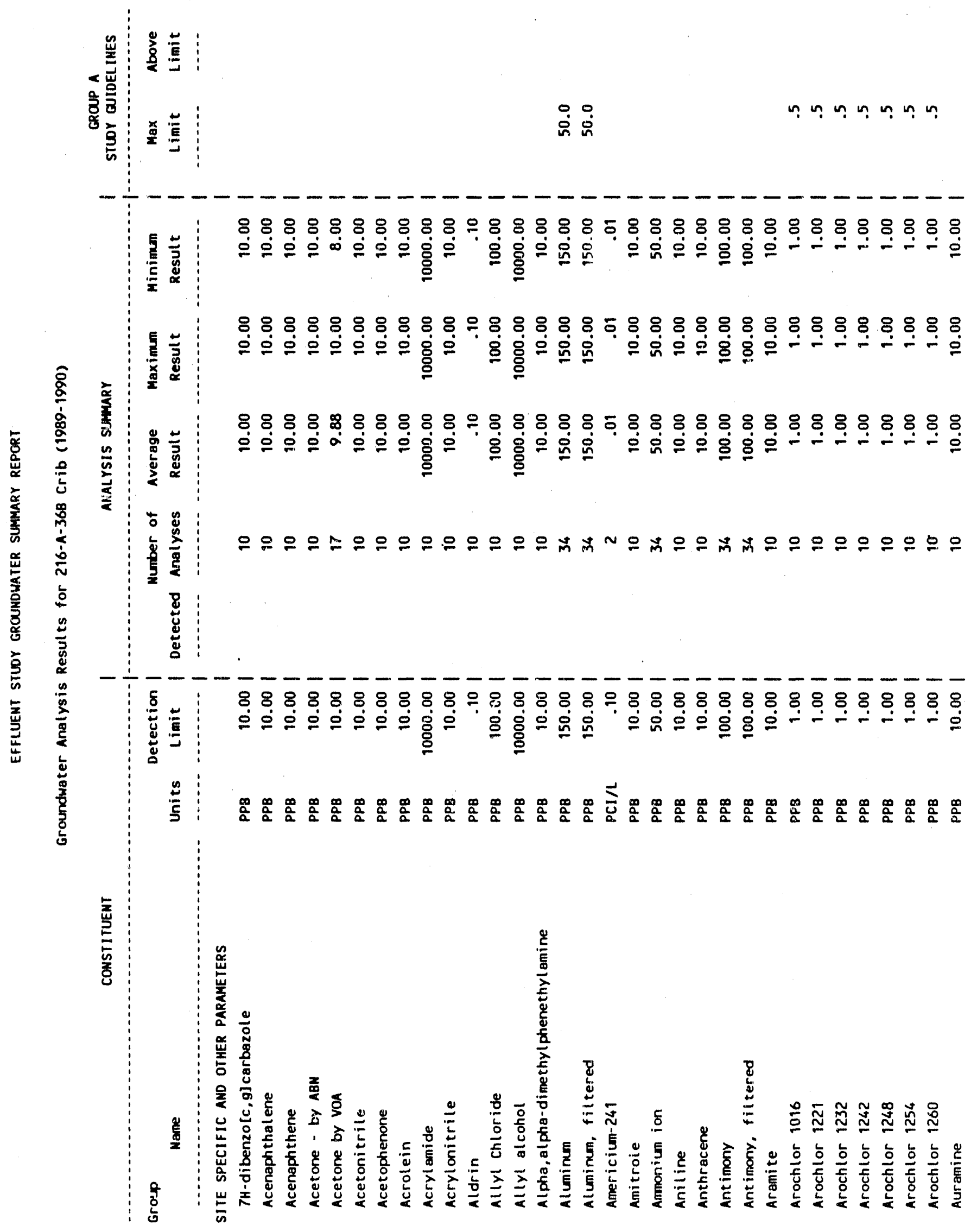


章管:

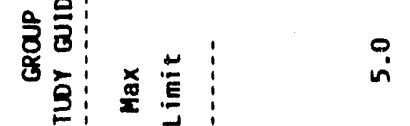

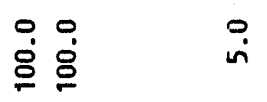

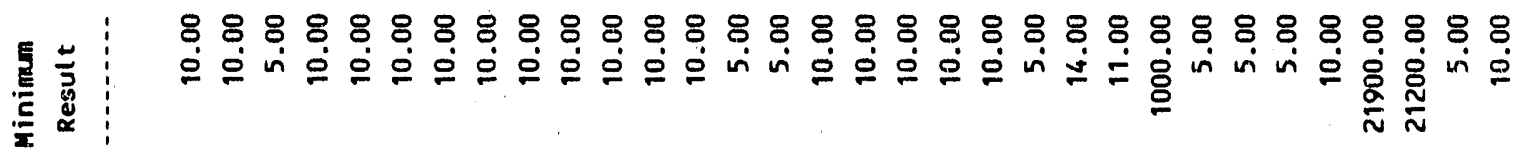

눈 产苟

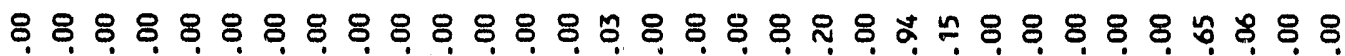
@ீ

焉

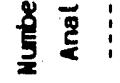

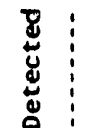

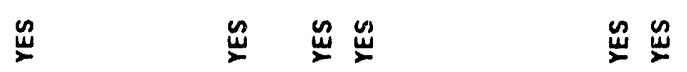

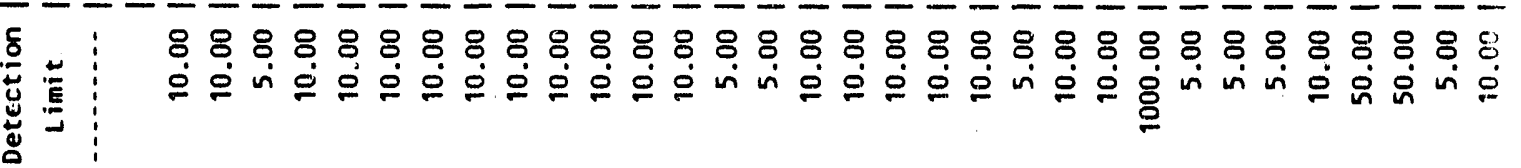

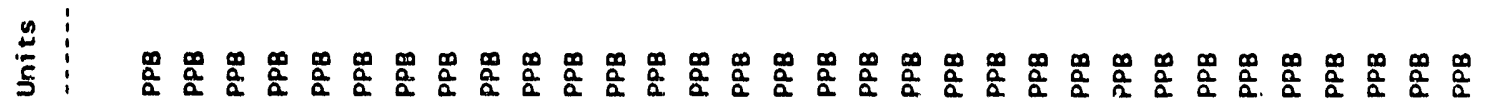

$\vdots$
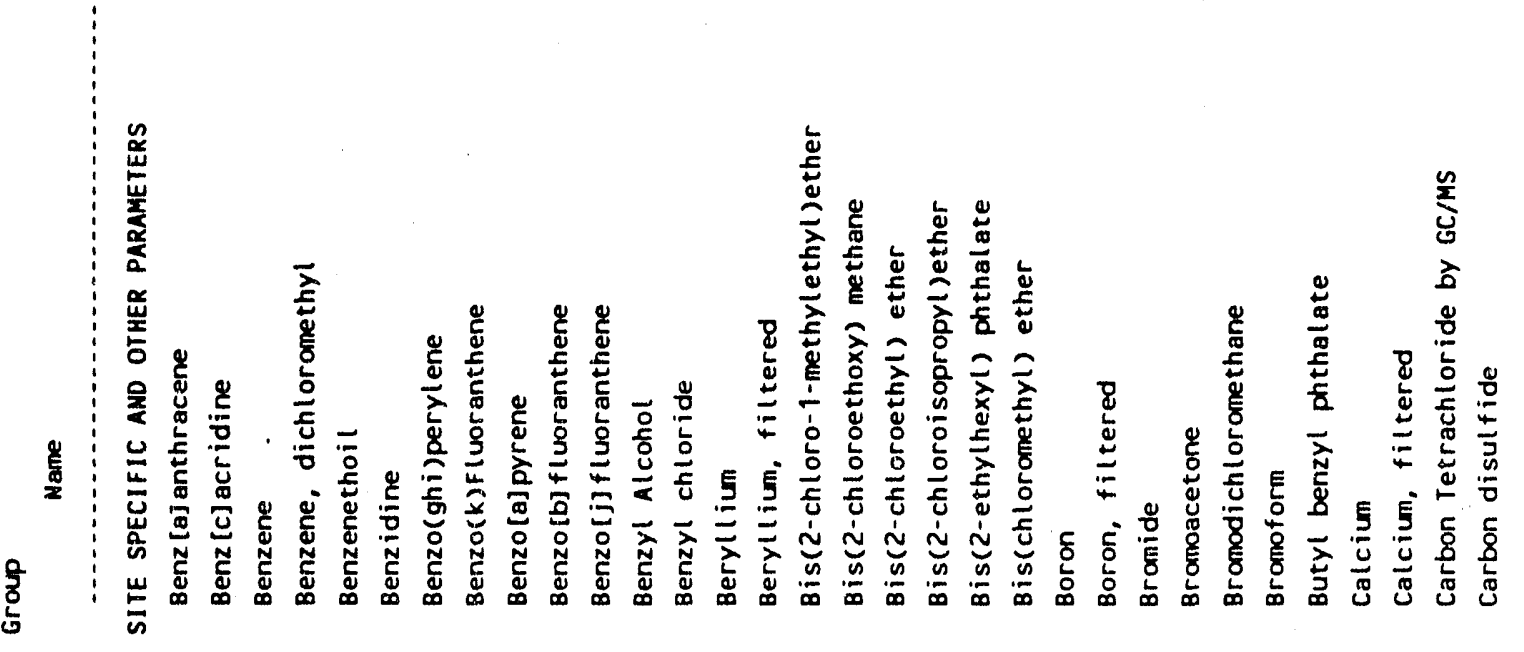


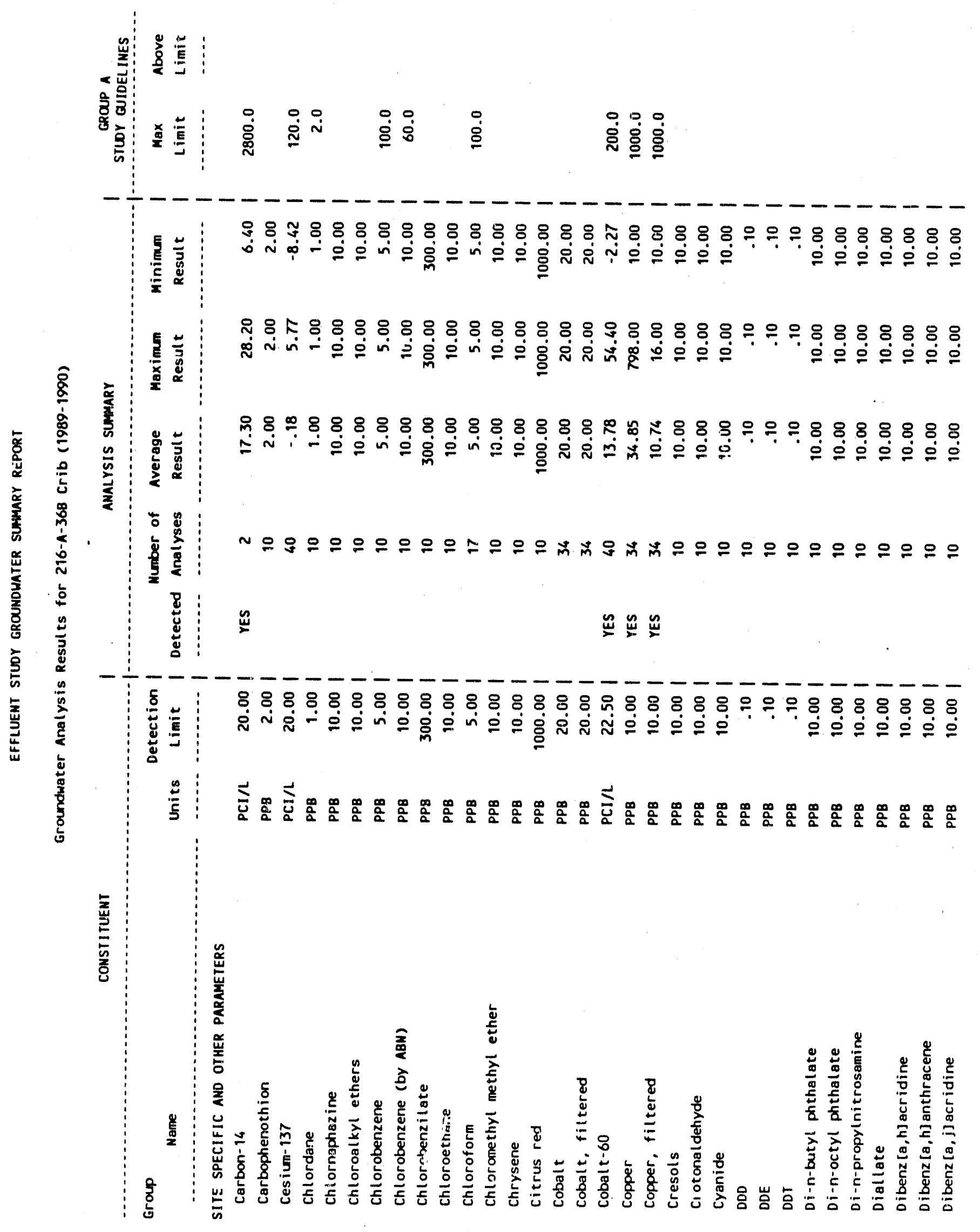




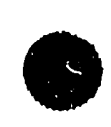

熟管:

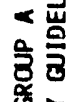

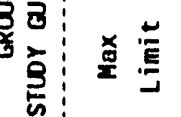

$\stackrel{\circ}{8}$

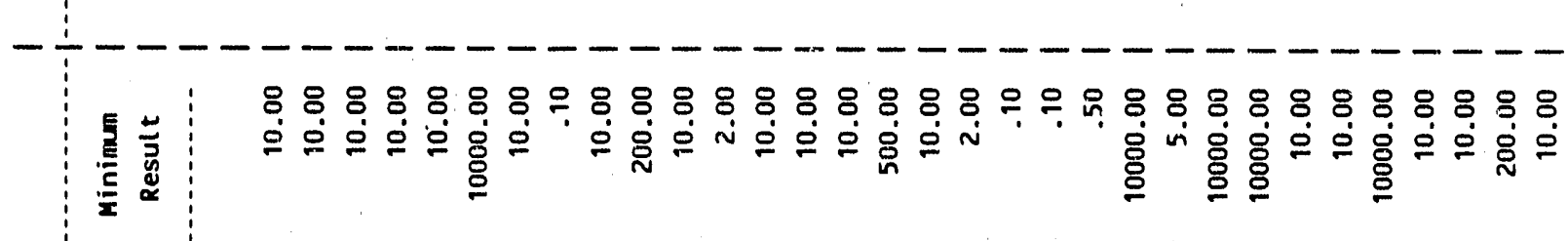

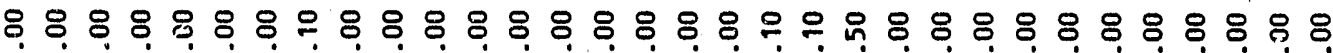

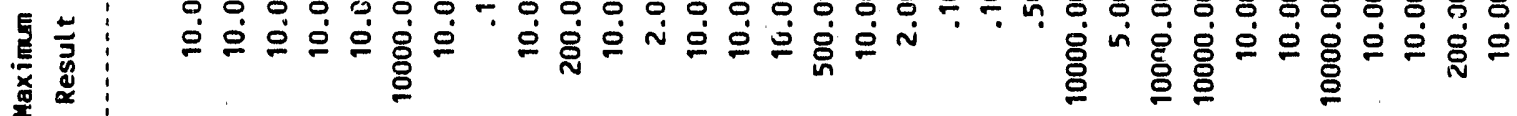

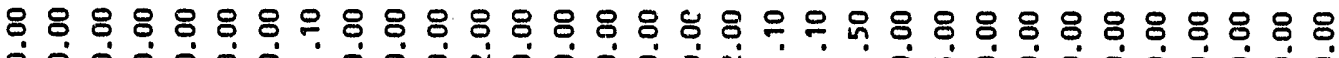

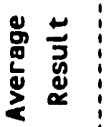

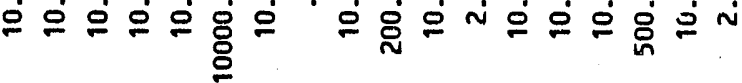
宊的宊 to

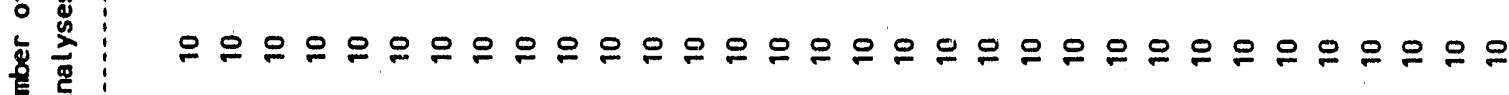
$\frac{5}{2}$

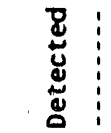

高

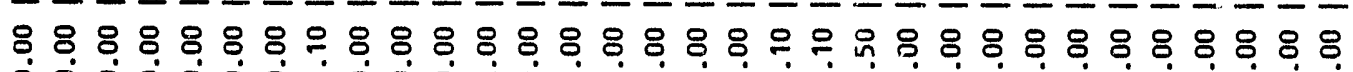

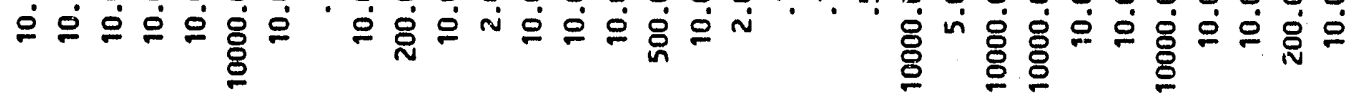

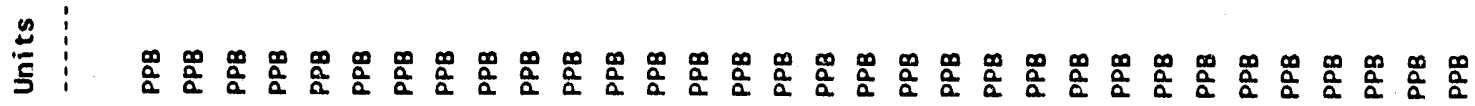

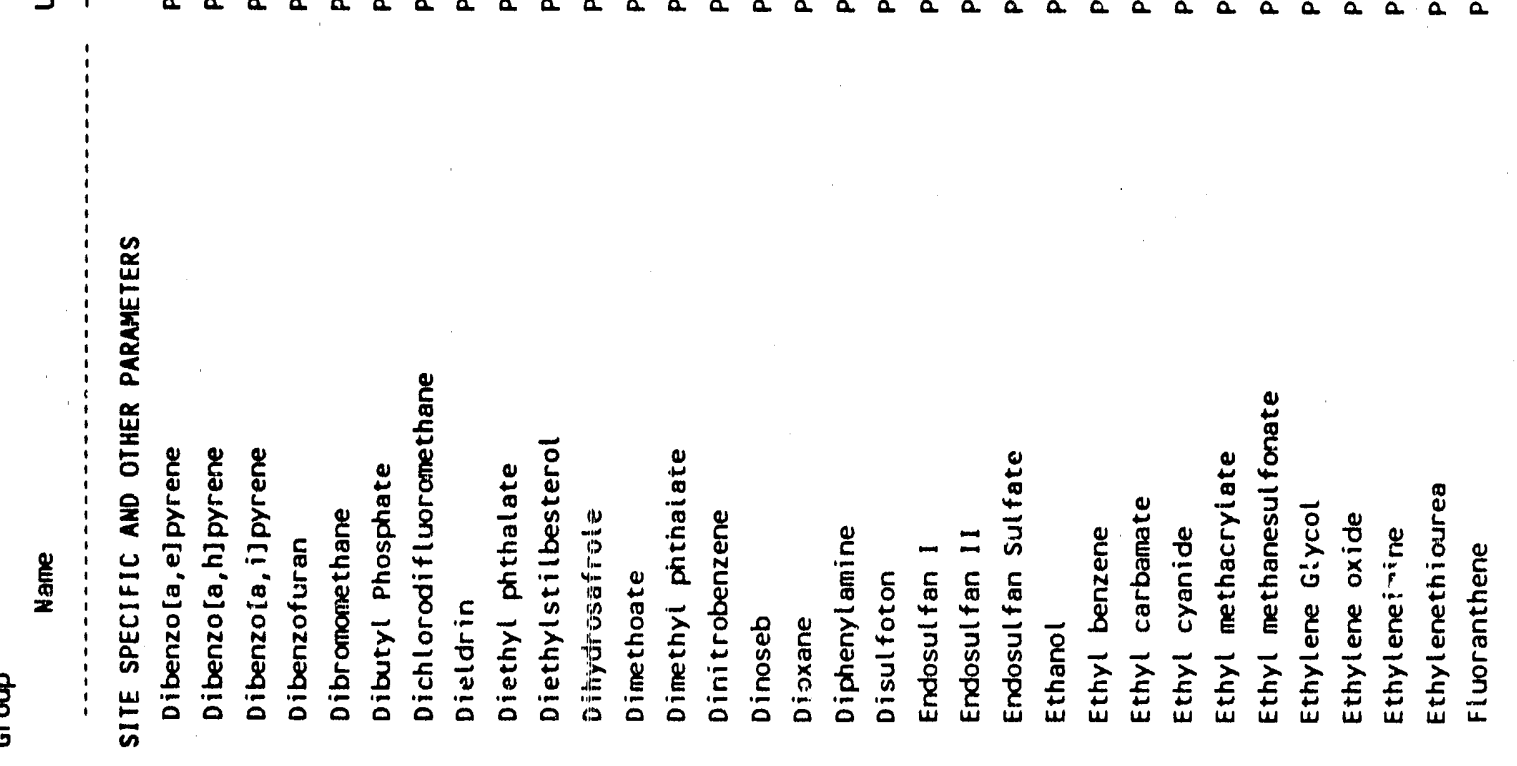


WHC-EP-0366

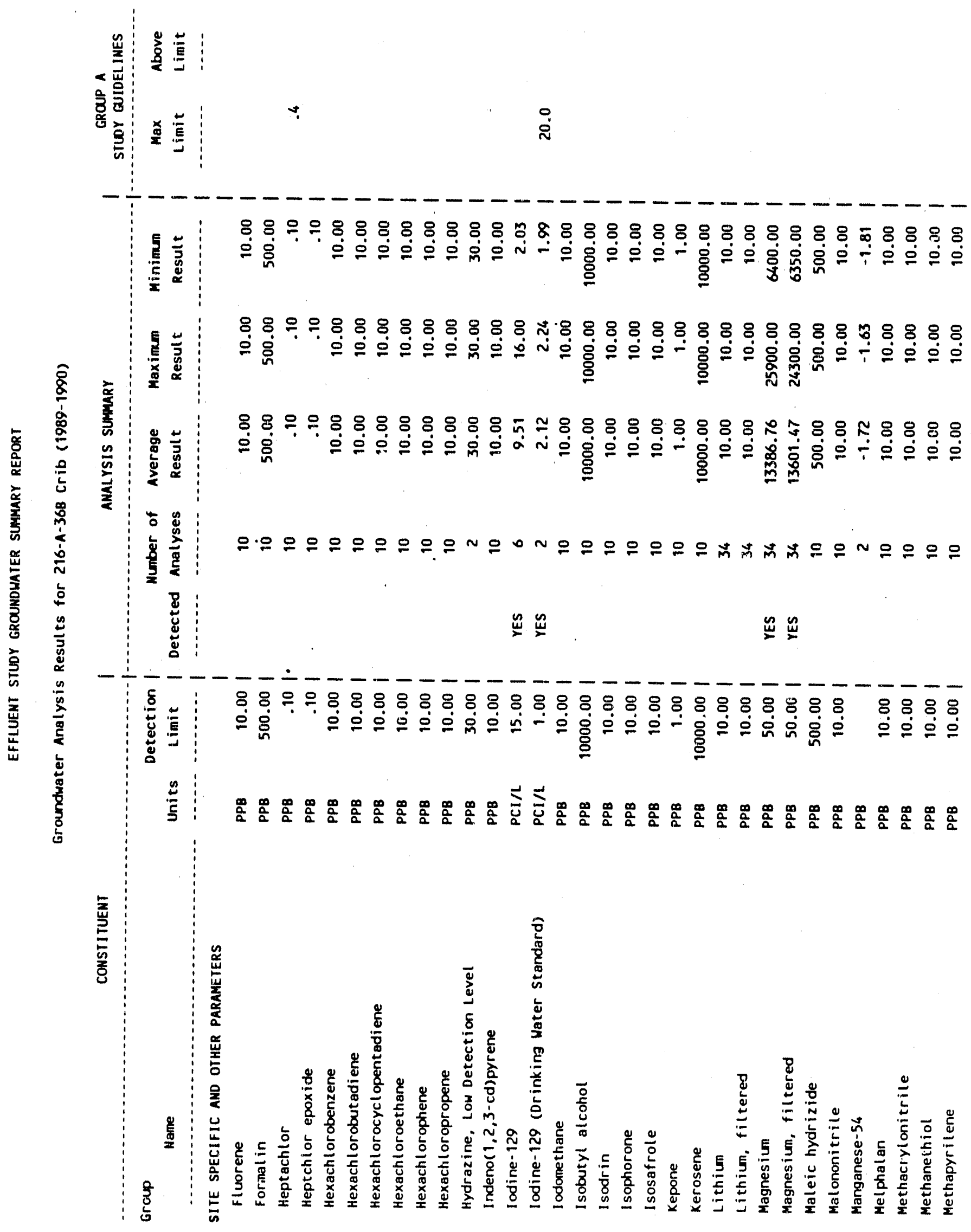


WHC-EP-0366

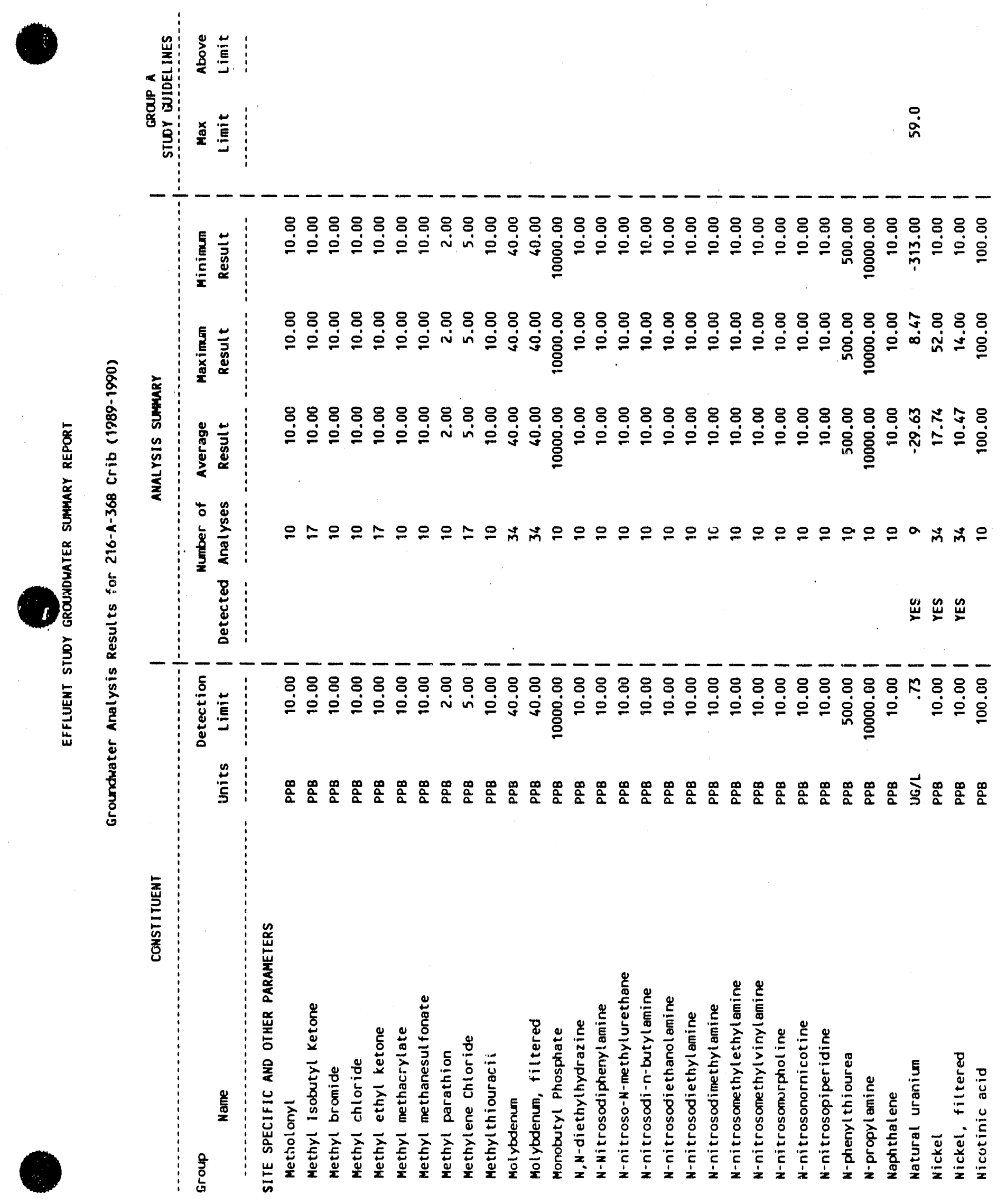




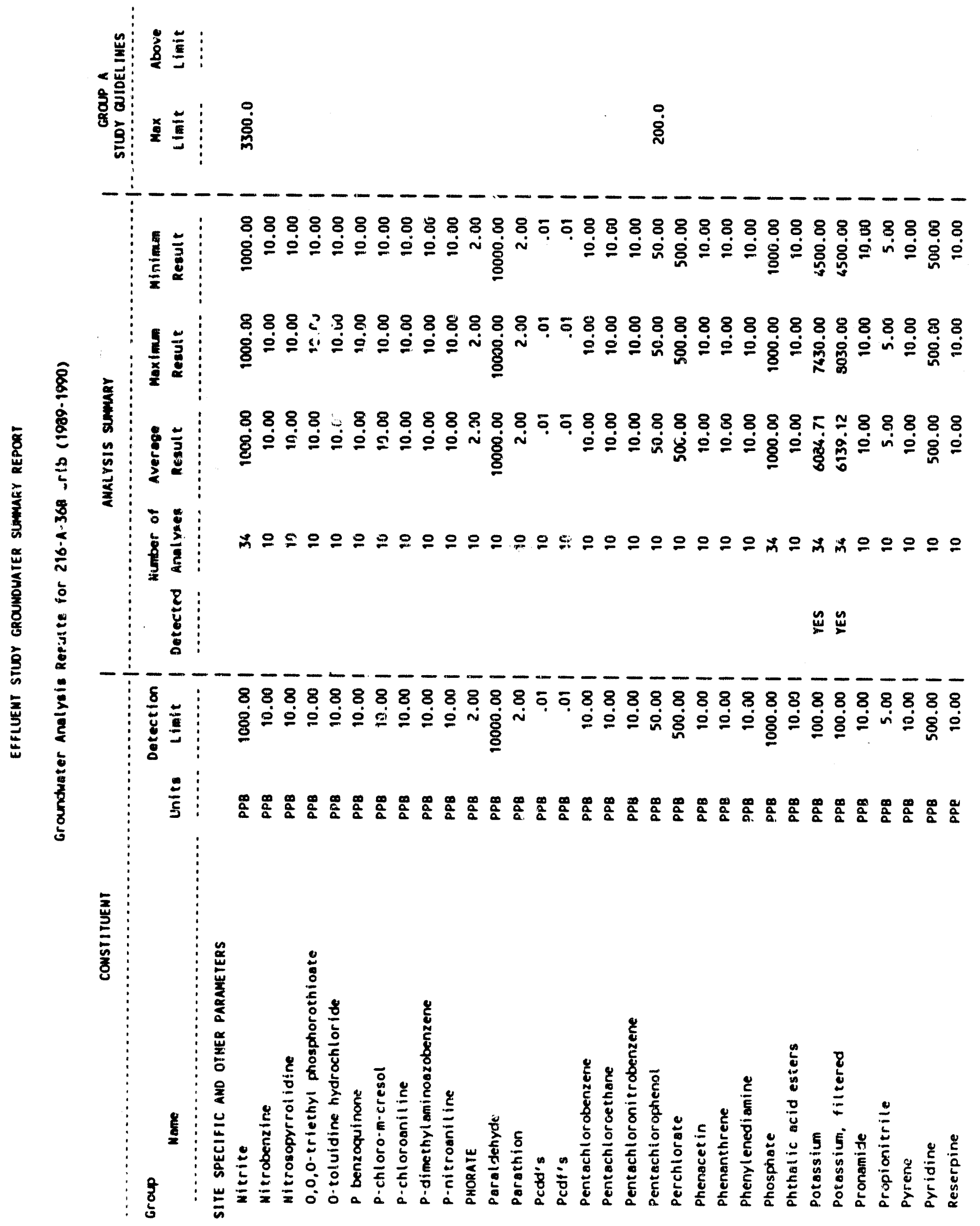


WHC-EP-0366

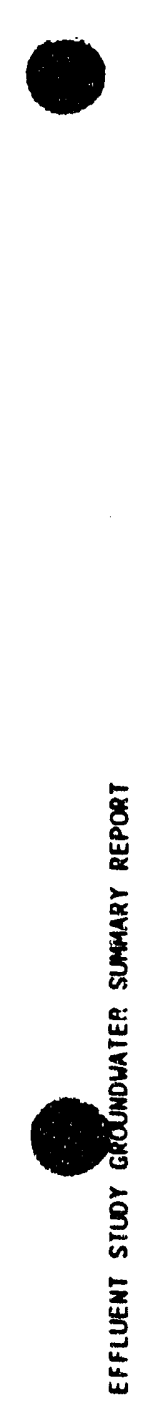

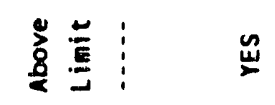

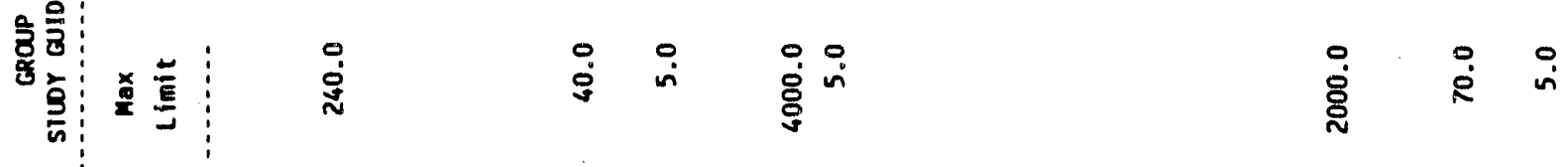

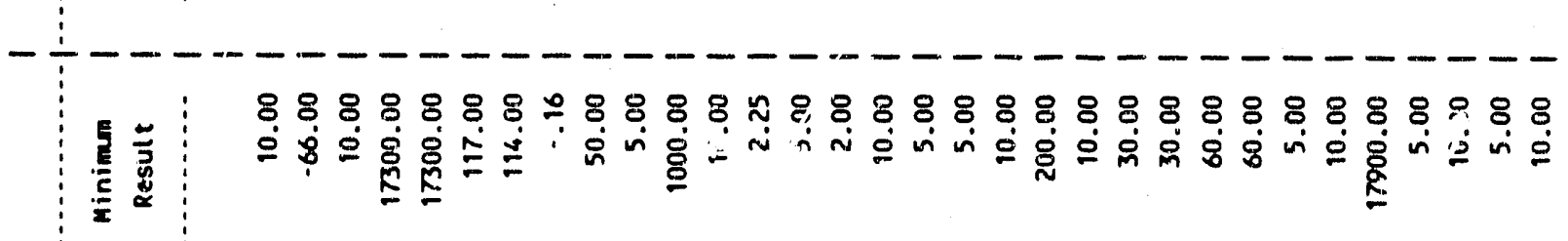

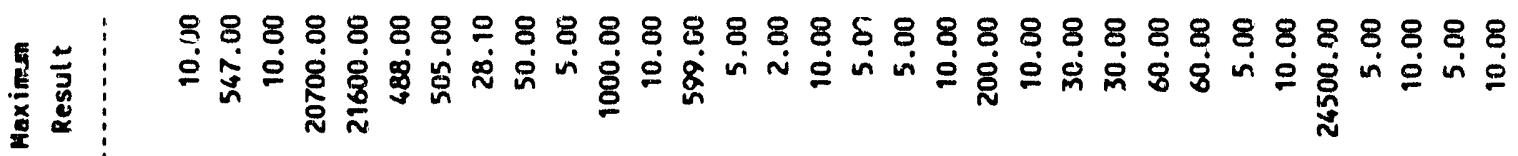

8 \%

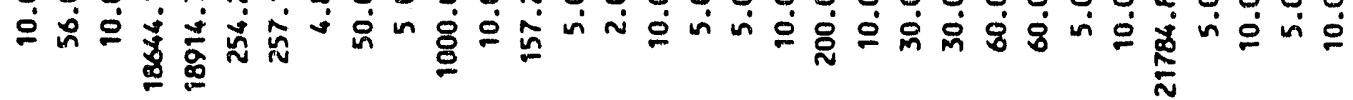

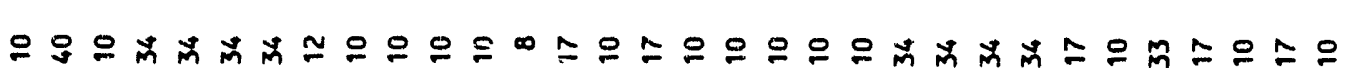

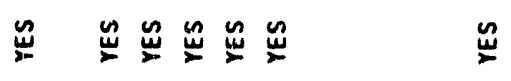

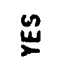

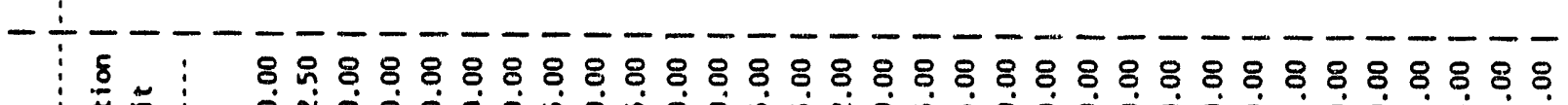

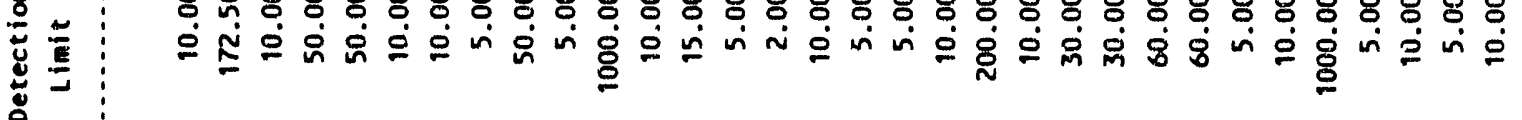

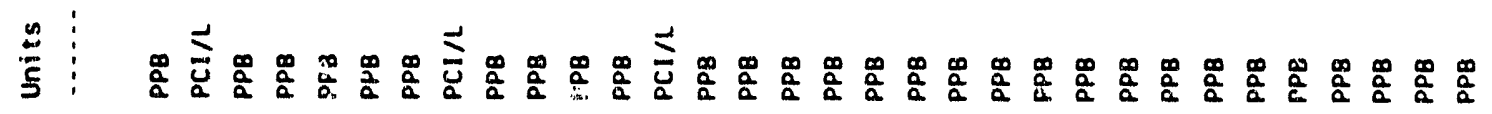

: 
WHC-EP-0366

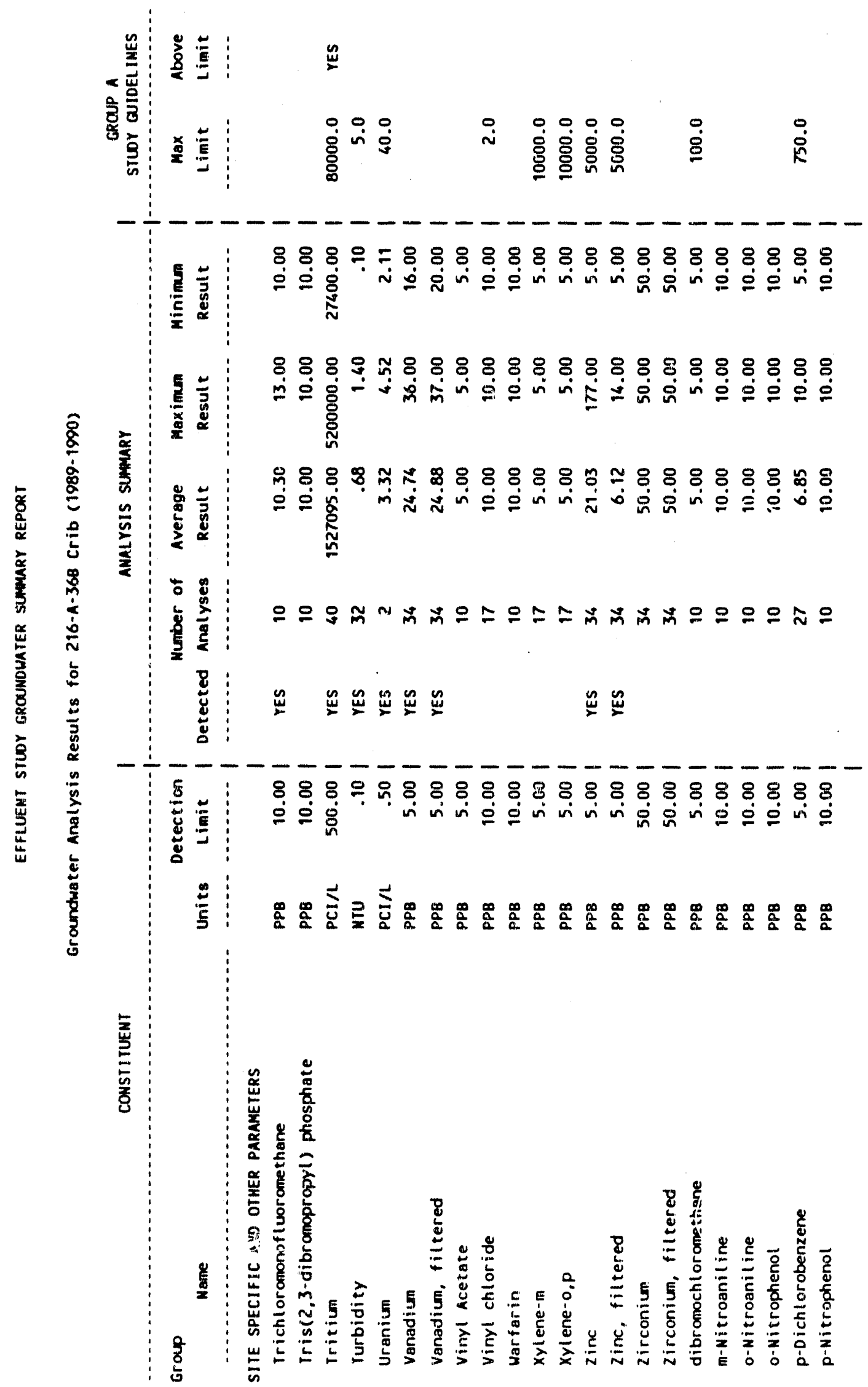



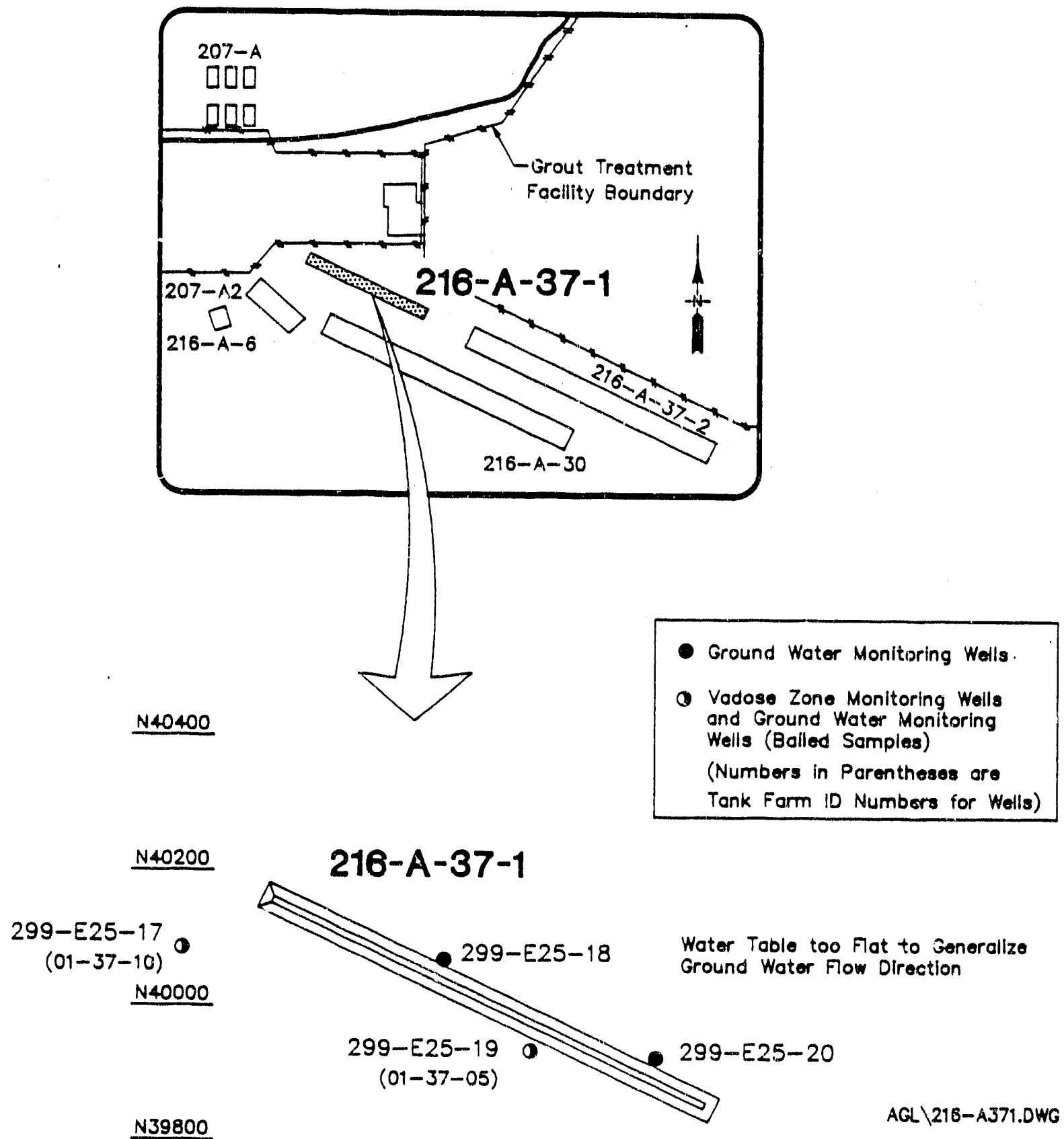

N39800

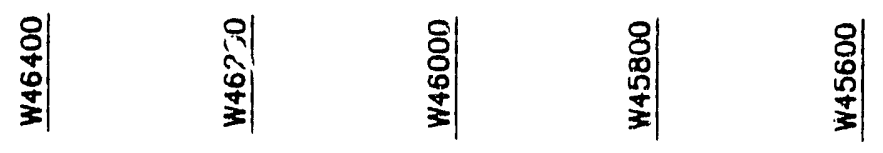

Well Location and Site Map for 216-A-37-1 Crib 
WHC-EP-0366

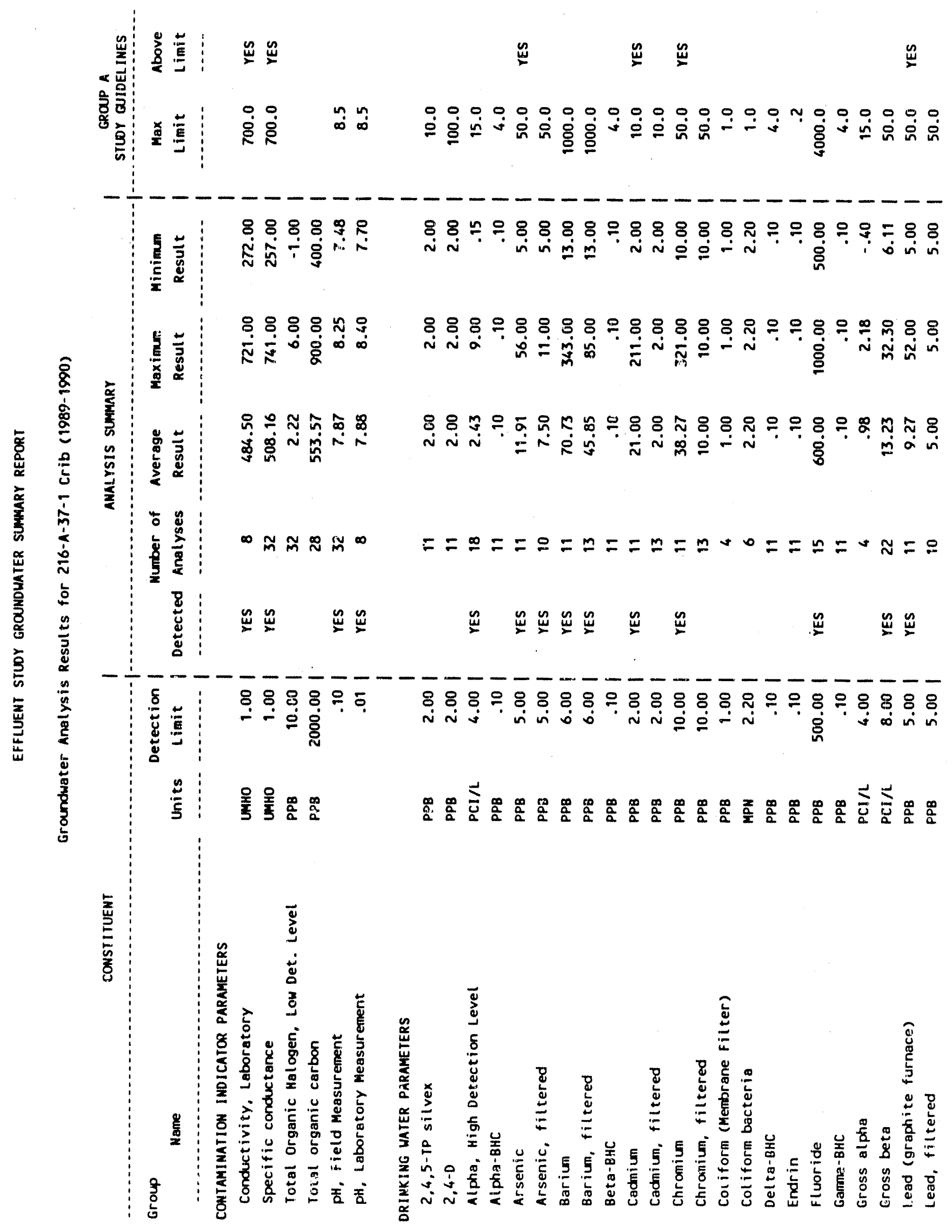


WHC-EP-0366

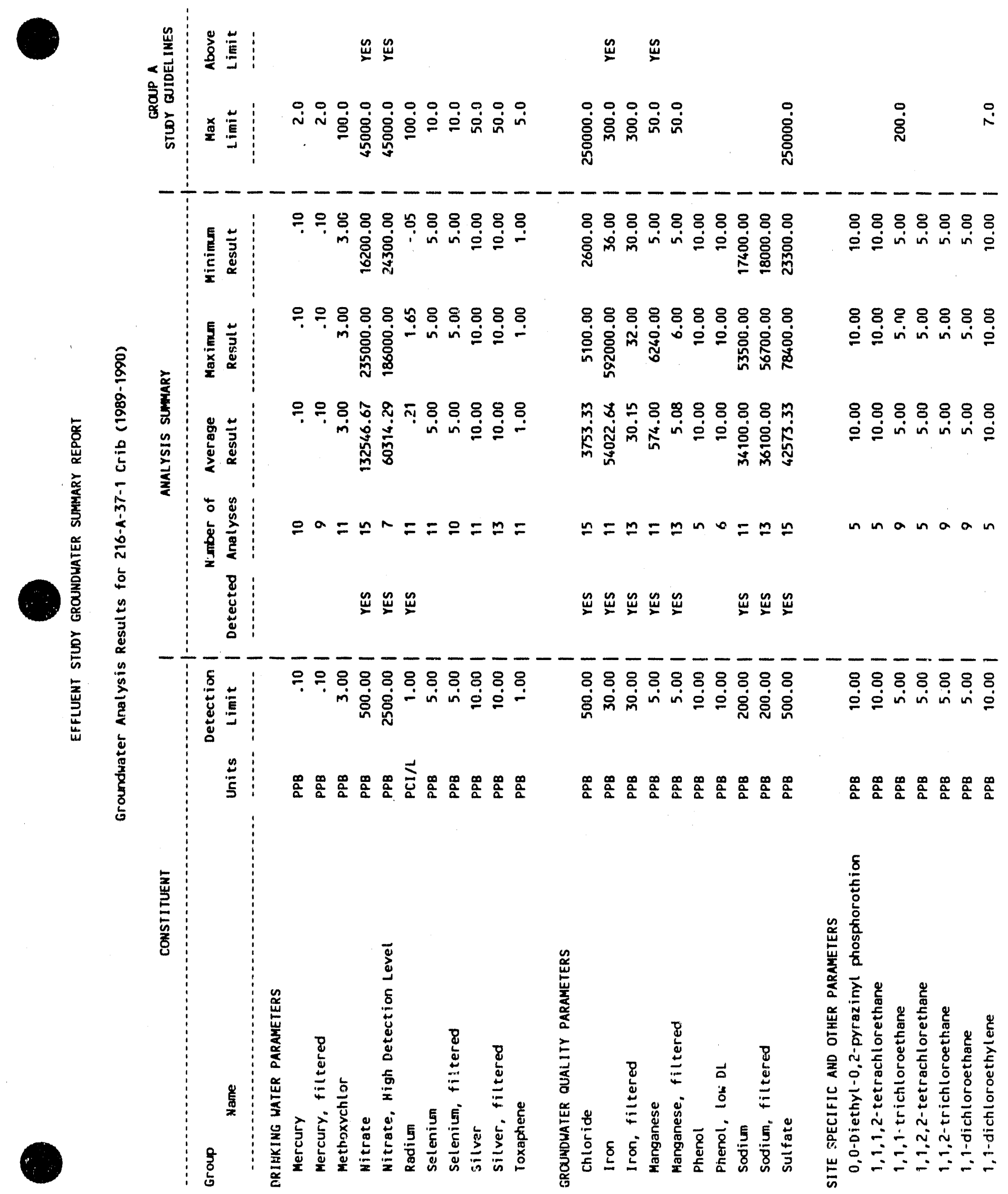


WHC-EP-0366
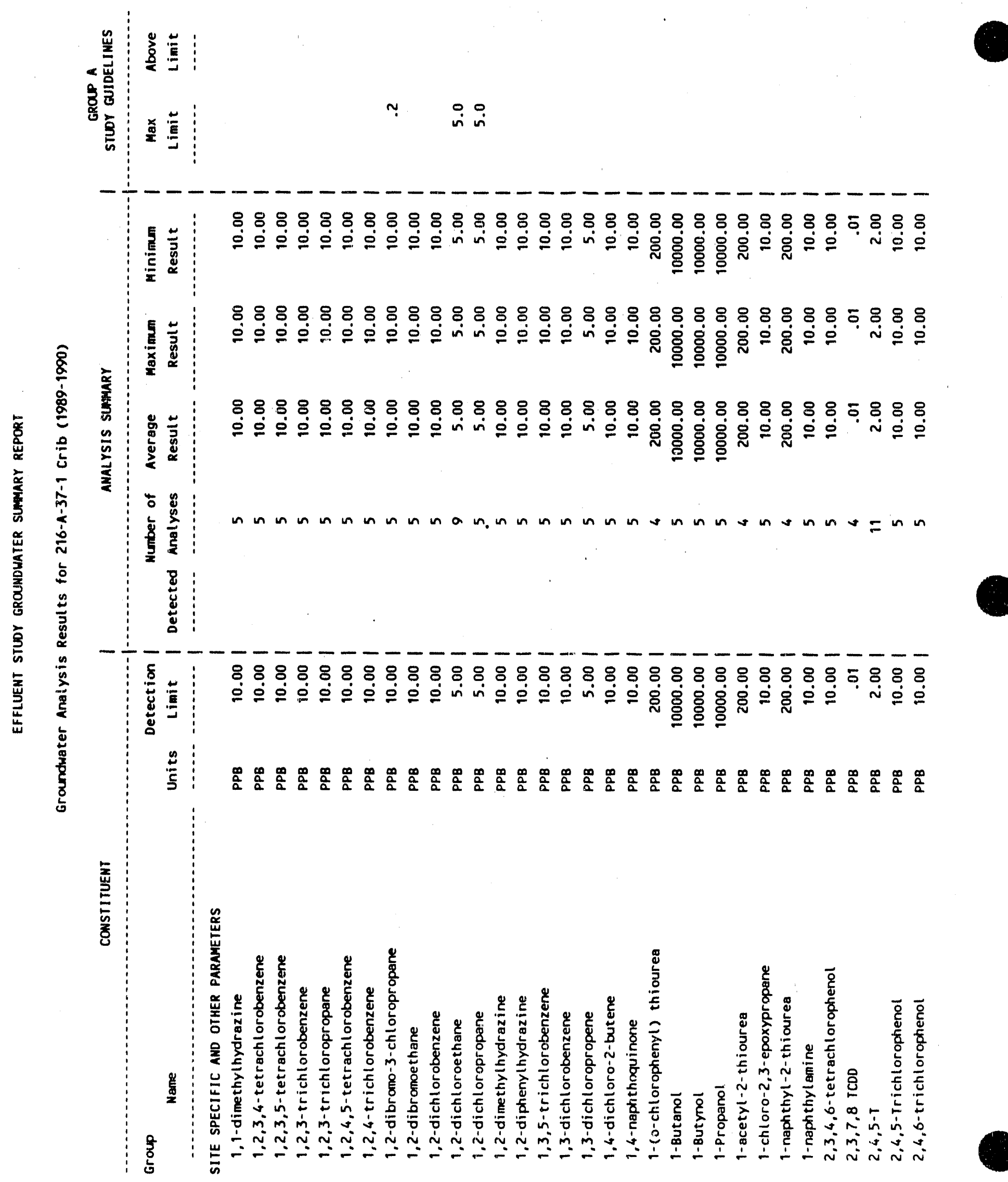
WHC -EP-0366

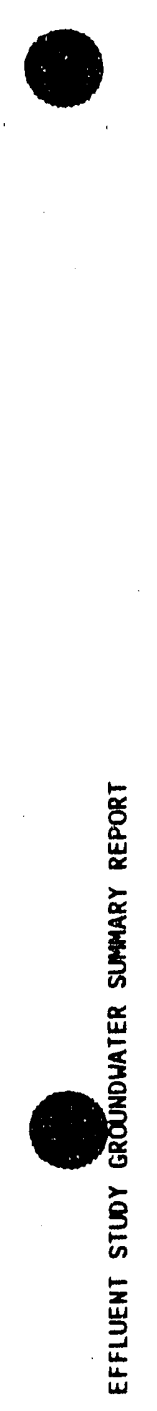

\section{要:}

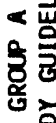

希蔮

铻言

88888888888888888888888888888888

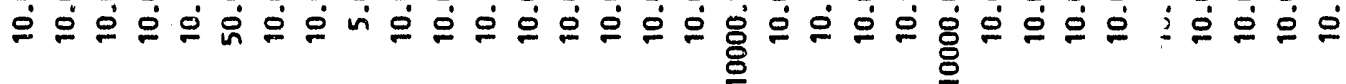

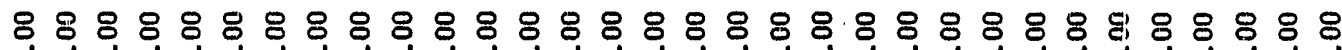

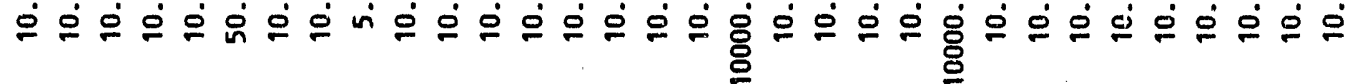

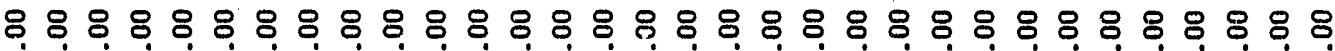
ஹ் 兽

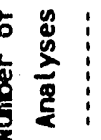

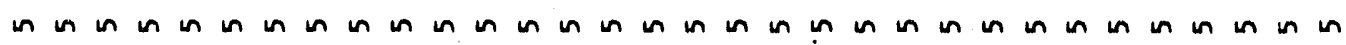
胥

8888888.888888888888880888088808

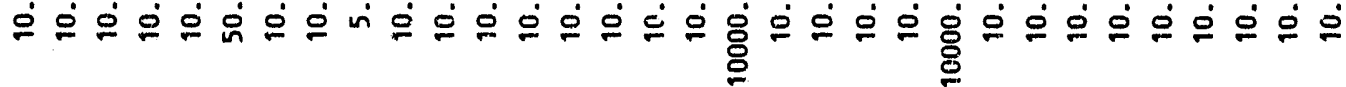

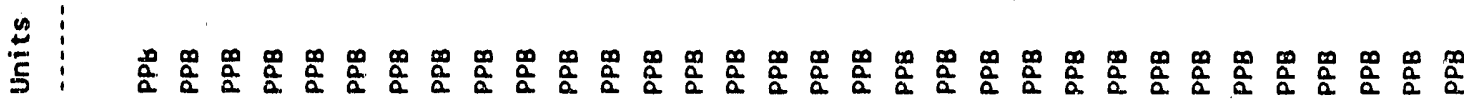

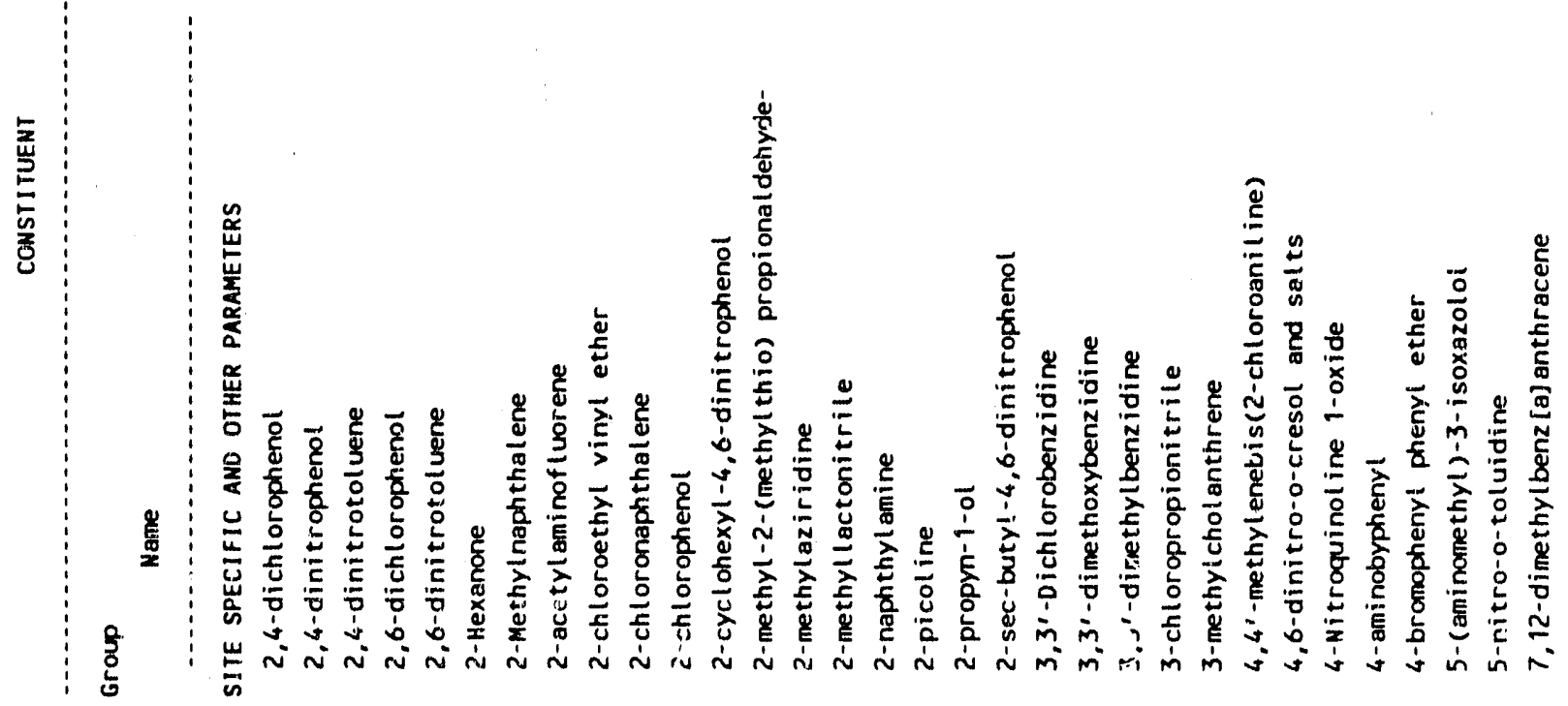


WHC-EP-0366

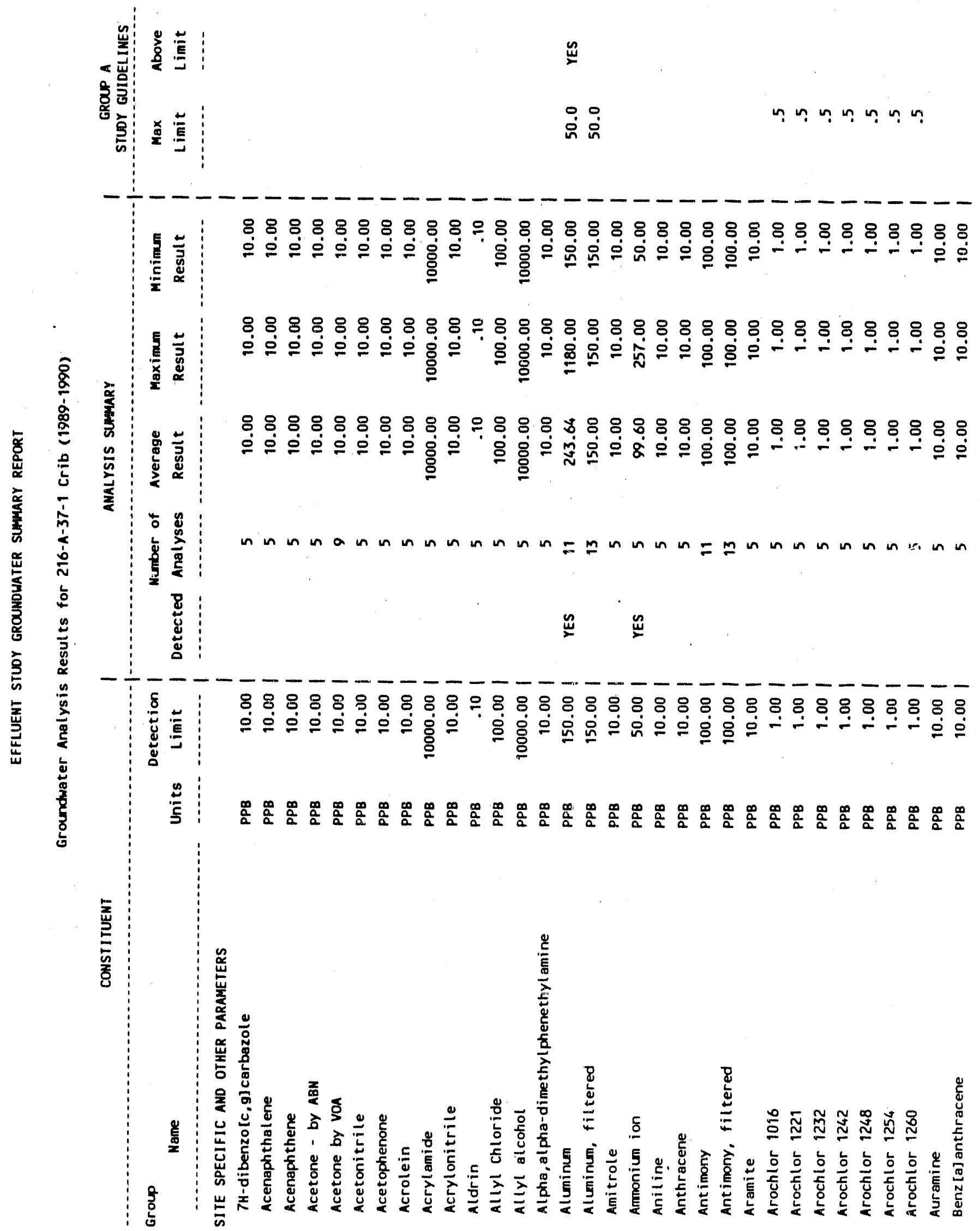


WHC-EP-0366

\section{薏}

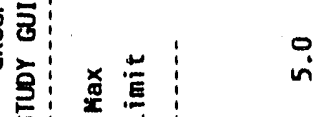

官官

88888888888888888888888888888888

产

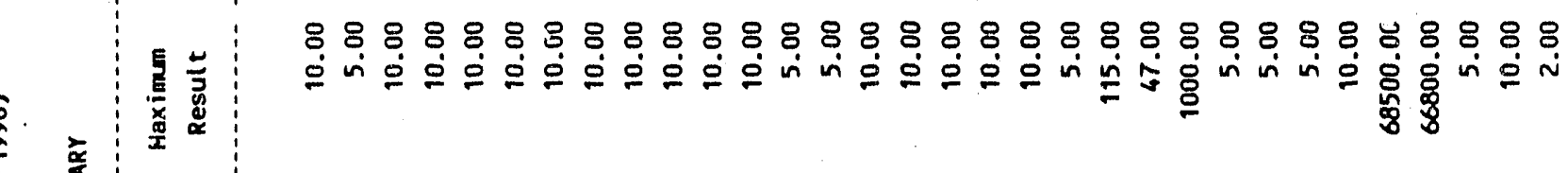

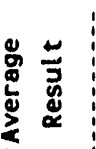

88888888888888888888 స த்

\begin{tabular}{l:|l}
4 \\
\hline
\end{tabular}

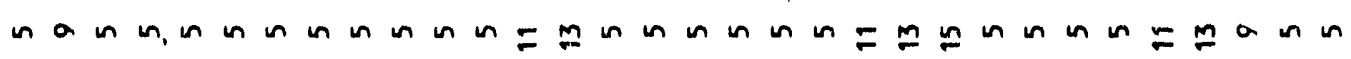

点

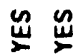

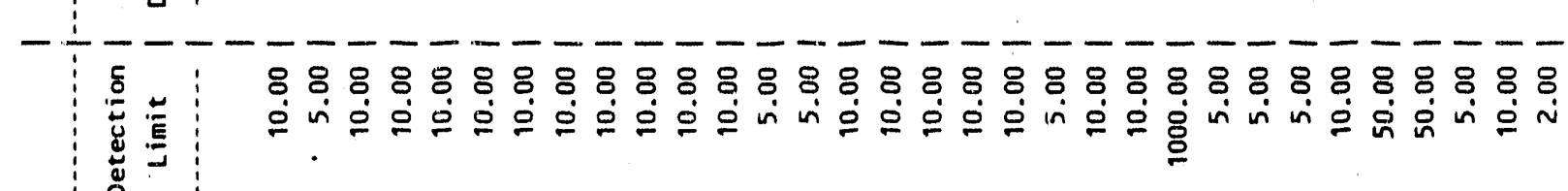

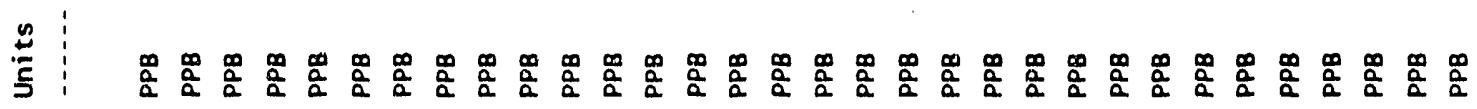

马

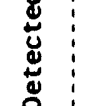


WHC-EP-0366

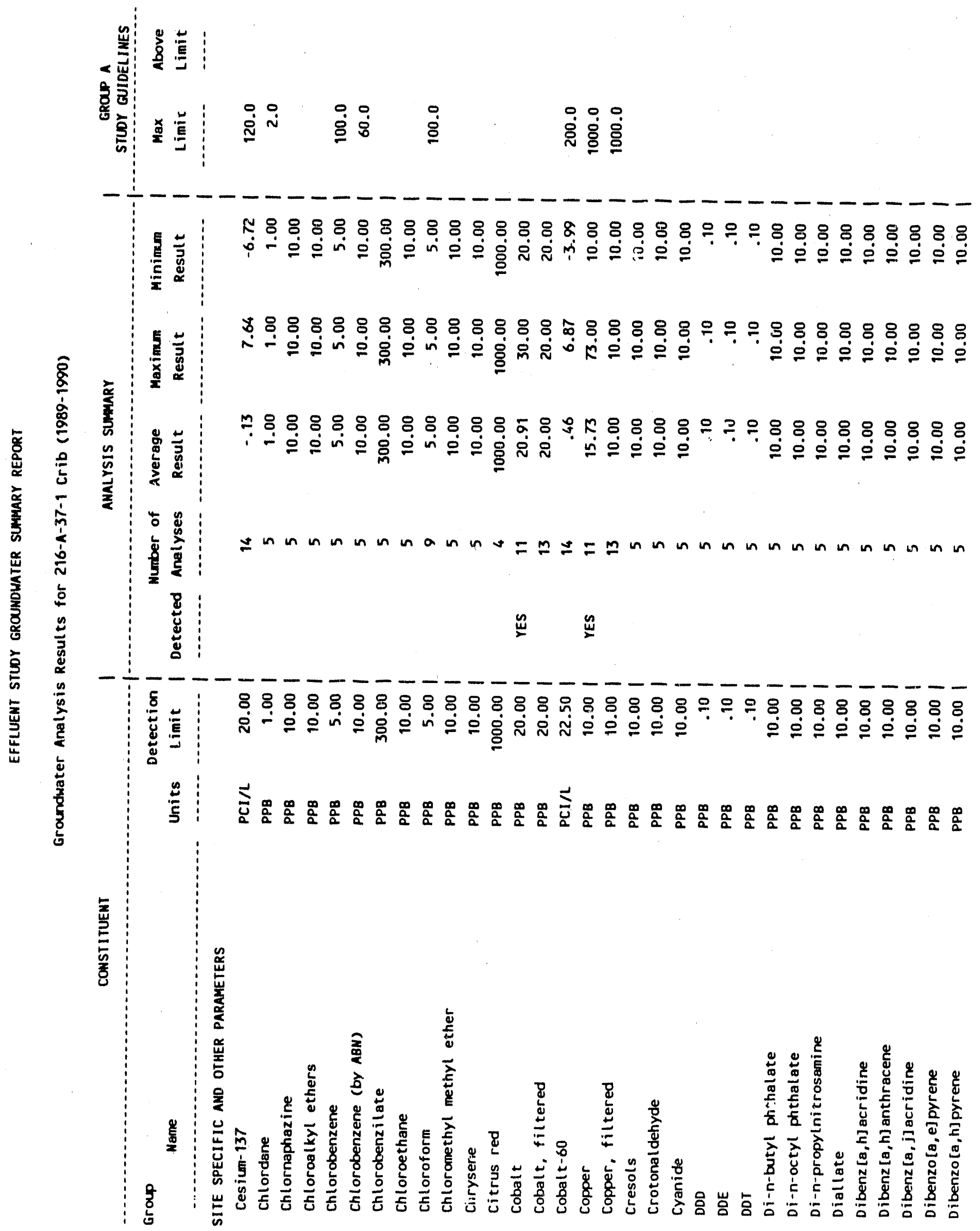




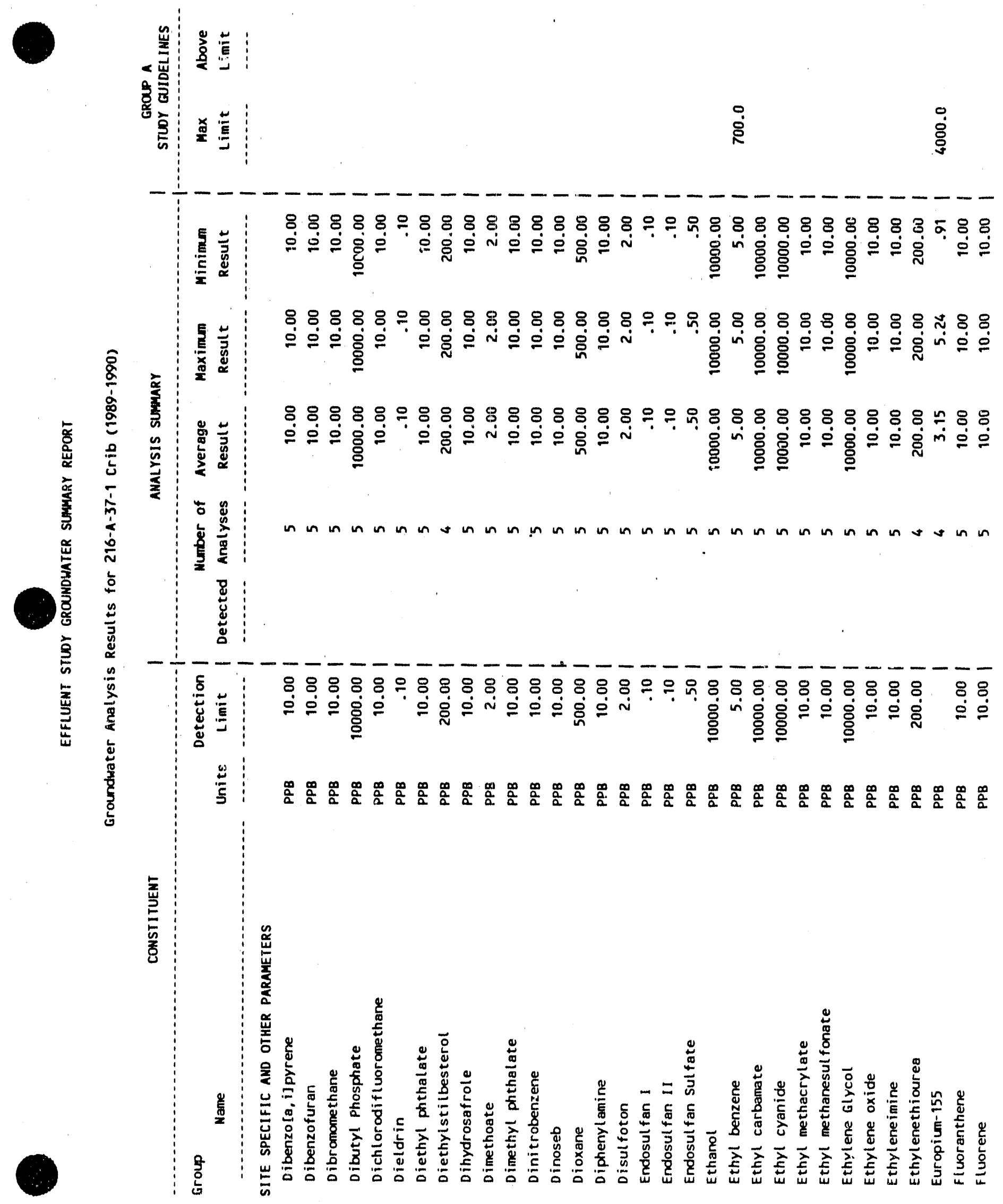


WHC-EP-0366

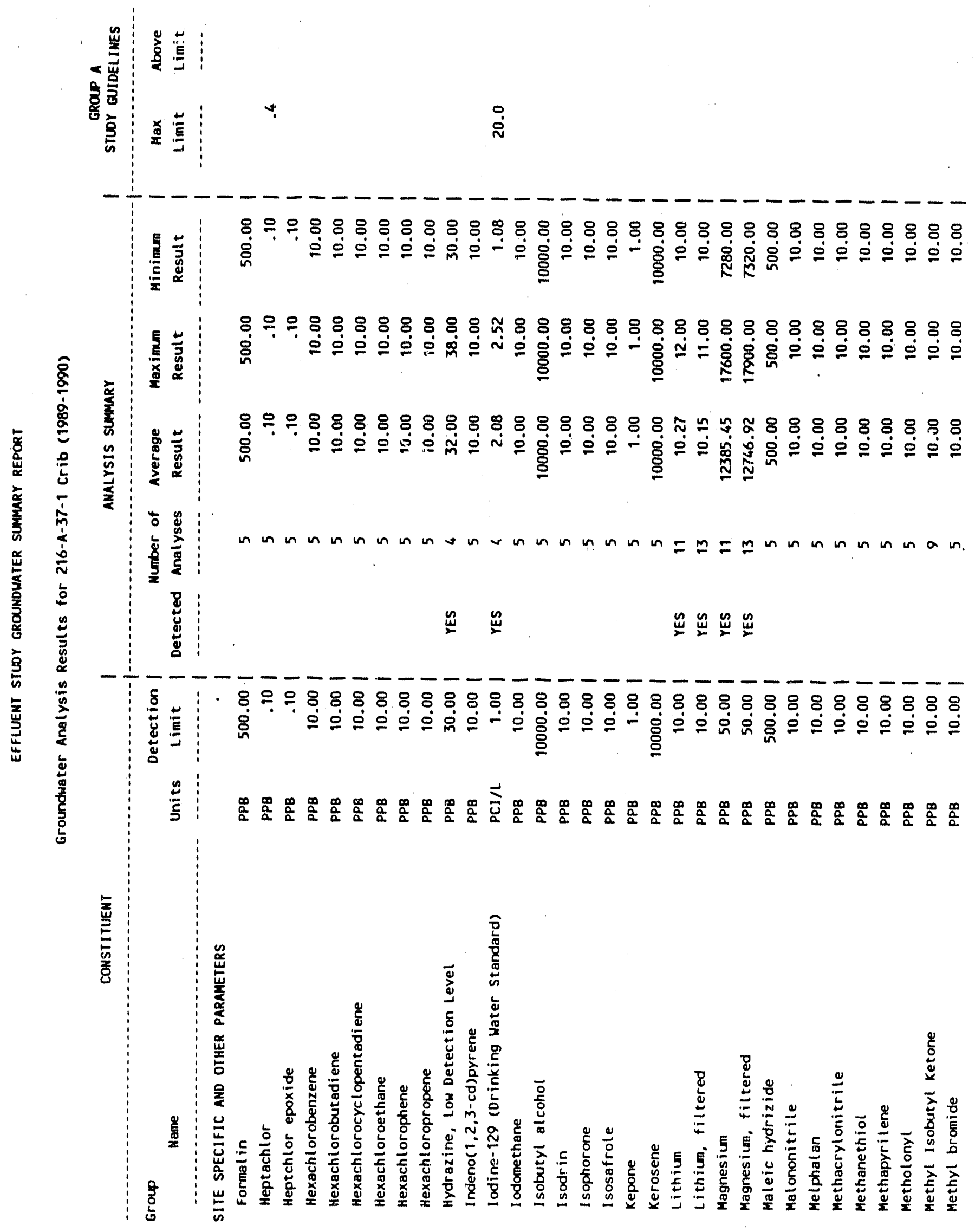




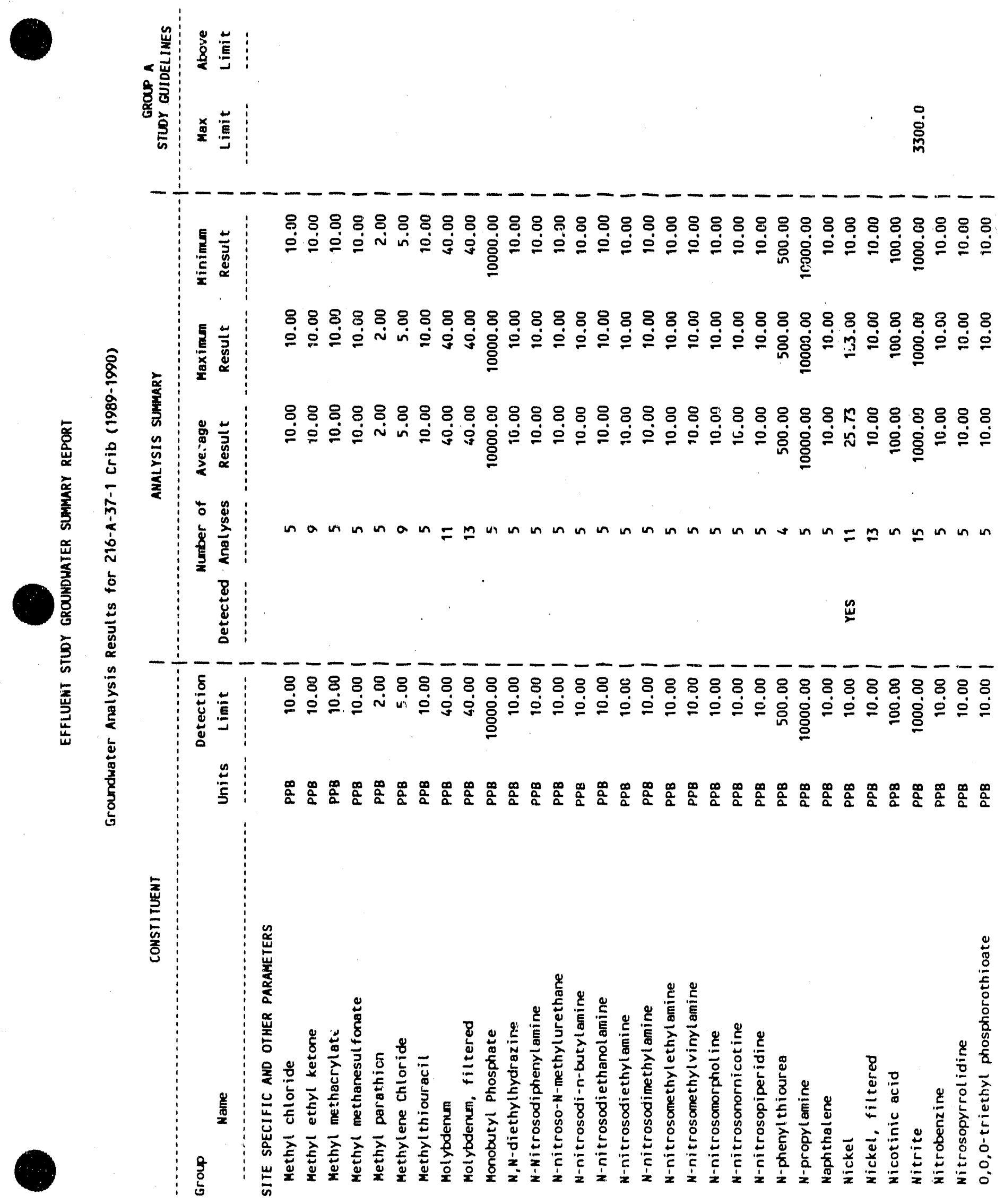


WHC-EP-0366

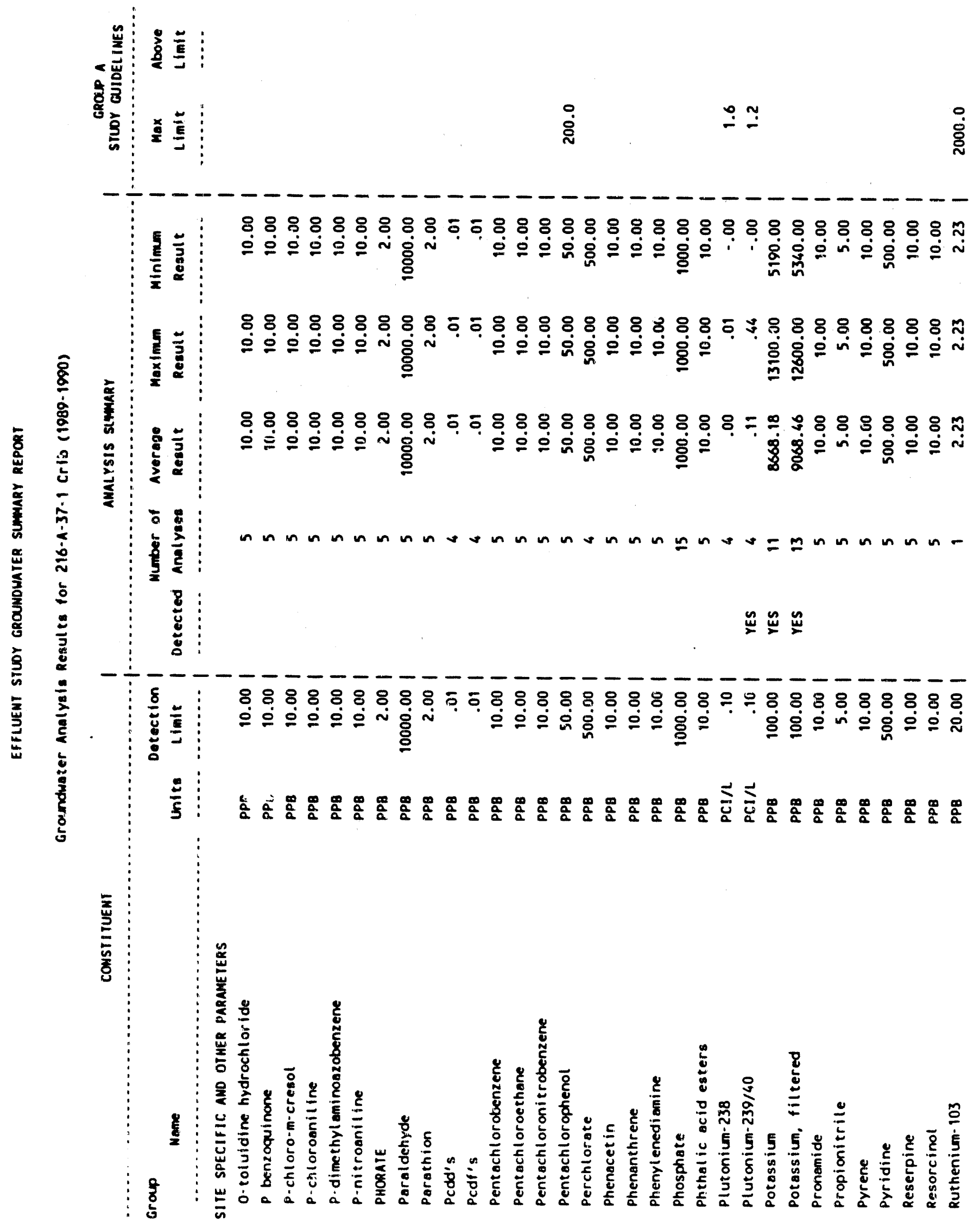


WHC - EP-0366

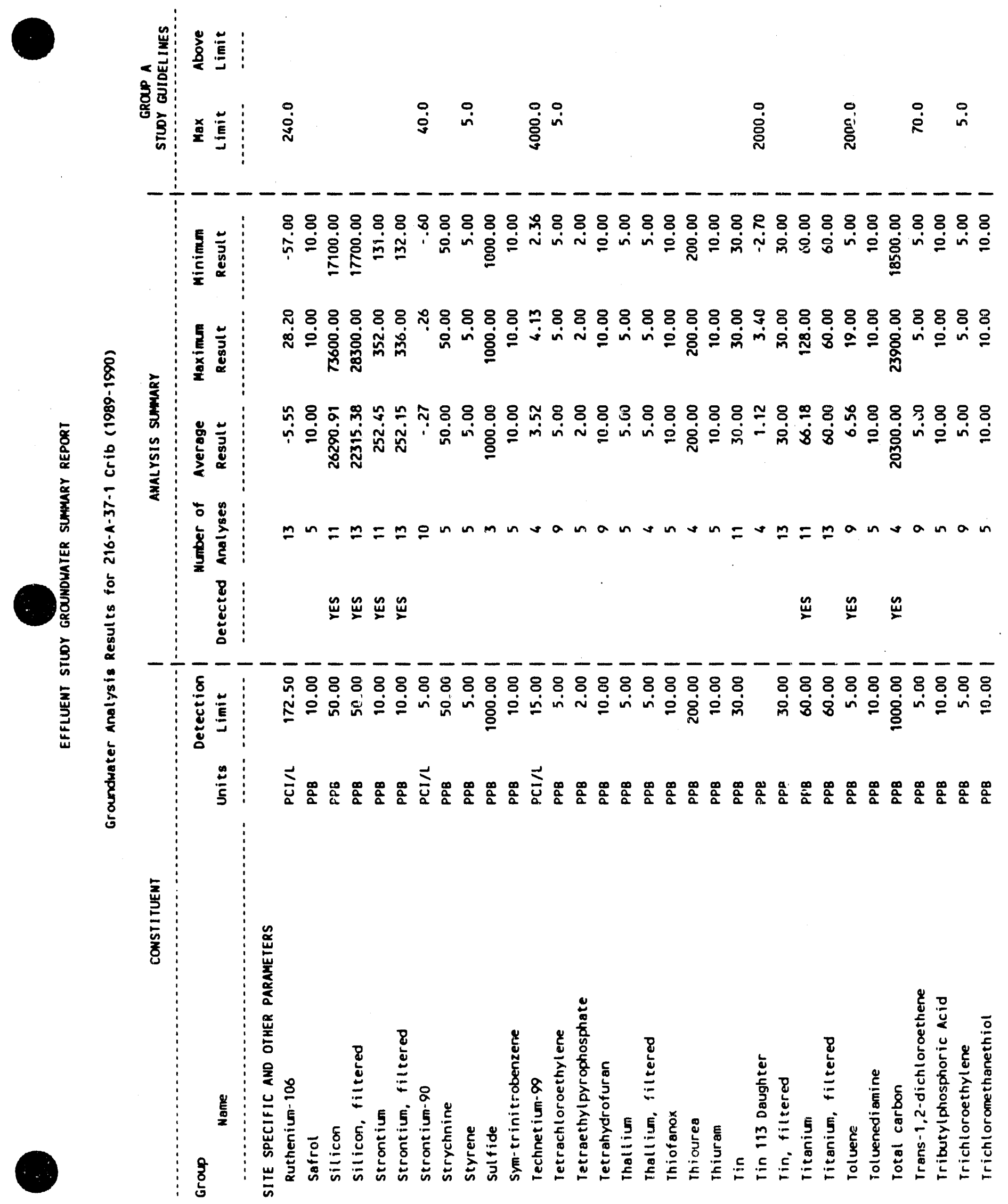


WHC-EP-0366

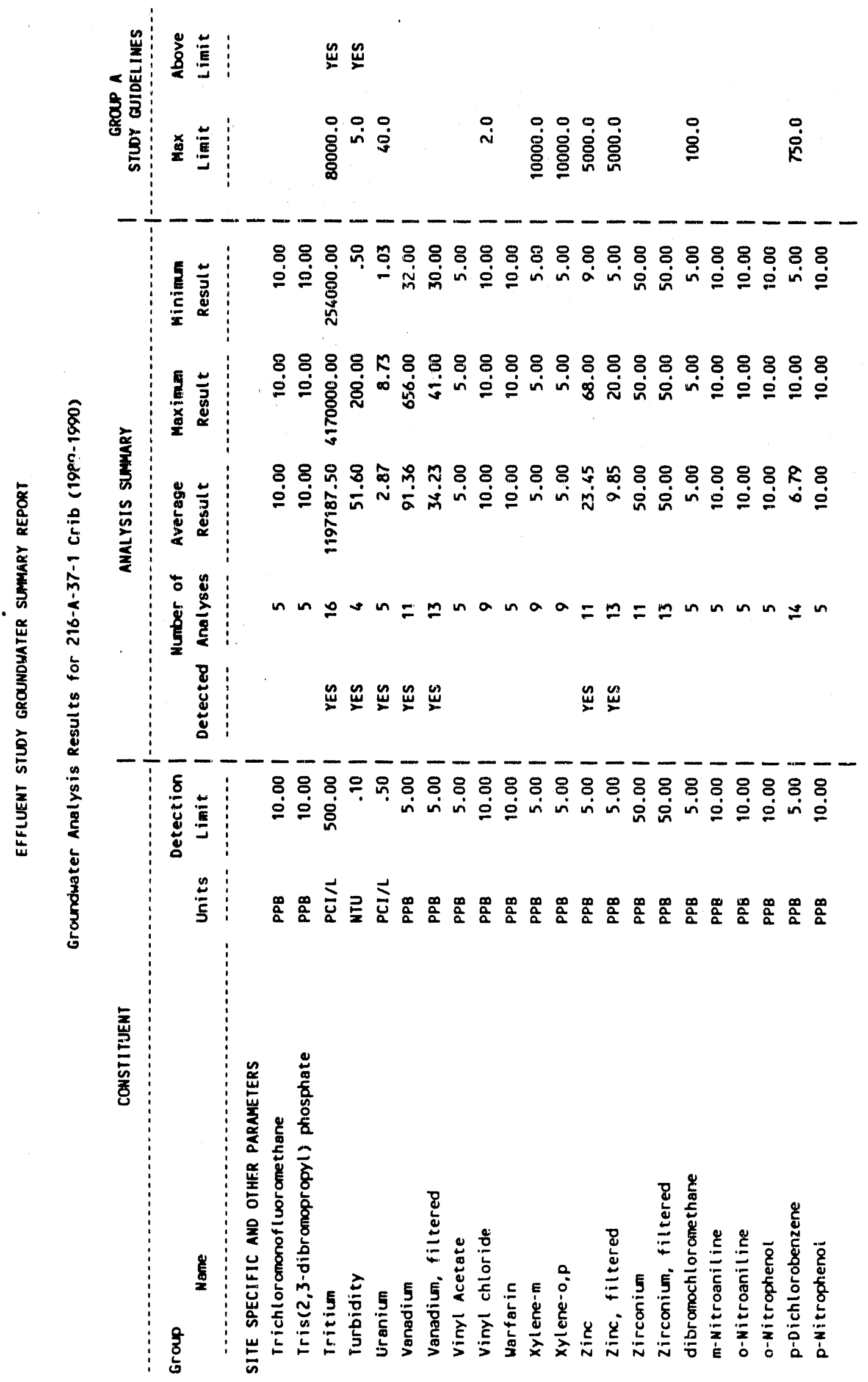




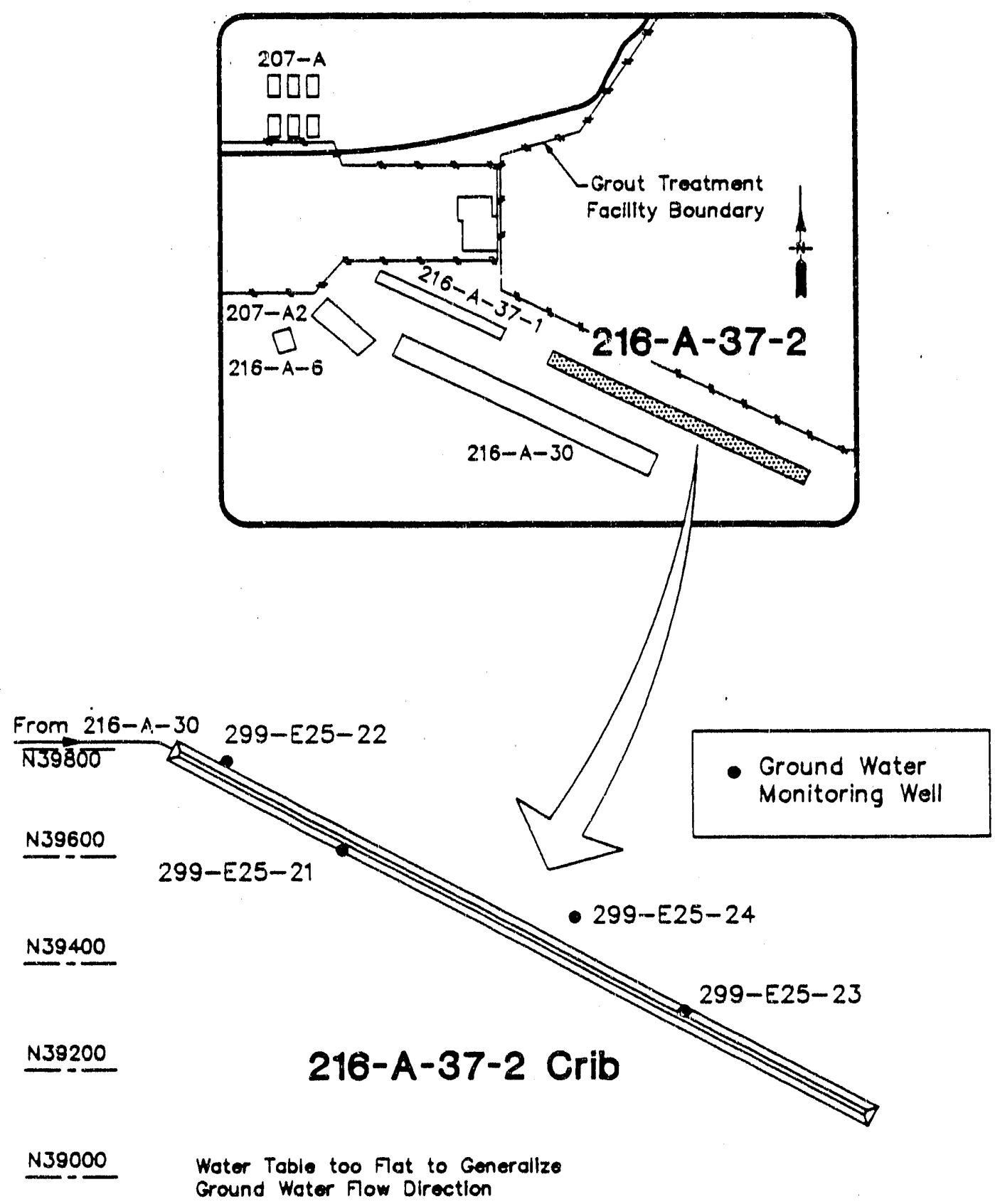

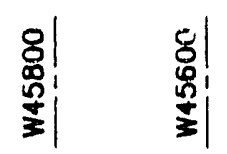
인
윯|
인
0
0
$\vdots$
$\vdots$
$\vdots$
AGL \216-A372.DWG 
WHC -EP-0366

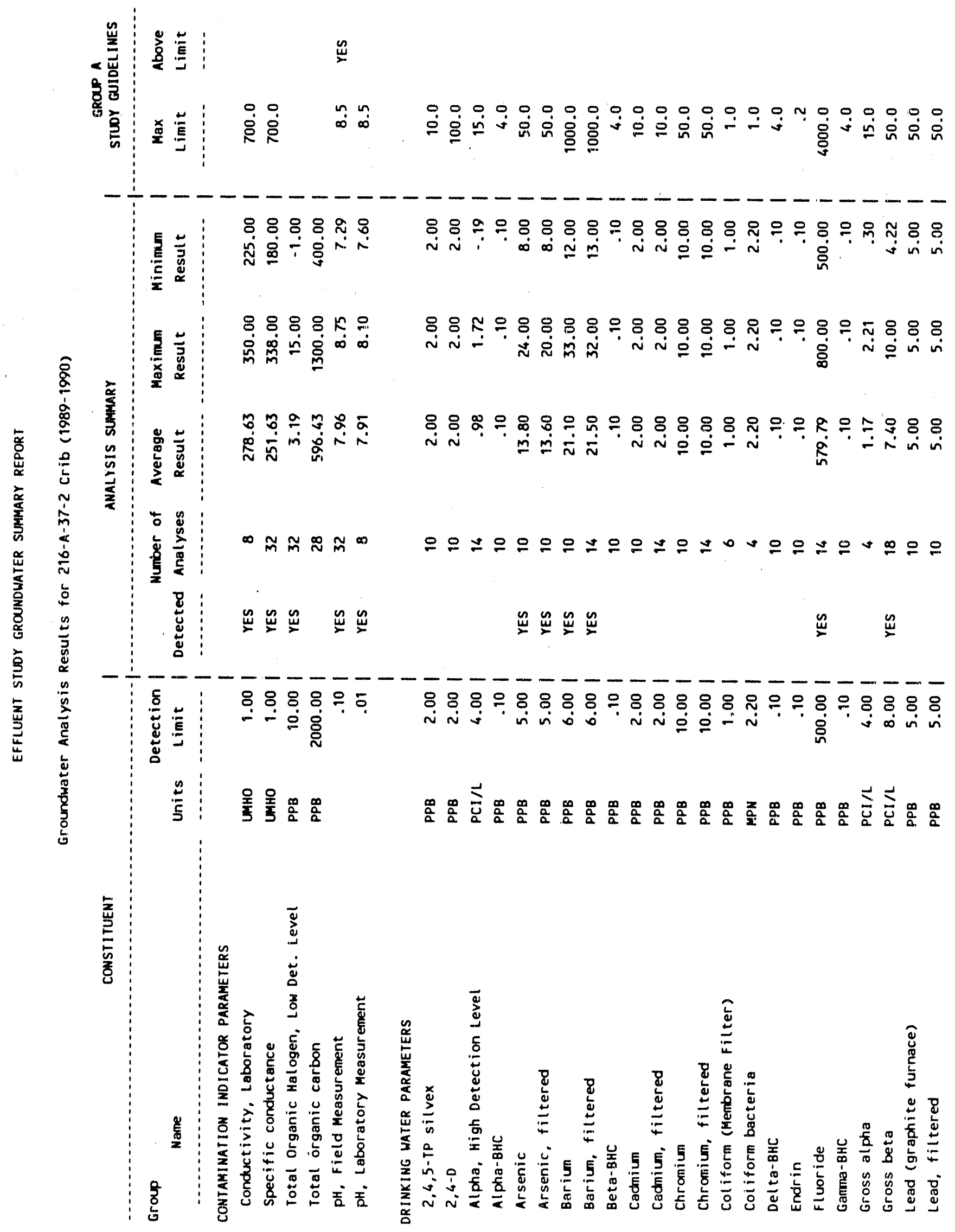


WHC-EP-0366

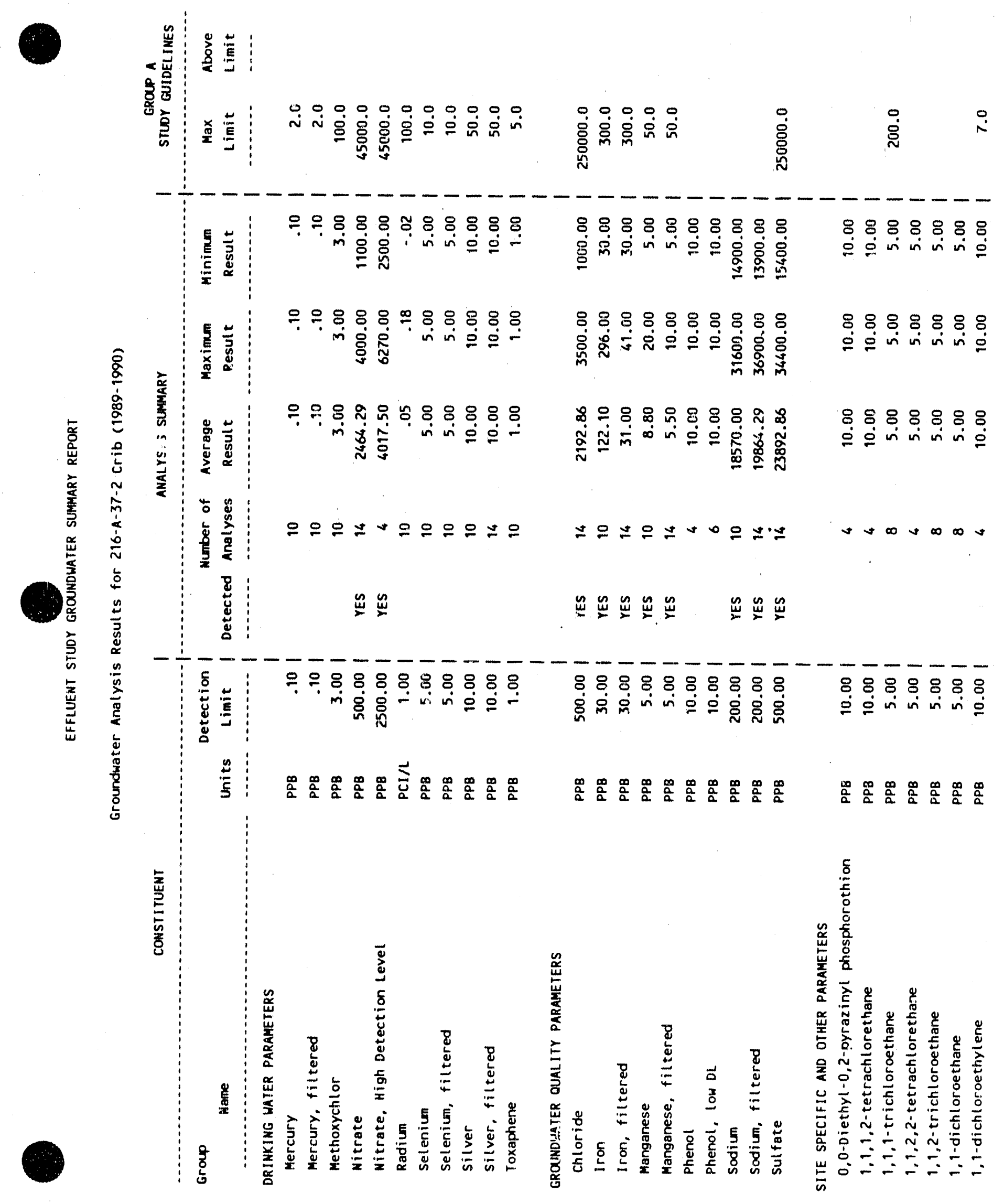


WHC -EP-0366

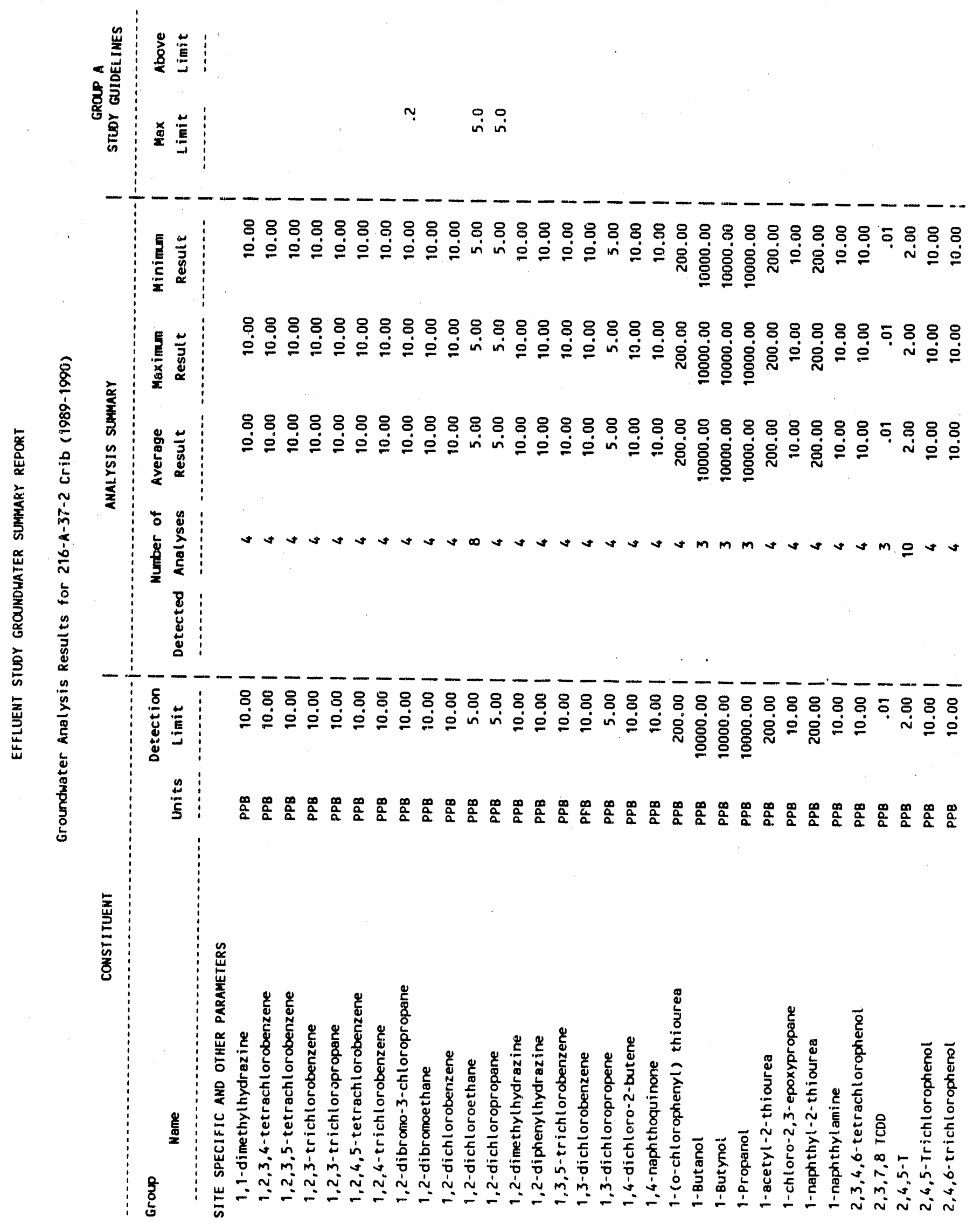


WHC-EP-0366

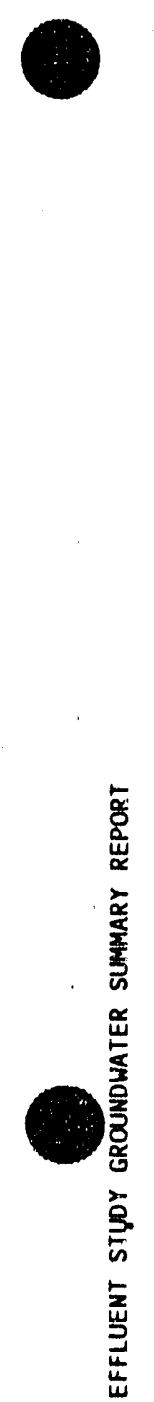

葛:

웅

总 $-\frac{i}{i}$

产

88888888888888888888888888888880

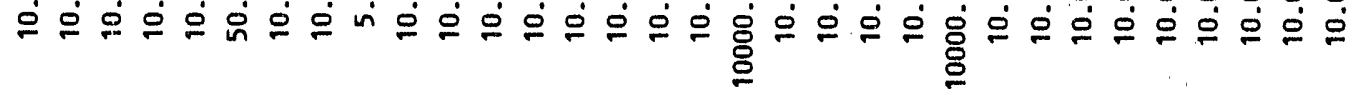

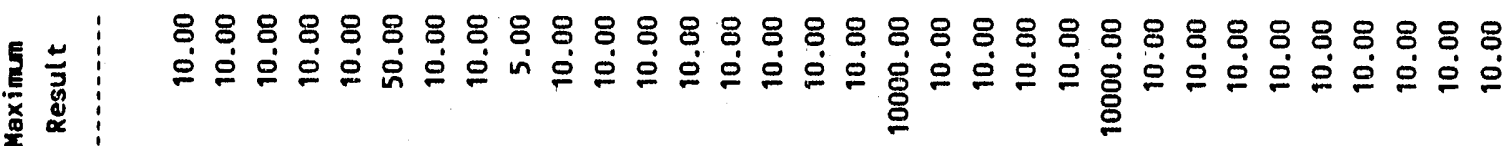

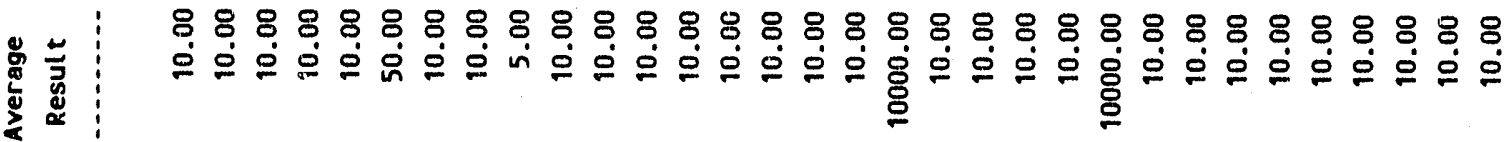

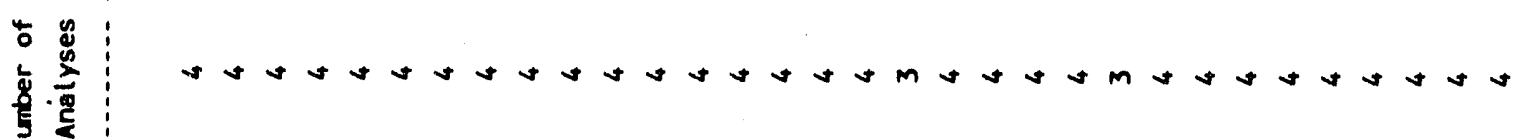

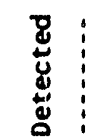

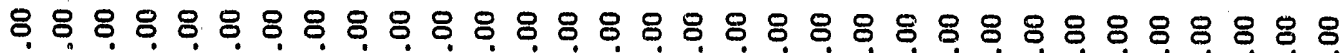

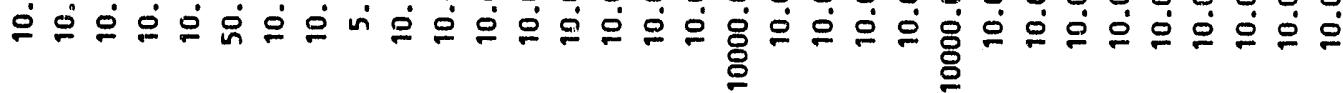

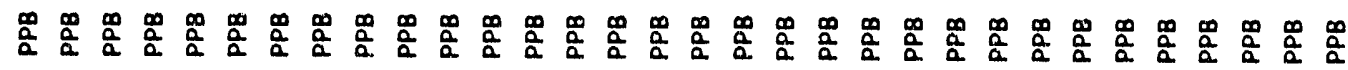

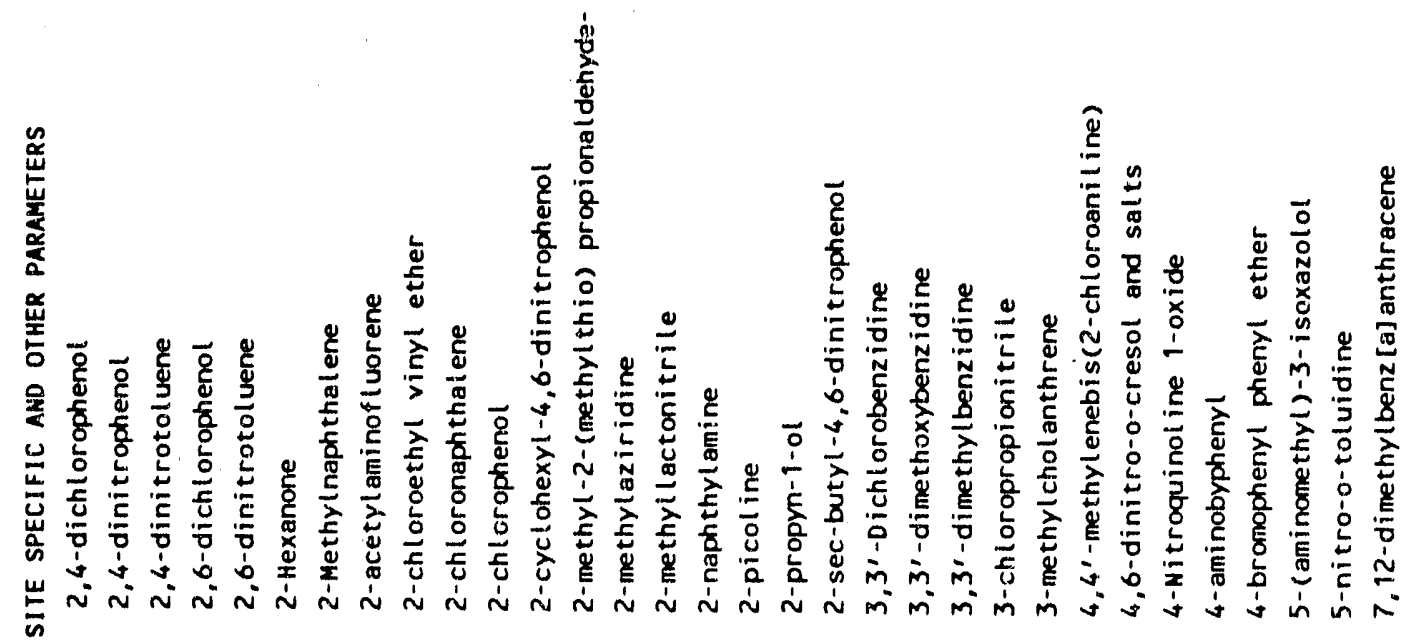


WHC-EP-0366

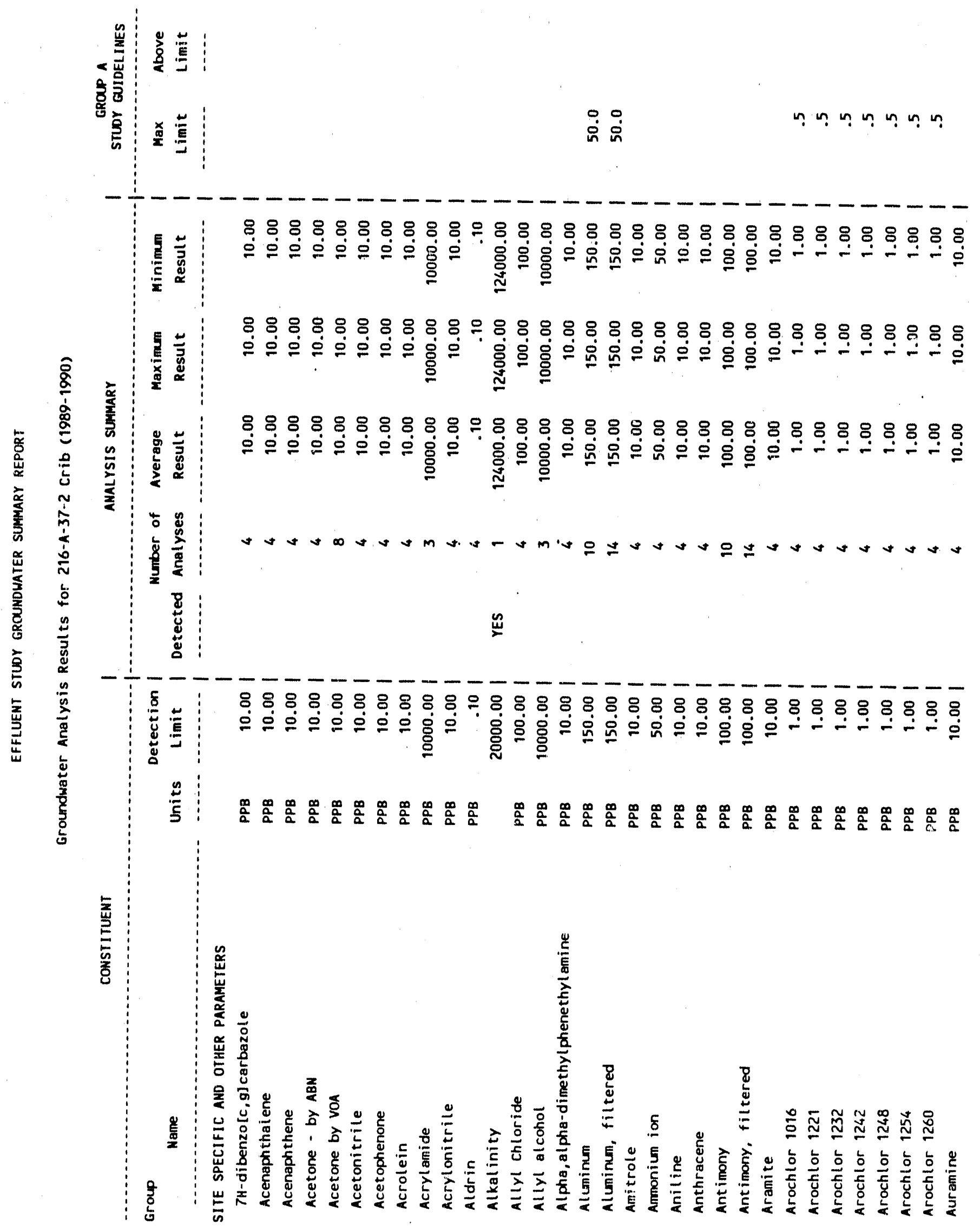


WHC-EP-0366

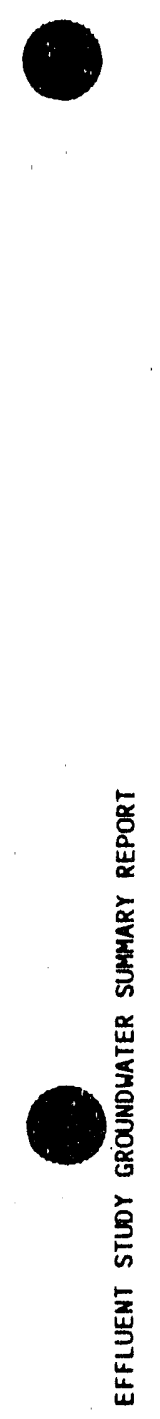

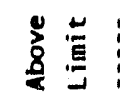

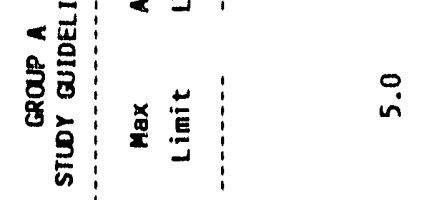

$\dot{\dot{g}} \dot{\dot{g}}$

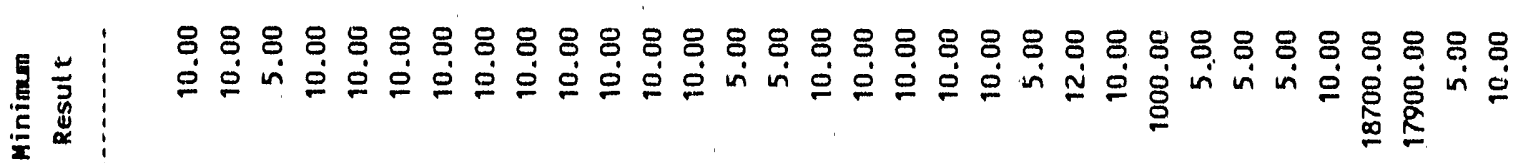

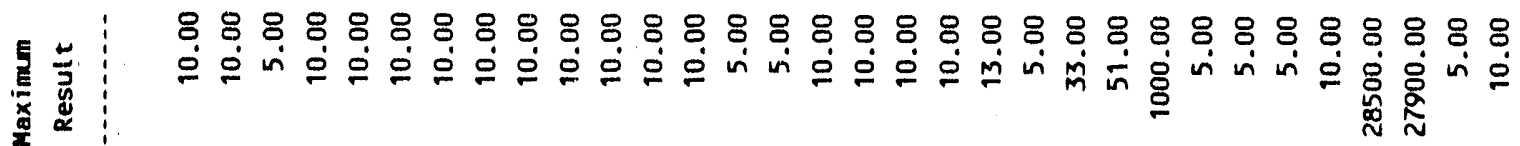

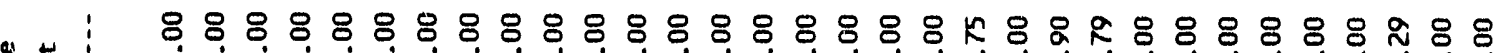

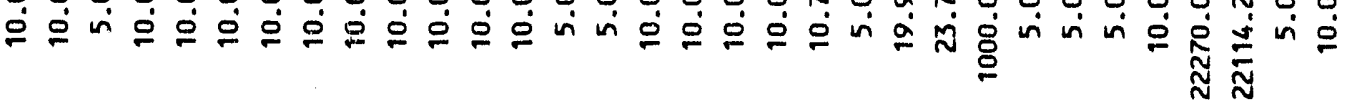

范

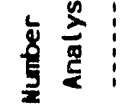

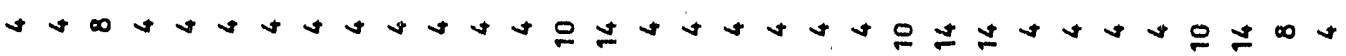

:

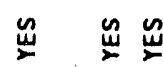

$\stackrel{\mathscr{m}}{\dddot{m}}$

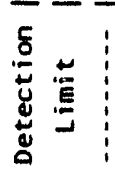

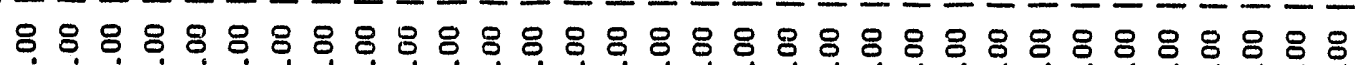

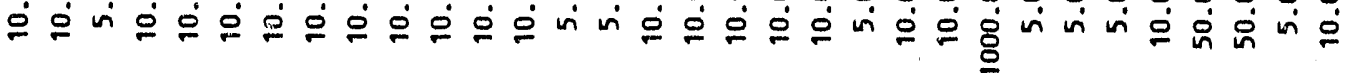

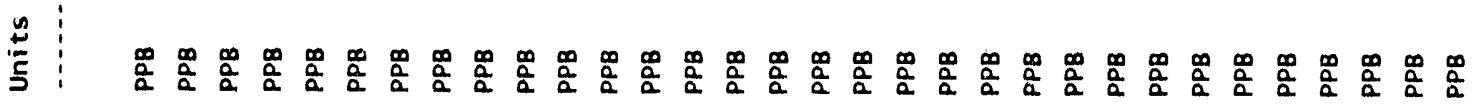

密

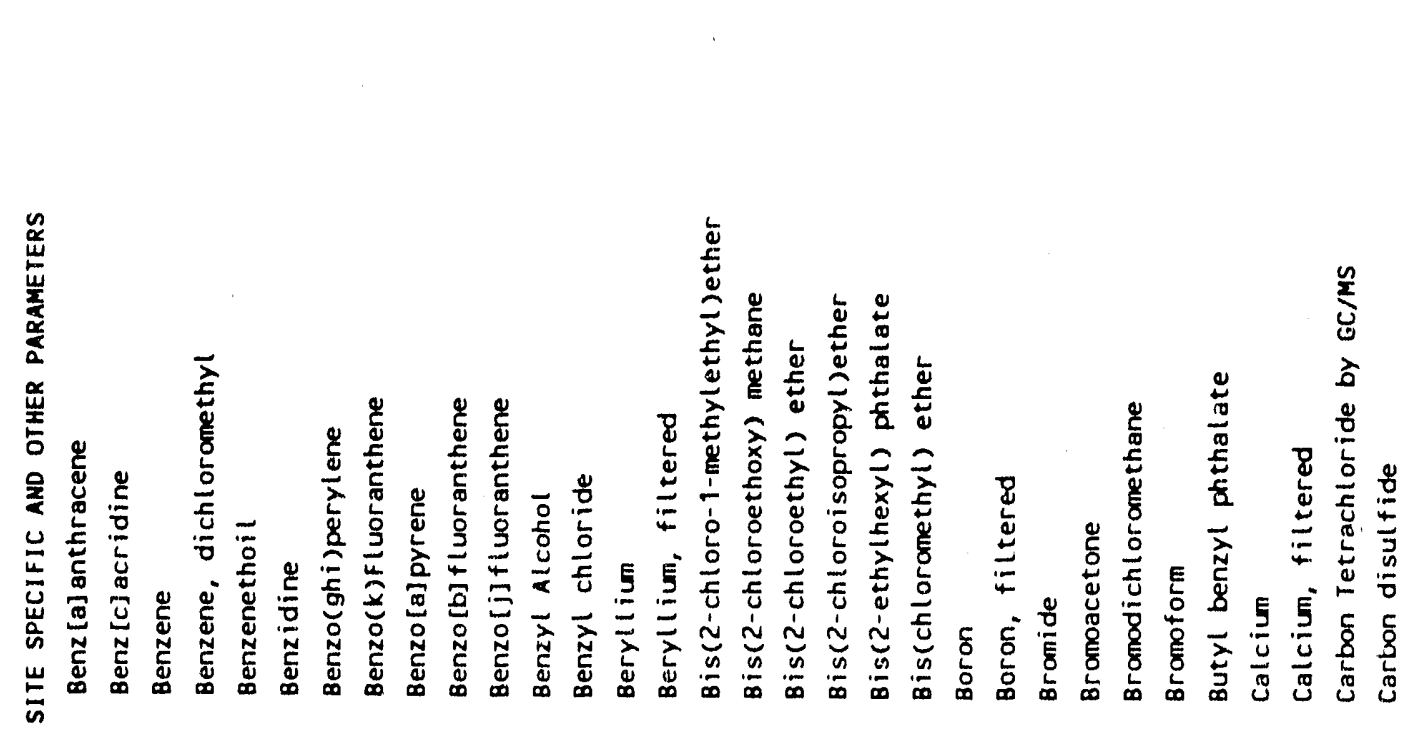




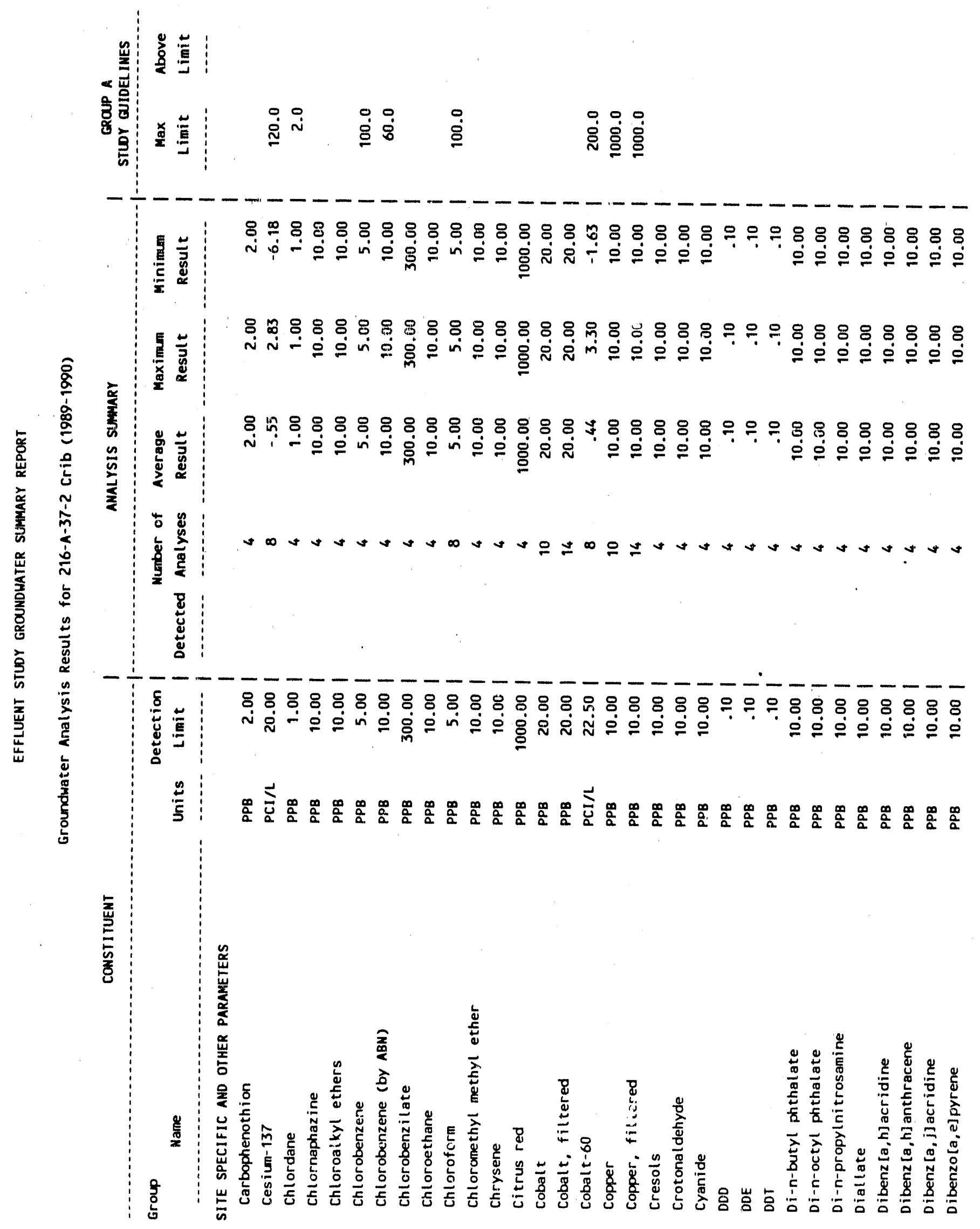




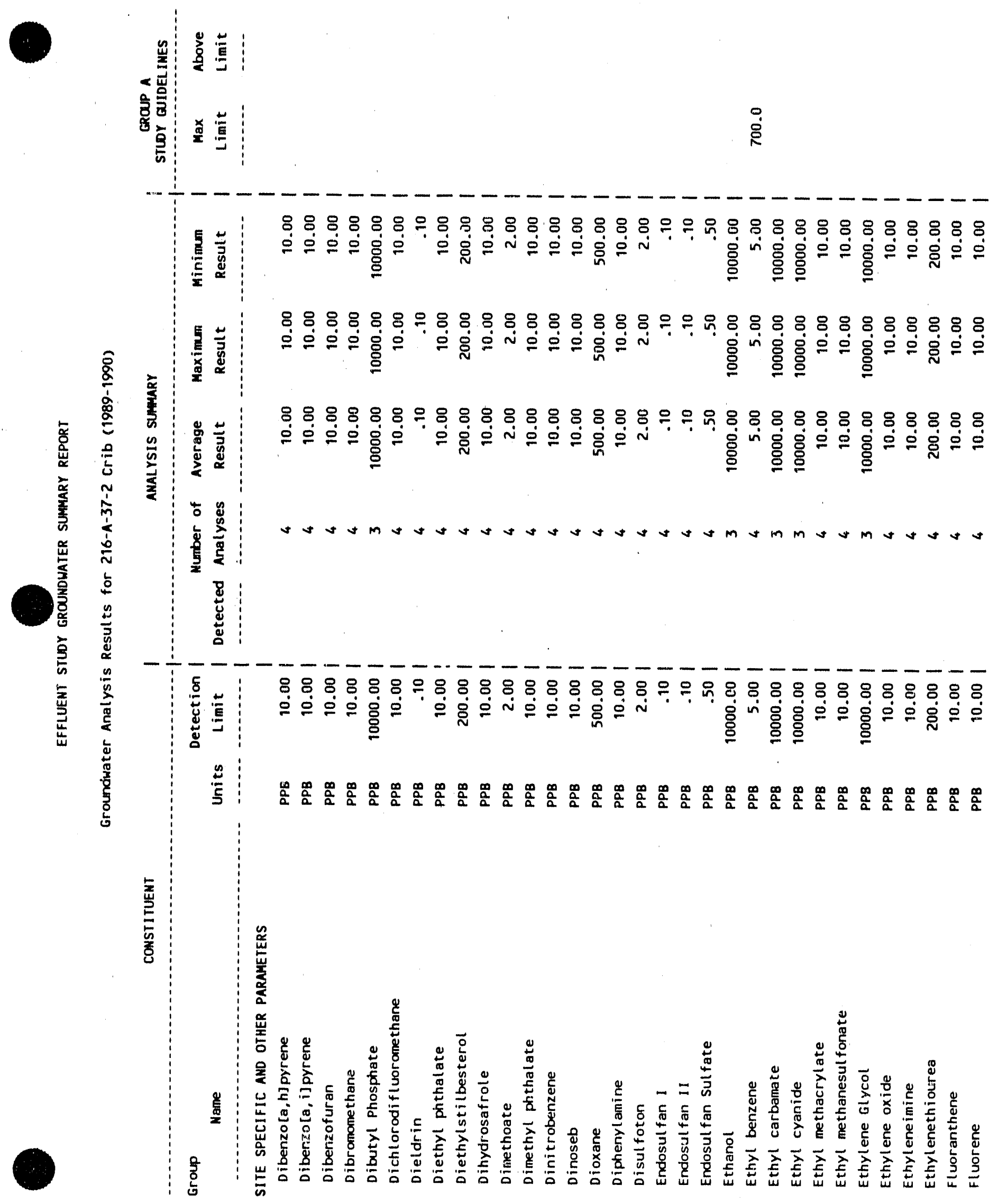


WHC-EP-0366

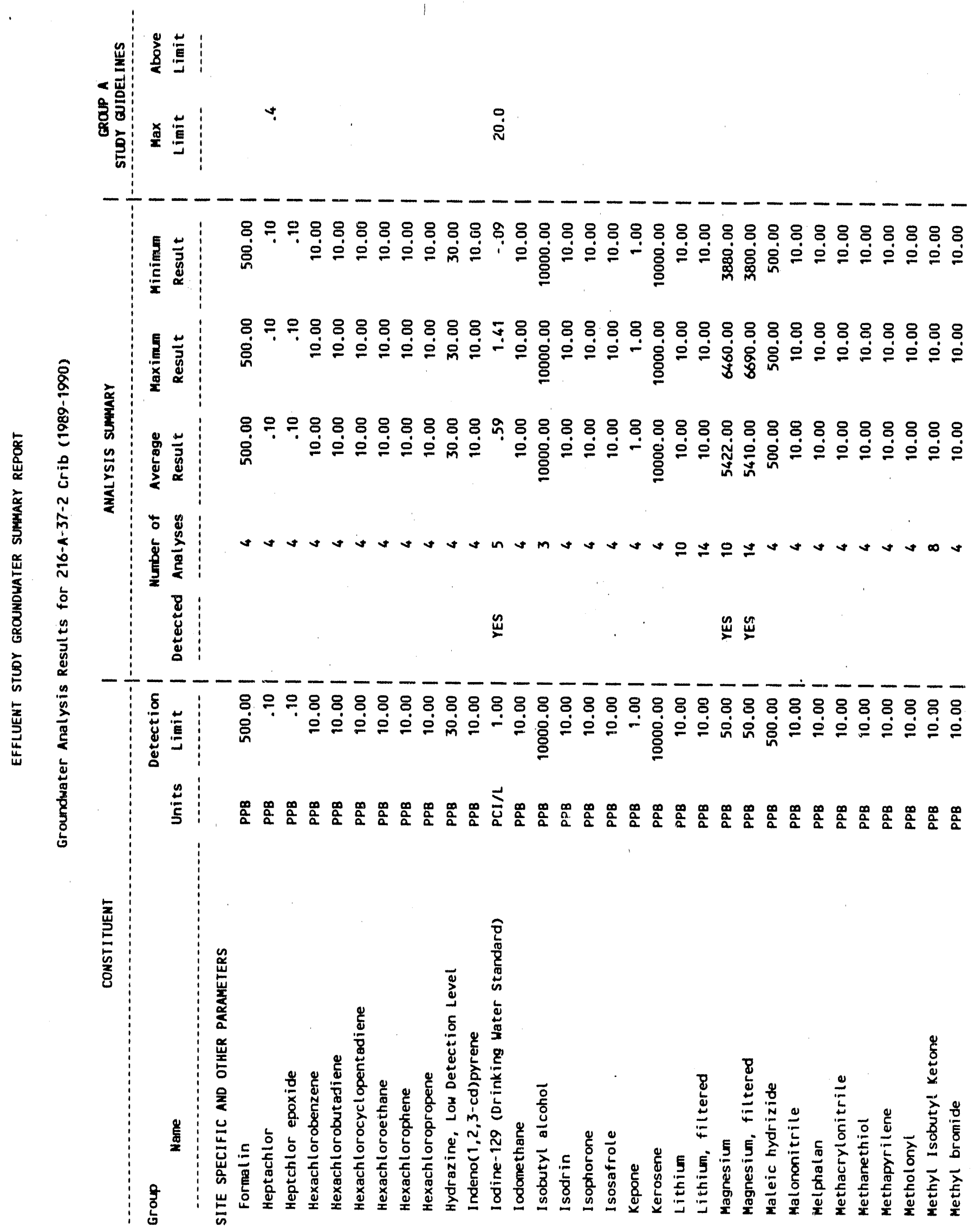



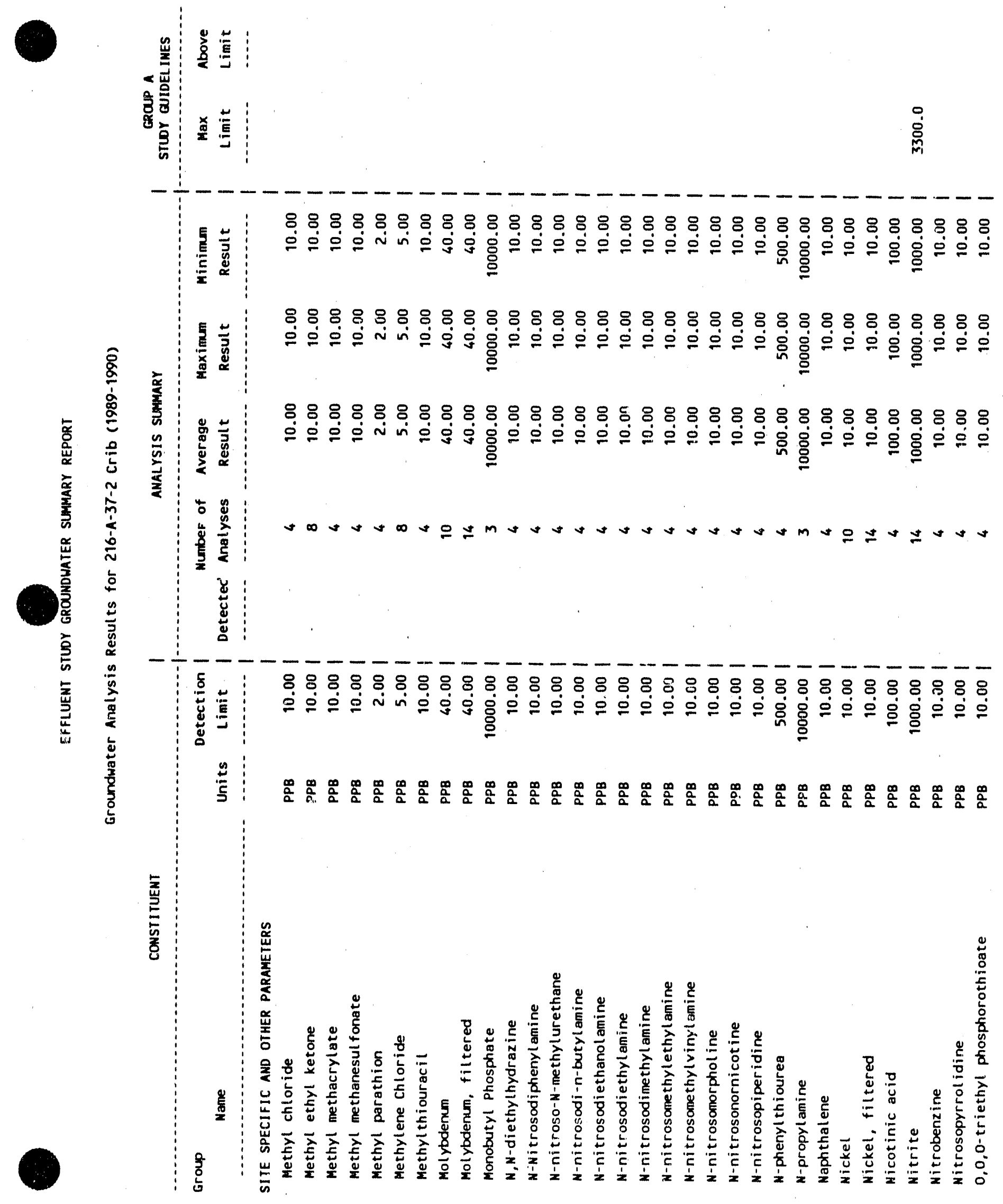


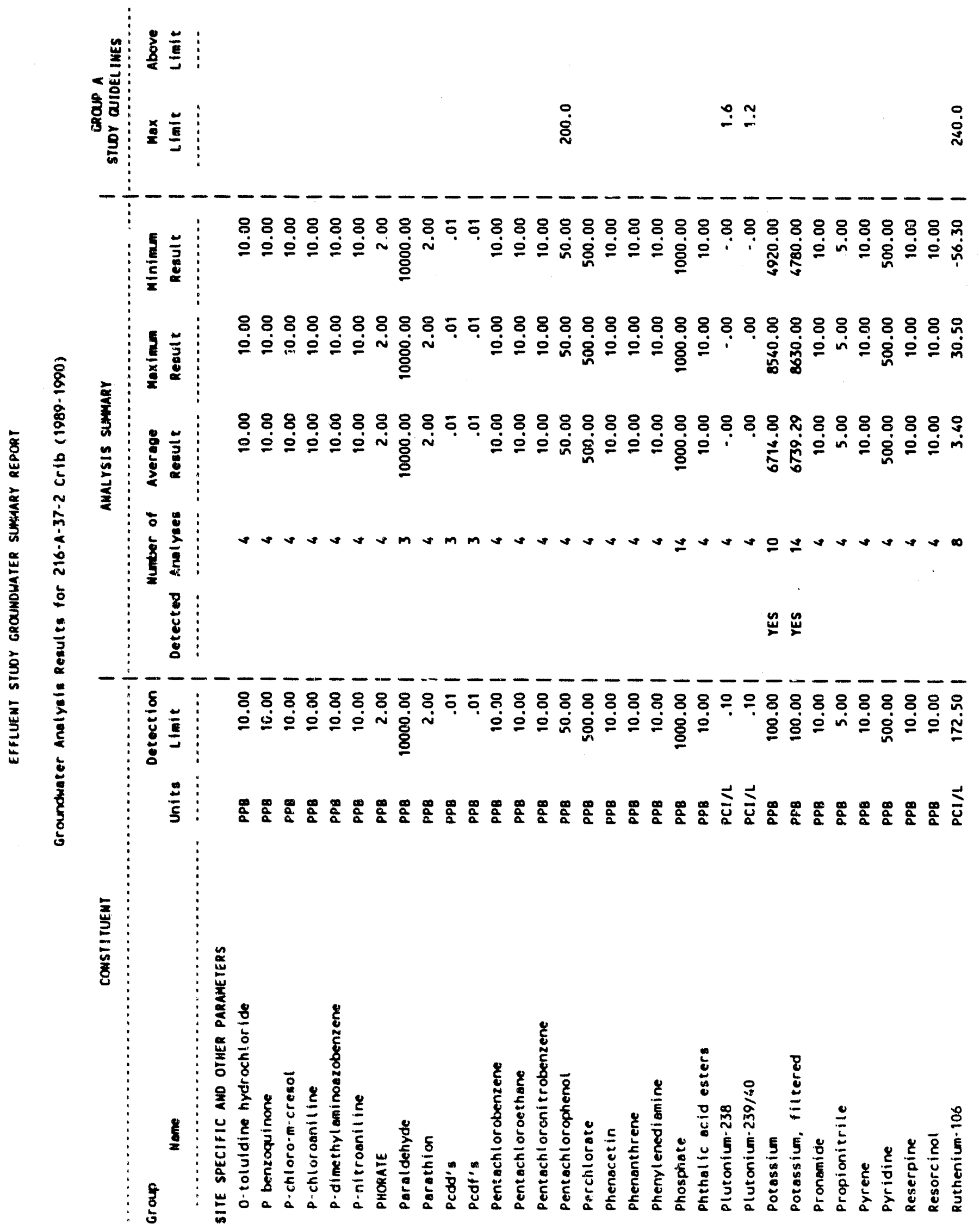


WHC -EP-0366

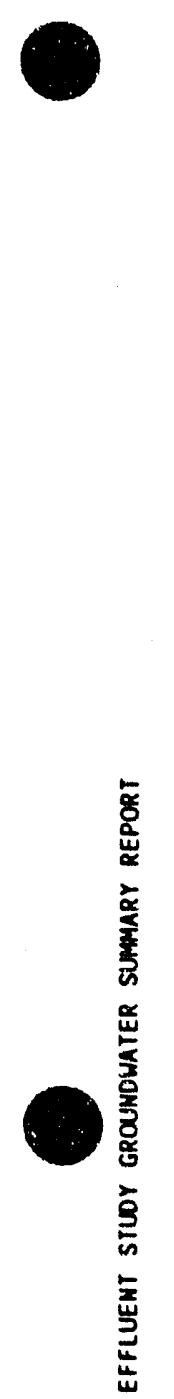
然:
$\stackrel{\leftrightarrow}{\rightleftharpoons}$
守

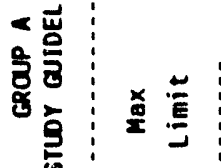

家

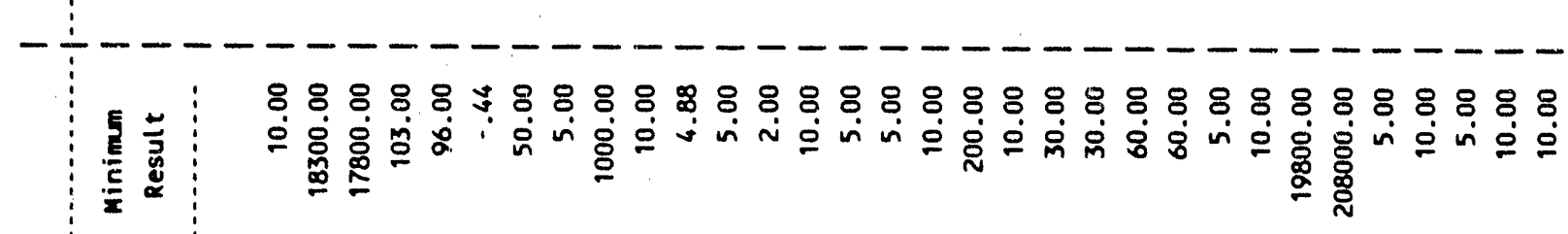

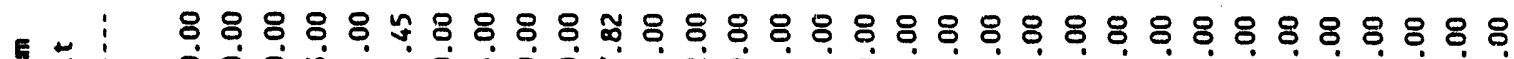

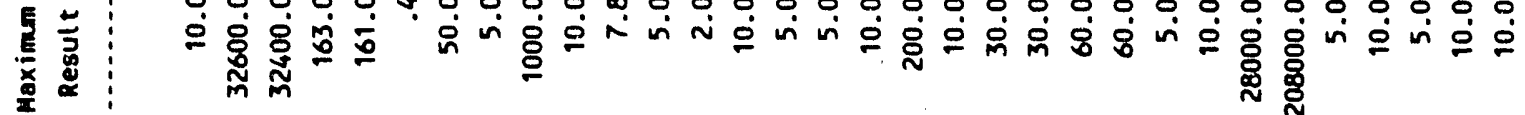

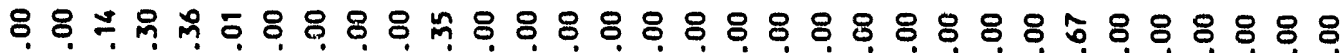

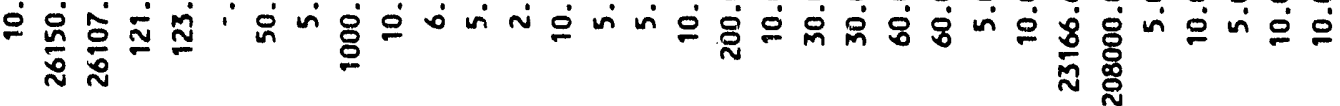

$\frac{1}{5}$

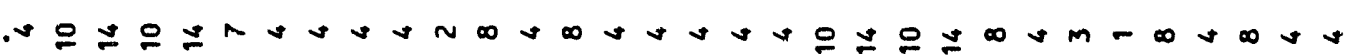

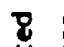

:

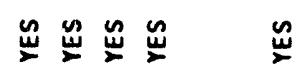

$\stackrel{\mathscr{m}}{\longleftarrow}$

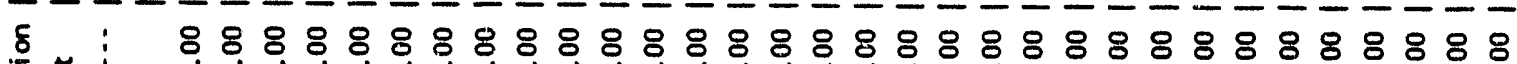

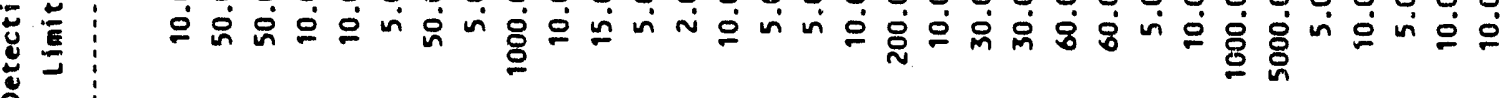

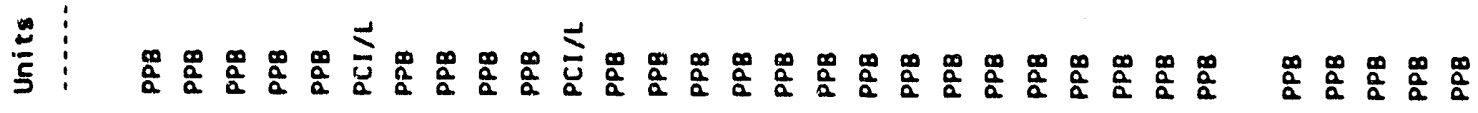

密

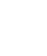

$$
\text { . }
$$

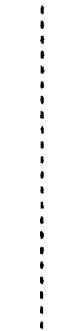

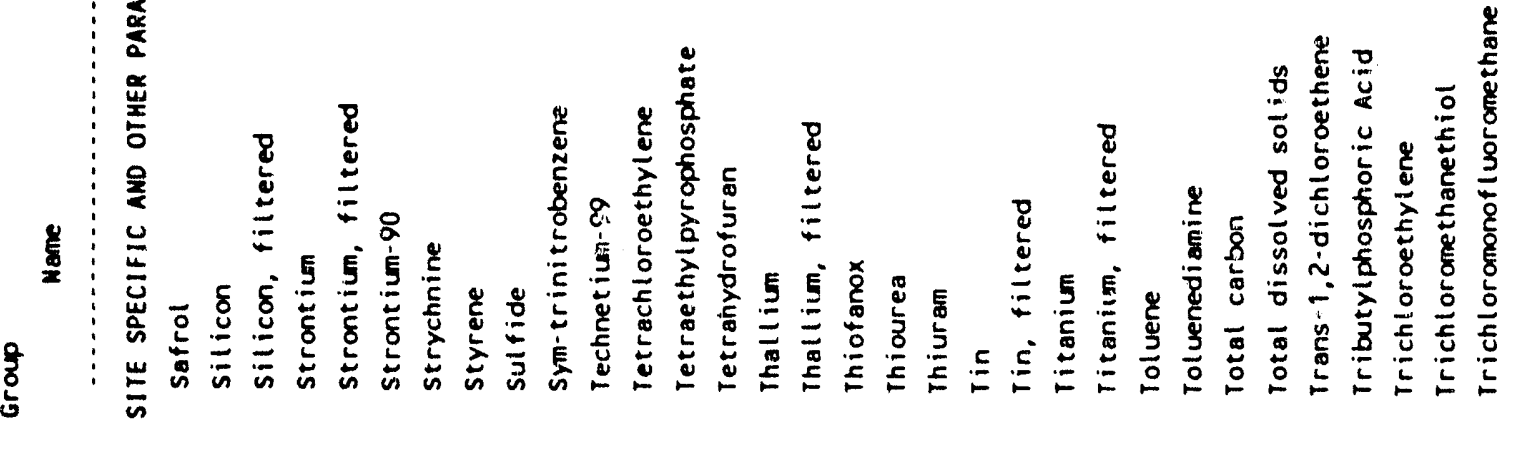


WHC-EP-0366

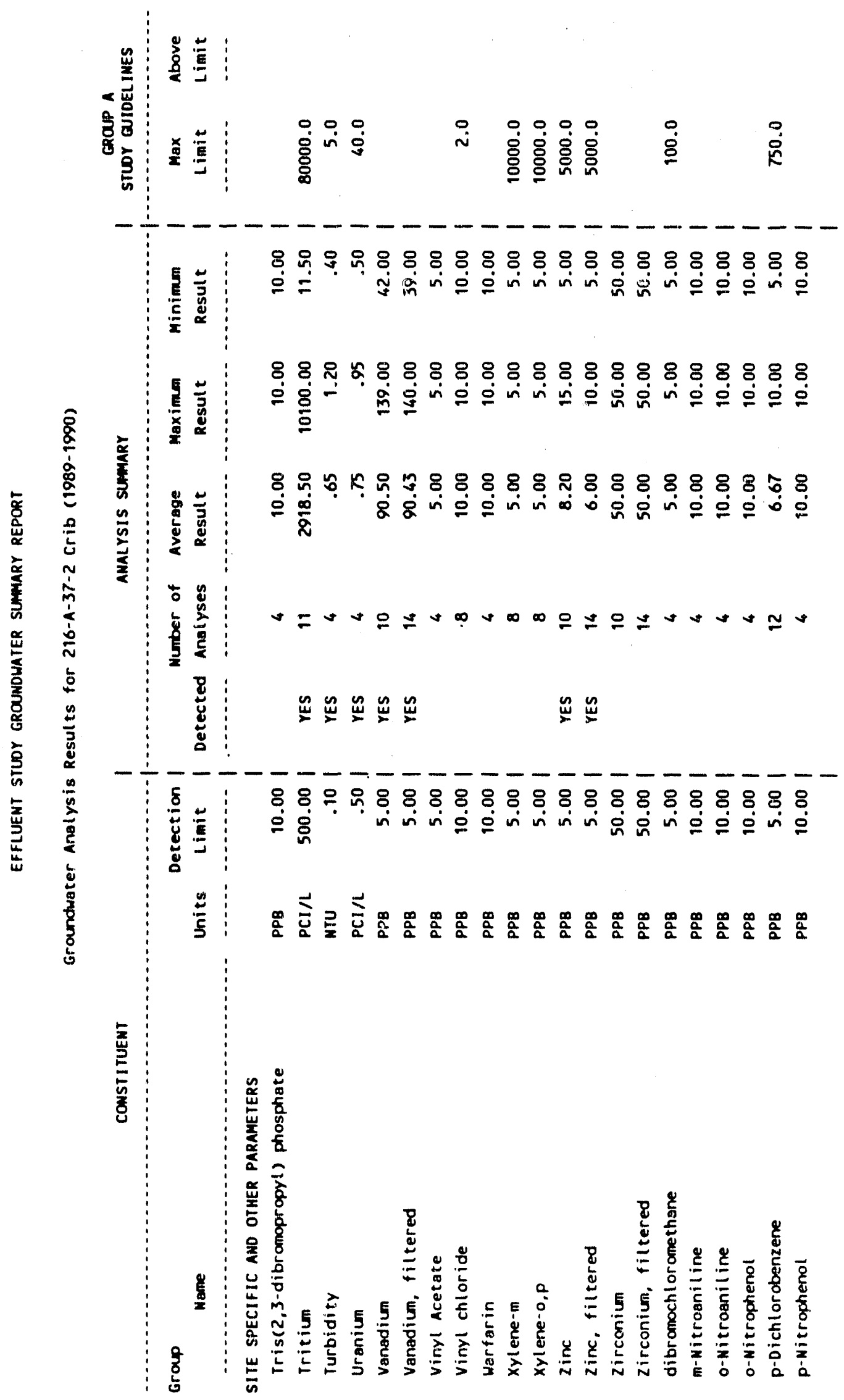




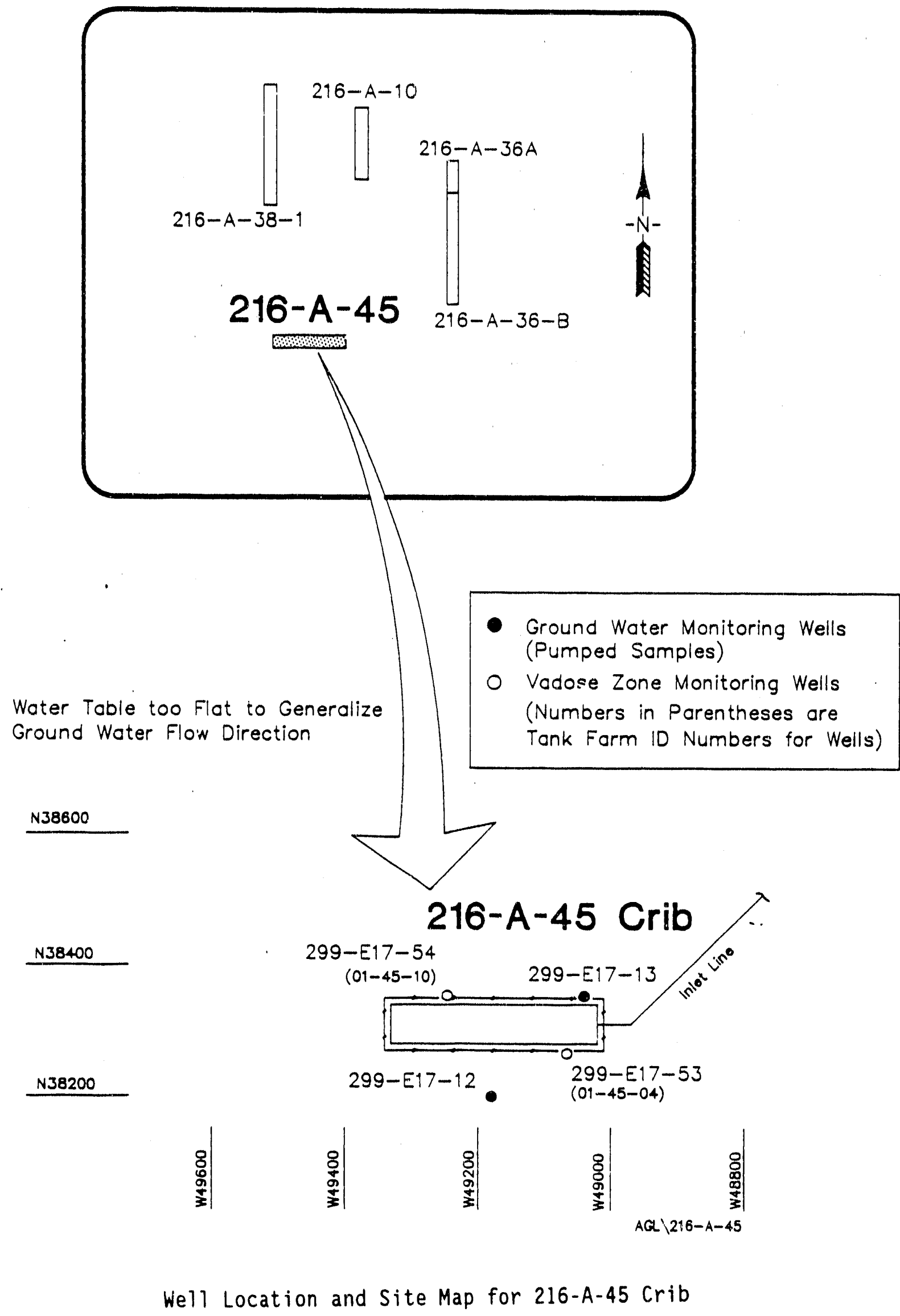


WHC -EP-0366

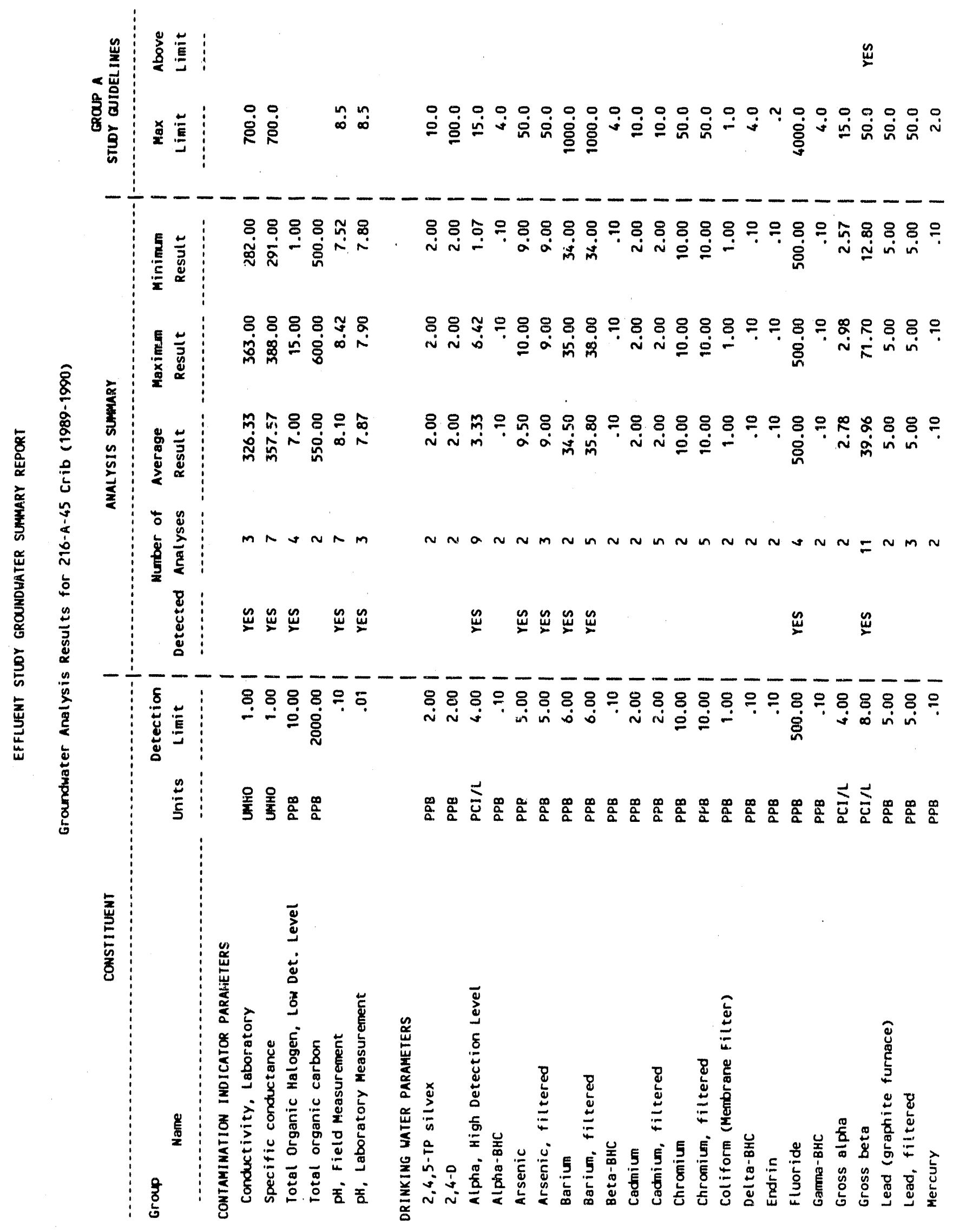


WHC-EP-0366

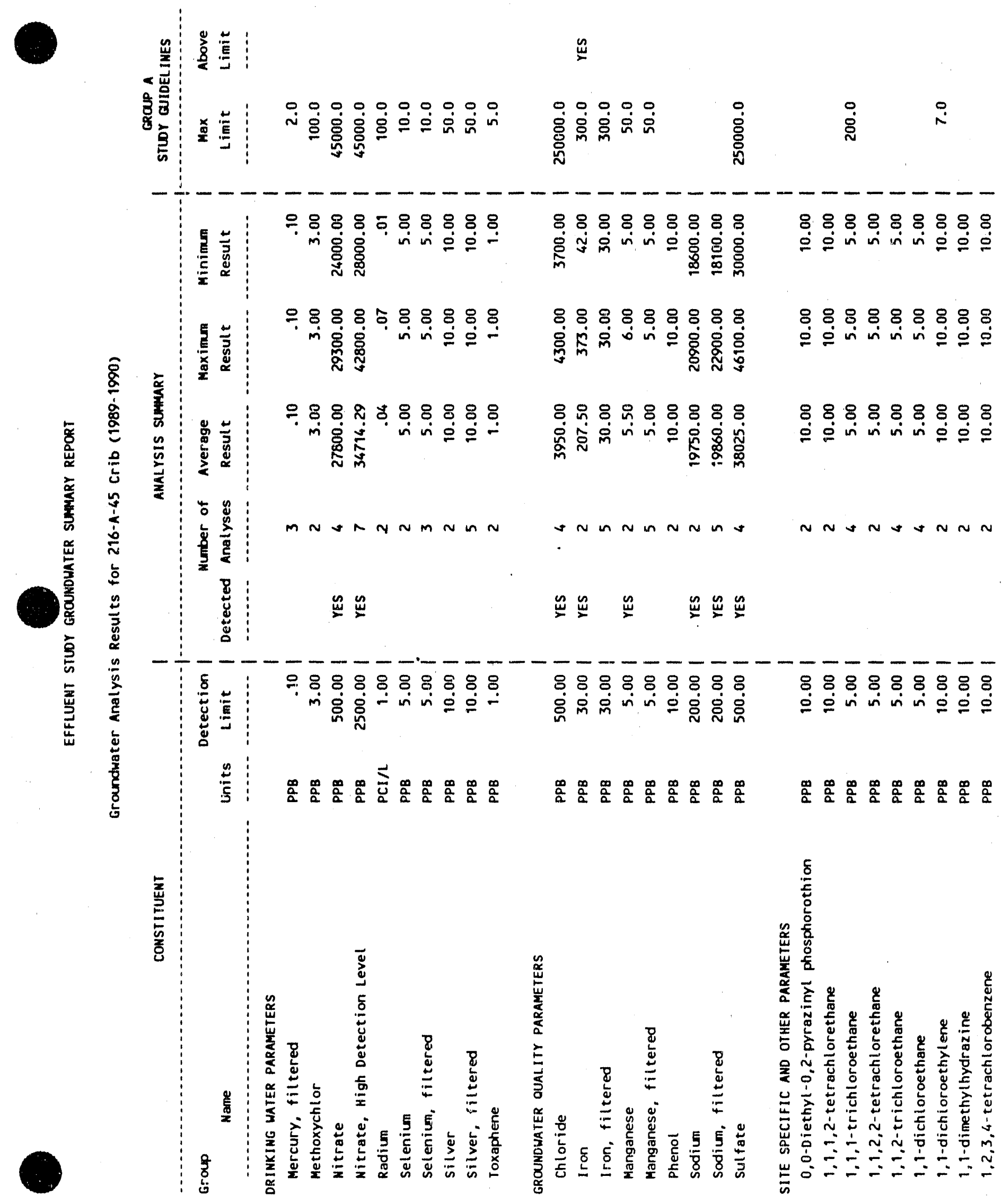


WHC-EP-0366
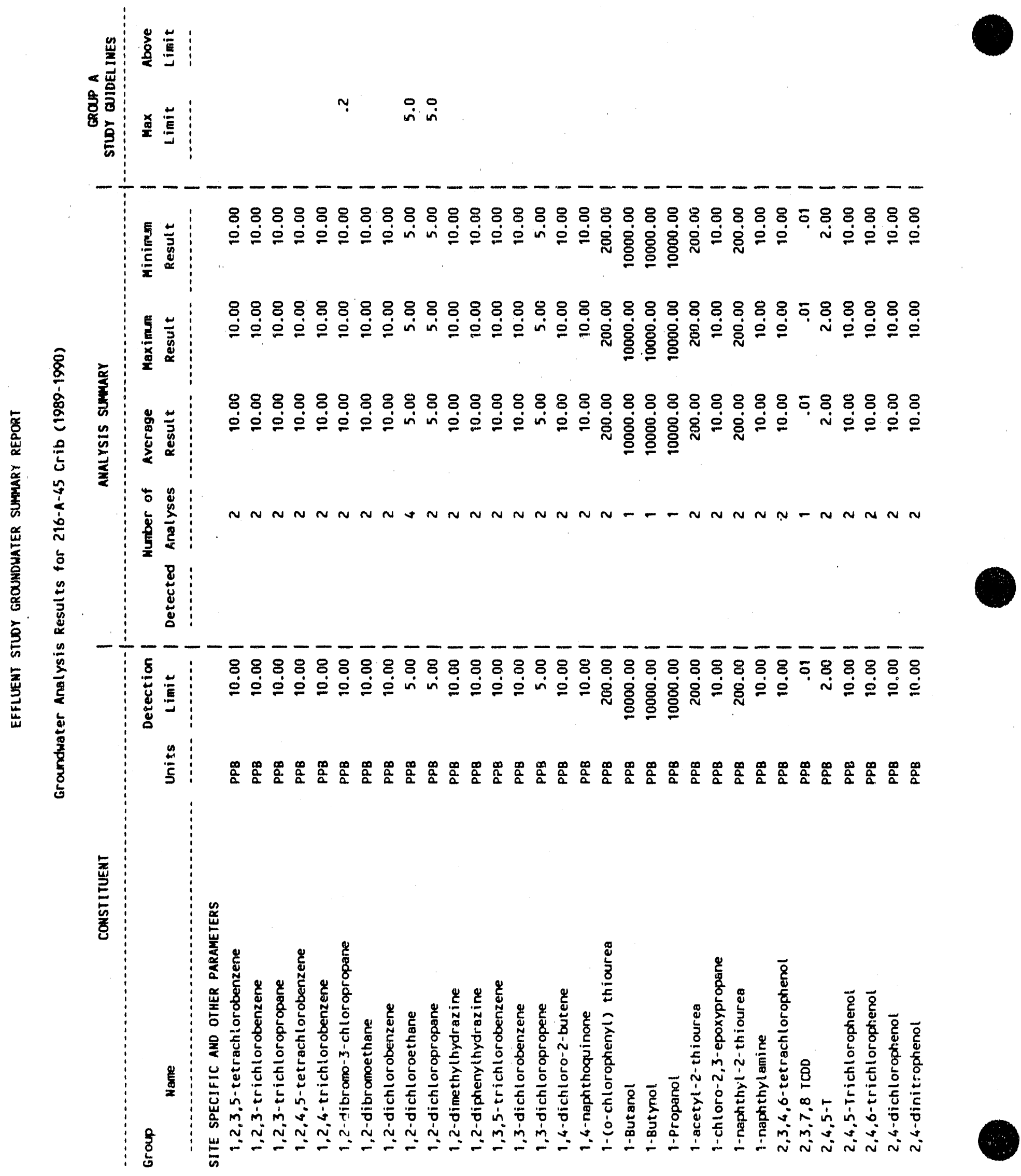
告:

政

s:

高蒙

毫套

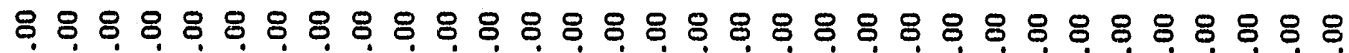

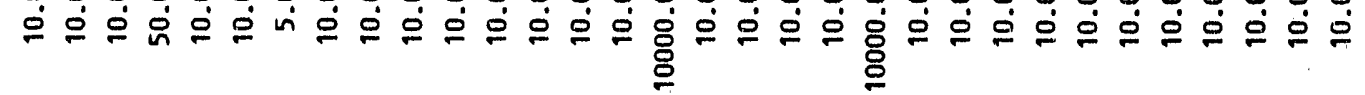

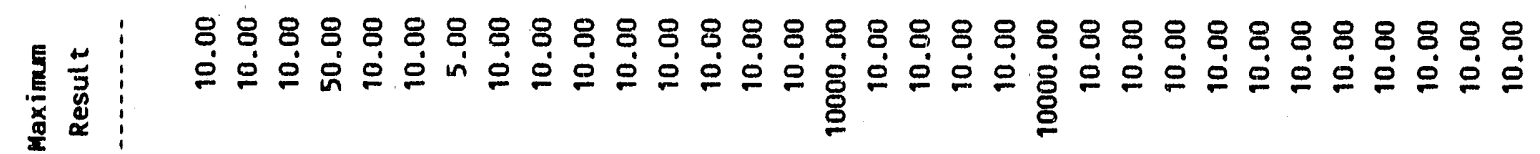

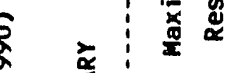

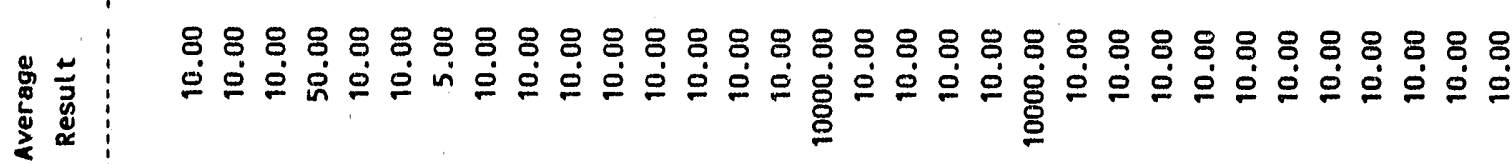

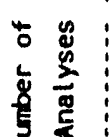

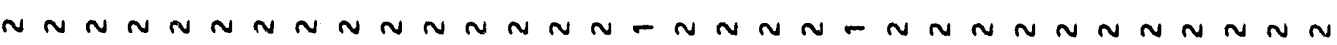
焉

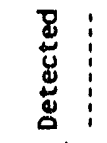

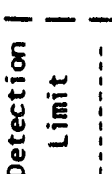

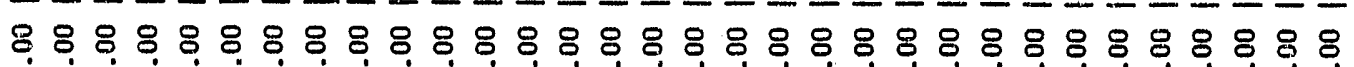

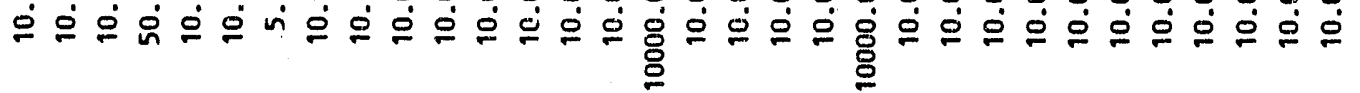

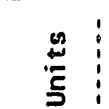

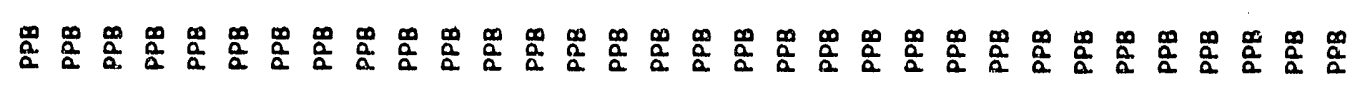
预 5 :

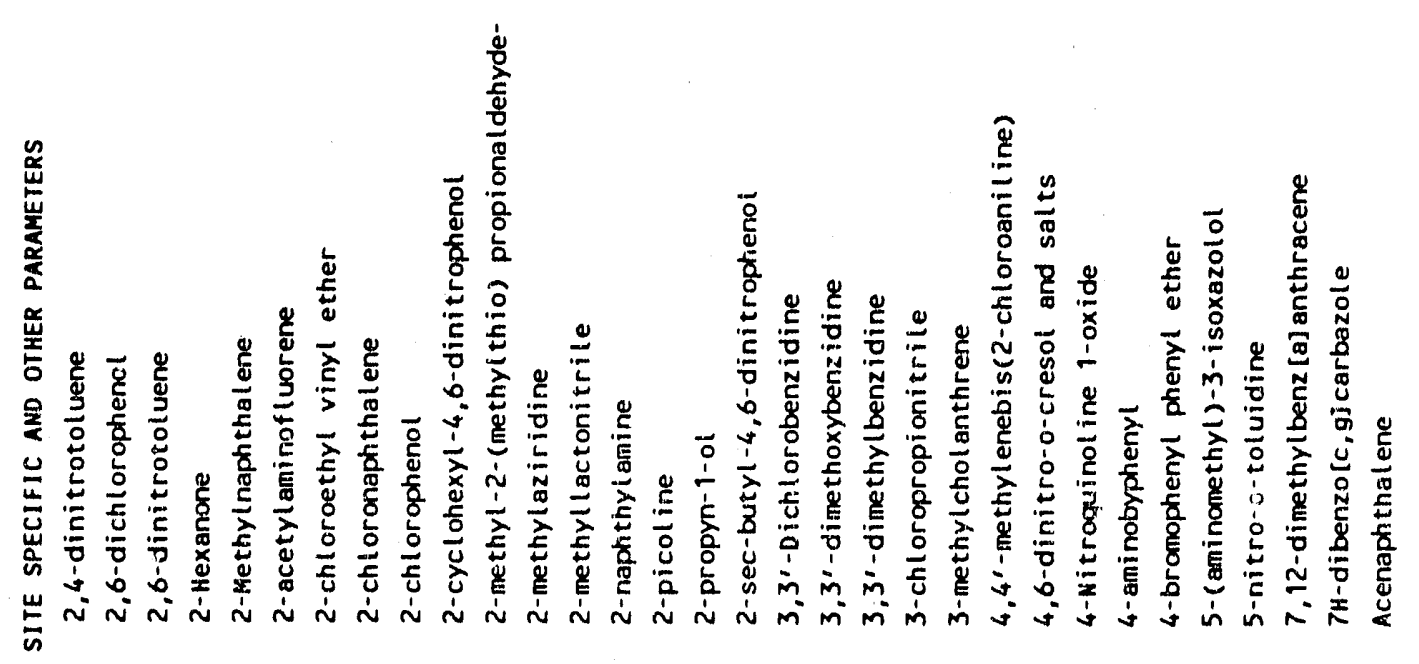


WHC-EP-0366

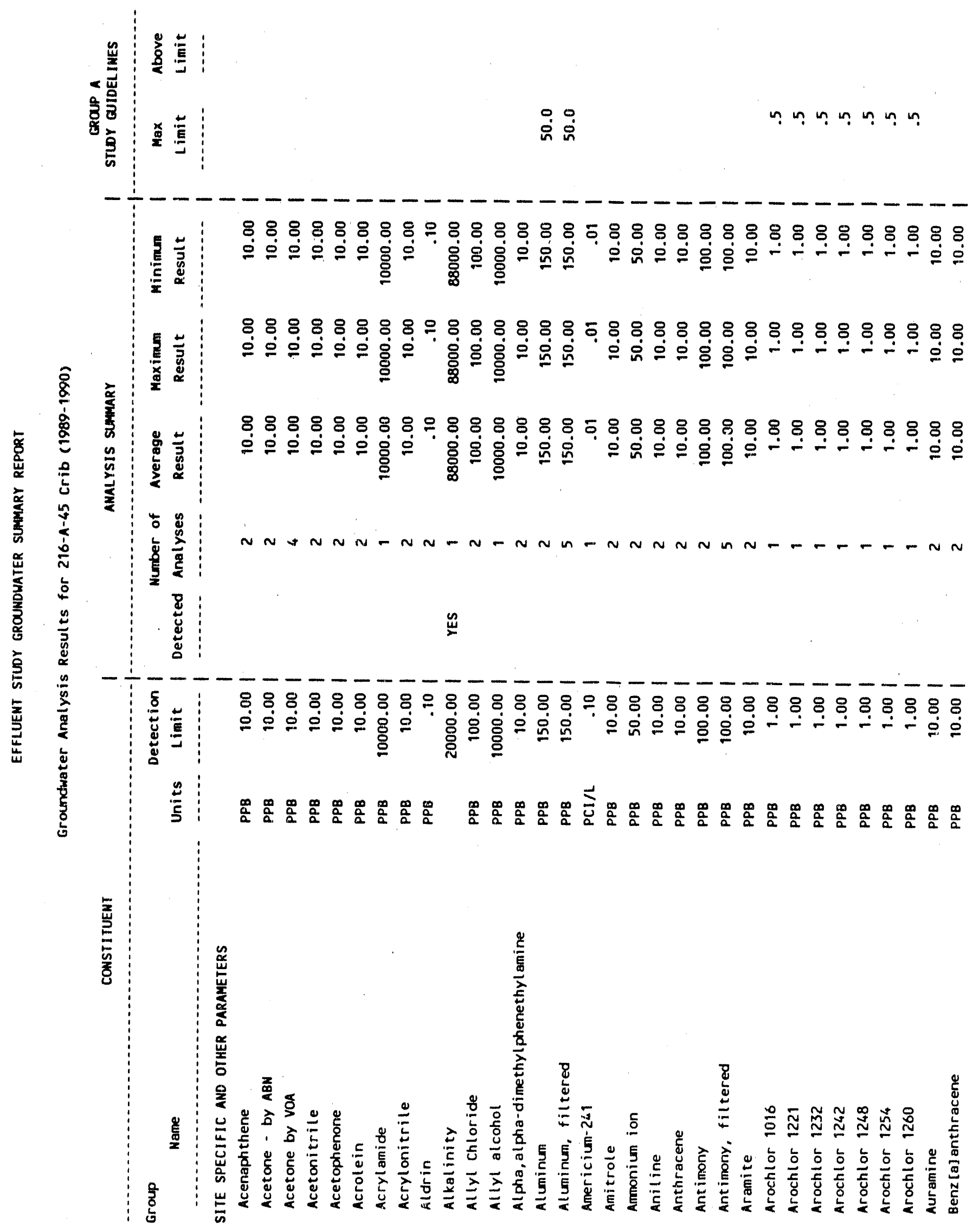




\section{(ni:}

践

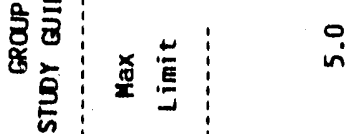

号

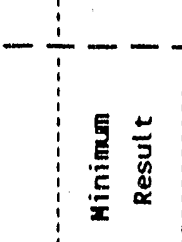

8888888888888888888888088088088

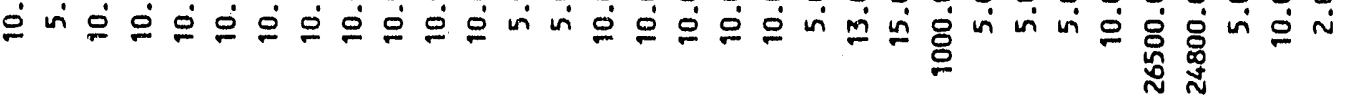

是苛

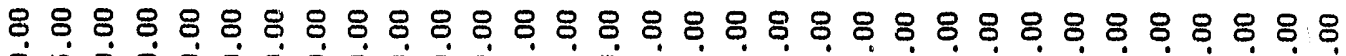

崖

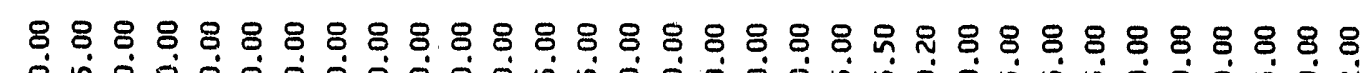

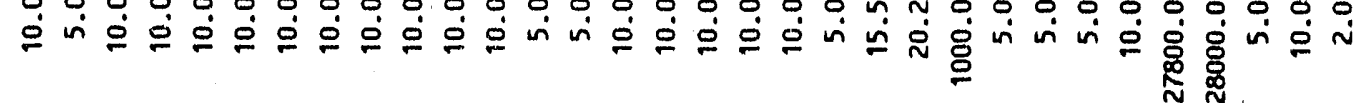

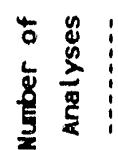

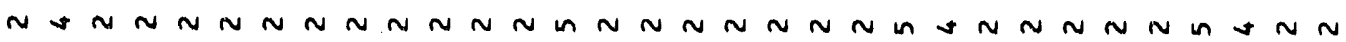

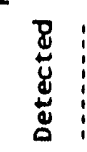

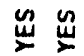

出蒡:

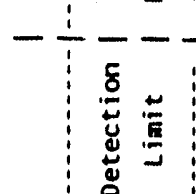

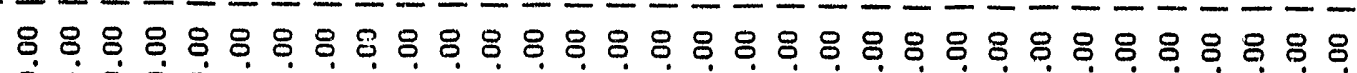

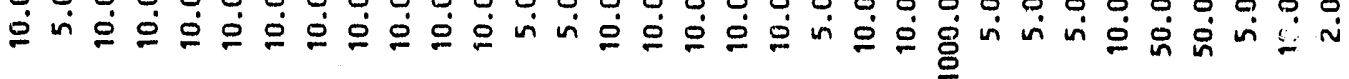

$\stackrel{n}{5}$

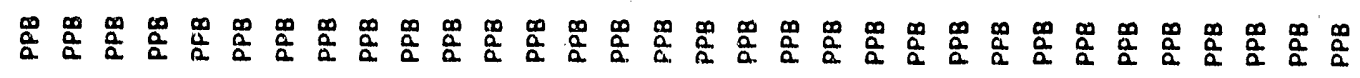

苞<smiles>C1CCCCC1</smiles>

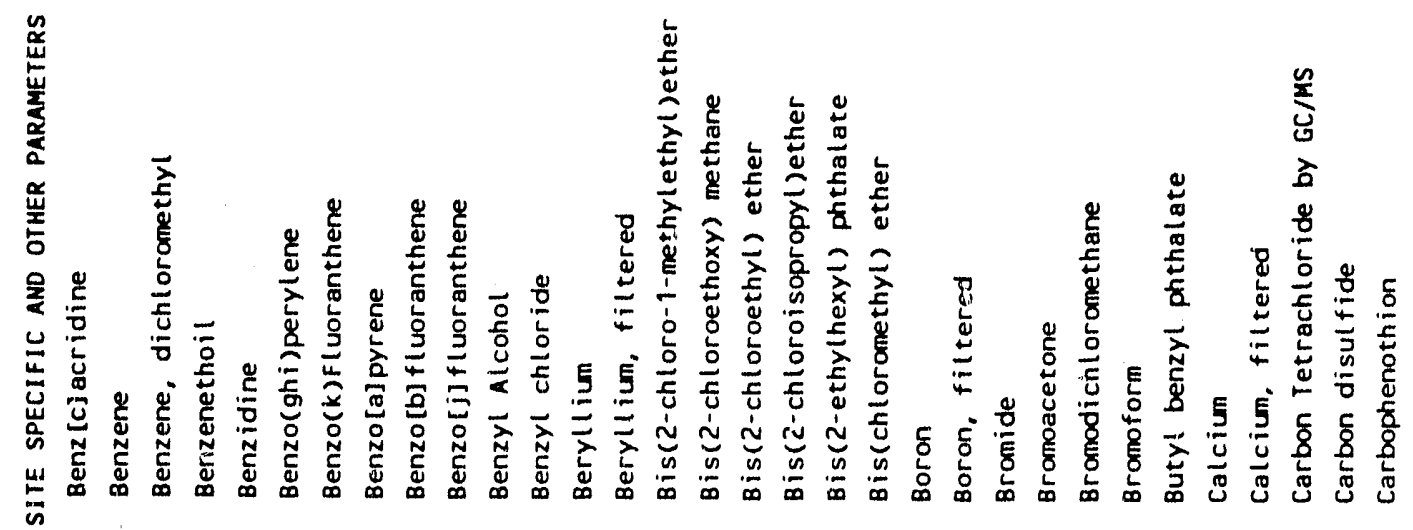


WHC-EP-0366

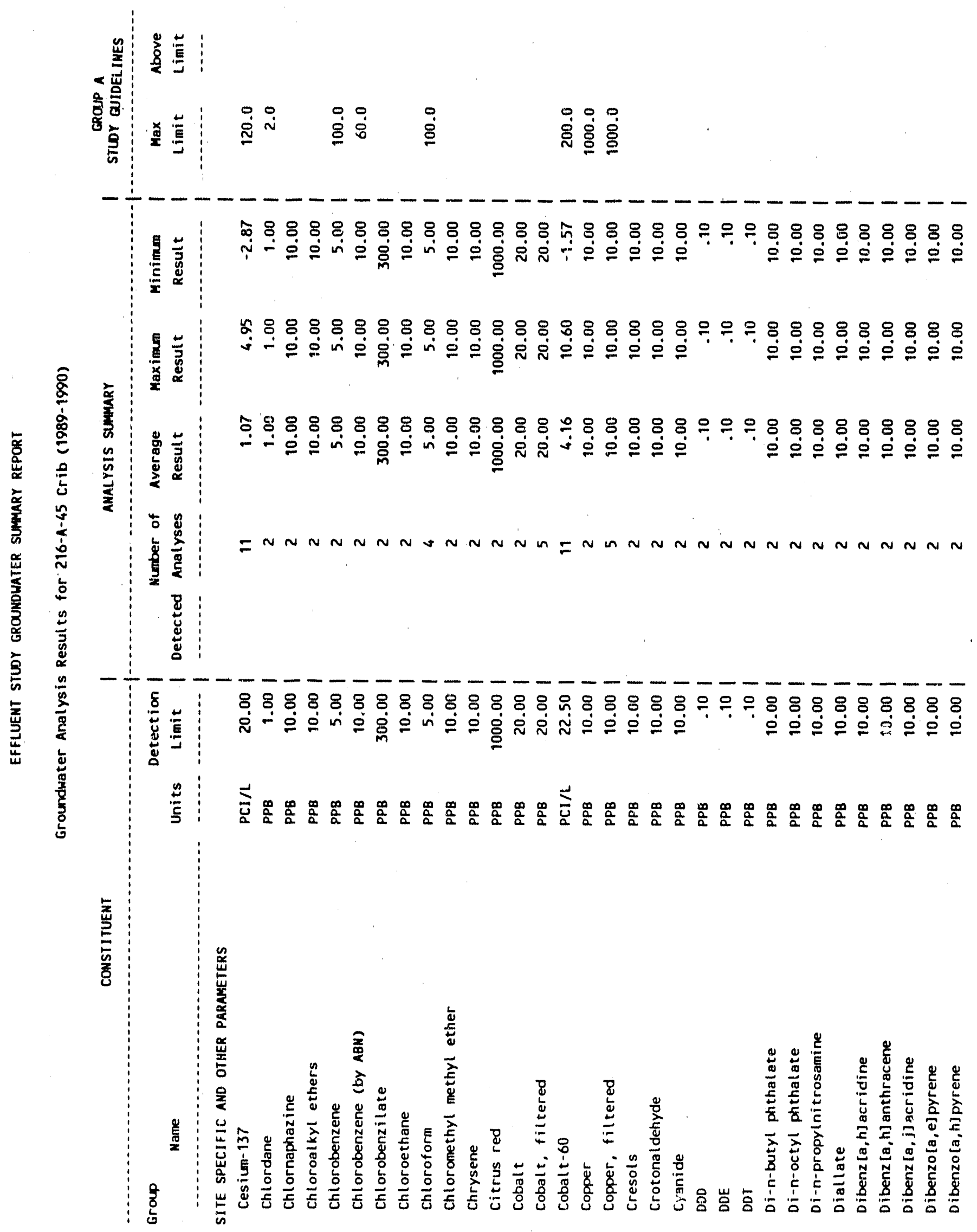


照高:

范

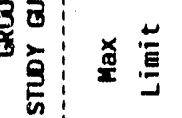

$\stackrel{\circ}{\grave{2}}$

官

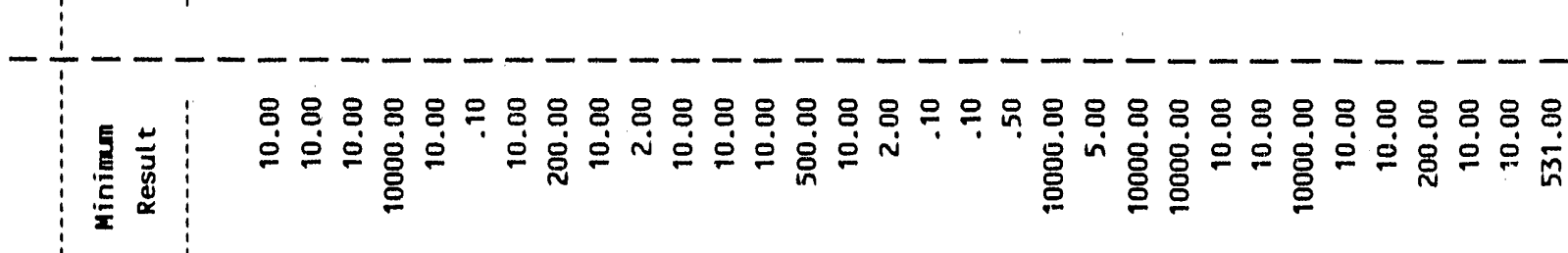

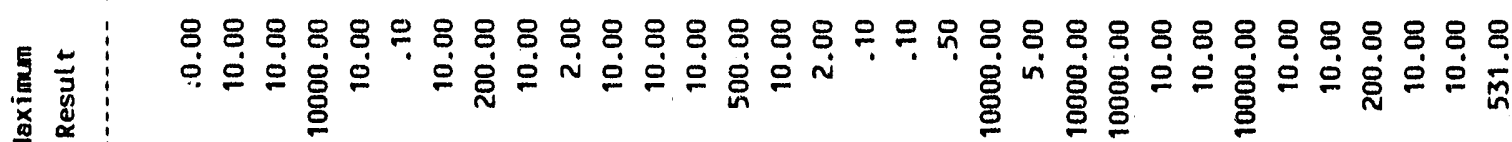

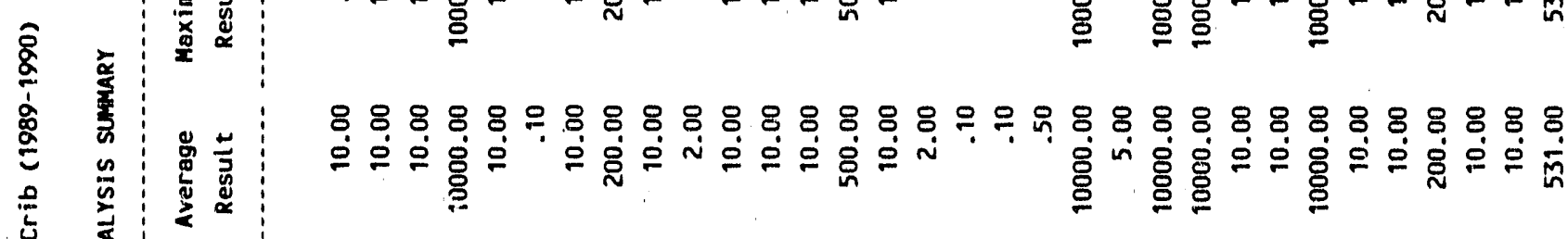
\begin{tabular}{l:l}
\hline \\
0
\end{tabular} 焉空

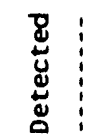

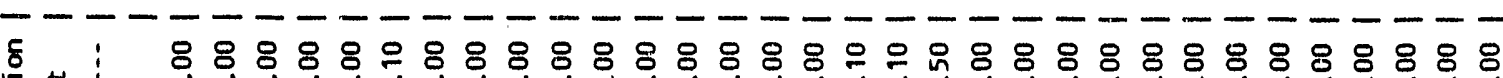

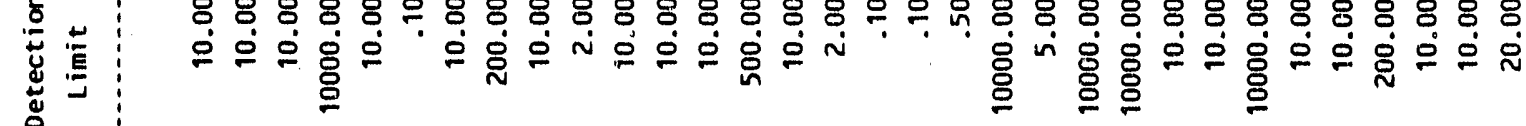

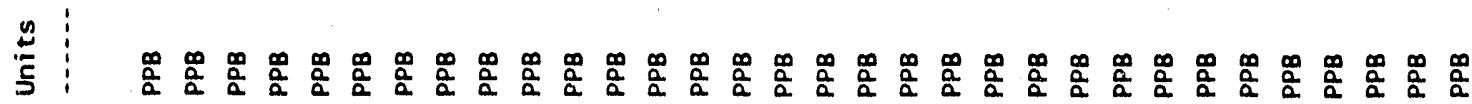


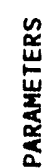

:

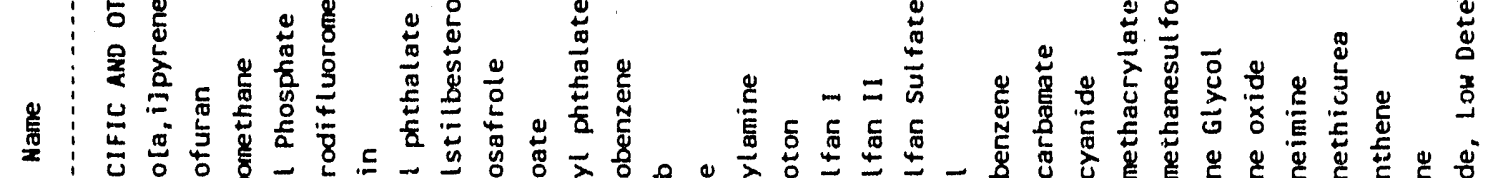

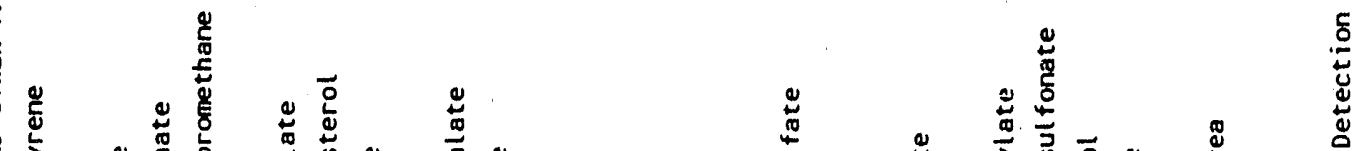

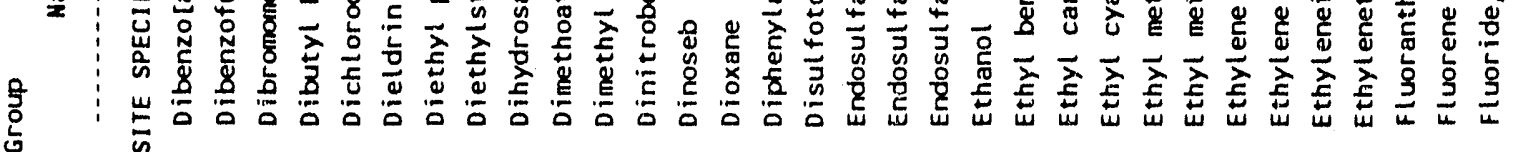




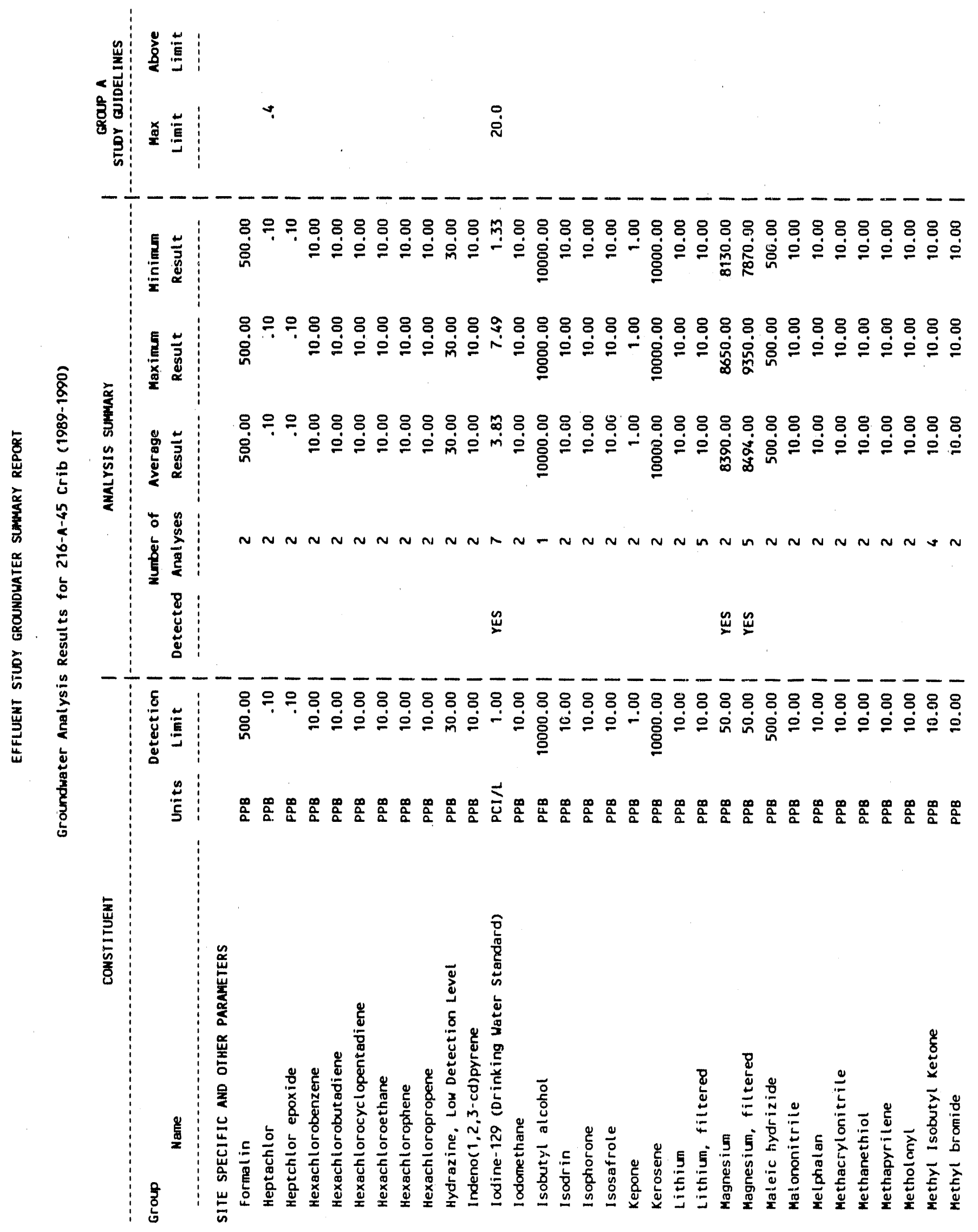



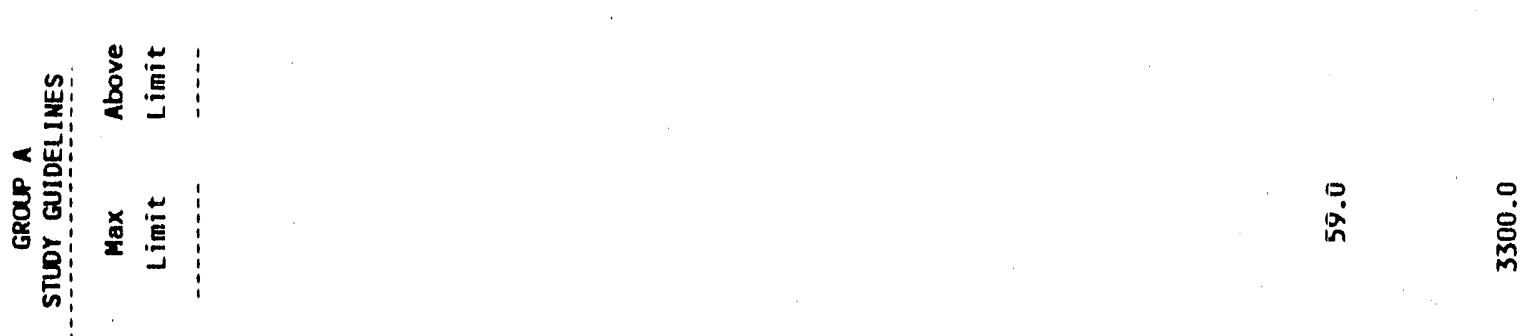

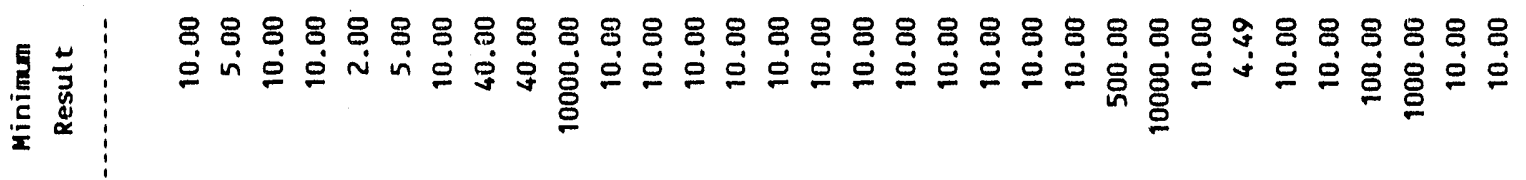

过

8 K $8888888888888888888888 \% 888888$

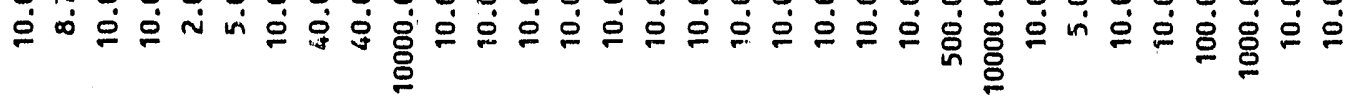

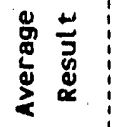

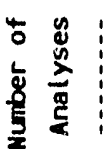

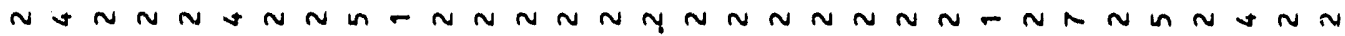

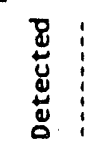

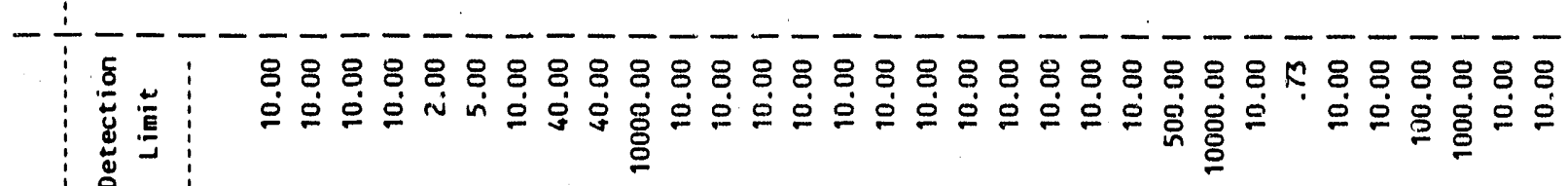

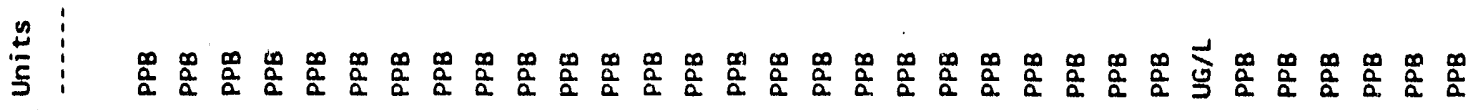

일

委

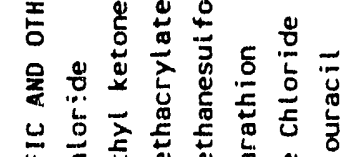

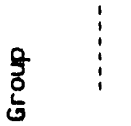

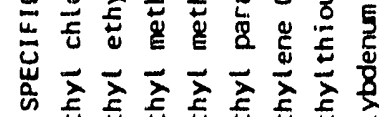

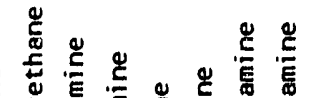

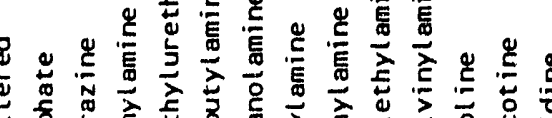


WHC-EP-0.366

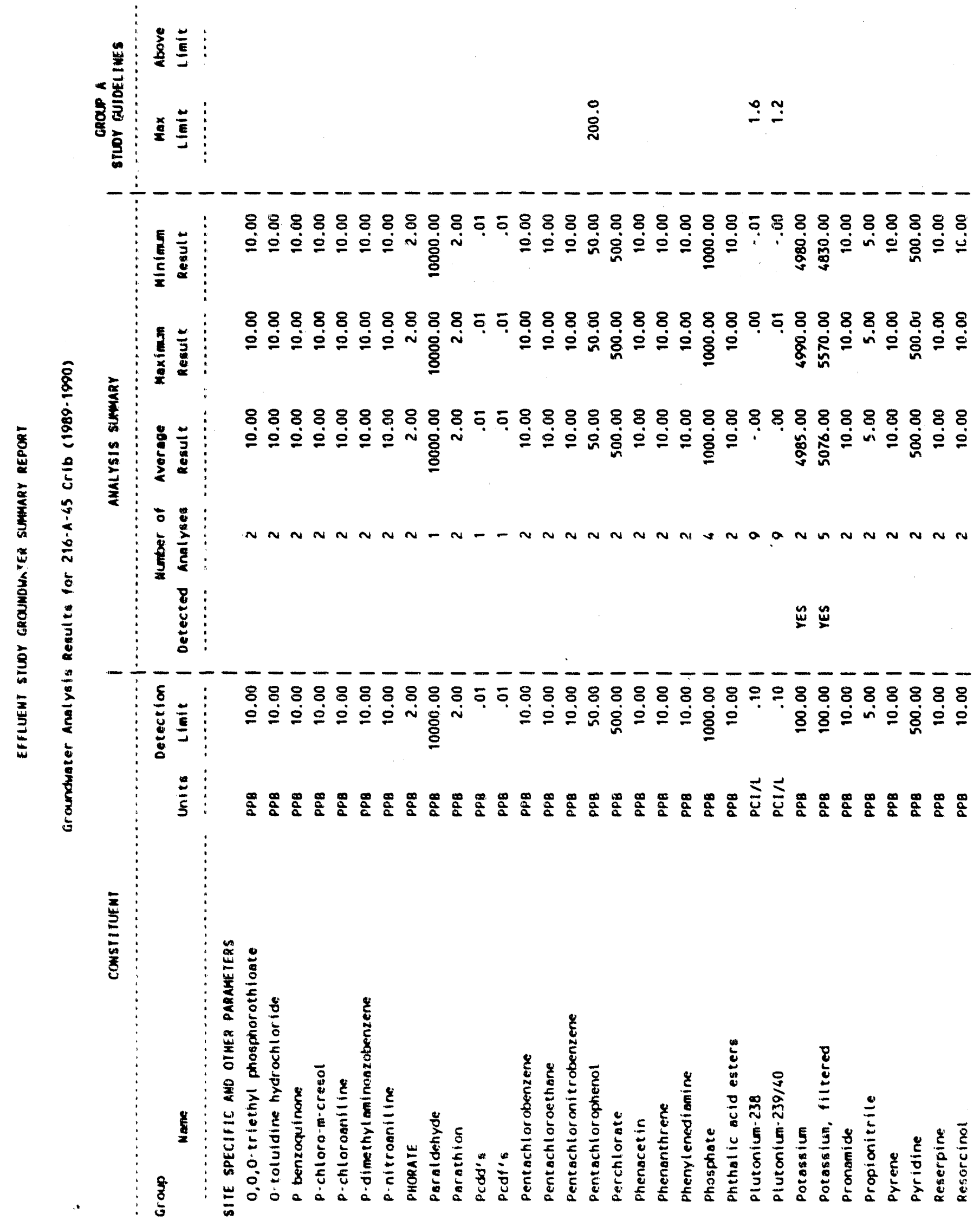




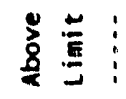

$<\overrightarrow{4}$

客:

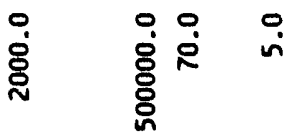

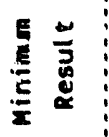

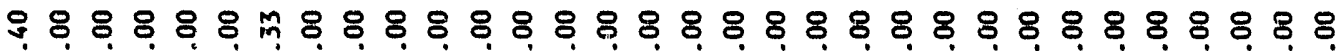
ஸे 08888898888888888888888888888888

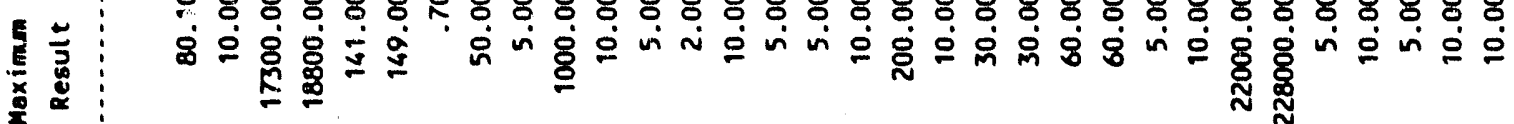

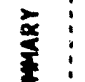

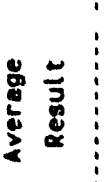

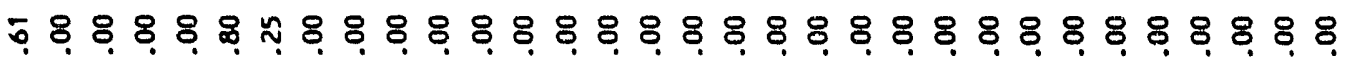

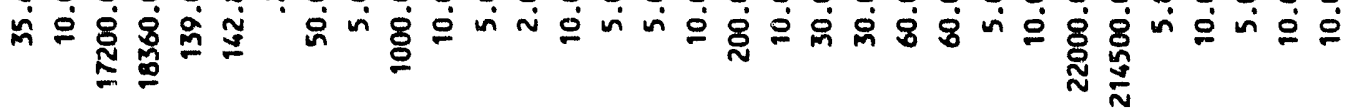

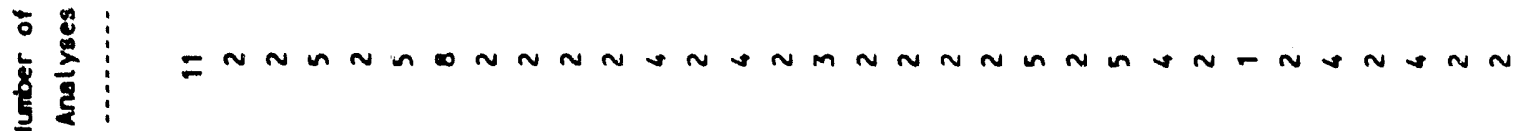
苞

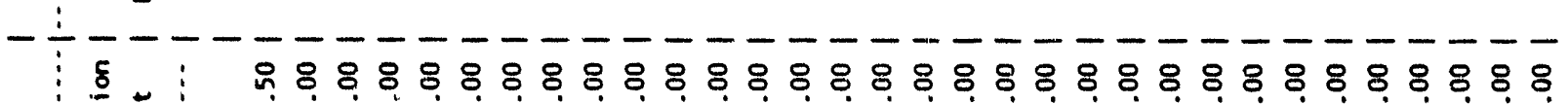

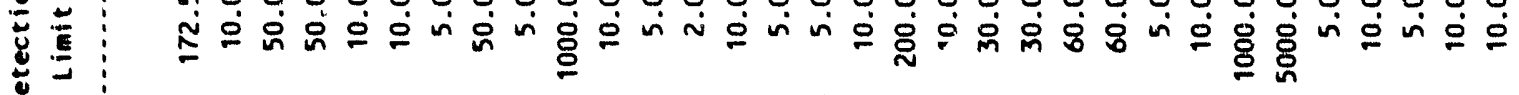
ร 
WHC-EP-0366

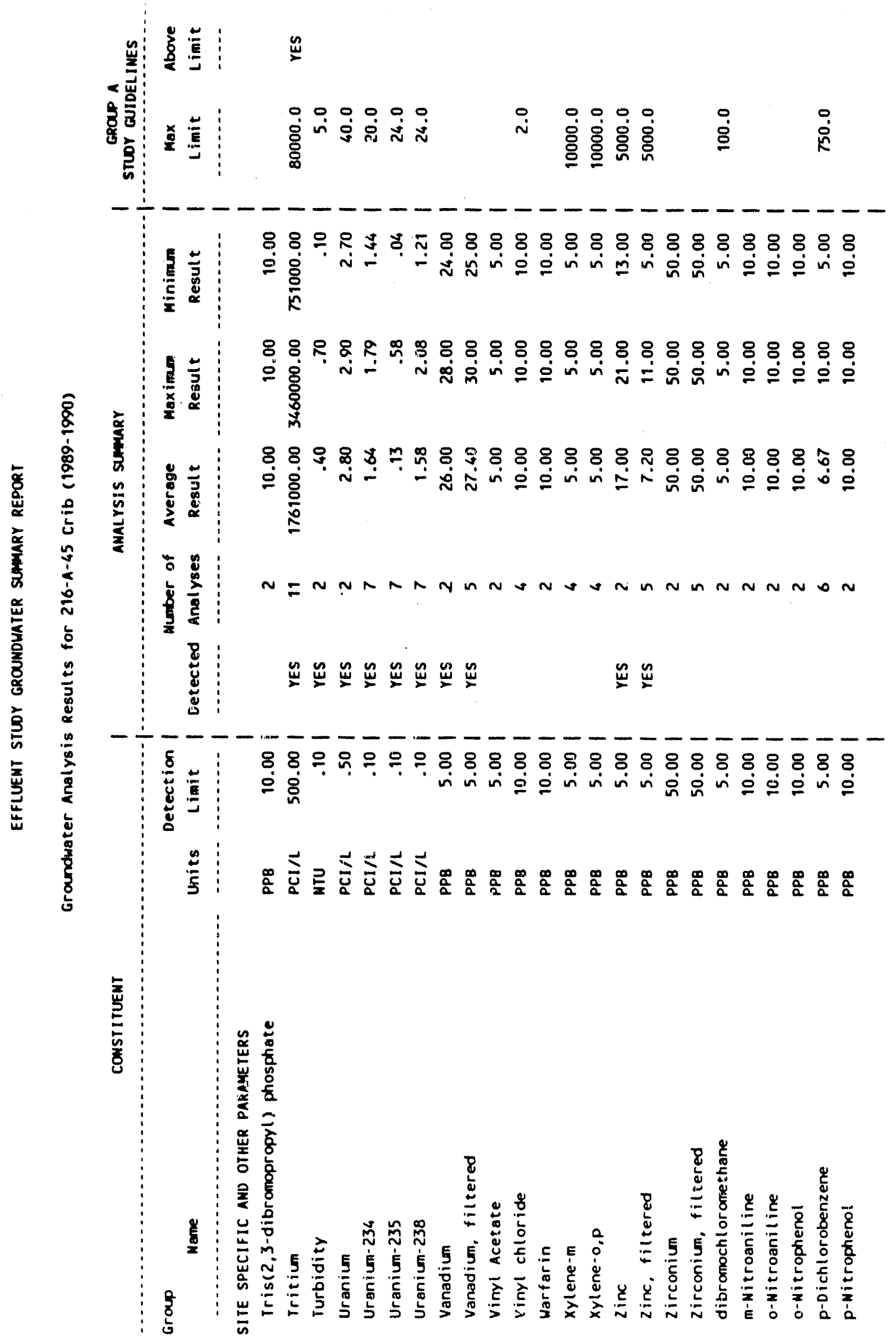


WHC -EP-0366
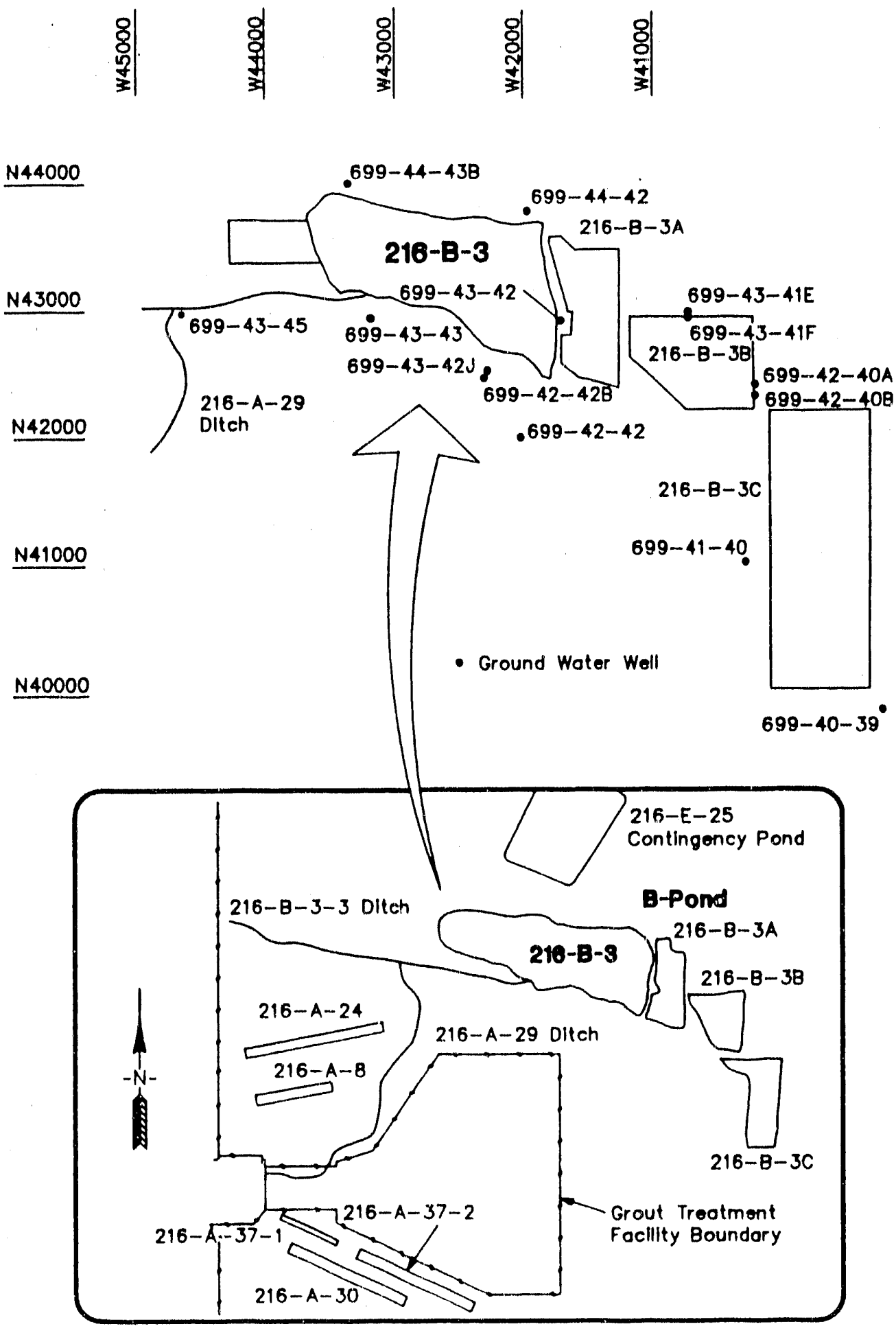

Aa $\longdiv { 2 1 6 - 8 - 3 }$

Well Location and Site Map for 216-B-3 Pond System 
WHC-EP-0366

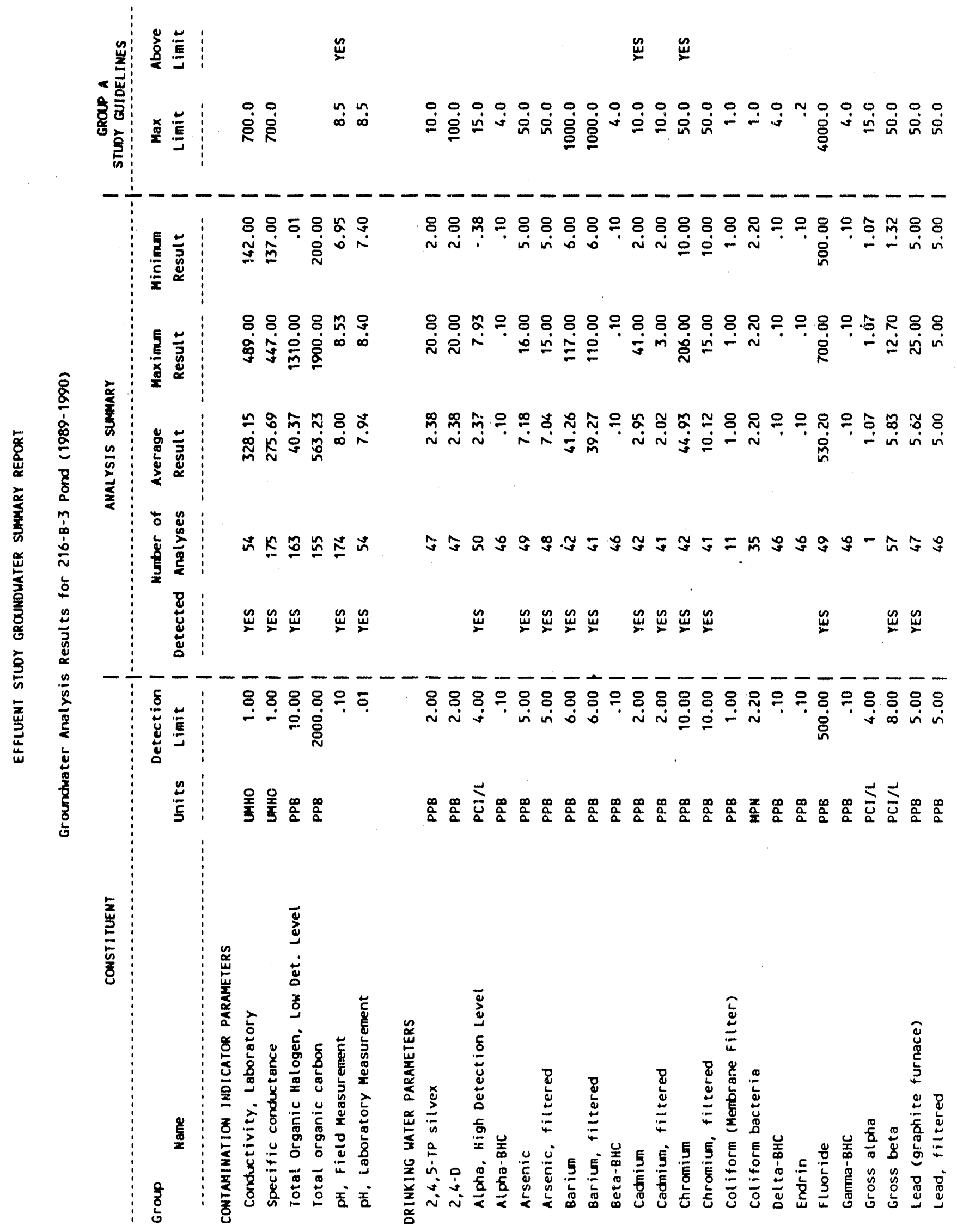




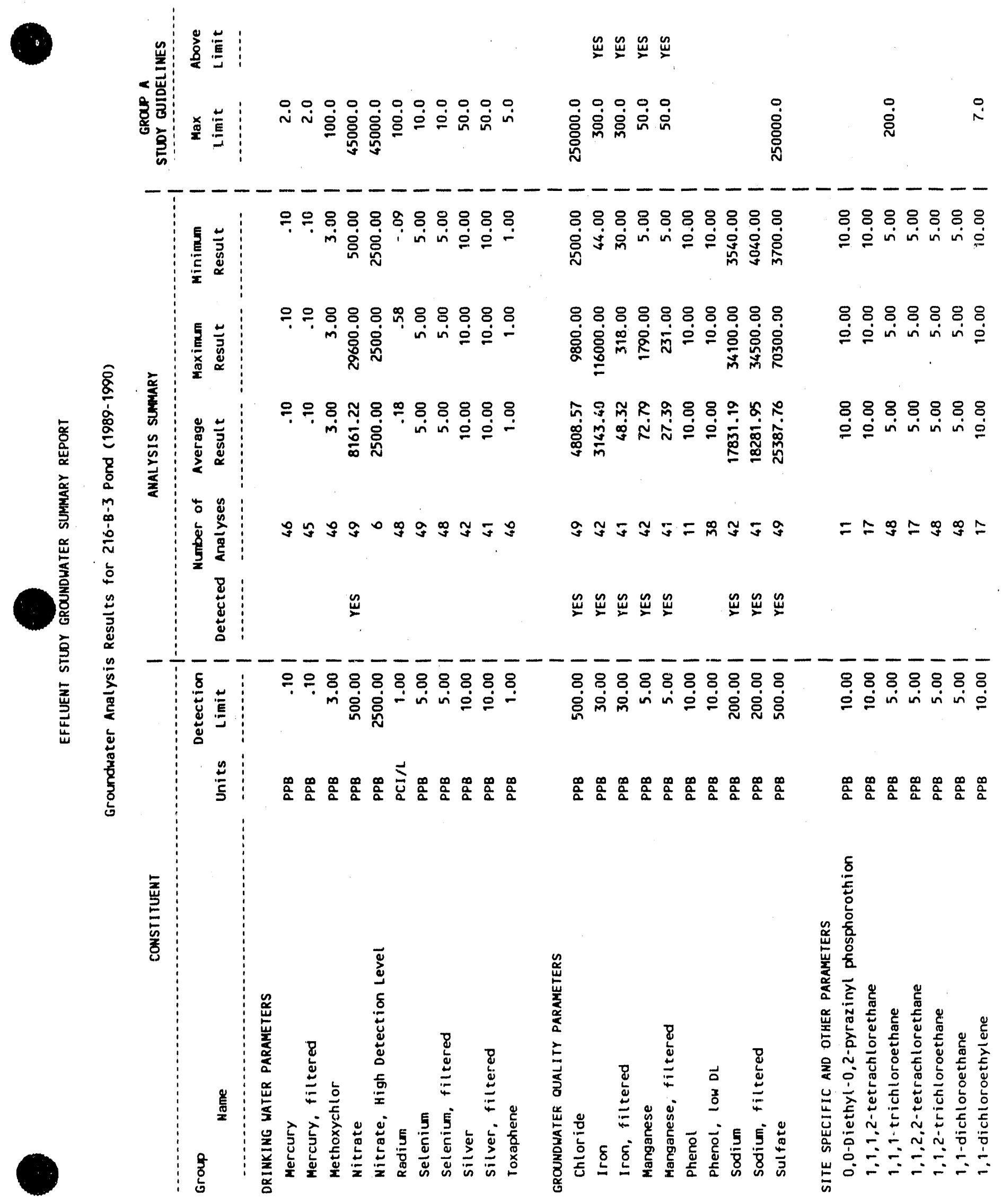


WHC-EP-0366

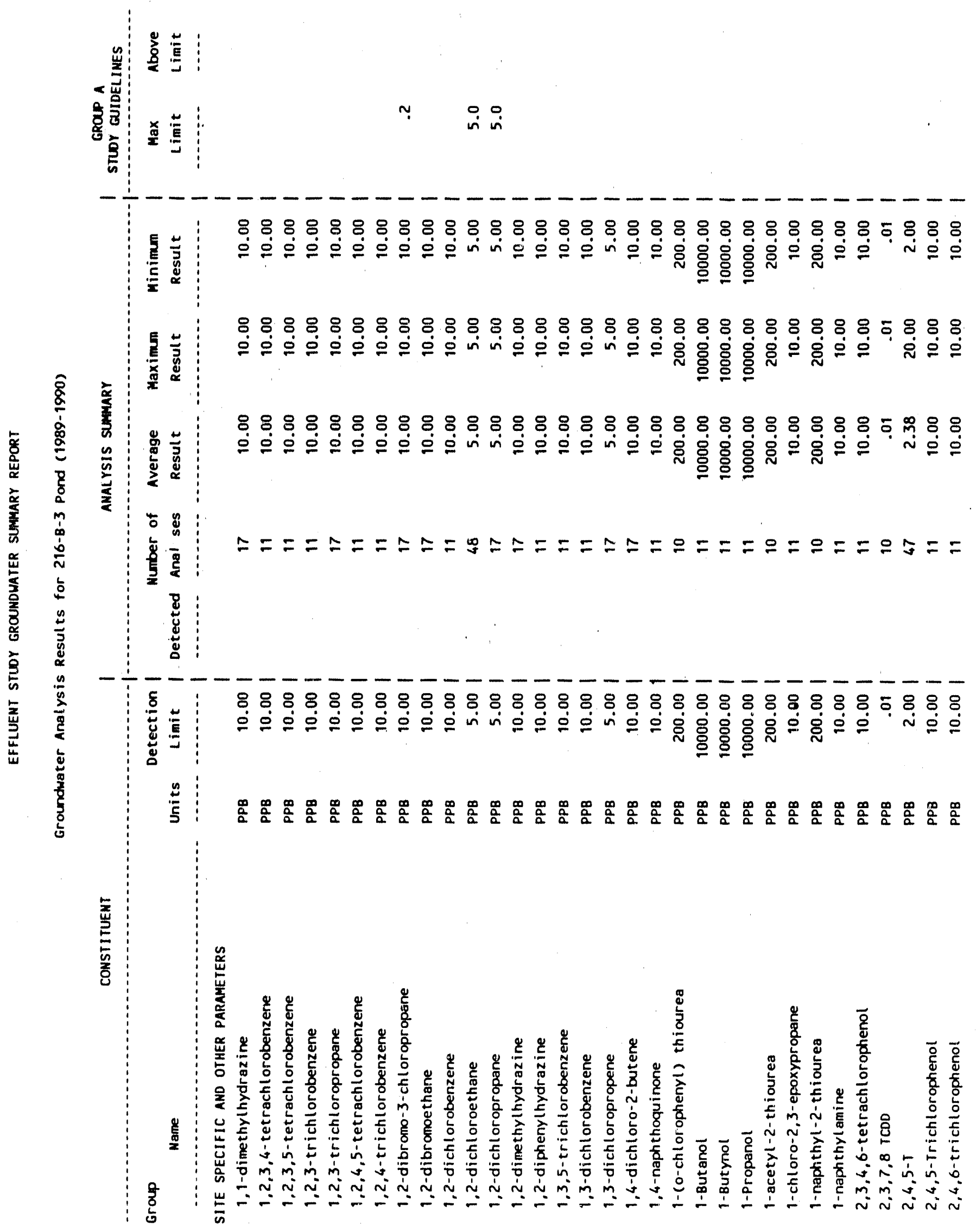


WHC-EP-0366
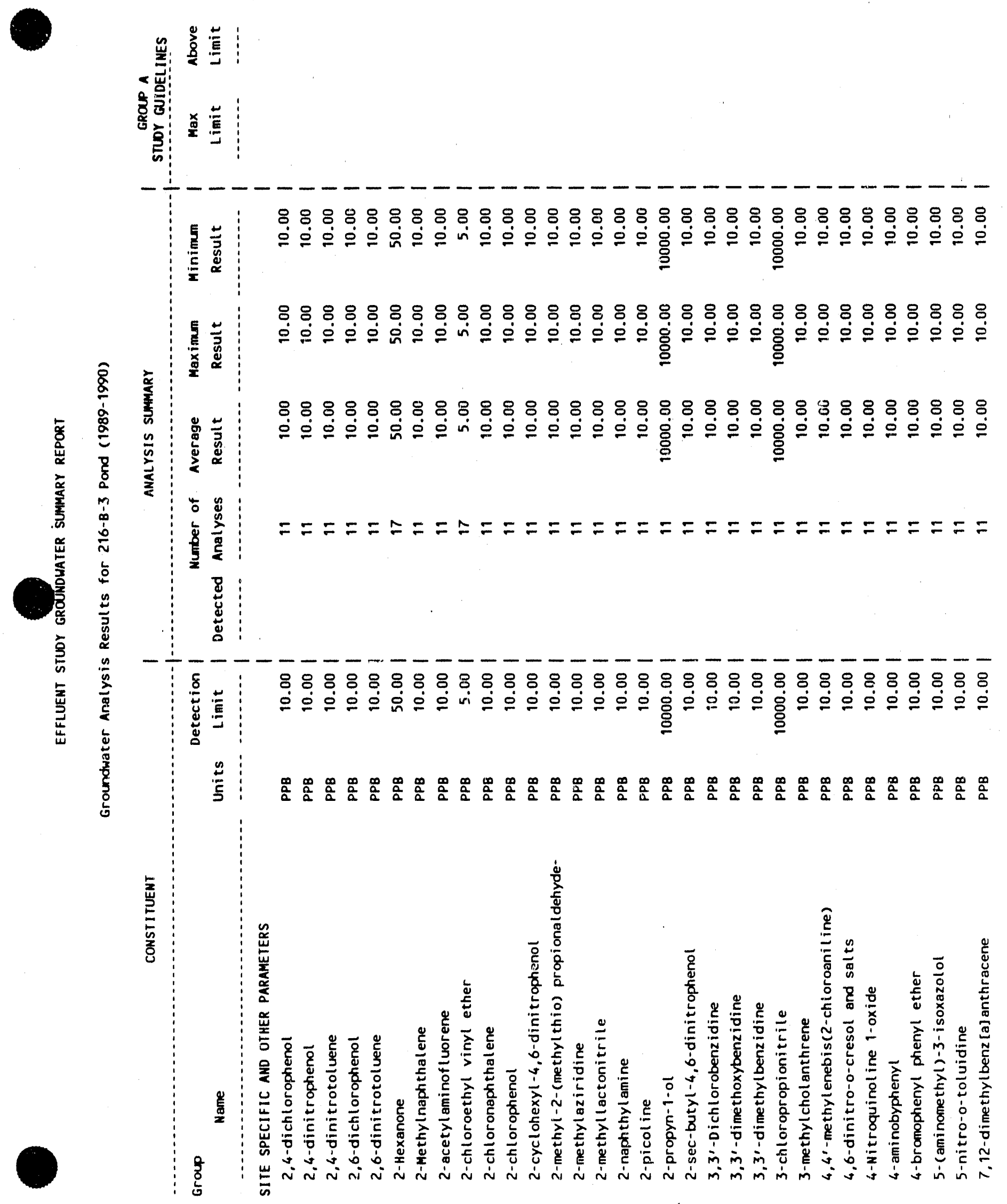


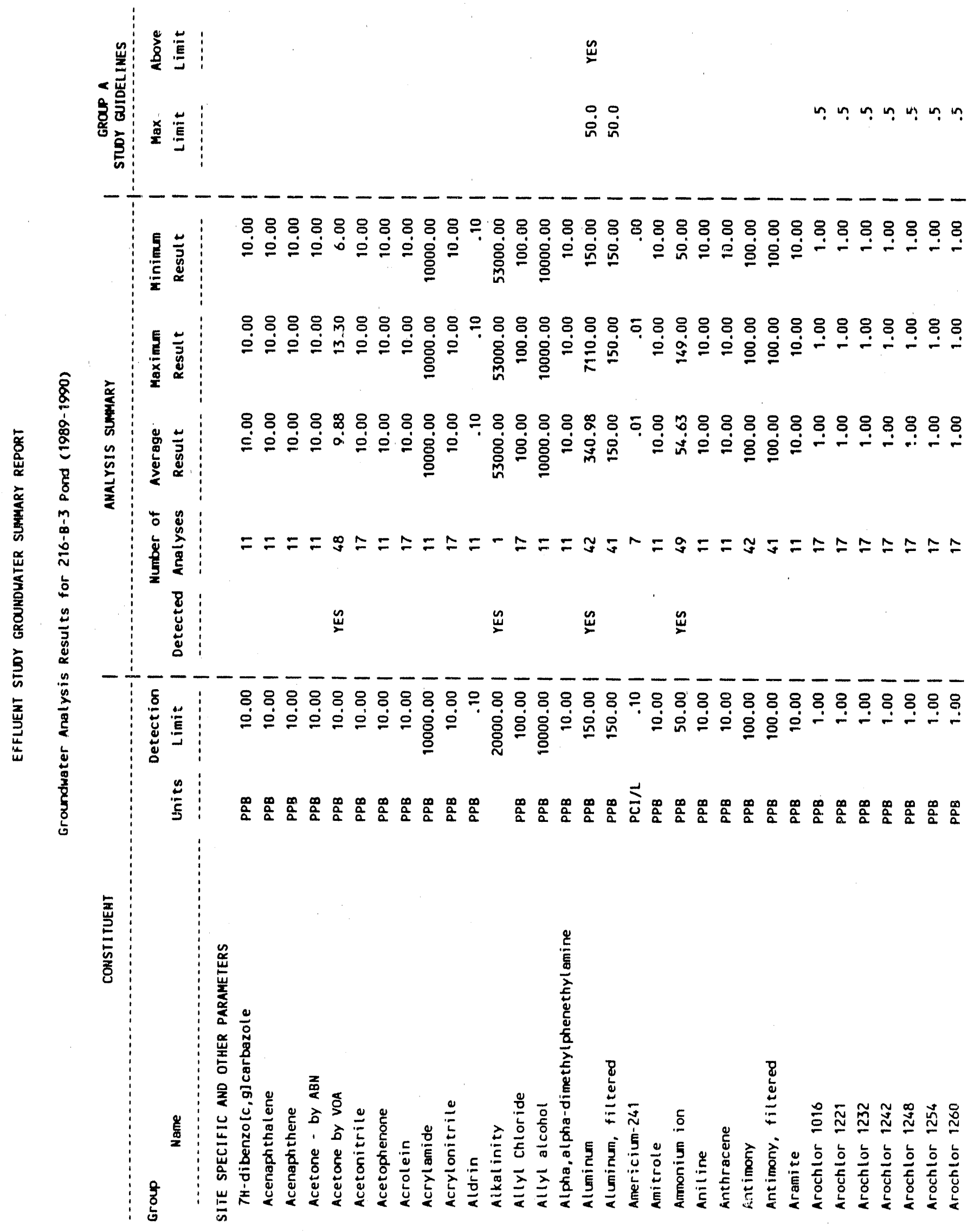




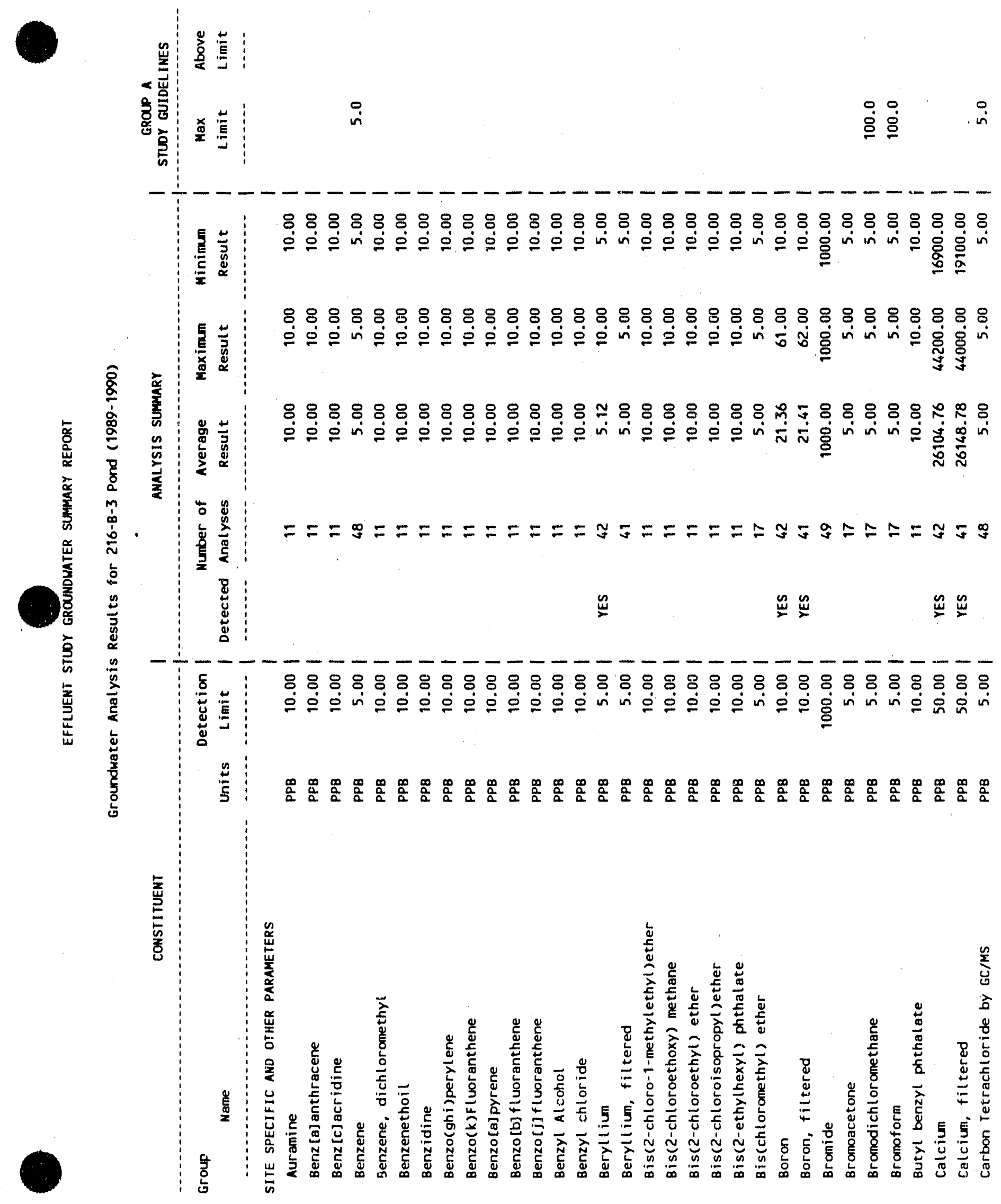




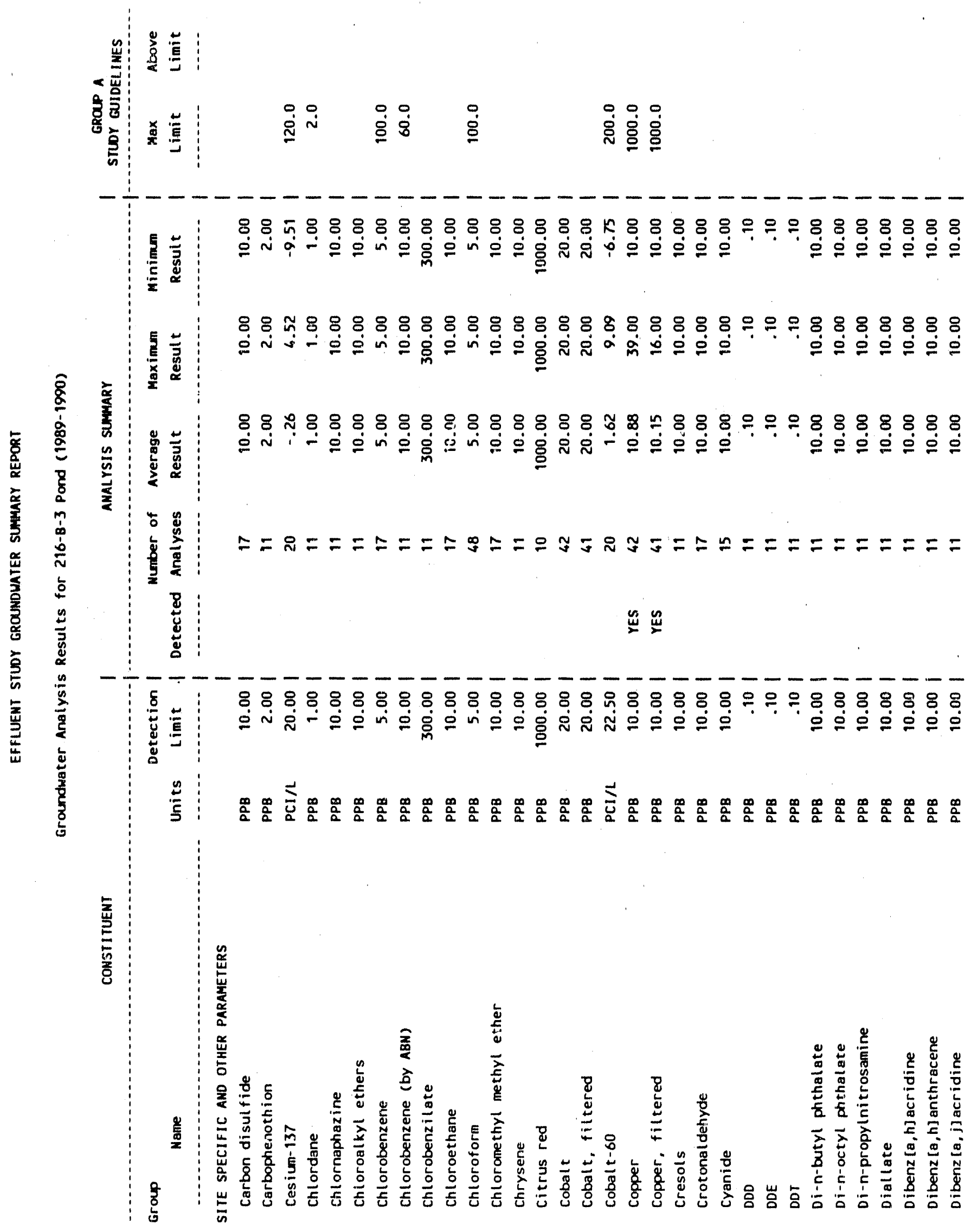




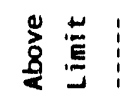

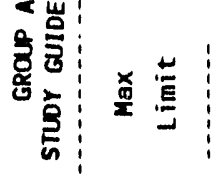

$\stackrel{\dot{0}}{\circ}$

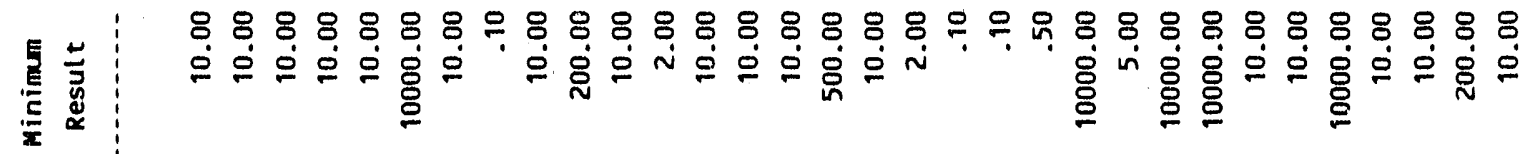

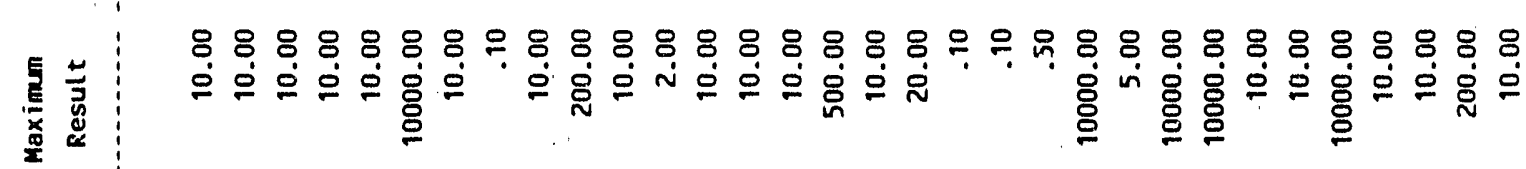

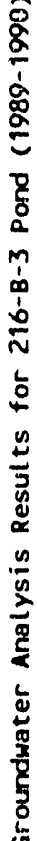

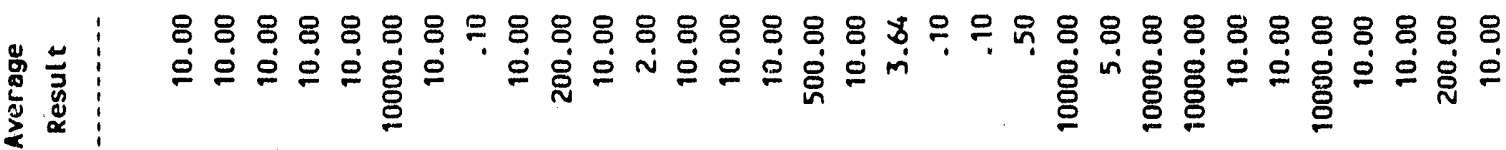
t: 8 :

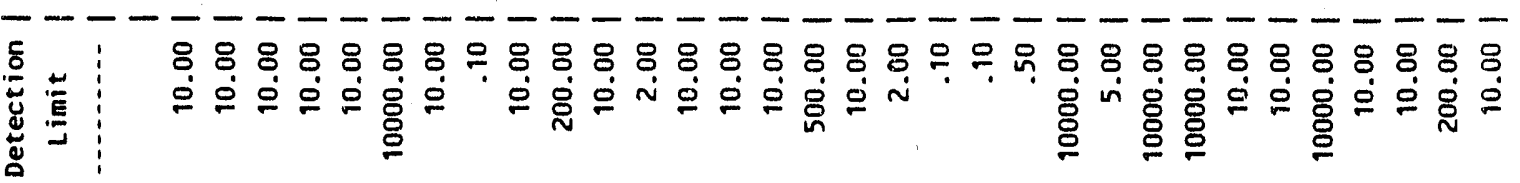

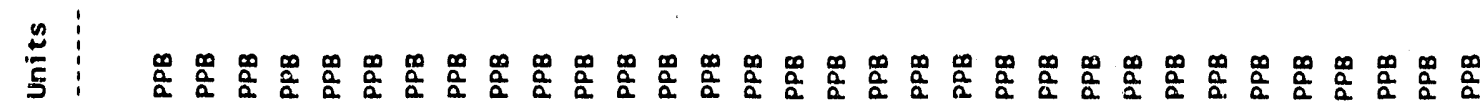
密 5

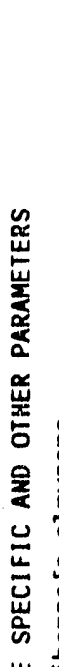

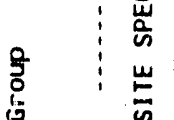

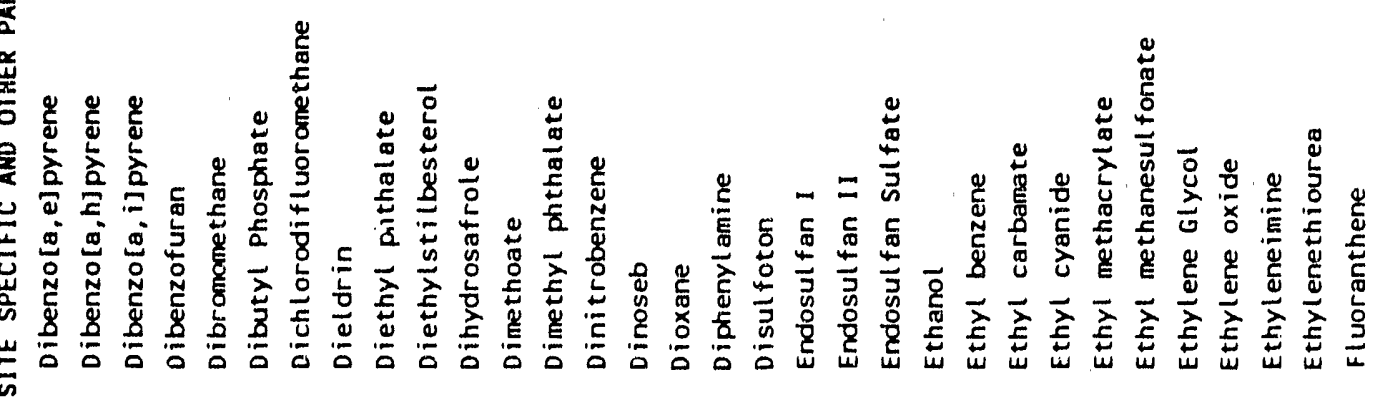




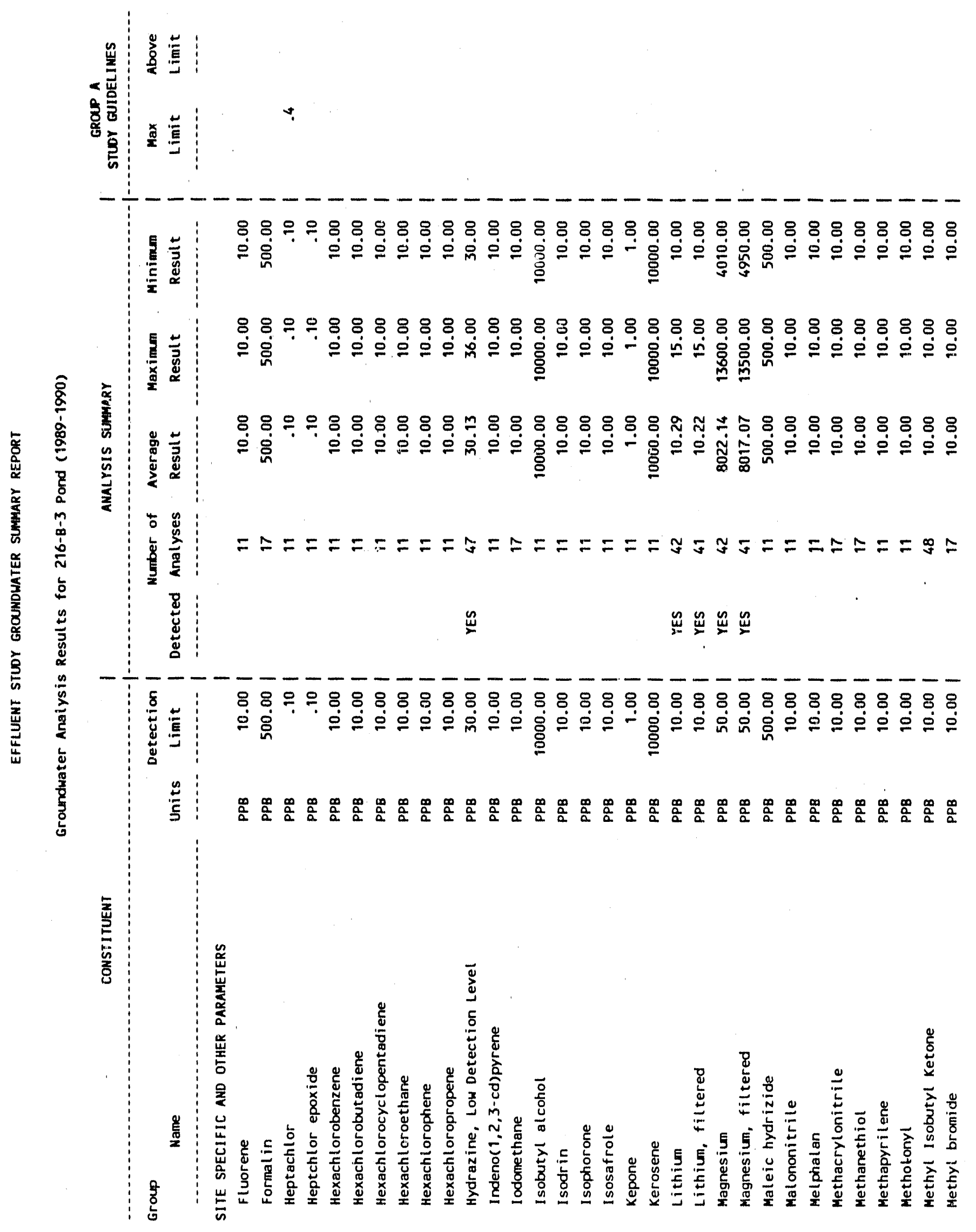


茶:

政

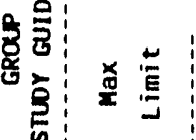

묘

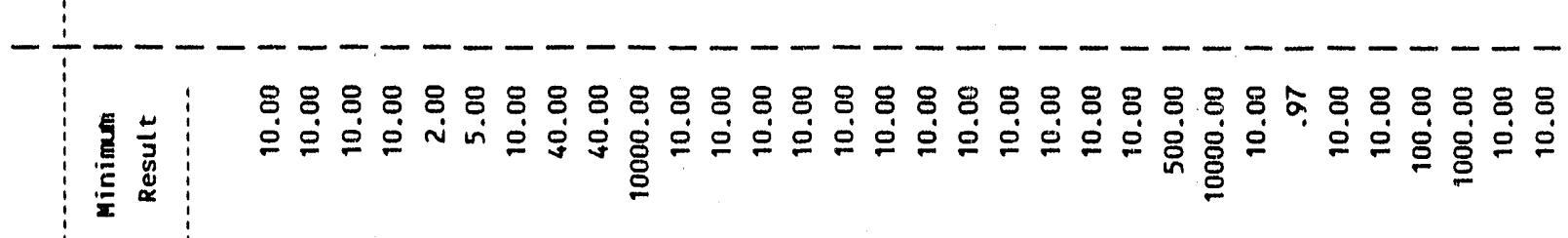

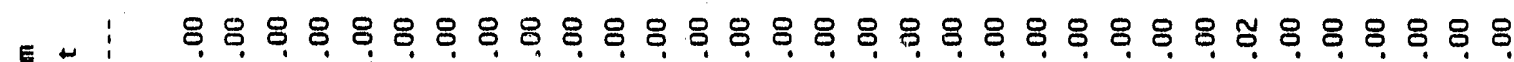

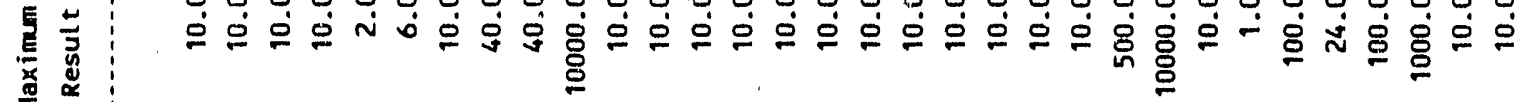

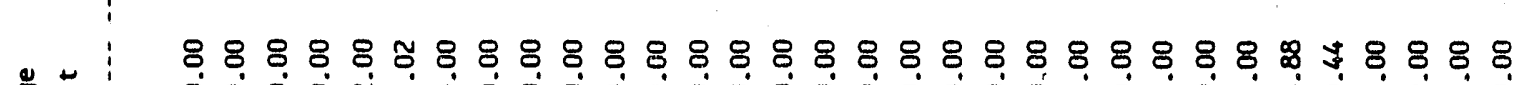

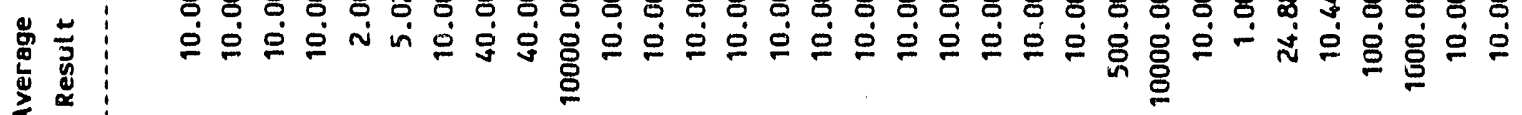

:

常

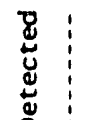

$\stackrel{\mathscr{m}}{2}$

出出出

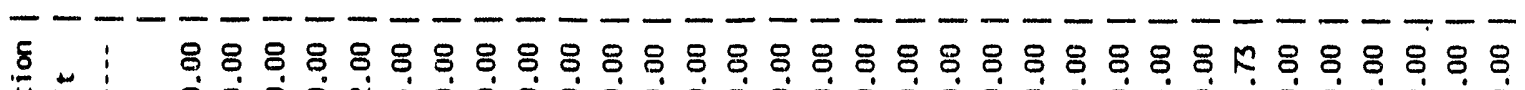

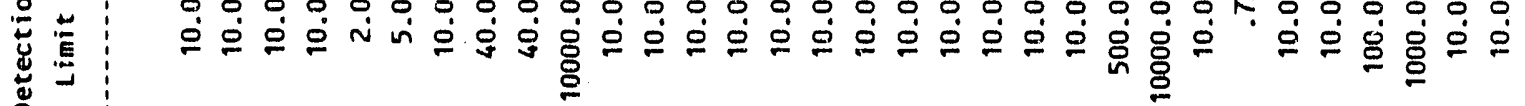

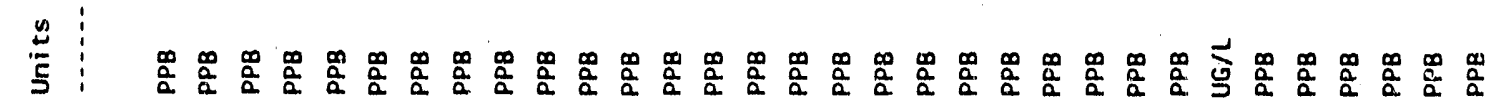

氙

密

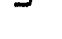

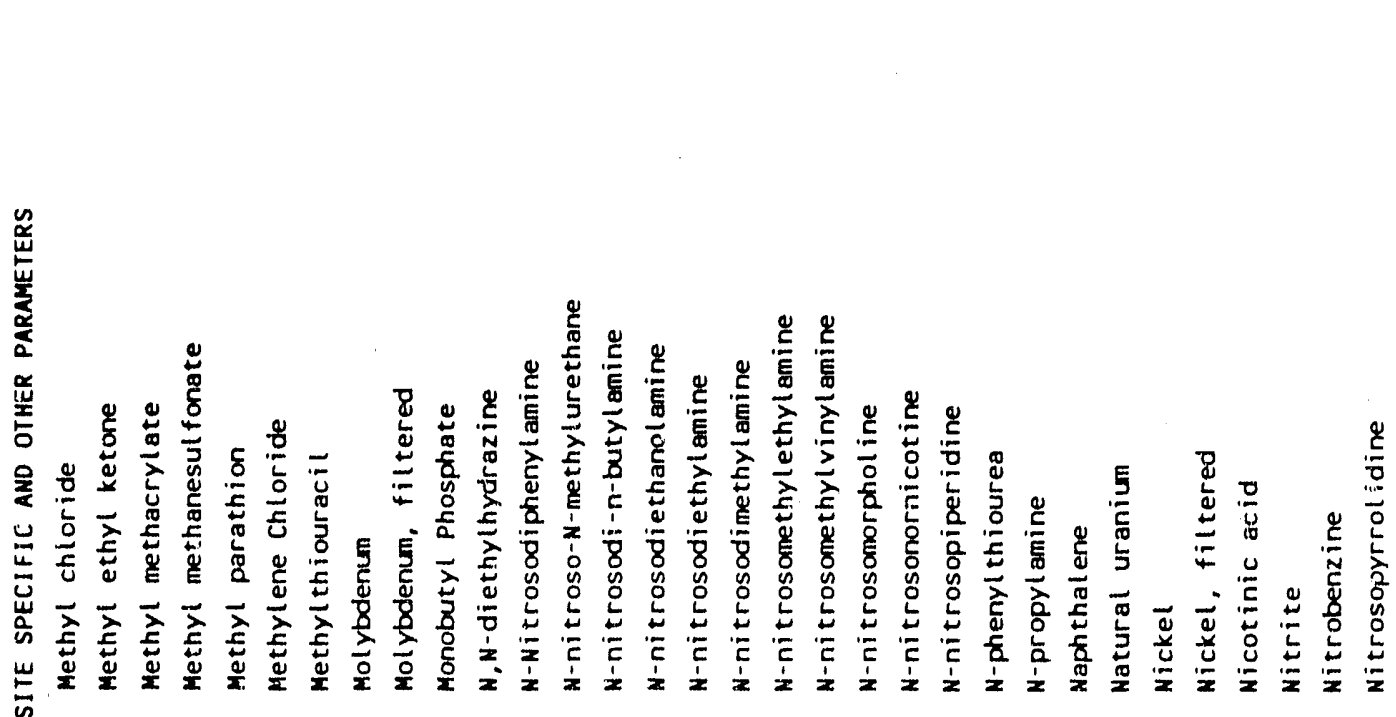



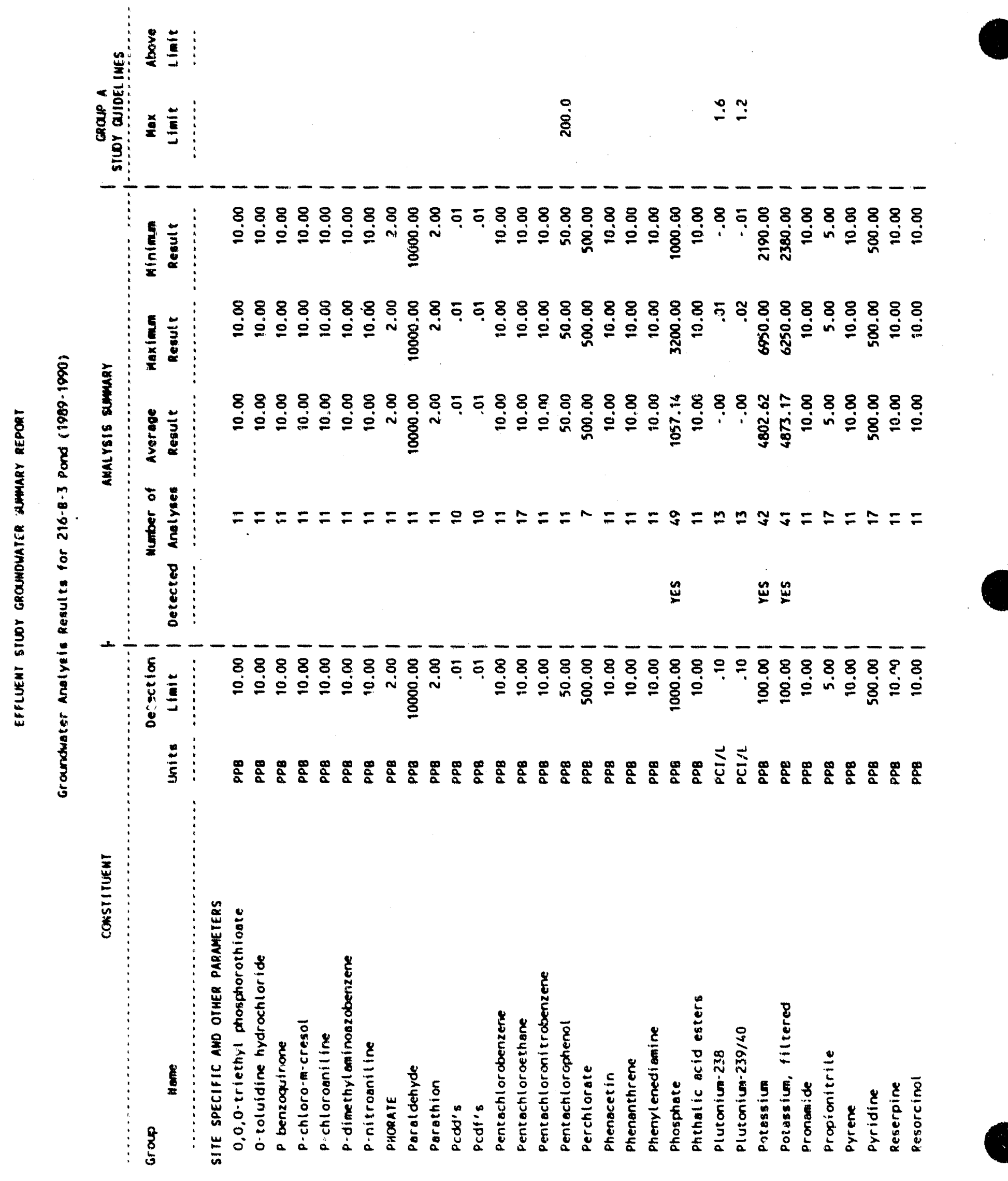
WHC-EP-0366

:

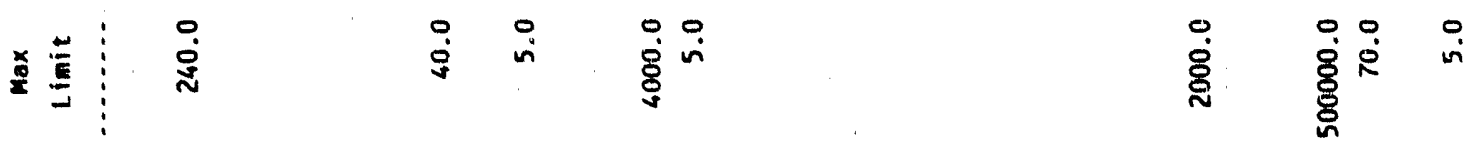

重言

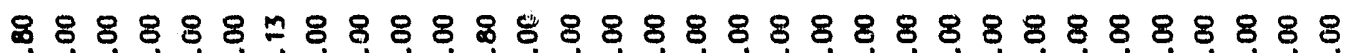

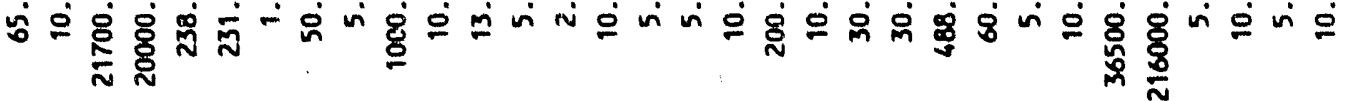

요 8 \%

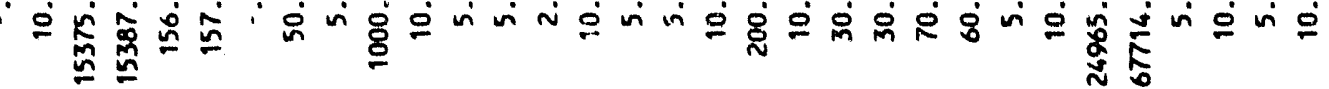
起

$\div 8:$

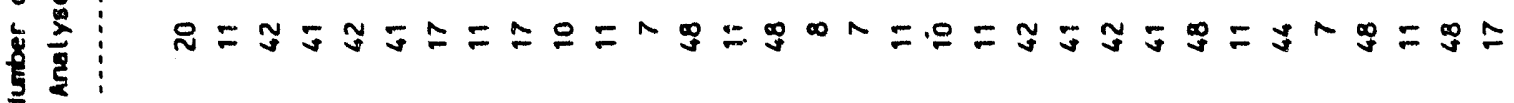

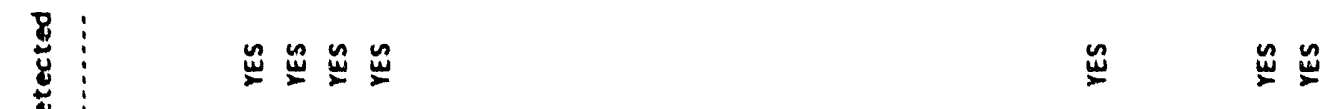

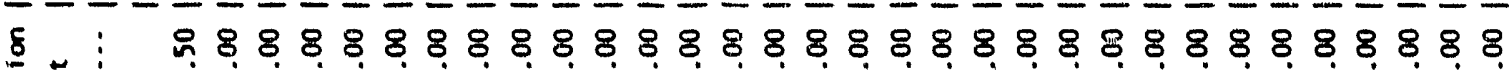
过 I

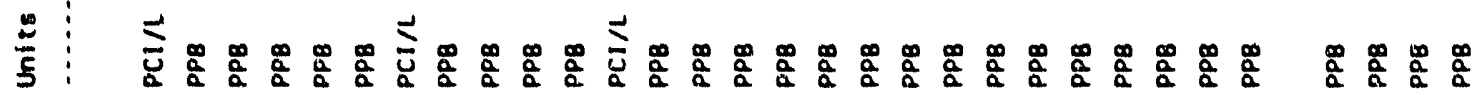
롱

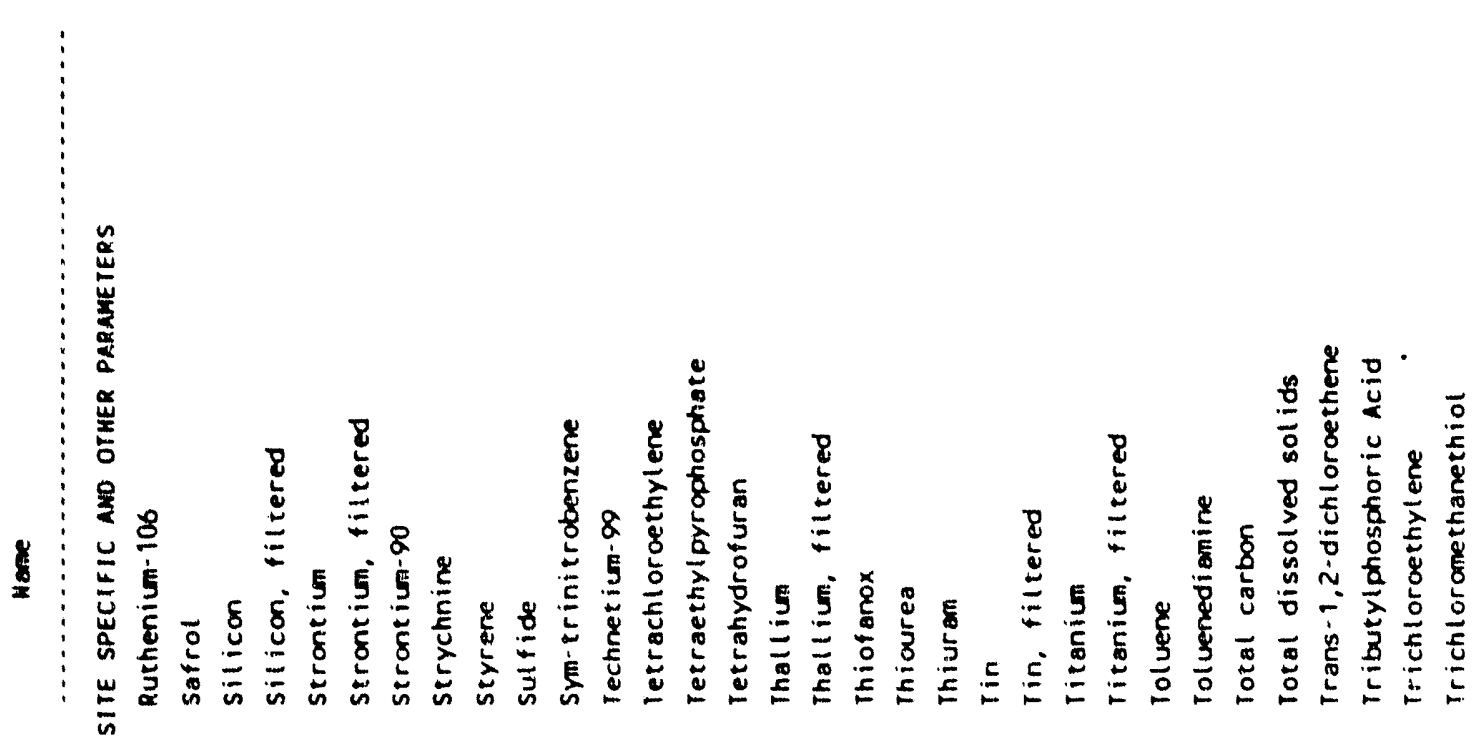


WHC-EP-0366

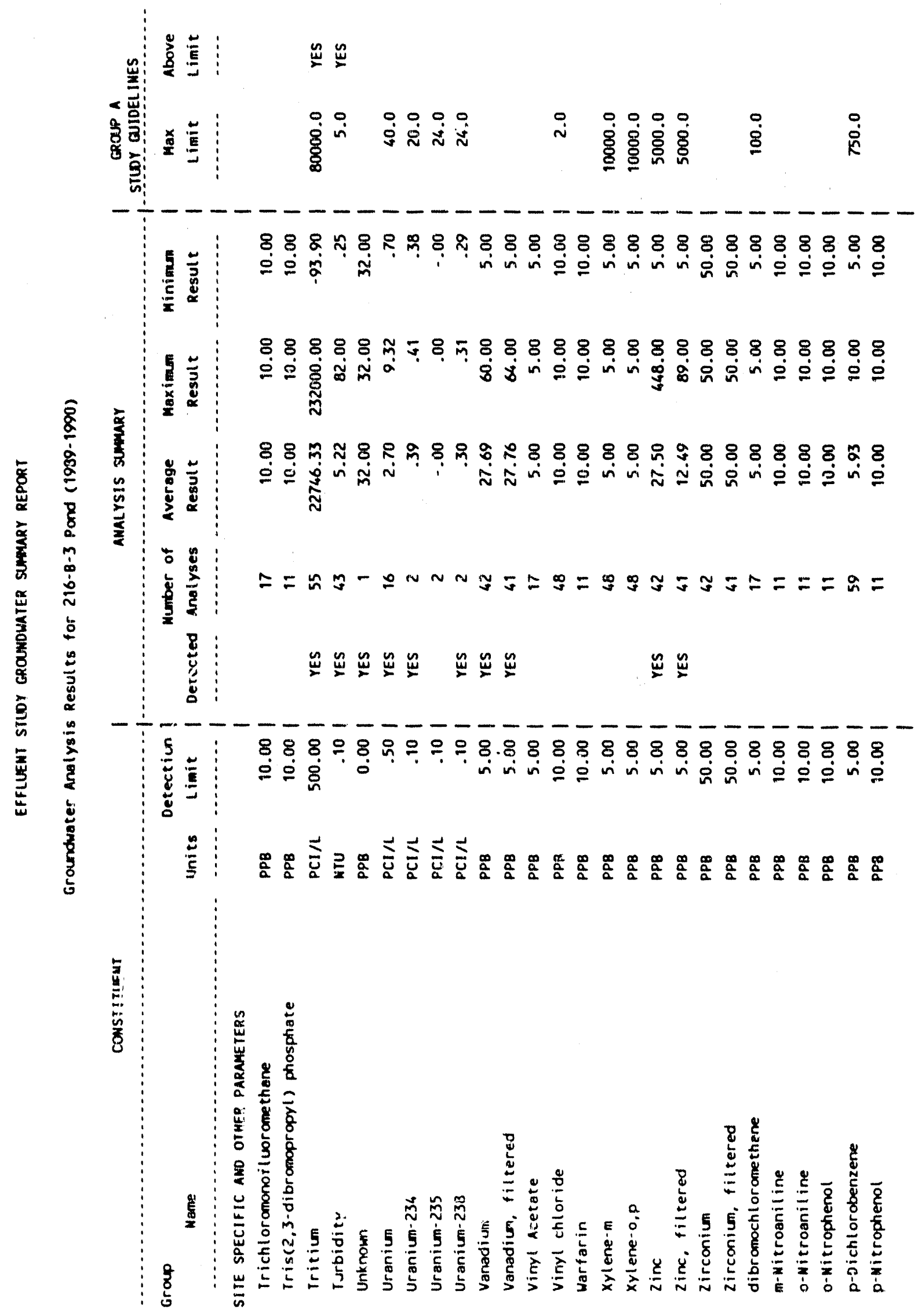




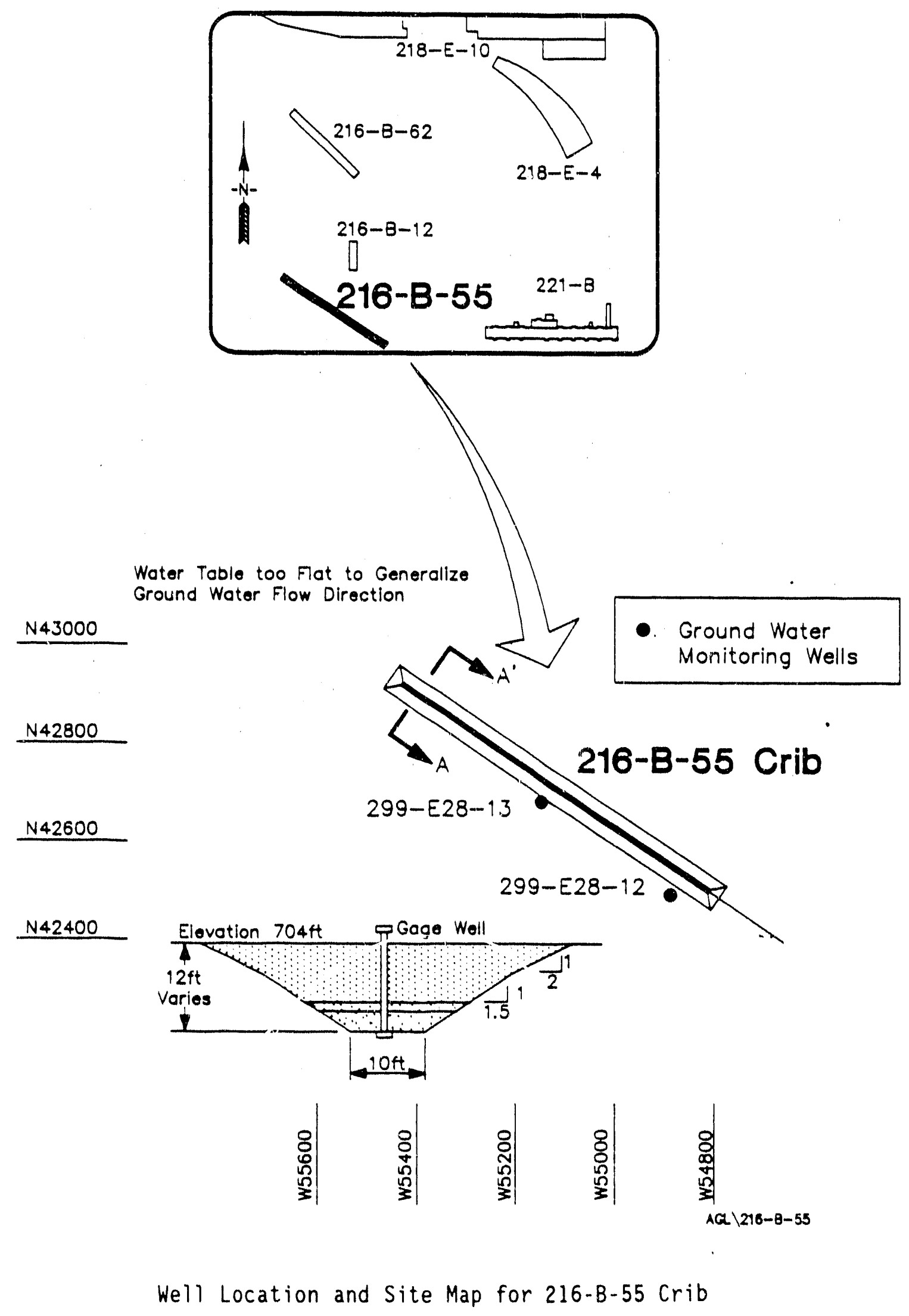


WHC-EP-0366

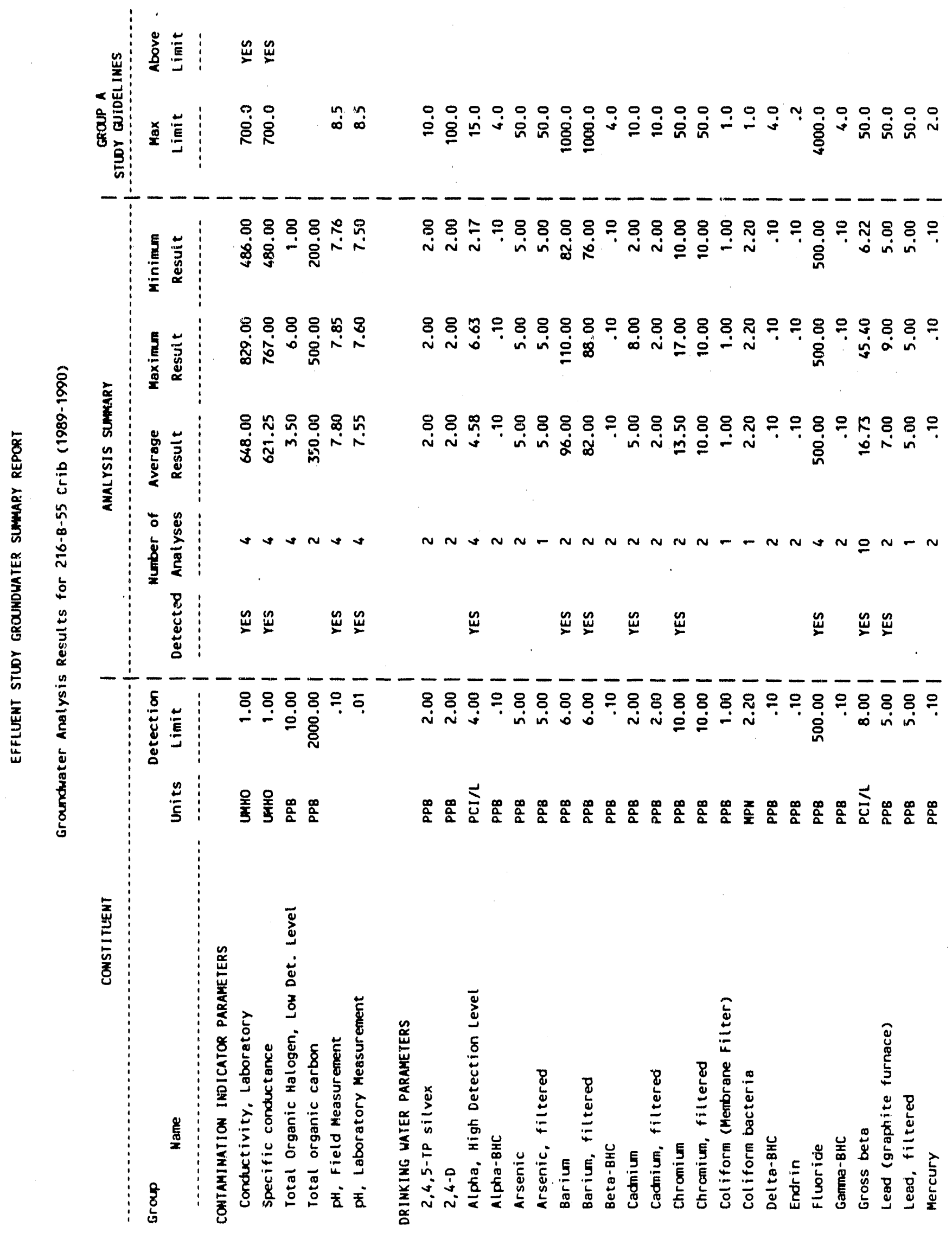


WHC-EP-0366

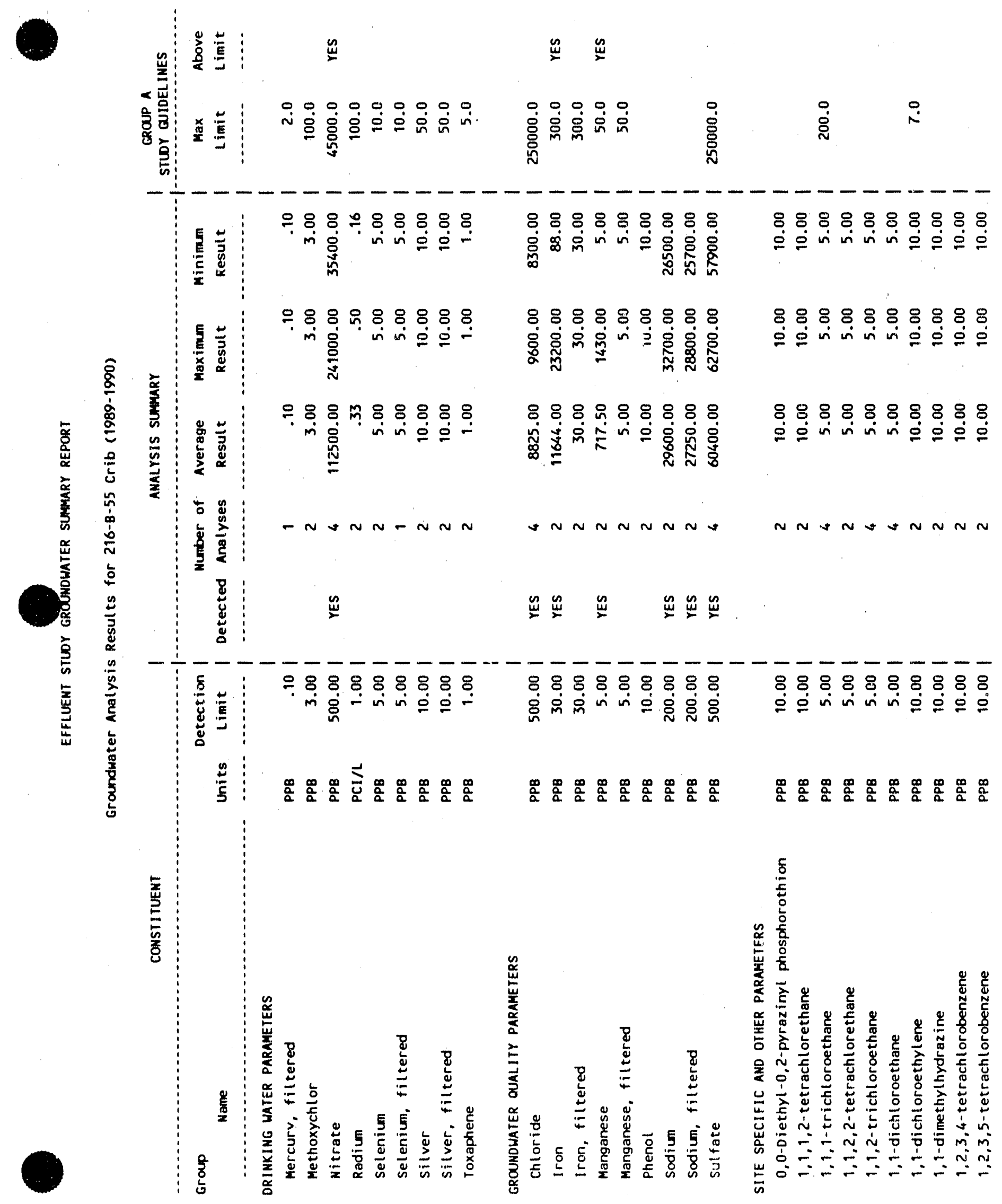


WHC-EP-0366

\section{章毫:}

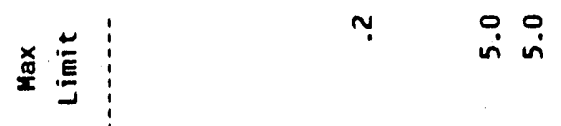

高喜

88888888888888888888888885888888

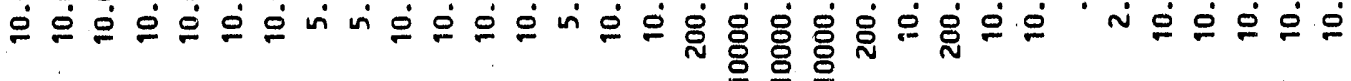

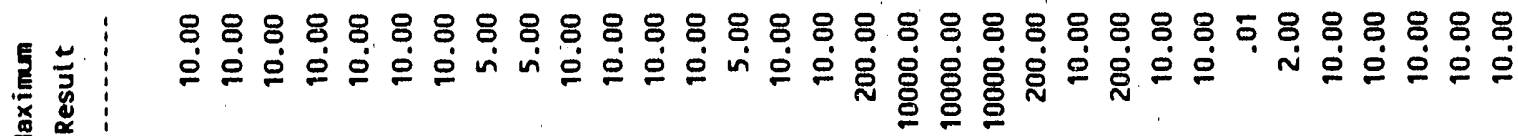
希

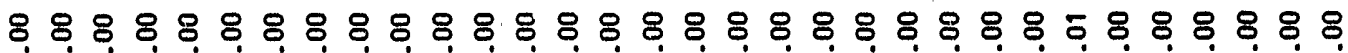
若 Lo 焉要

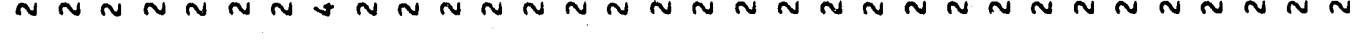
(a)

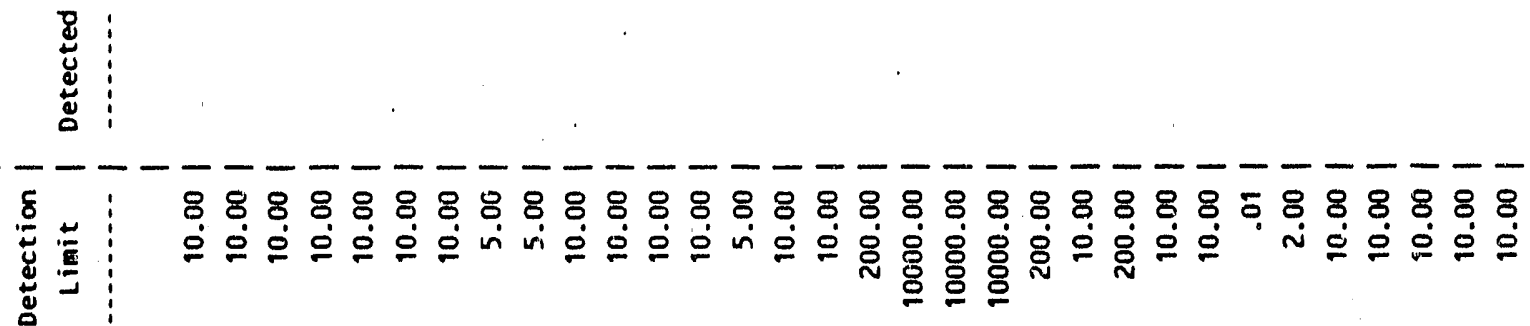
旁:

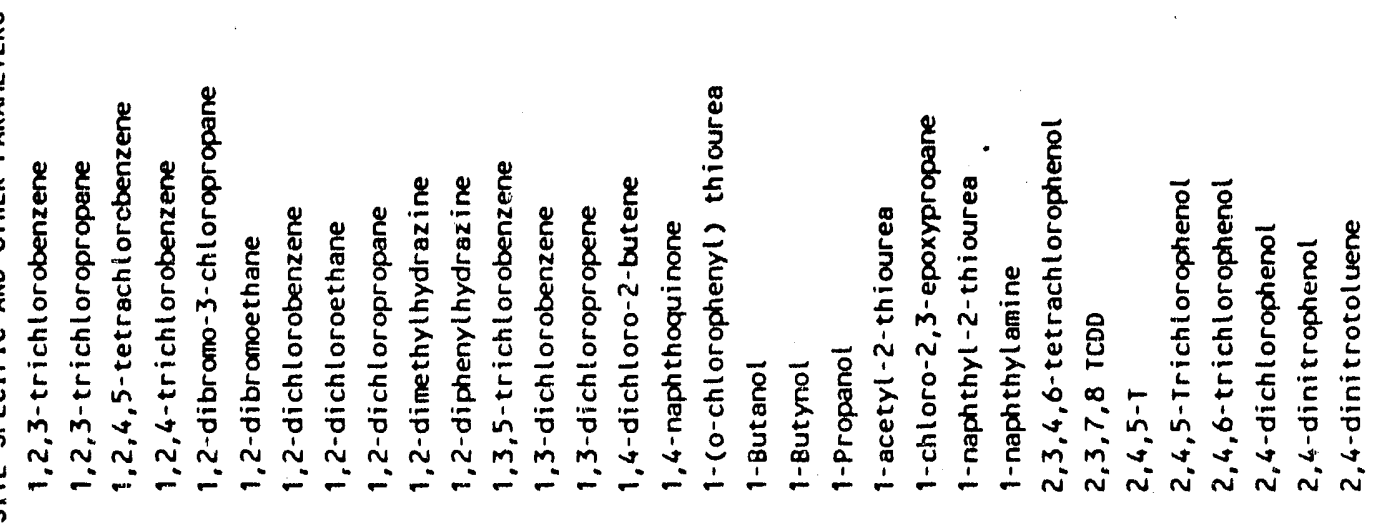


WHC-EP-0366

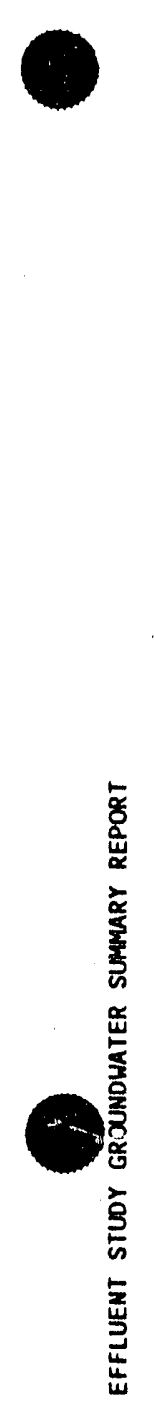

葛

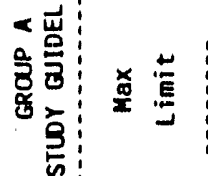

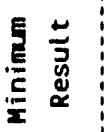

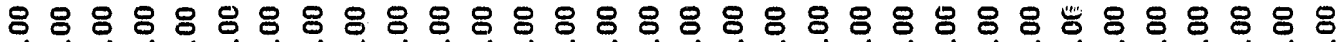

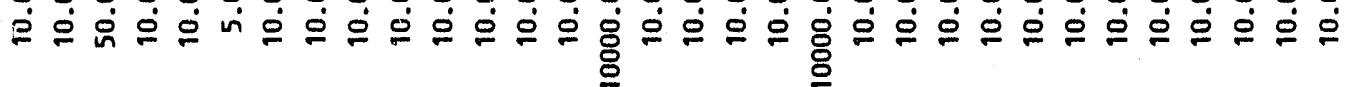

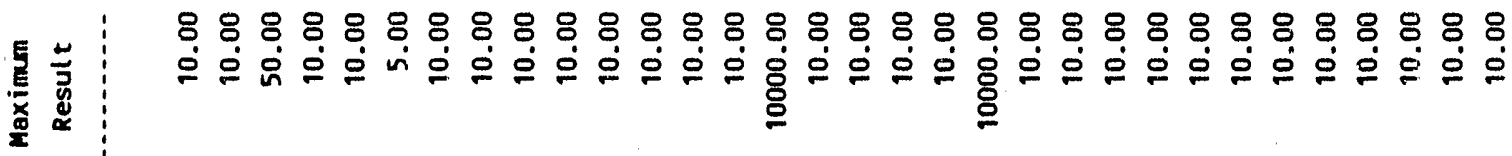

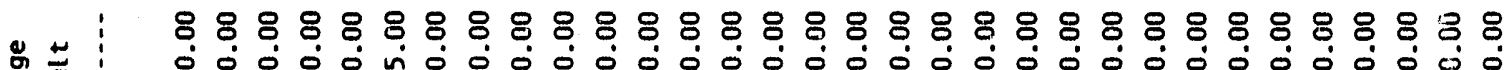

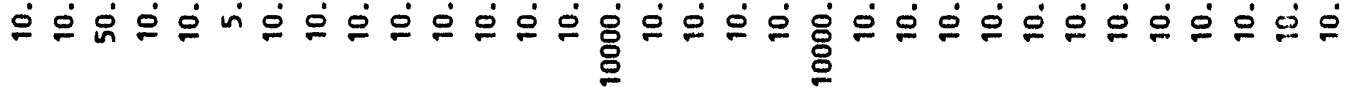

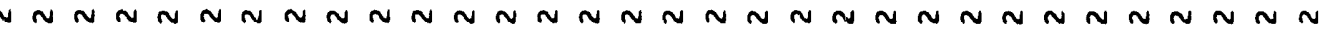

$\frac{\square}{0}$ 原 :

$-\frac{1}{\vdots}$

尊

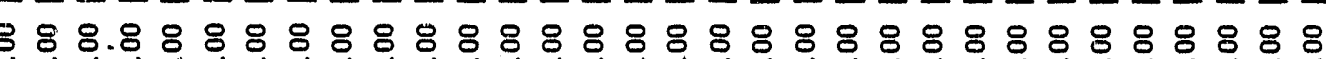

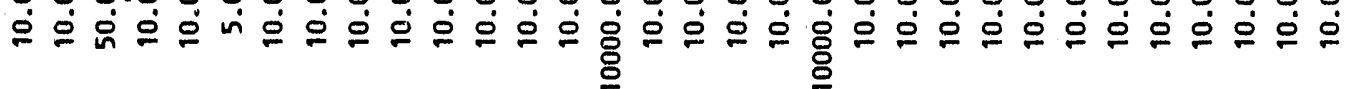

$\stackrel{2}{5}:$

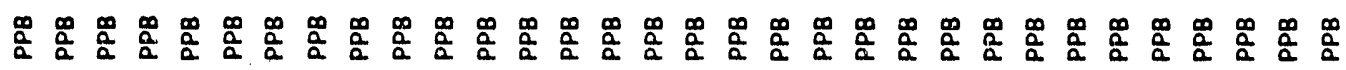

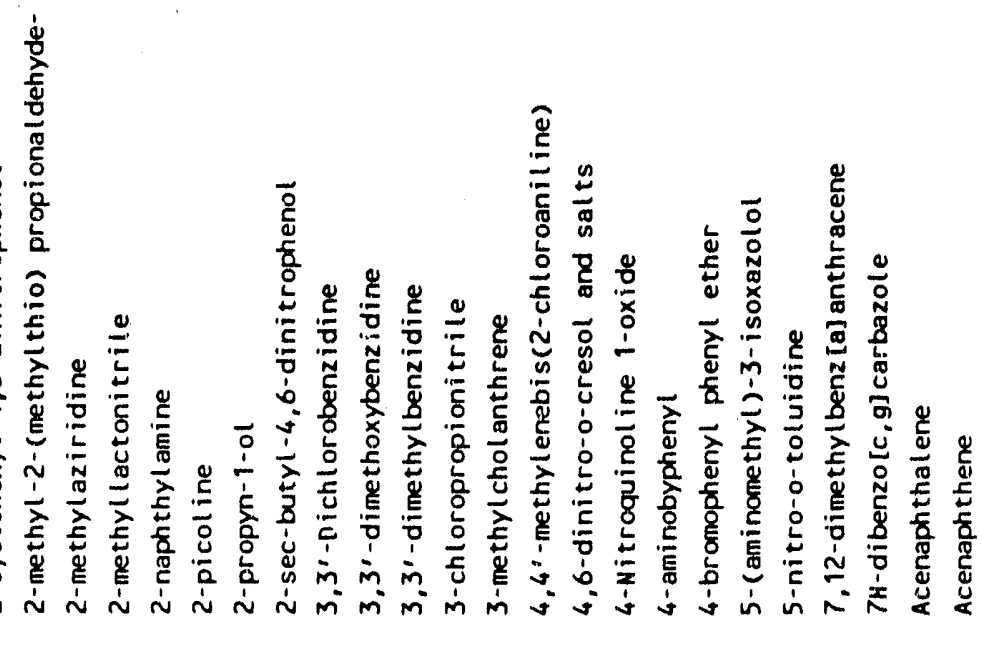


WHC-EP-0366

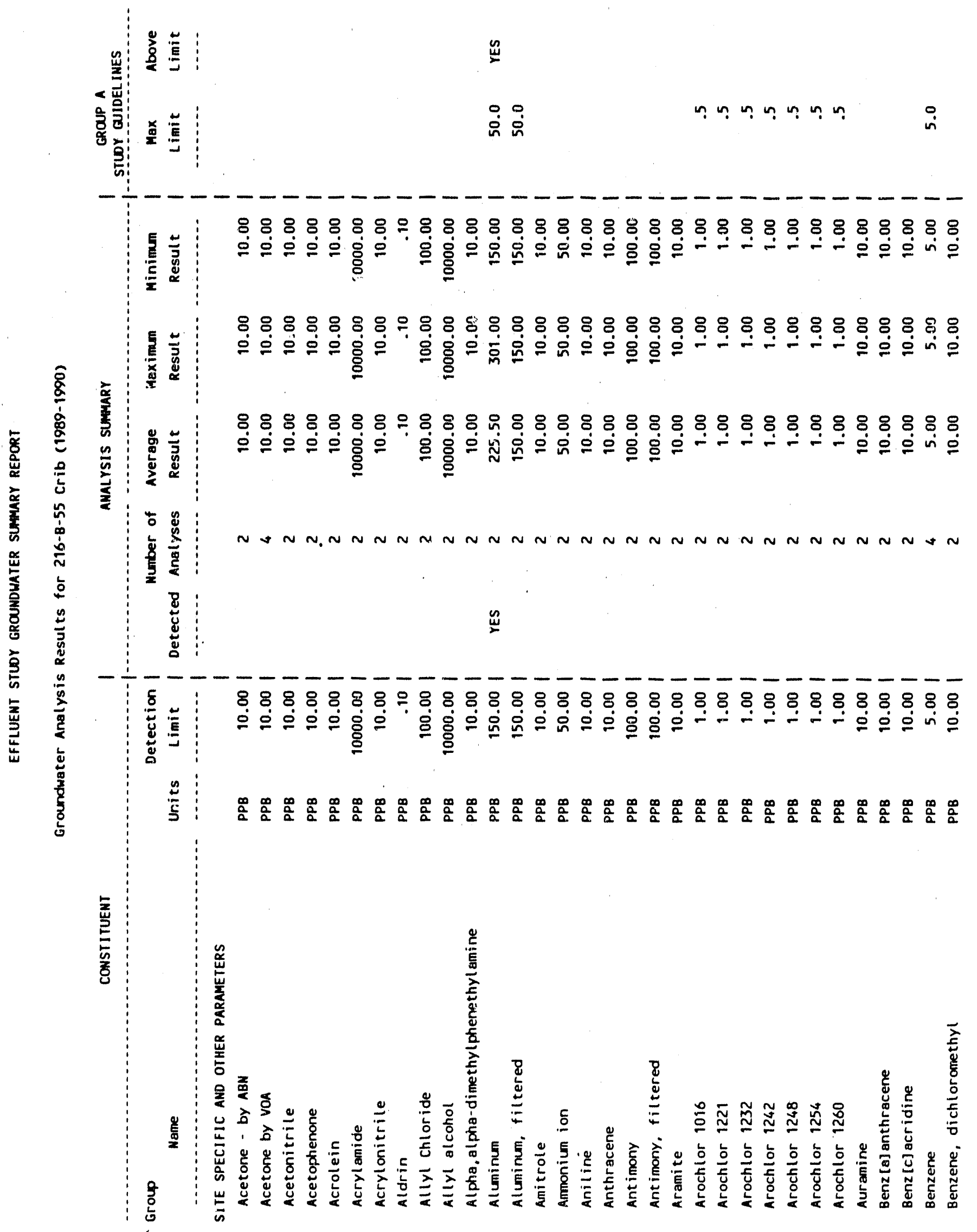




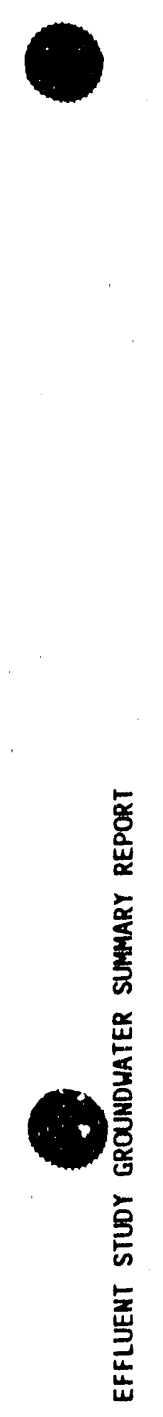

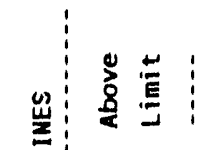

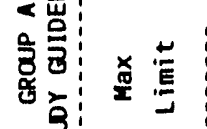

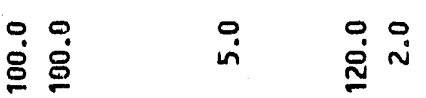
$-\frac{1}{1}$

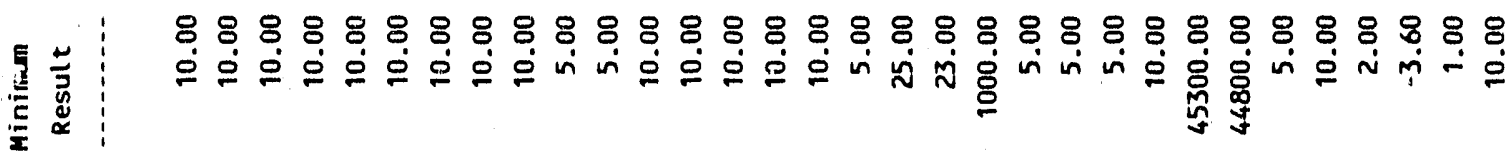

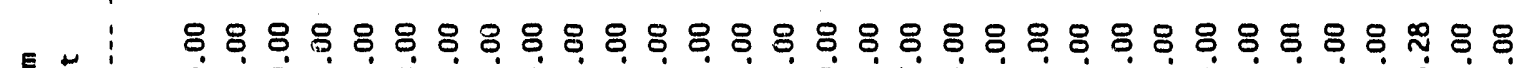
重 $\quad$ @

$88888888888888988808888888888 \kappa 88$

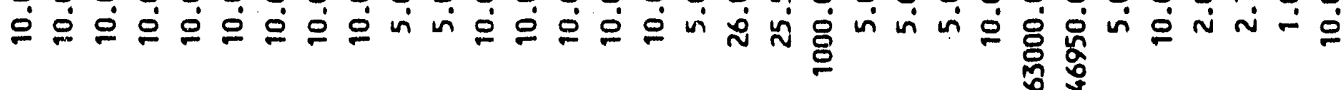

兽蔫 $4:$

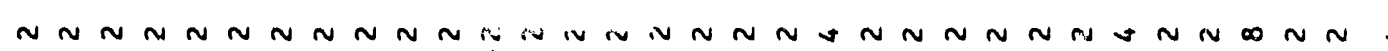

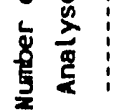
总 :

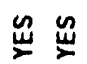

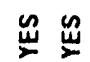

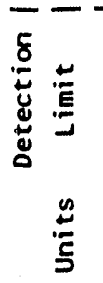

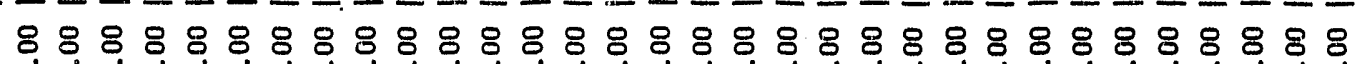

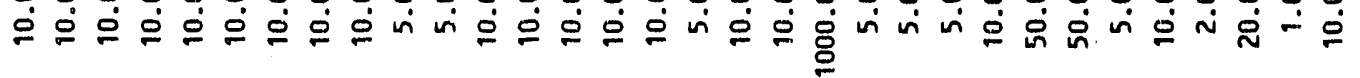

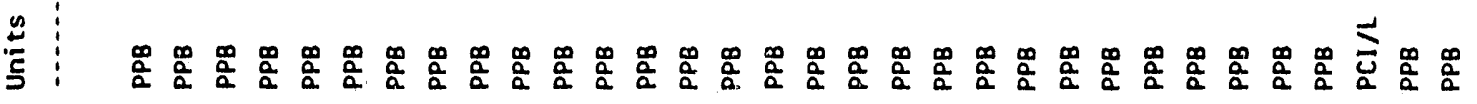

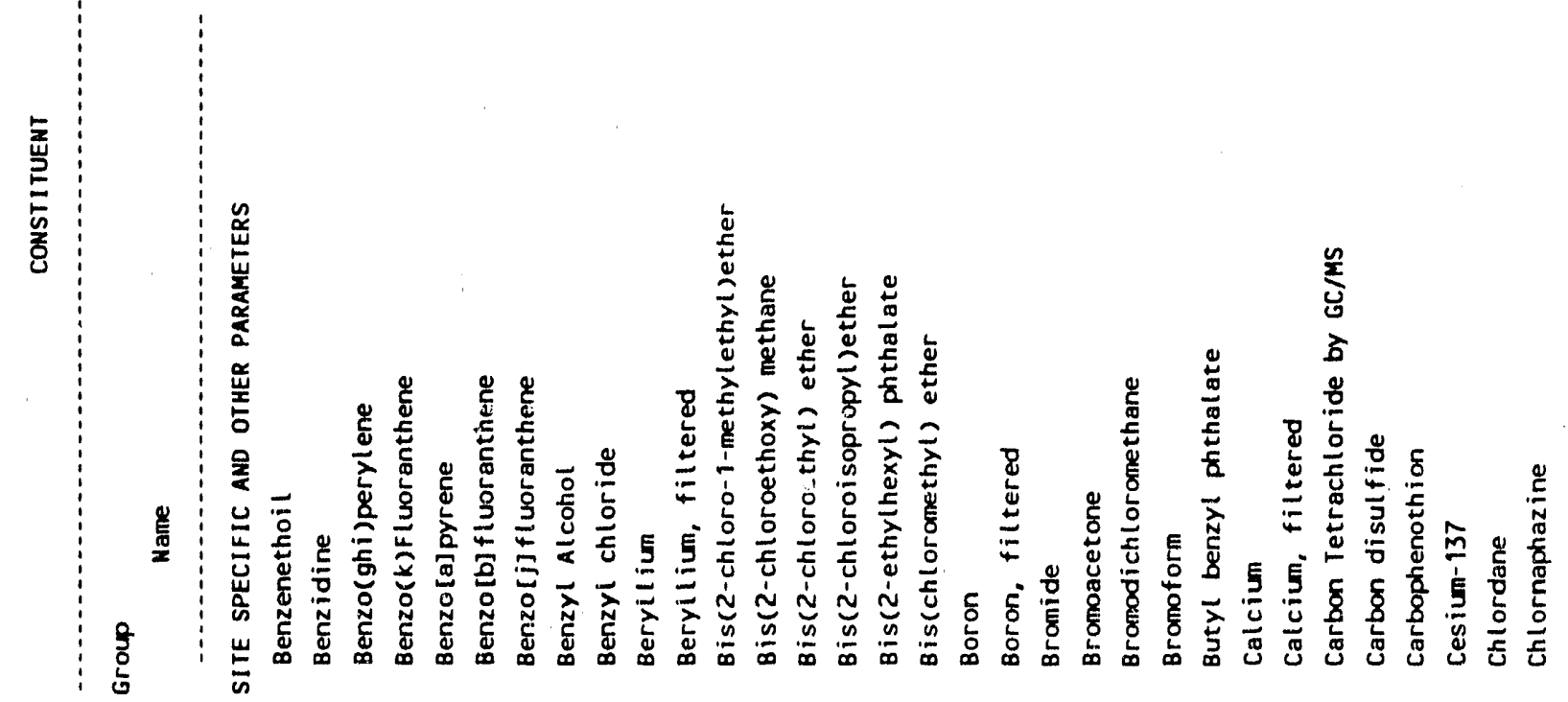


WHC -EP-0366

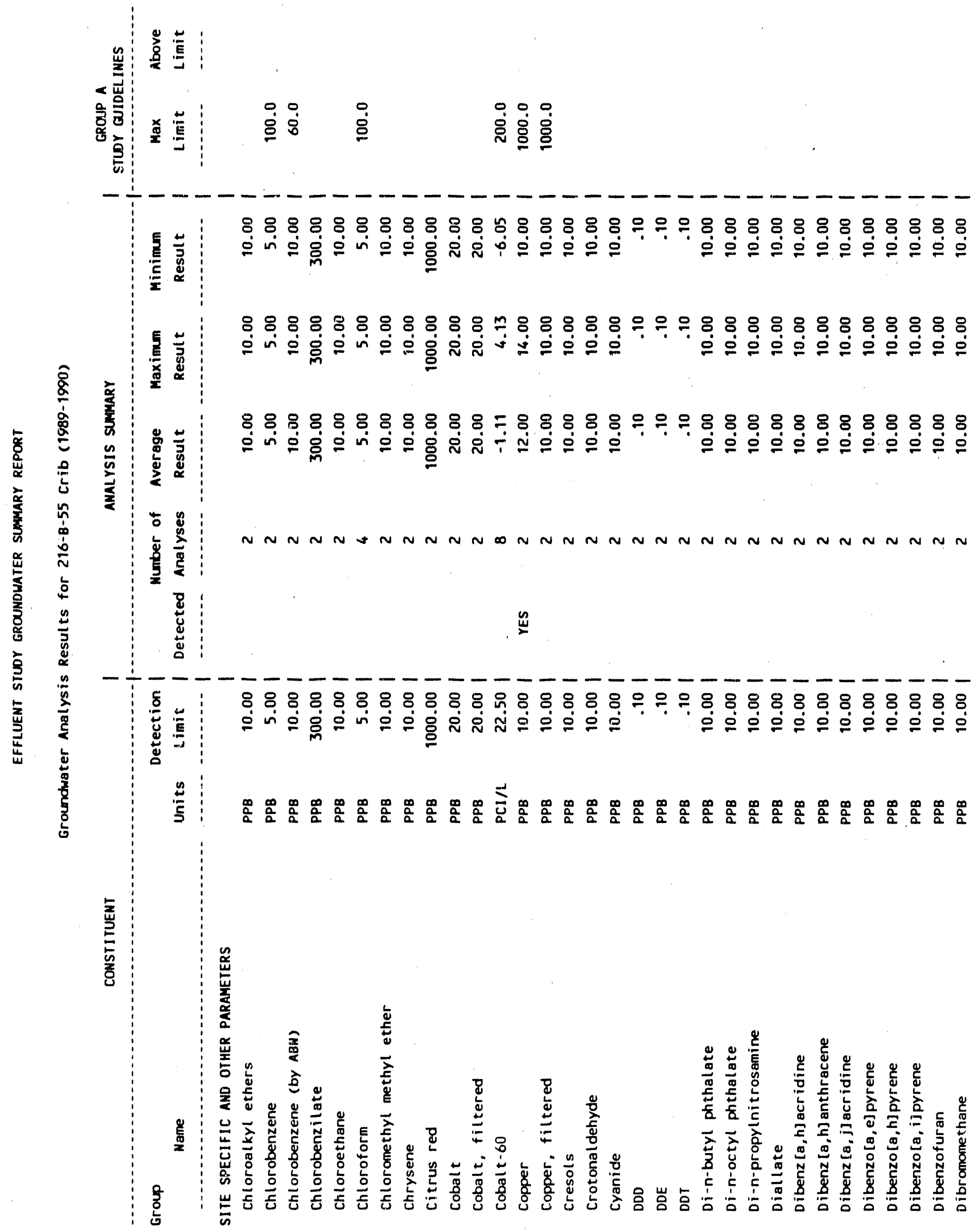




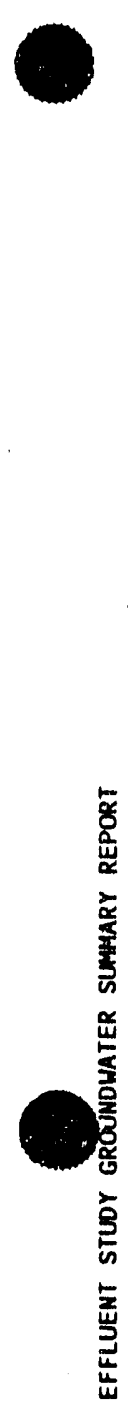

营:

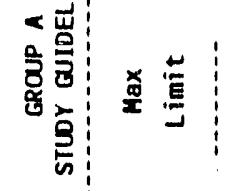

옹

:

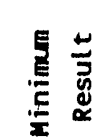

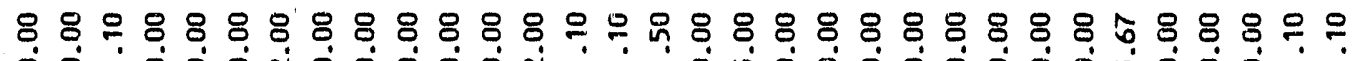

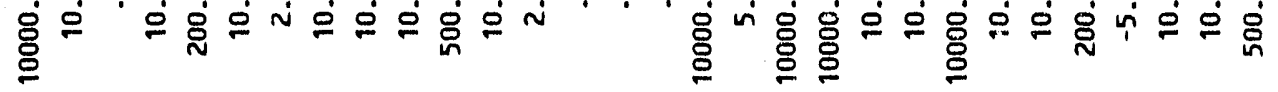
\begin{tabular}{l|ll}
\hline & 8 & 8
\end{tabular}

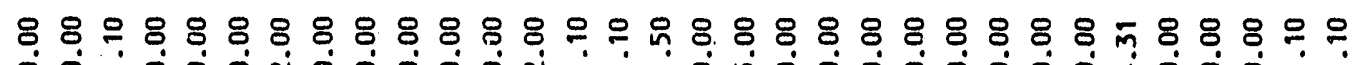
㞭苛

实

ठ்

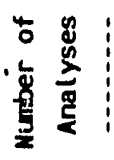

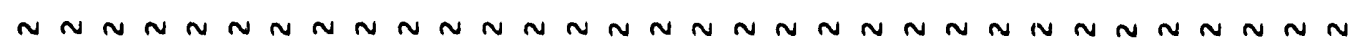

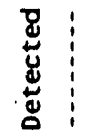

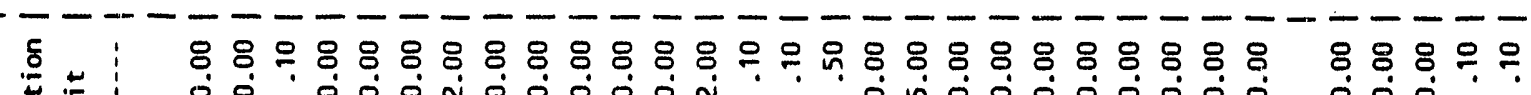
蒁

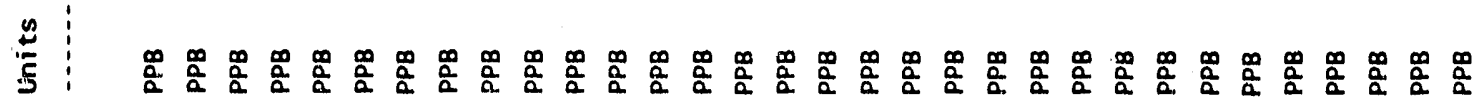
롤

密

足

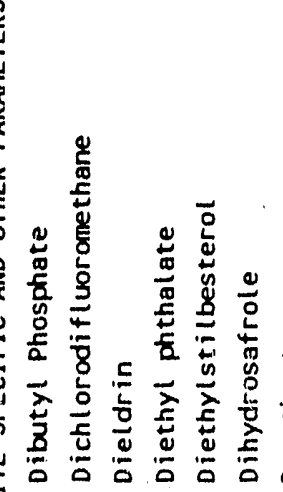

s

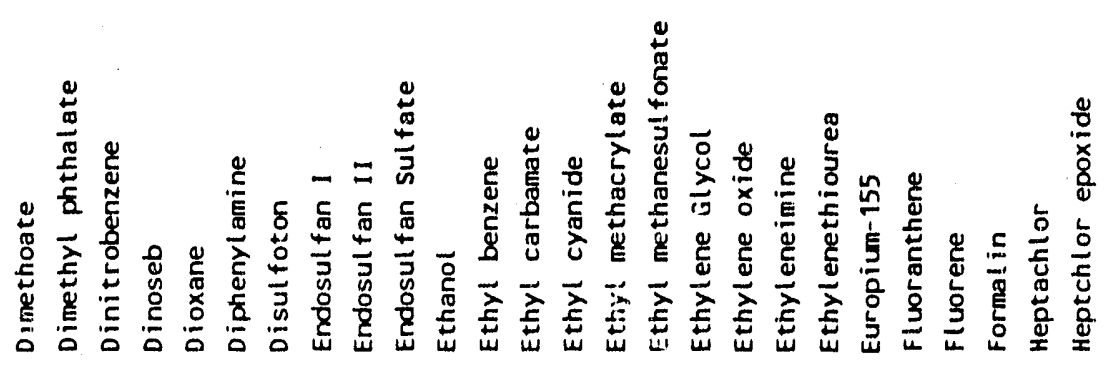


WHC-EP-0366

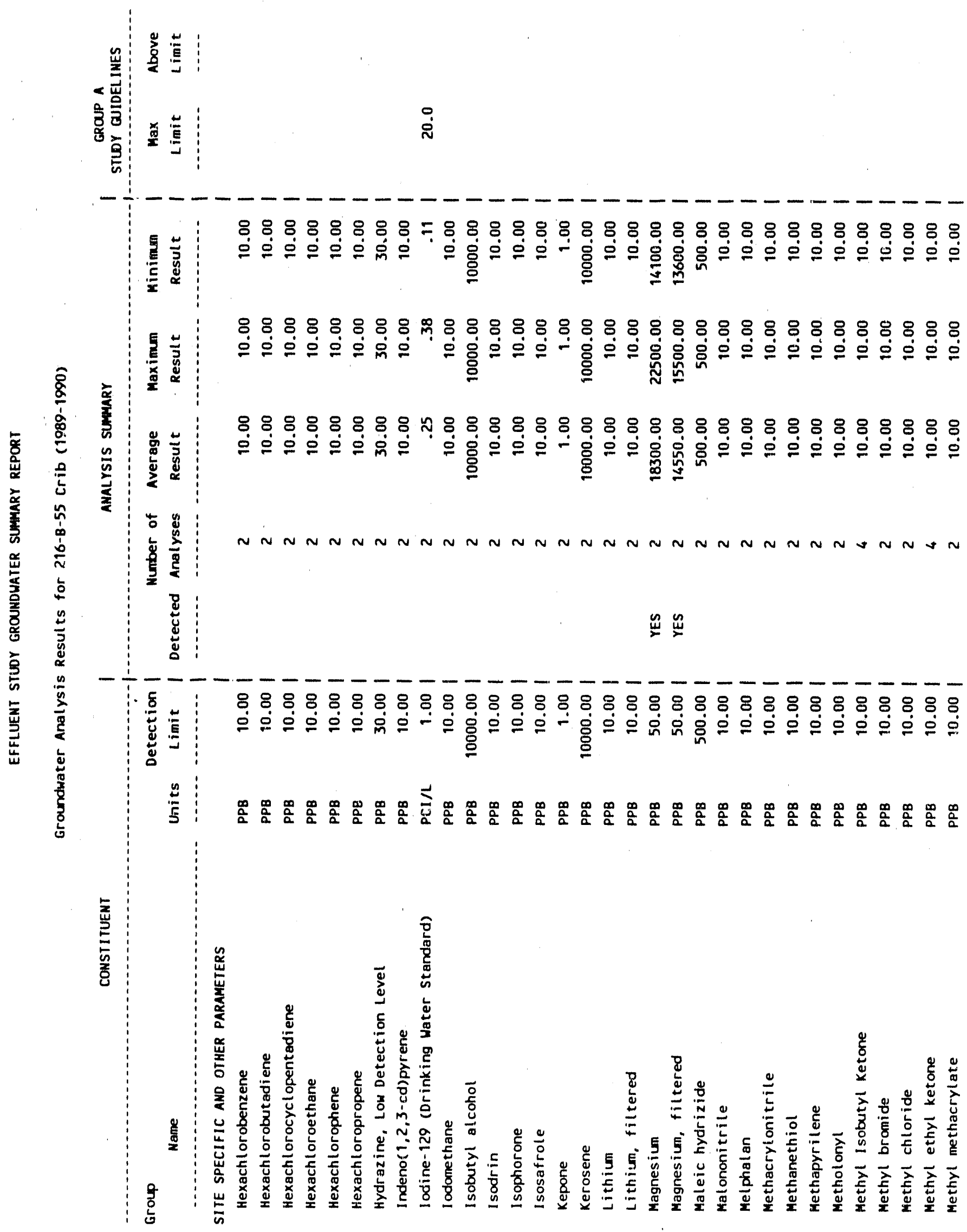




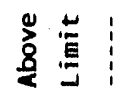

恼

:

疋

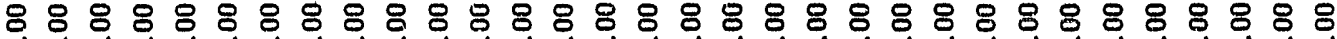

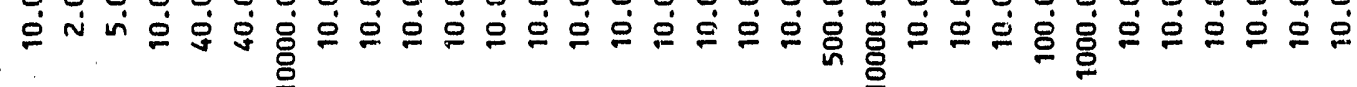

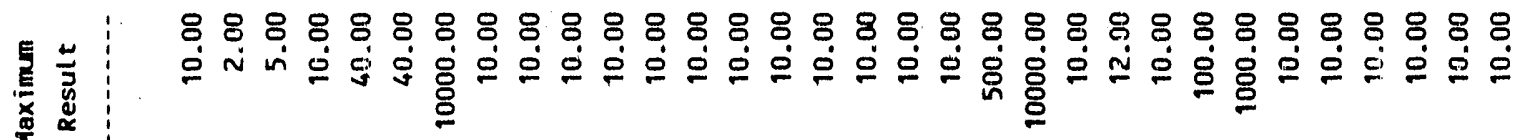

88888888888888888888888888888888 ஹ่

兽苛

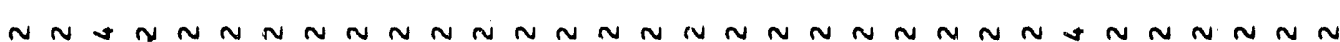

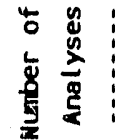

$n \sin n \sin n \sin$ ஹ

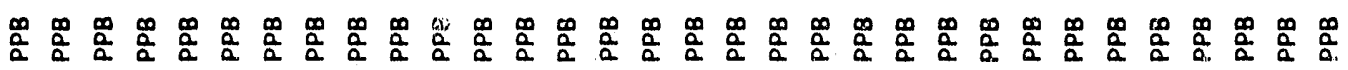

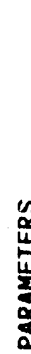

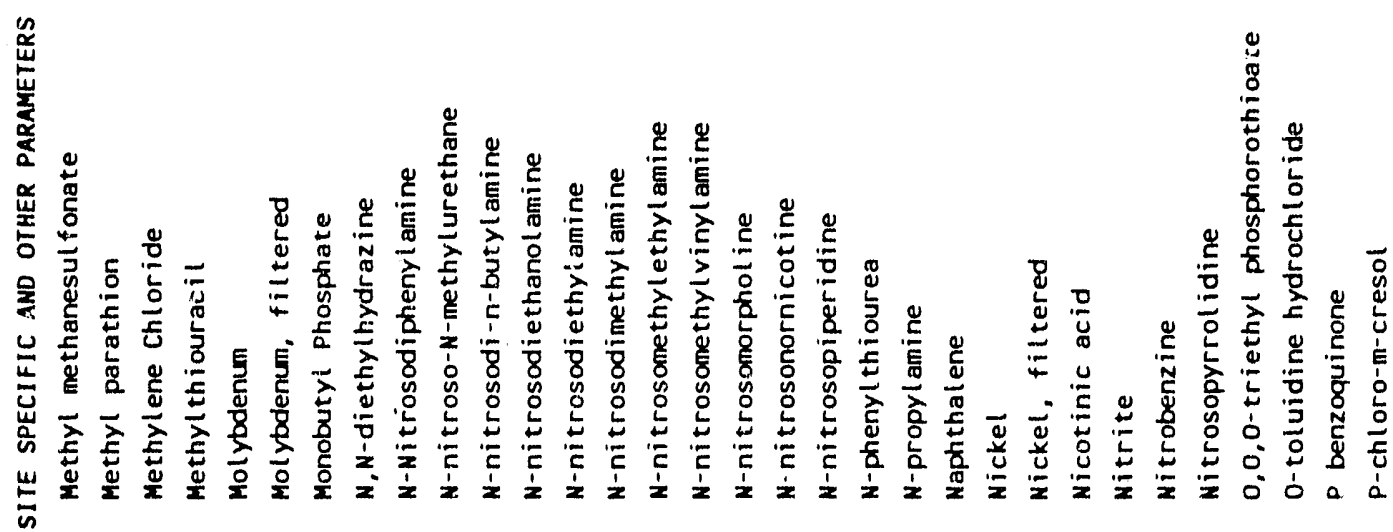




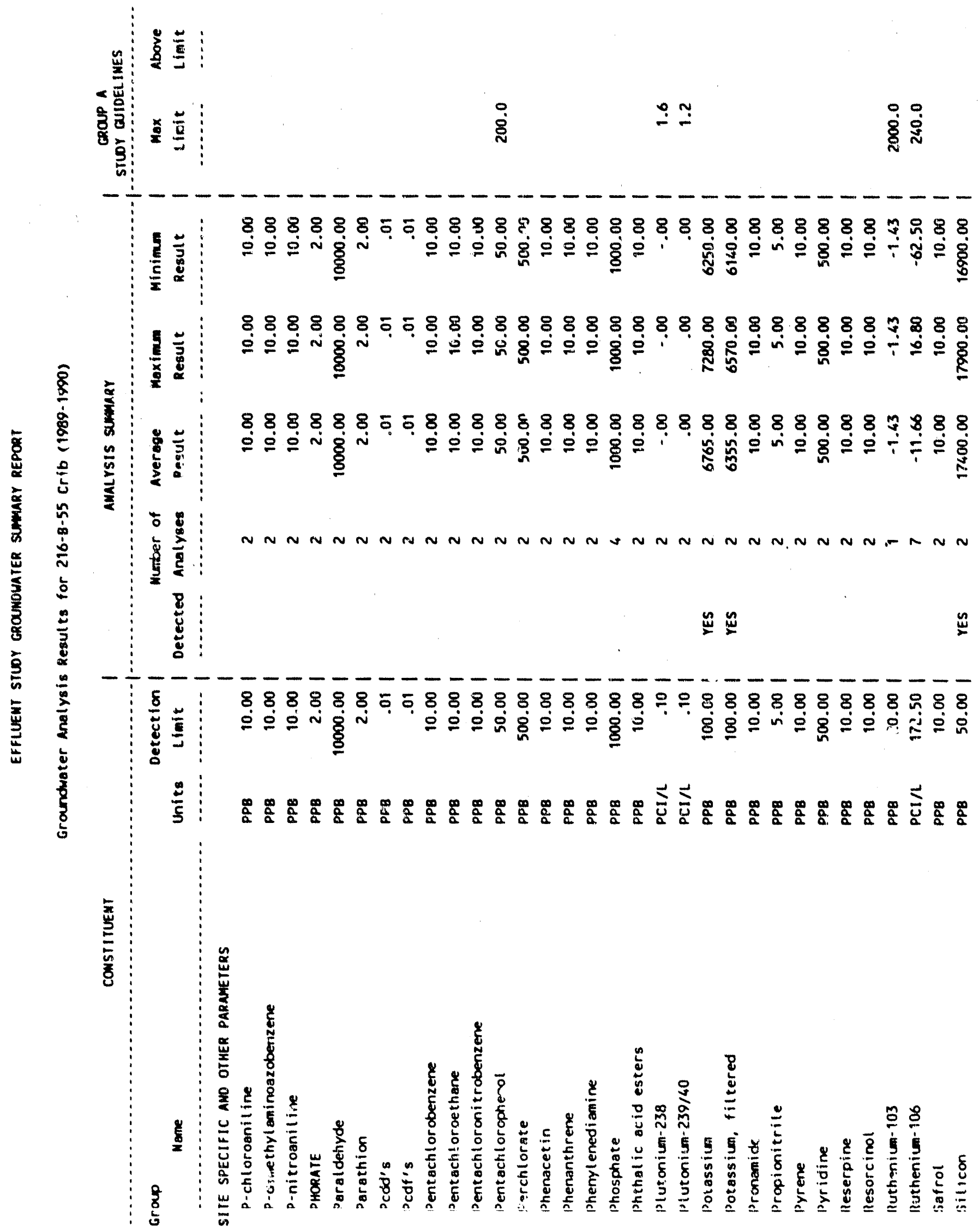




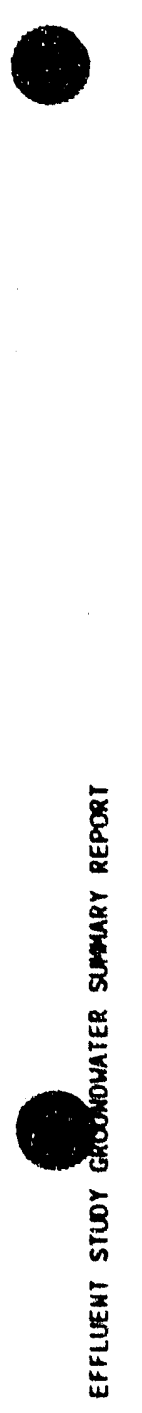

\section{疍}

资:

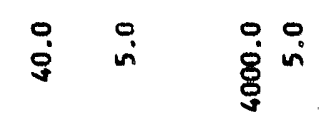

客

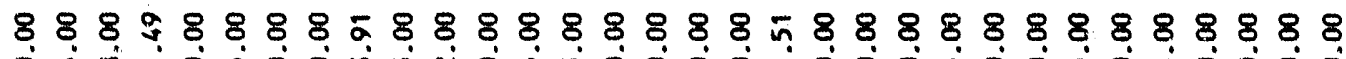

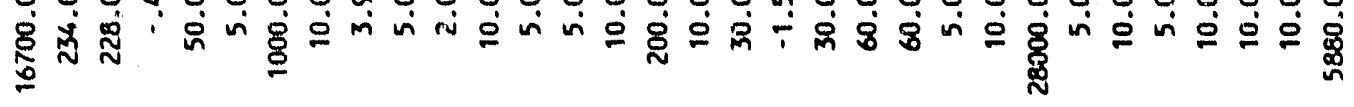

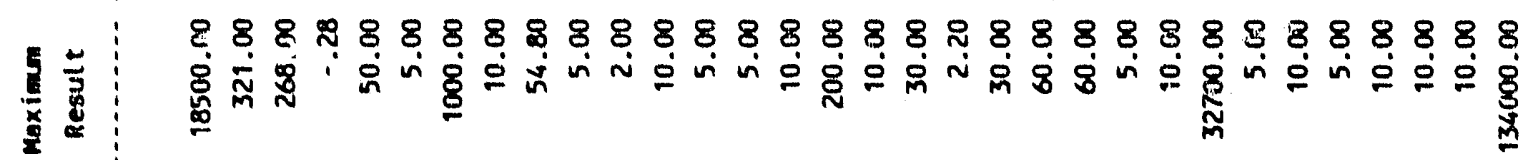

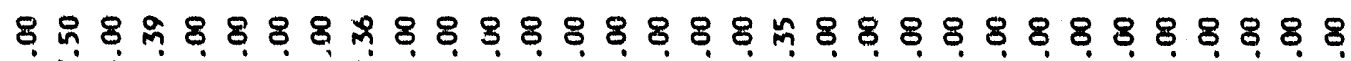
尊芦

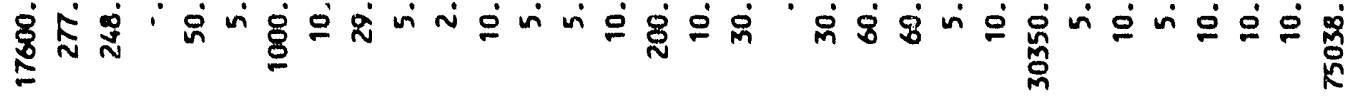

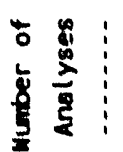

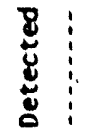

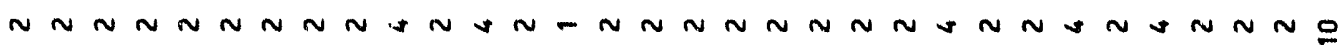

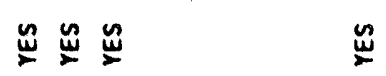

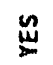
$\stackrel{?}{\dddot{2}}$ ב⿱艹 888888888888888888 8 $888888888 \overline{8}$

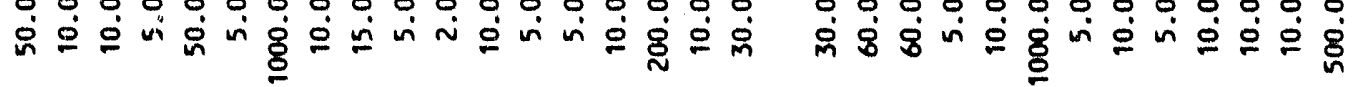

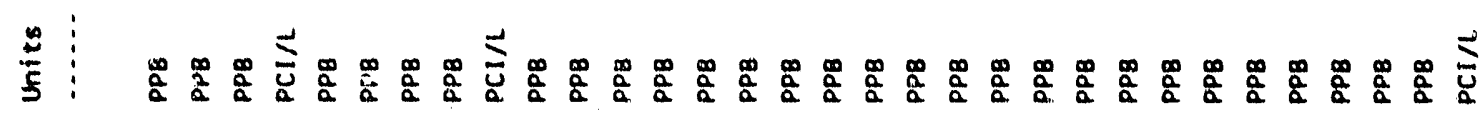

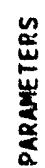

$\vdots: \frac{a}{2}$

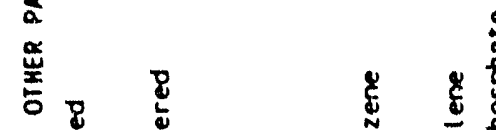

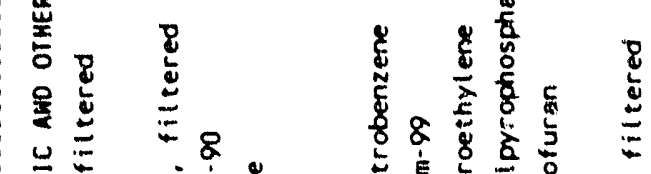
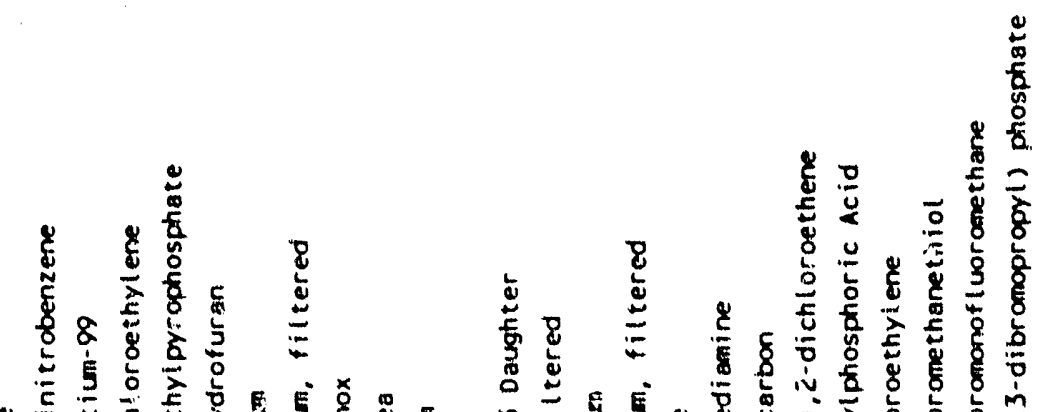
ด 
WHC-EP-036E

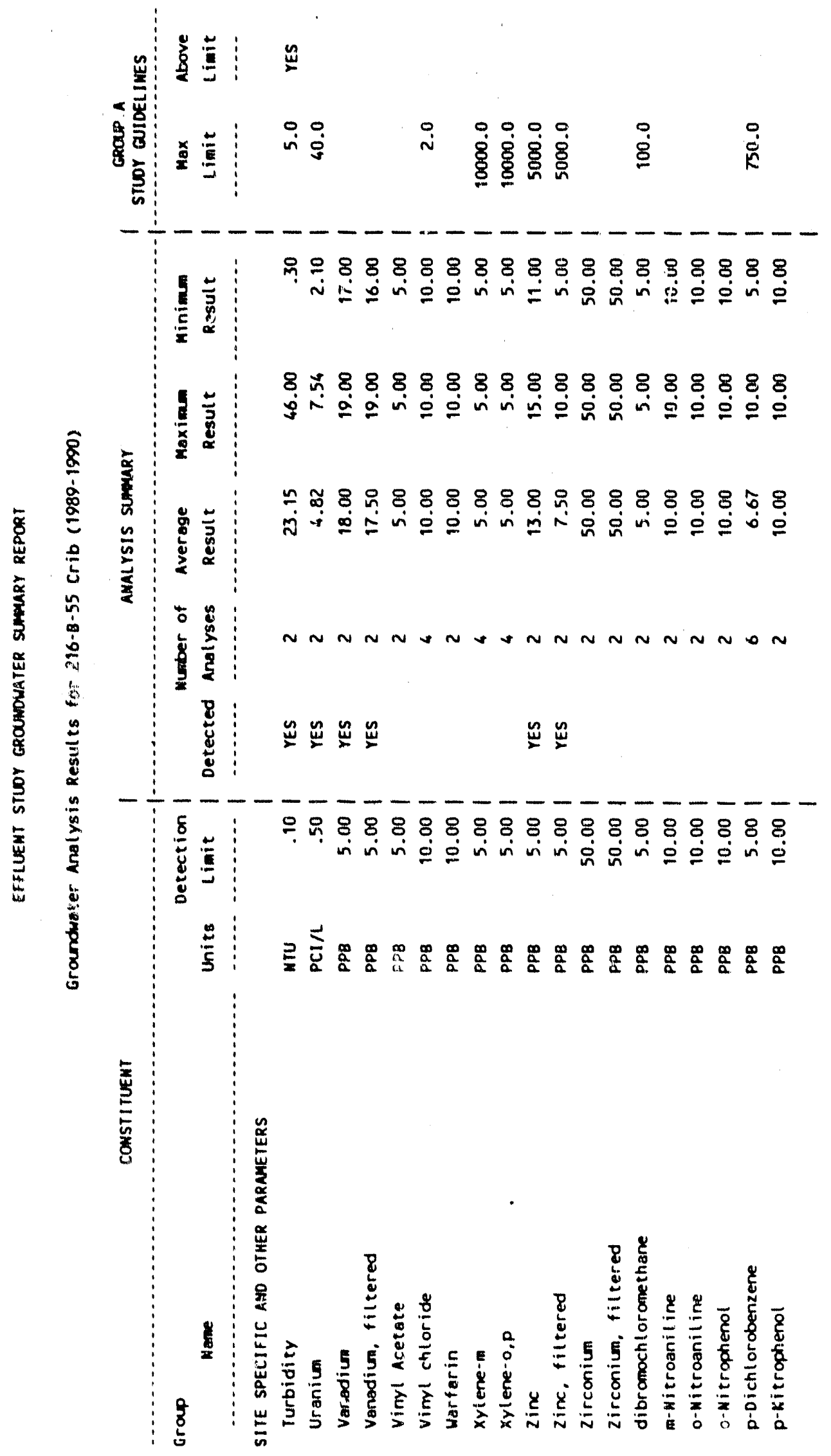



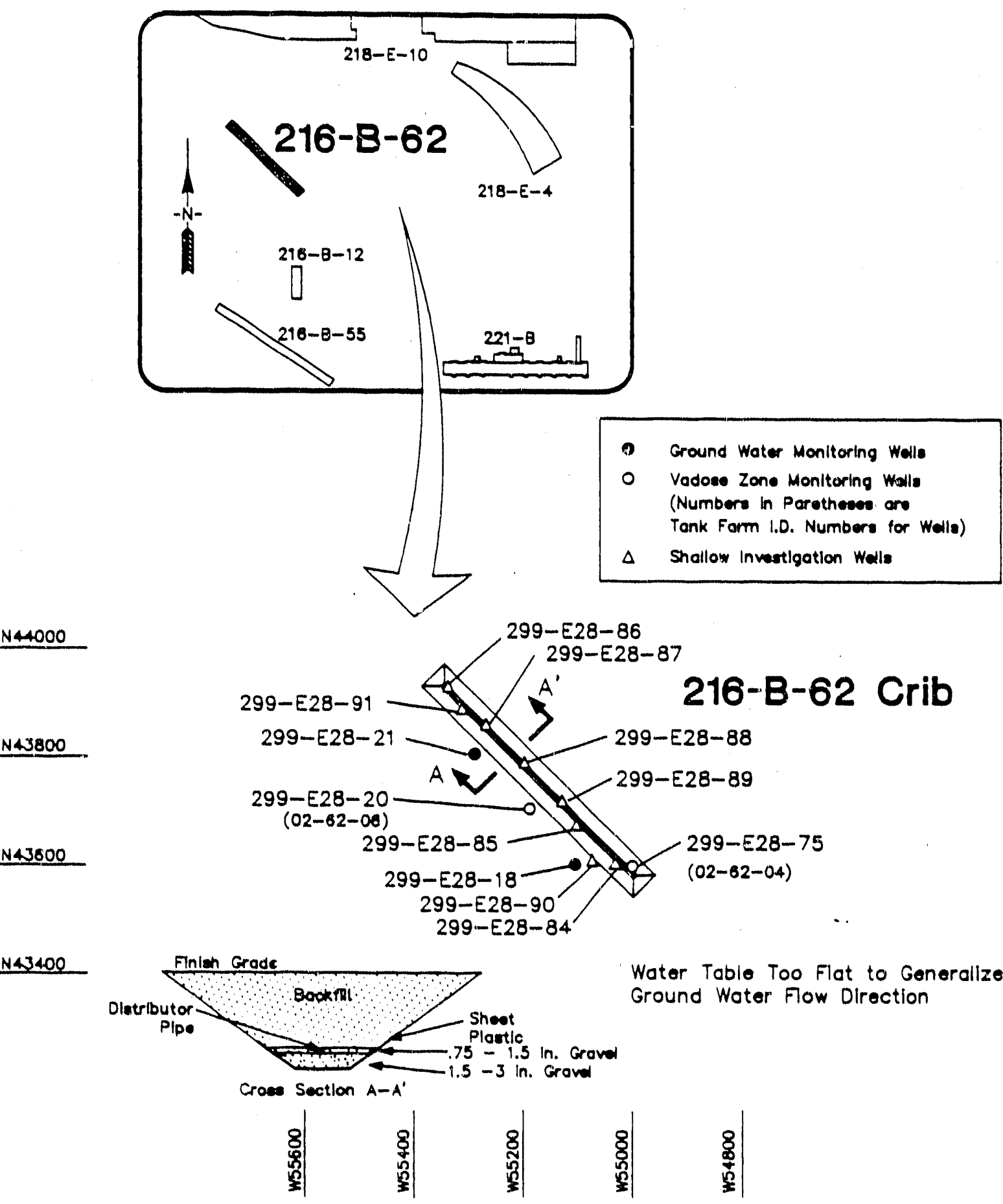


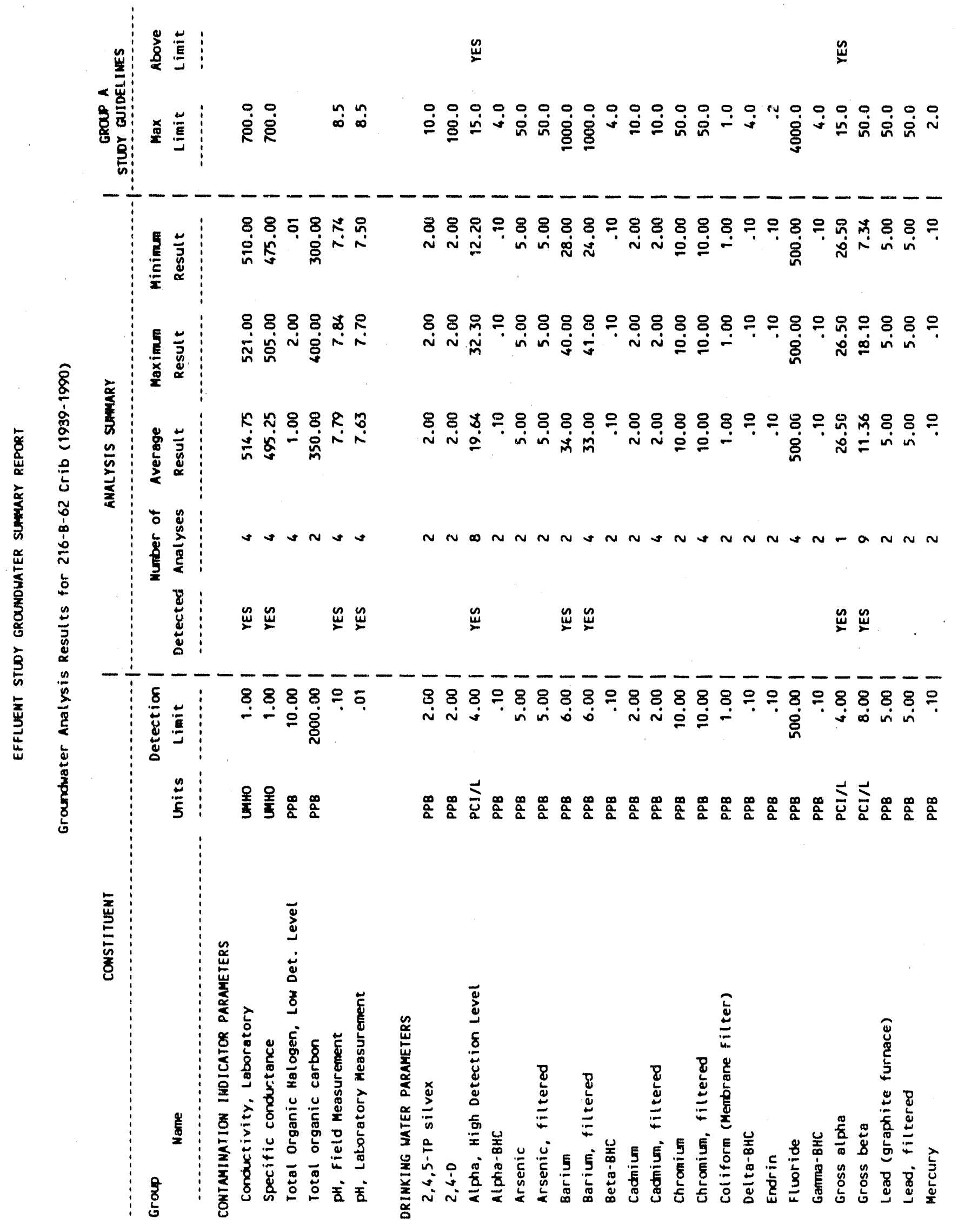


WHC-EP-0366

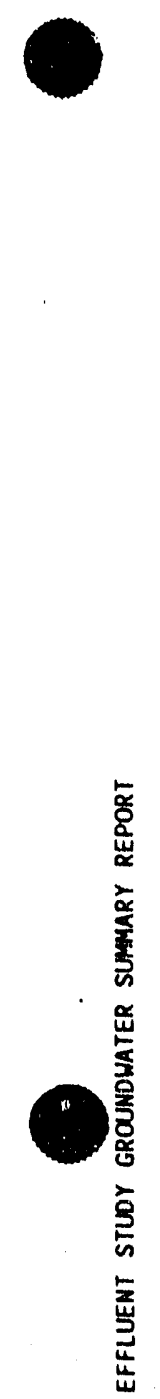

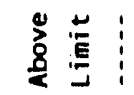

$\stackrel{\dddot{m}}{\sim}$

$<\overrightarrow{\mathrm{u}}$

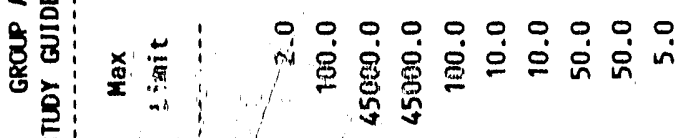

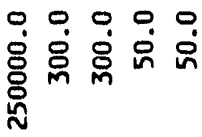

0
8
8

$\stackrel{\circ}{\stackrel{0}{N}} \stackrel{0}{\circ}$

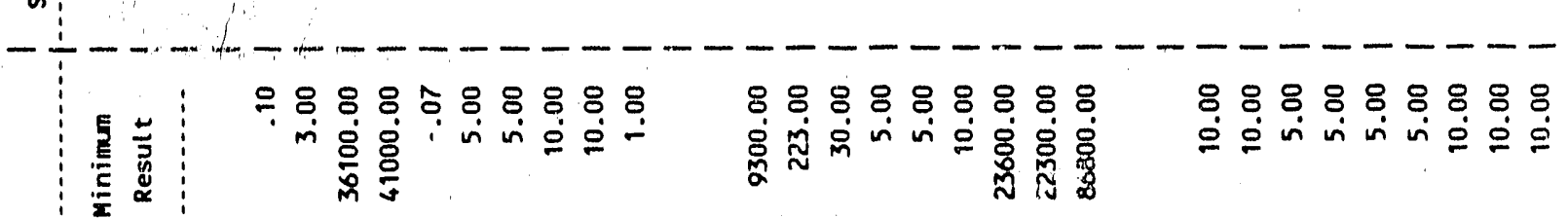

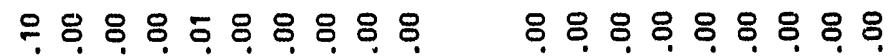

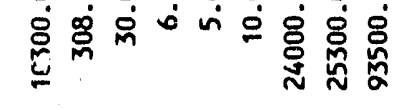

888888888

镸苛

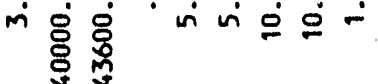

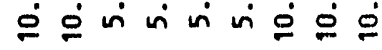

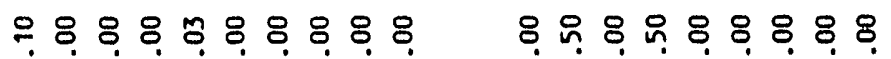

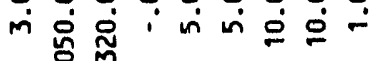
总

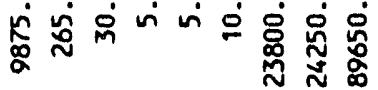

888888888

苞蓠

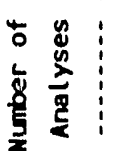

$n n \operatorname{mnn} n \operatorname{mn} n$

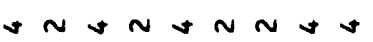

$n n+n \rightarrow \operatorname{snn}$

\&

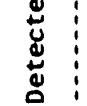

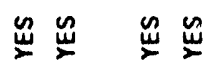

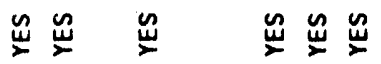

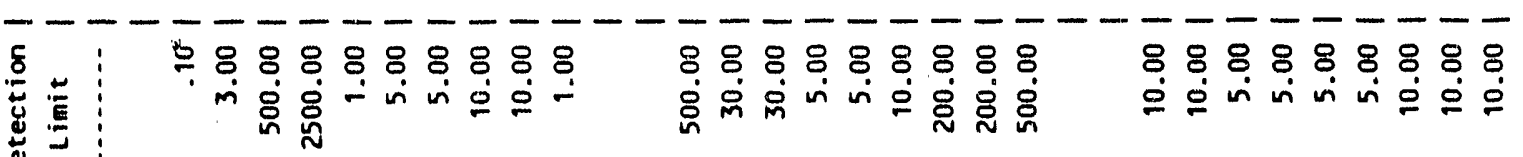

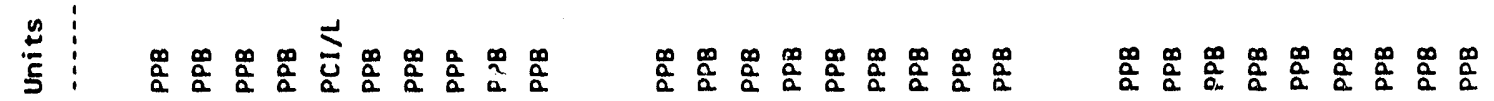
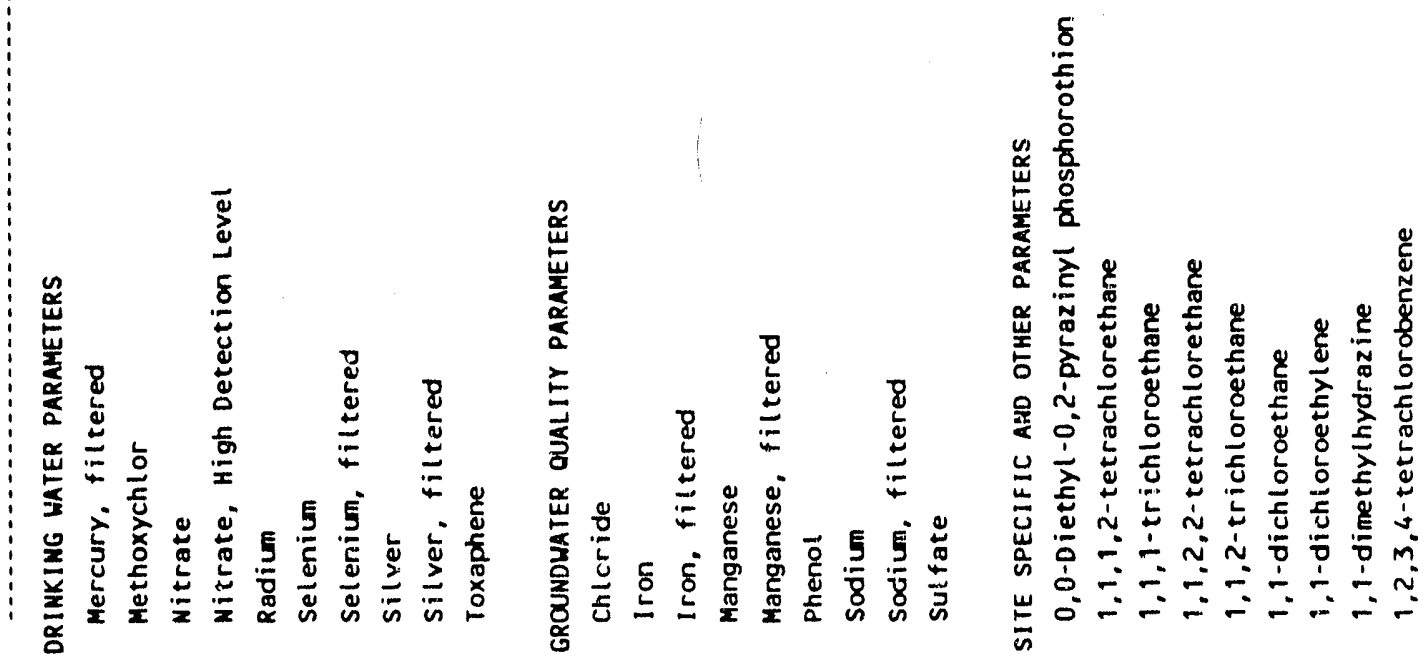


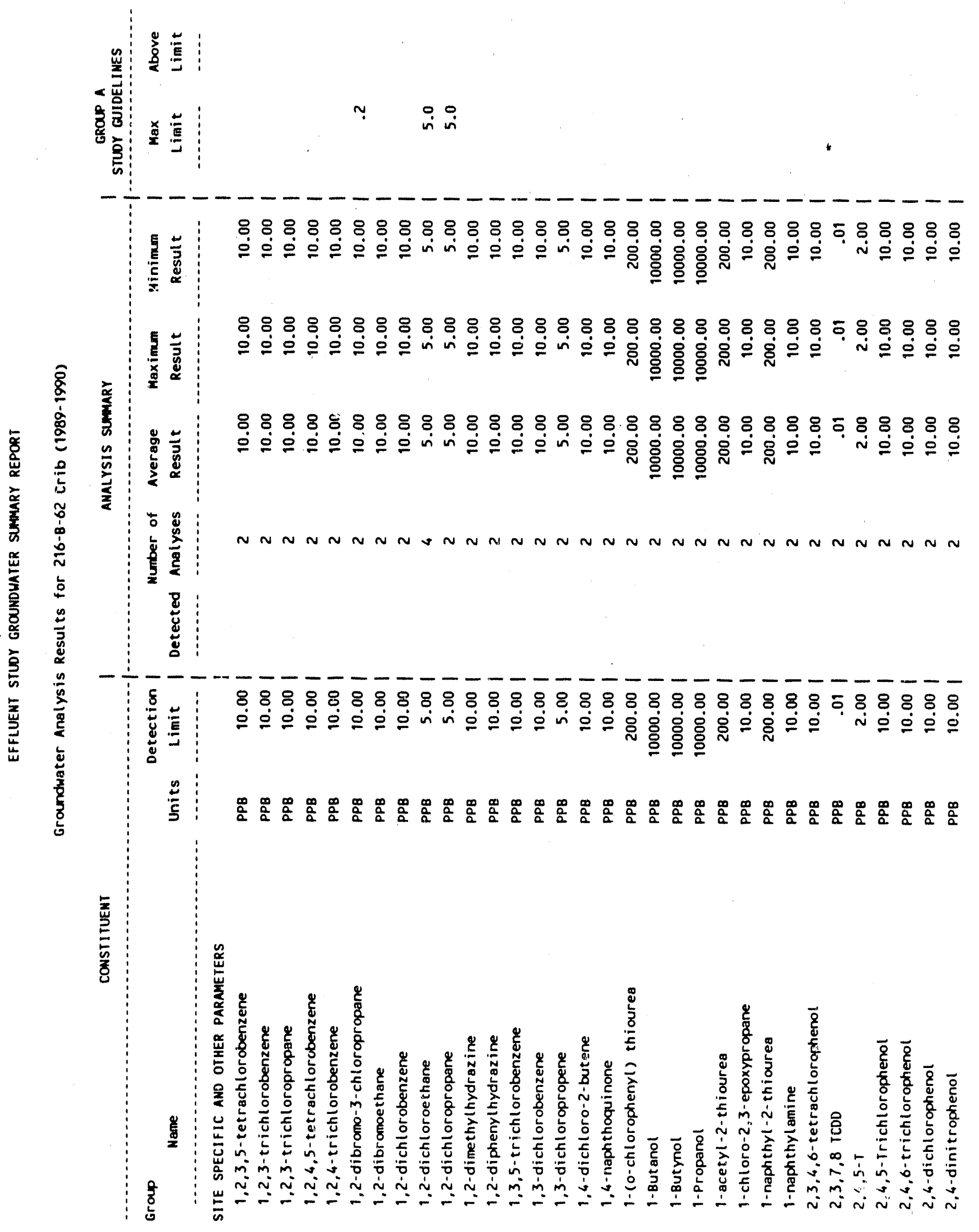


蒙:

政

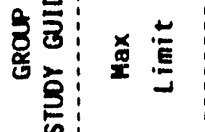

\begin{tabular}{r} 
\\
\hdashline \\
$\vdots$ \\
e
\end{tabular}

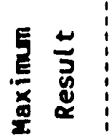

88888888888888888888088888888888

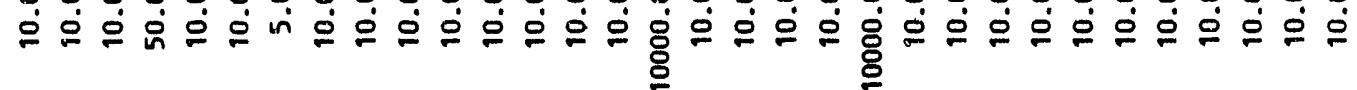

8888888888888888888888888888888

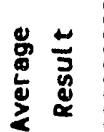

88888888888888888888888888888888

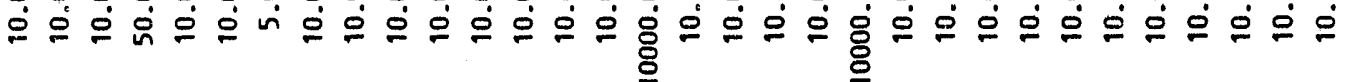

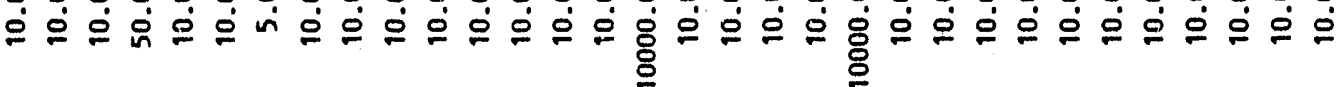

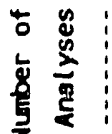

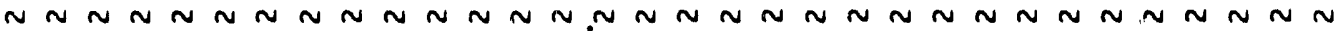
( :

 这 8888888888888888888888888888888

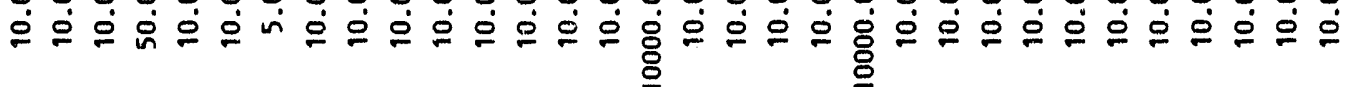
$\stackrel{m}{\stackrel{2}{5}}$

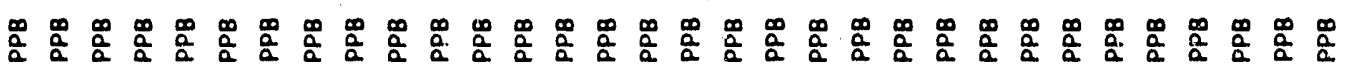

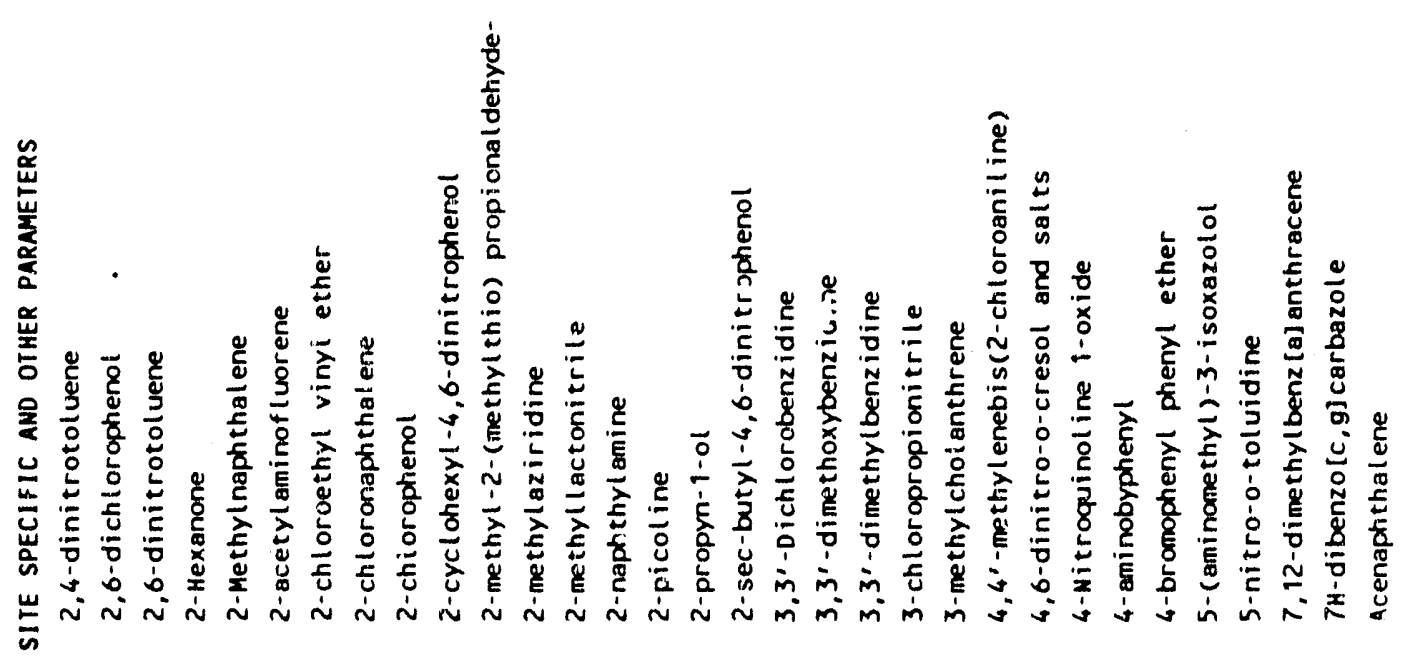


WHC-EP-0366

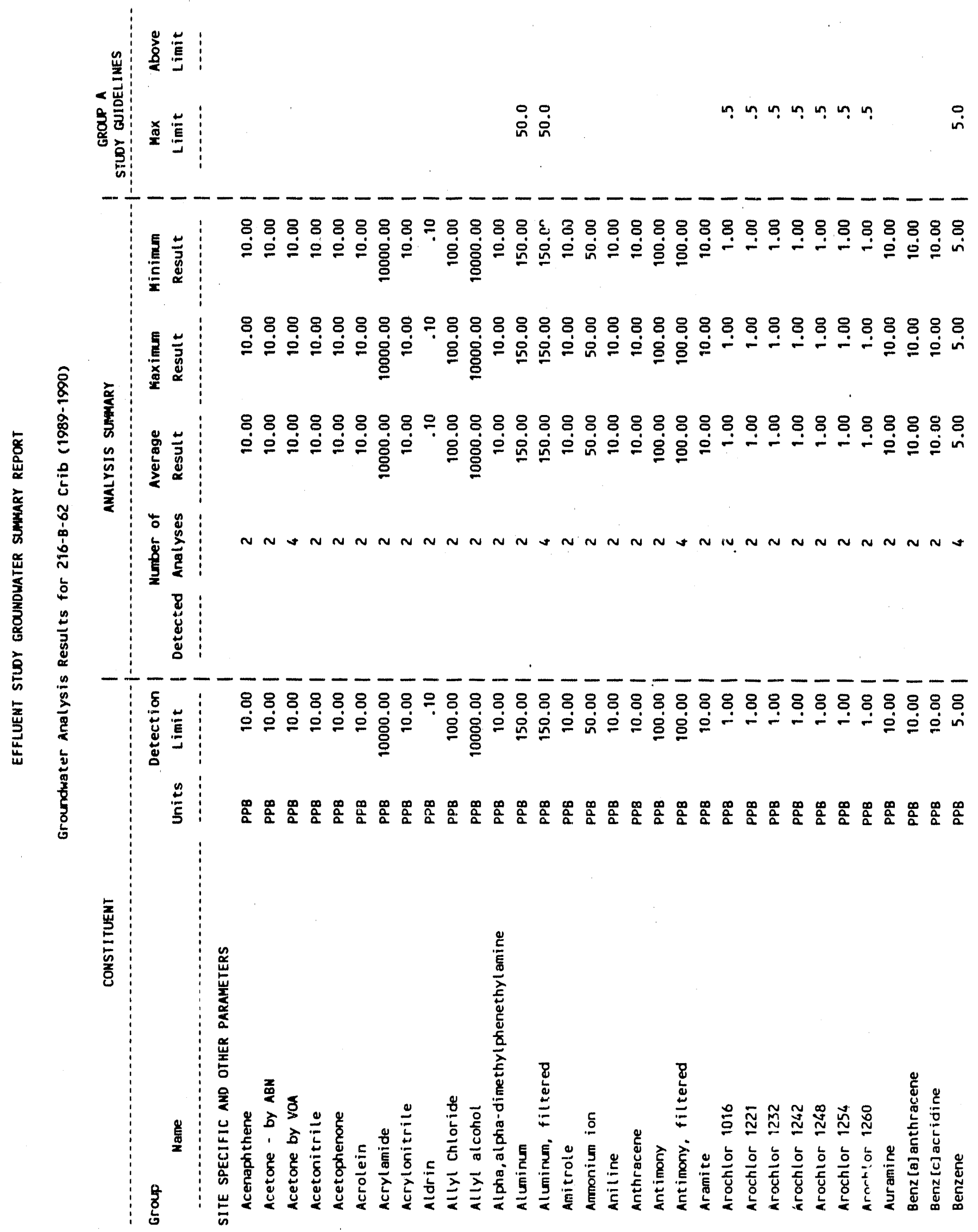




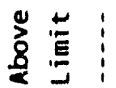

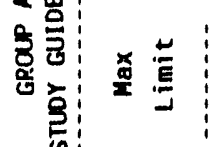

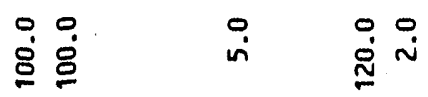

88888888888888888888888838888818

重

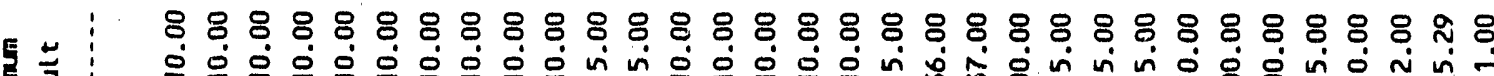

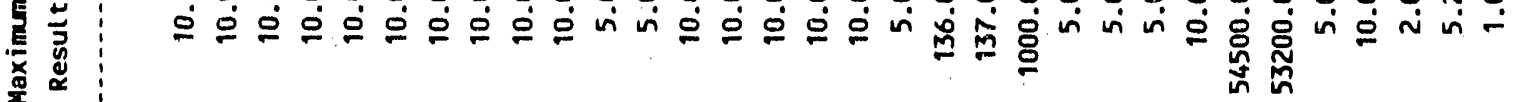

4 $\quad 8888088888888888080$

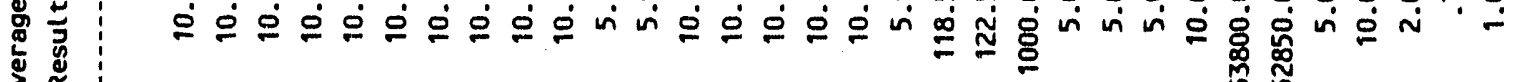

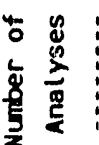

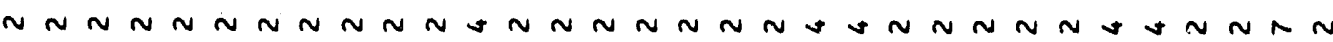

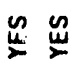

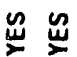

:

$-\frac{1}{1}$

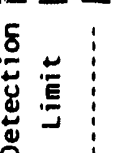

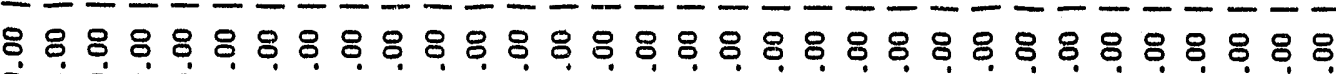

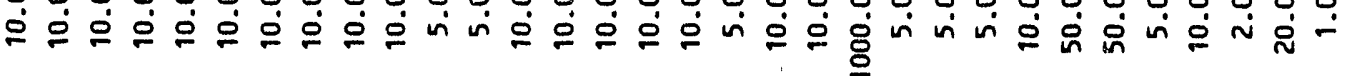

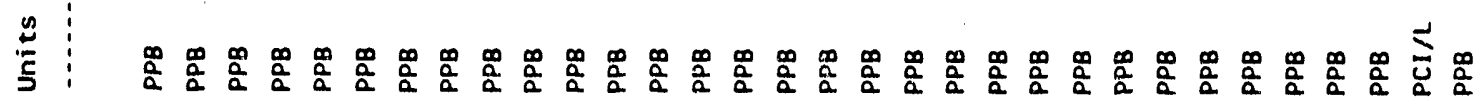

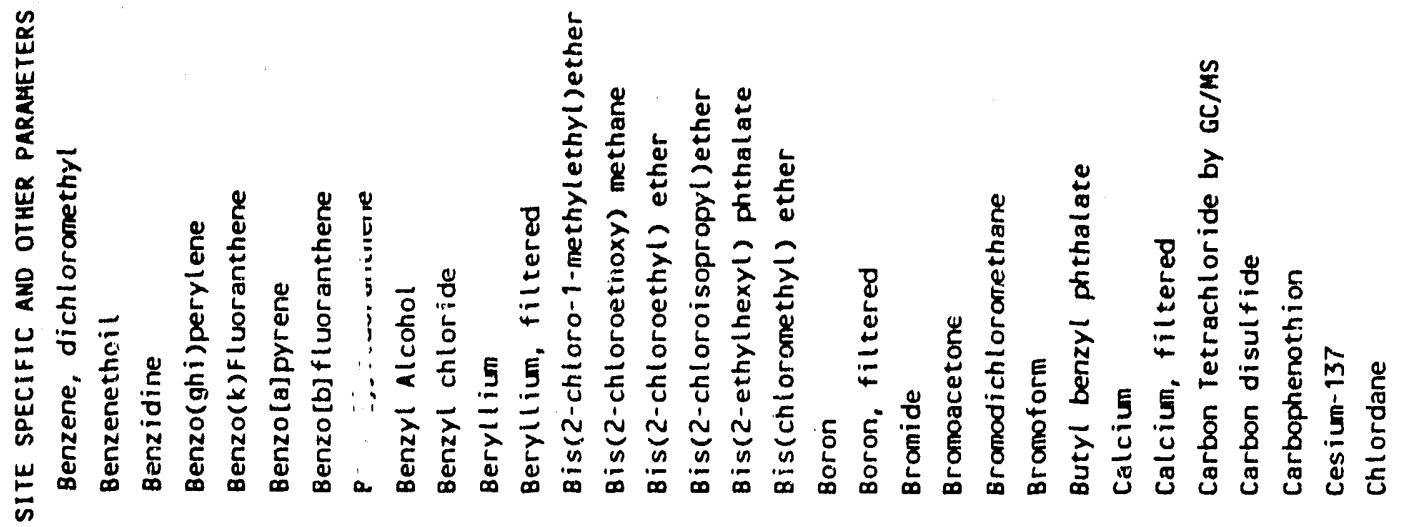


WHC-EP-0366

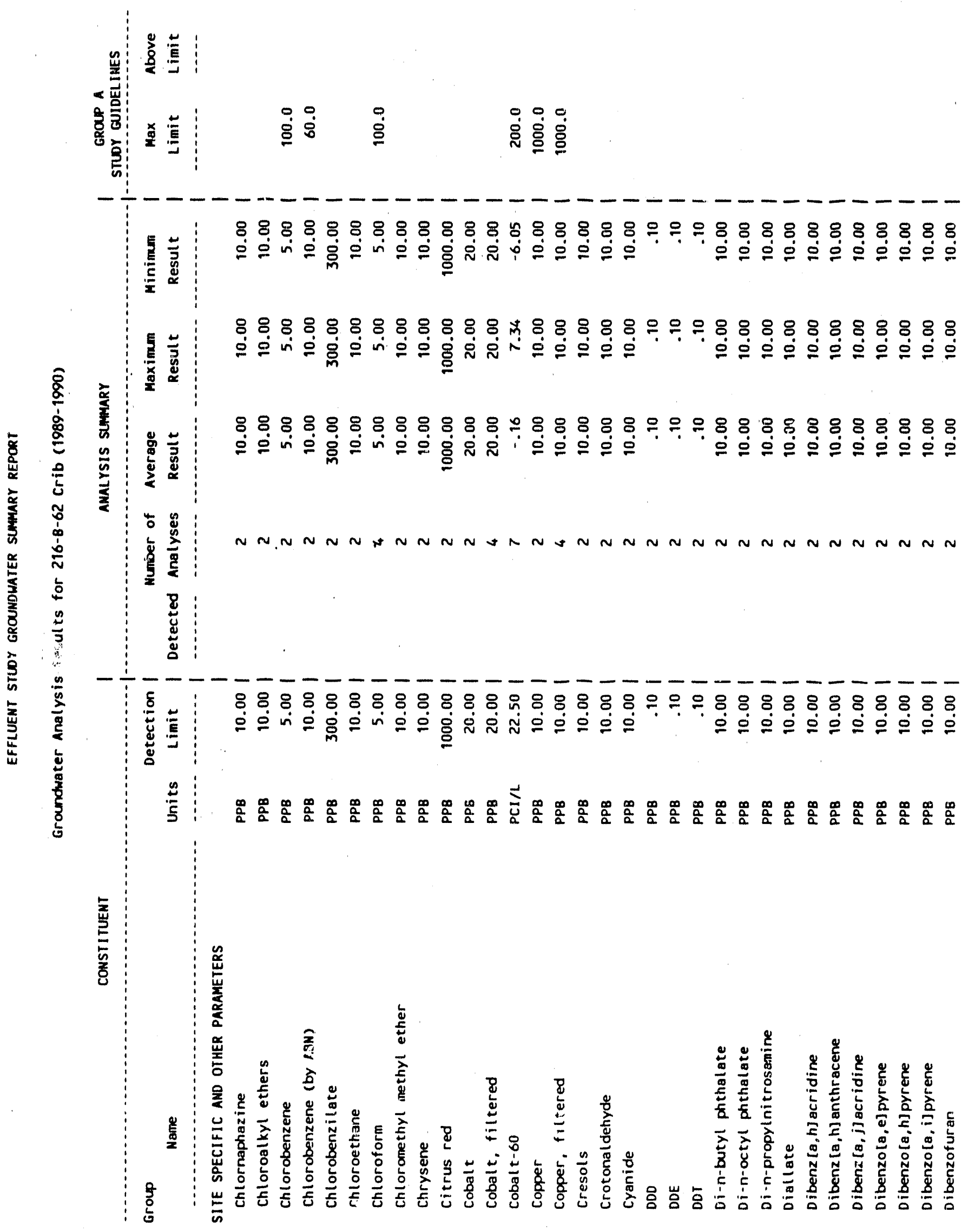




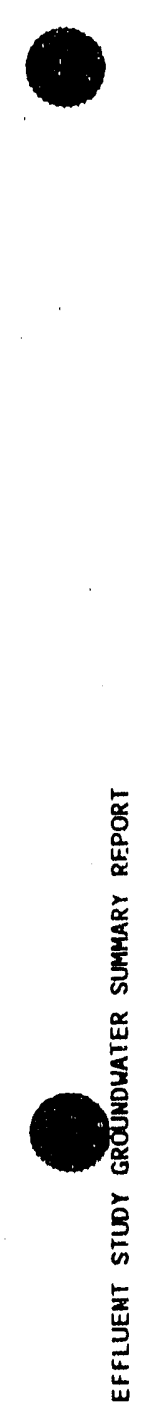

葛:

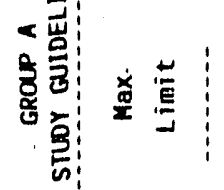

$\stackrel{\circ}{\dot{8}}$

$\stackrel{\circ}{\stackrel{8}{8}}$

$\checkmark$

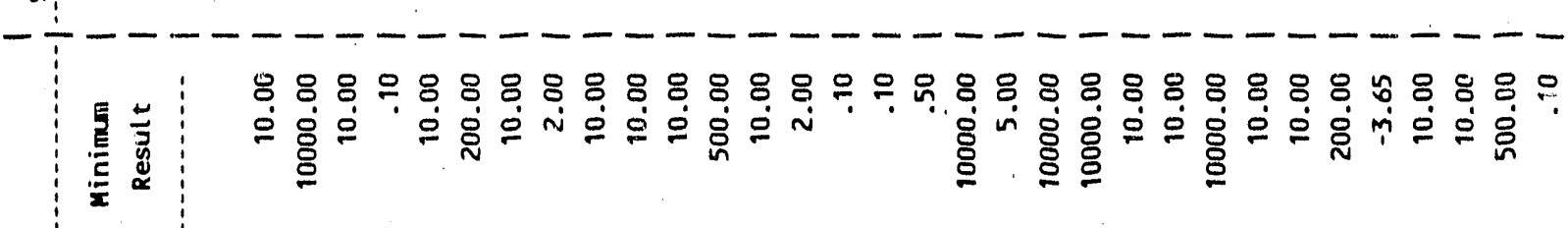

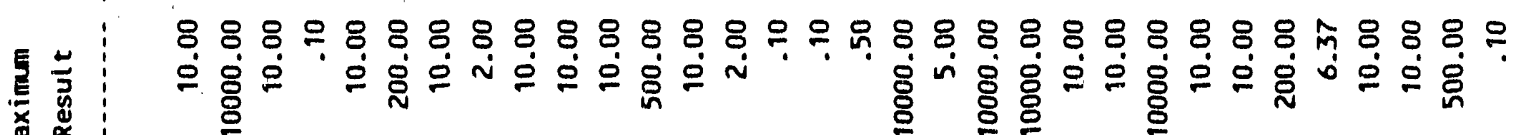

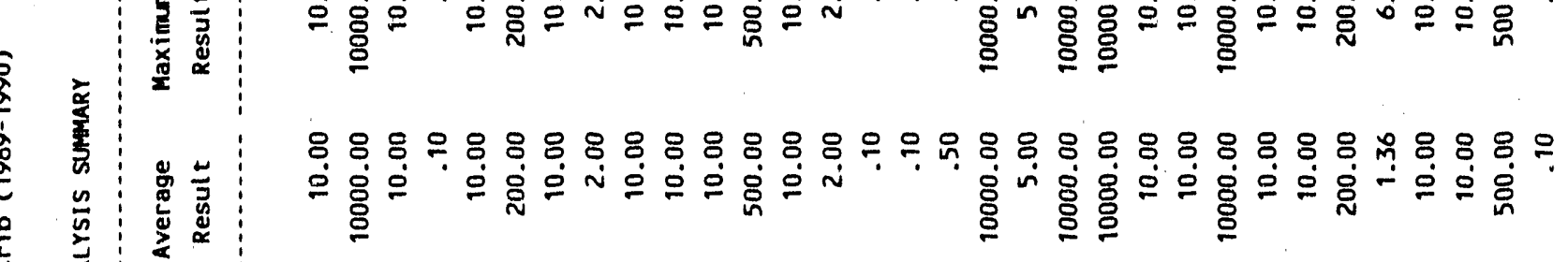

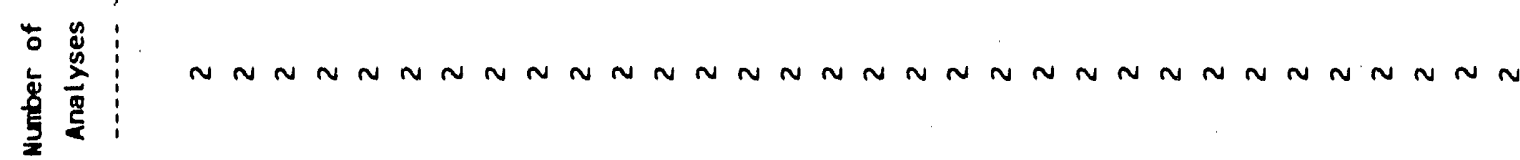

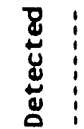

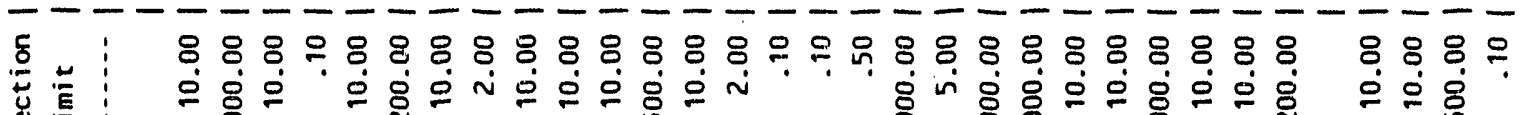

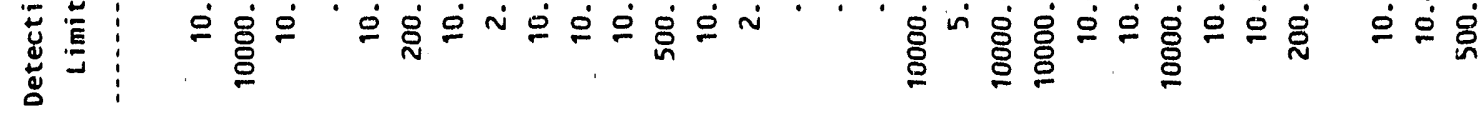

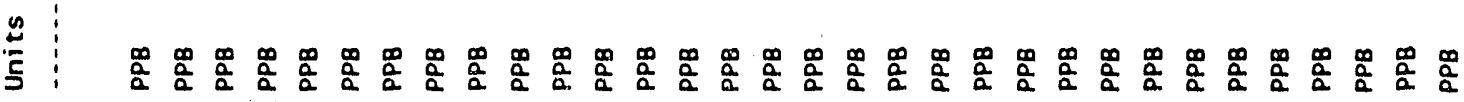

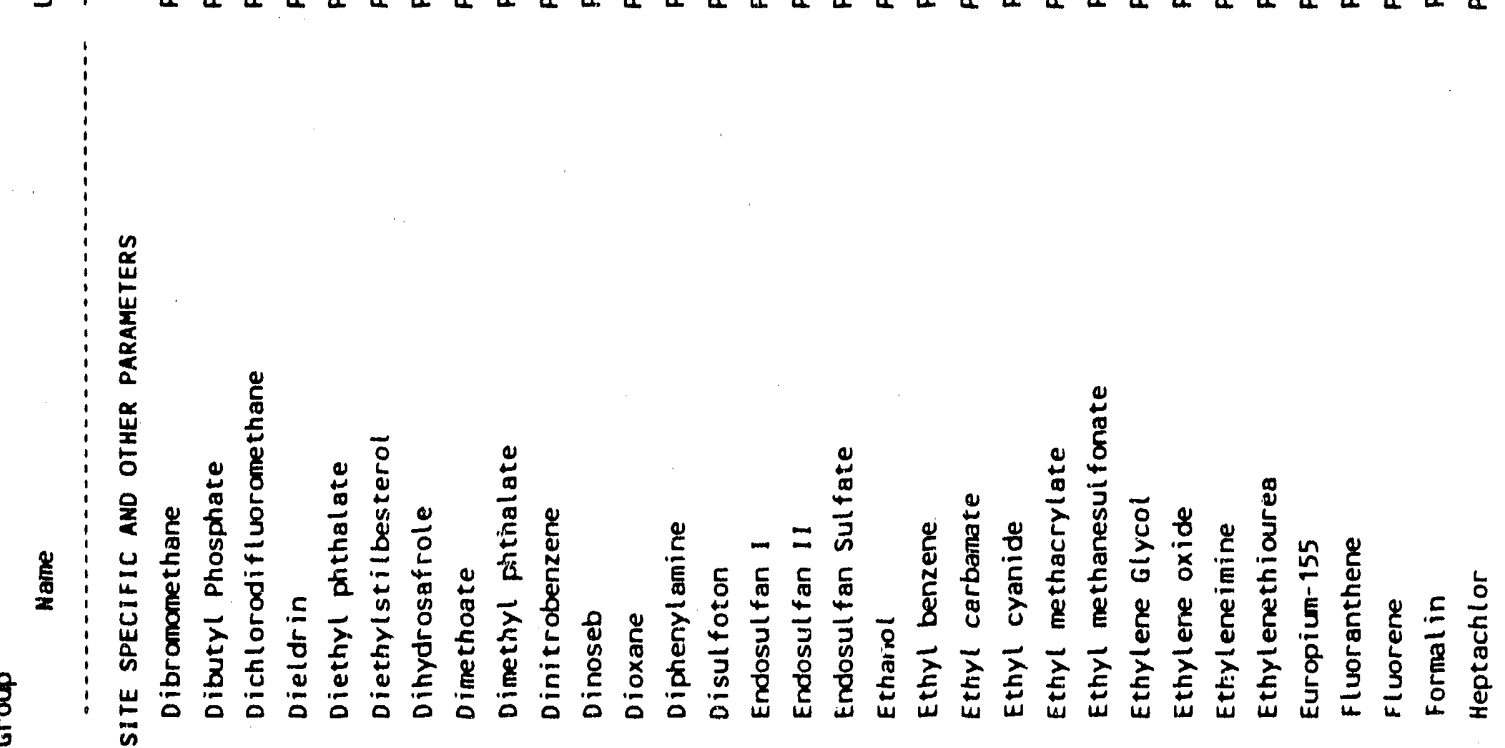




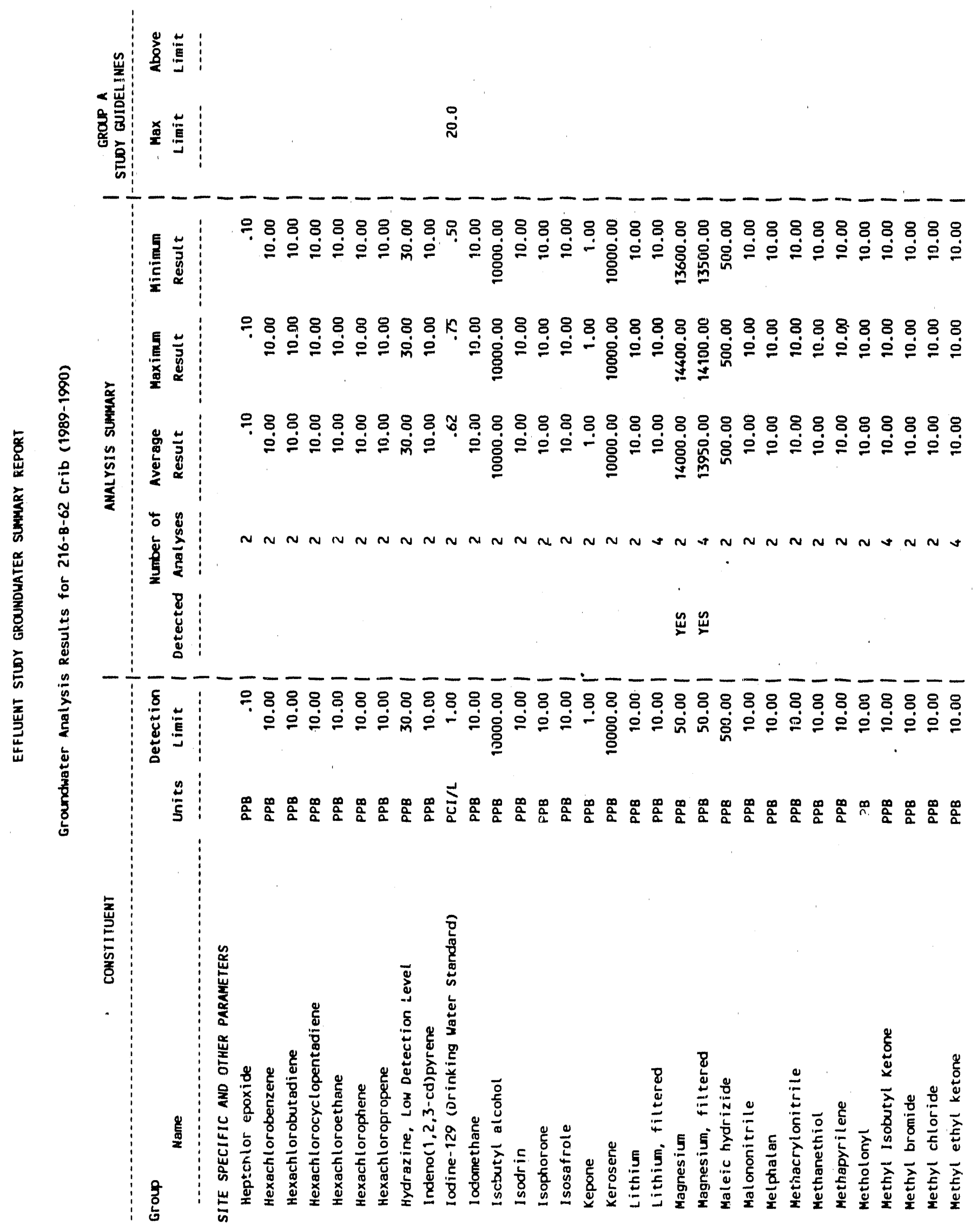




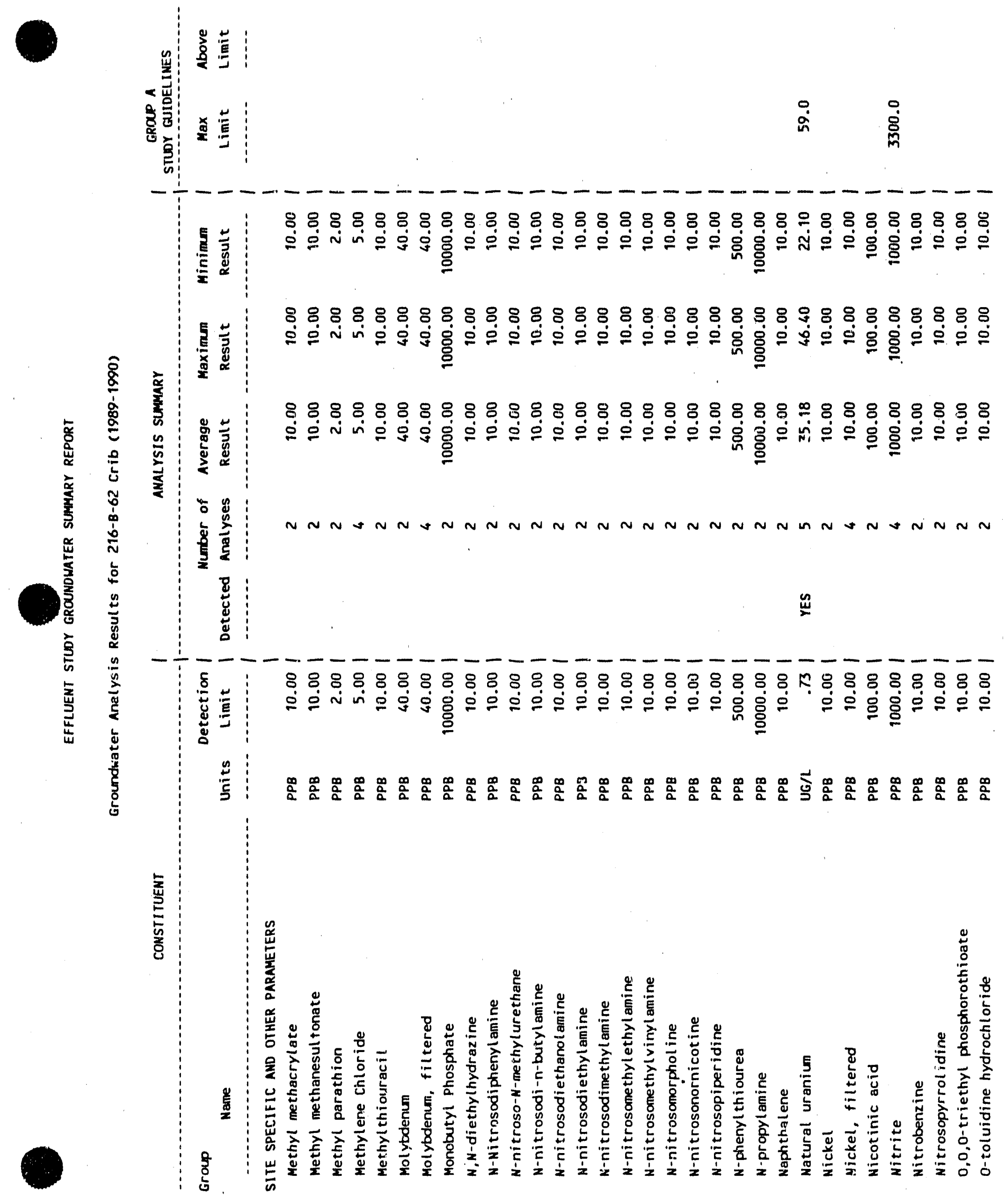



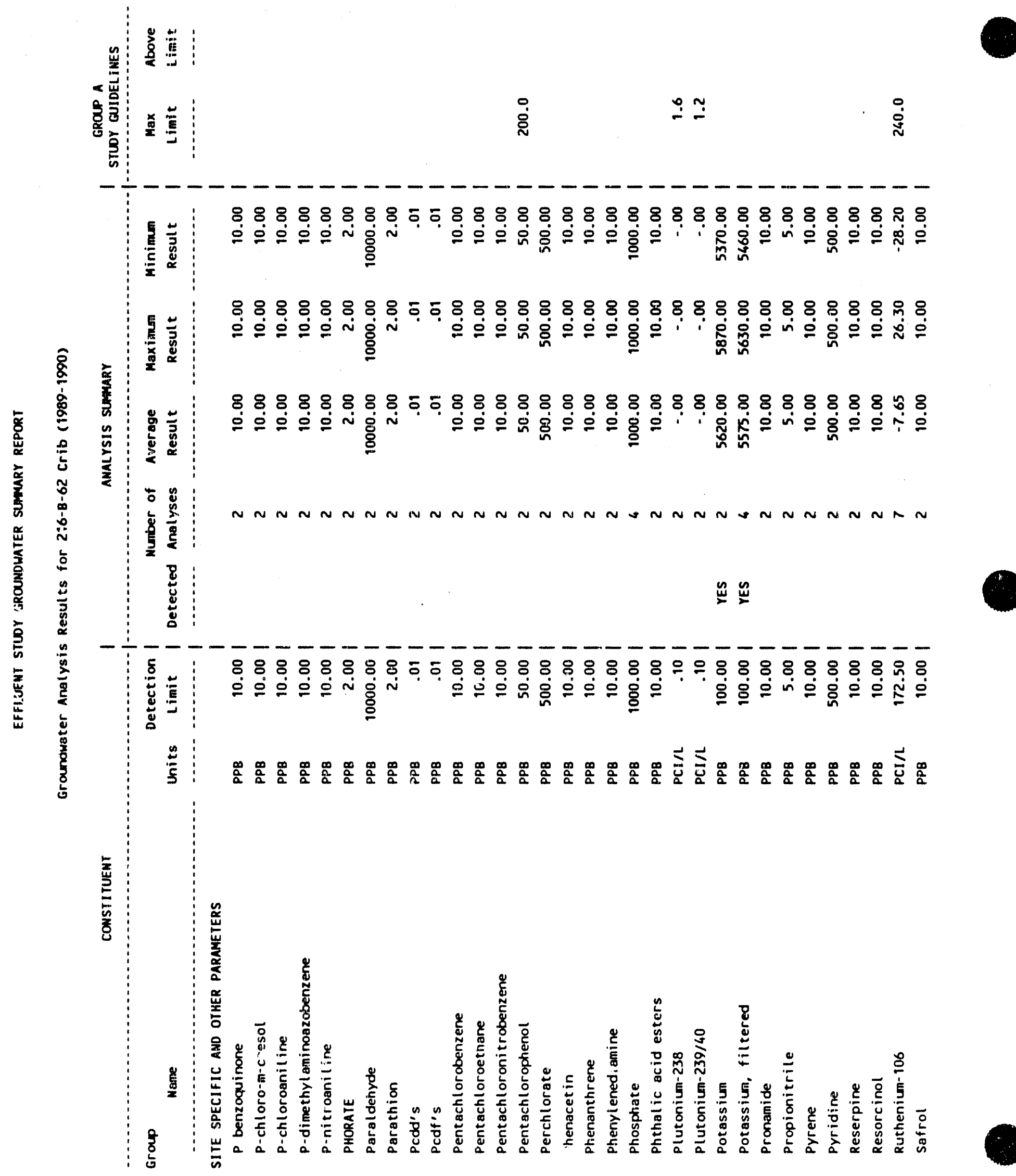
WHC -EP-0366

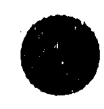

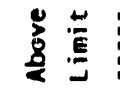

然:

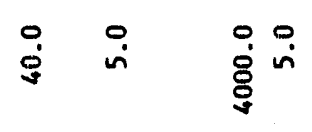

悹 悹

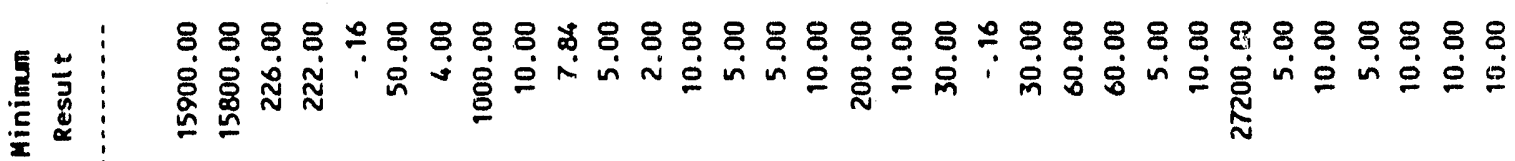

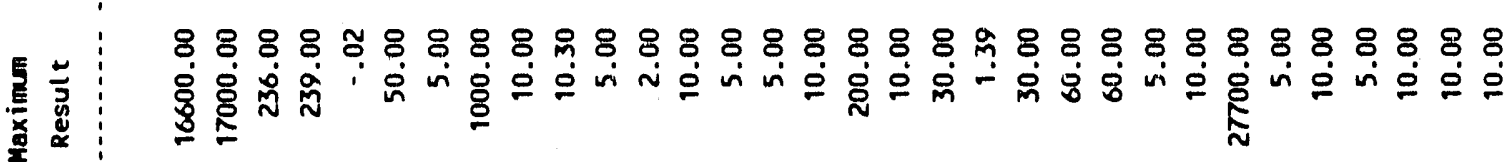

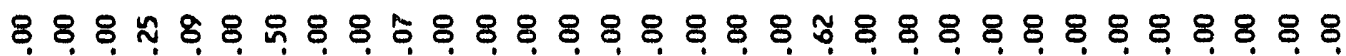

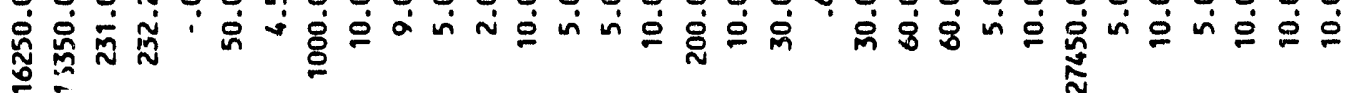

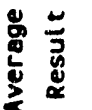

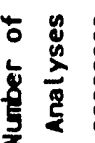

\&:

离

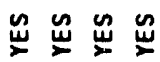

$\stackrel{\Perp}{\Sigma}$

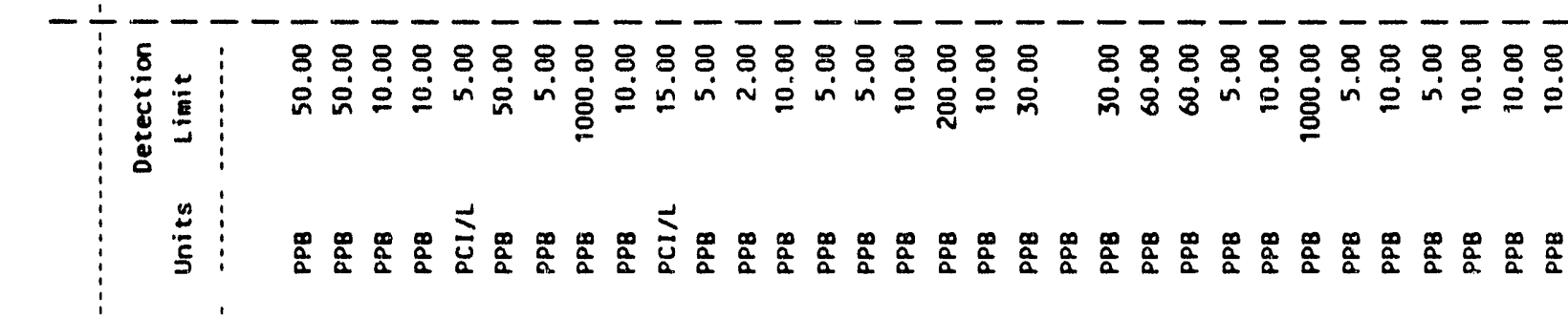

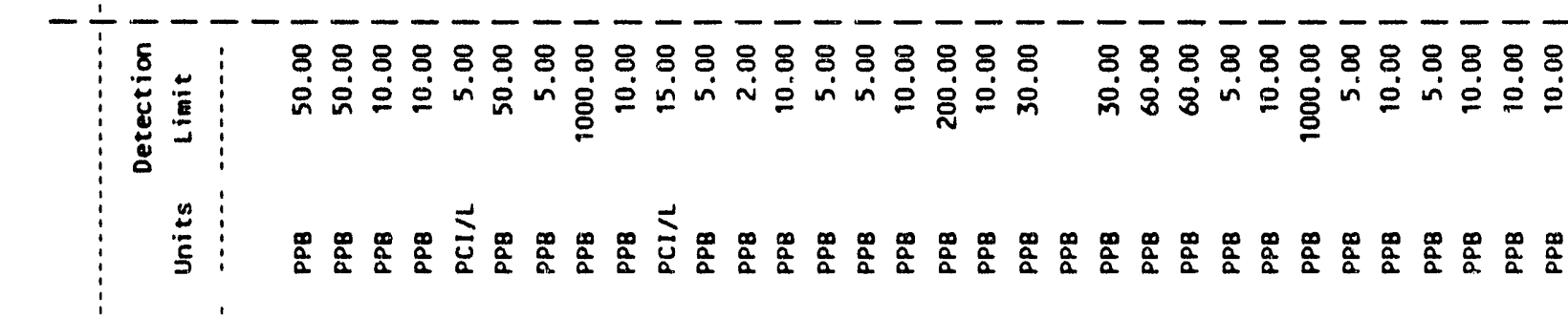

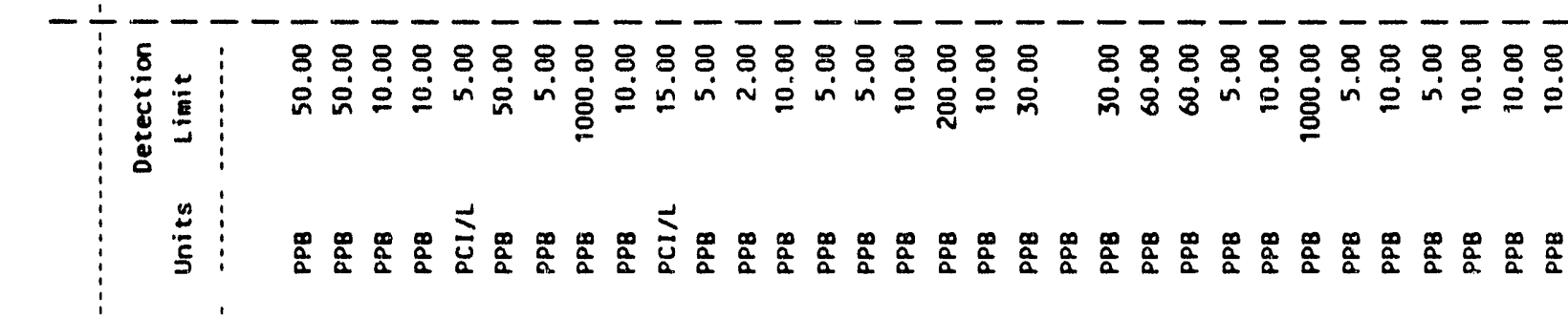

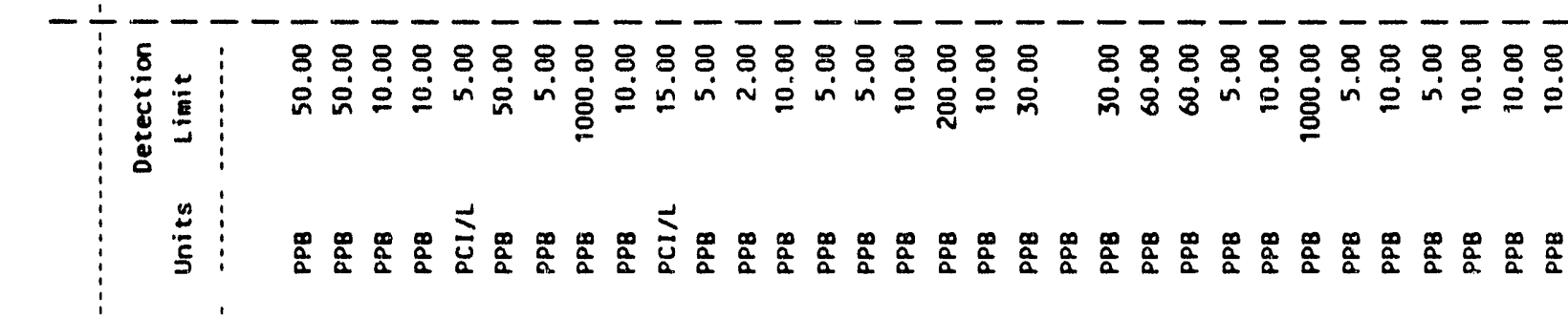

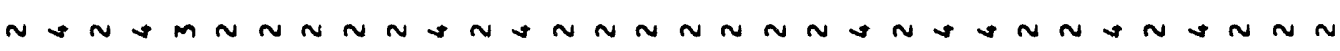

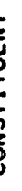

(

원

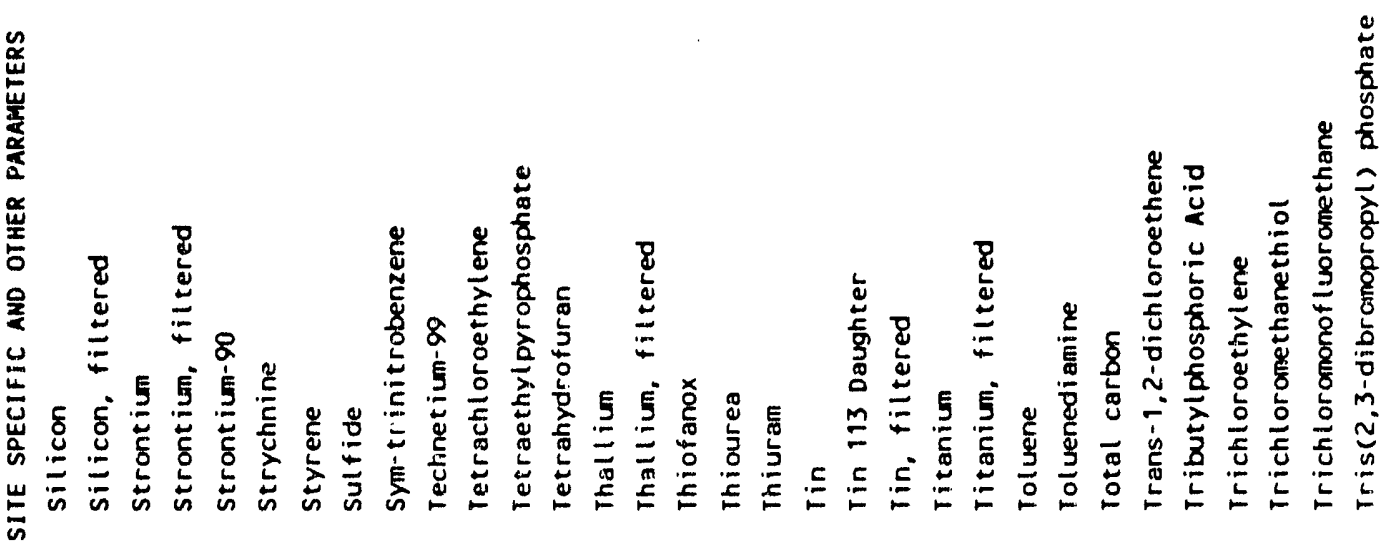




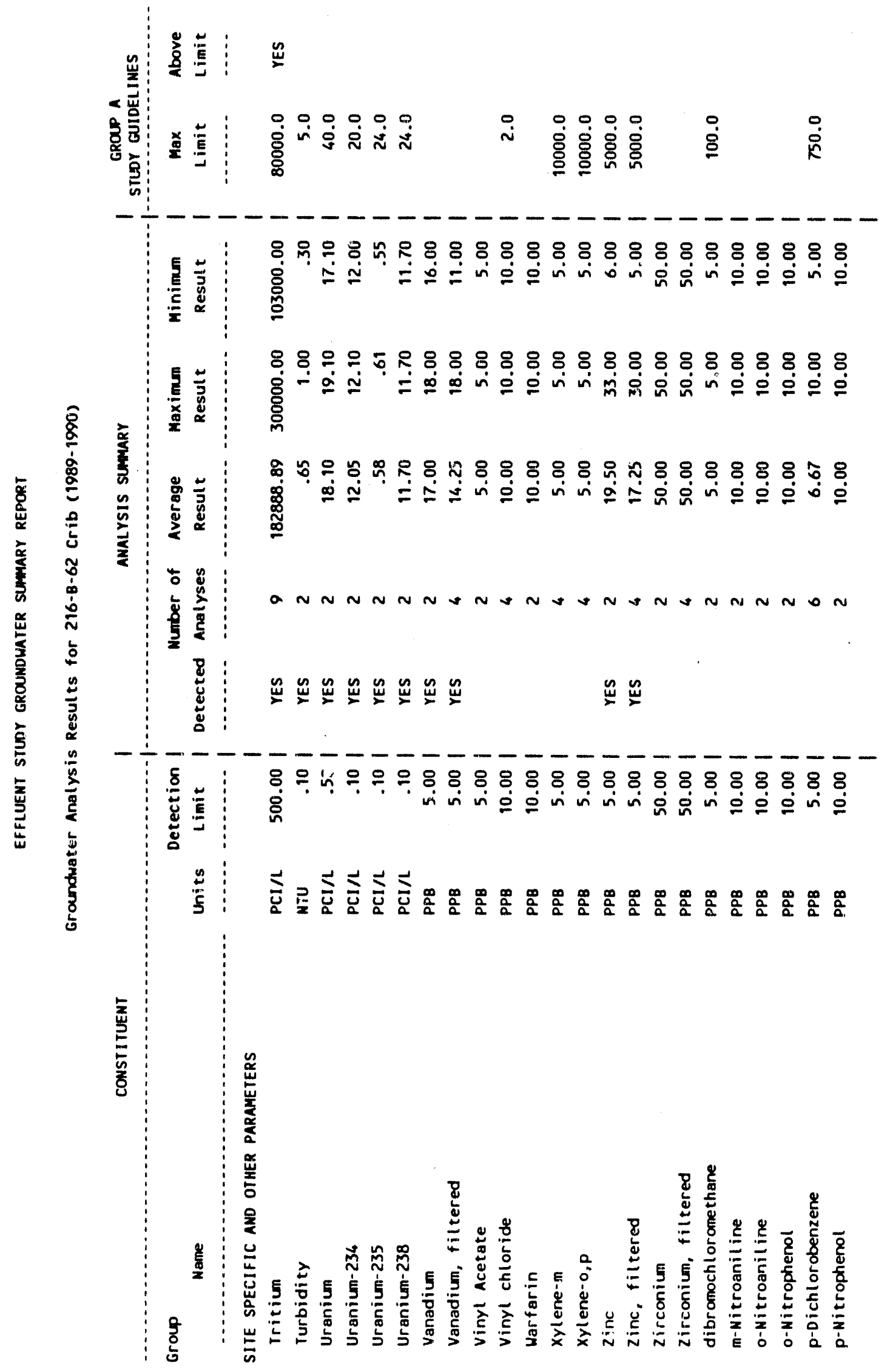


147900
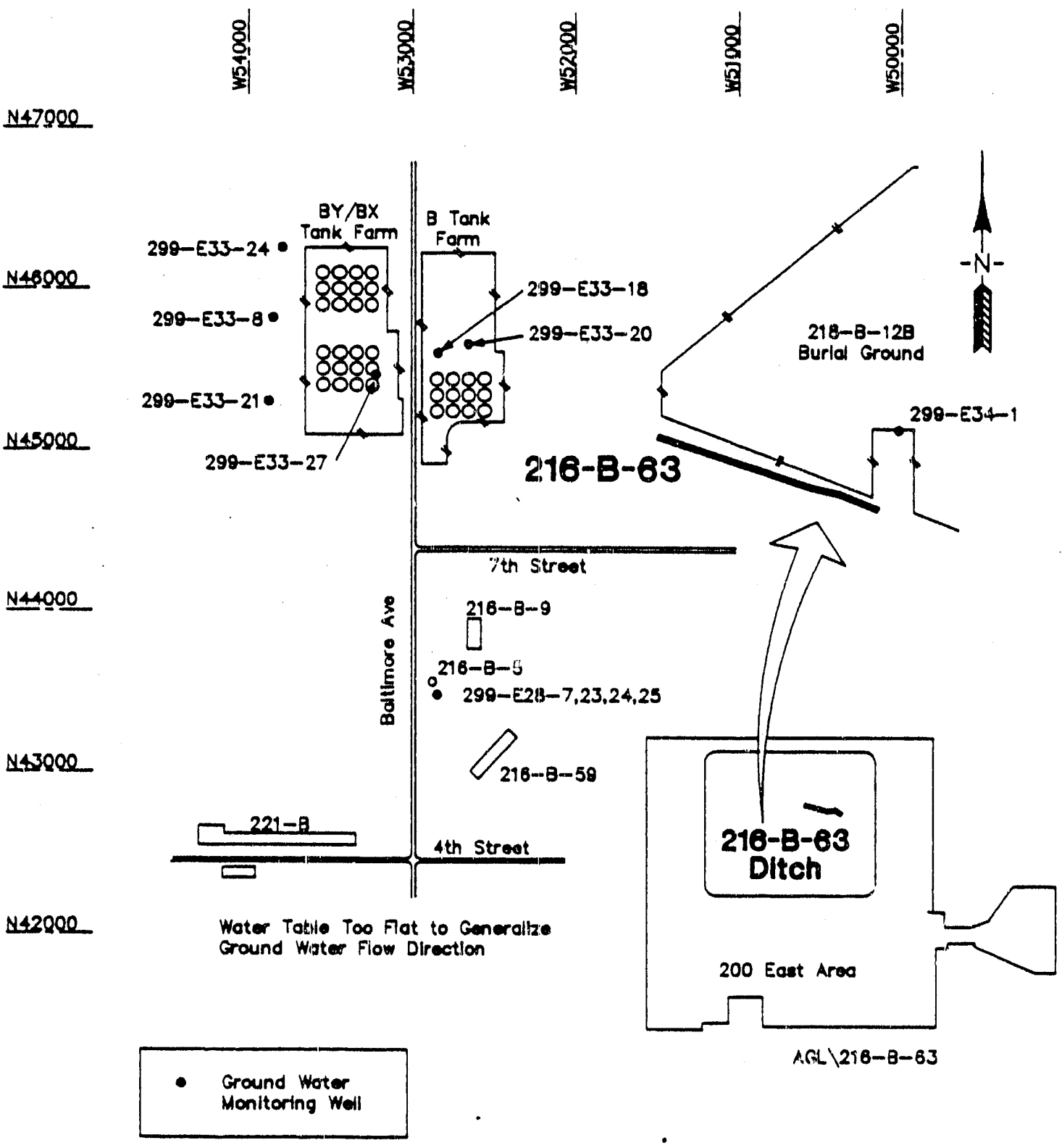

We $1 \mathrm{i}$ Location and Site Map for 216-C-63 Ditch 


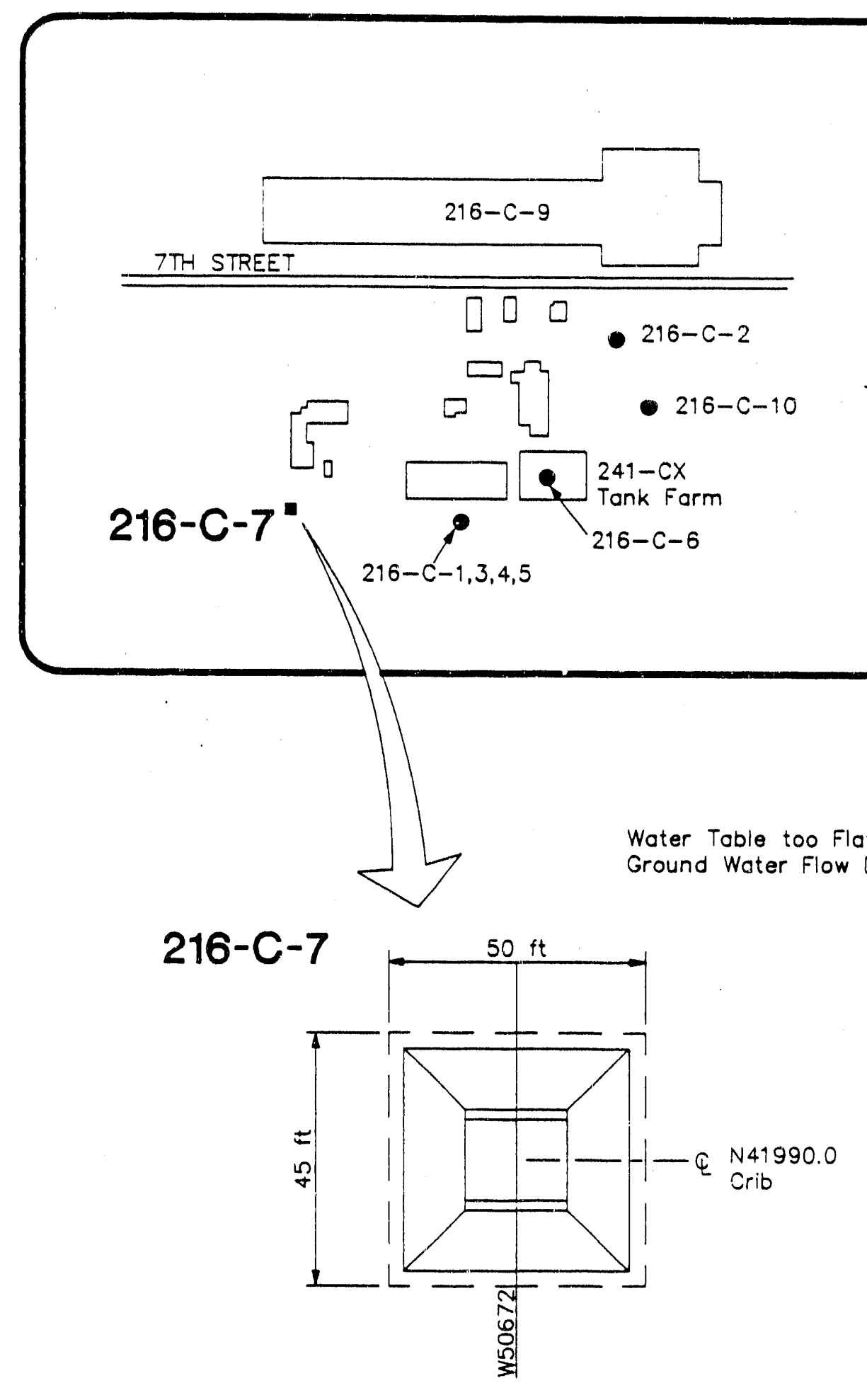

AGL $\backslash 216-\mathrm{C}-7$

Well Locat ion and Site Map for 216-C-7 Crib 


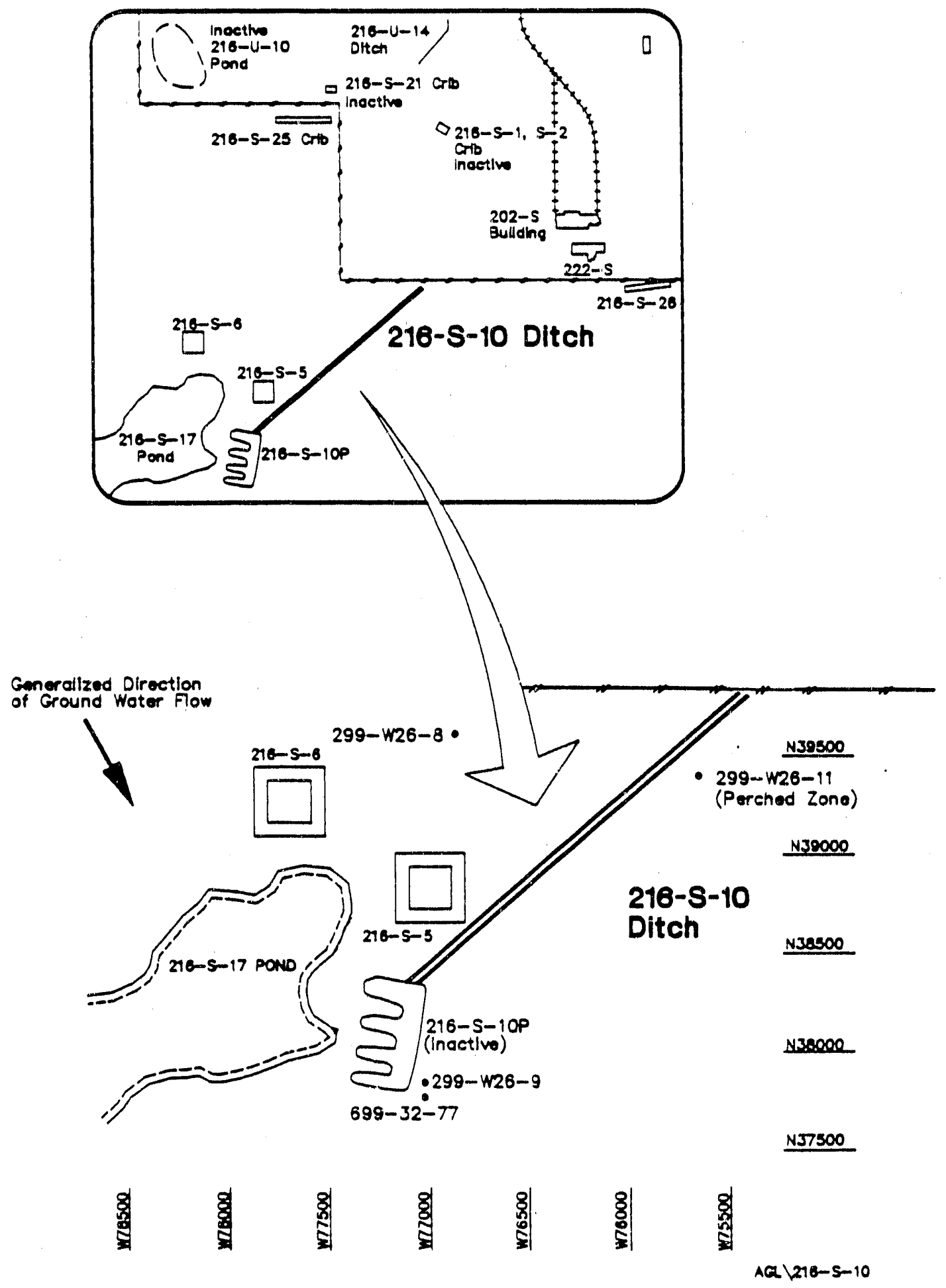

We11 Location and Site Map for 216-S-10 Ditch 
WHC-EP-0366

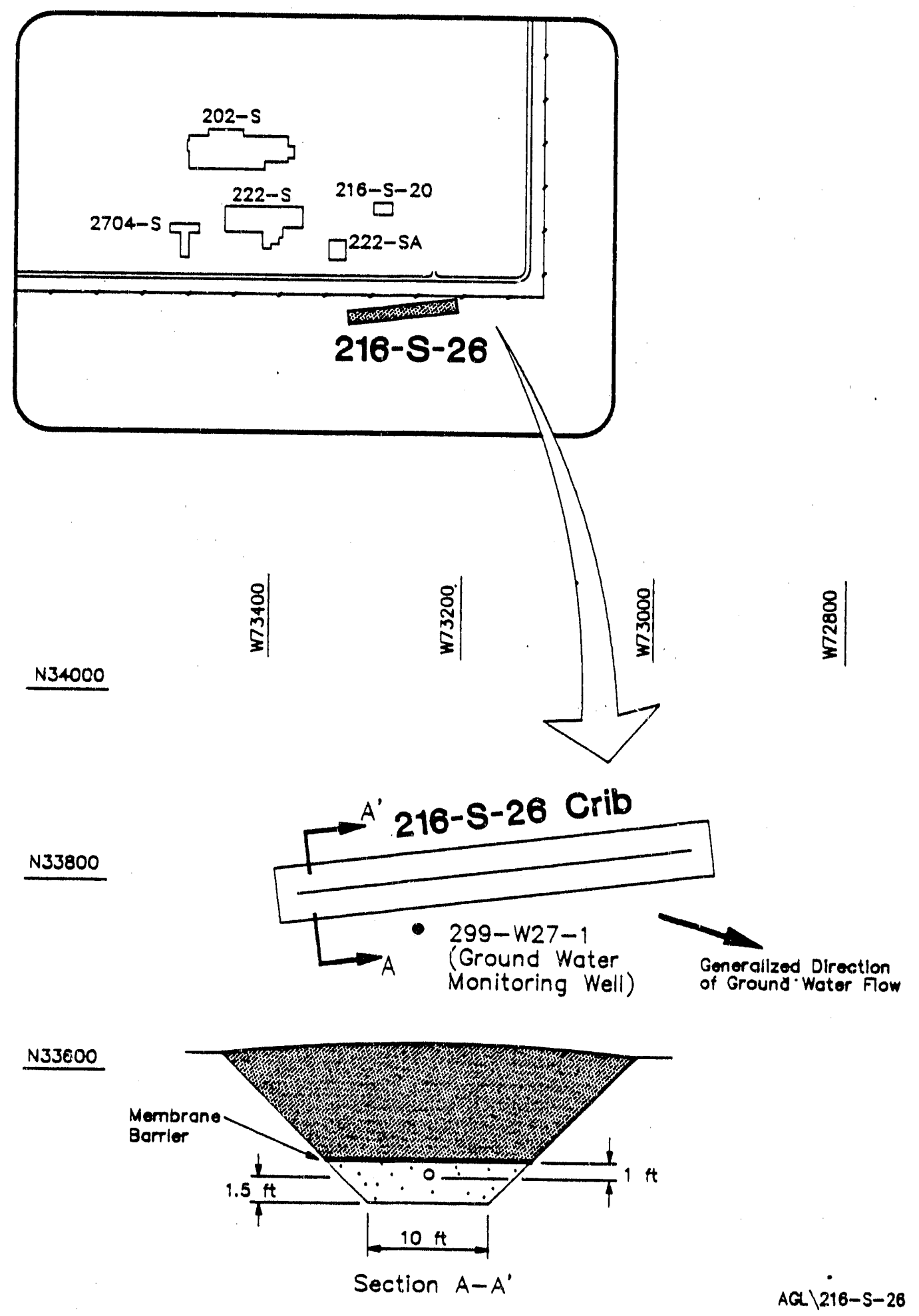

We11 Location and Site Map for 216-S-26 Crib 
WHC - EP-0366

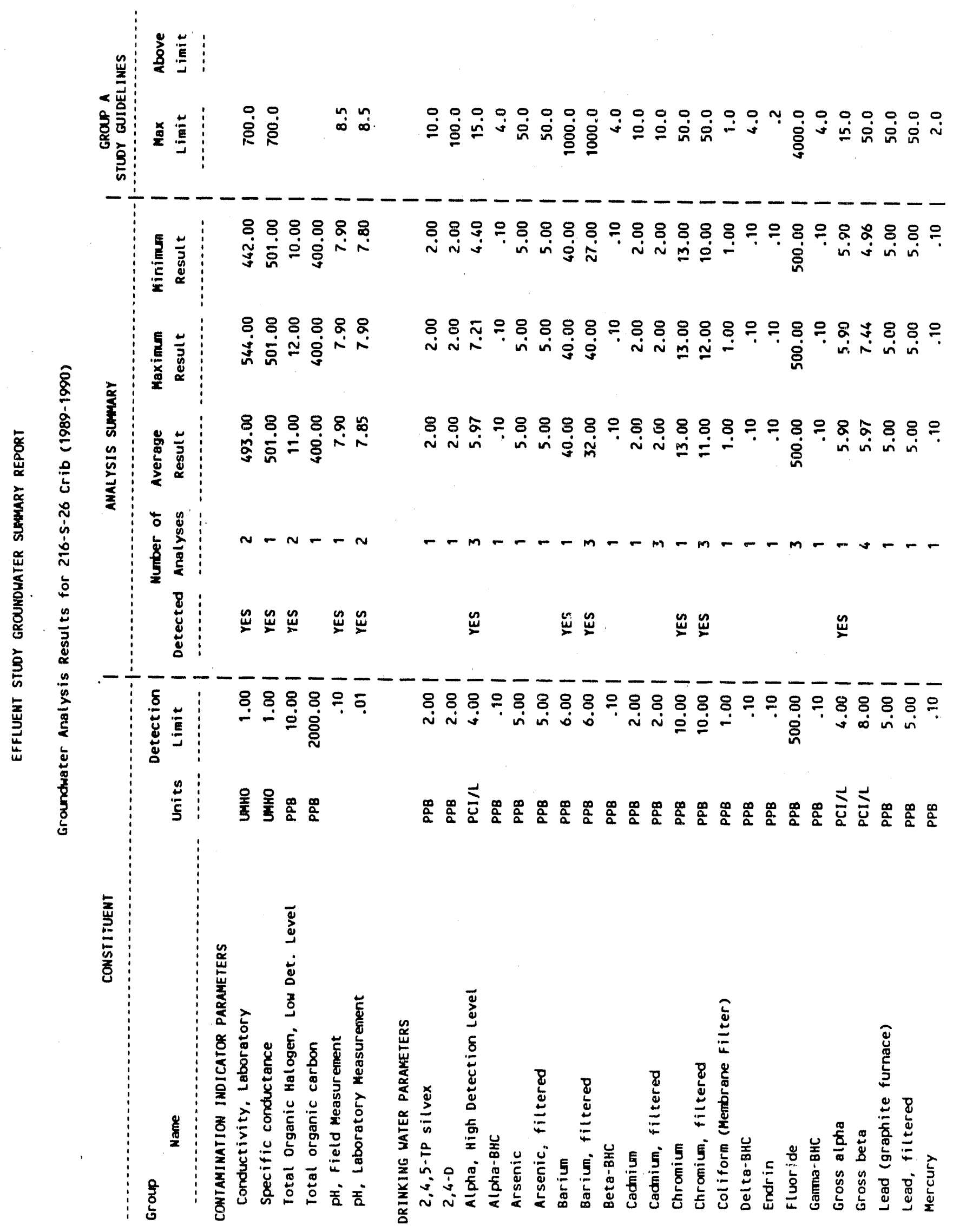




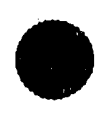

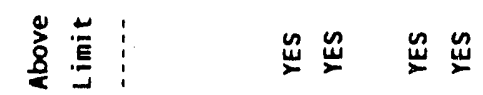

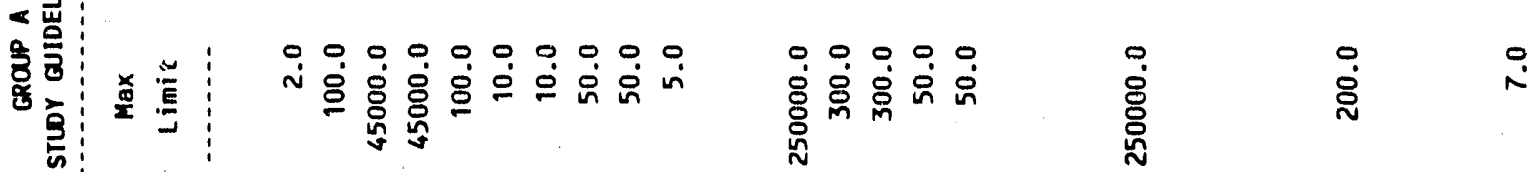

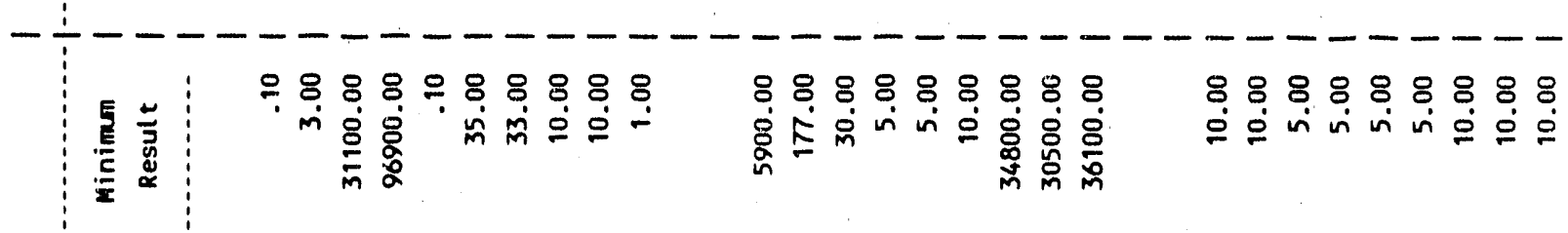

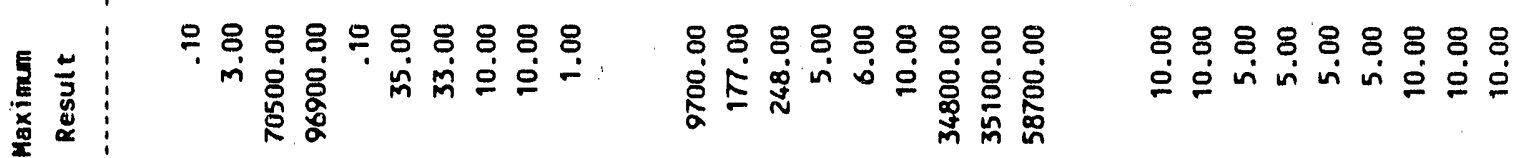

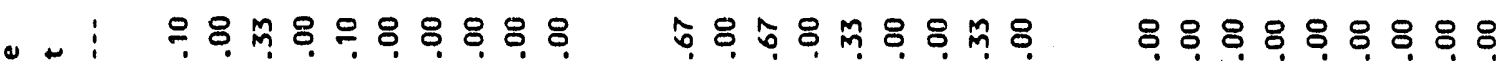

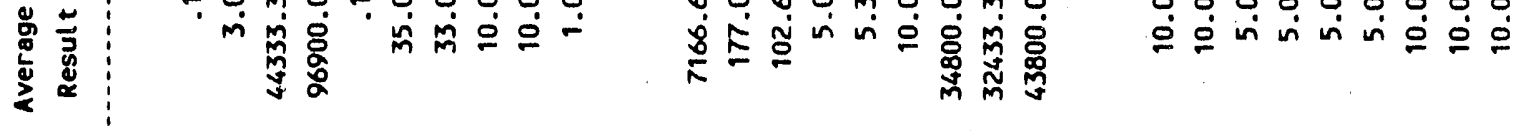

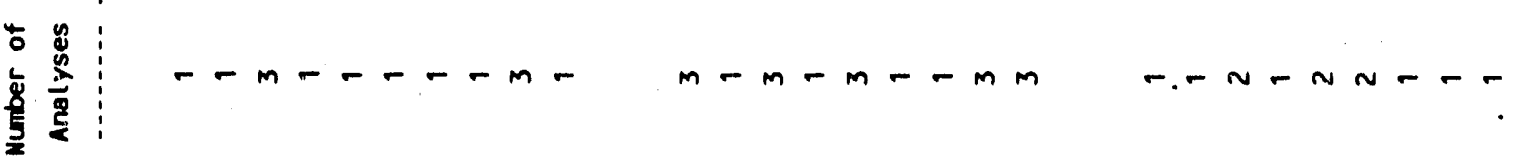

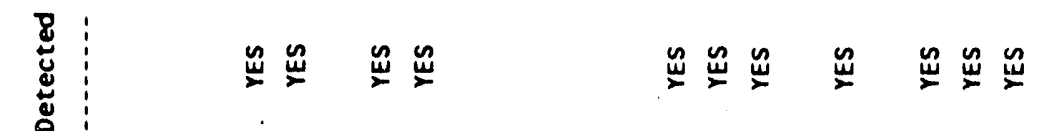

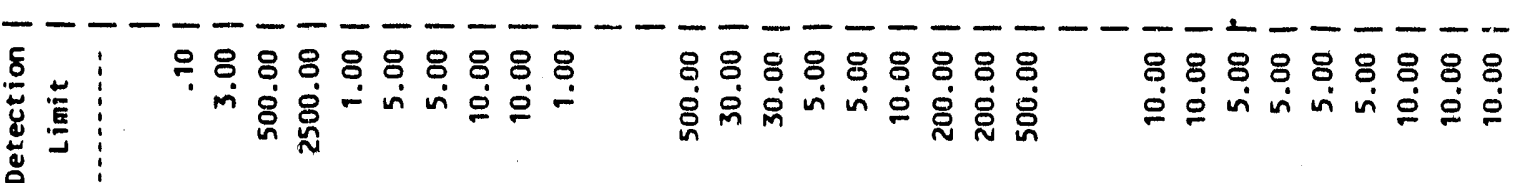

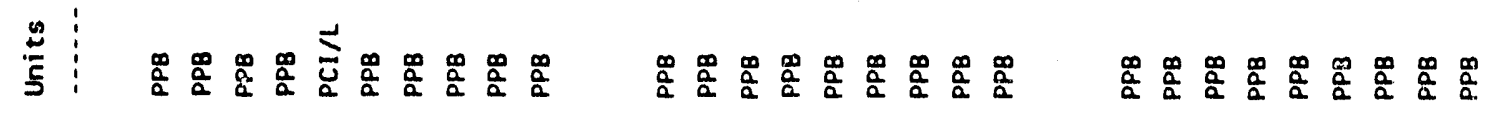

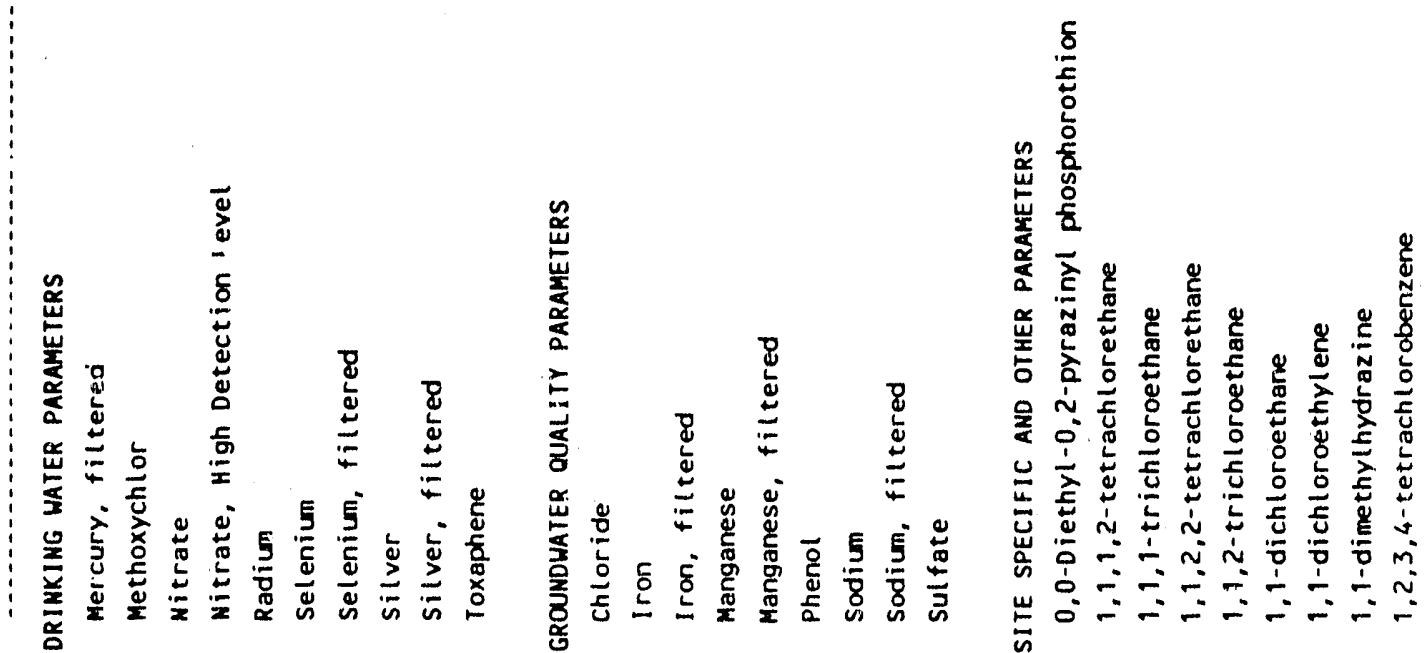




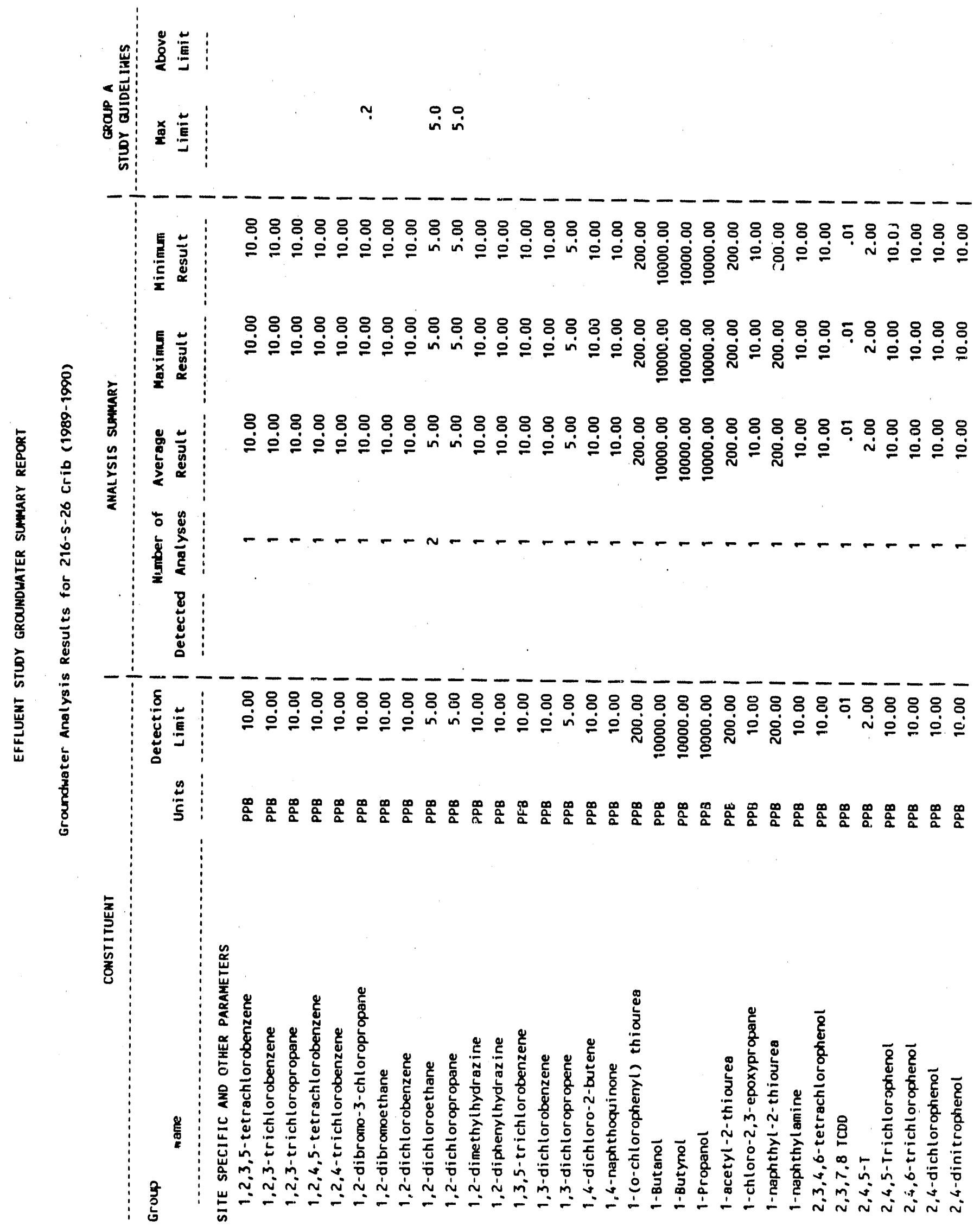


WHC-EP-0366

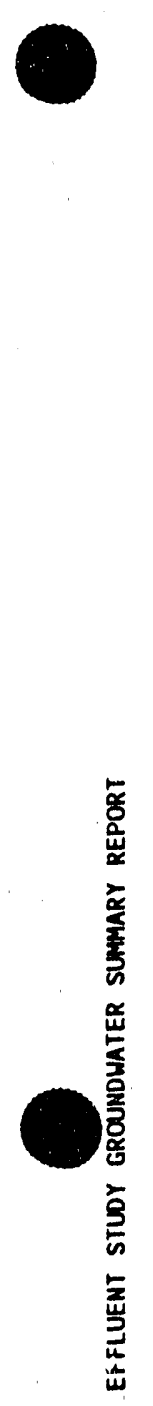

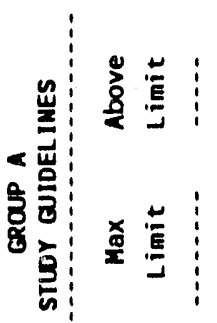

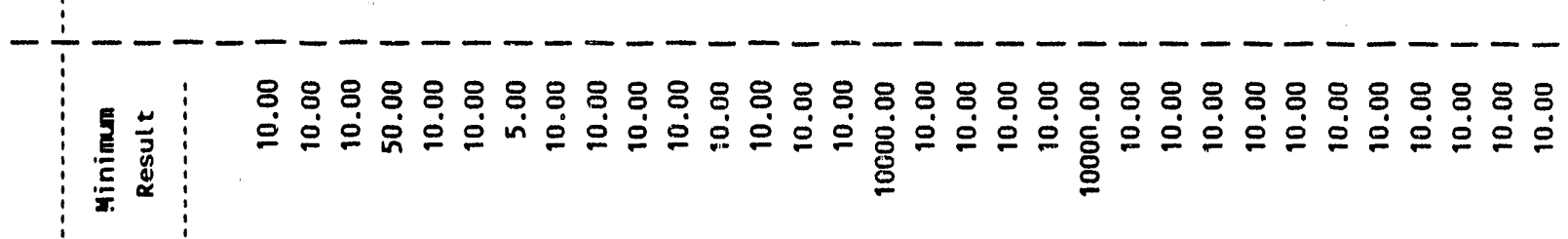

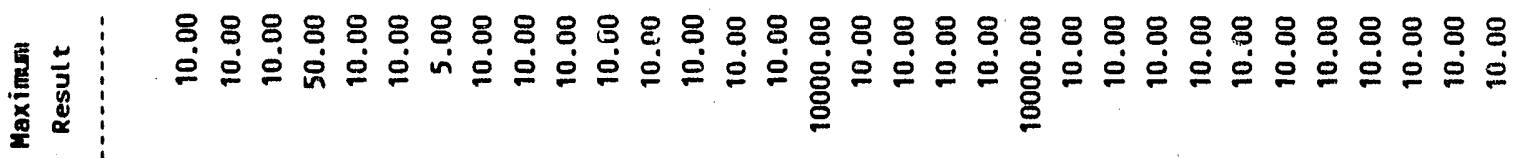

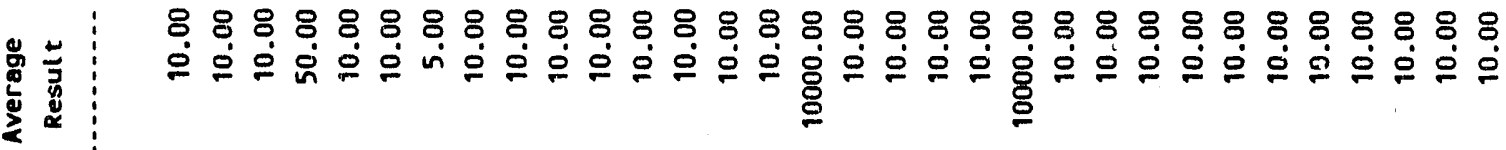

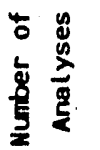

\&

:

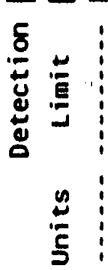

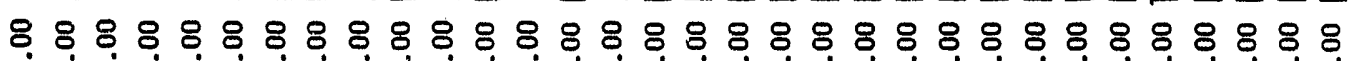

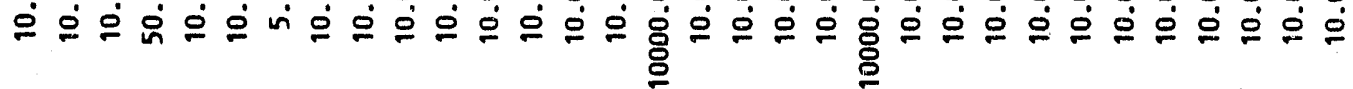

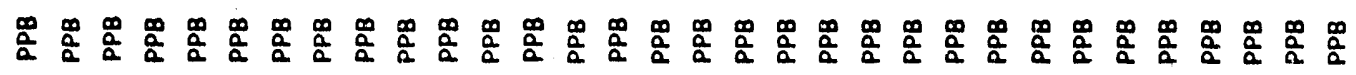

逽

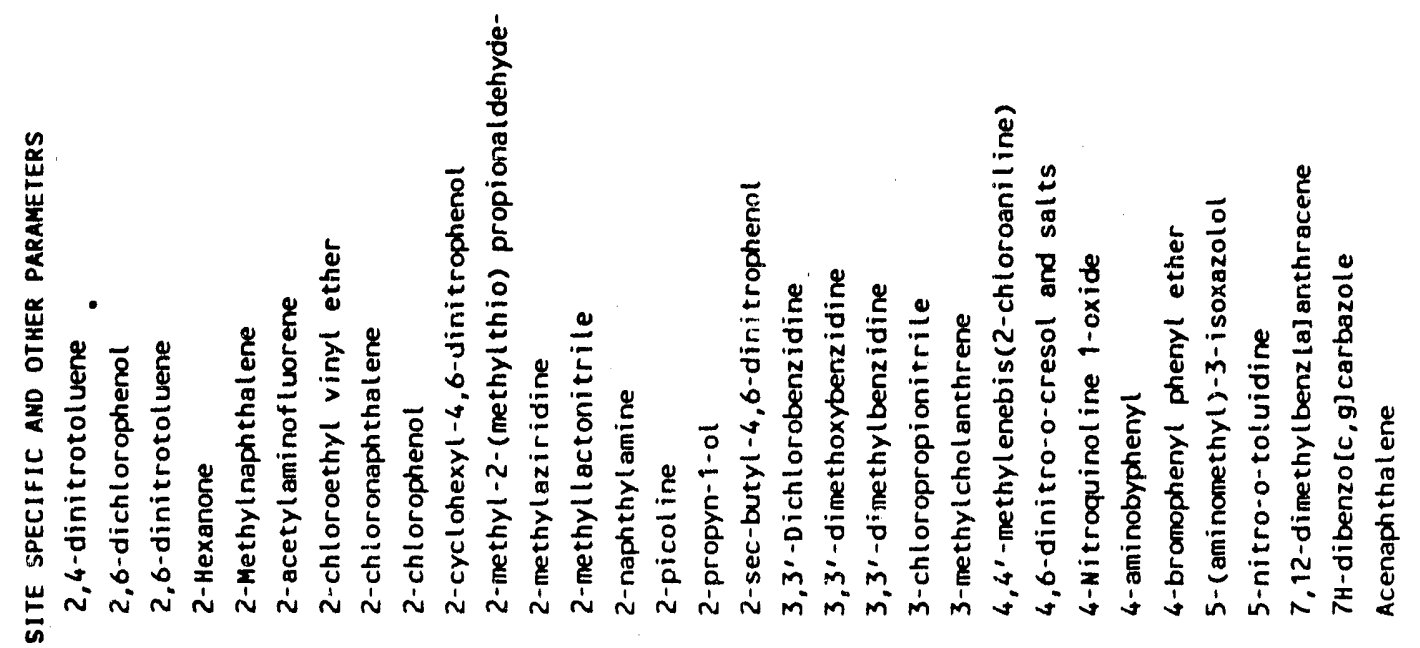


WHC-EP-0366

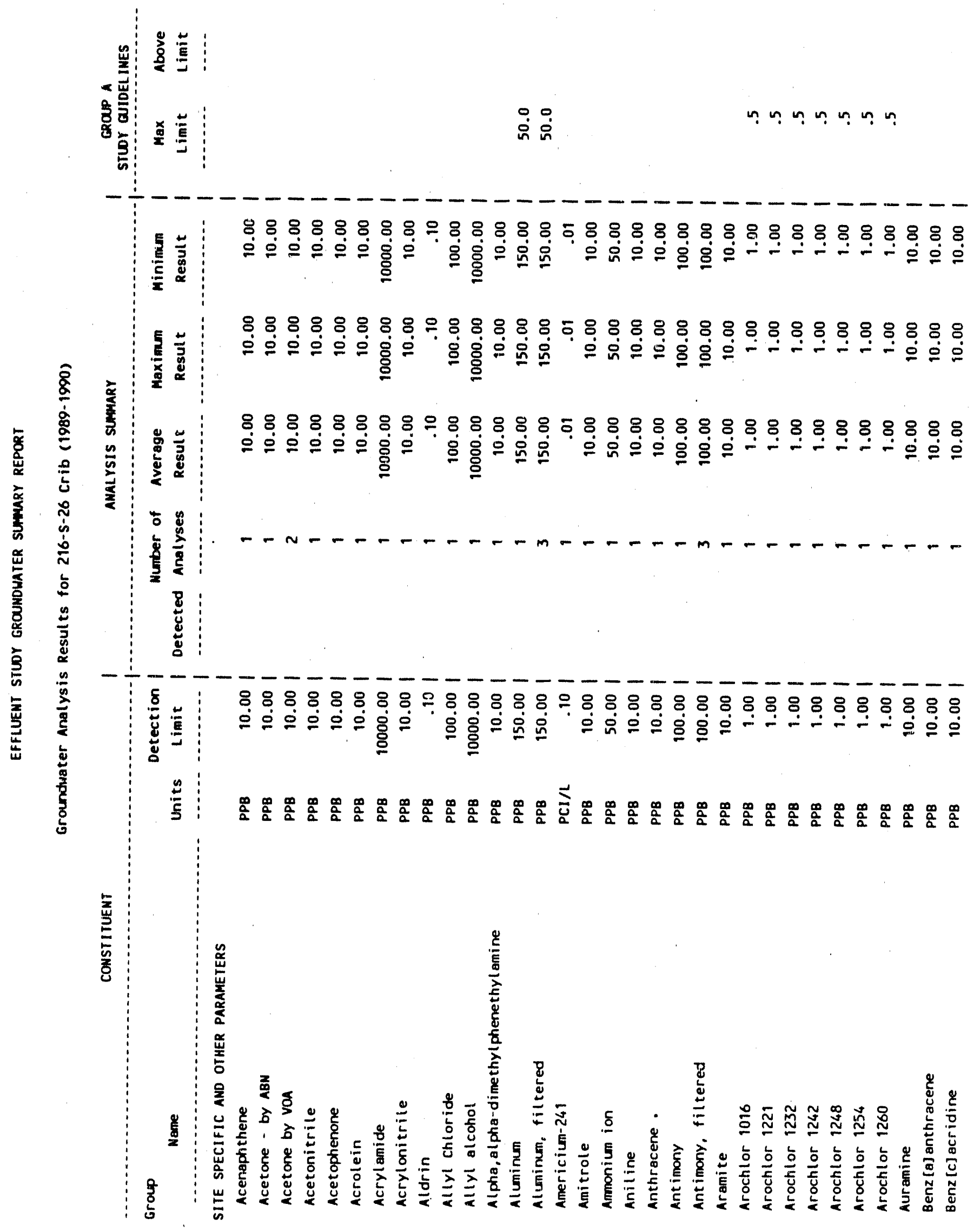




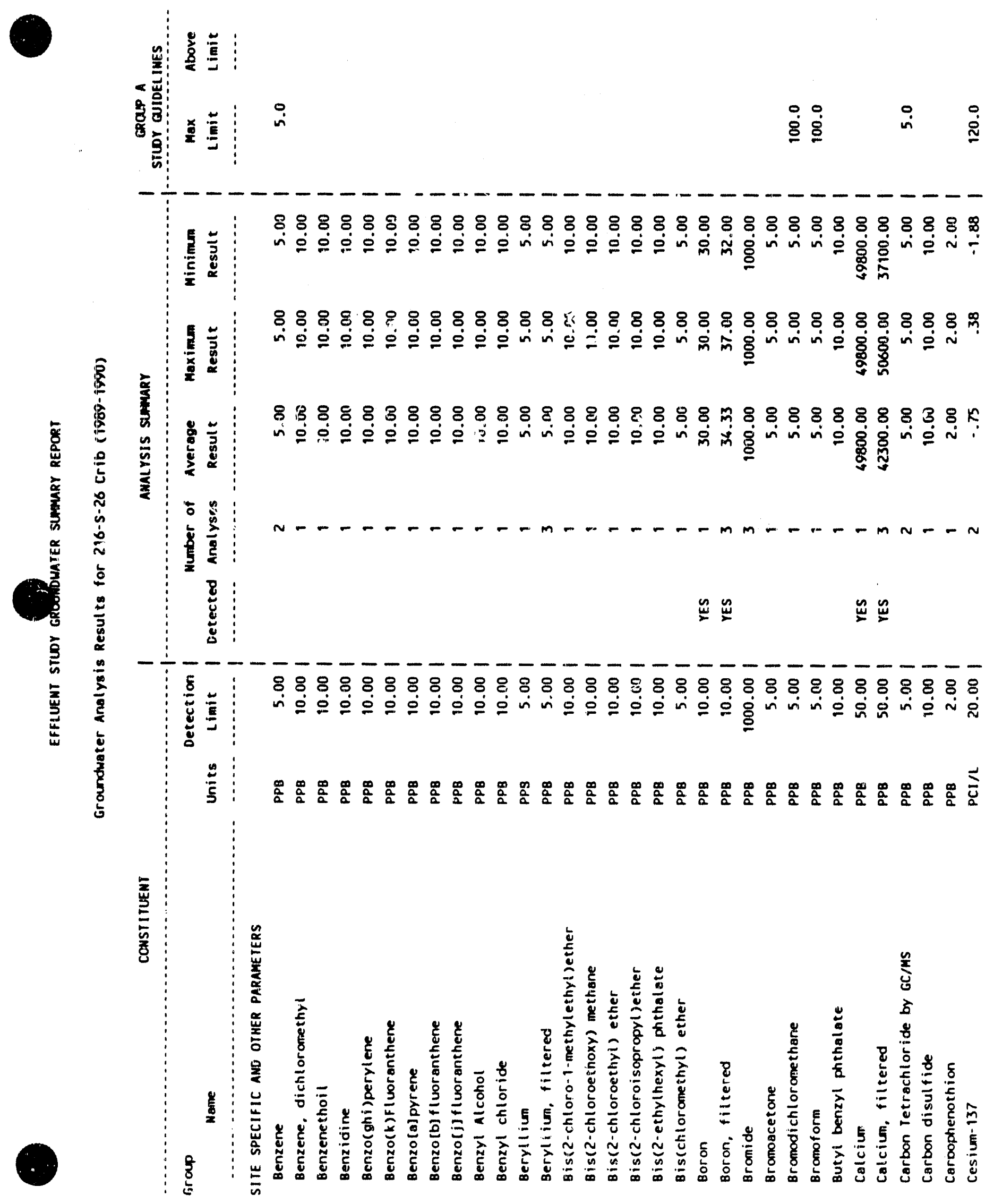


WHC-EP-0366

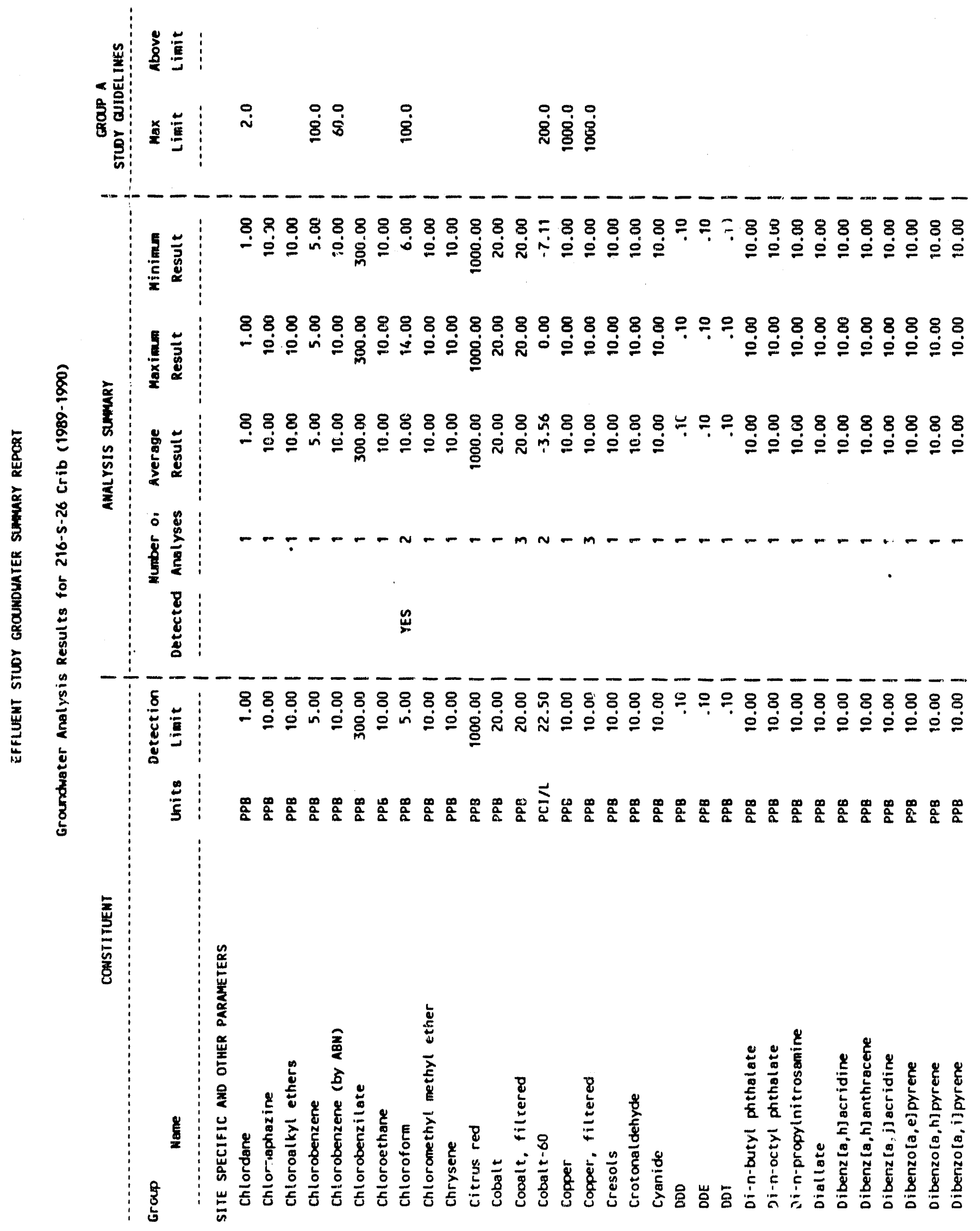




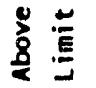

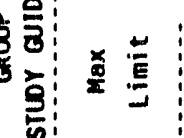

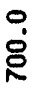

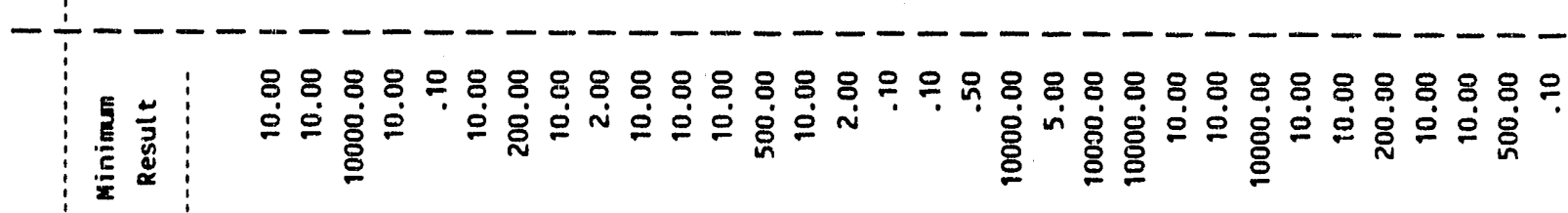

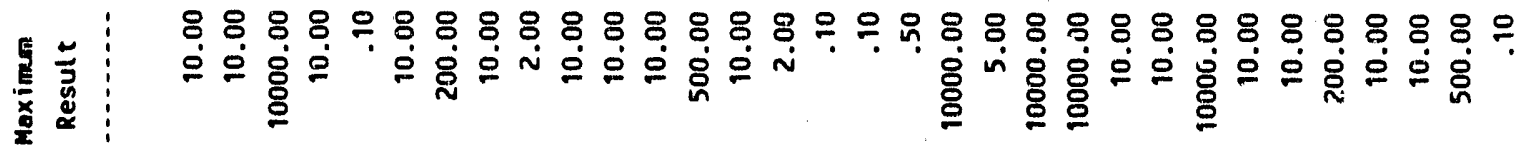

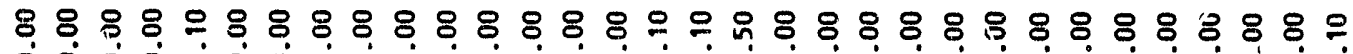
@ᄋ

¿

\%

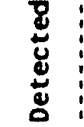

$-\frac{\vdots}{\vdots}$

峞

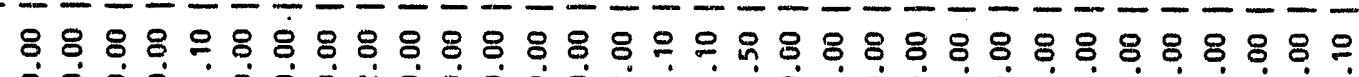
த்

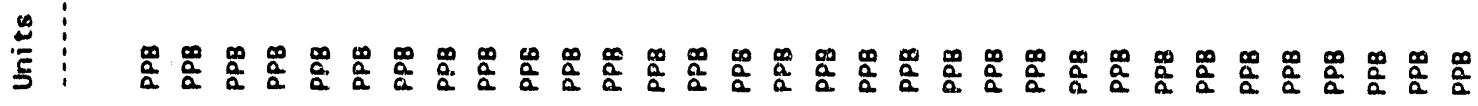

窟

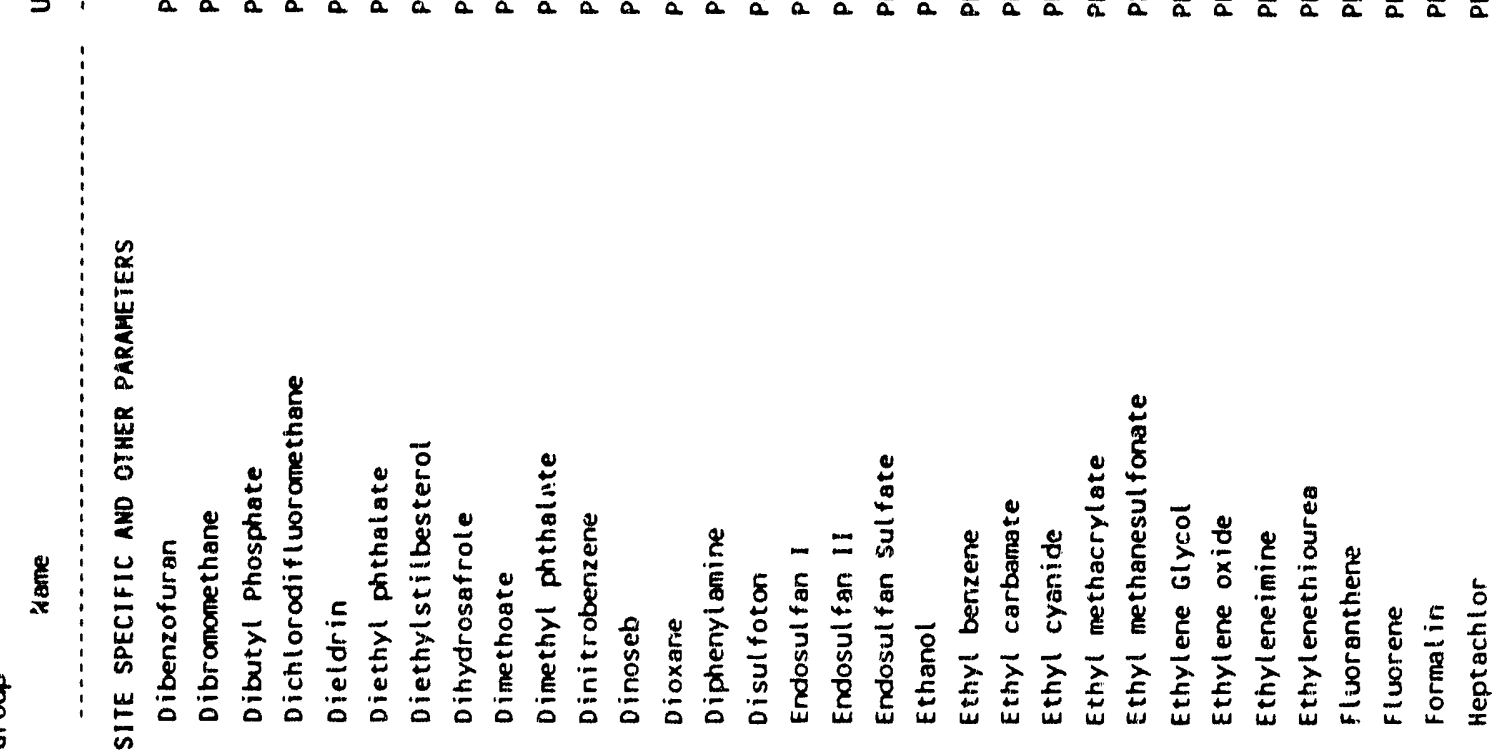




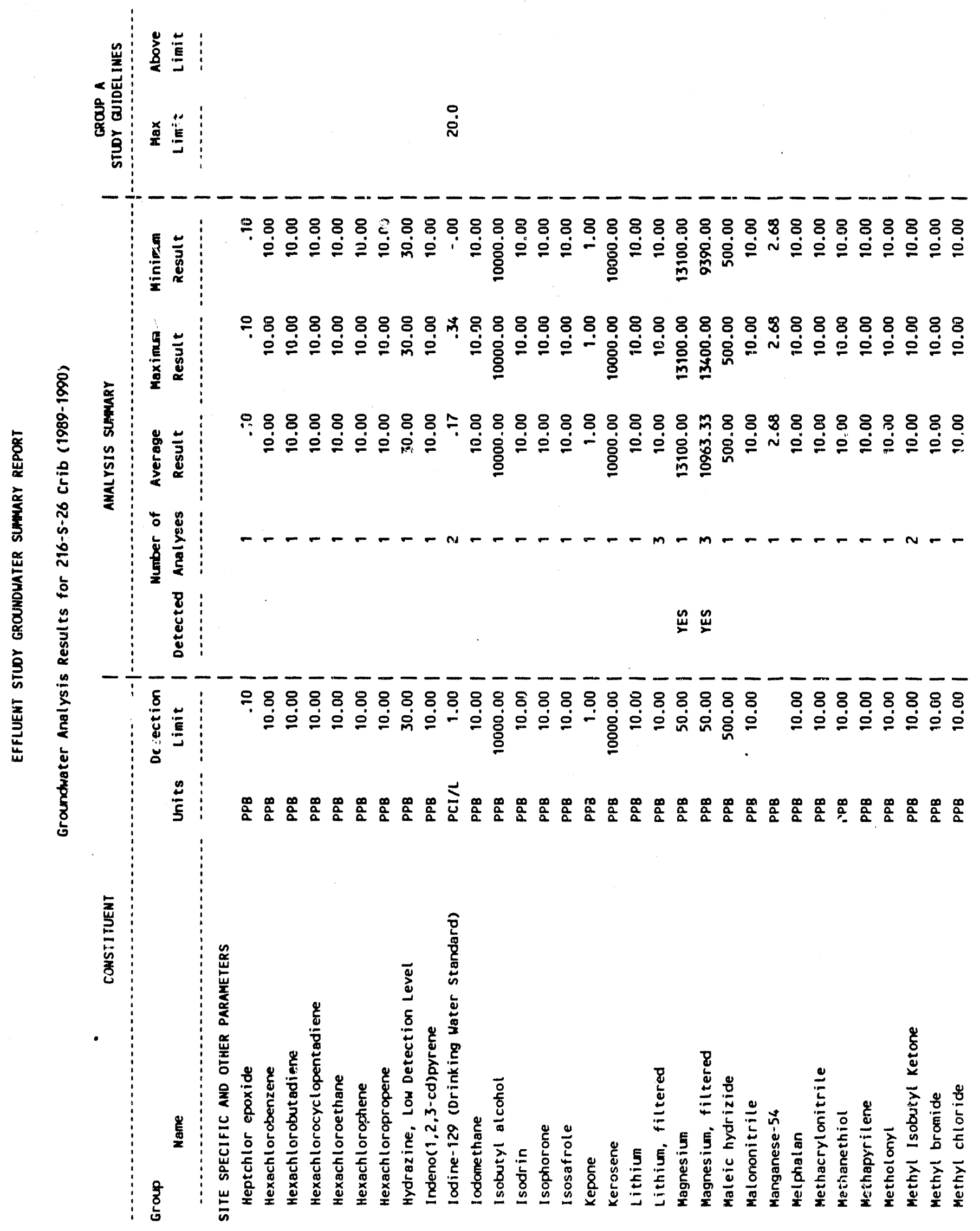




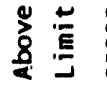

รูำ

崖:

in

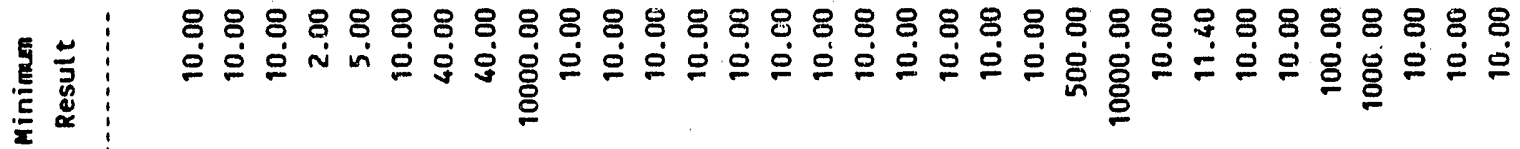

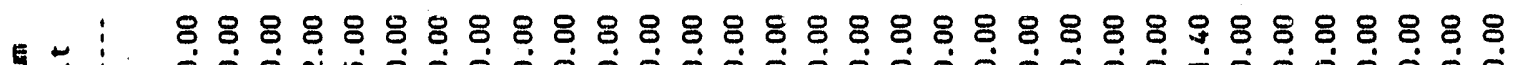

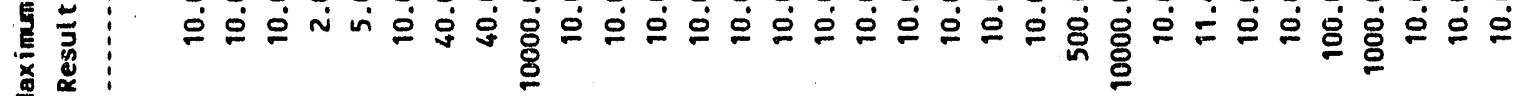

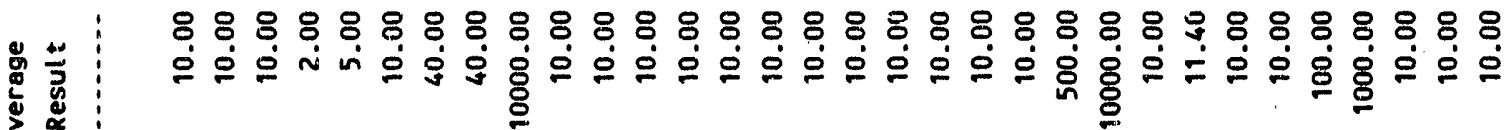

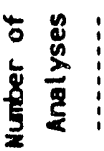

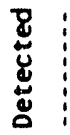

N

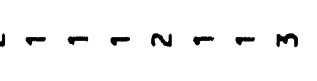

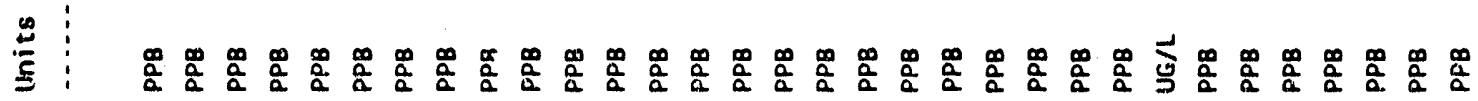

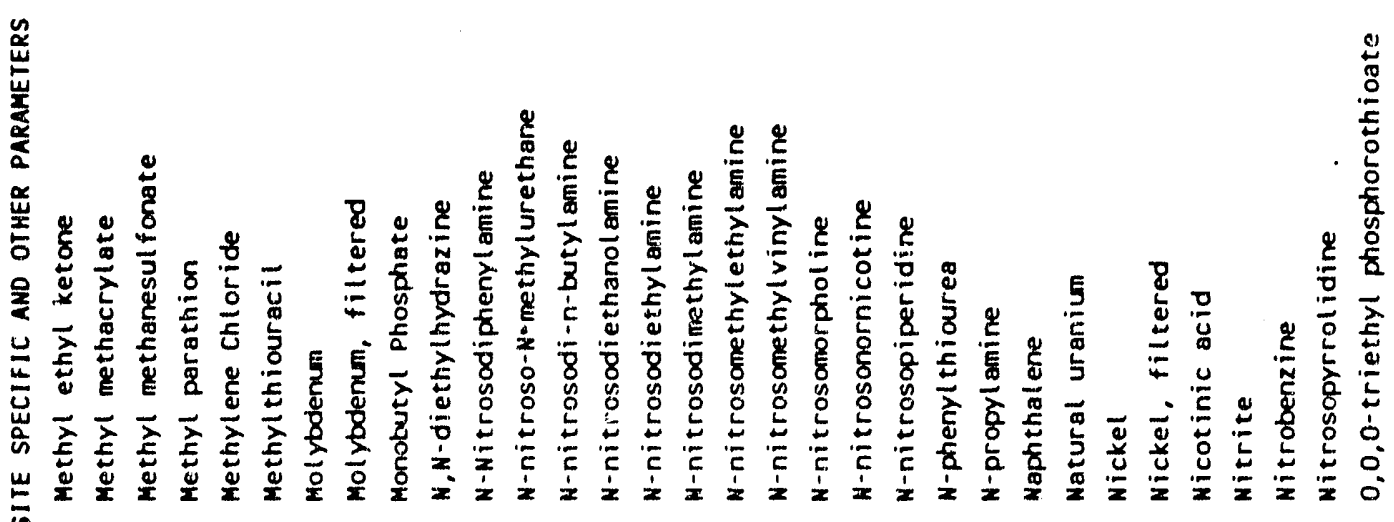



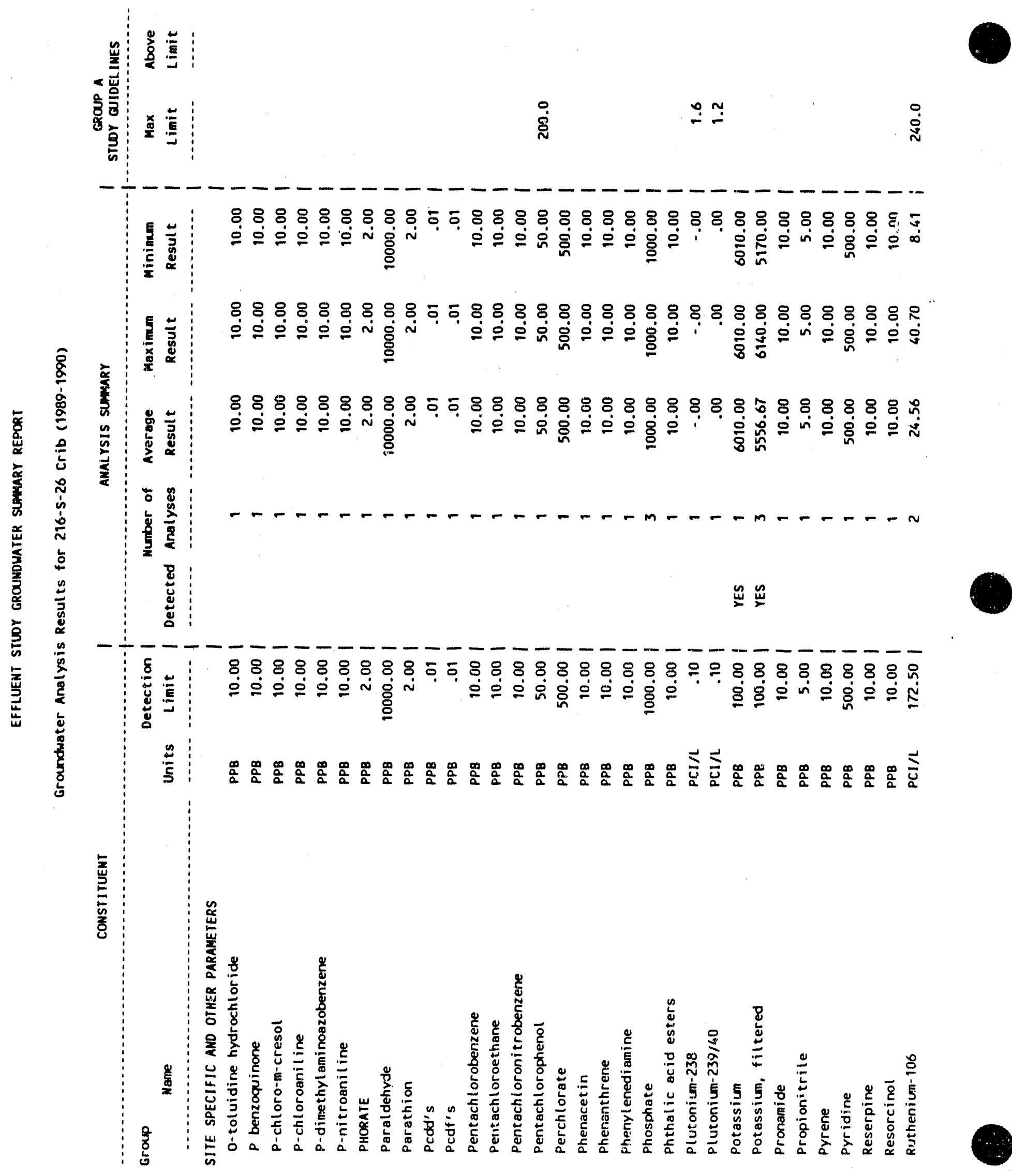
WHC-EP-0366

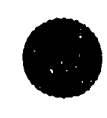

高

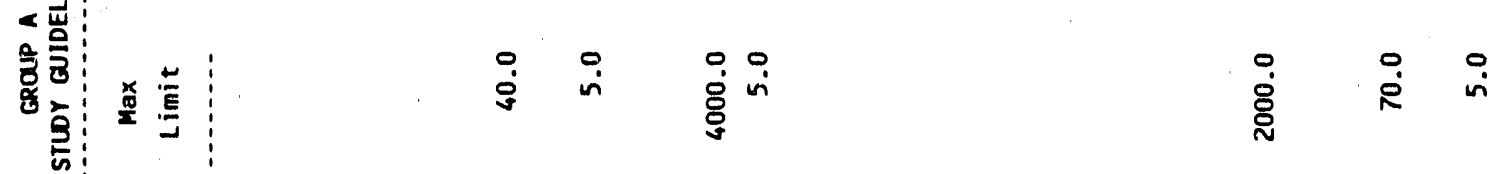

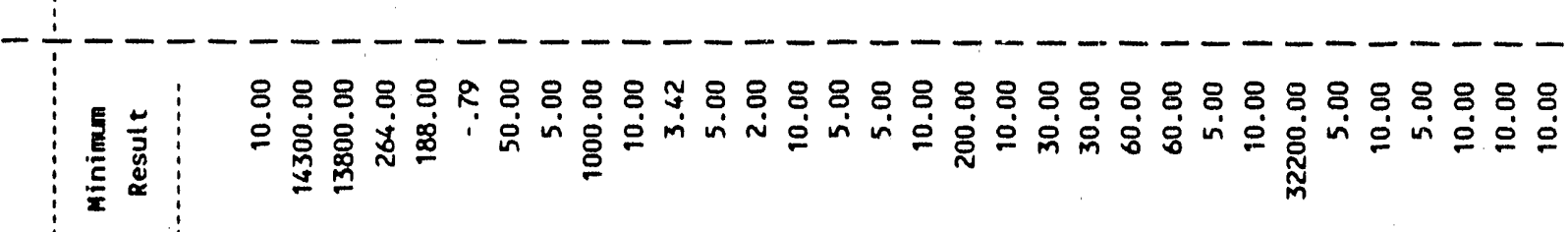

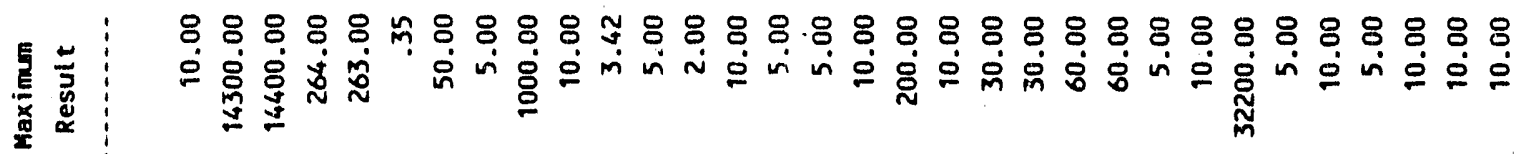

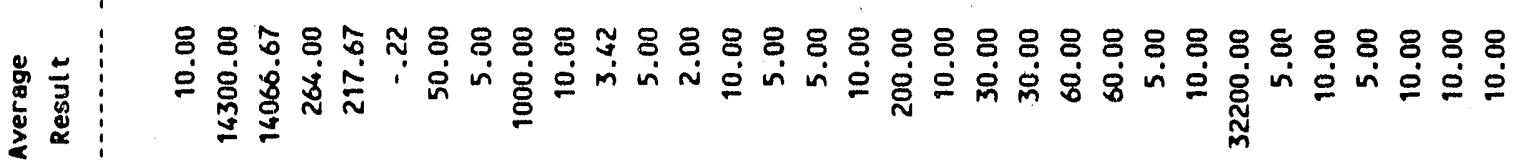

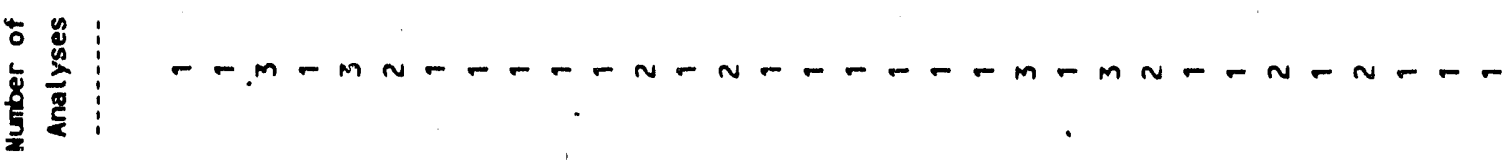

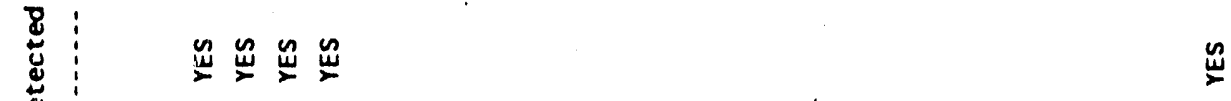

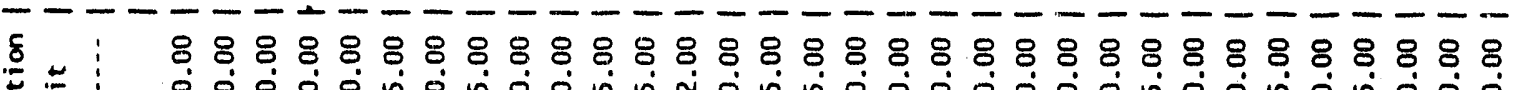

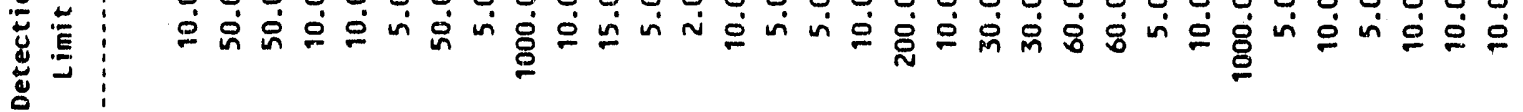

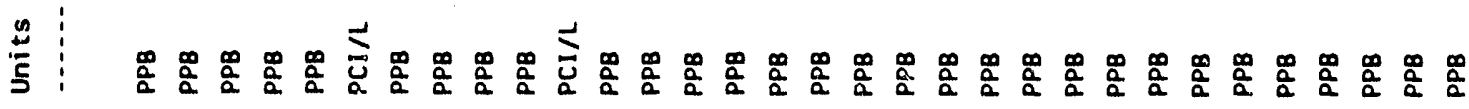
苨

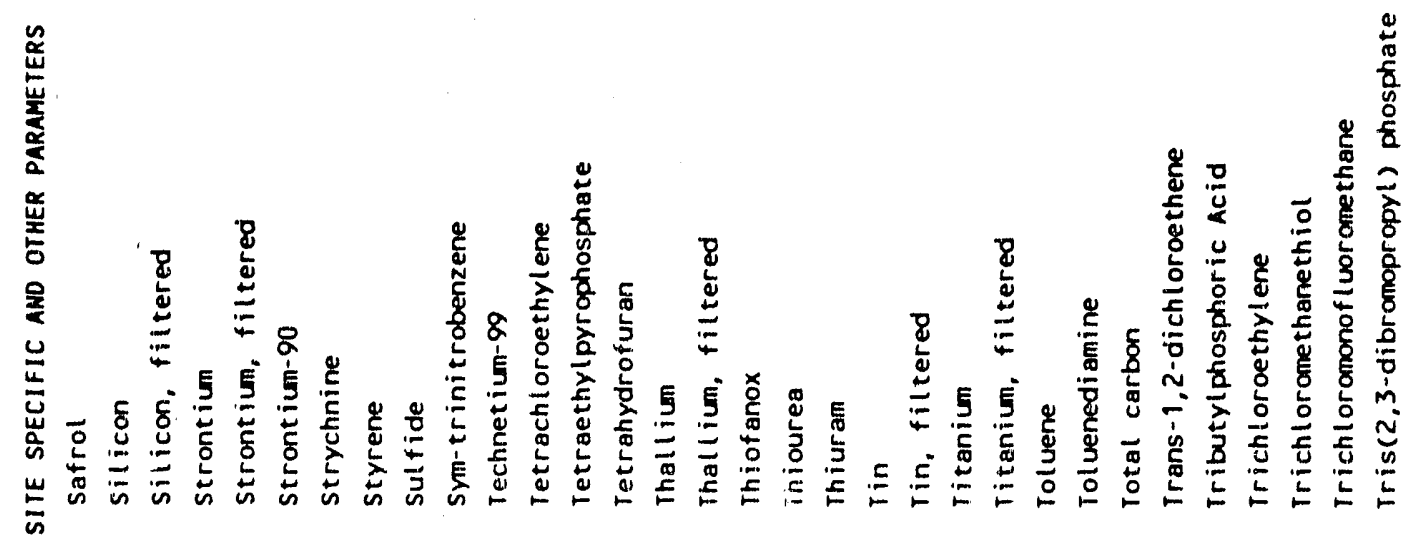




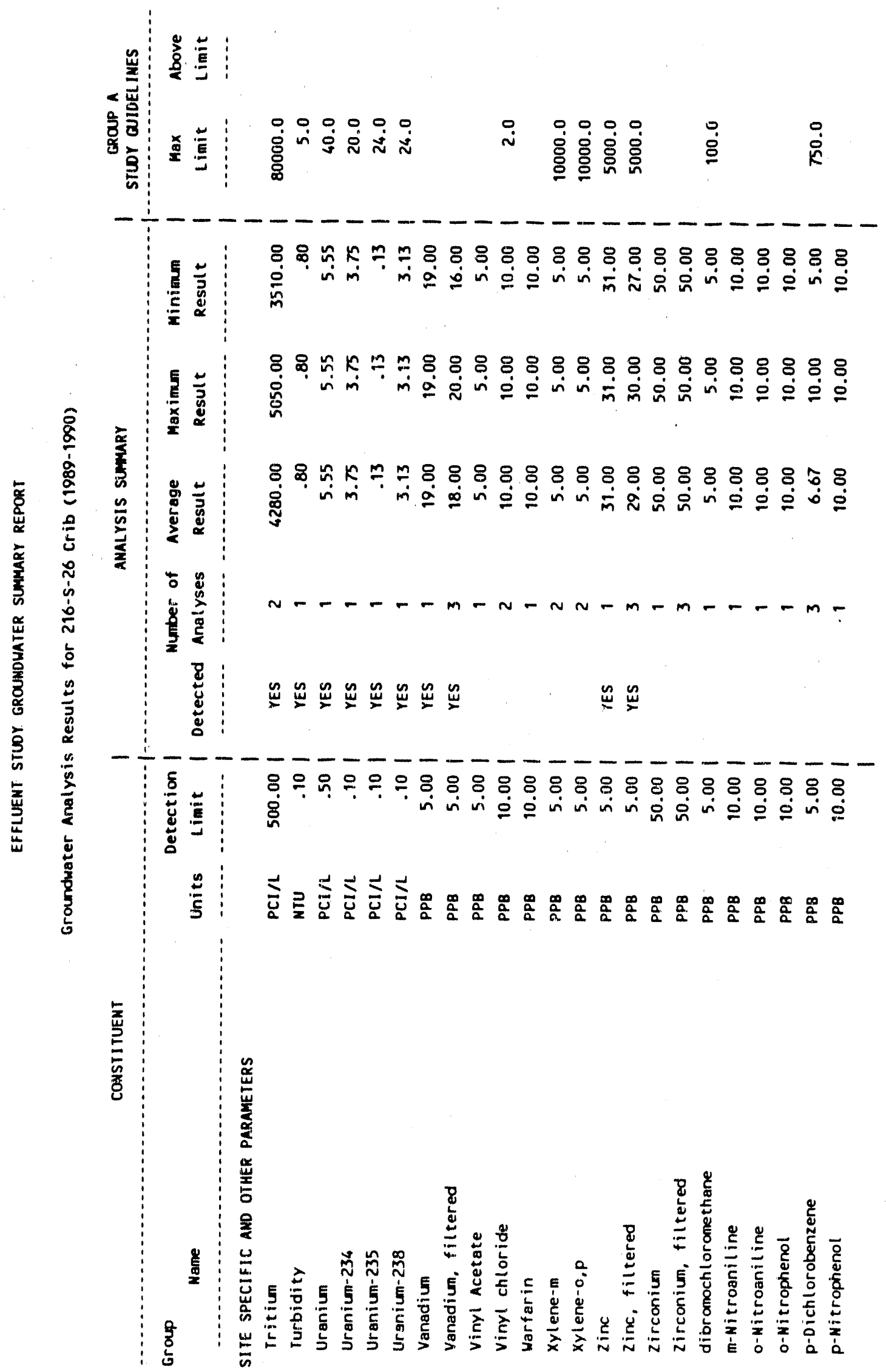




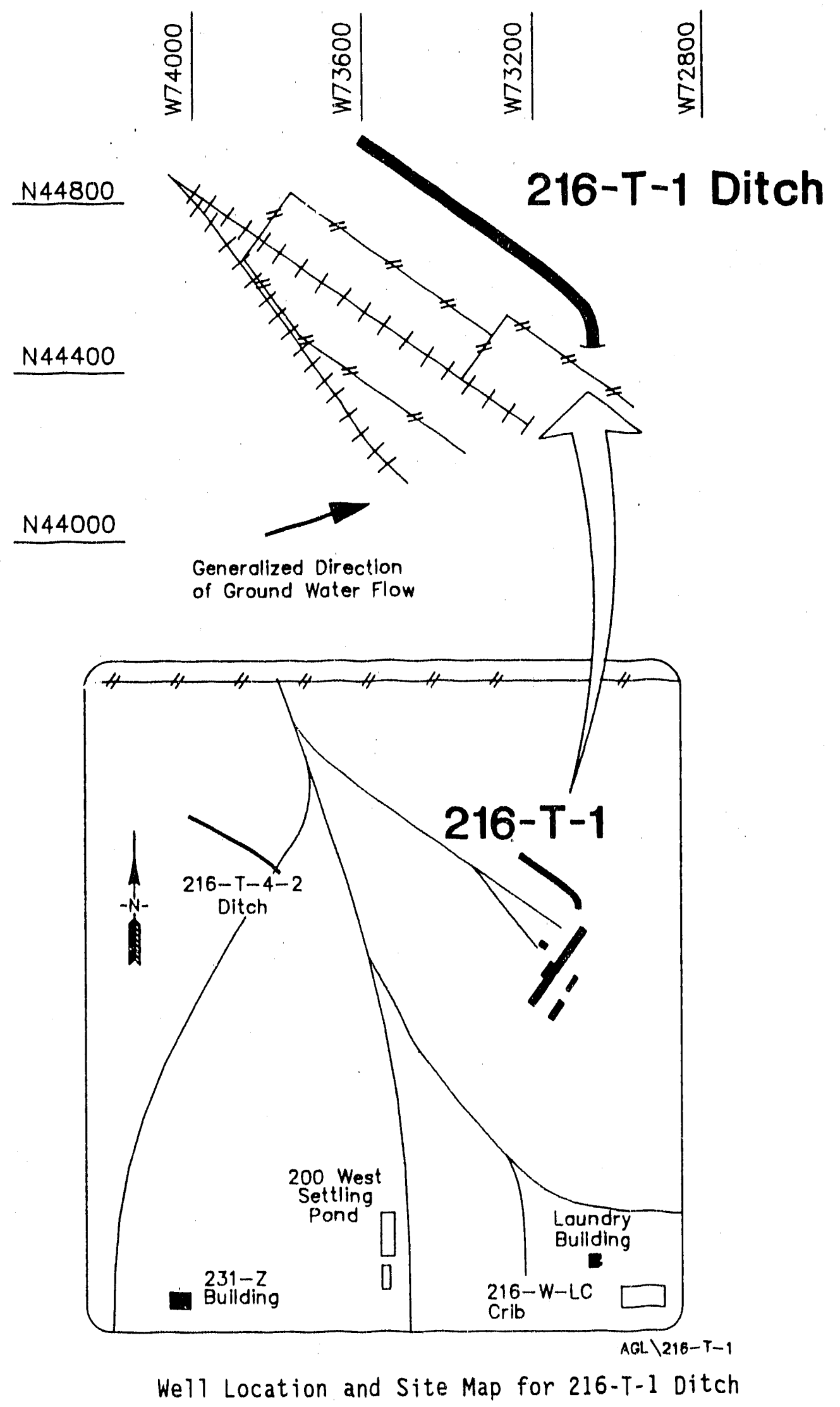


WHC-EP-0366

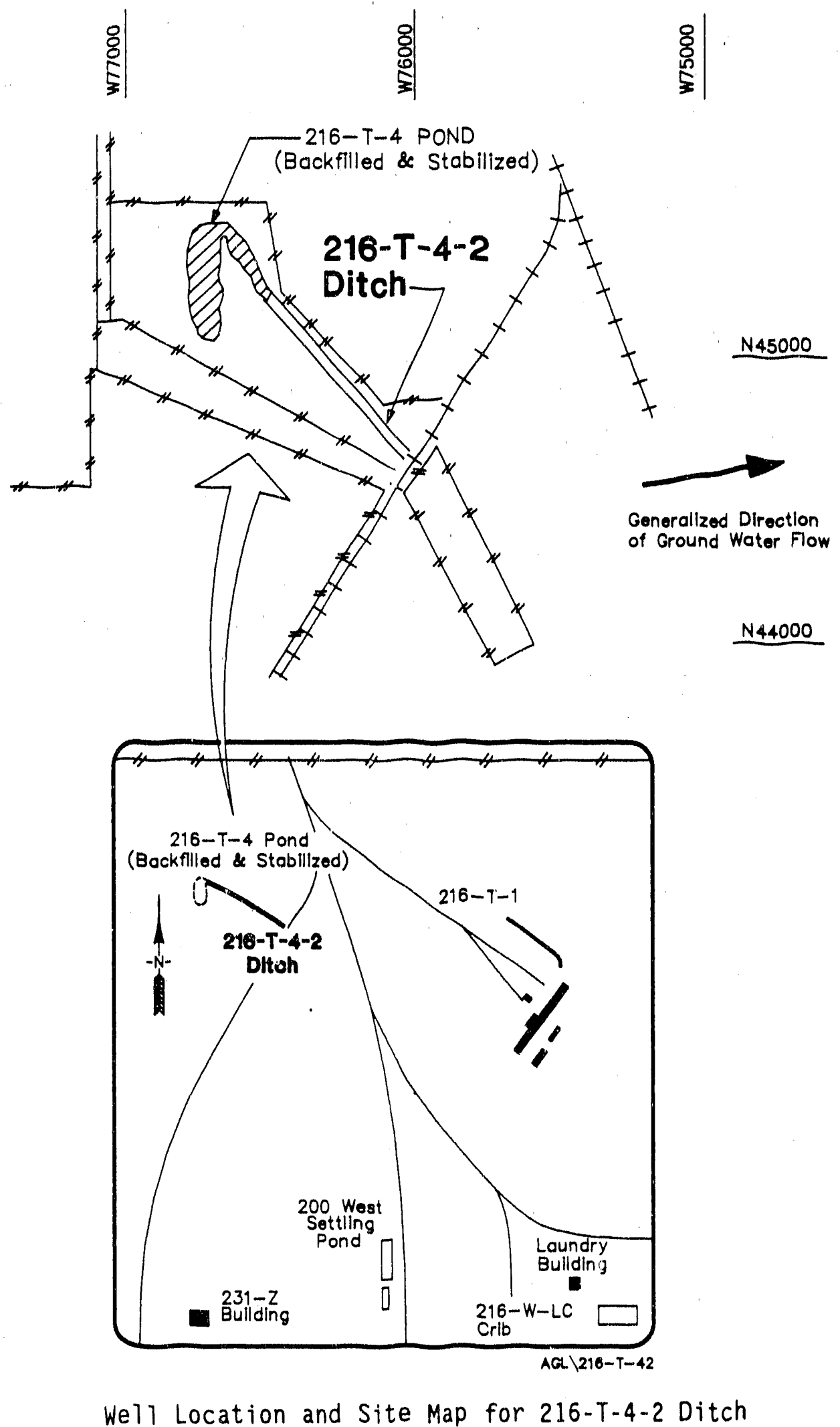




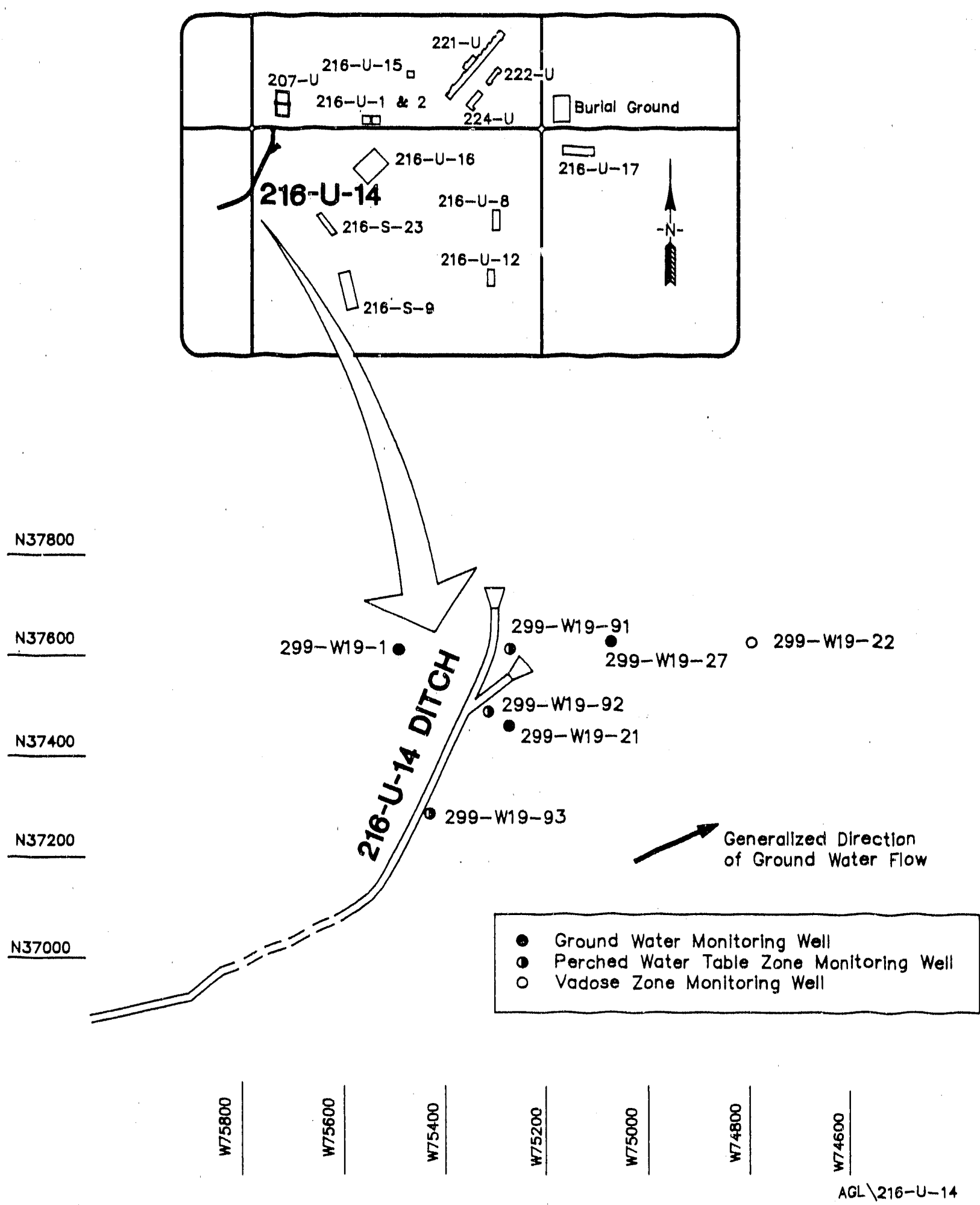

Wel1 Location and Site Map for 216-U-14 Ditch 
WHC-EP-0366

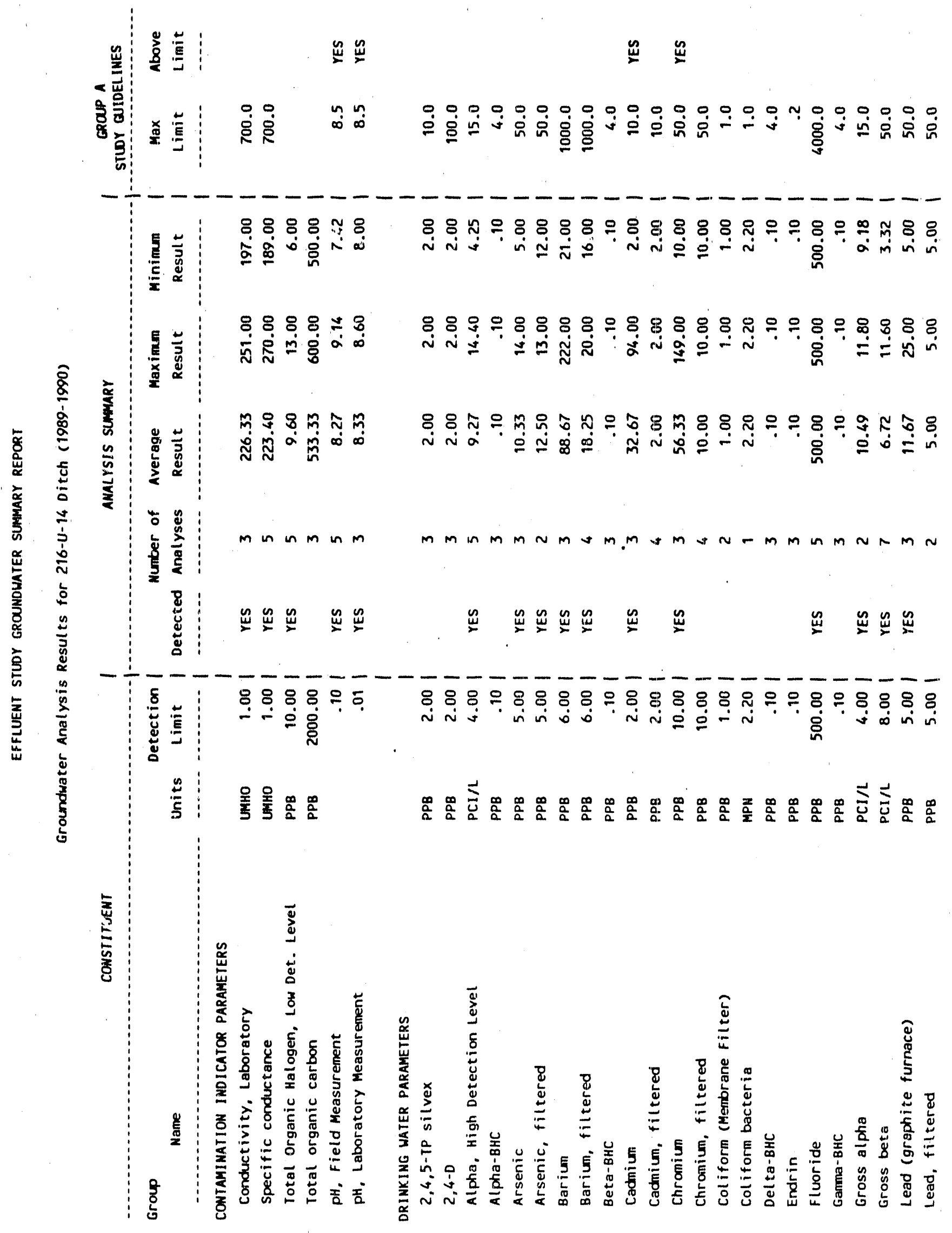


WHC-EP-0366

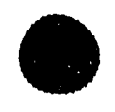

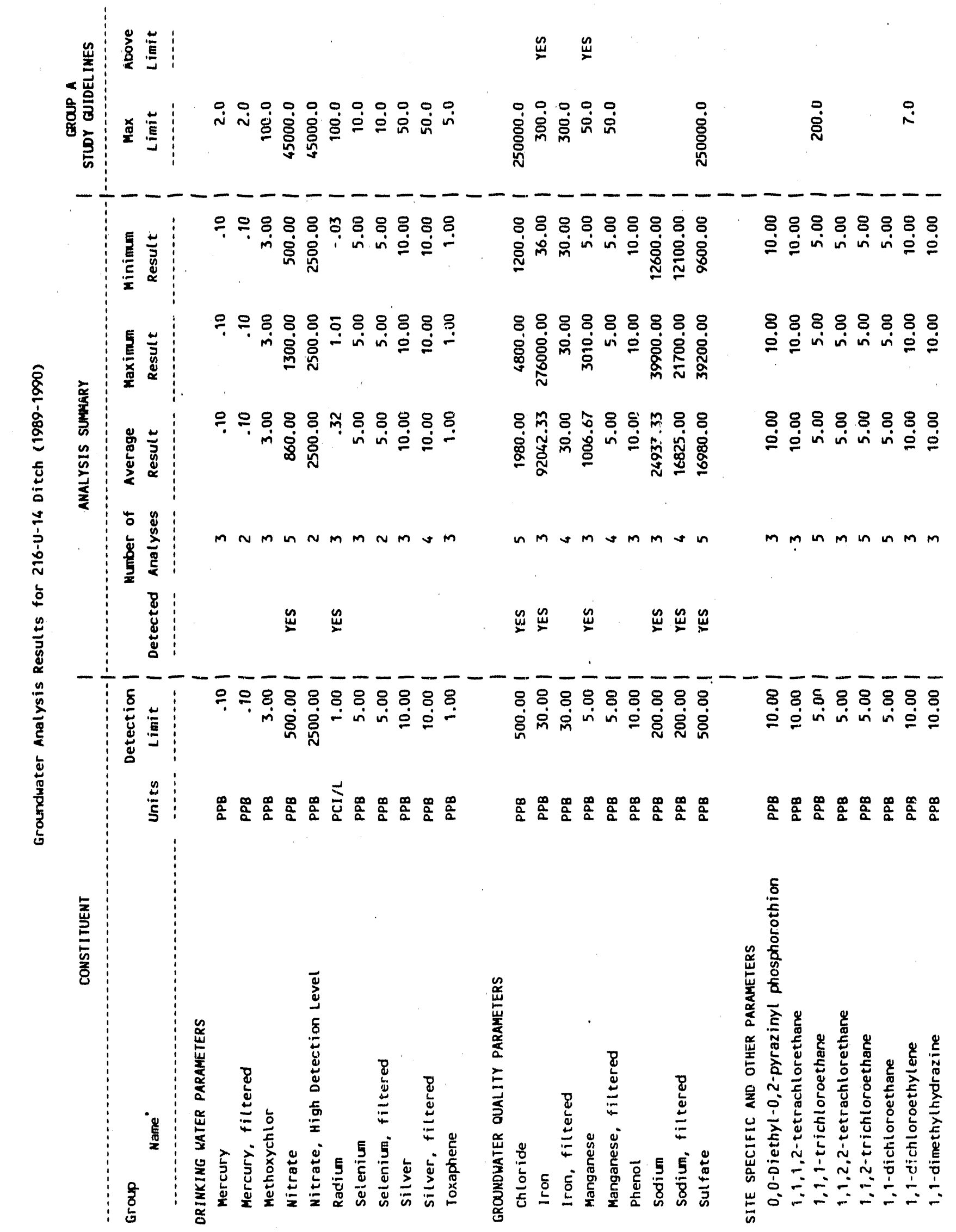




$$
\text { WHC-EP-0366 }
$$

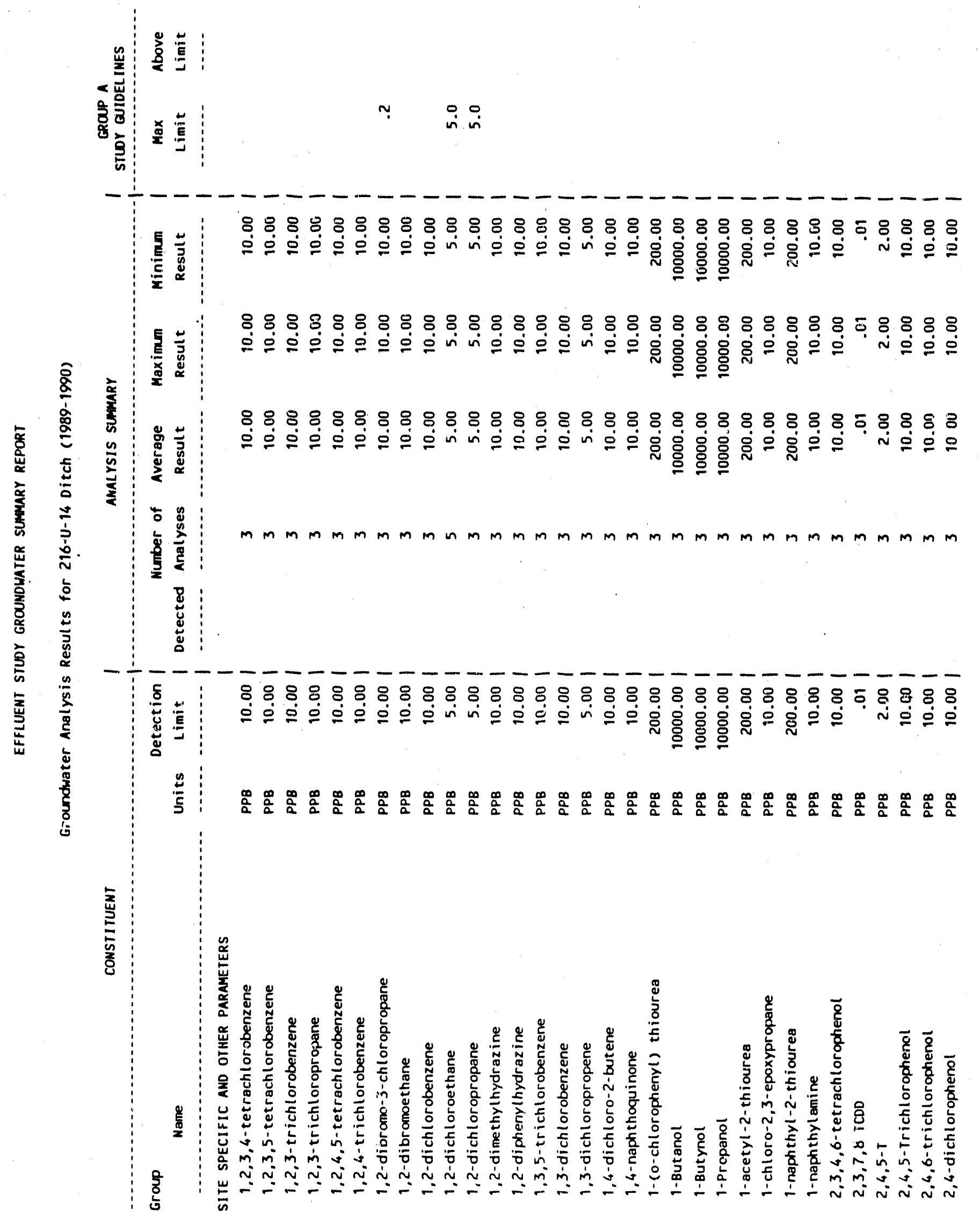


WHC -EP-0366

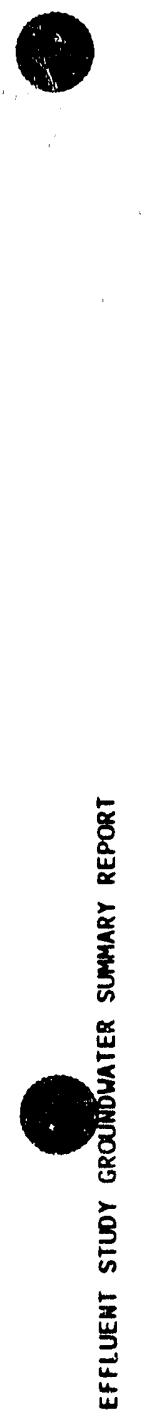

草:

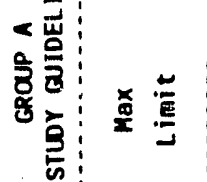

$-\frac{1}{1}$

$\frac{5}{\tilde{E}}$

88888888888888888888888888888888

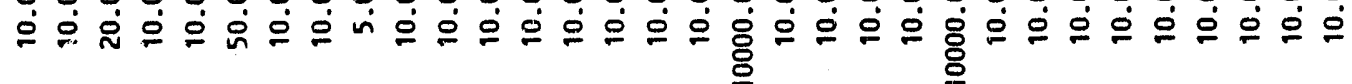

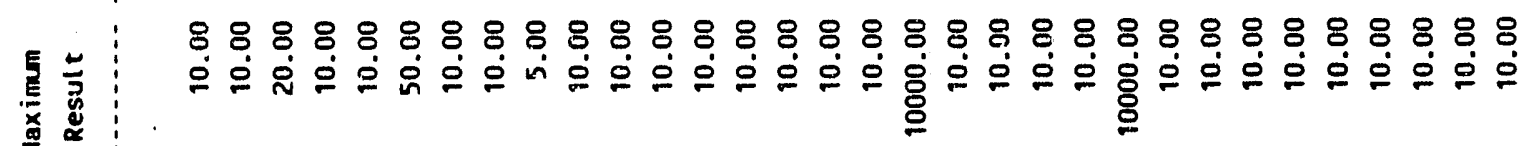

488888888888888888888888888888888

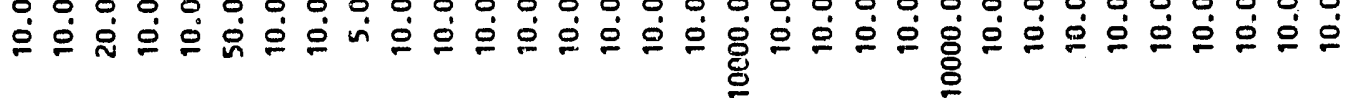
$\sum$

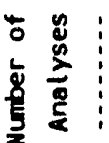

$m m-m m m m m m m, m m m m m m m m m m m m m m m m m m m m m m$

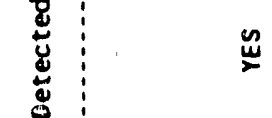

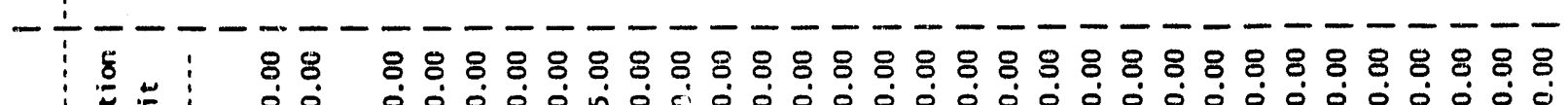

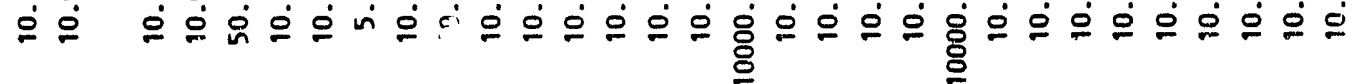
ป

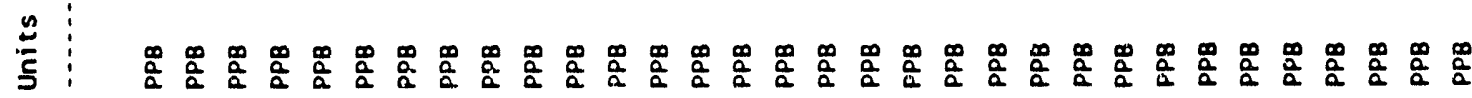

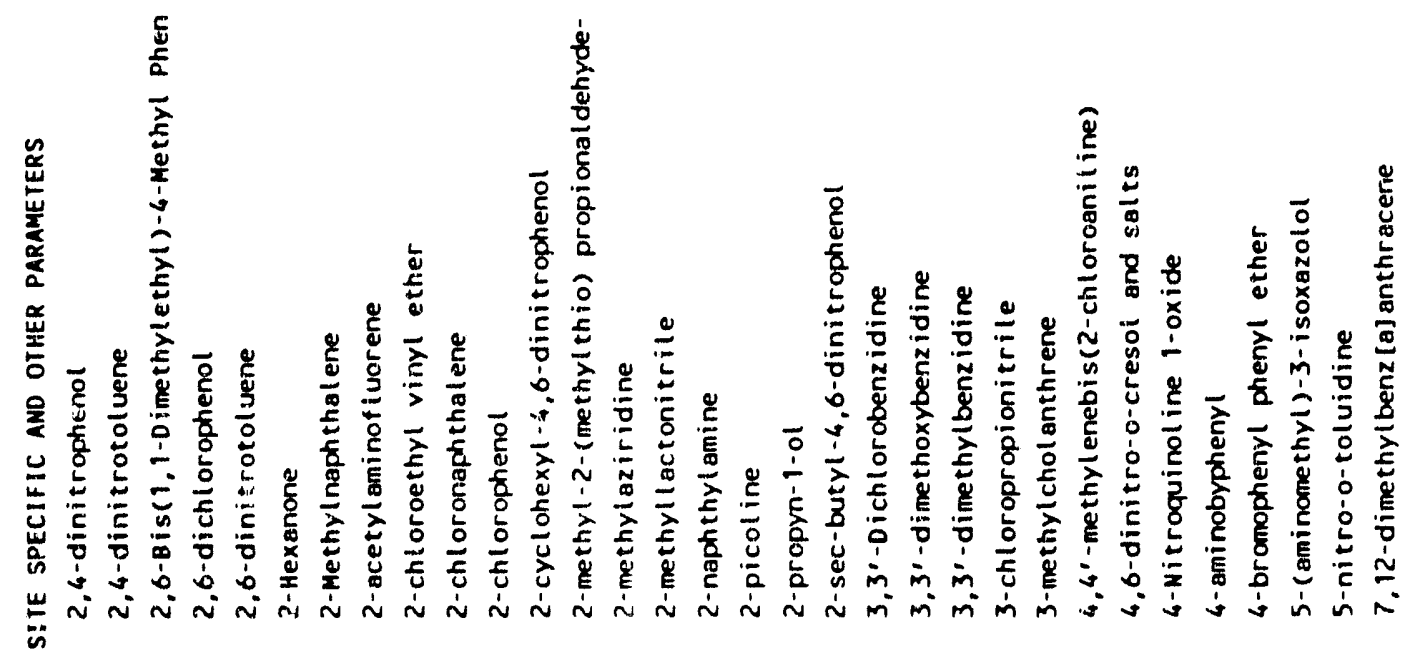




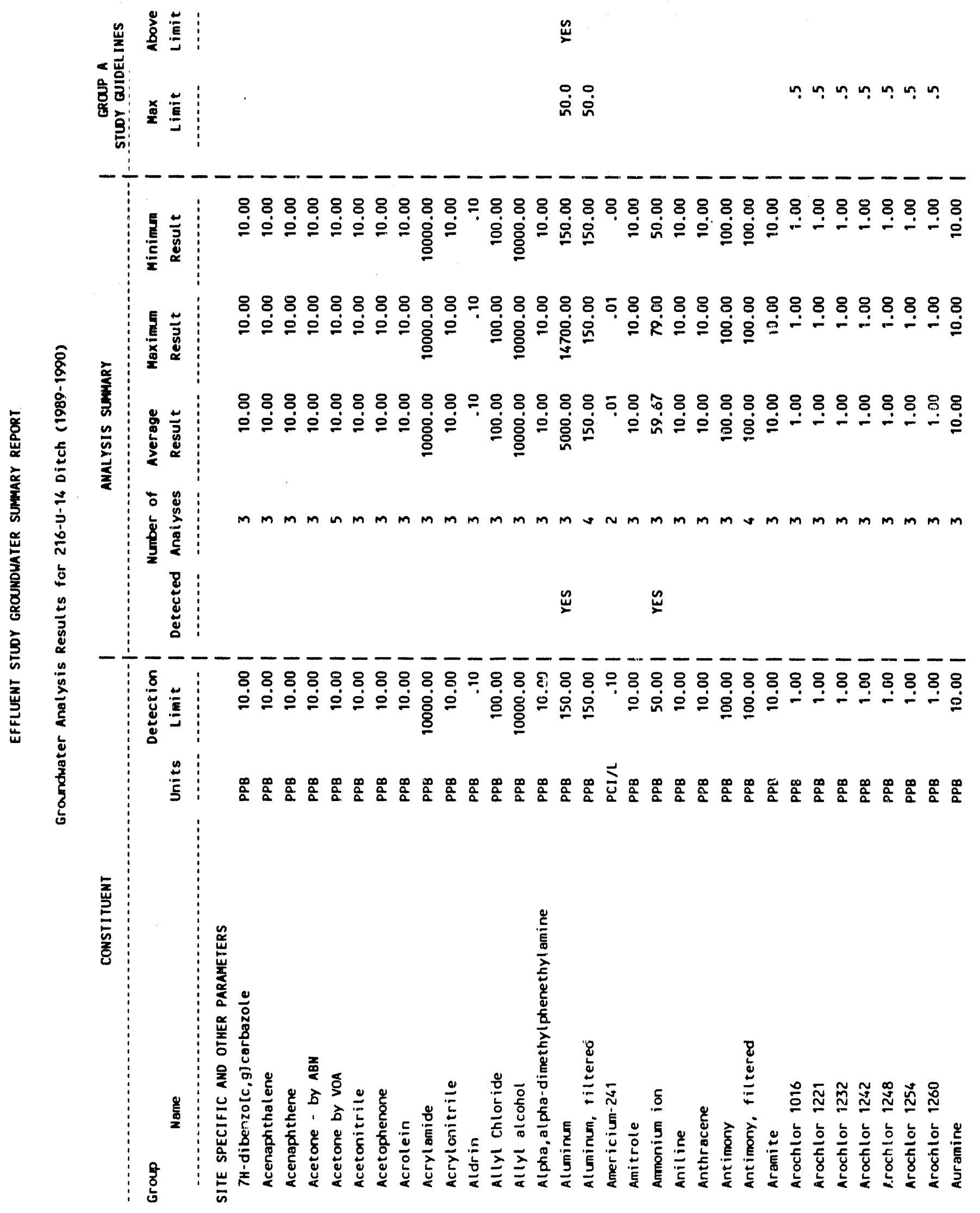


ชั้

<

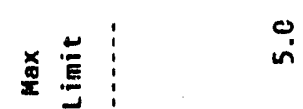

용

in

究

in

\section{$-\frac{1}{\vdots}$}

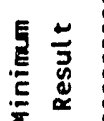

888888888080808088888888888080888 仓்

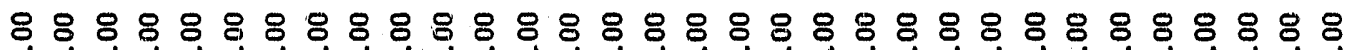

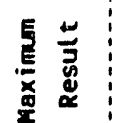

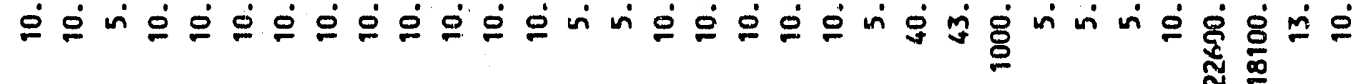

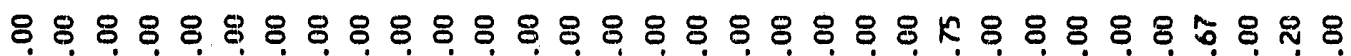

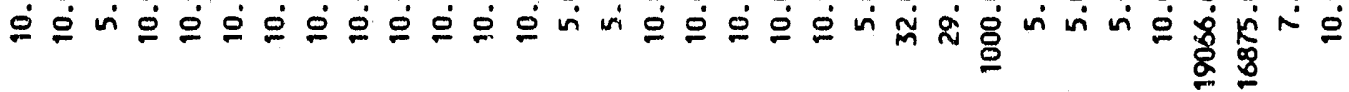

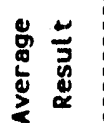

范

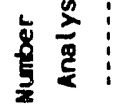

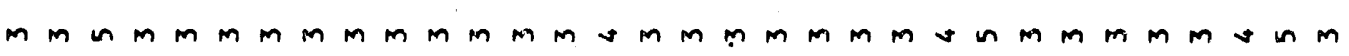

䓌

$\stackrel{\mathscr{M}}{\dddot{M}}$

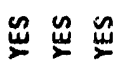

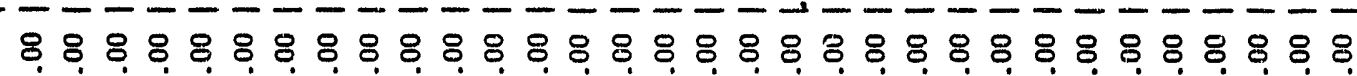

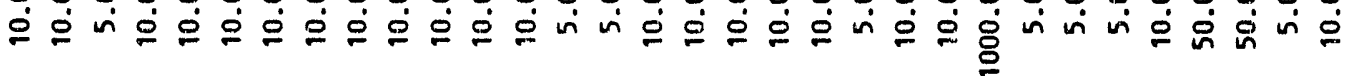

商

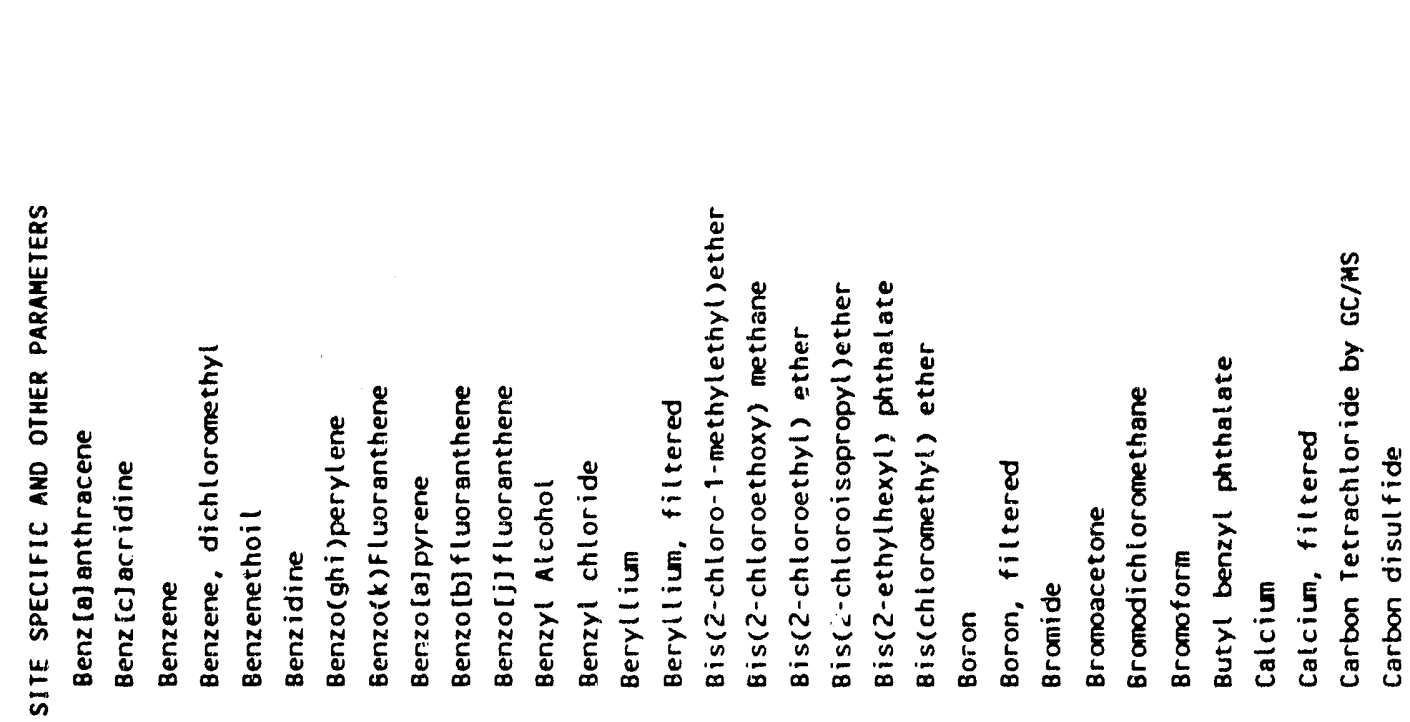




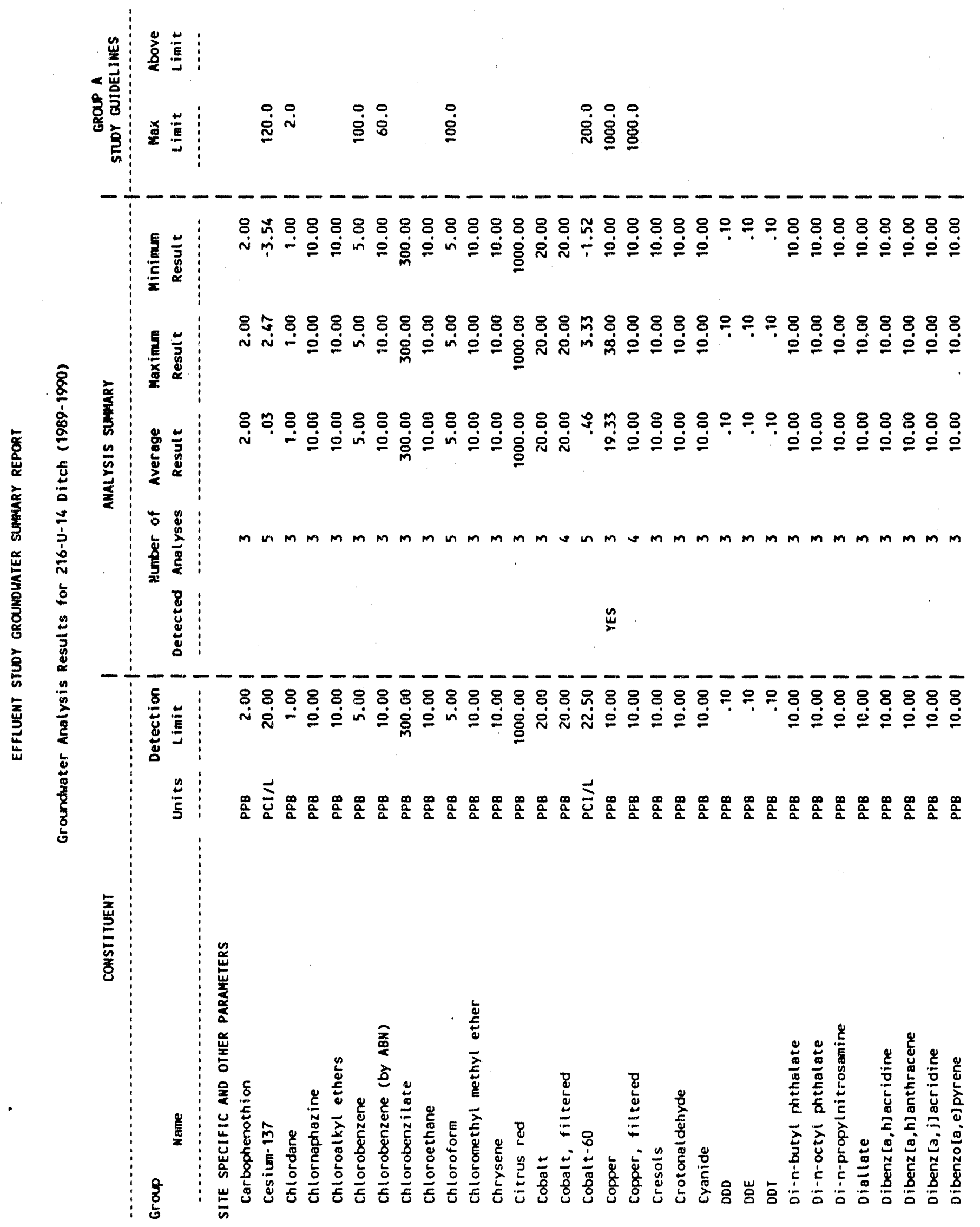




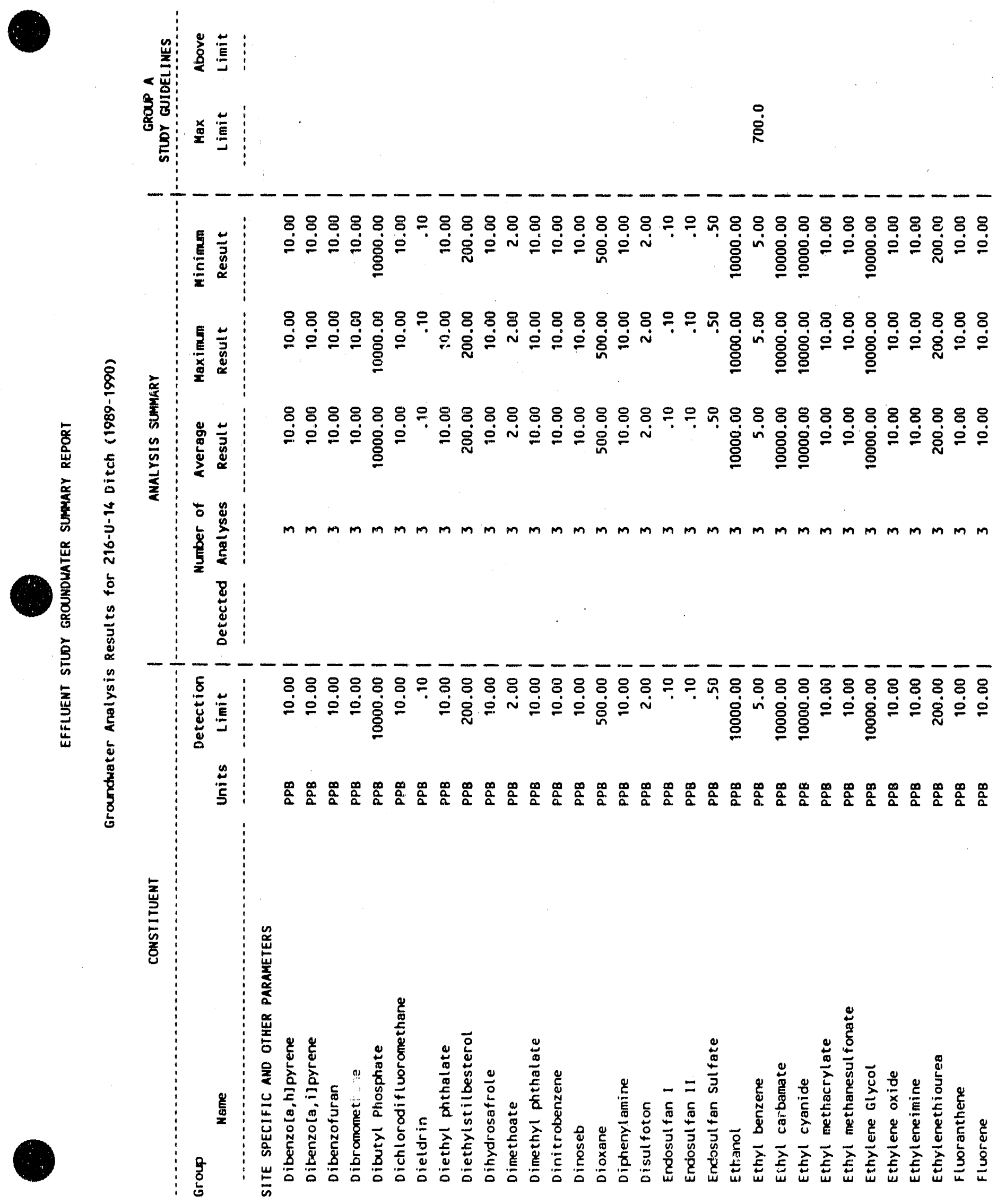




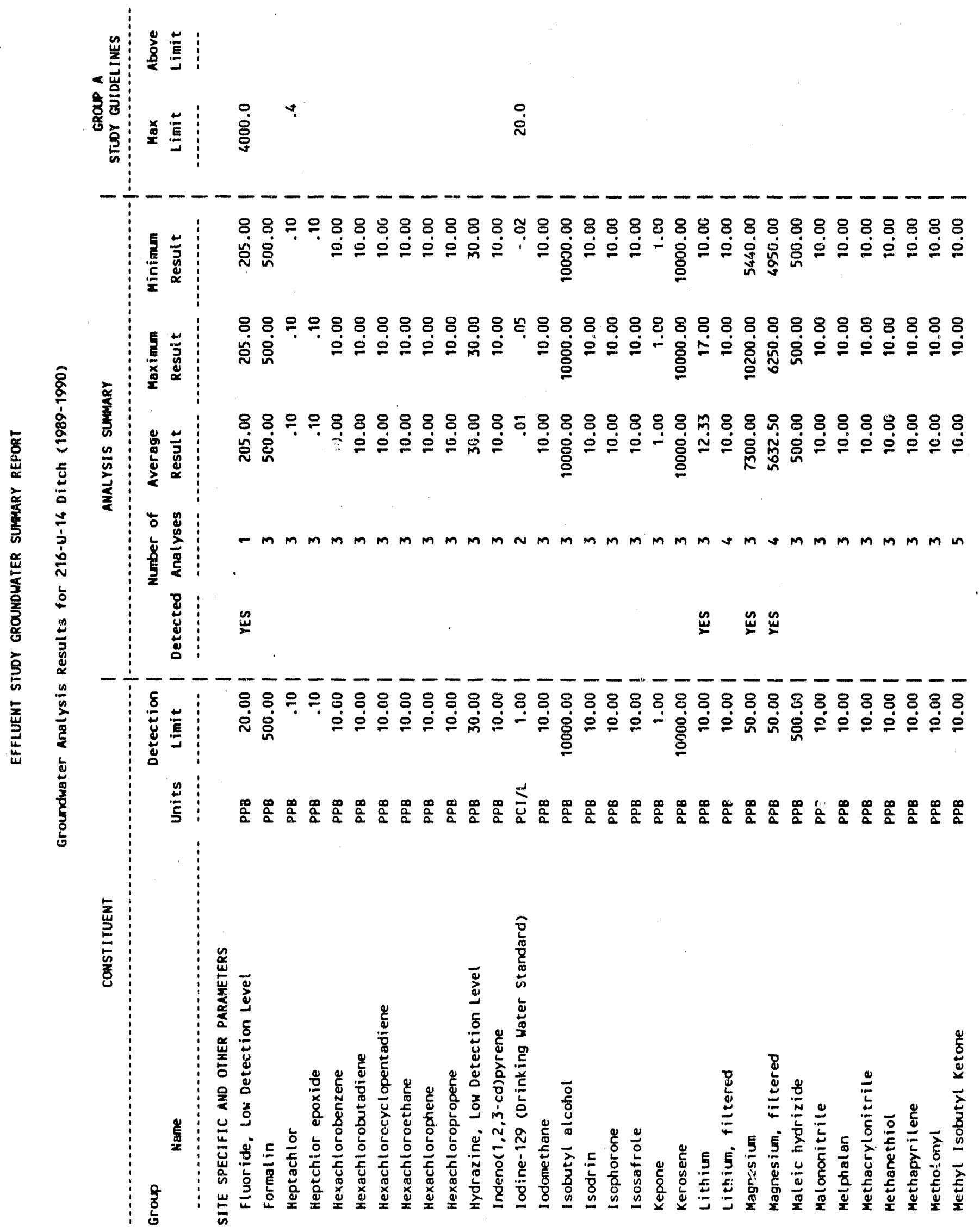


莫

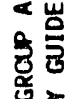

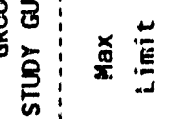

in

品

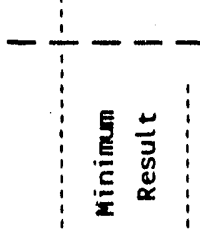

88888888888888888888880888088888

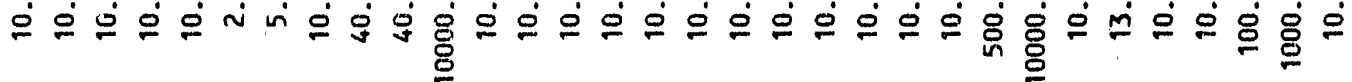

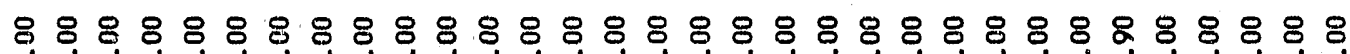

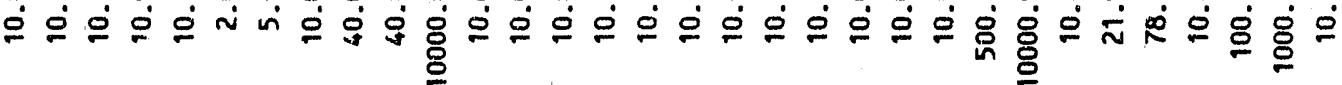

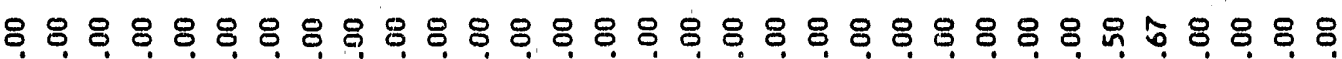

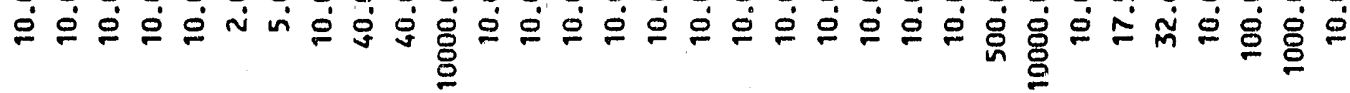

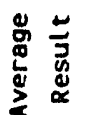

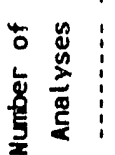

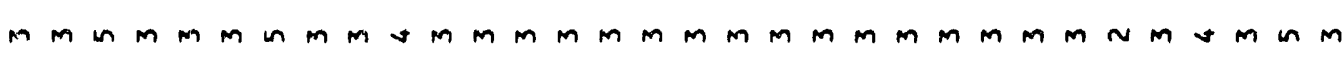

:

:

$\stackrel{\mathscr{w}}{\stackrel{\leftrightarrow}{x}}$

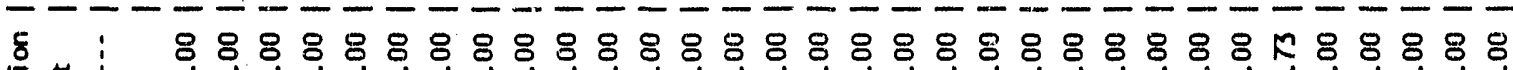

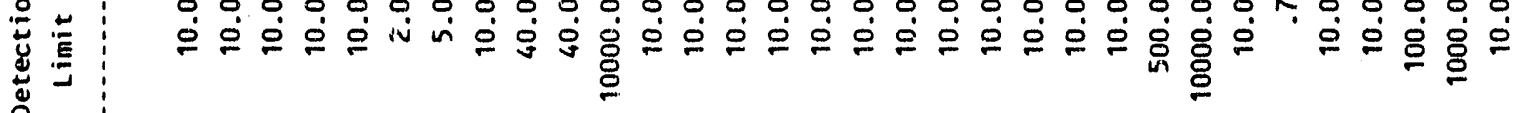
造

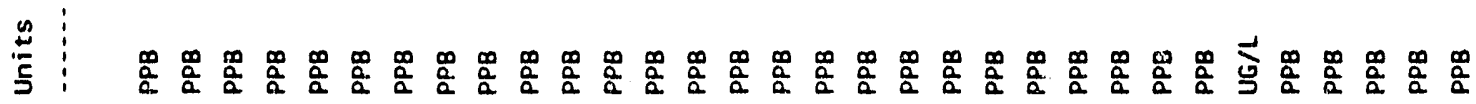

㺼

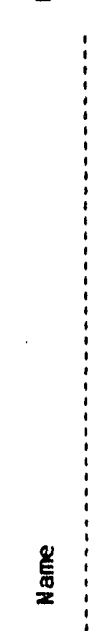

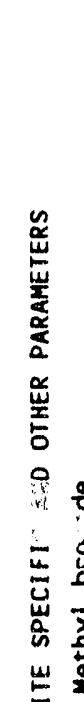

0
0

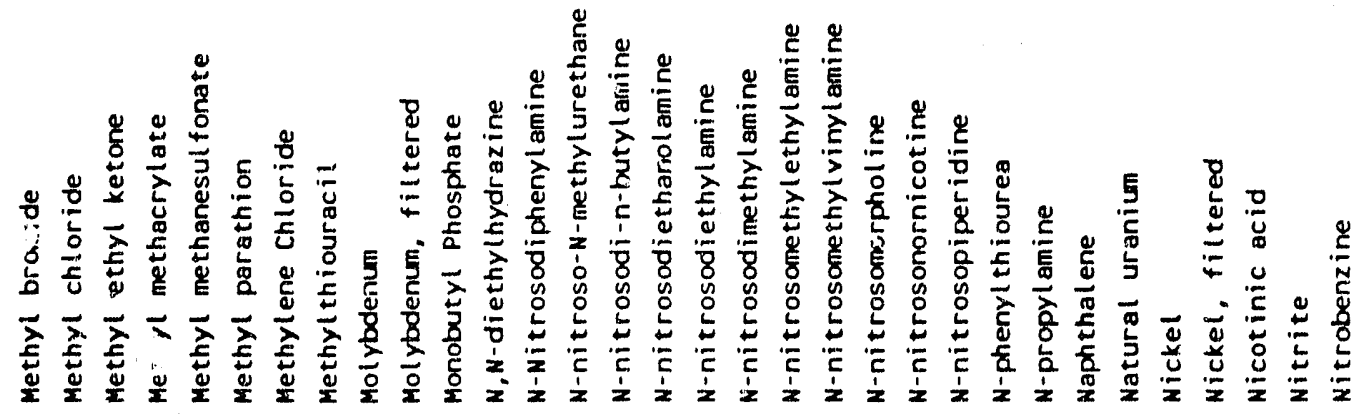




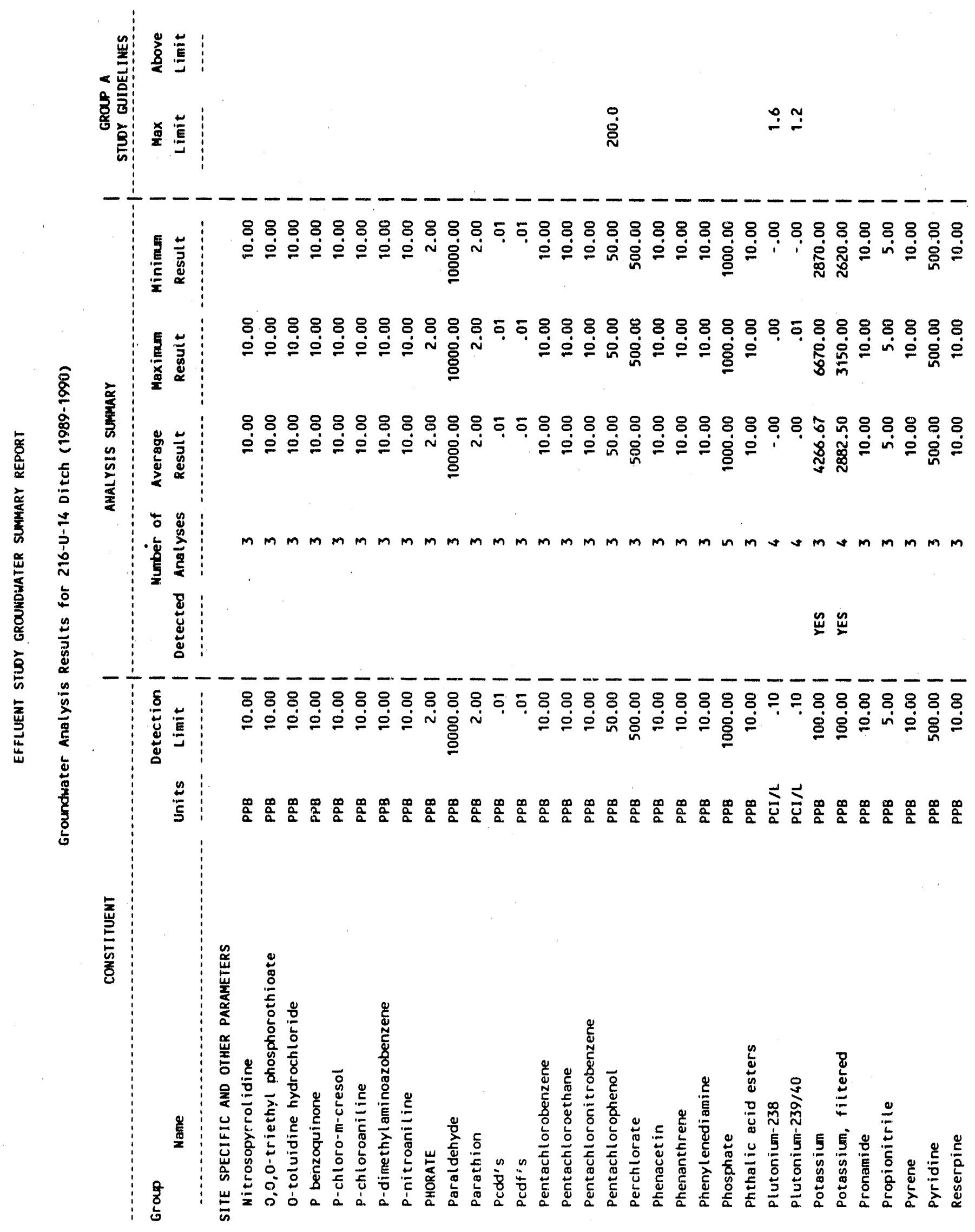


WHC-EP-0366

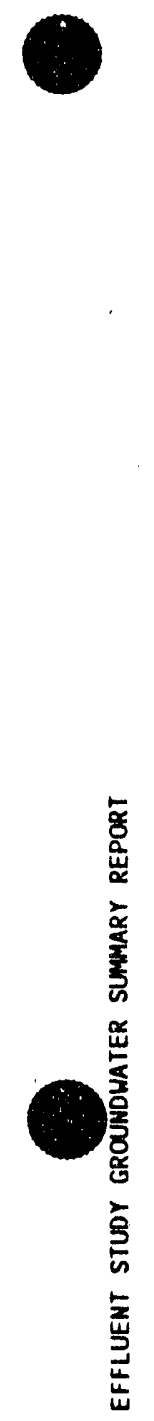

资:

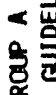

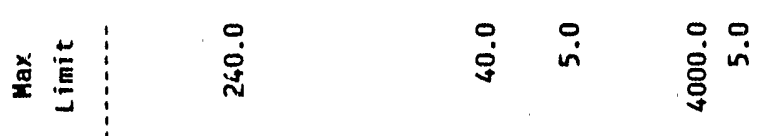

$a^{2}:$

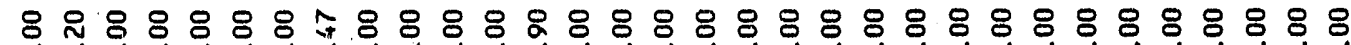

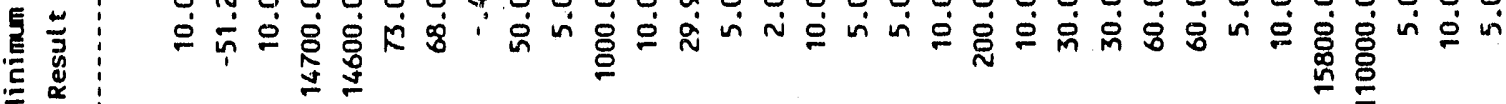

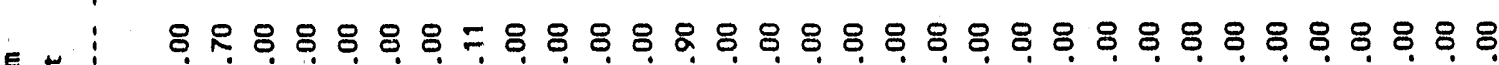

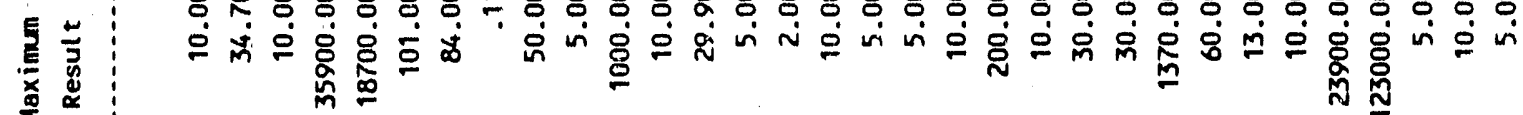

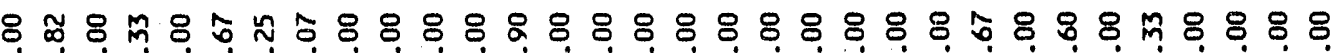

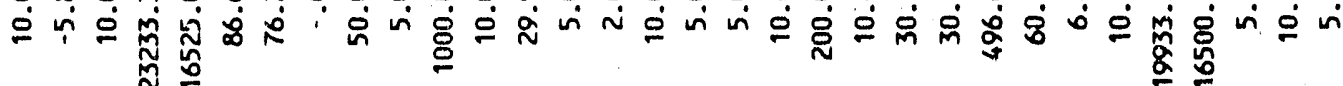

苋苛

to

焉

苞:

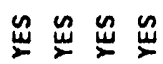

$\stackrel{\sharp}{\longleftarrow}$

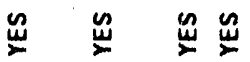

$-\frac{1}{i}$

总

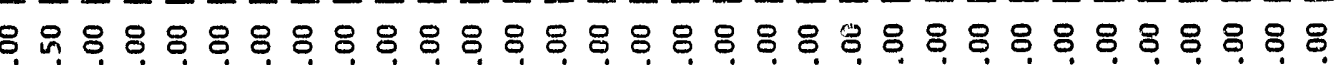

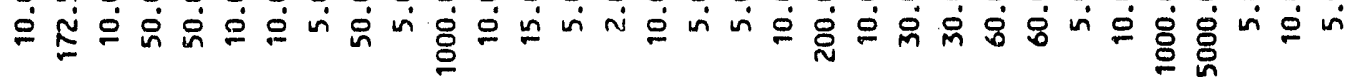

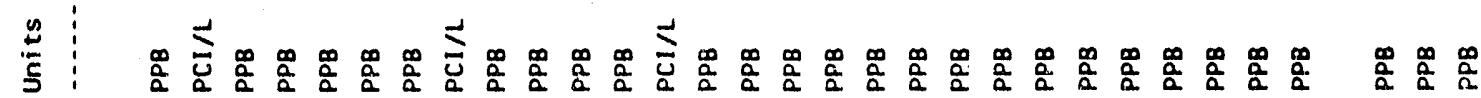

:

:

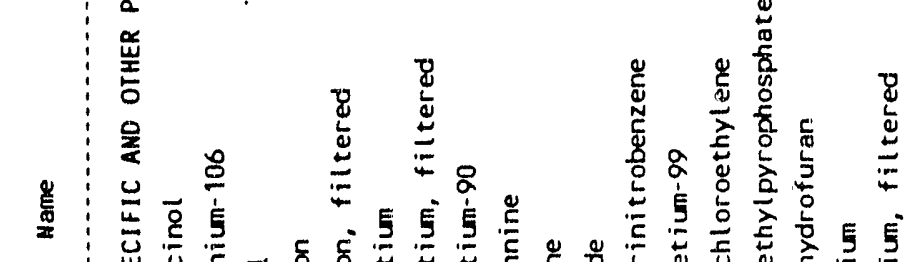

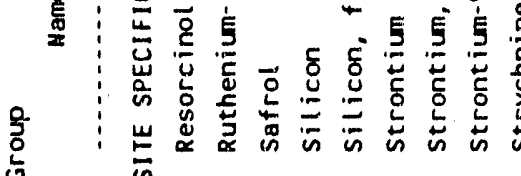

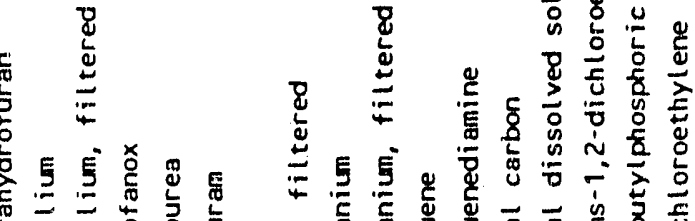

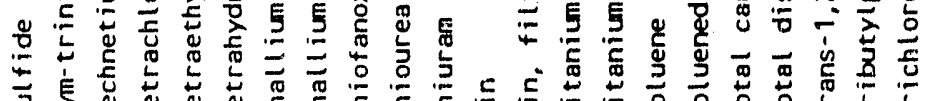


WHC-EP-0366

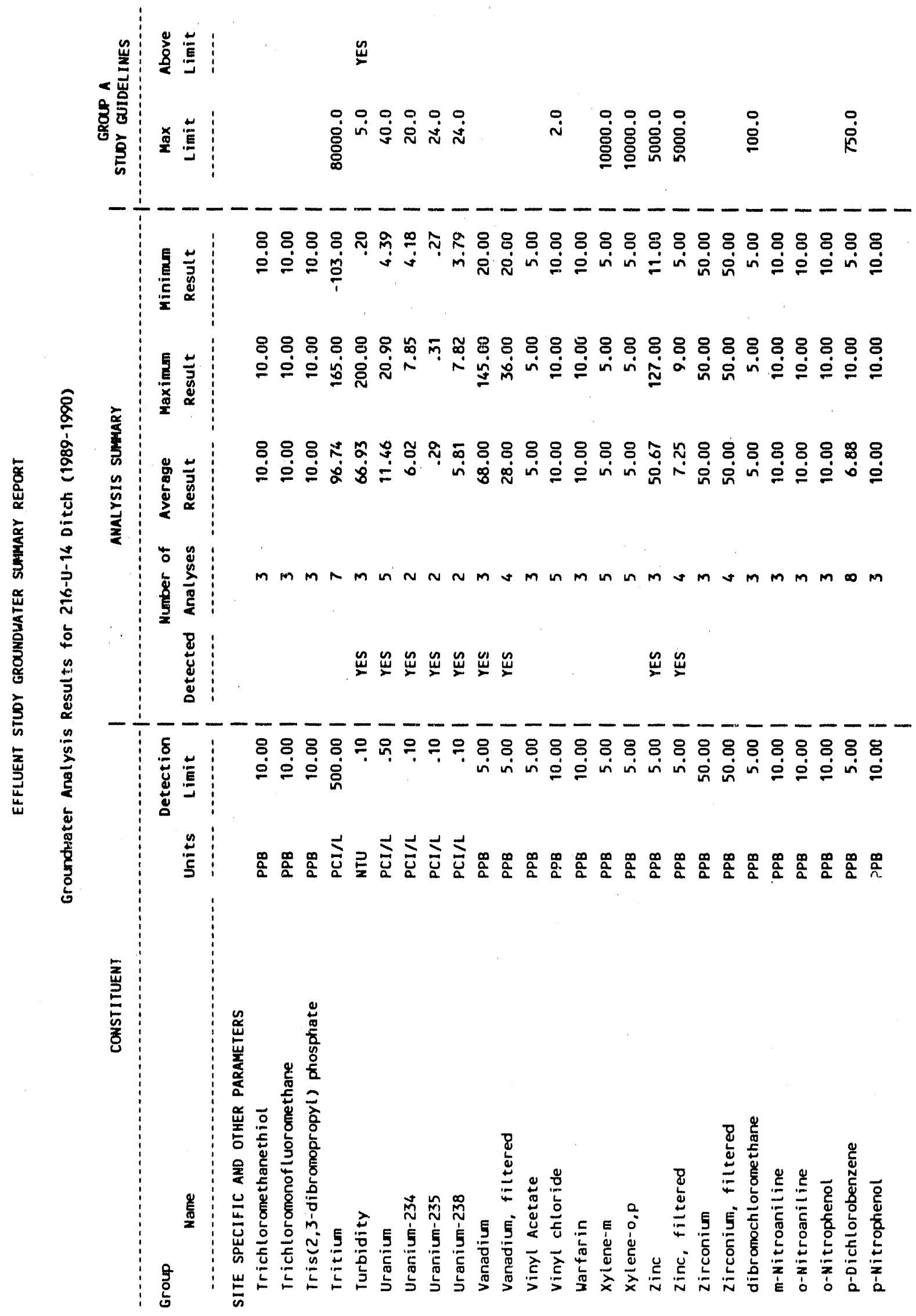




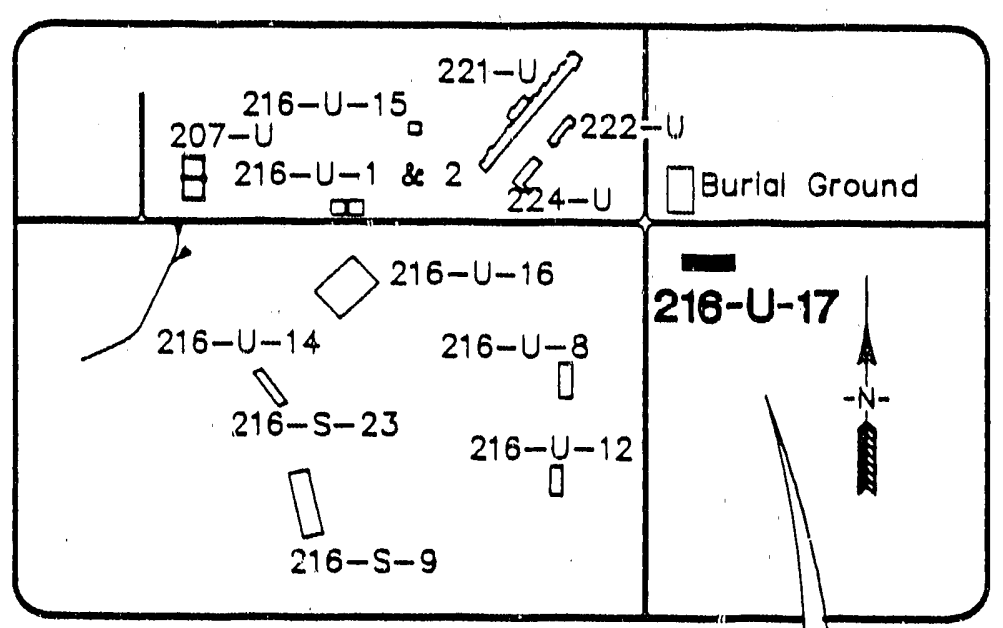

- Ground Water Monitoring Well (Pumped Somples)

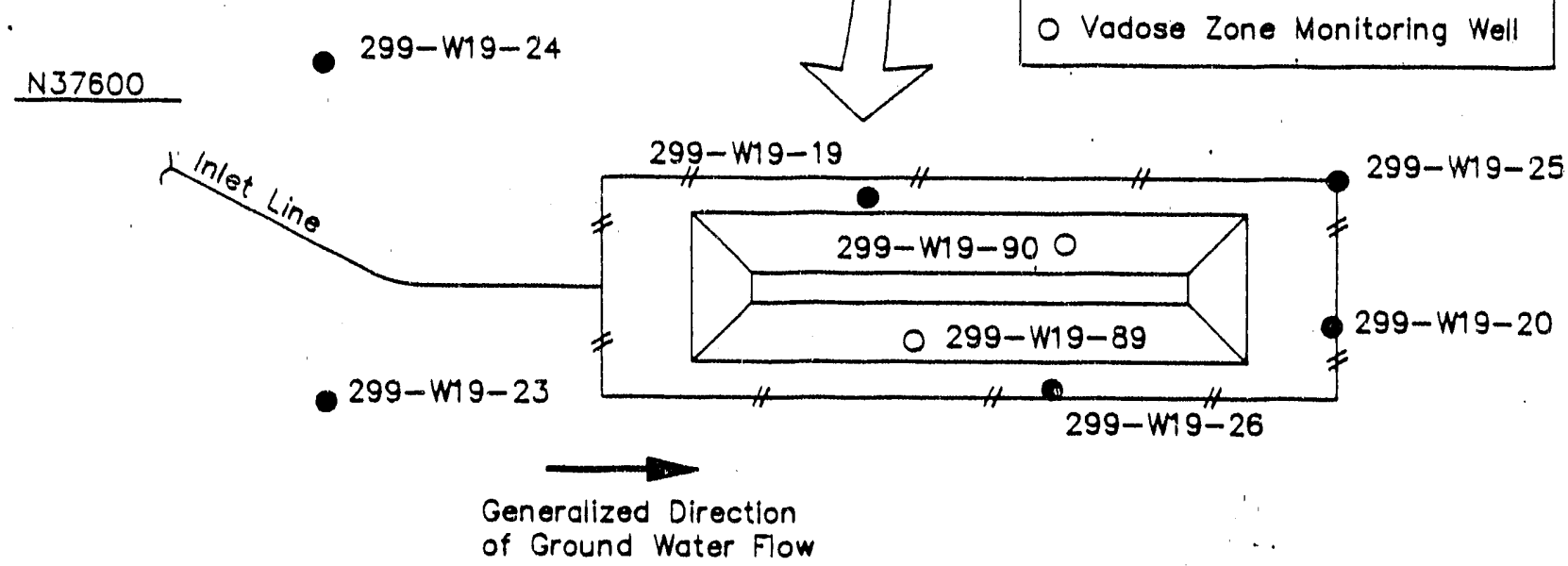

$\begin{array}{ll}N 37400 & 8 \\ \stackrel{8}{0} \\ \stackrel{1}{\xi}\end{array}$

$\frac{8}{8}$

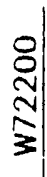

$A G L \backslash 216-U-17$

We11 Location and Site Map for 216-U-17 Crib 
WHC-EP-0366

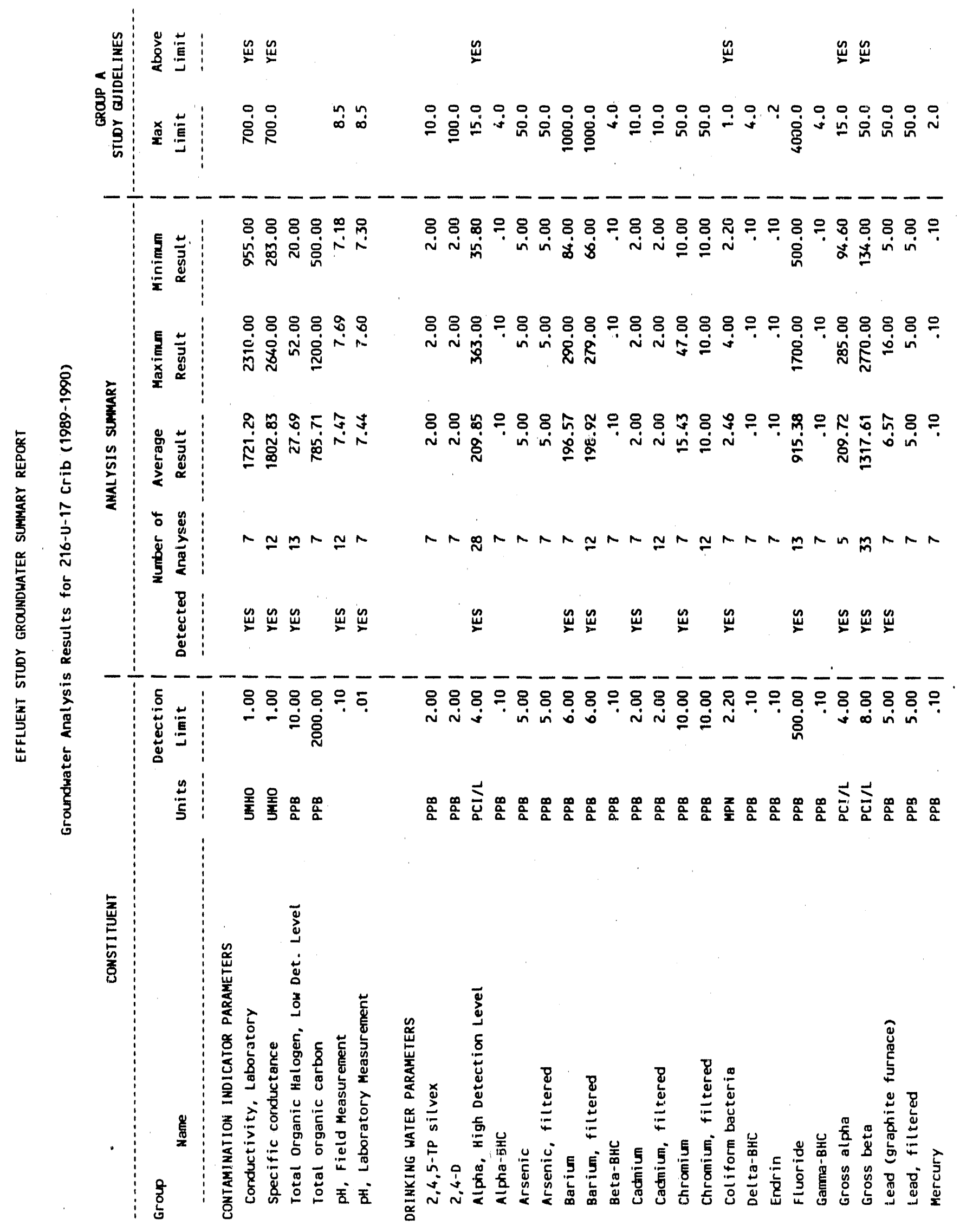




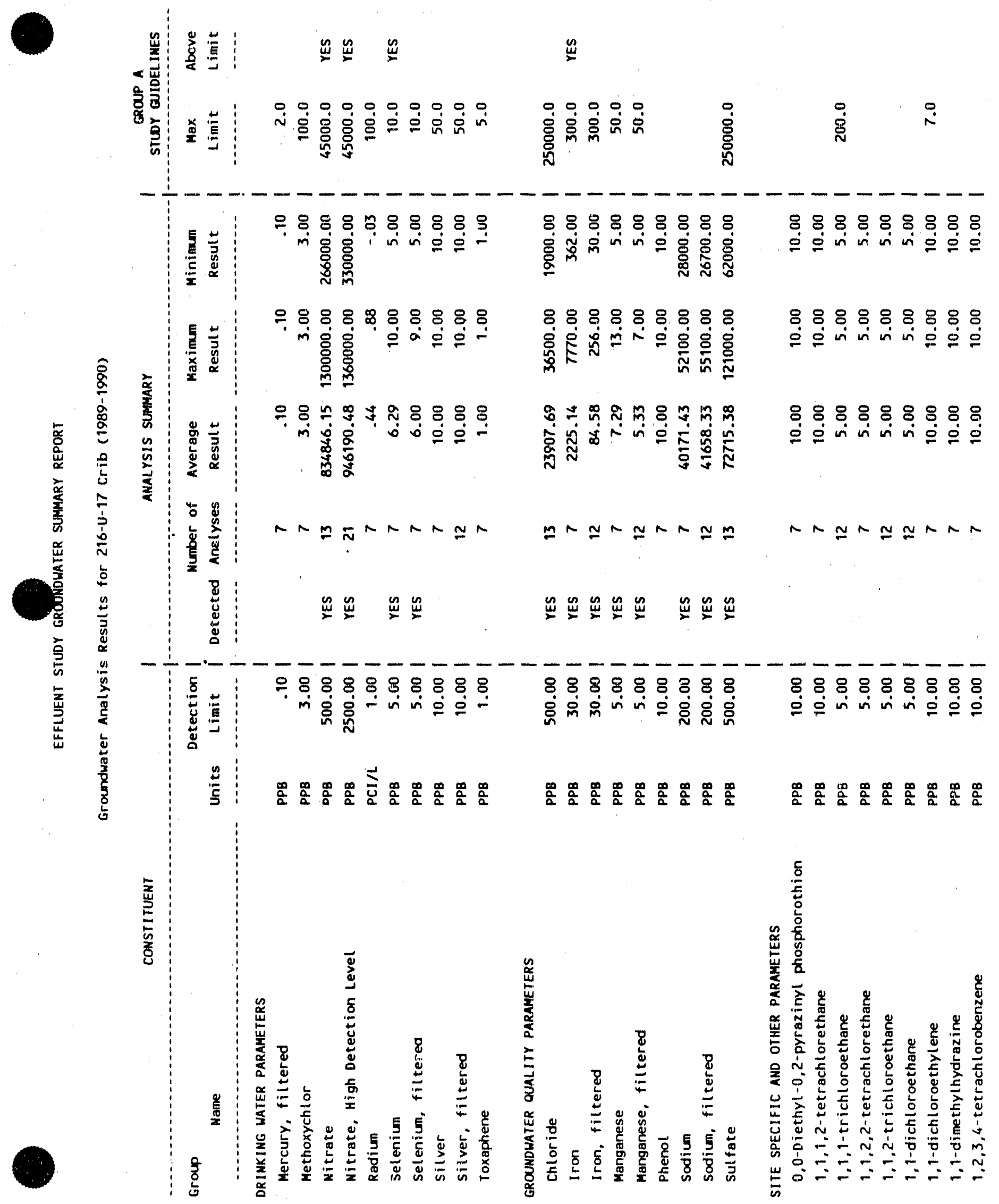


WHC-EP-0366
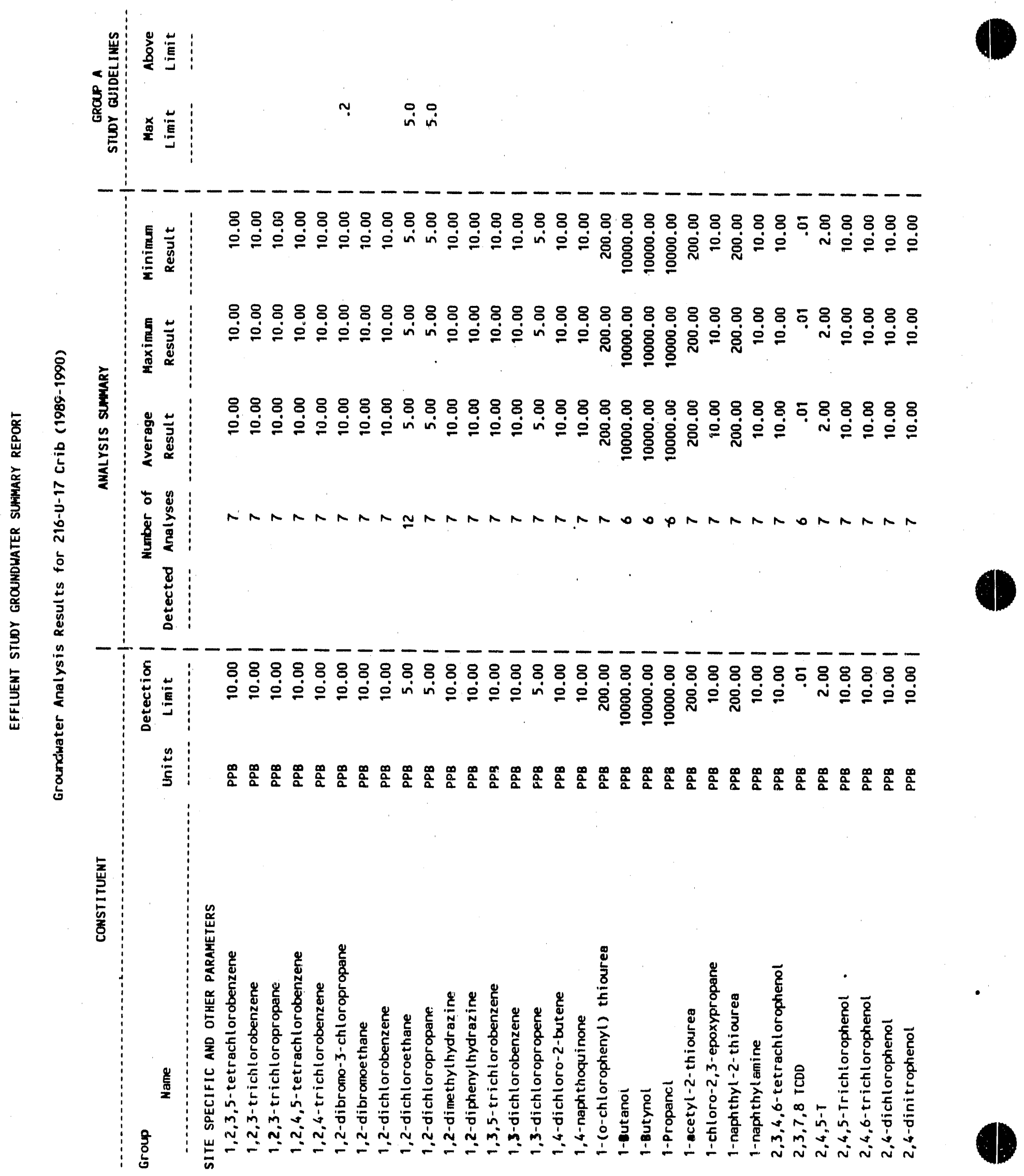
WHC-EP-0366

管旁:

象新

鰙

88888888888888888880888888888888

焉营

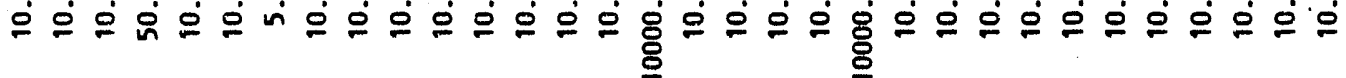

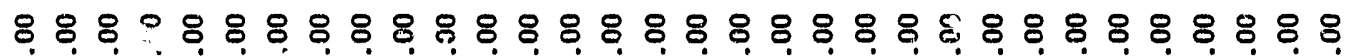

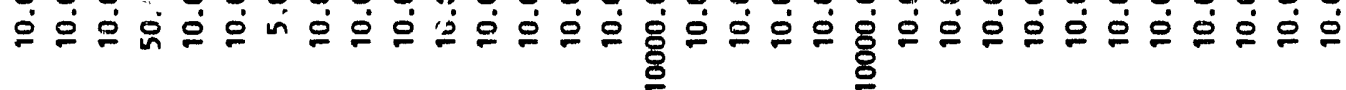

8888888888888888888888888888888

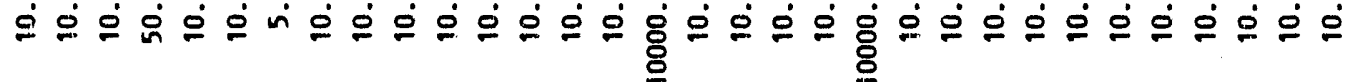

峞

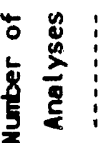

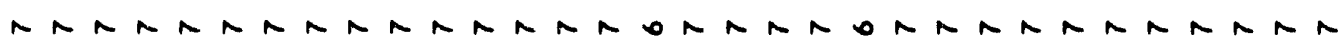

8

悹

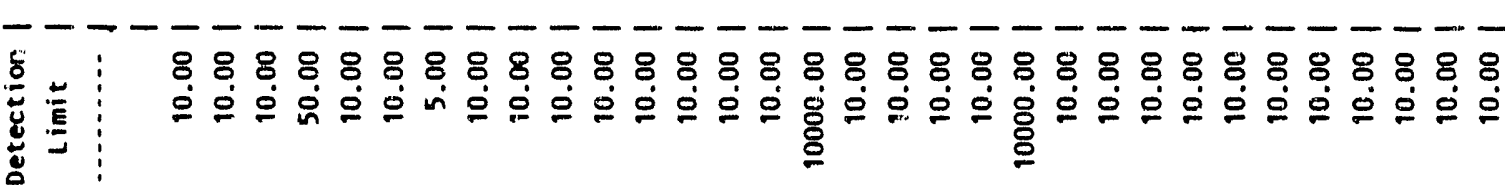

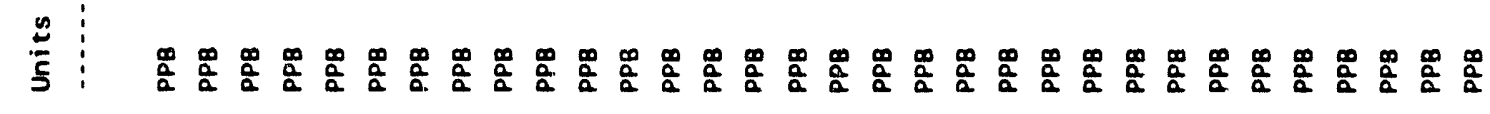

$$
\text { 氧 }
$$
(1)

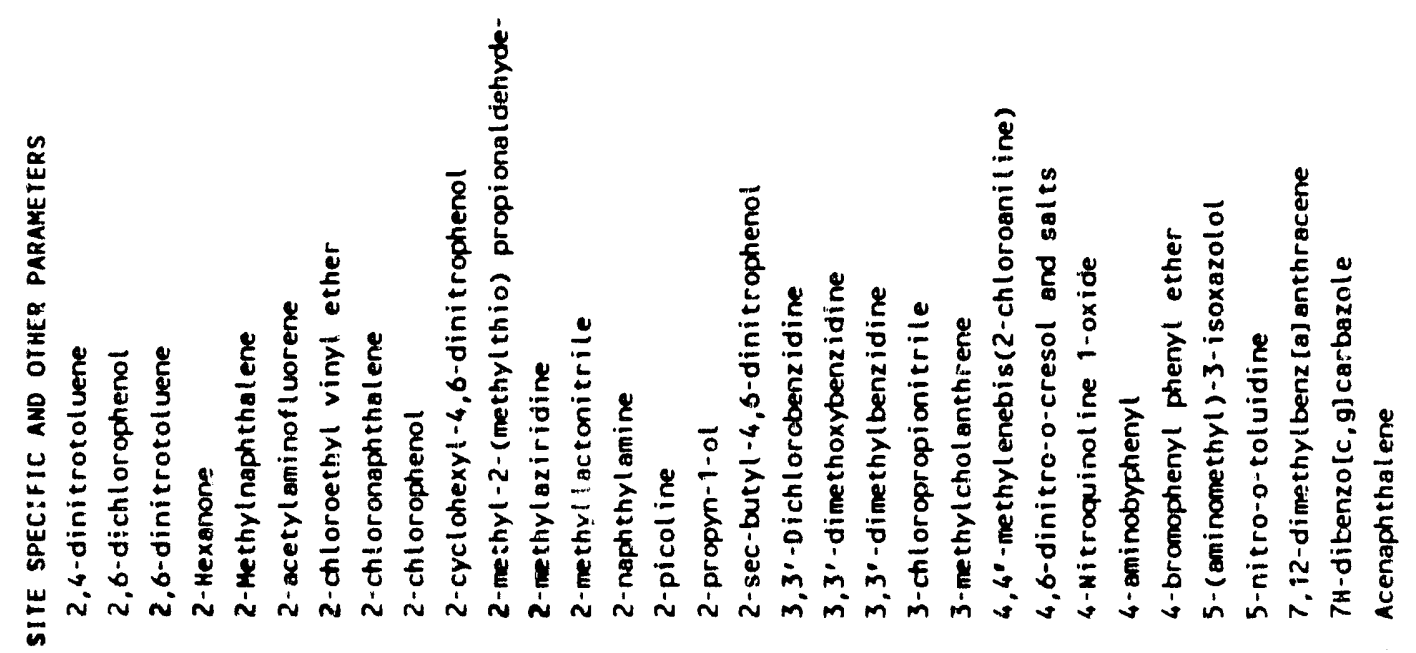




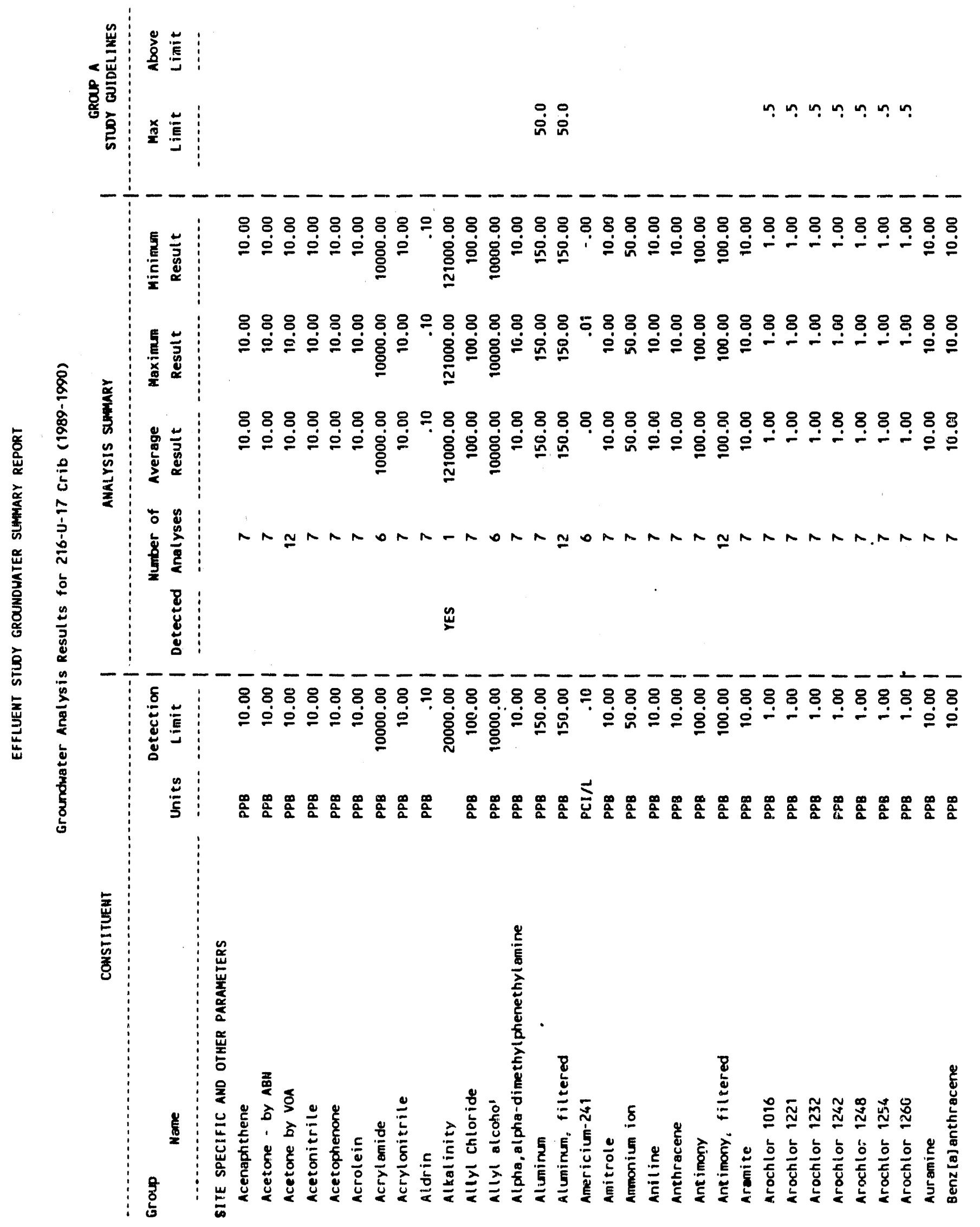



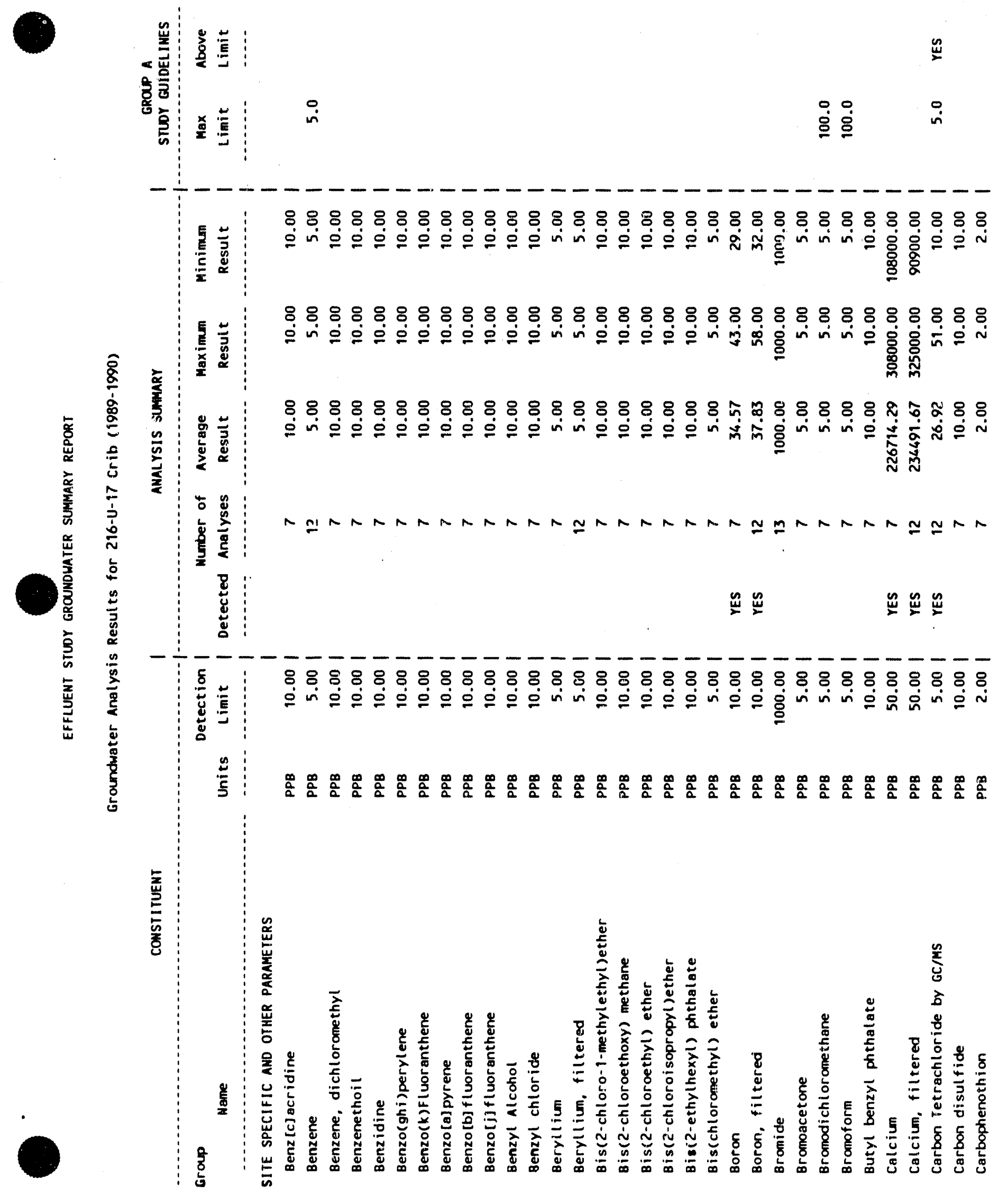

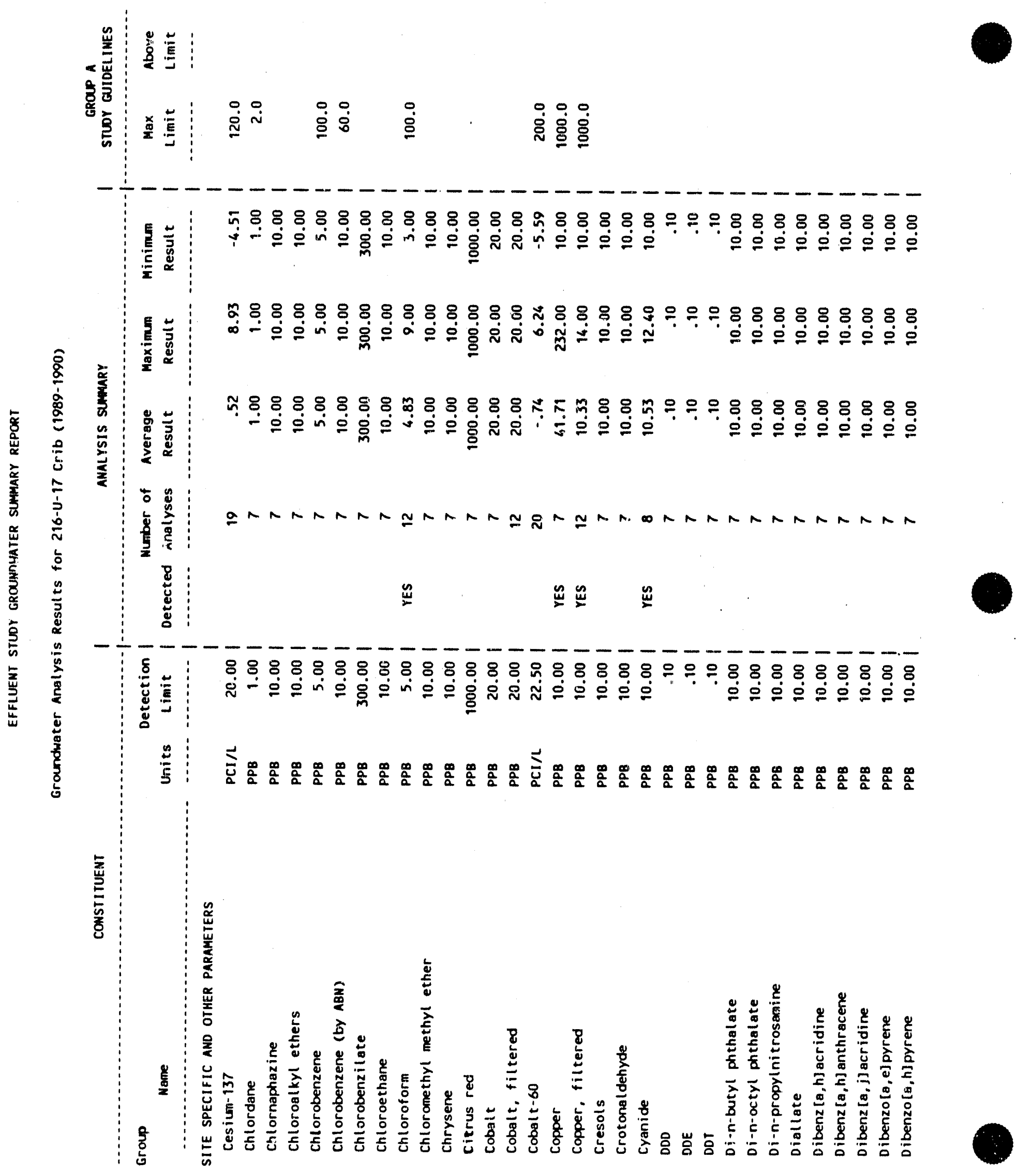


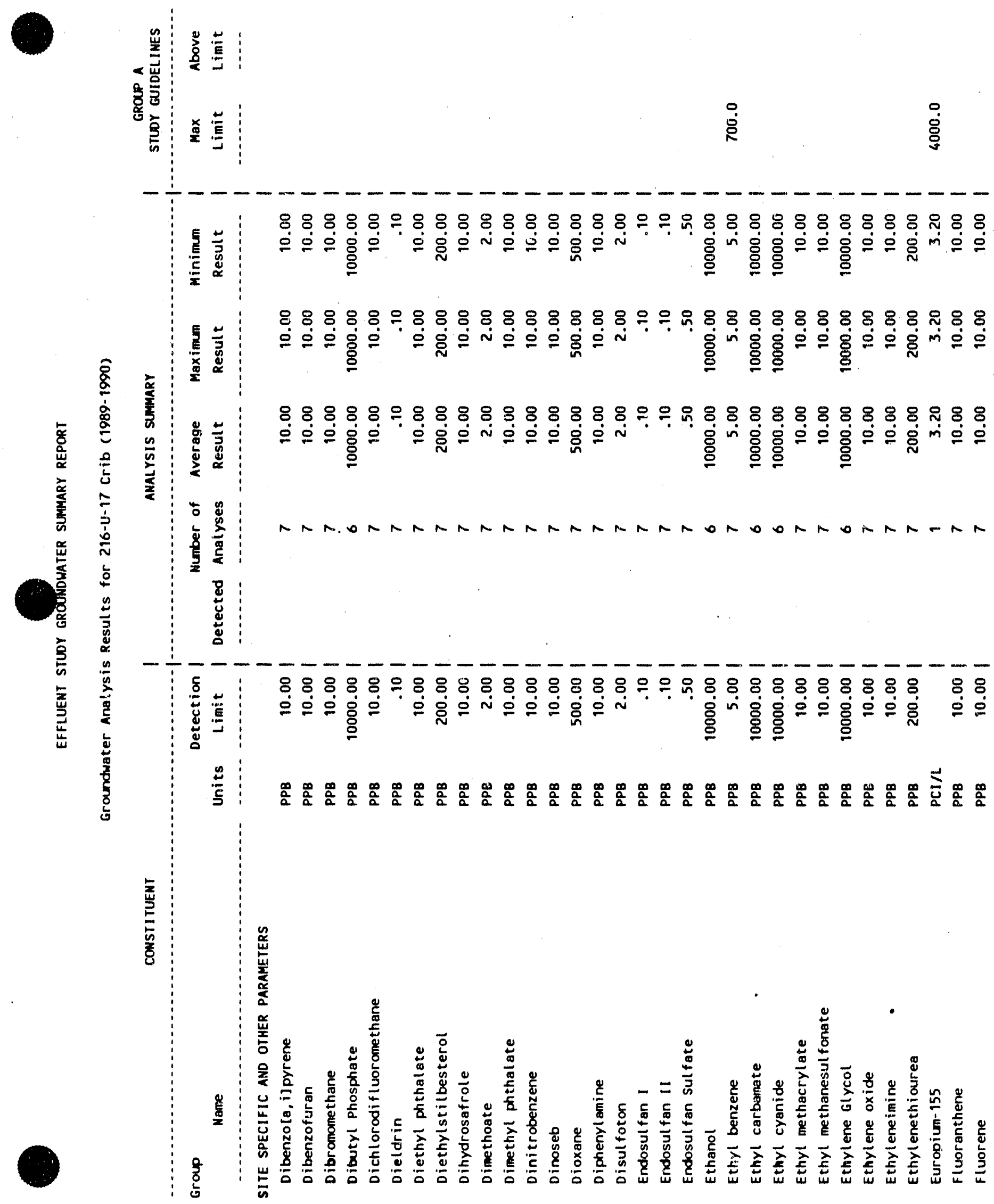




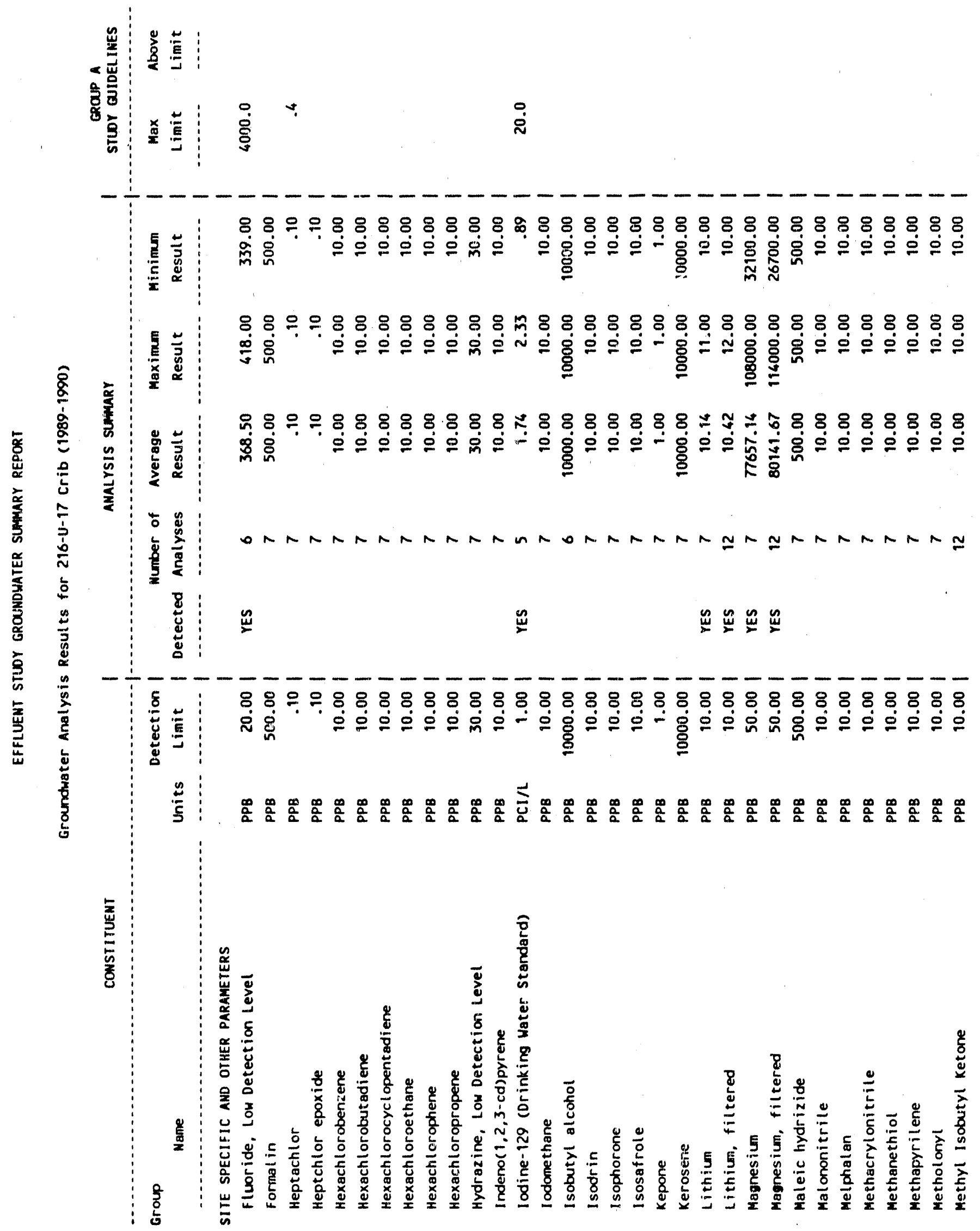




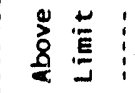

客完

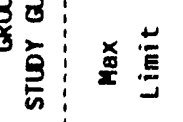

宊 官

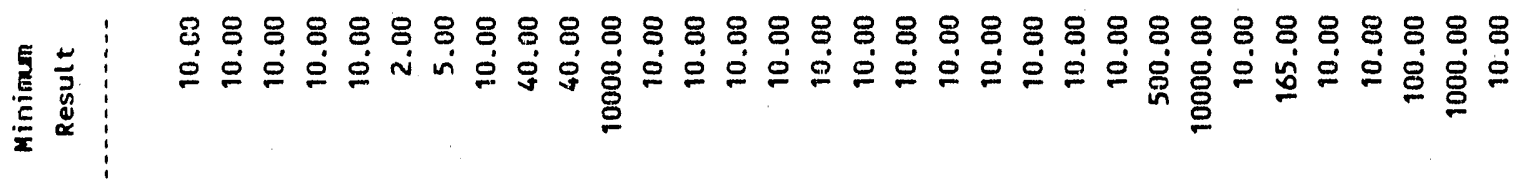

5 8888888888888888888888888888888

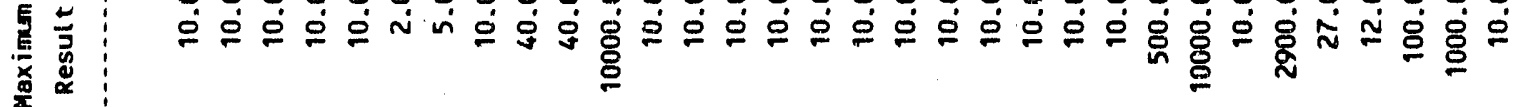

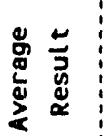

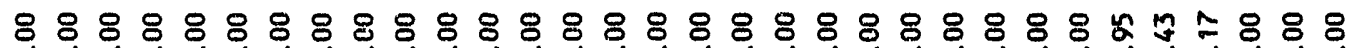
ㅇㅇㅇㅁ 它尊 2

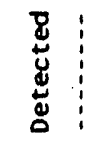

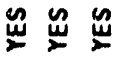

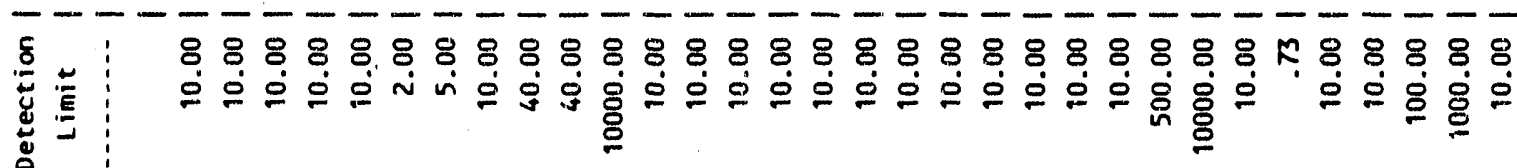

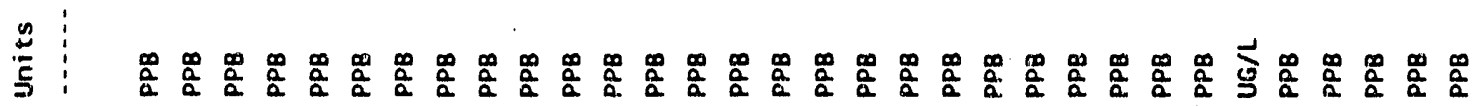




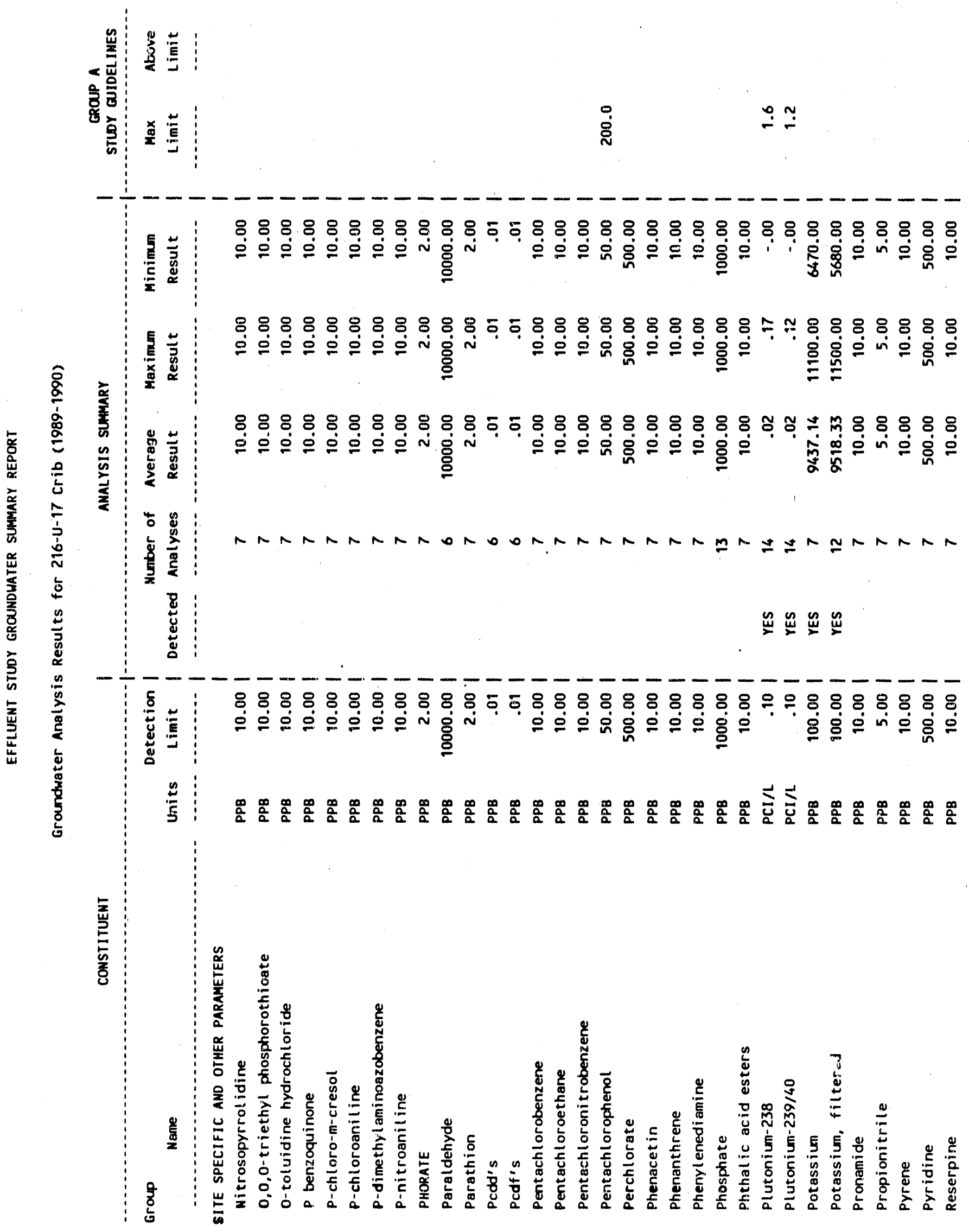


WHC-EP-0366

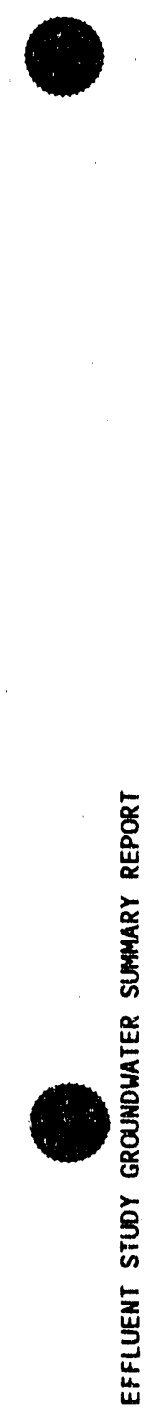

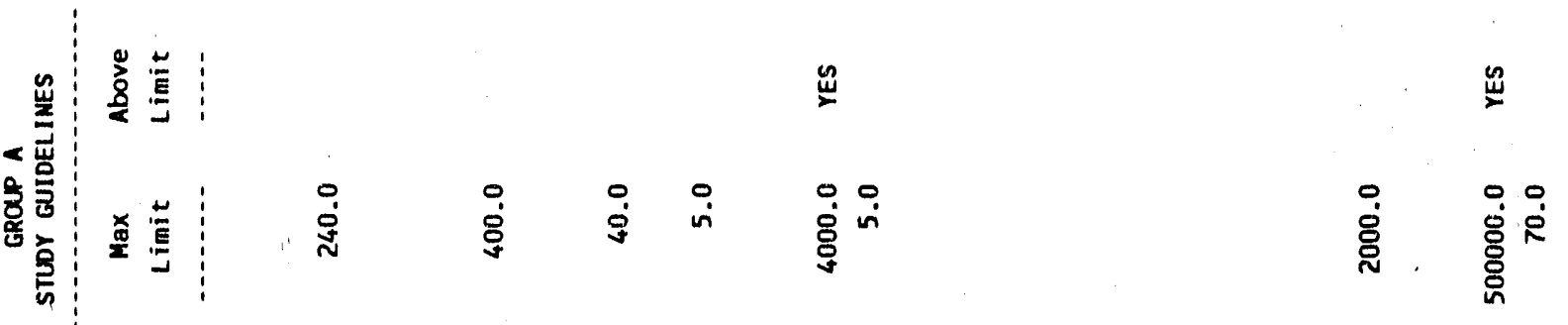

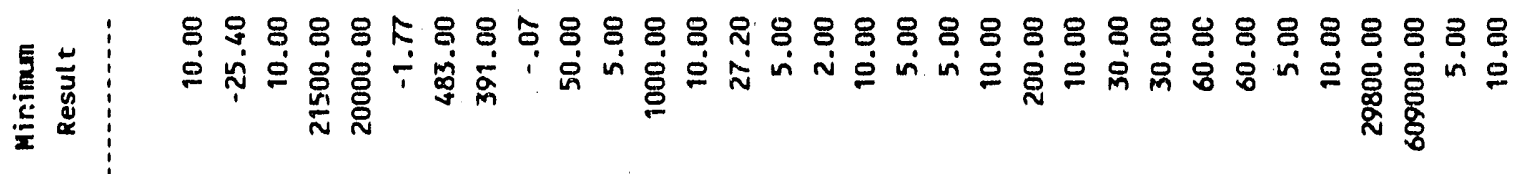

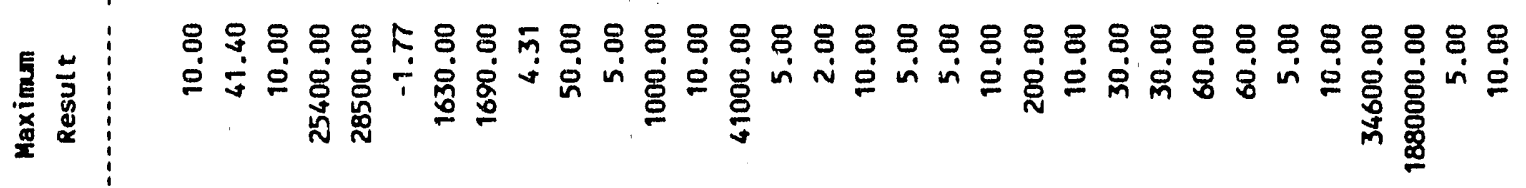

兽言

复:

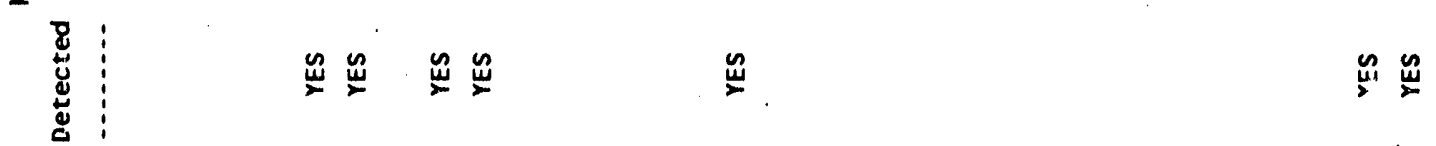

遥

突 $\quad$ 焉

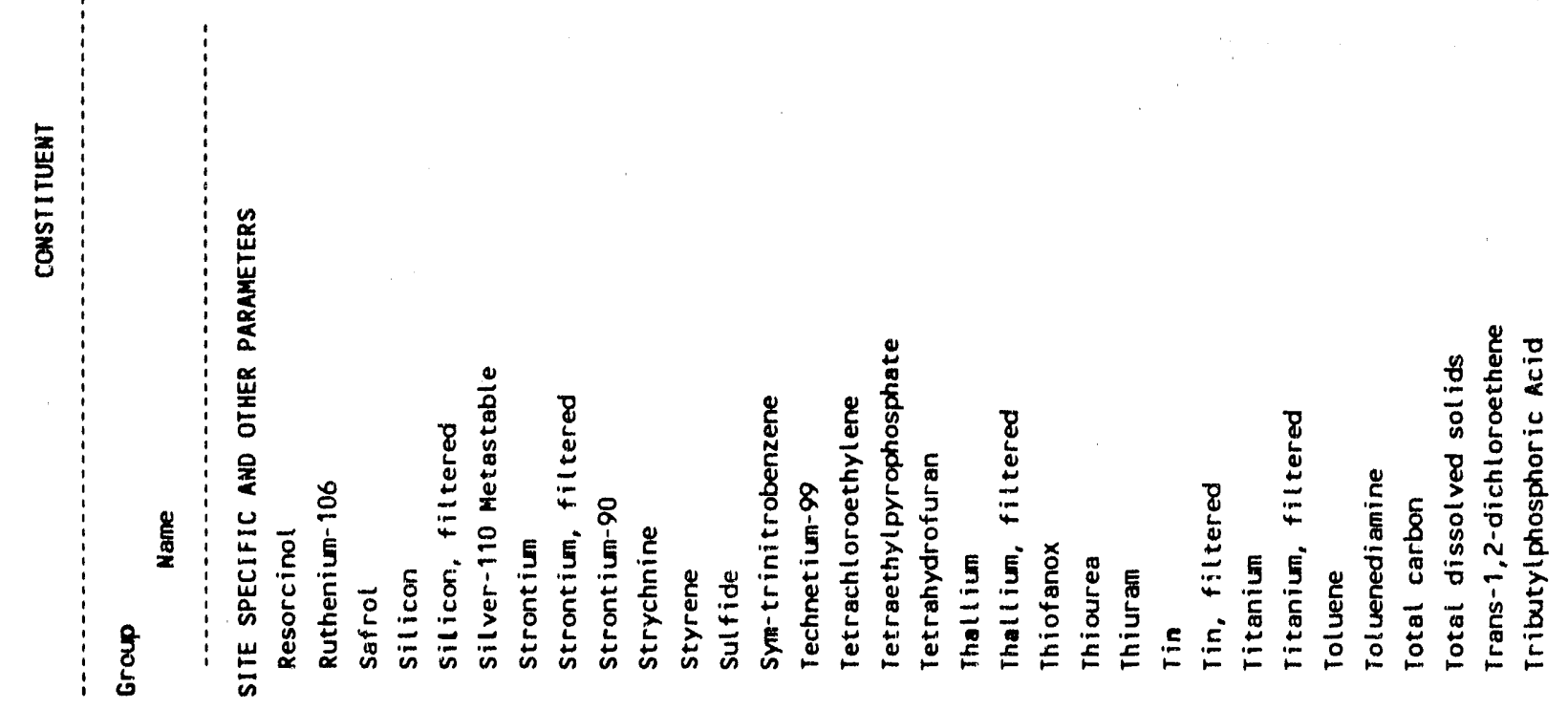




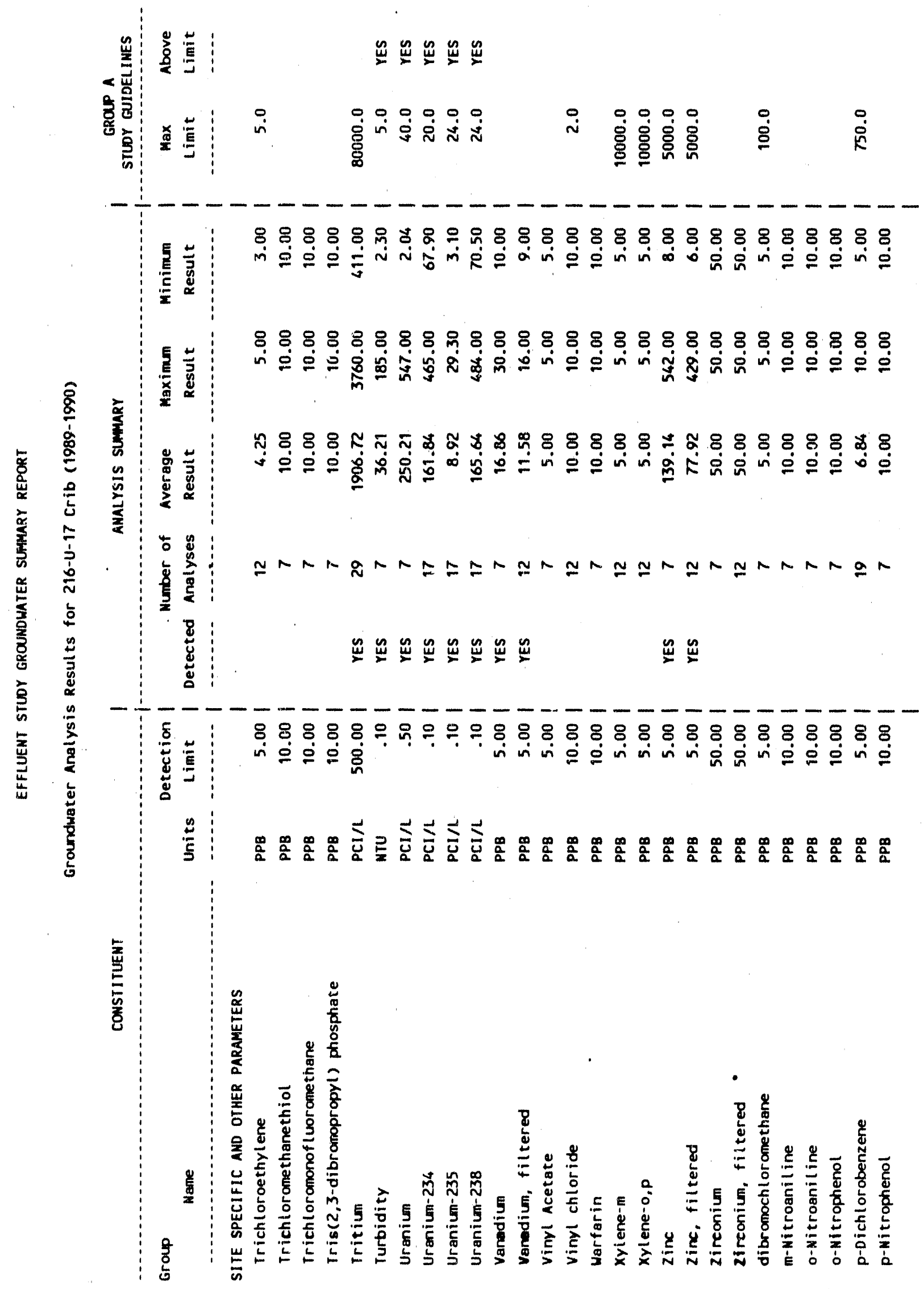


WHC-EP-0366

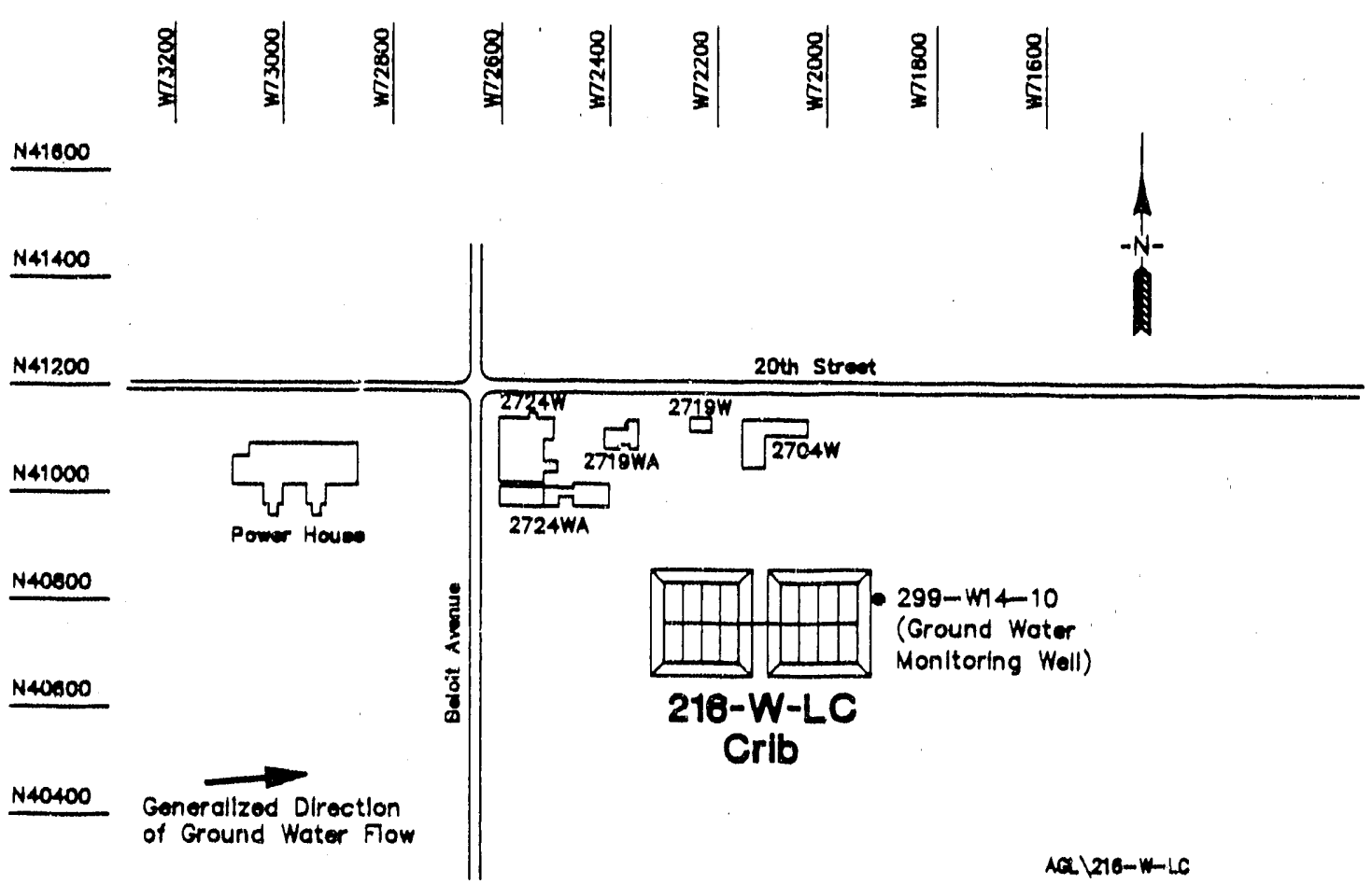

We 11 Location and Site Map for 216-W-LC Crib 
WHC -EP-0366

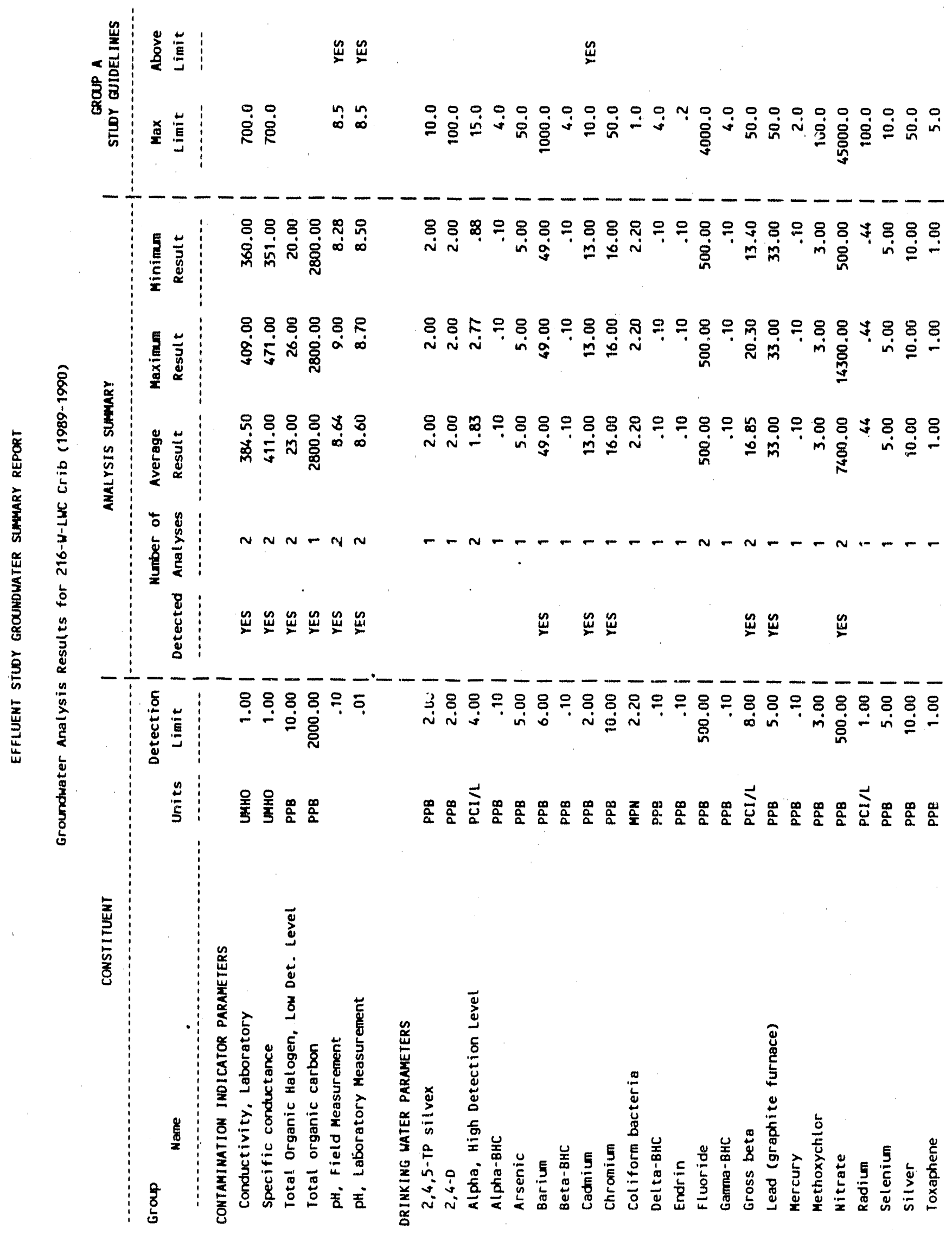


WHC-EP-0366

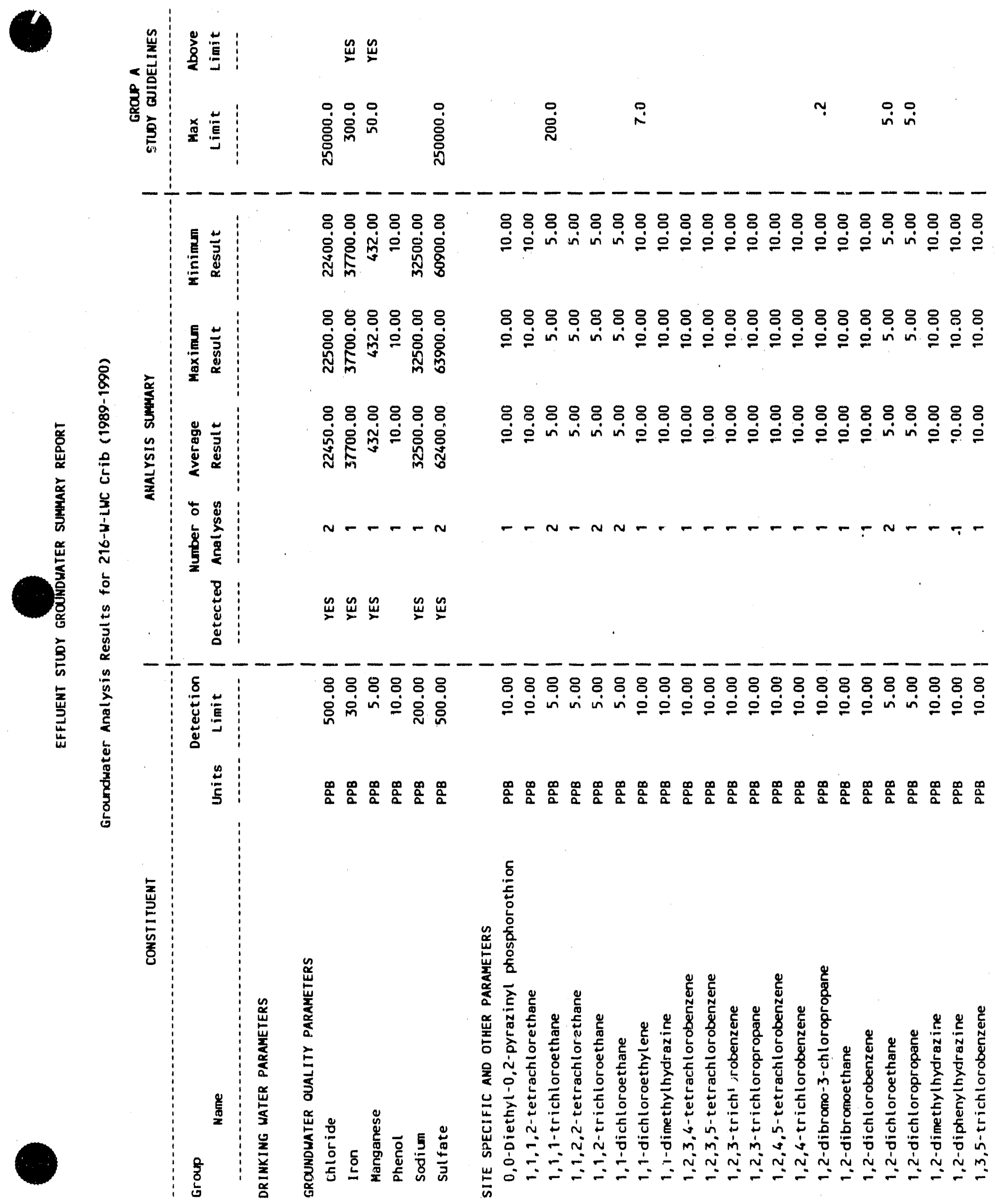


WHC-EP-0366

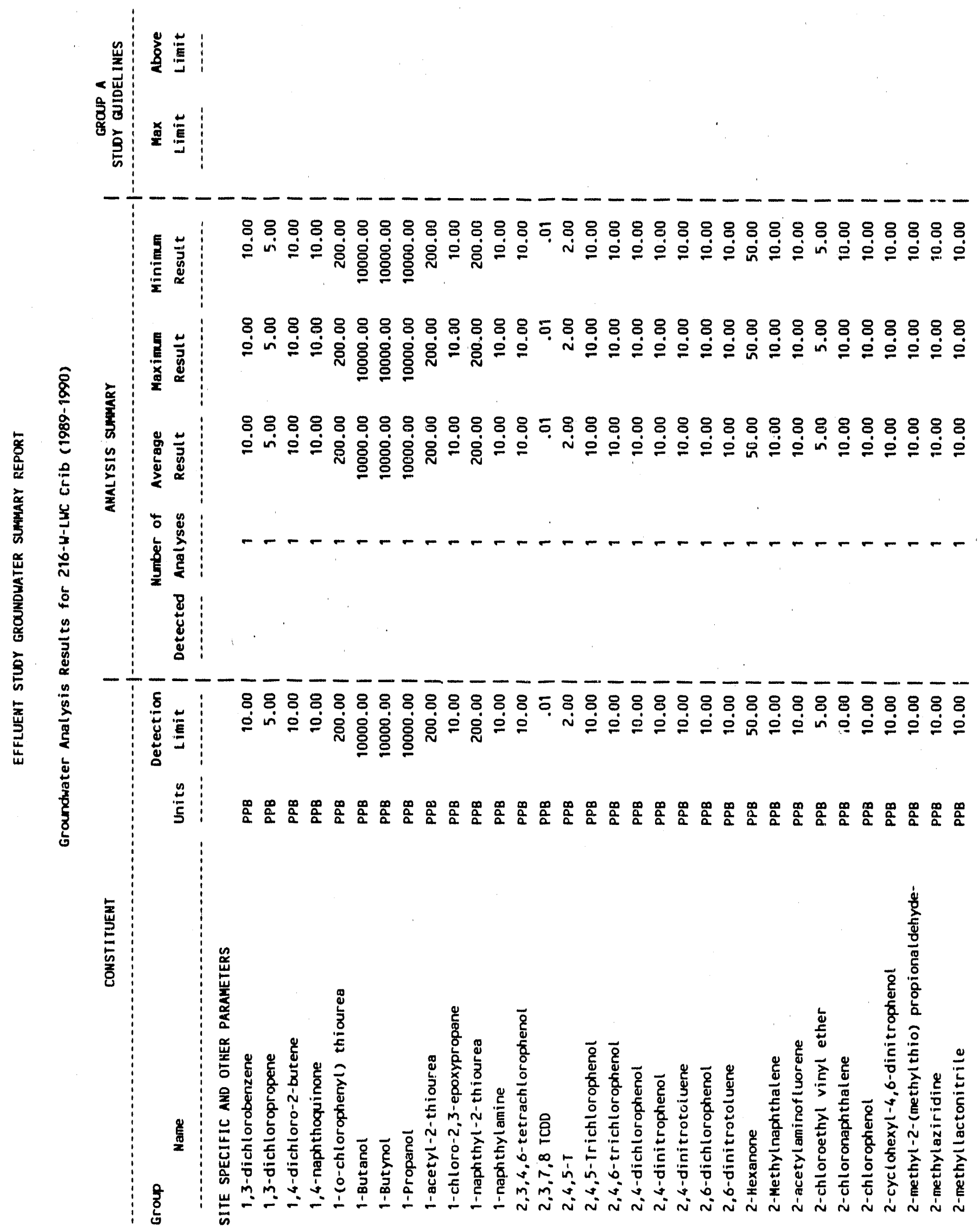


WHC-EP-0366

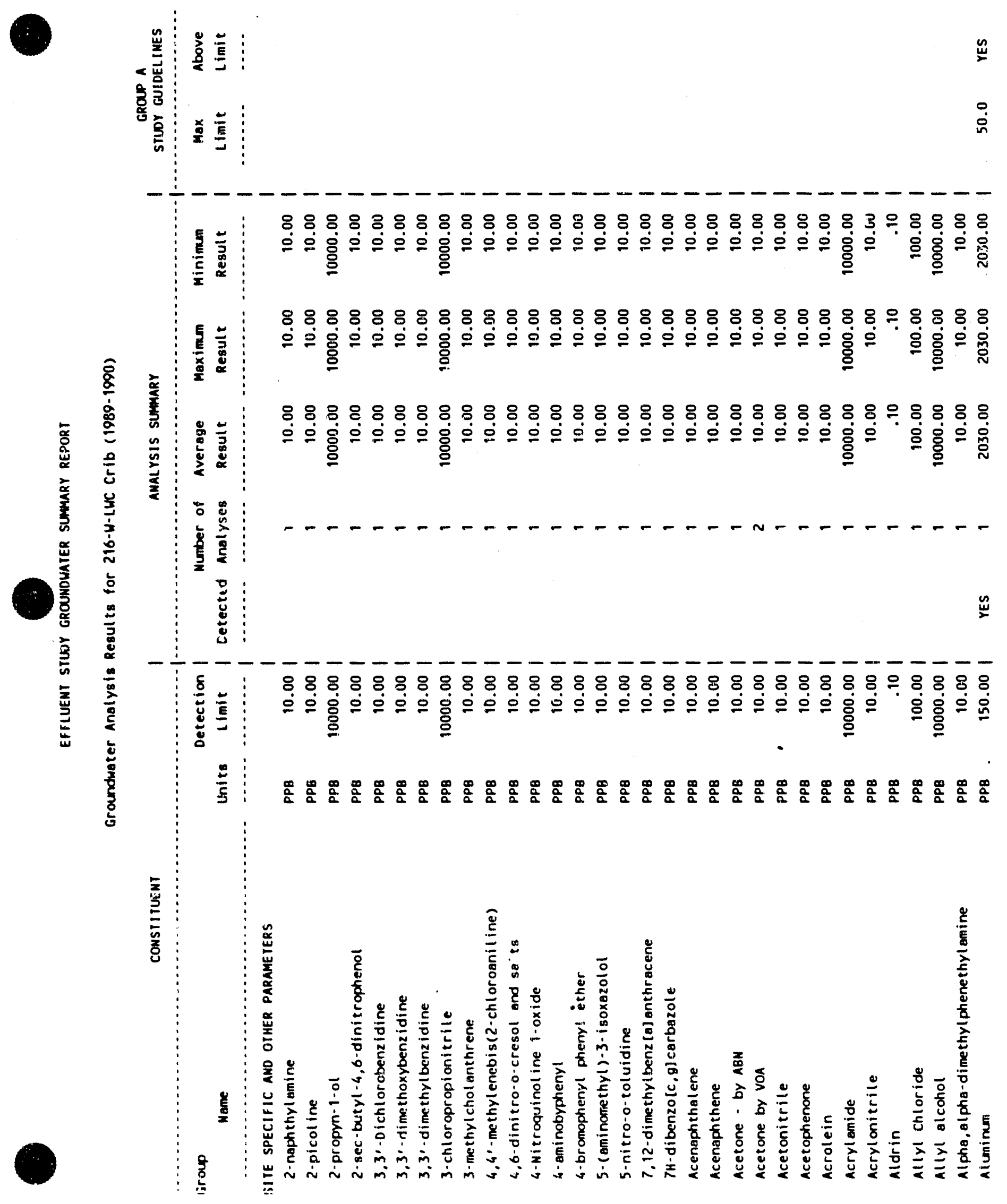


WHC-EP-0366
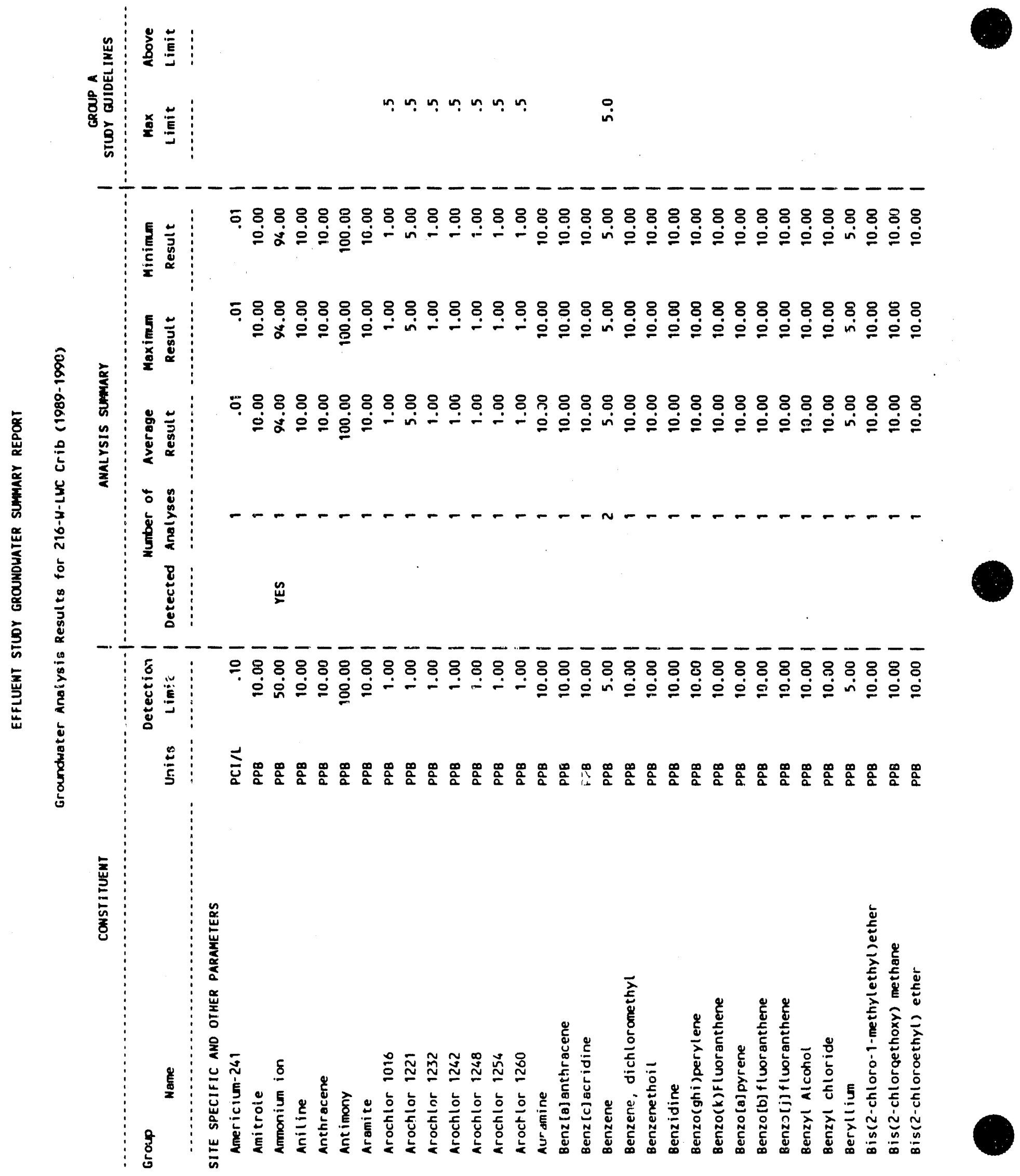
WHC-EP-0366

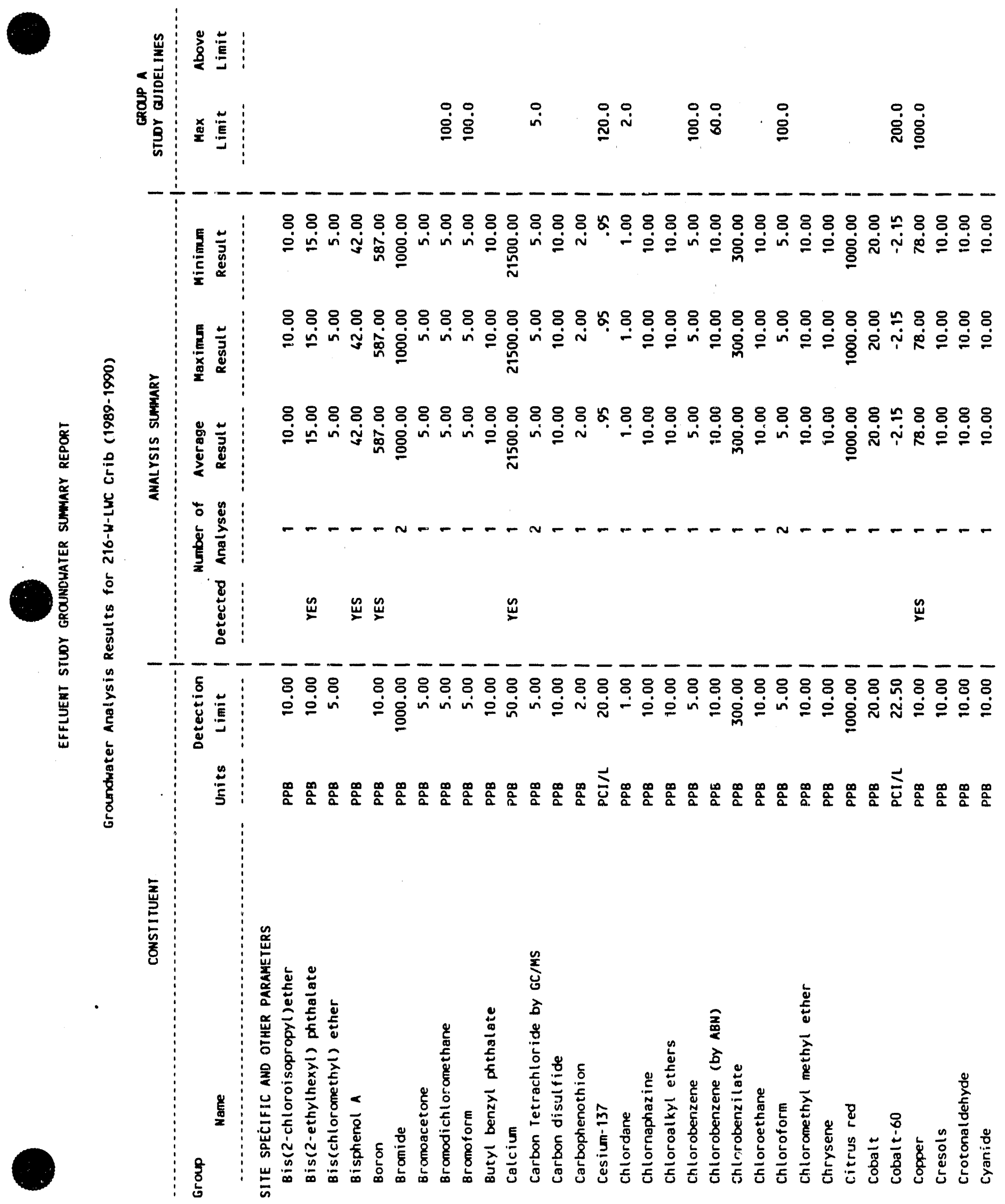



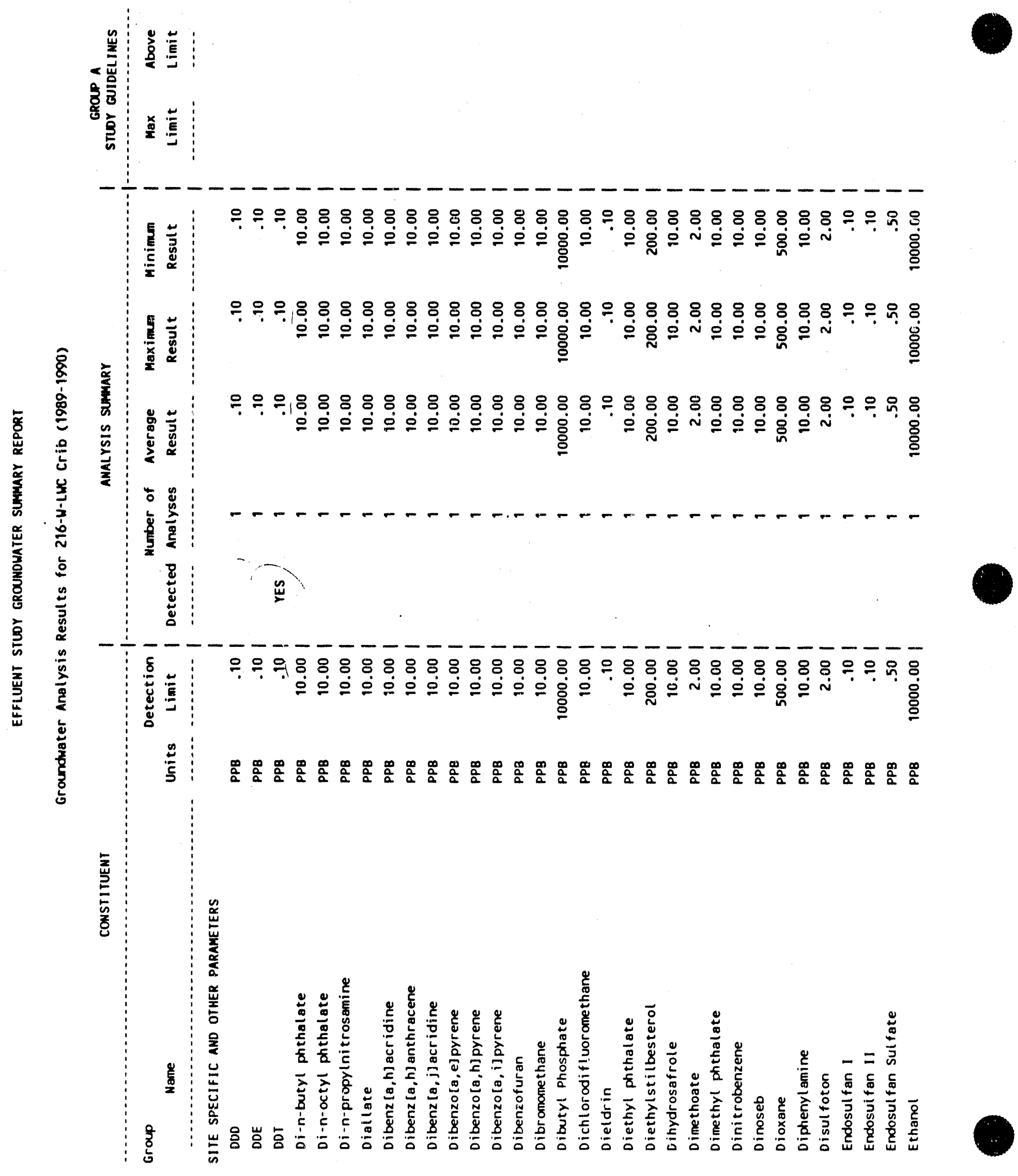
WHC-EP-0366

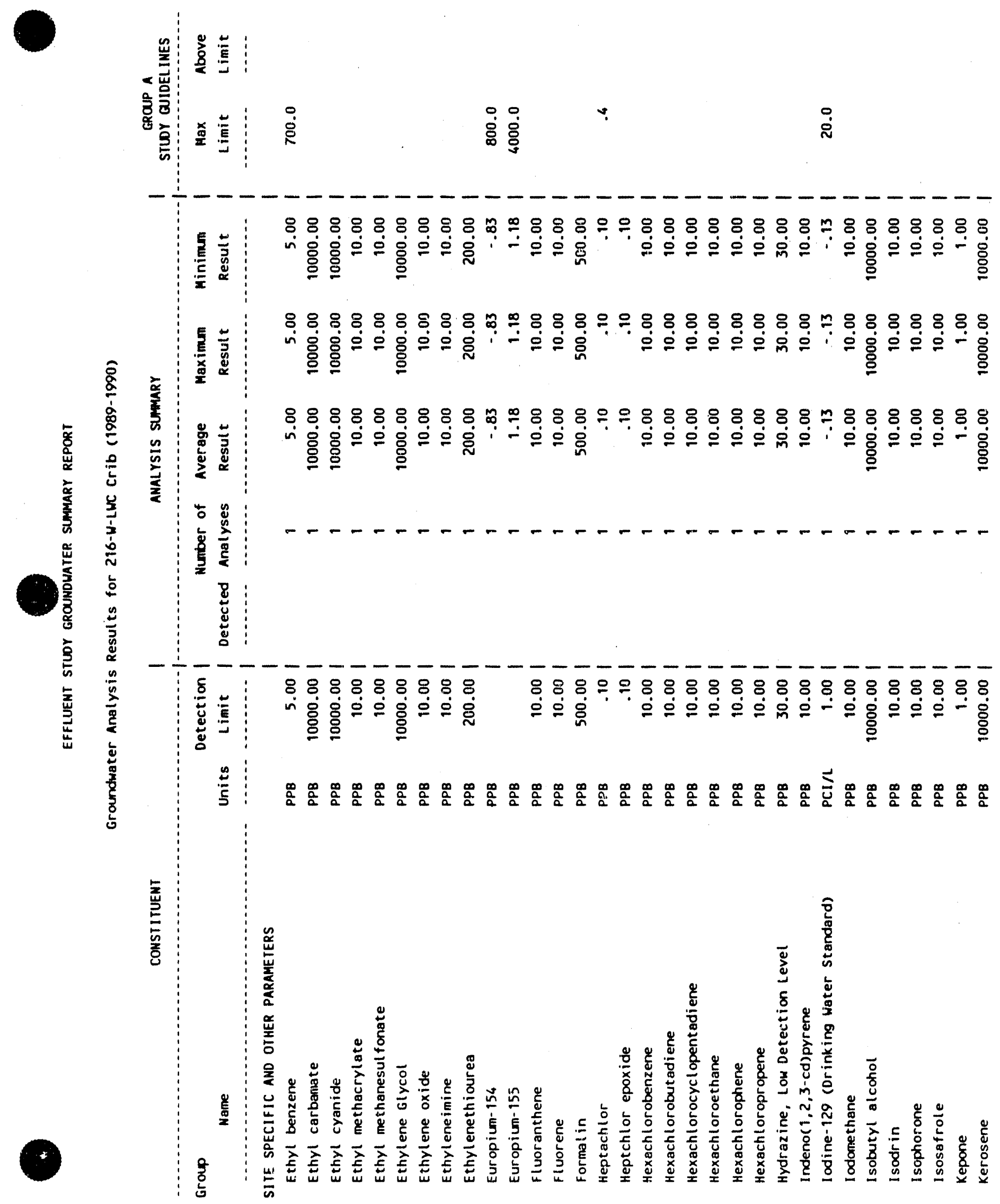


WHC-EP-0366

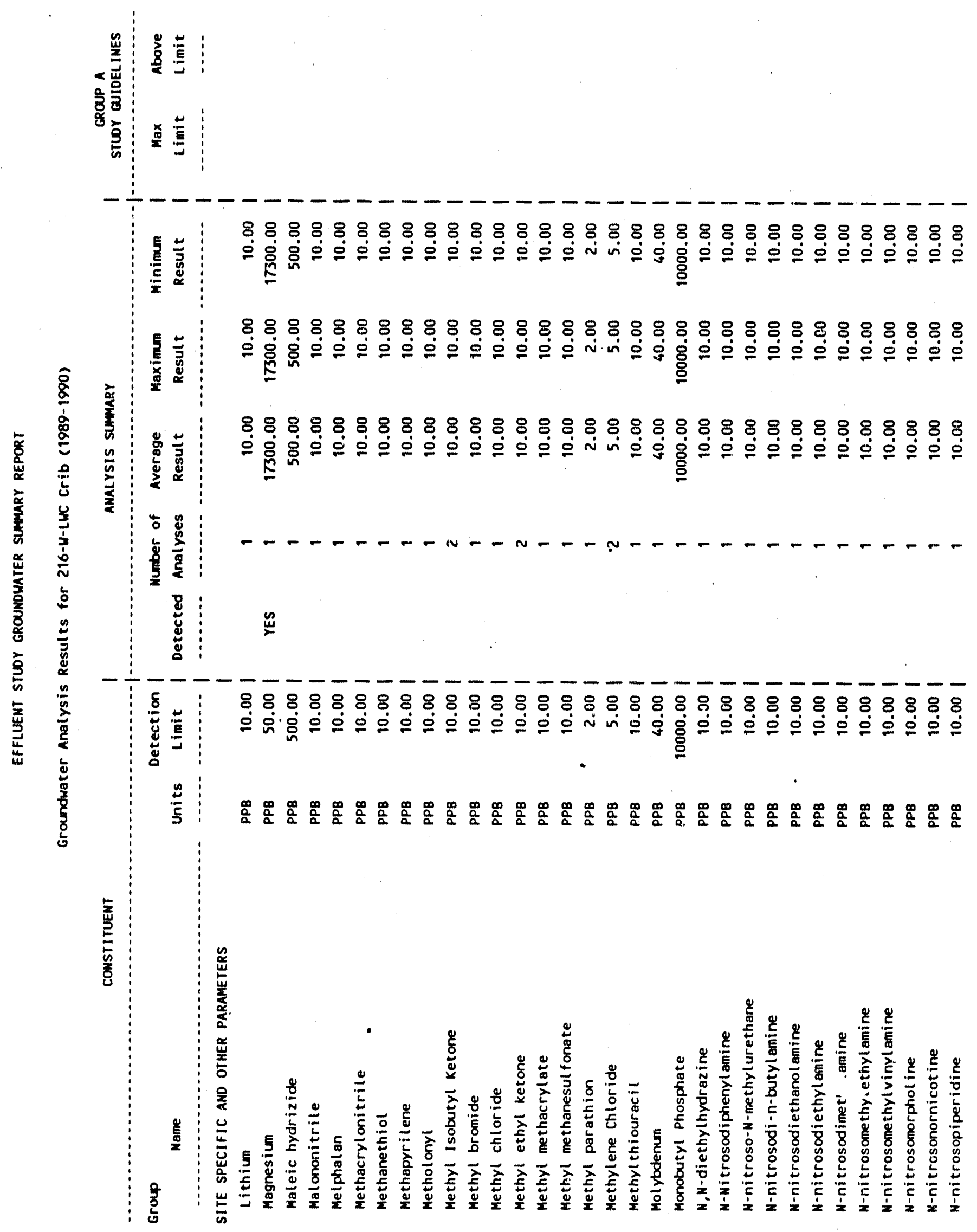




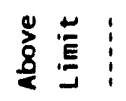

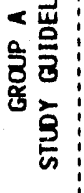

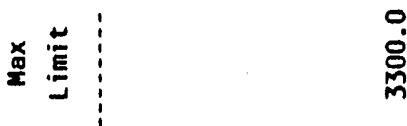

$\stackrel{\circ}{\dot{0}}$

$\stackrel{0}{\dddot{n}}$

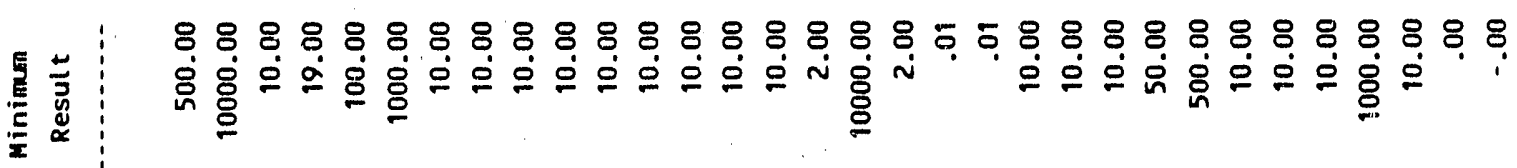

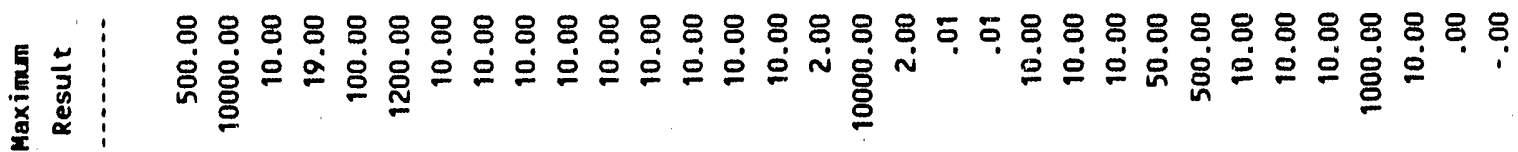

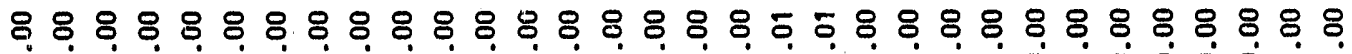

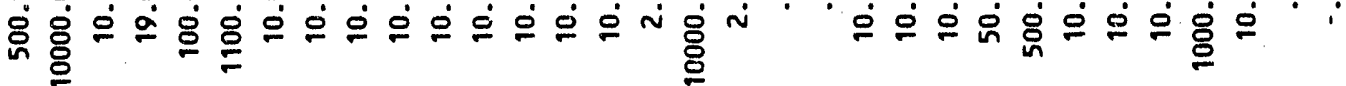

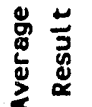

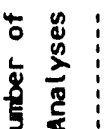

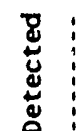

$\stackrel{\sim}{*} \stackrel{\mathscr{w}}{2}$

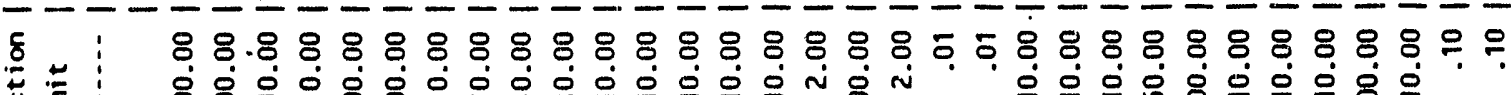

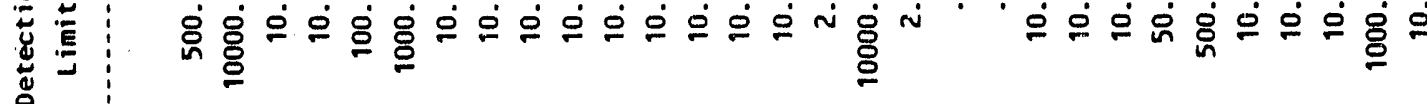

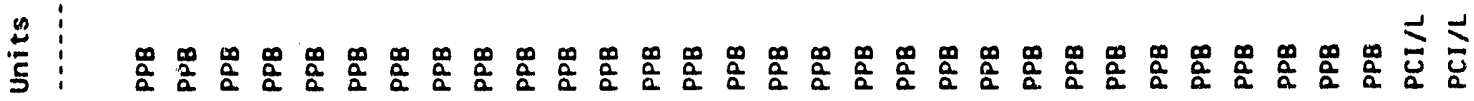

홀 
WHC -EP-0366

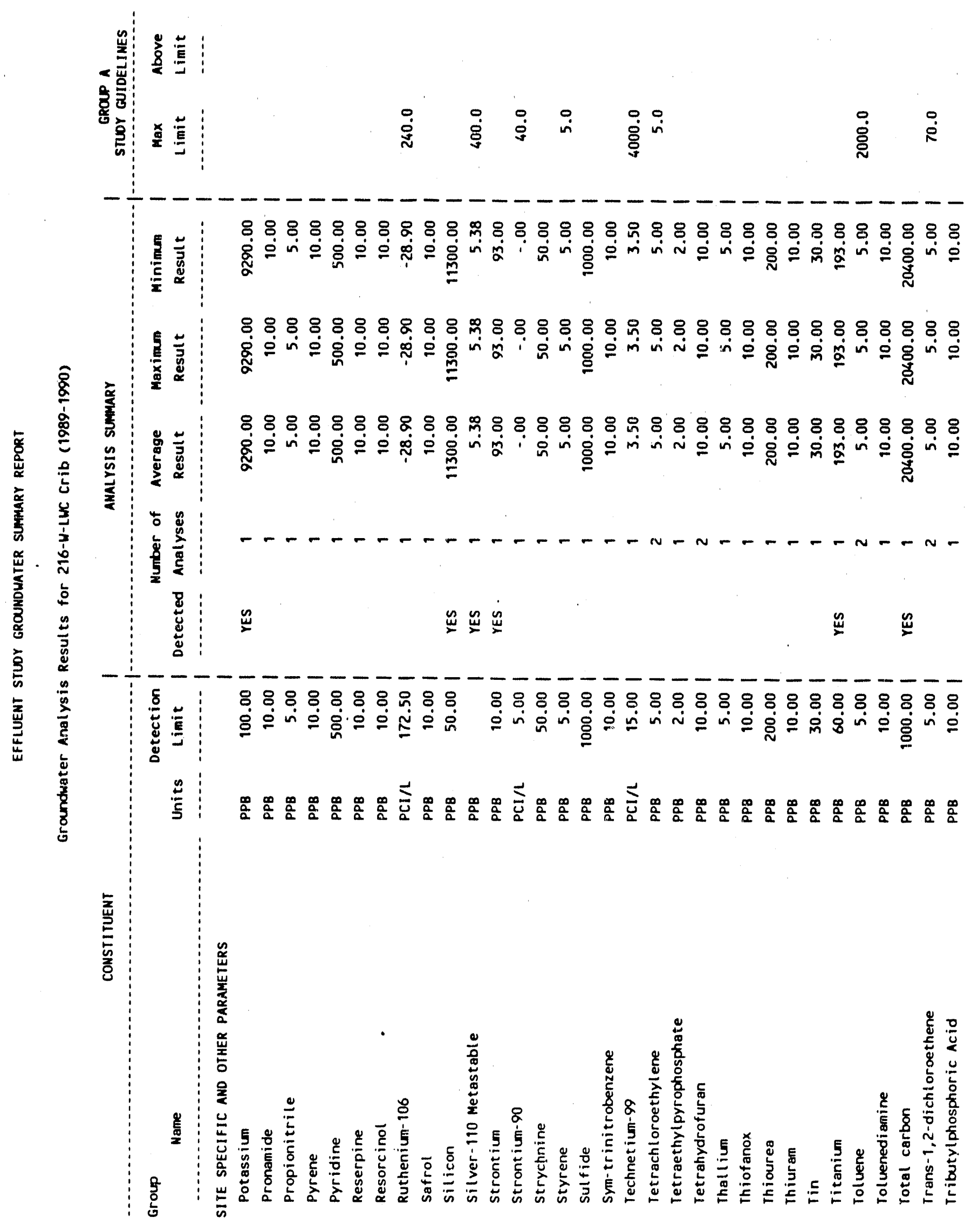


WHC-EP-0366

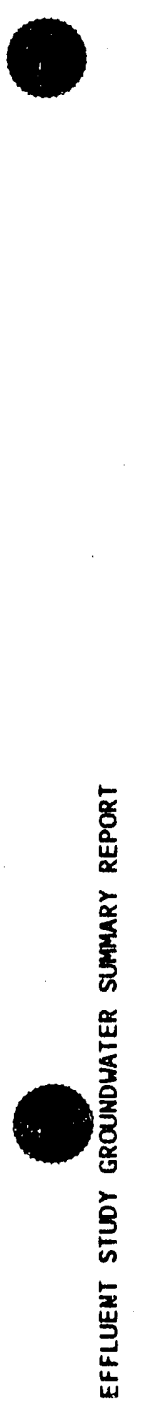

资:

을

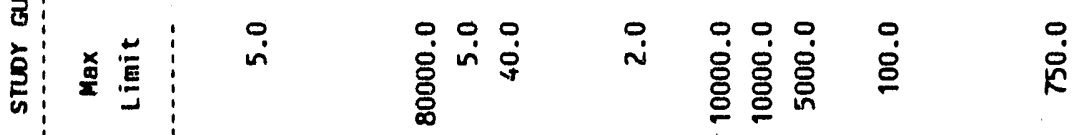

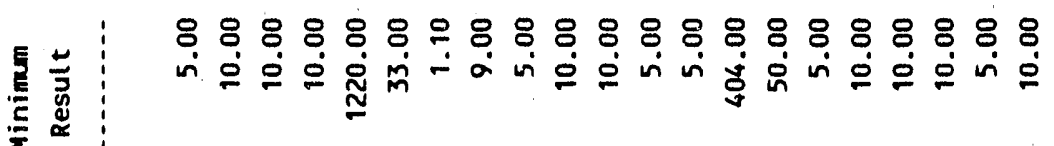

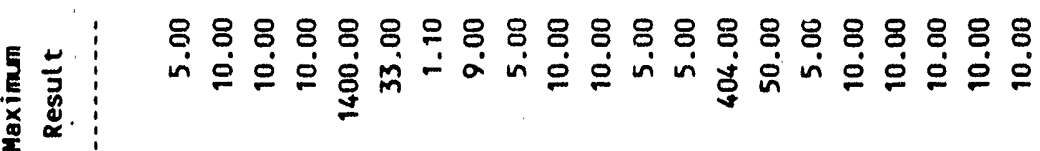

888888988888888888858

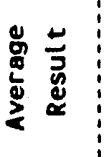

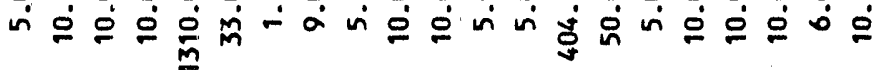

范

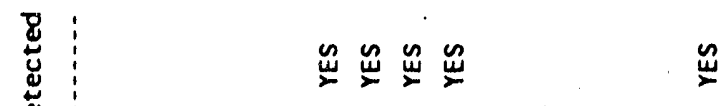

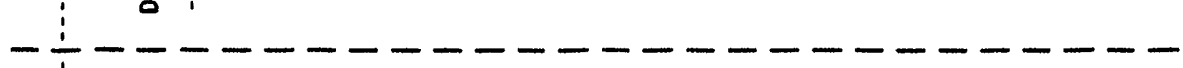

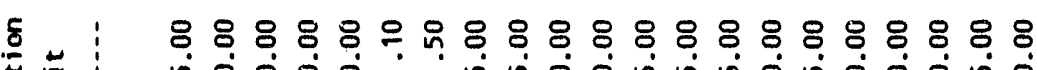

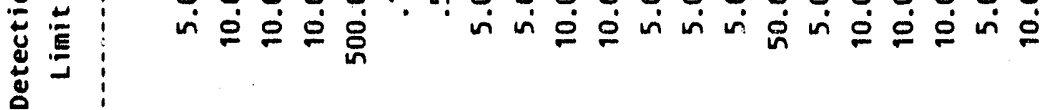

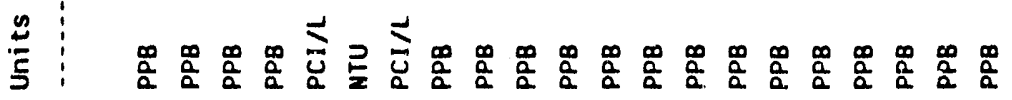

苑

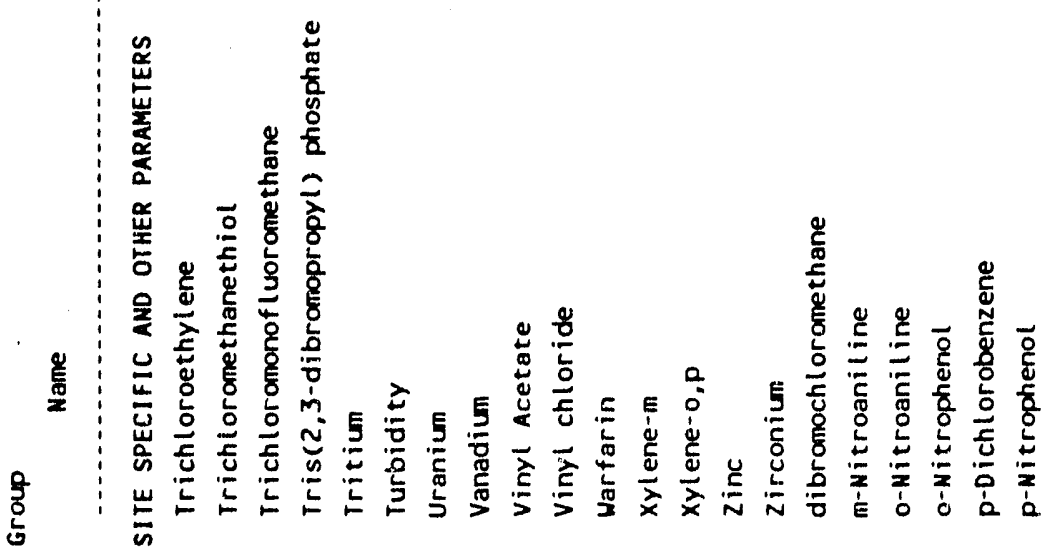




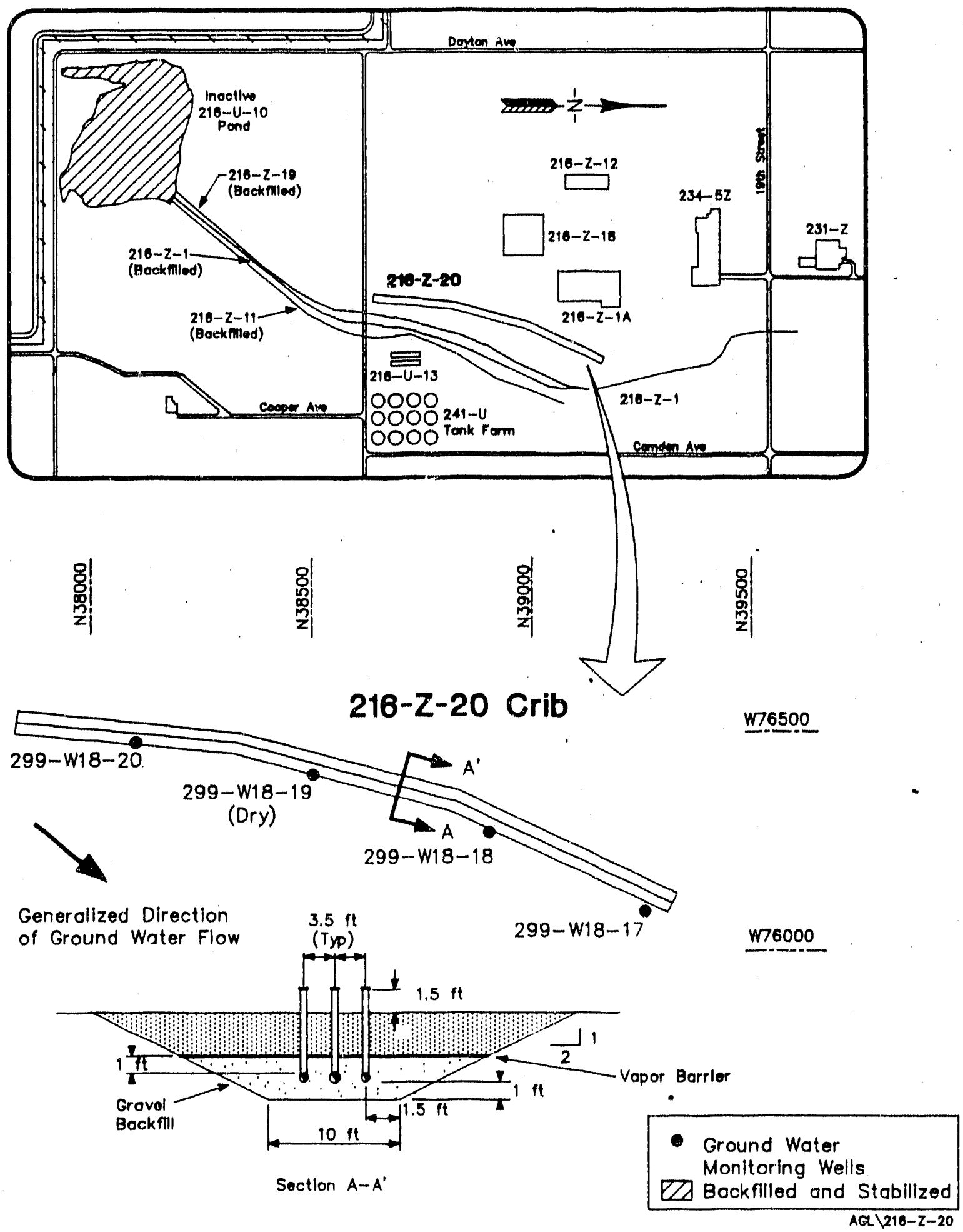

Well Location and Site Map for 216-2-20 Crib 
WHC-EP-0366

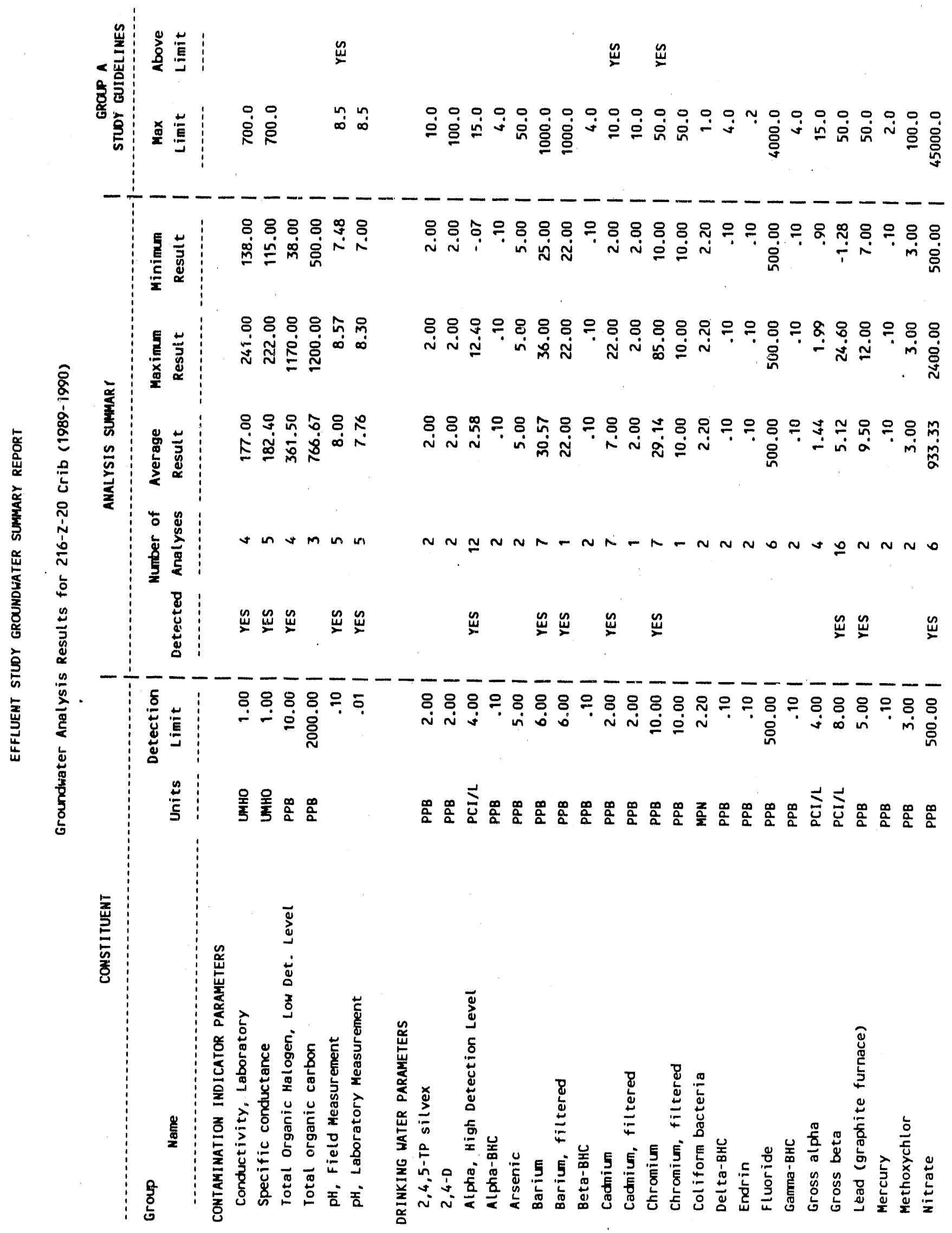


WHC-EP-0366

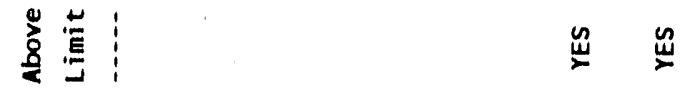

曾

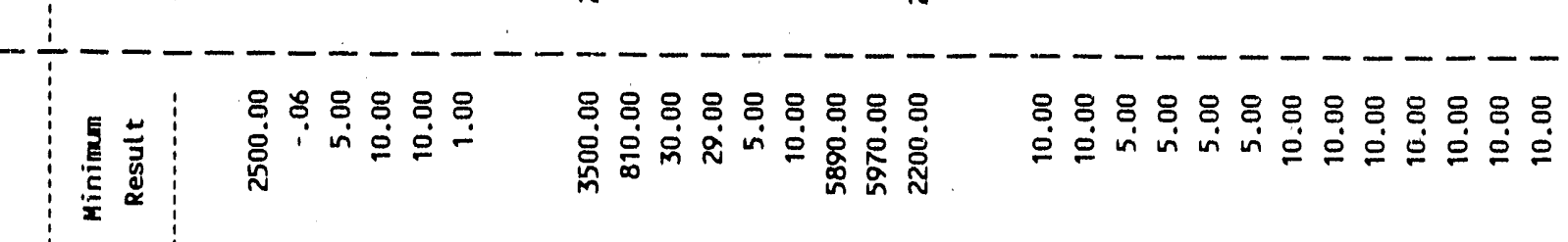

E

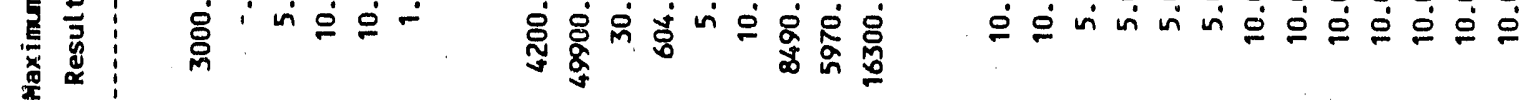

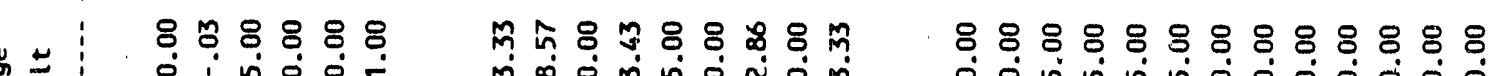

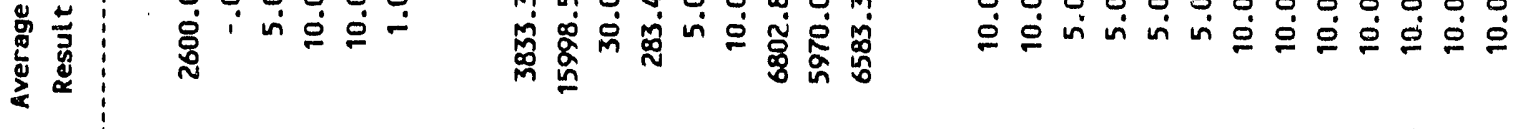

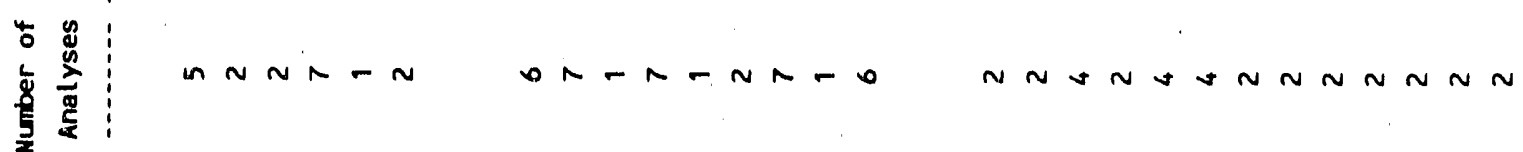

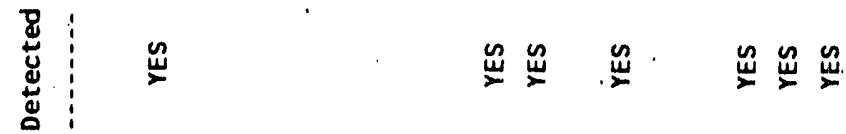

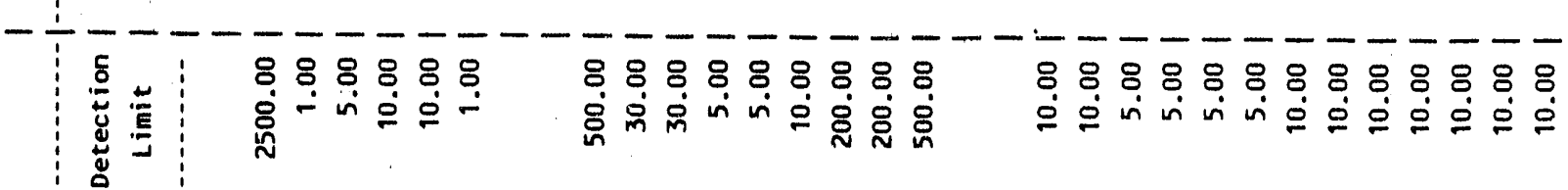

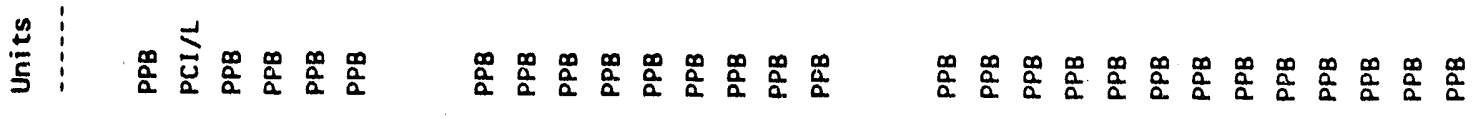

氢

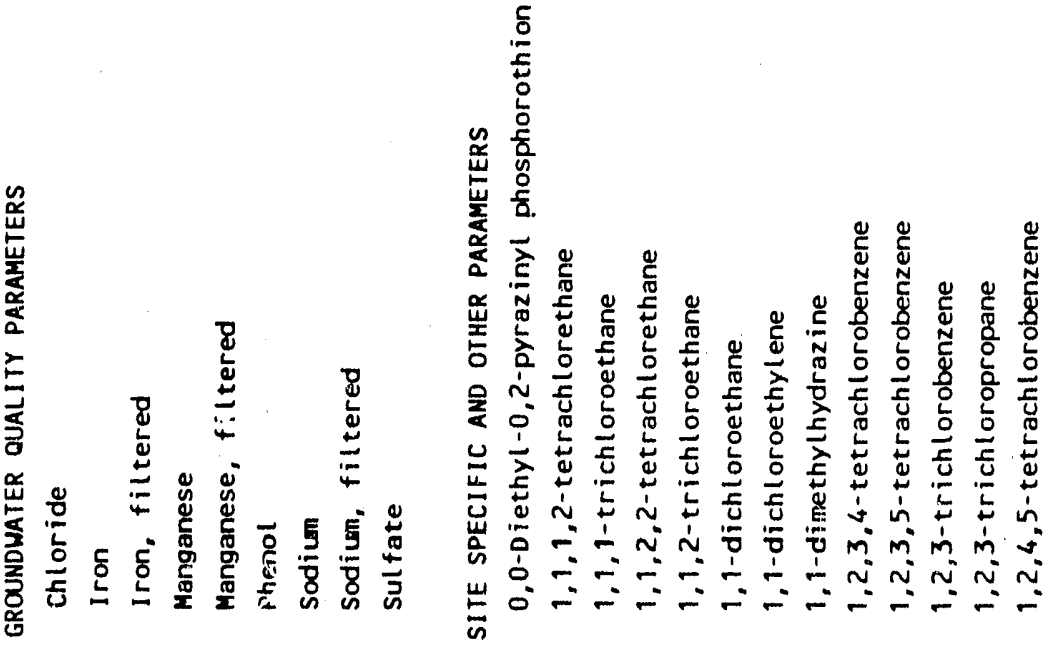



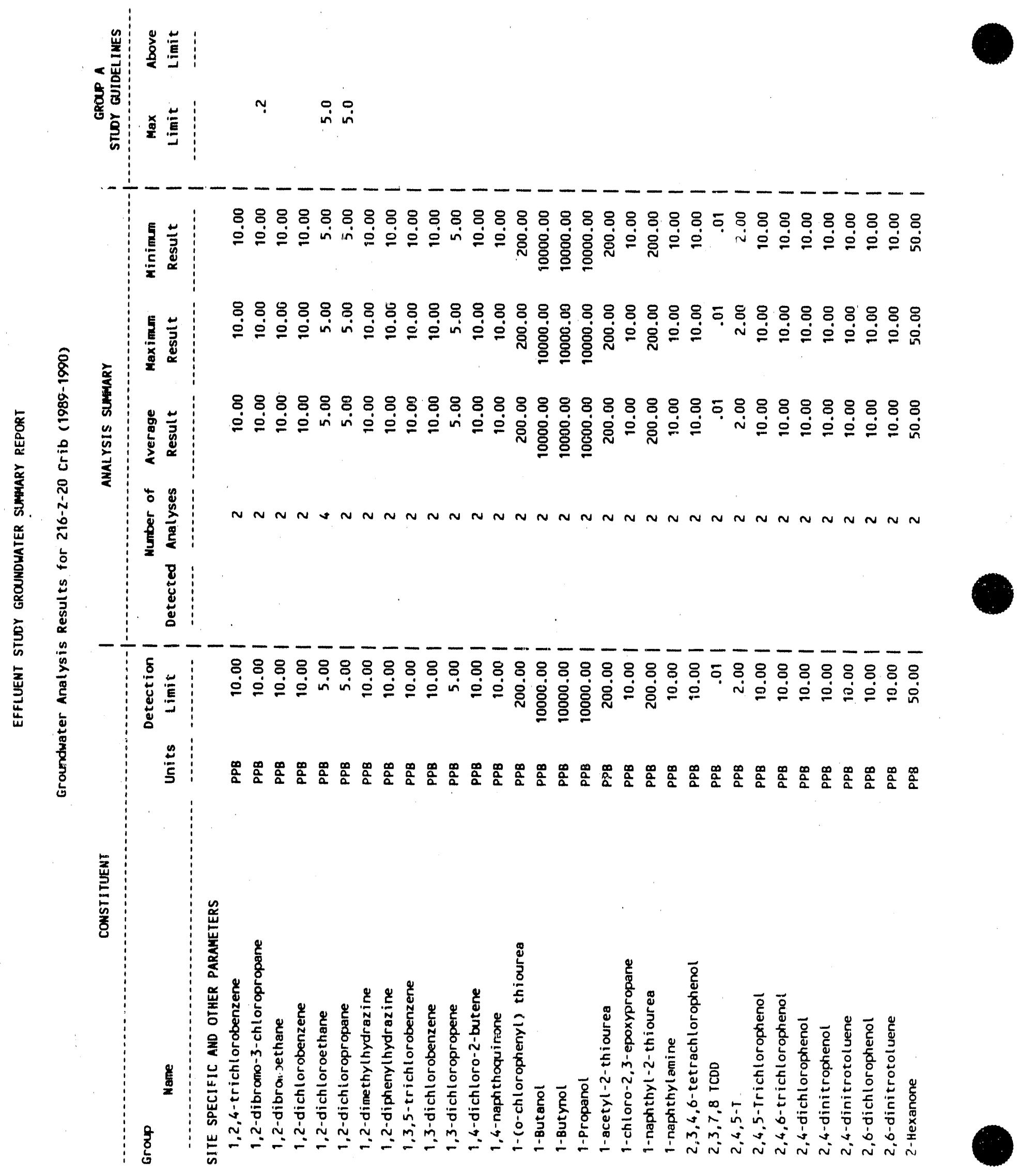
蒙

总

莹

8888888888888888888888888888808

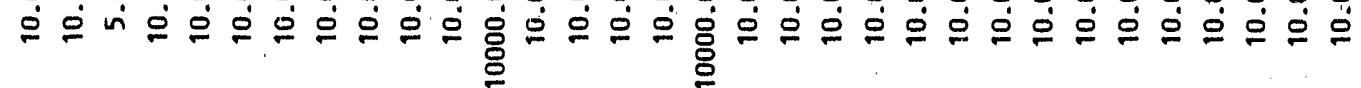

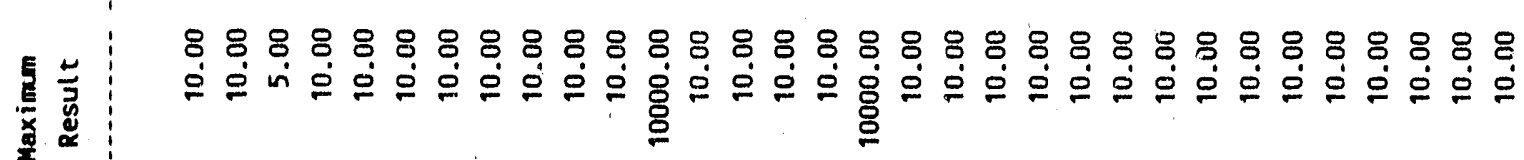

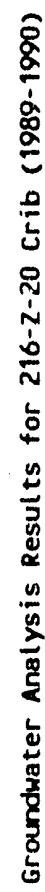

8888888888888888888888880888888 ن்

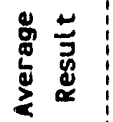

t०

:

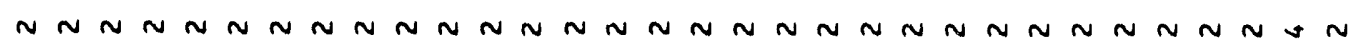
政

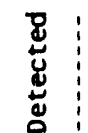

880808808888088888888888880888

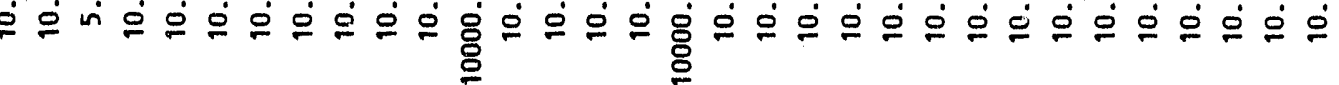

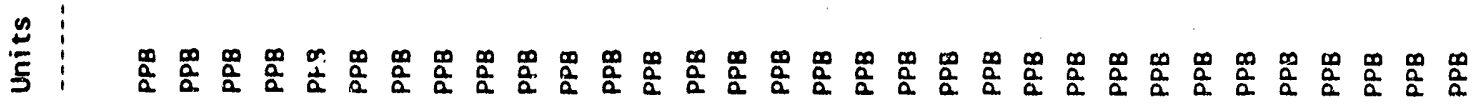

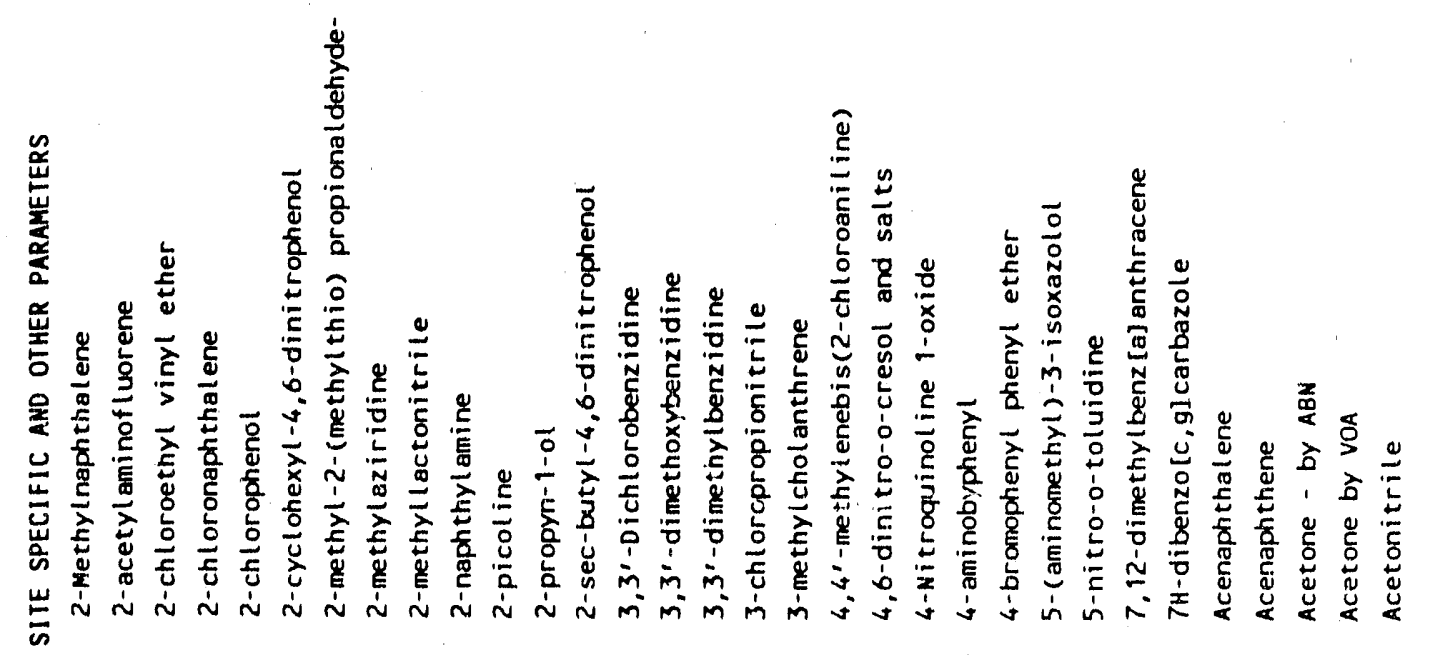


WHC-EP-0366

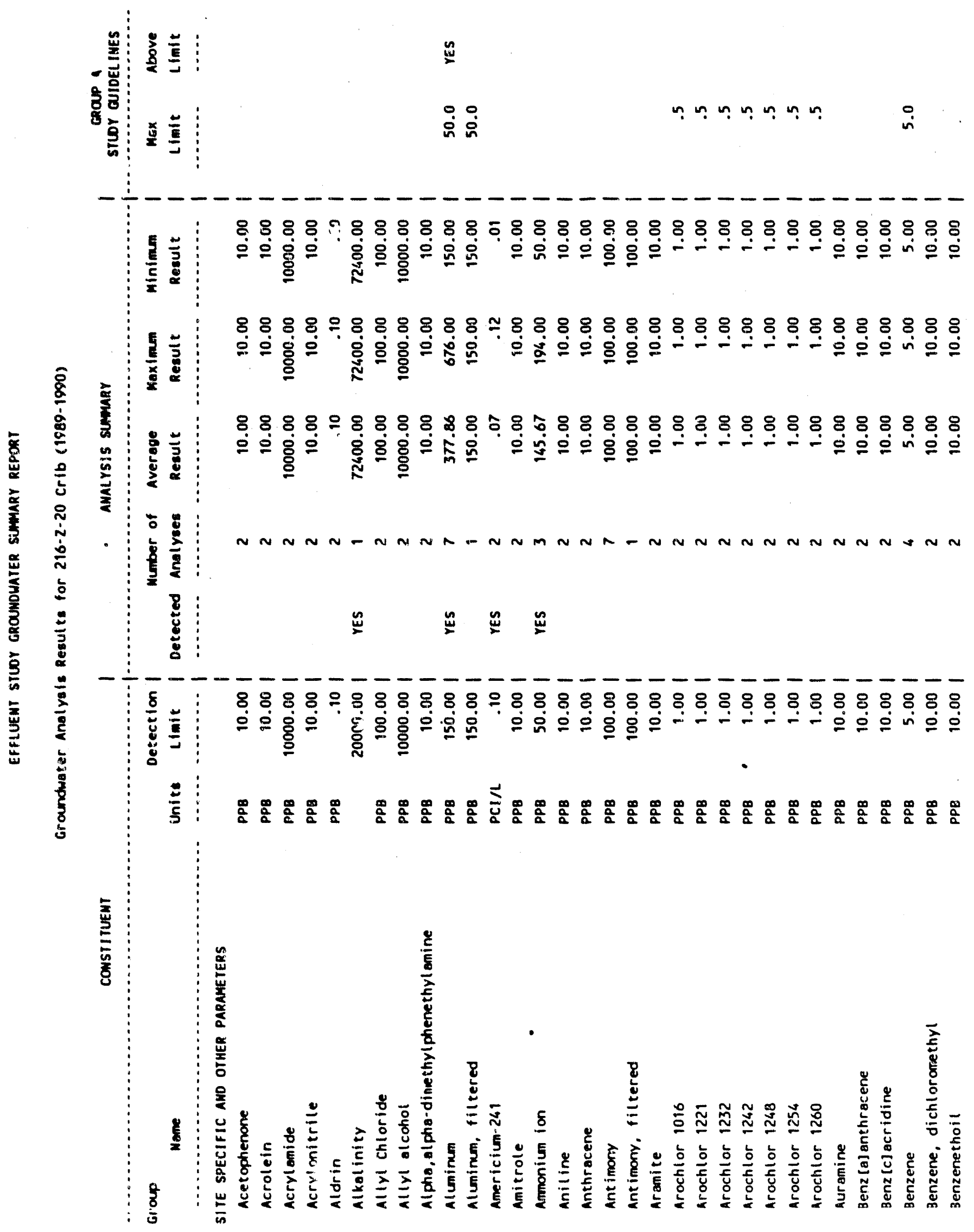




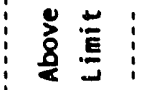

串:

毫

官 :

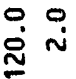

$-\frac{1}{i}$

产

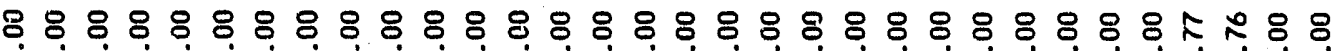

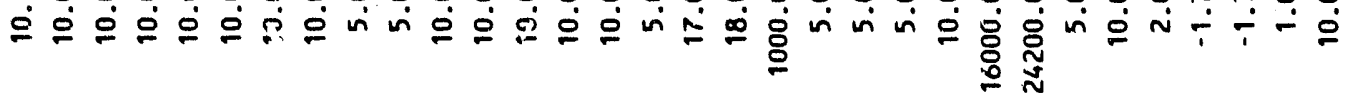

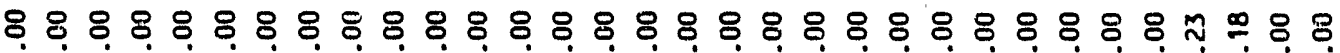

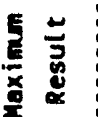

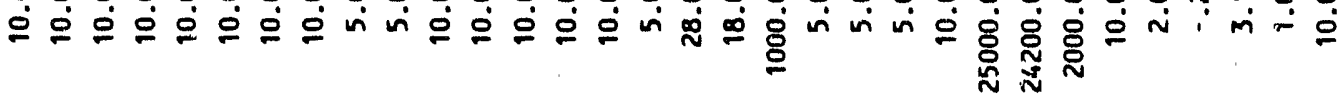

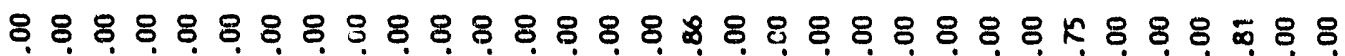
范莣

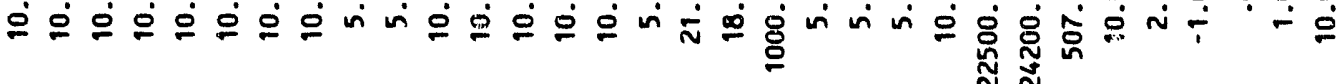

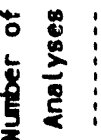

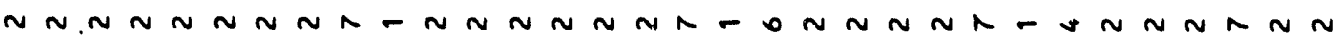

8

:

$\stackrel{\sim}{2} \stackrel{\sim}{2}$

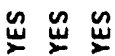

8

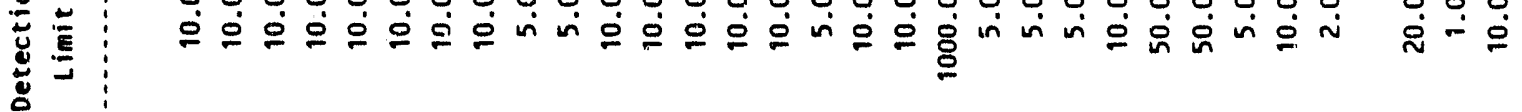

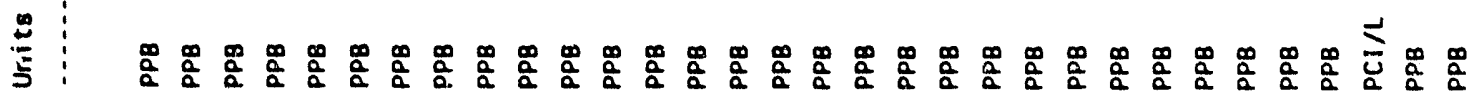

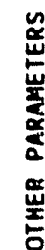
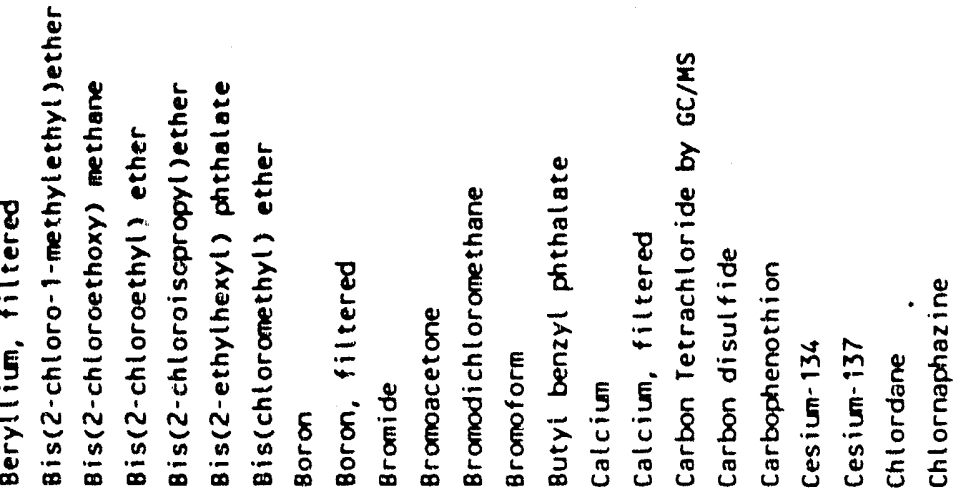
WHC-EP-0366

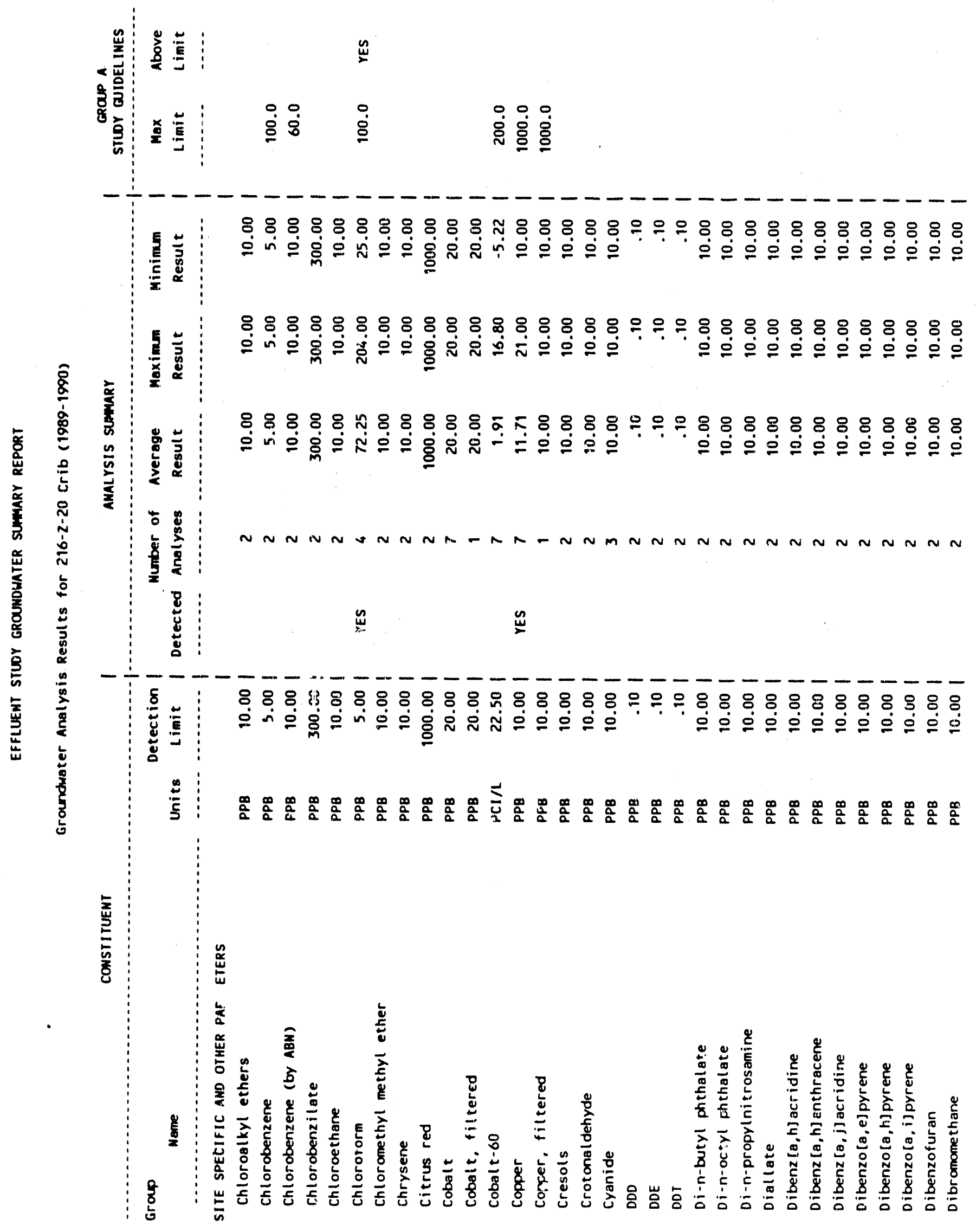




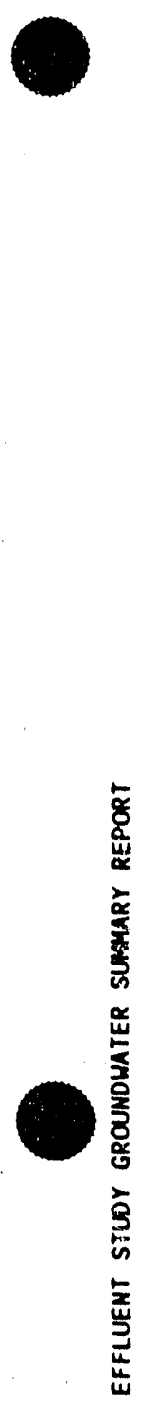

意亭:

究

旁

$\stackrel{9}{\circledR}$

$\checkmark$

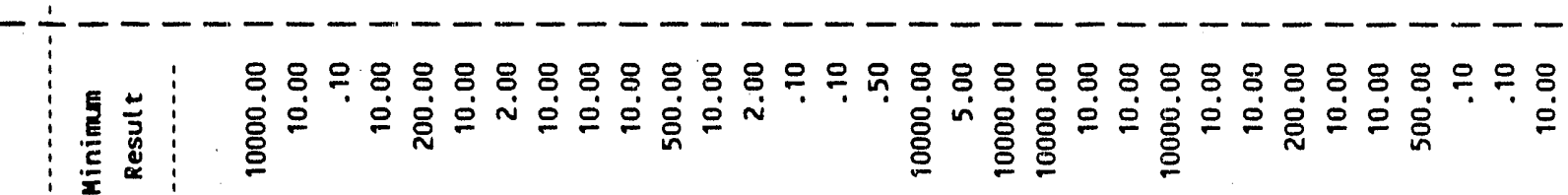

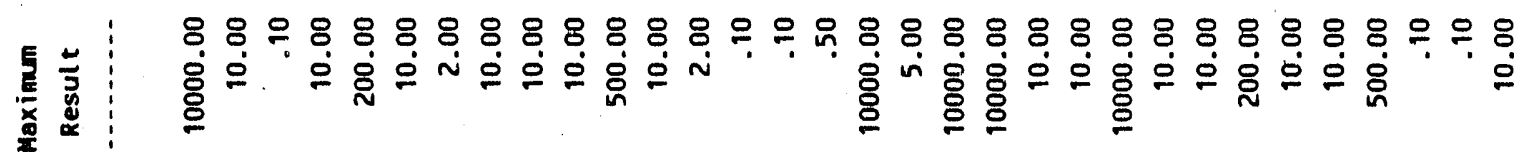

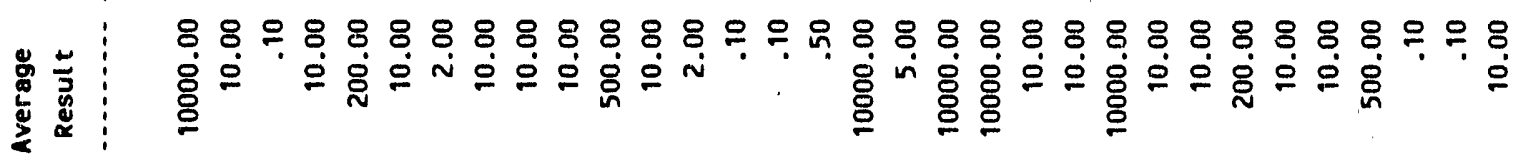

\begin{tabular}{l:l}
0 \\
0 \\
\hline
\end{tabular}

焉安

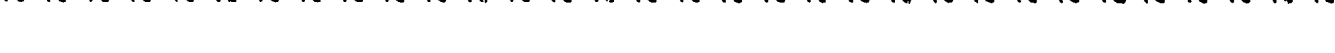

8

:

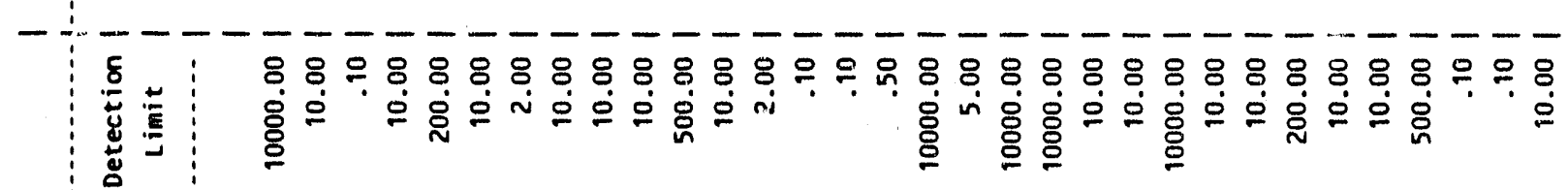

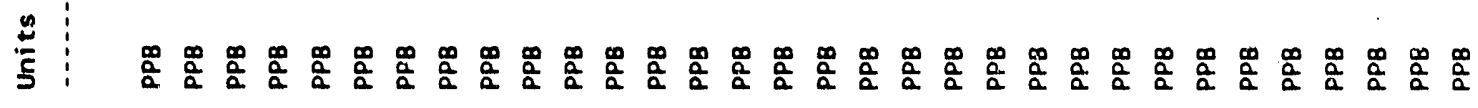

怘

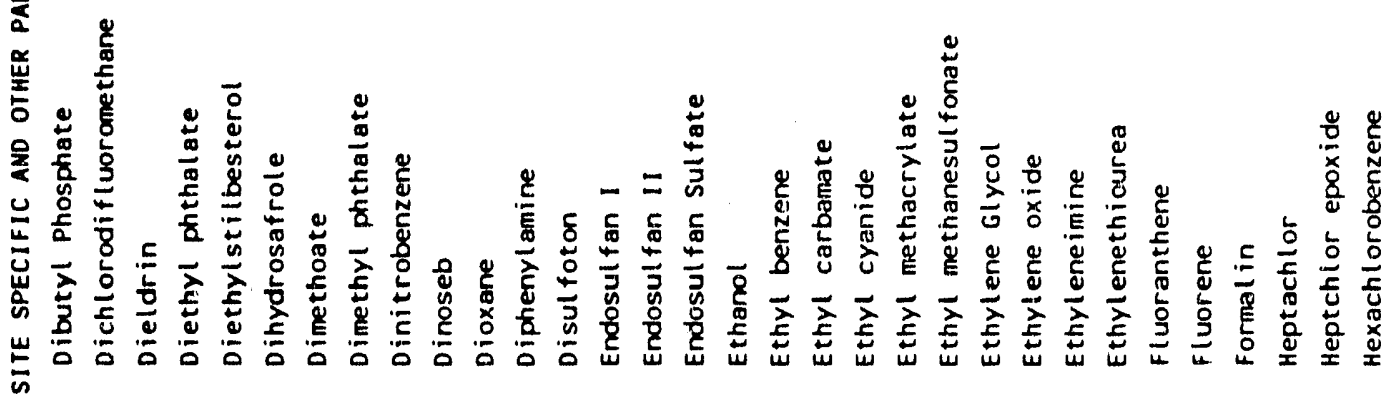




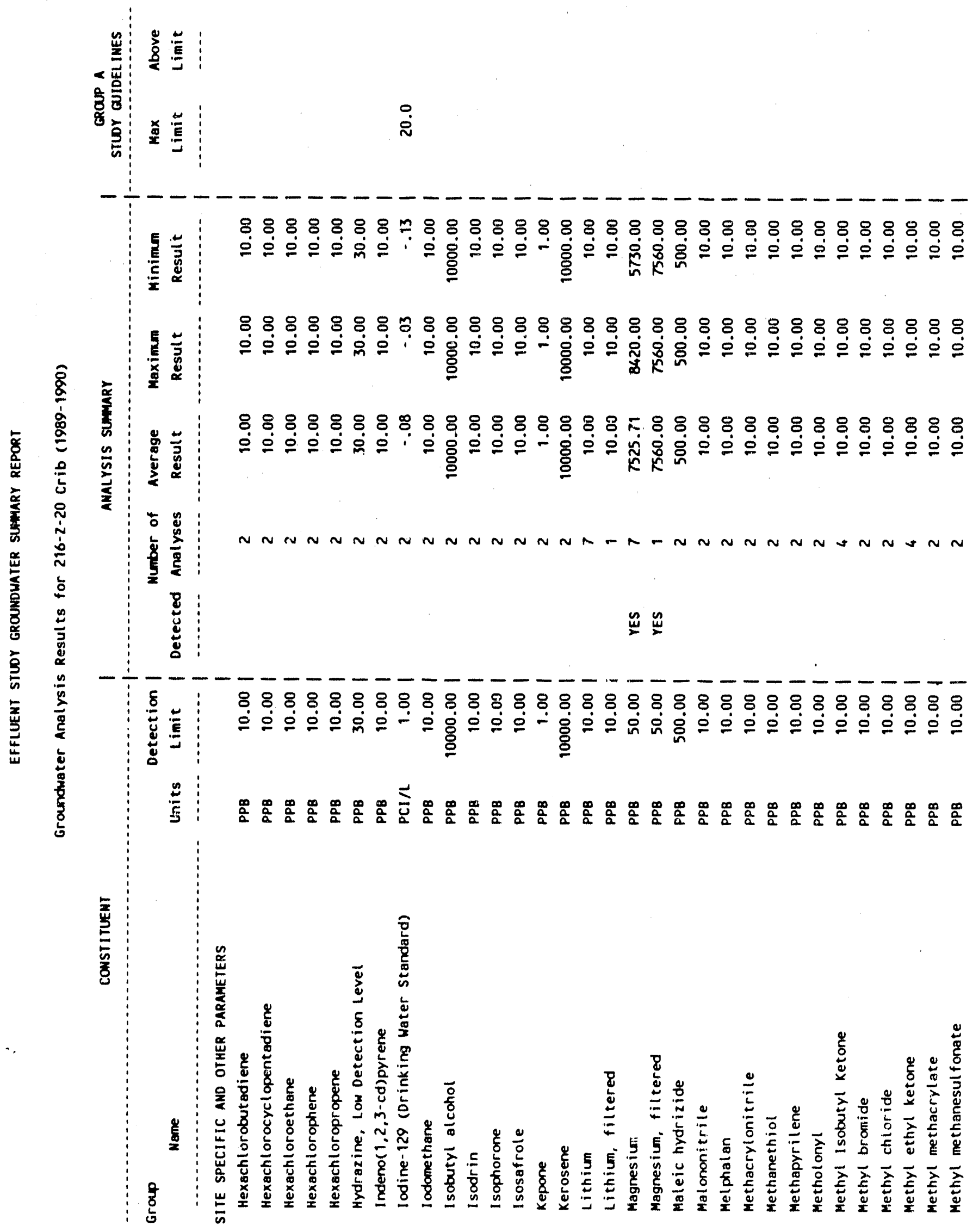




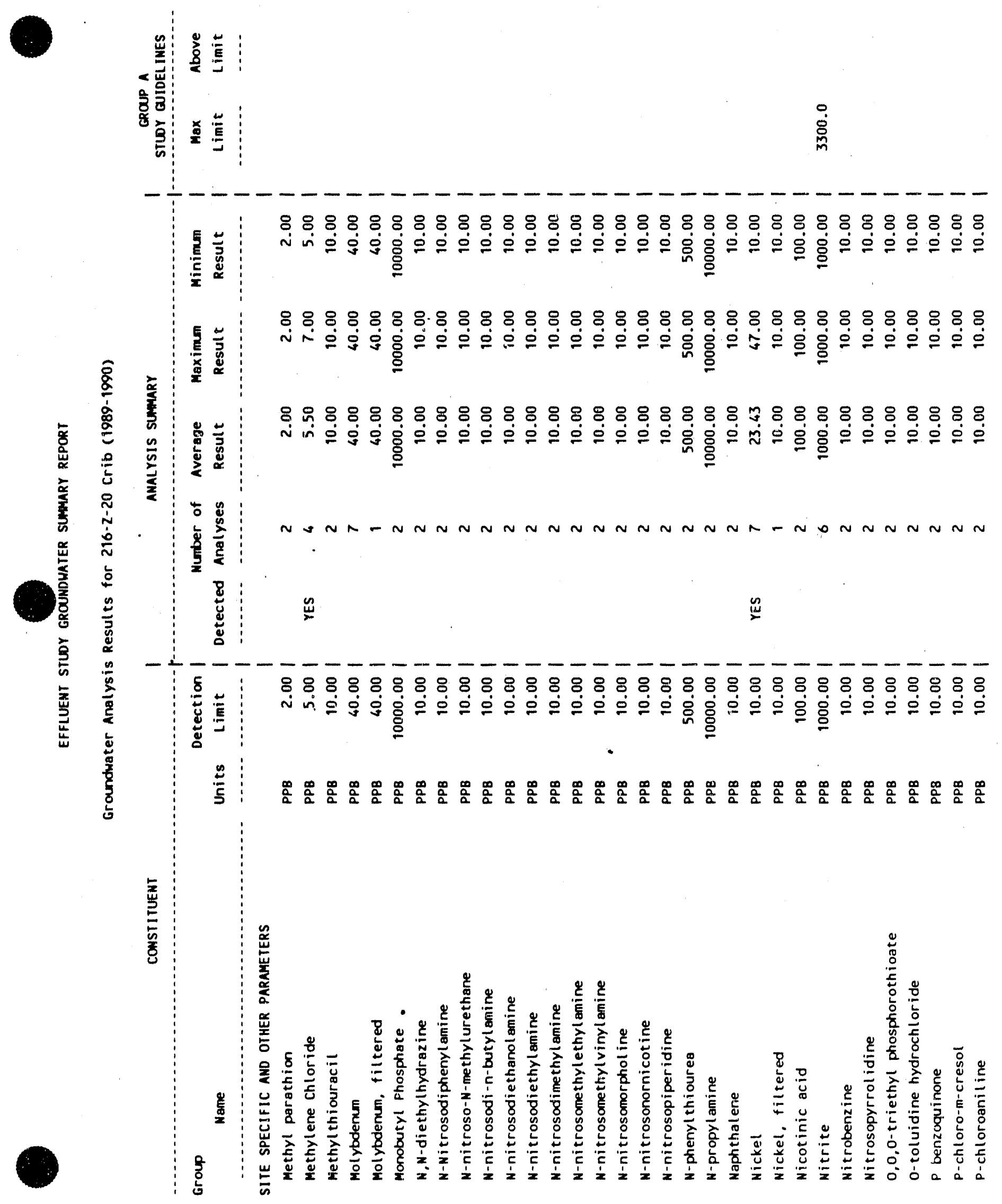




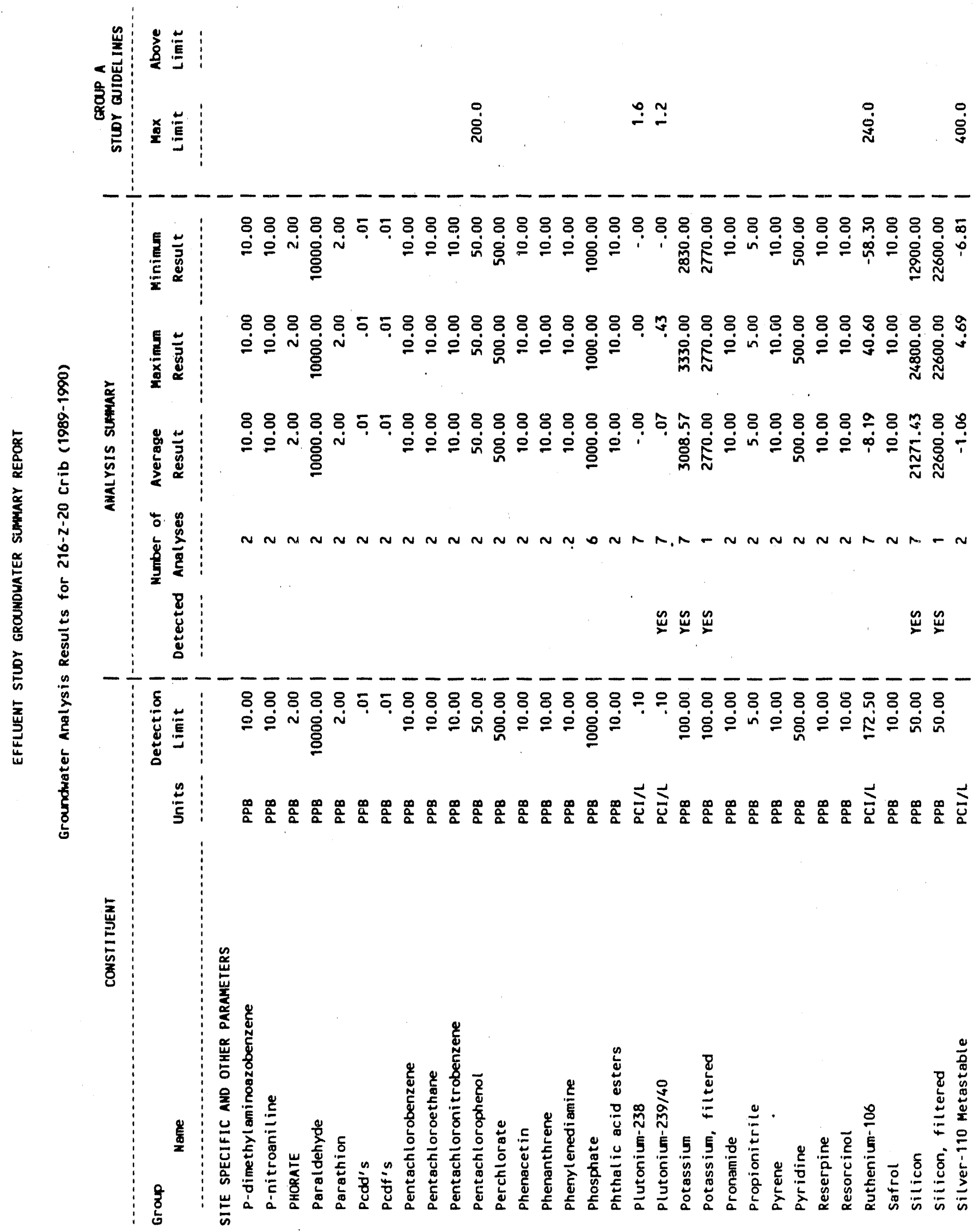


WHC-EP-0366

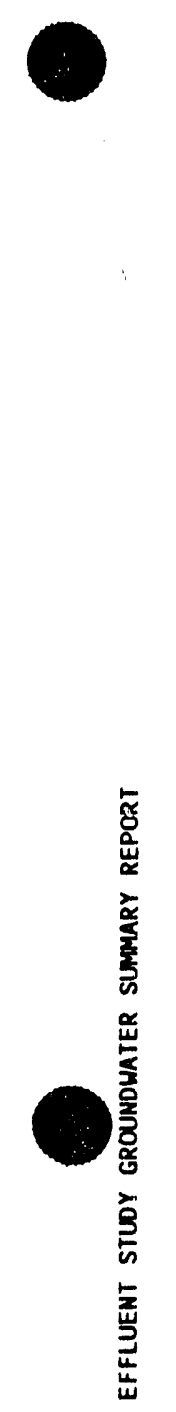

资:

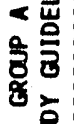

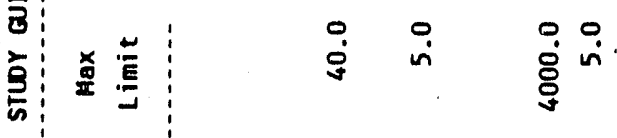

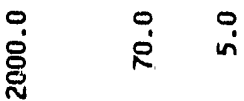

$\stackrel{\leftrightarrow}{\longleftarrow}$

$\stackrel{\mathscr{4}}{2}$

$-\frac{1}{\vdots}-0$

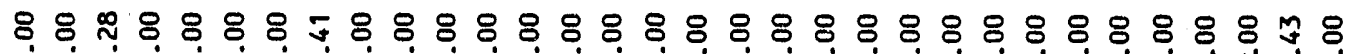

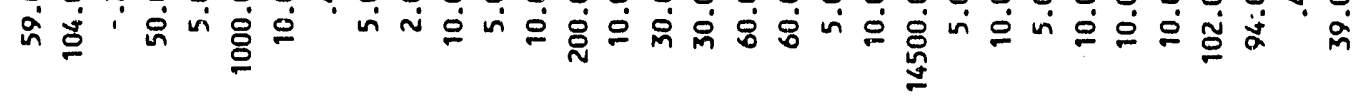

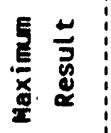

$88888889888888888888888888888=8$

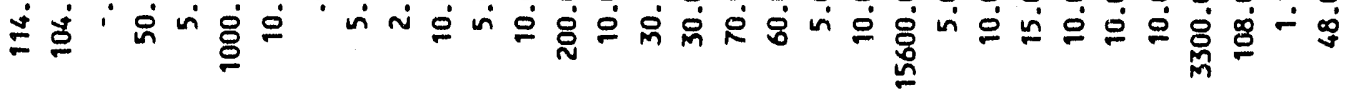

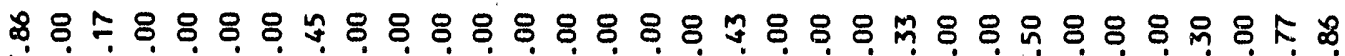

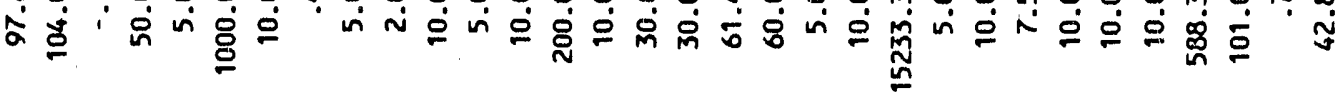

焉

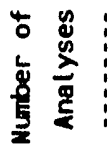

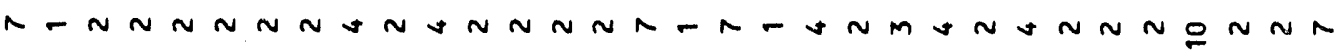

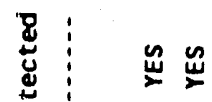

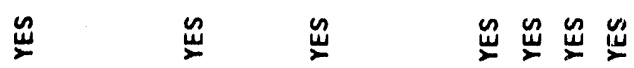

-

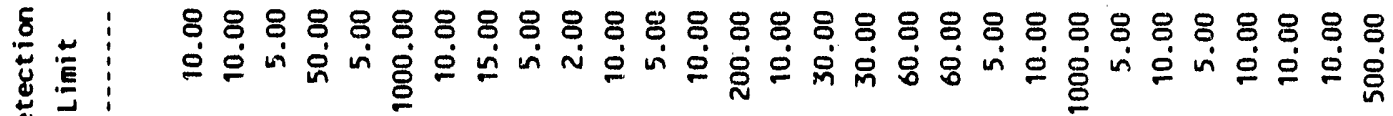

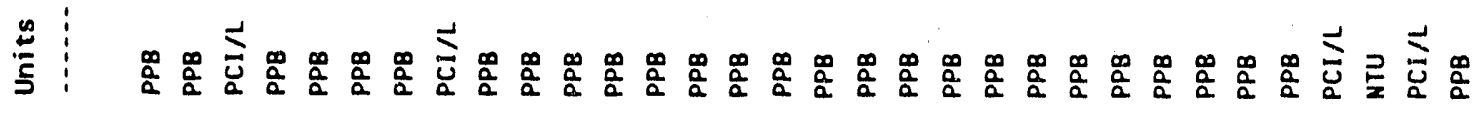

롤

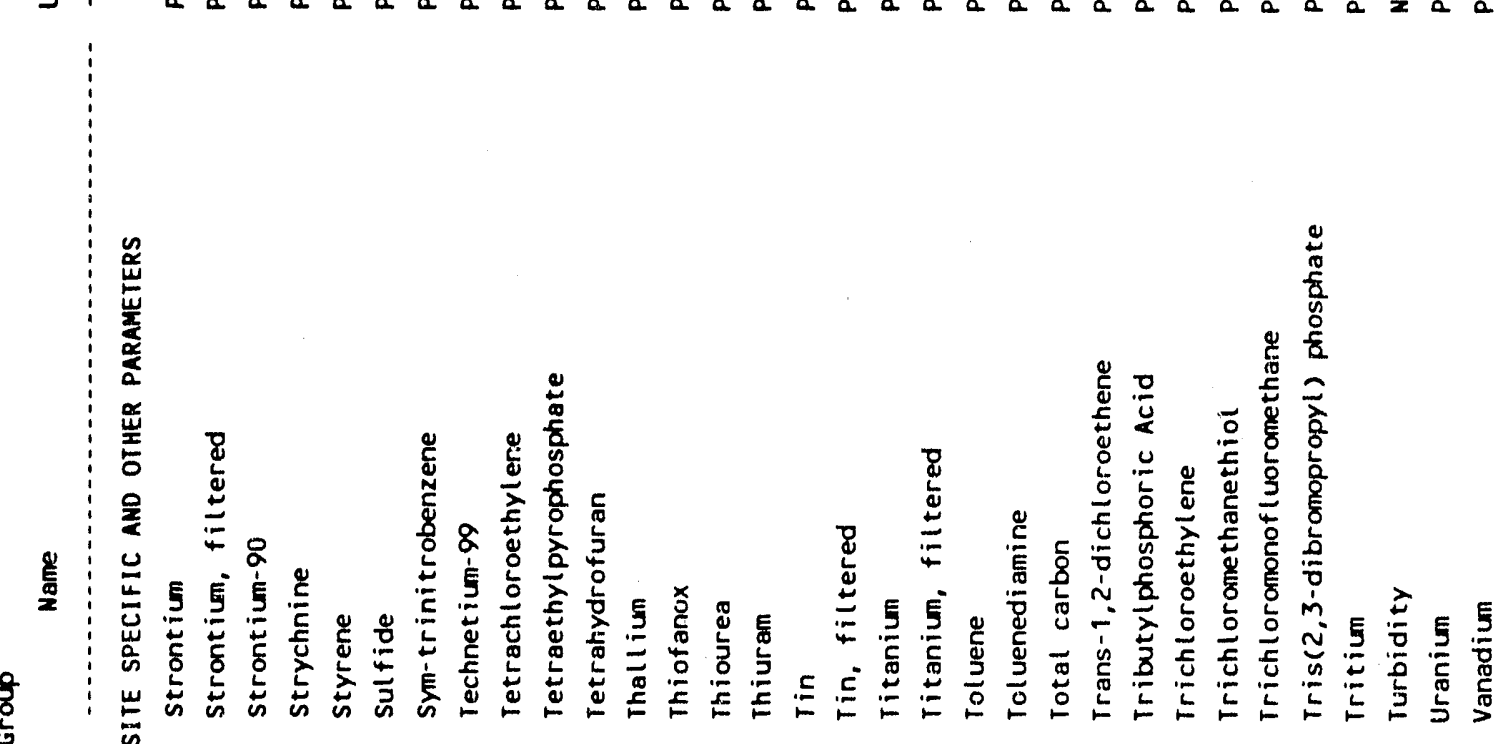


WHC-EP-0366

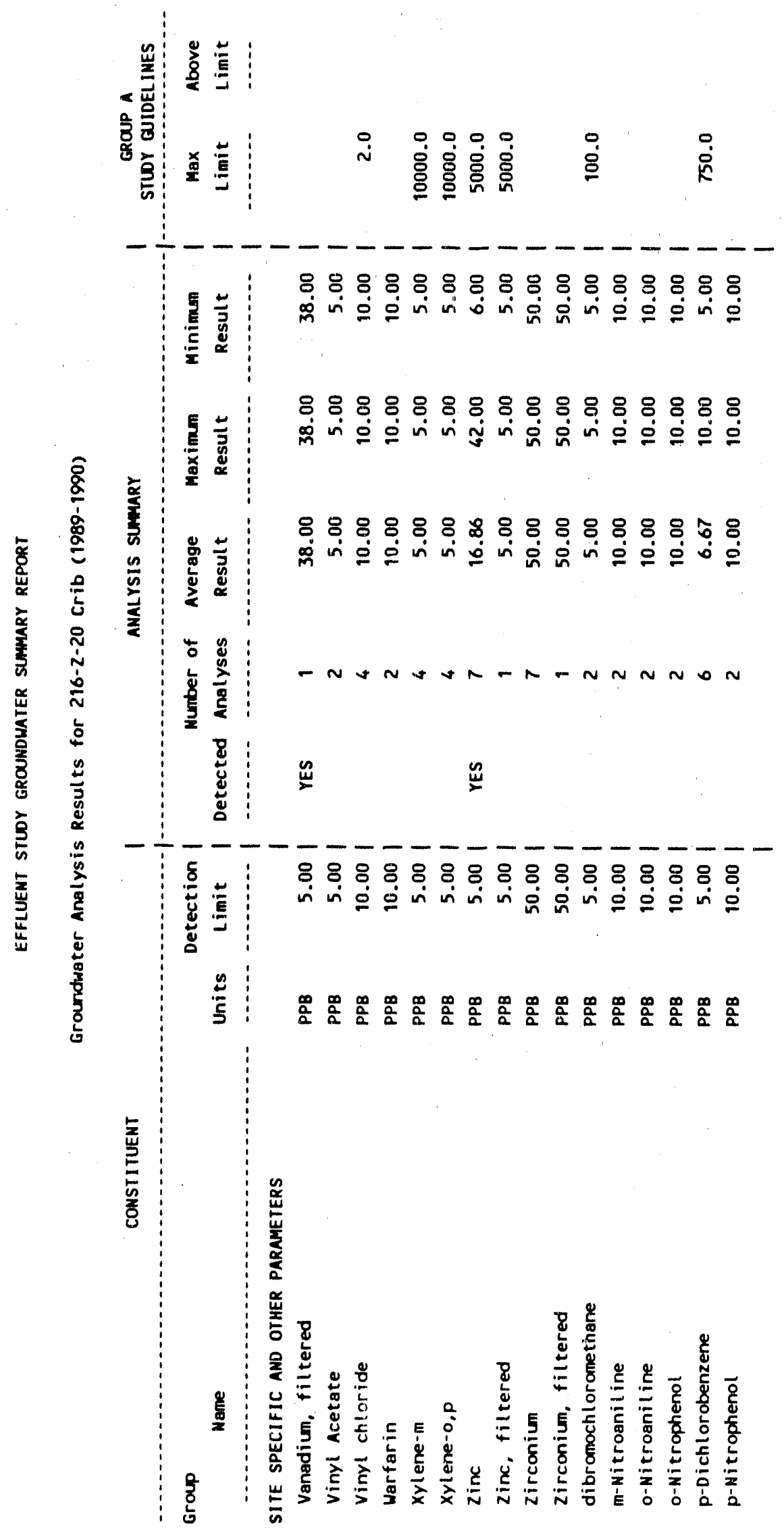


WHC-EP-0366

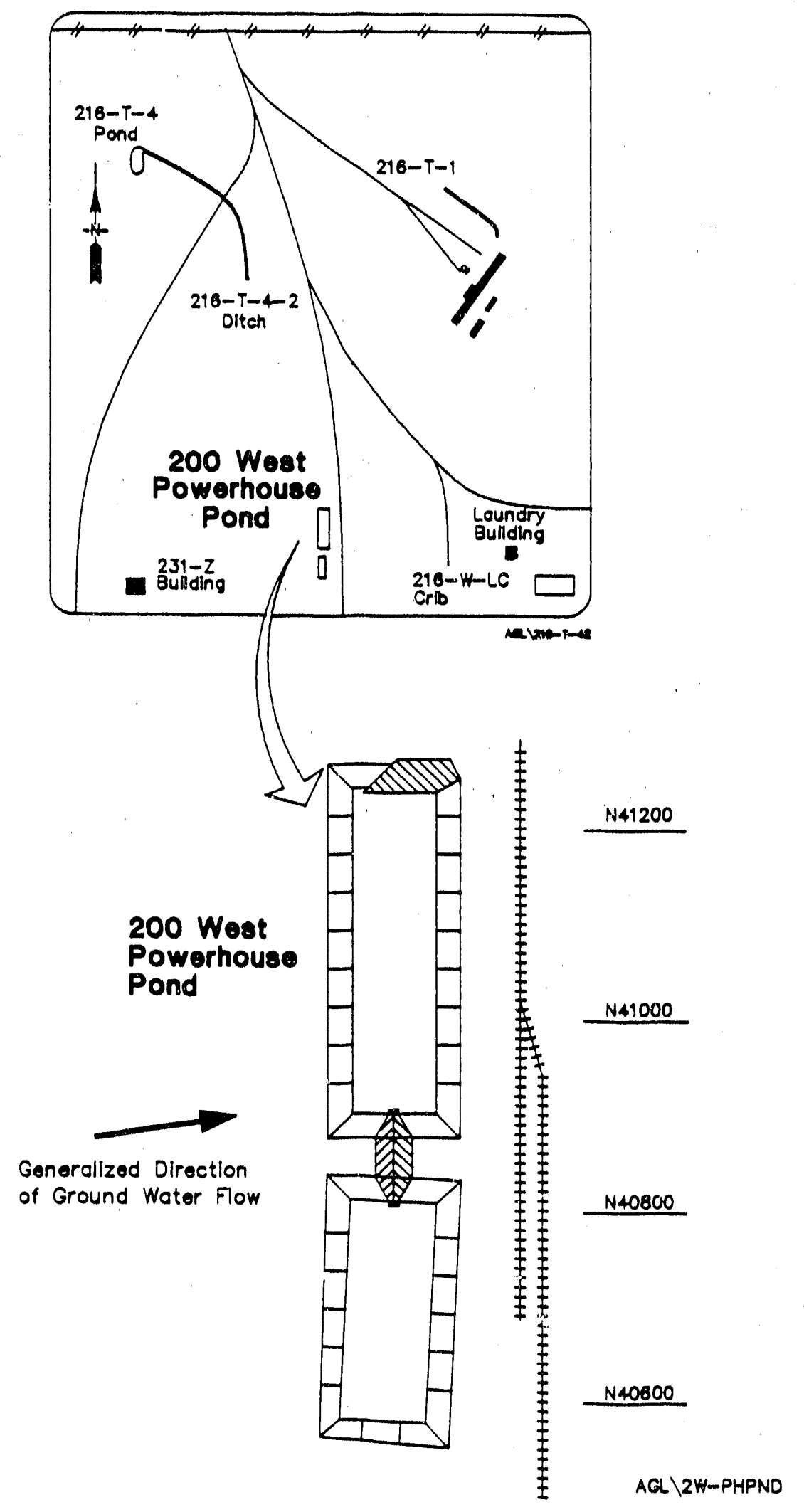

We11 Location and Site Map for 200-W Powerhouse Pond 


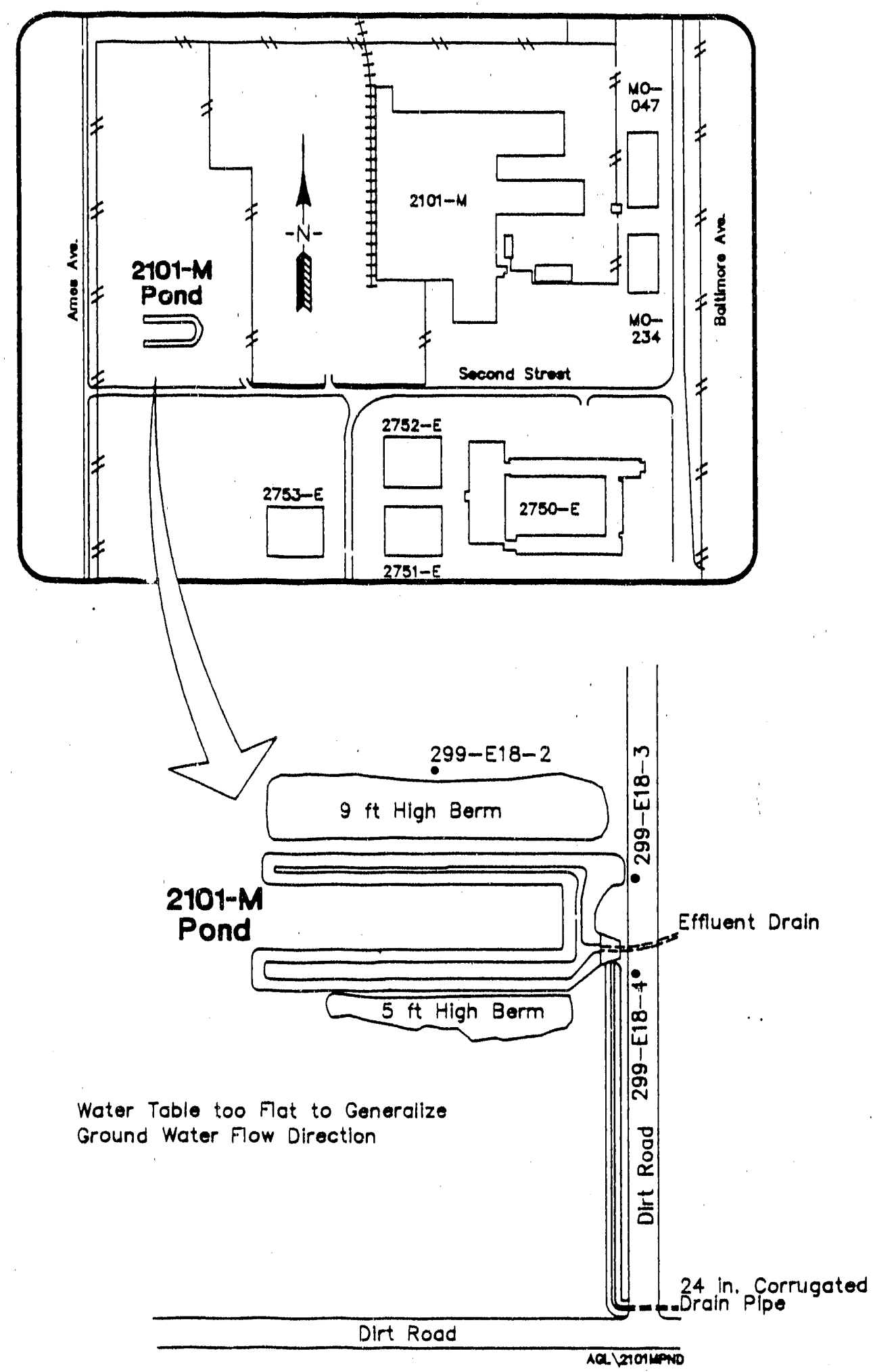

Well Location and Site Map for 2101-M Pond 


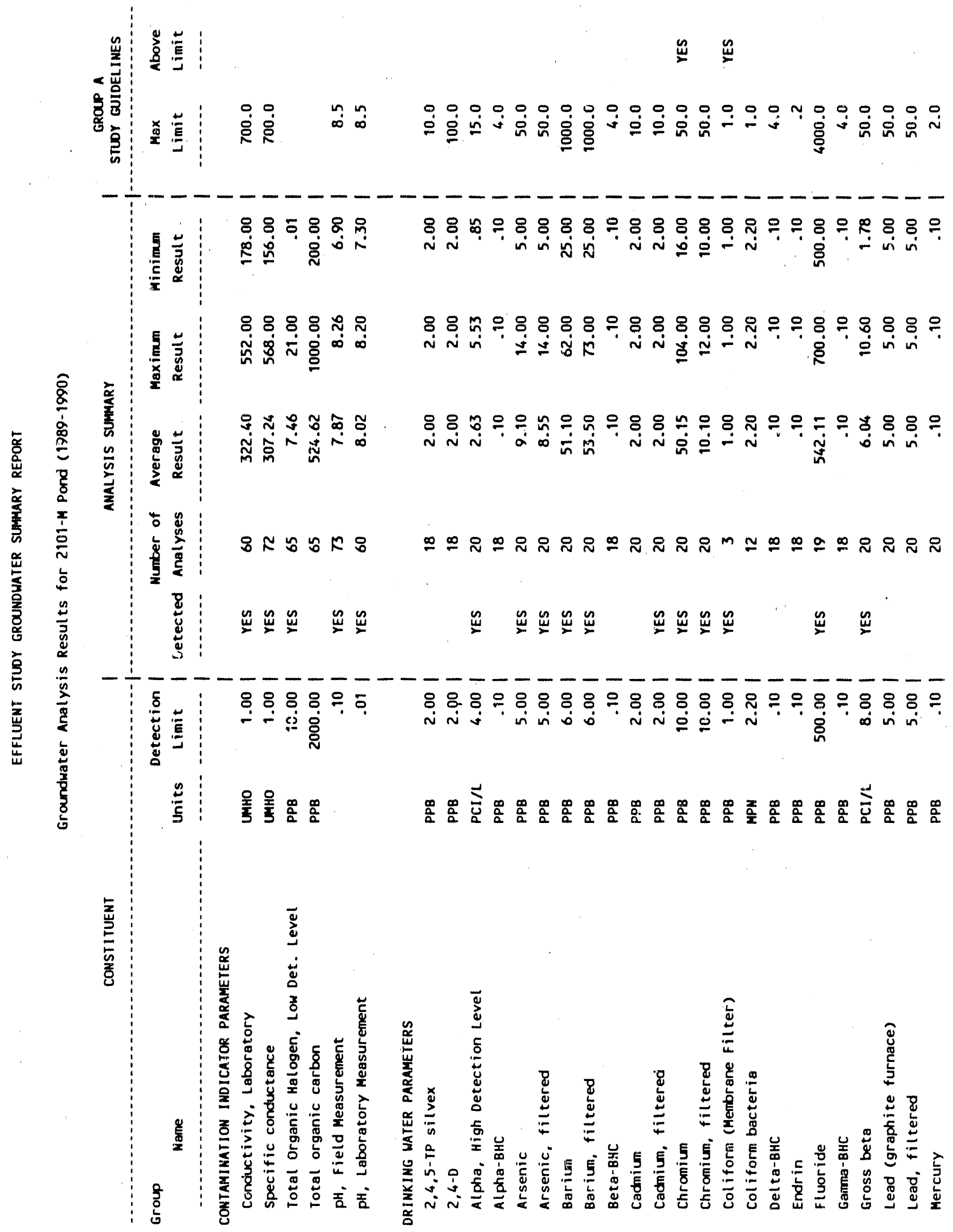


WHC-EP-0366

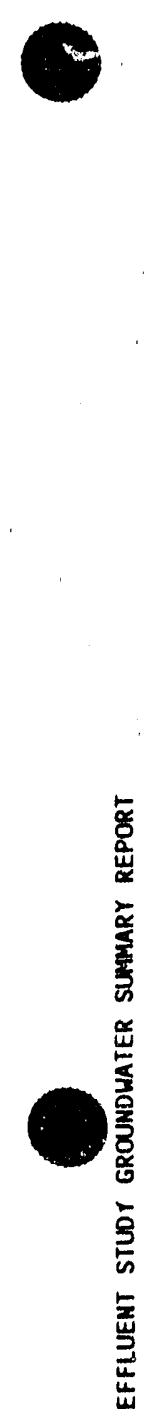

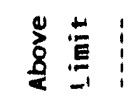

$\stackrel{\mathscr{m}}{\longleftarrow}$

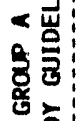

总

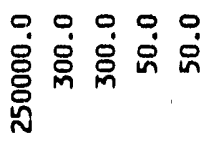

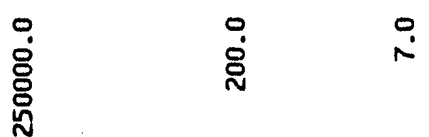

$-\frac{1}{1}$

童芦

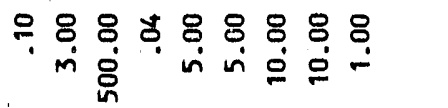

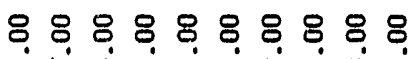

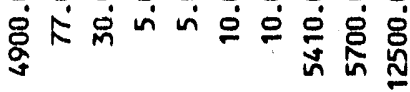

888888888

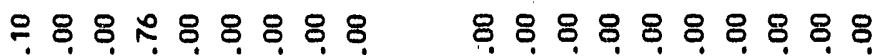

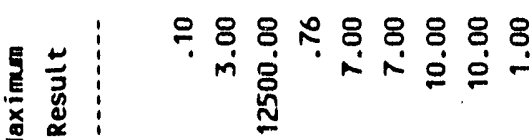

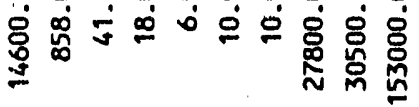

88888888

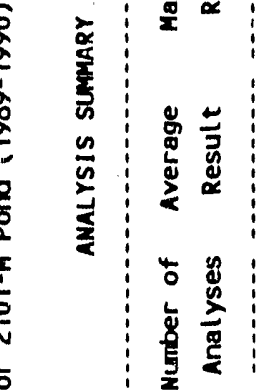

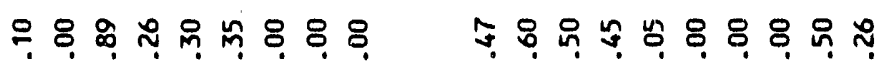

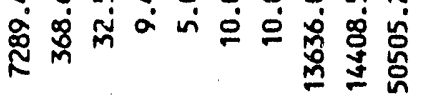

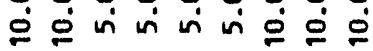

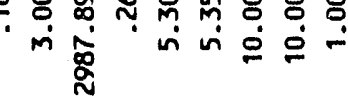

88888888 巳்

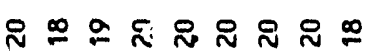

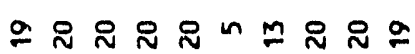

Un PNo gNA M

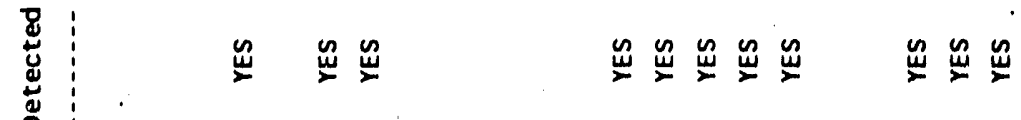

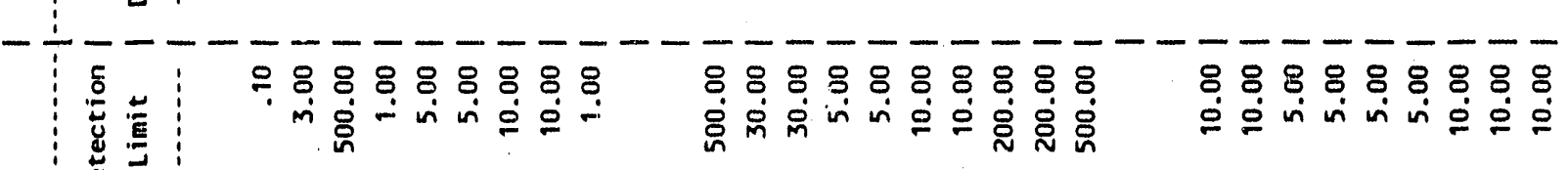

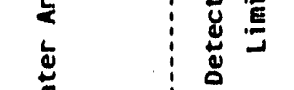

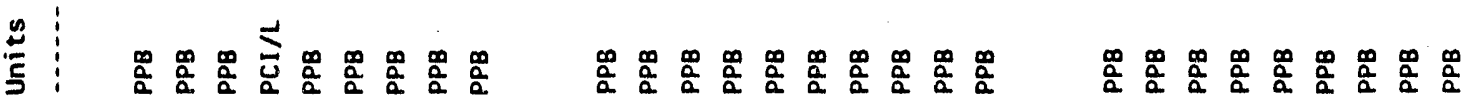

$$
\text { 告 }
$$

苞
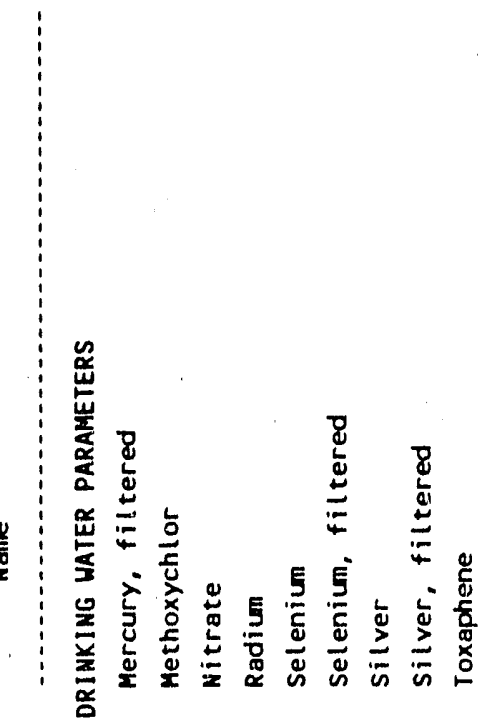

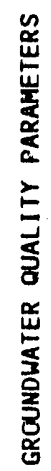
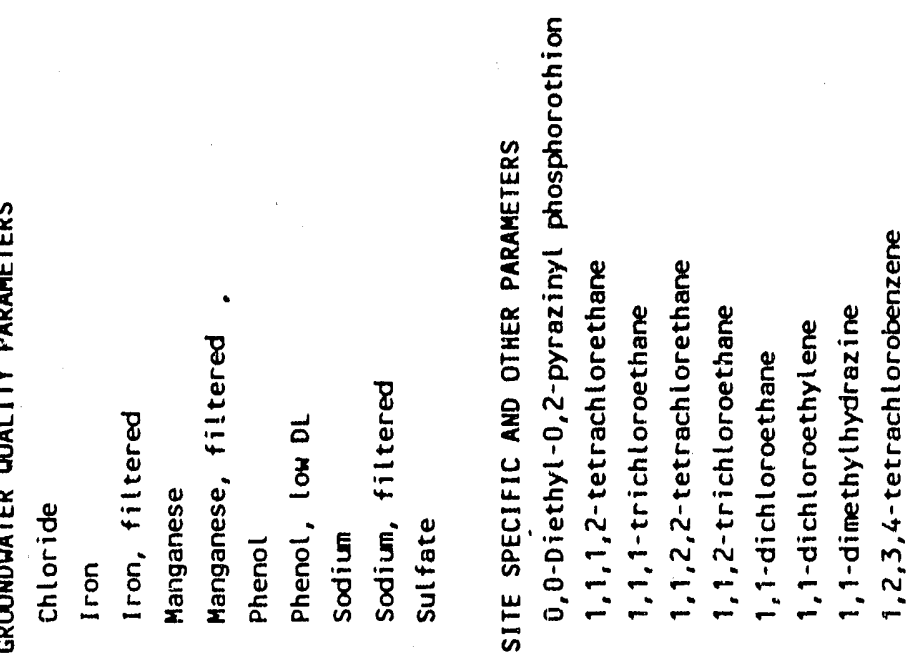
WHC-EP-0366
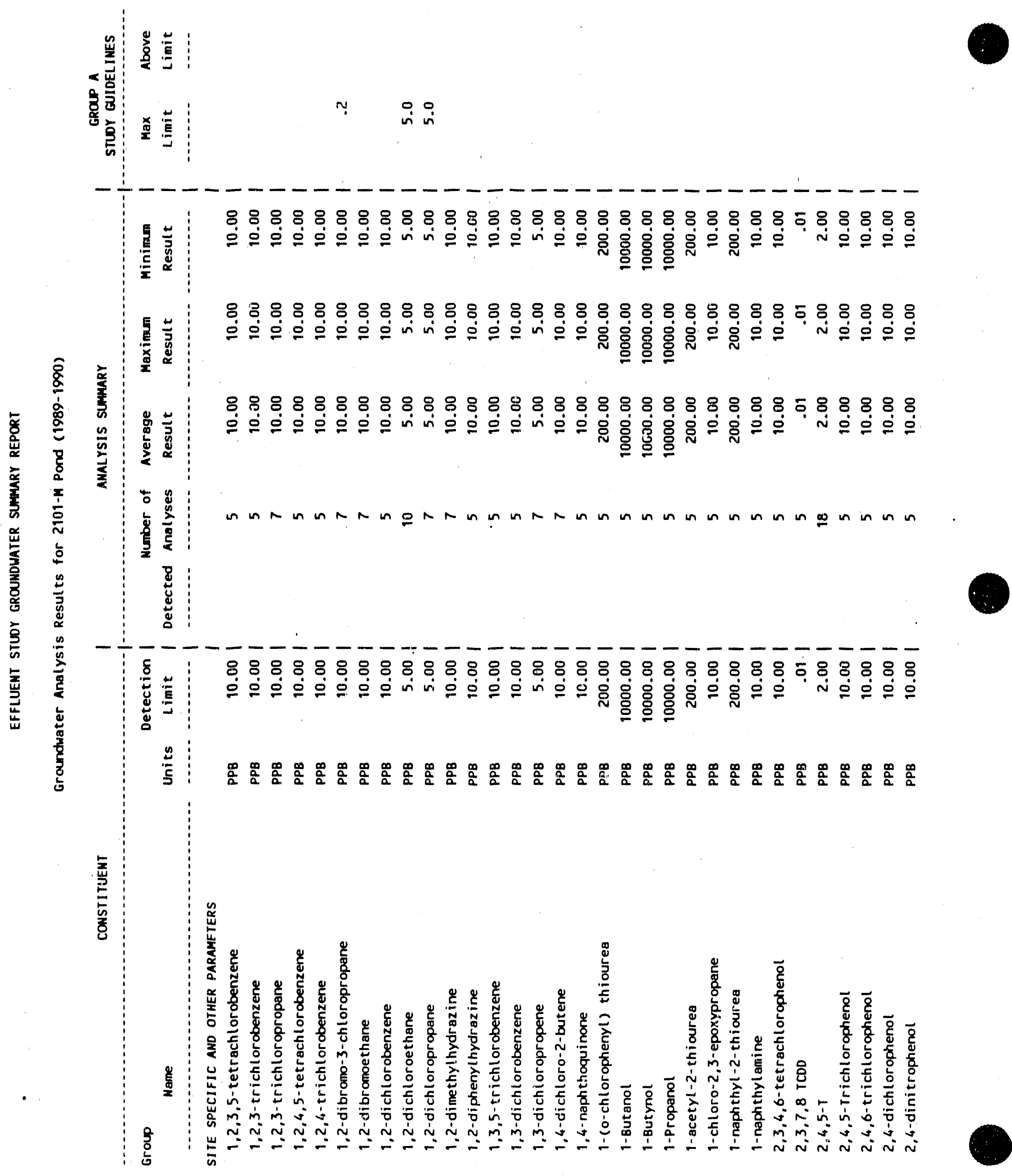
WHC-EP-0366

每:

s)

:

疽意

(

莀

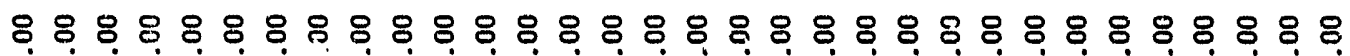

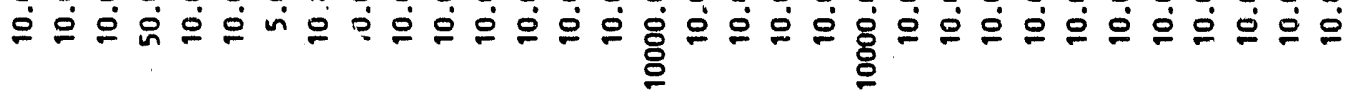

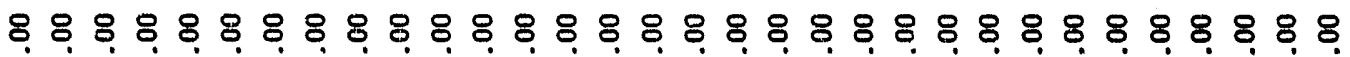

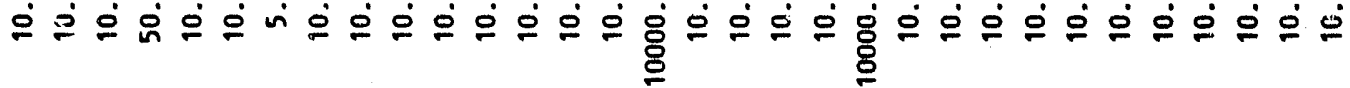

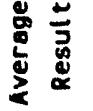

sis

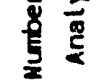

胥

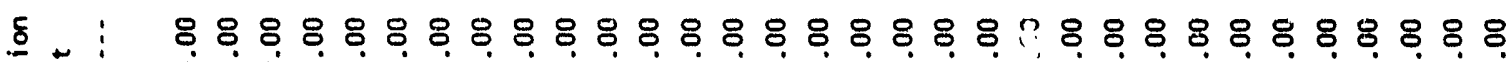

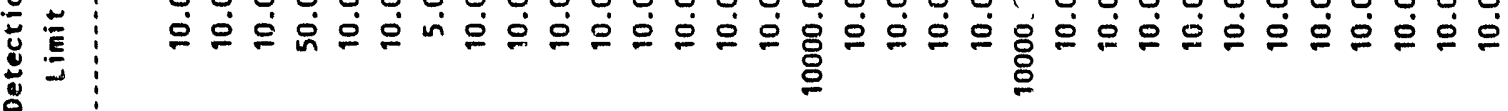

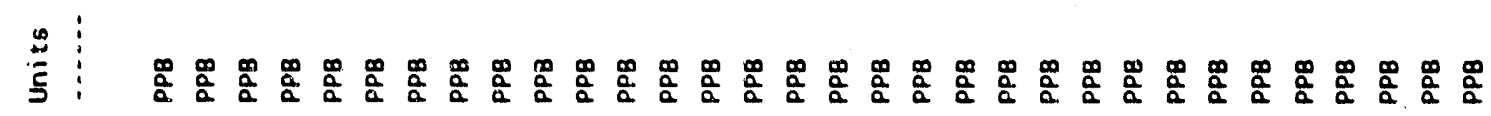

客

总

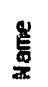

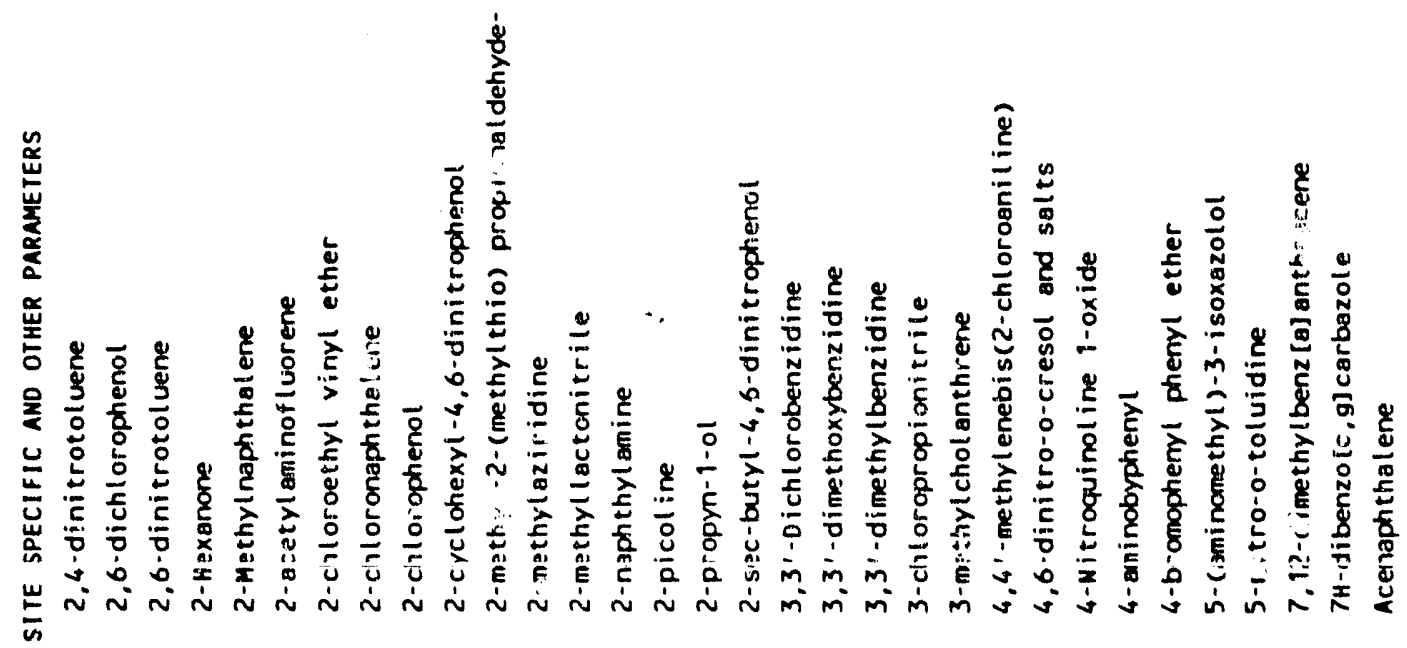


WHC -EP-0366
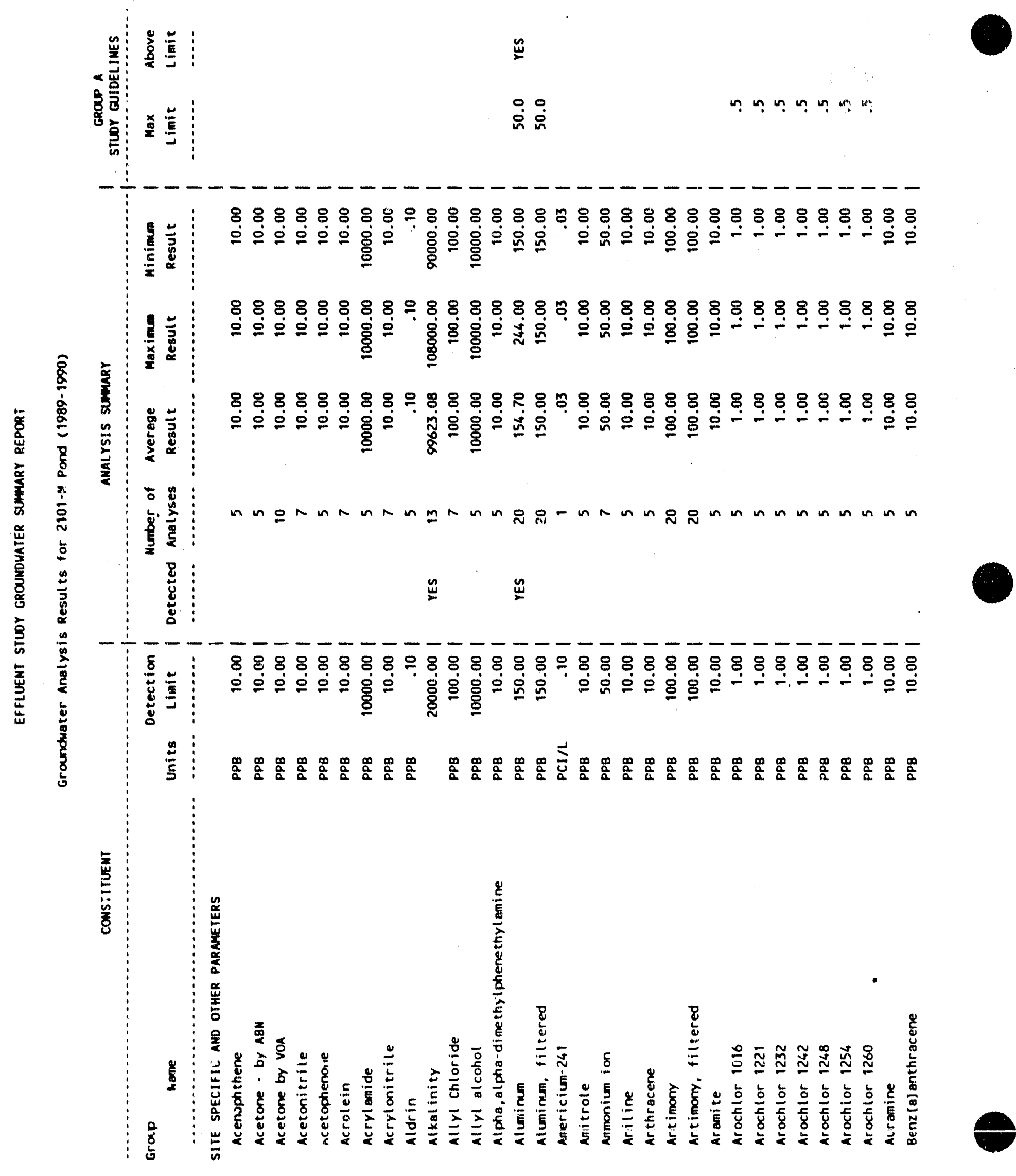


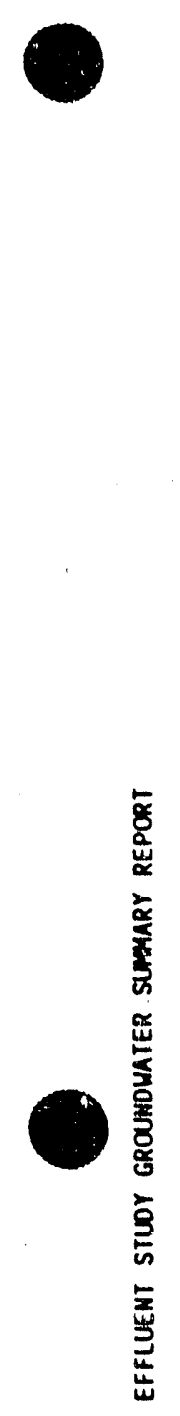

ifii:

த்

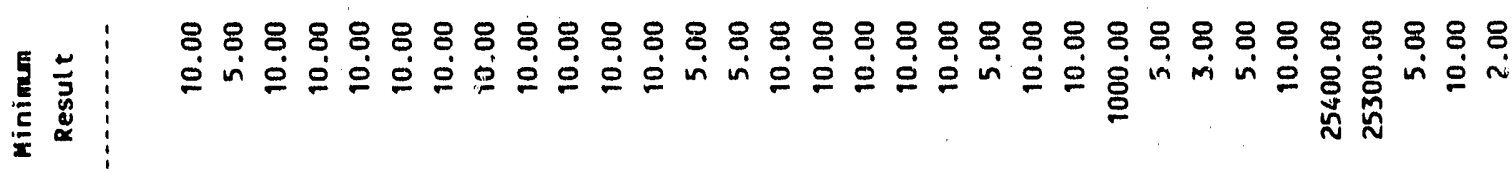

\begin{tabular}{l|ll}
\hline & 8
\end{tabular}

\&

:

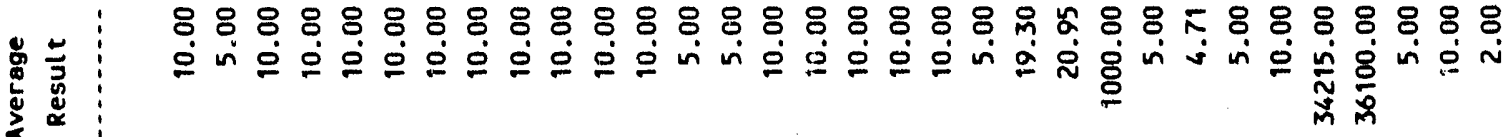

落

$8:$

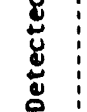

$\stackrel{n}{2}$

$\stackrel{\mathscr{m}}{\stackrel{4}{2}}$

$\stackrel{\mathscr{W}}{\underset{w}{2}}$

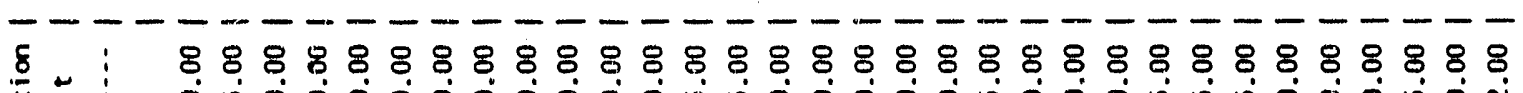
速至

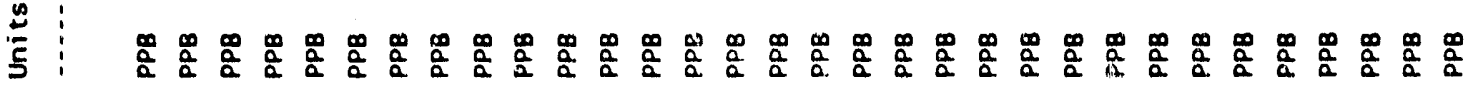

蜜

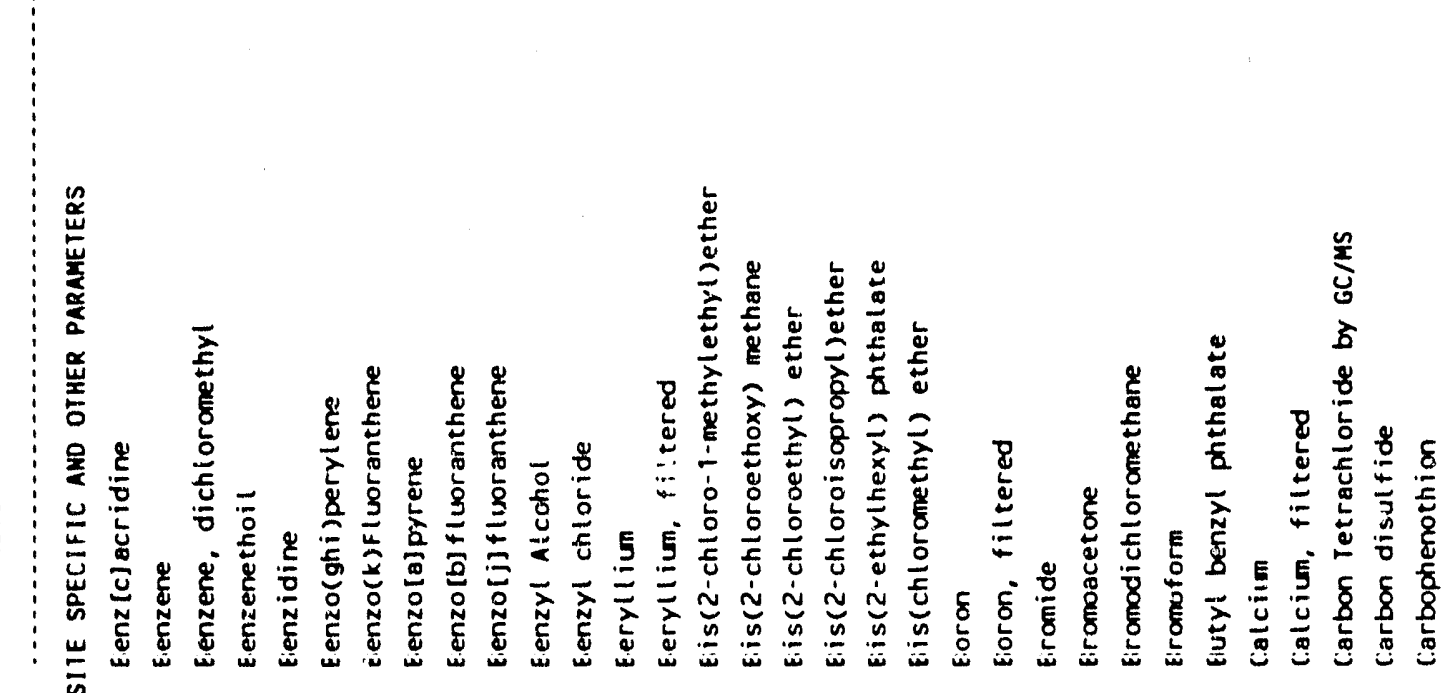


WHC-EP-0366

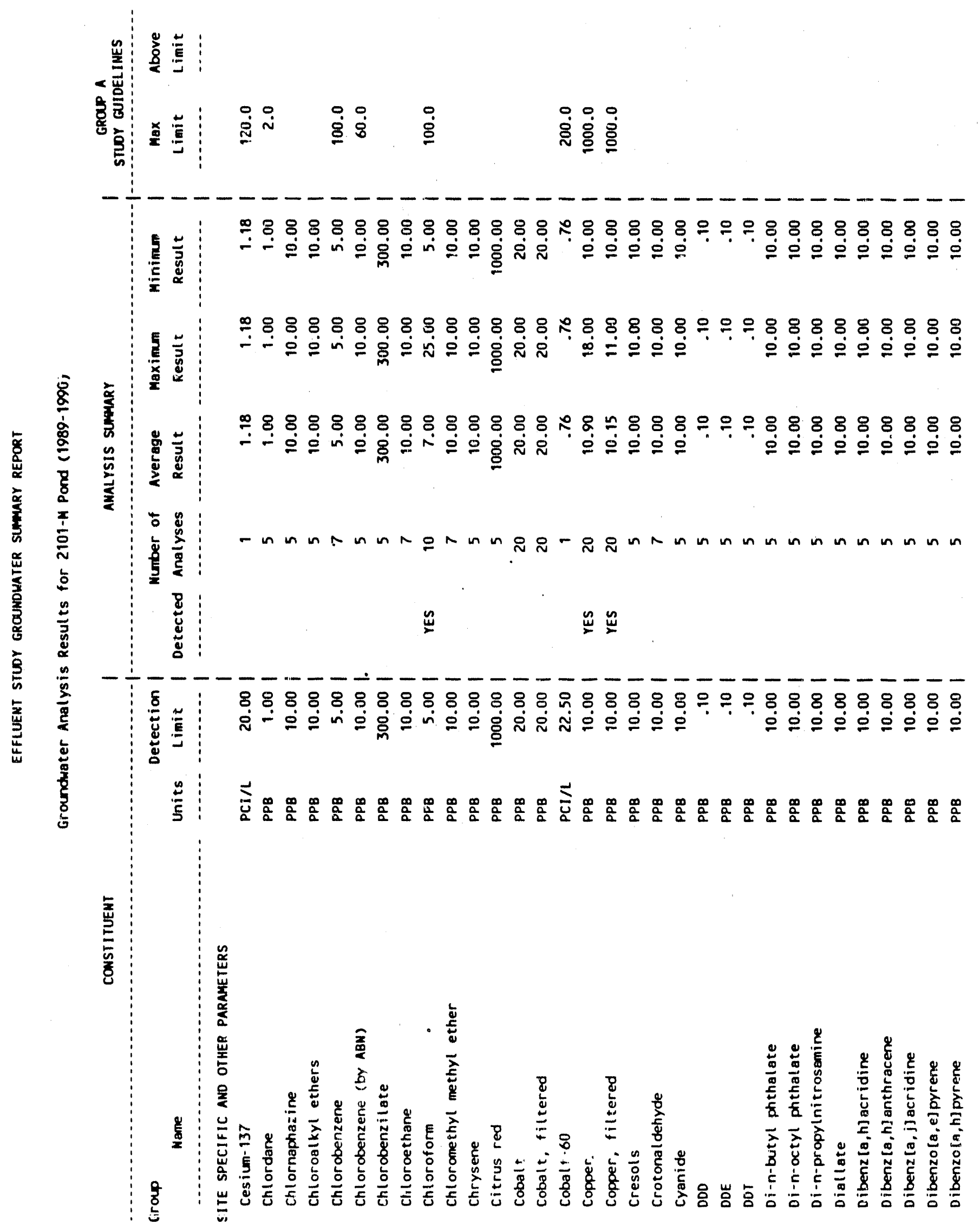


WHC-EP-0366

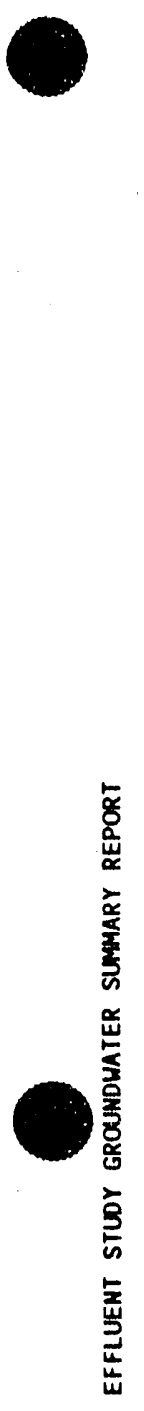

量喜

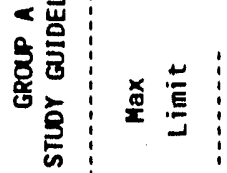

ஜ̊.

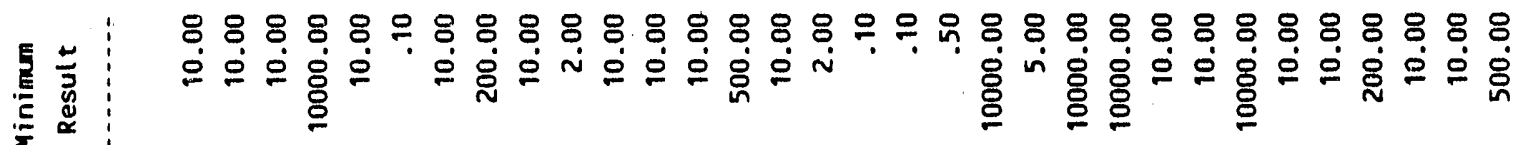

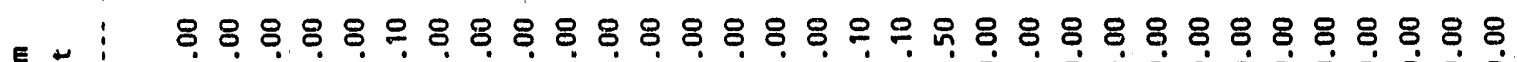

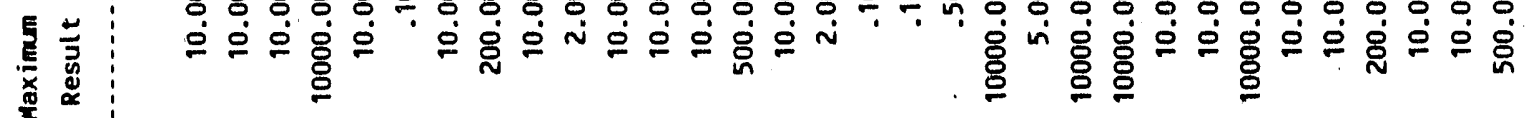

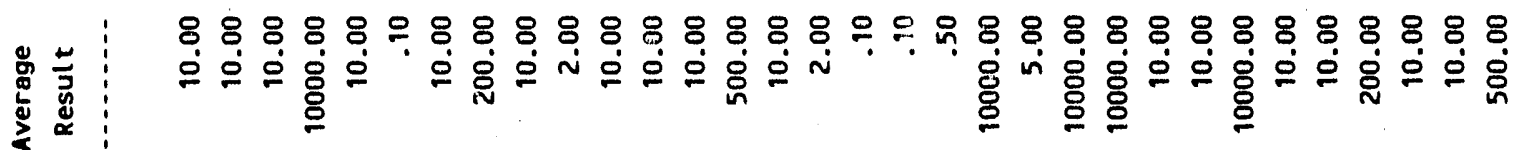

to 要

导:

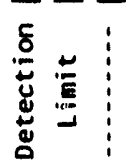

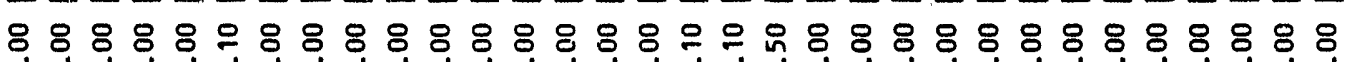

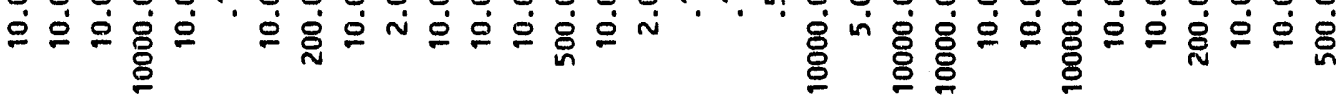

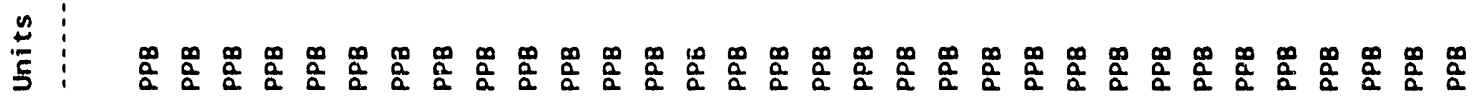

㟒

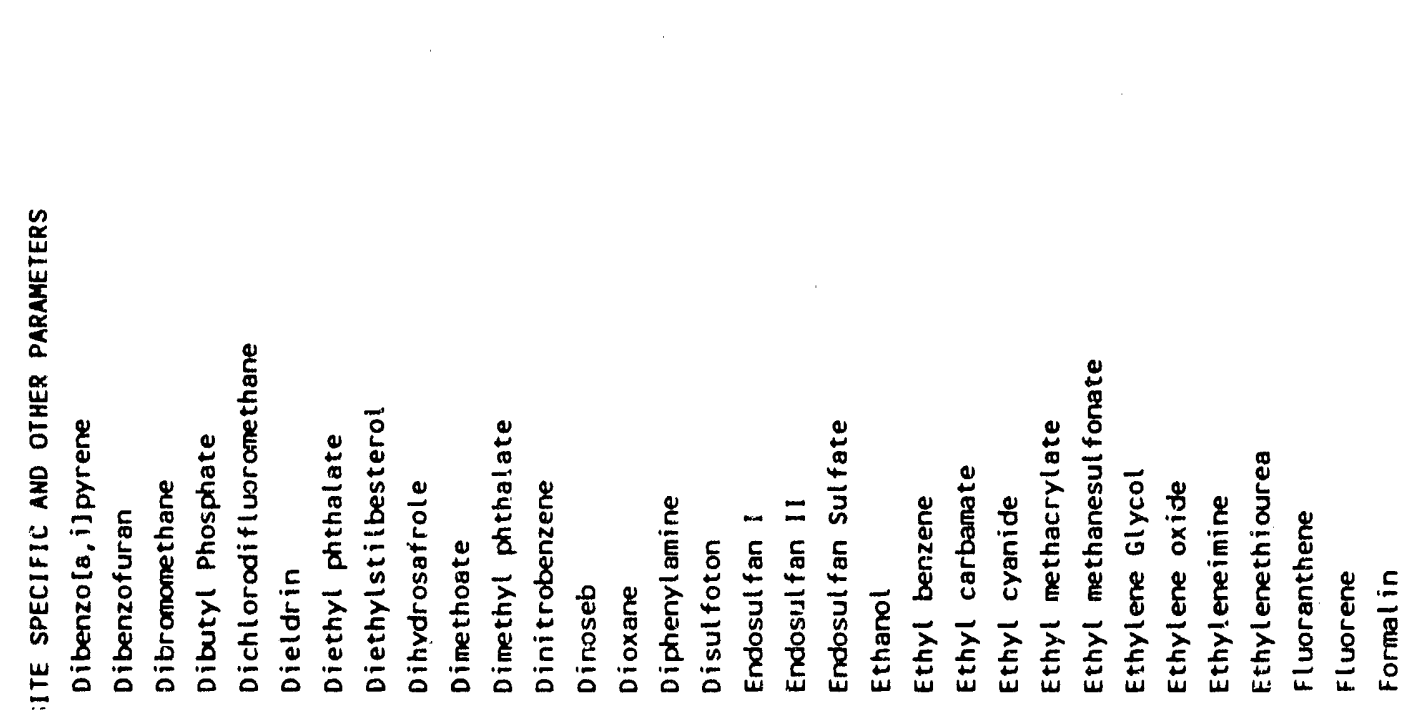




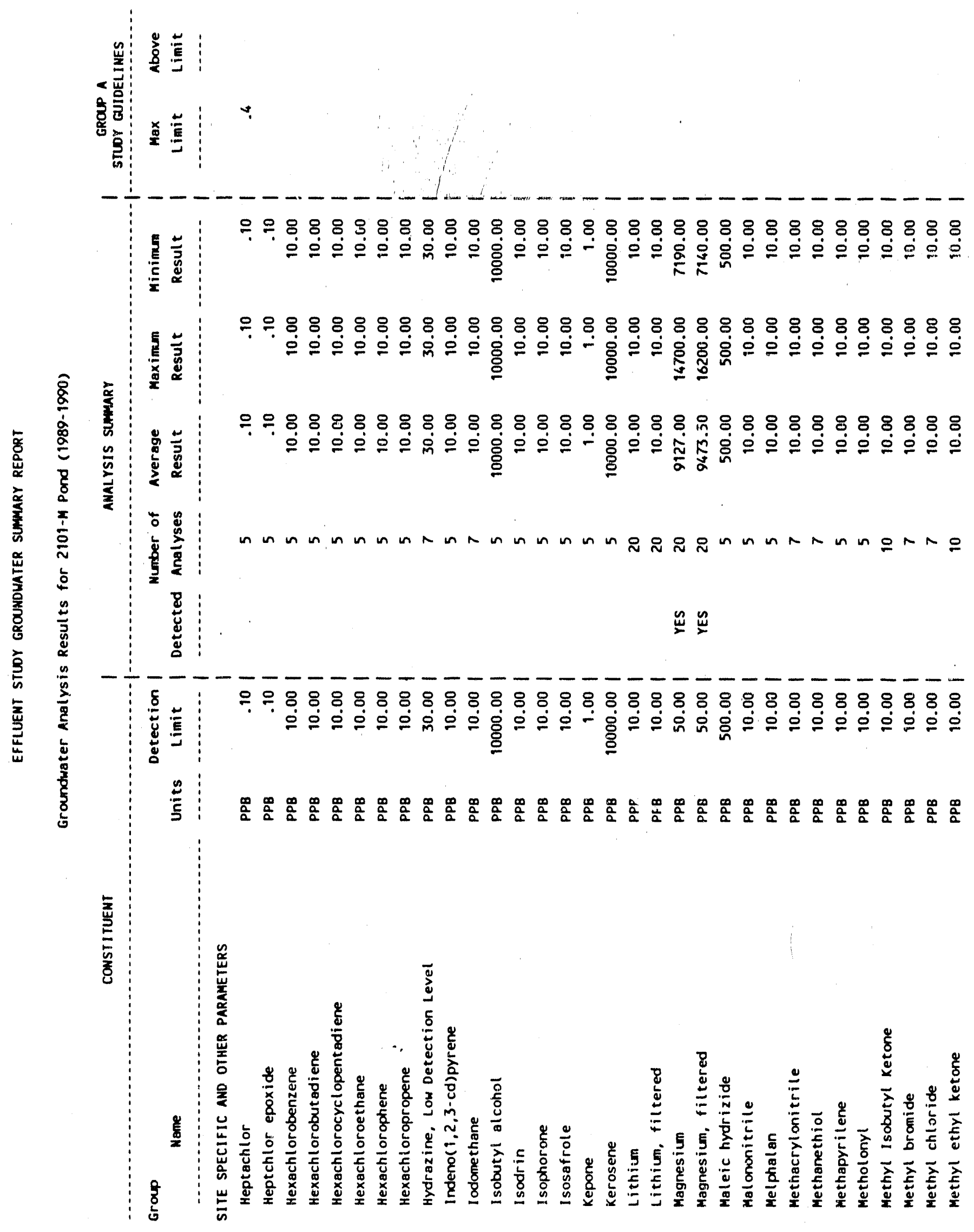


WHC-EP-0366

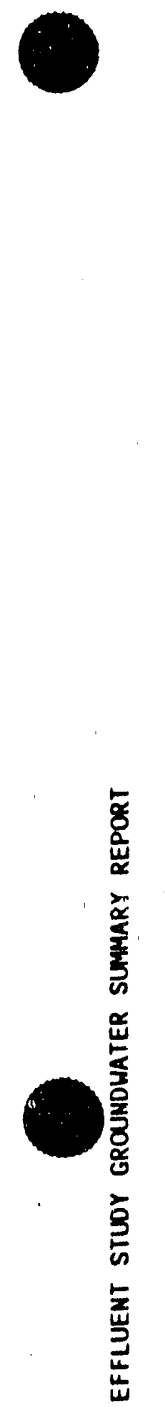

繁喜

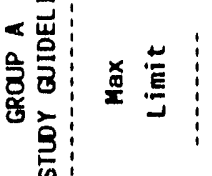

品

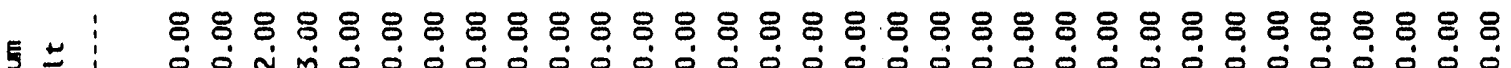
递

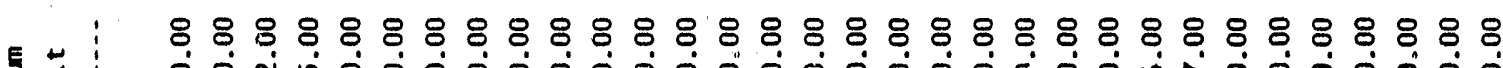

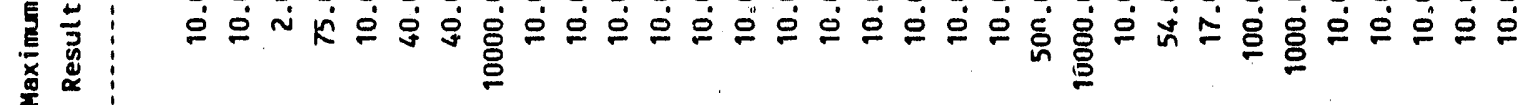

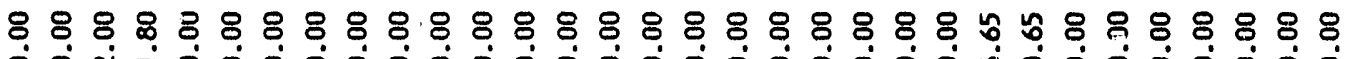

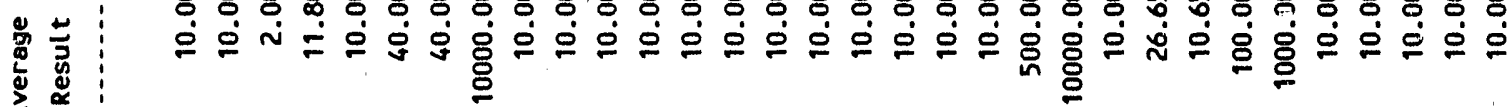

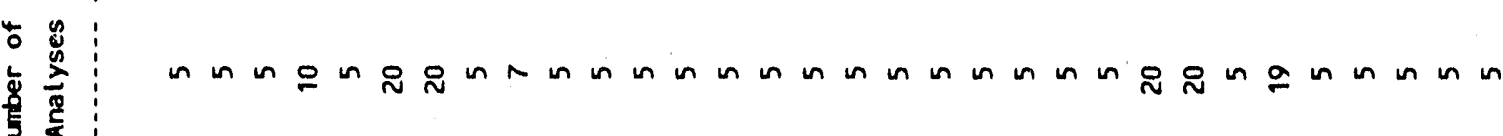

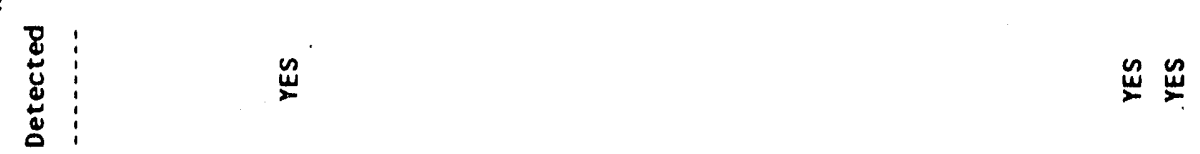

焉

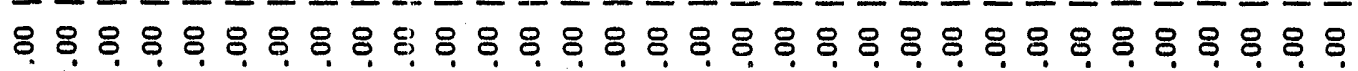
ஸ் ஸ்

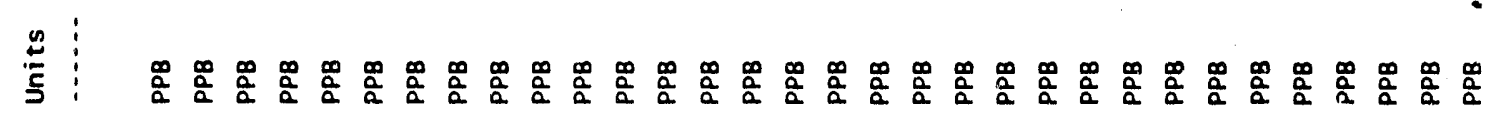

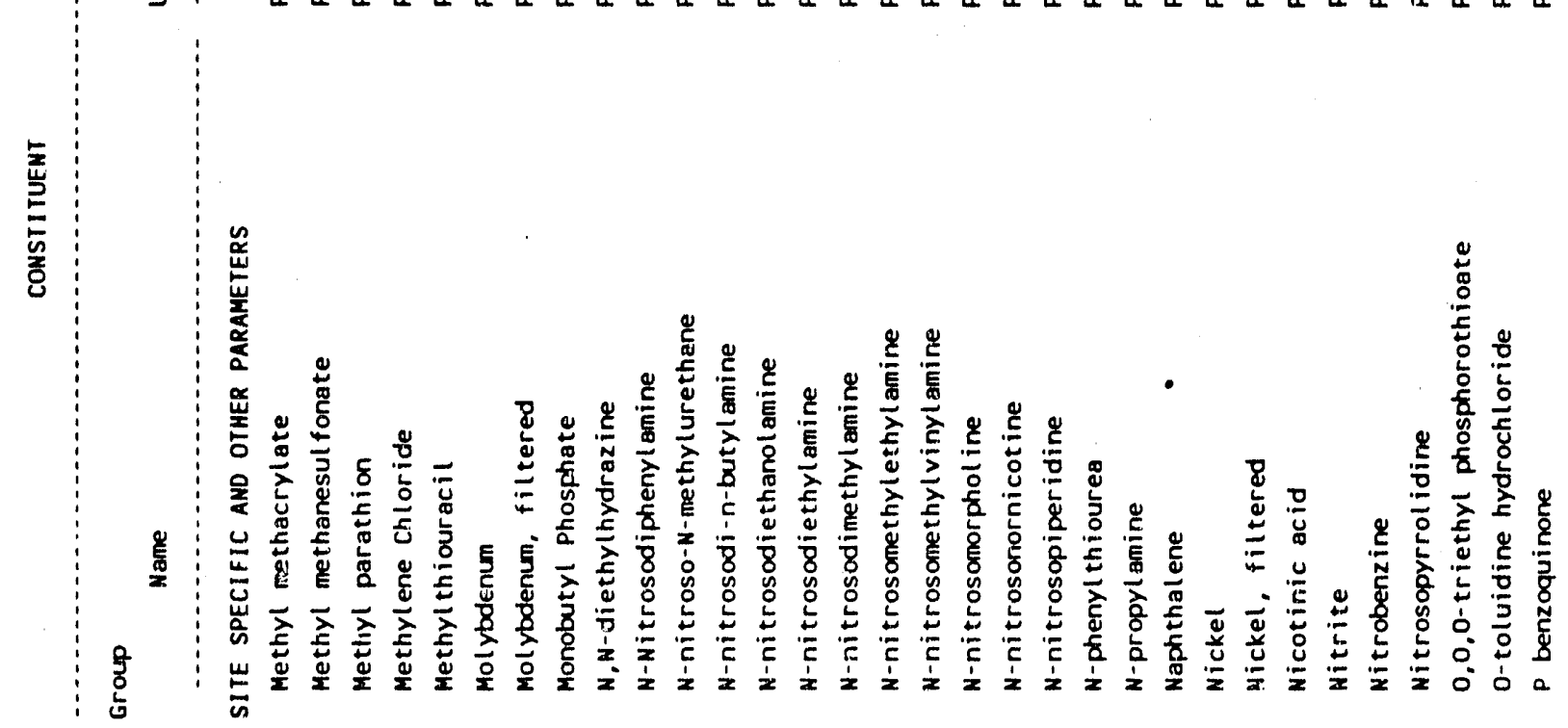




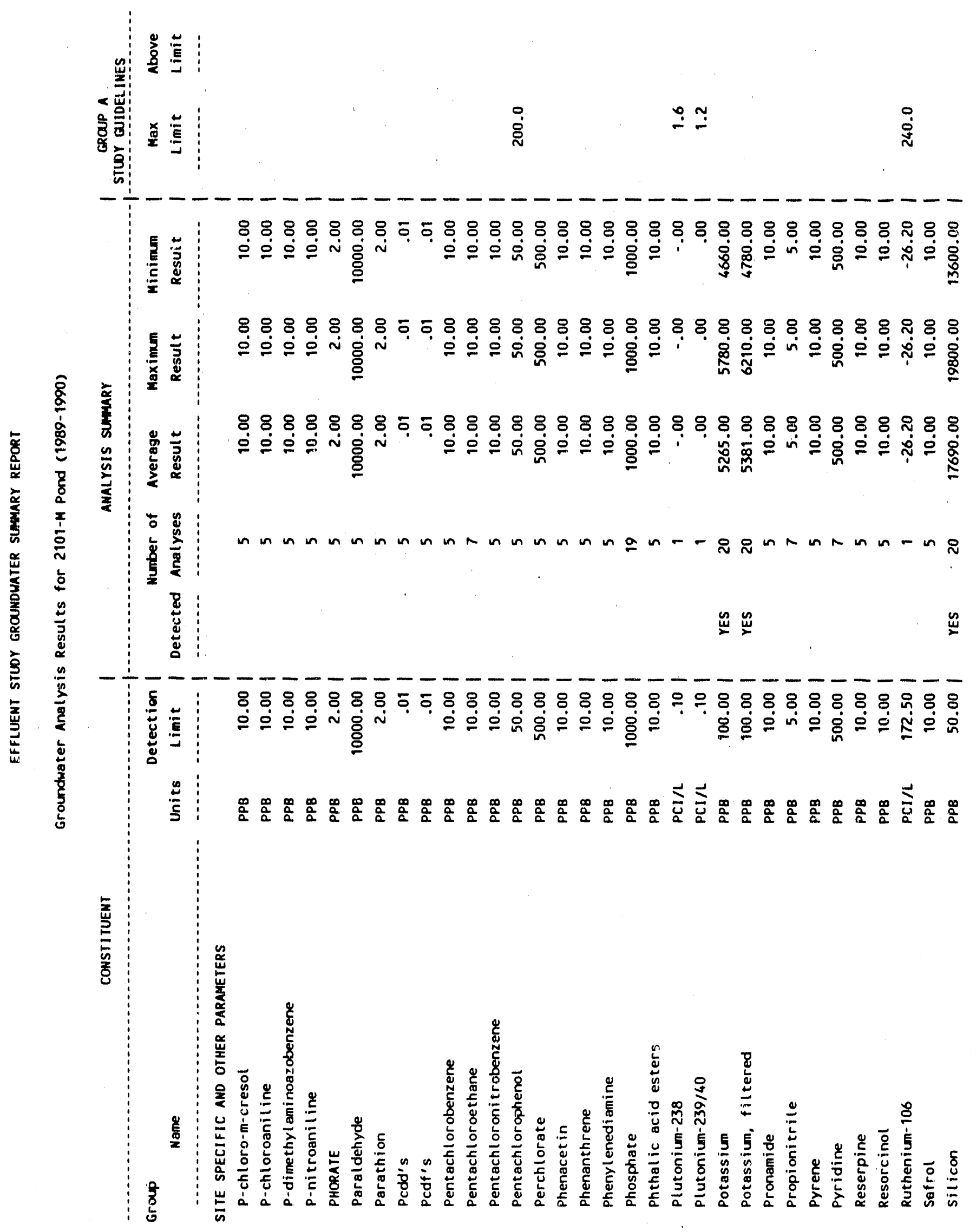


蒙

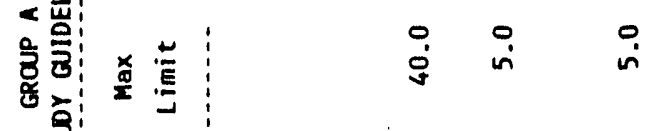

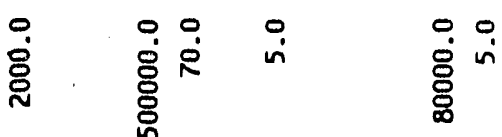

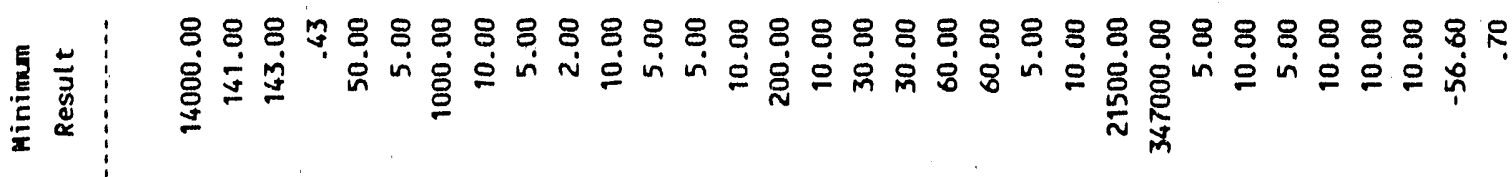

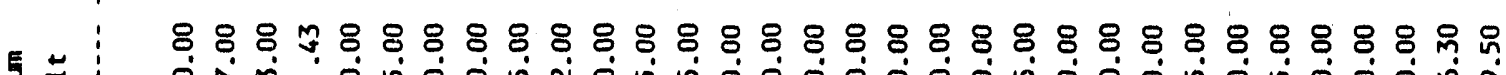

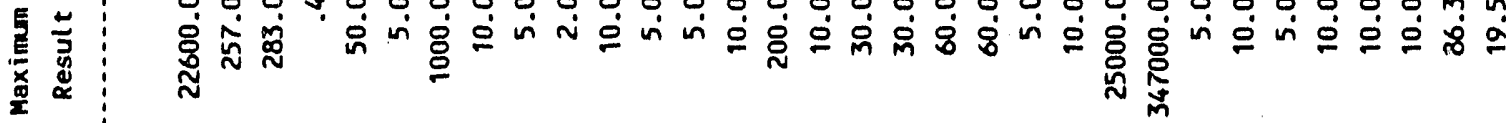

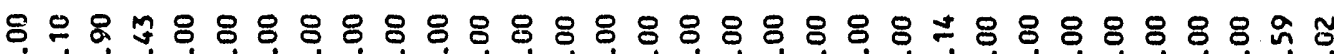
若苟

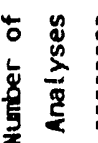

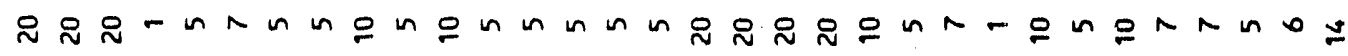

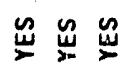

$\stackrel{\mathscr{w}}{\underline{w}}$

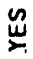

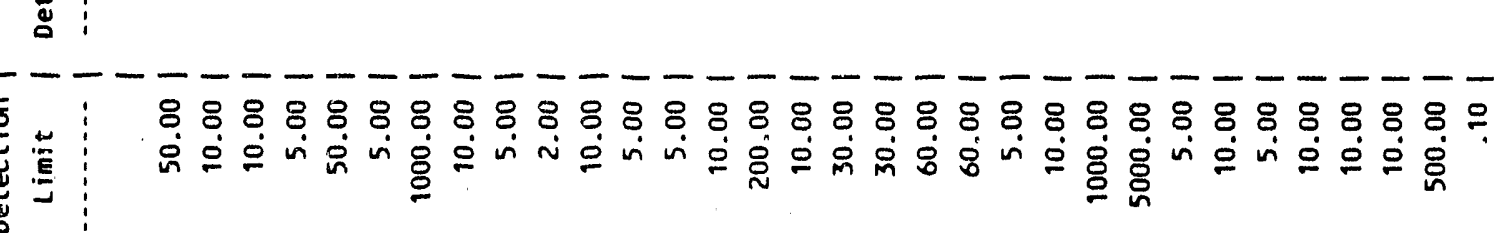

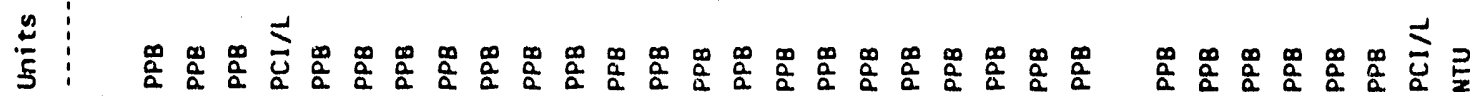

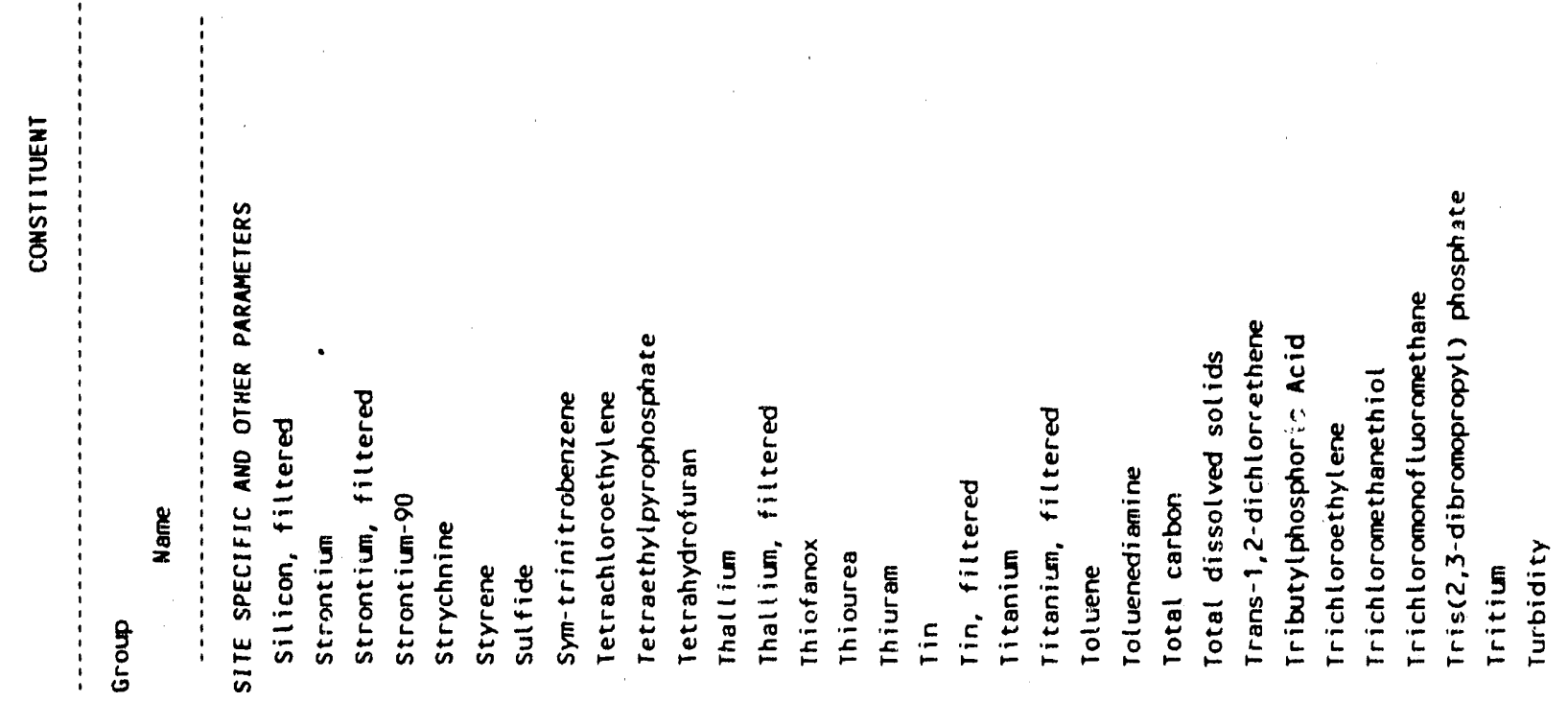


WHC-EP-0366

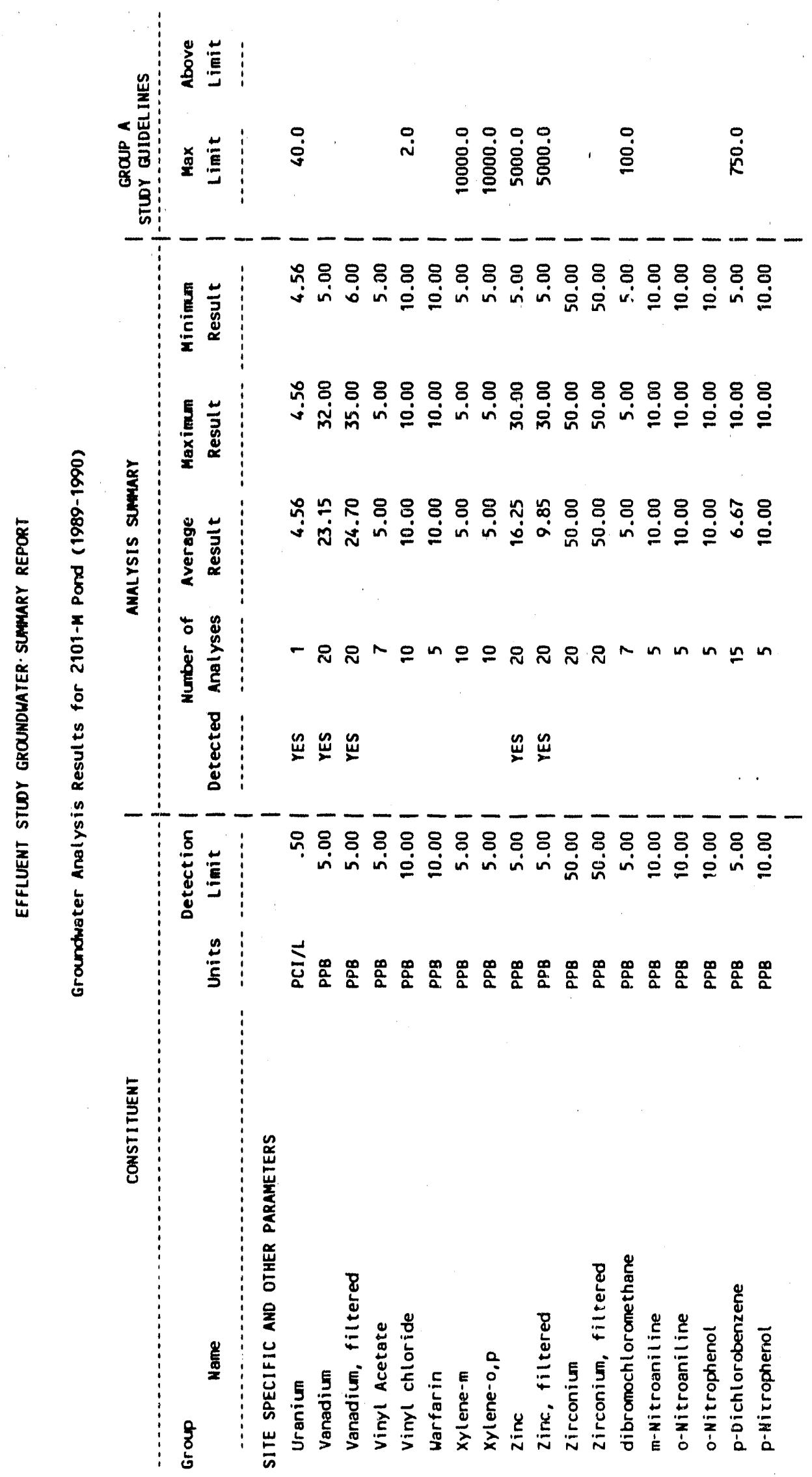


WHC-EP- 0366

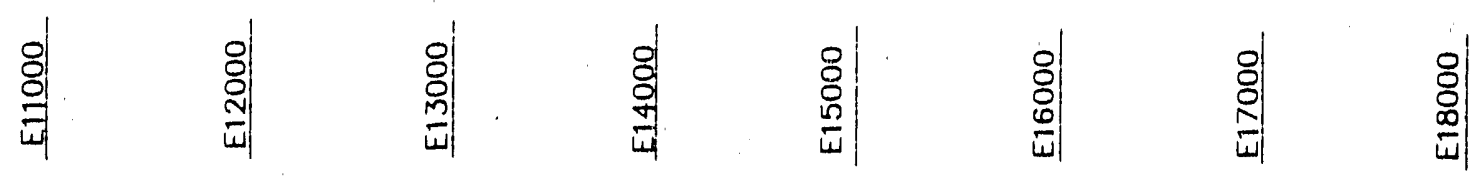

-699-S19-E13

N60000

- Ground Water

Monitoring Well

(Well Prefix 399-Xx-Xx)

- $1-18 \mathrm{~A}, 18 \mathrm{~B}, 18 \mathrm{C}$

N59000

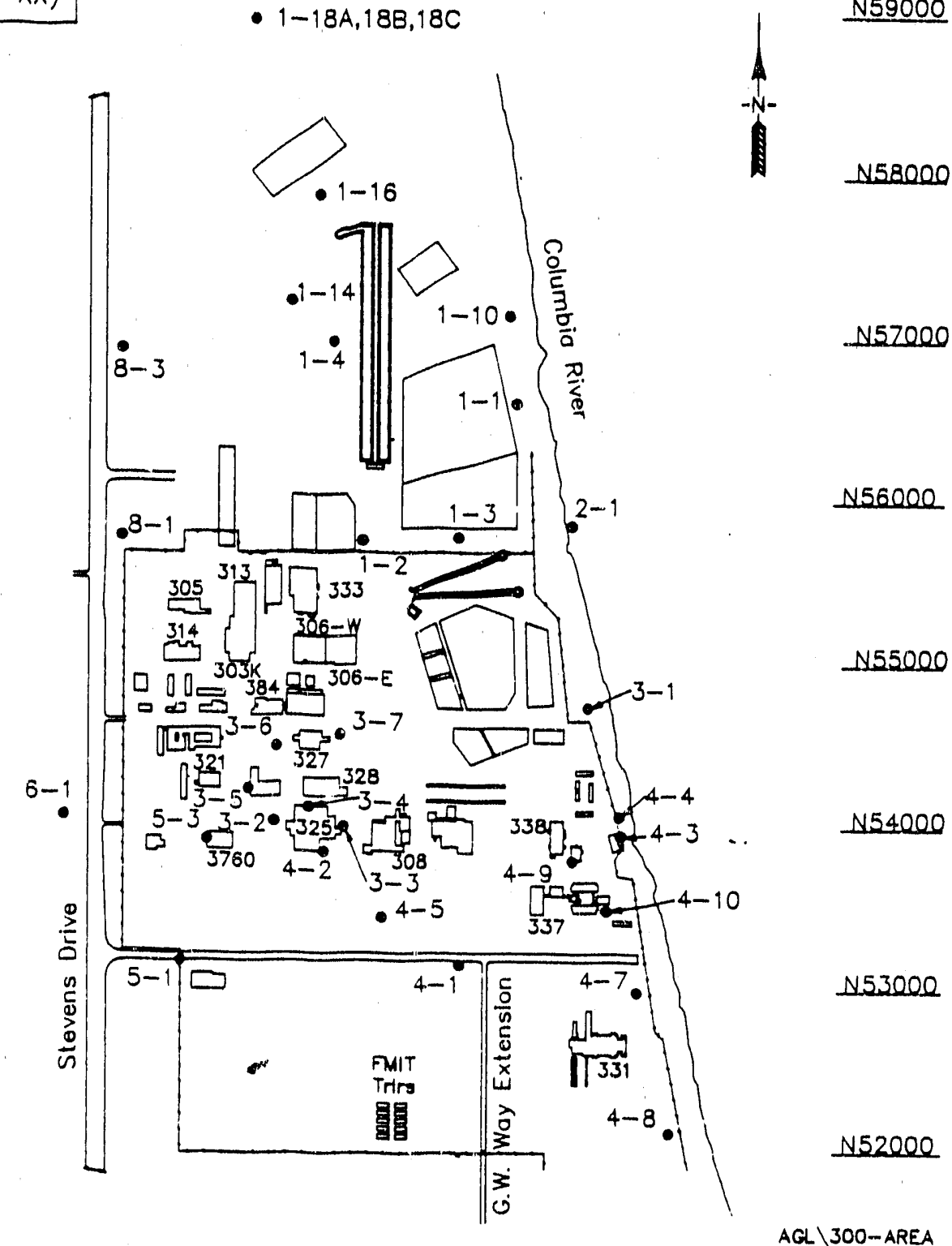

$+58000$

$\$ 57000$
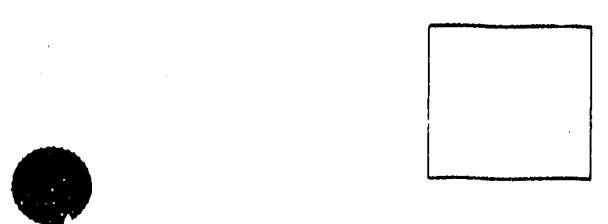

$\square$

8-2

. 5-2

Well Location and Site Map for 300 Area Process Trenches 
WHC-EP-0366

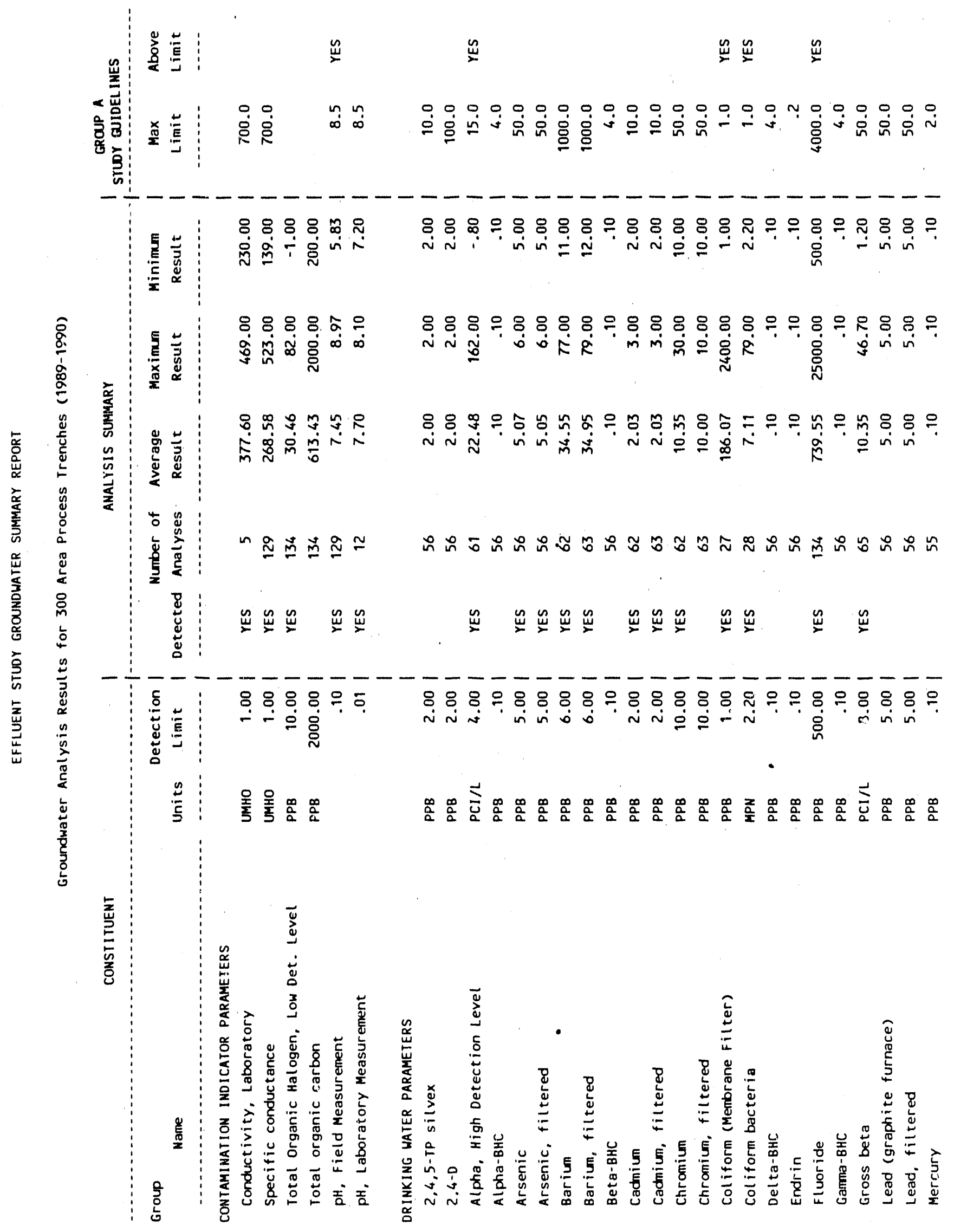


WHC-EP-0366

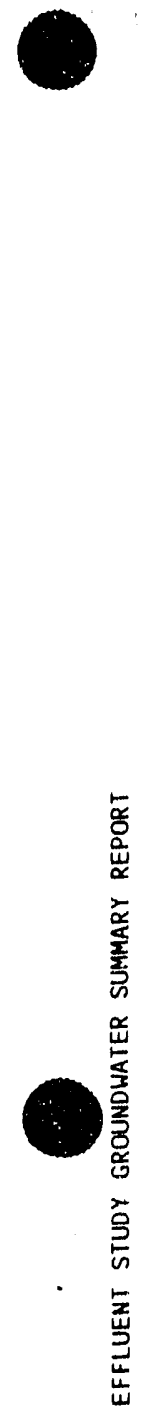

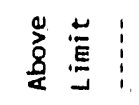

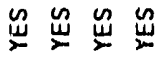

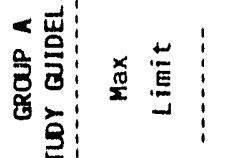

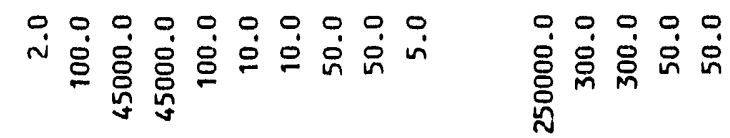

宫

$\stackrel{\dot{0}}{i} \stackrel{i}{i}$

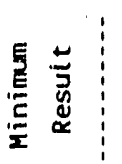

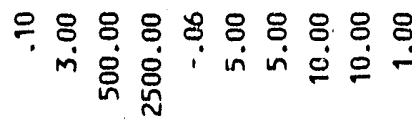

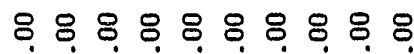

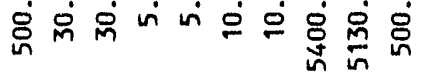

88888888

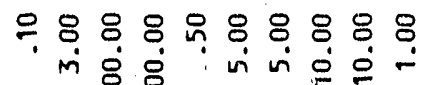

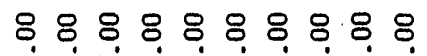

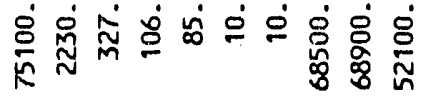

88888888

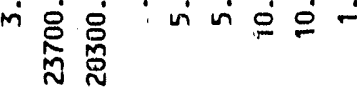

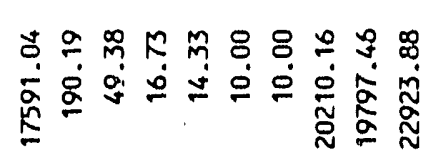

88888888

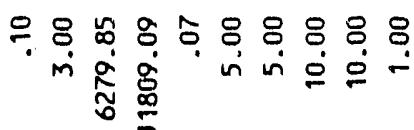

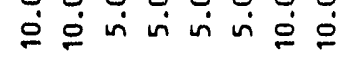

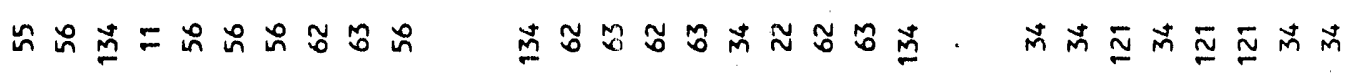

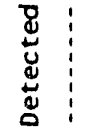
$\stackrel{\oiiint}{\check{\Psi}}$

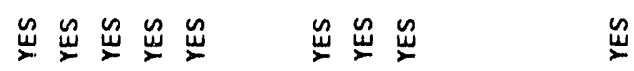

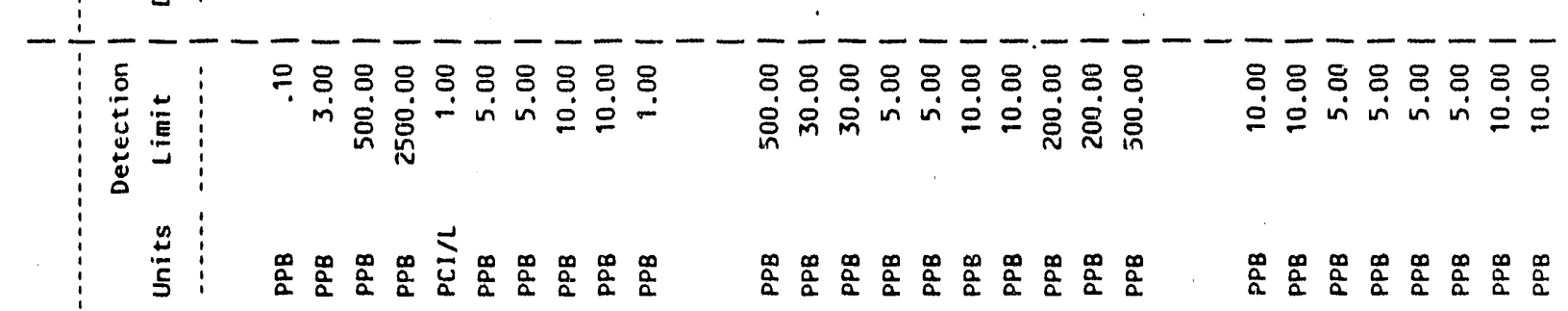

苨

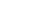
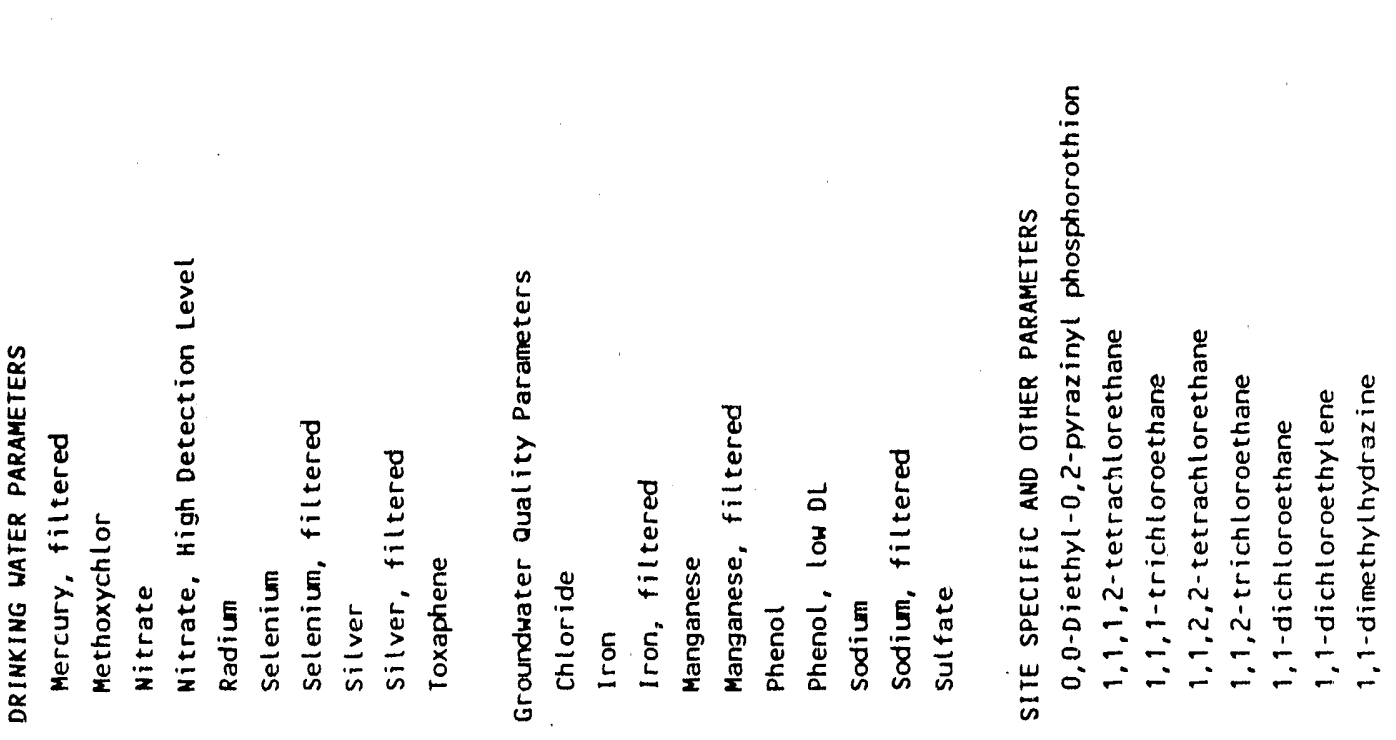


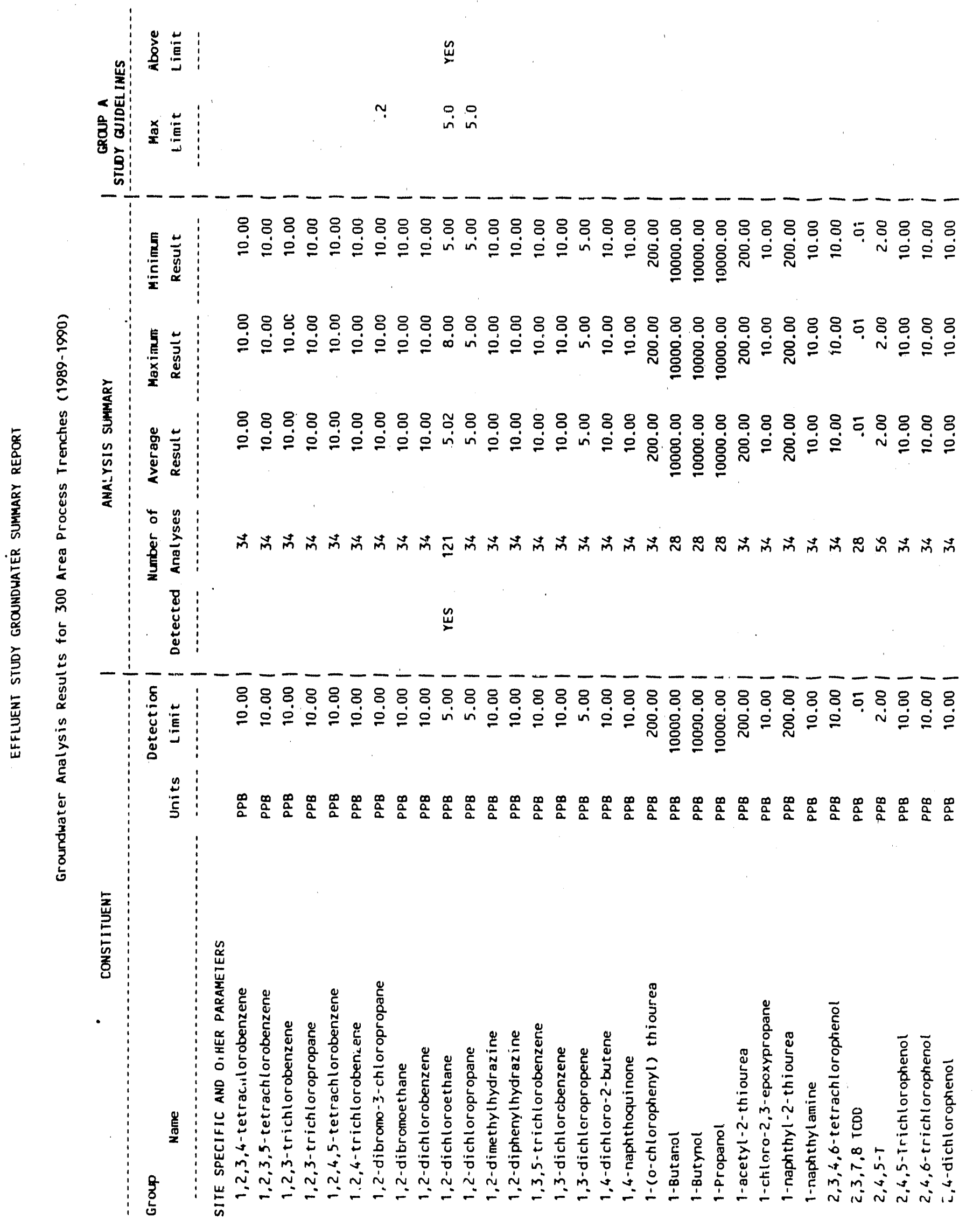


WHC - EP- 0366

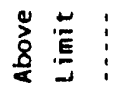

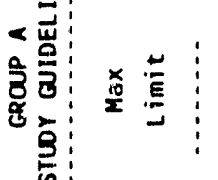

$E$
$\frac{E}{5}$
$\frac{5}{x}$

88888888888888888888888888888888

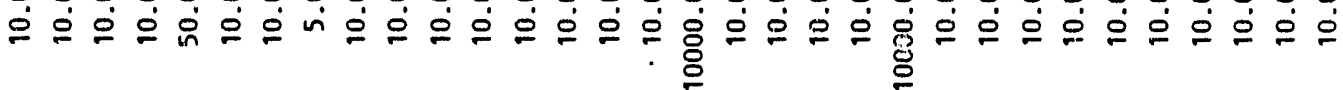

88888888888888888888888888888.88

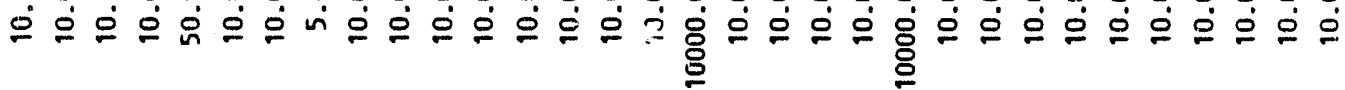

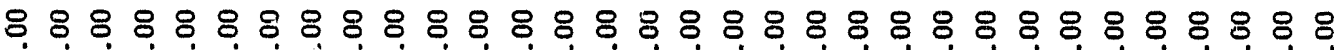

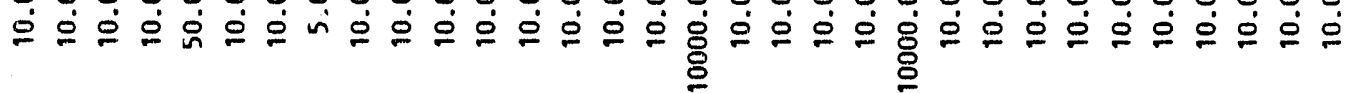
崖苛

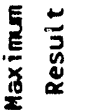

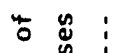

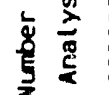

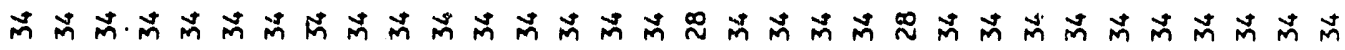

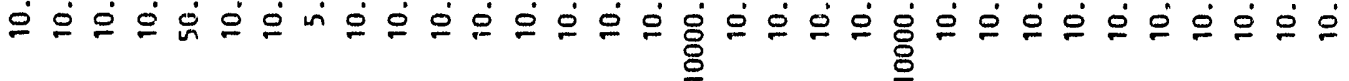

$\stackrel{n}{=}$

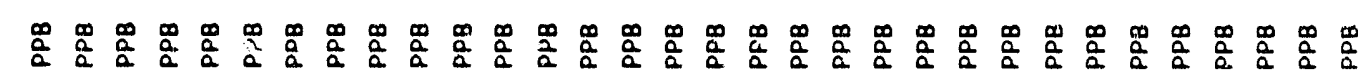

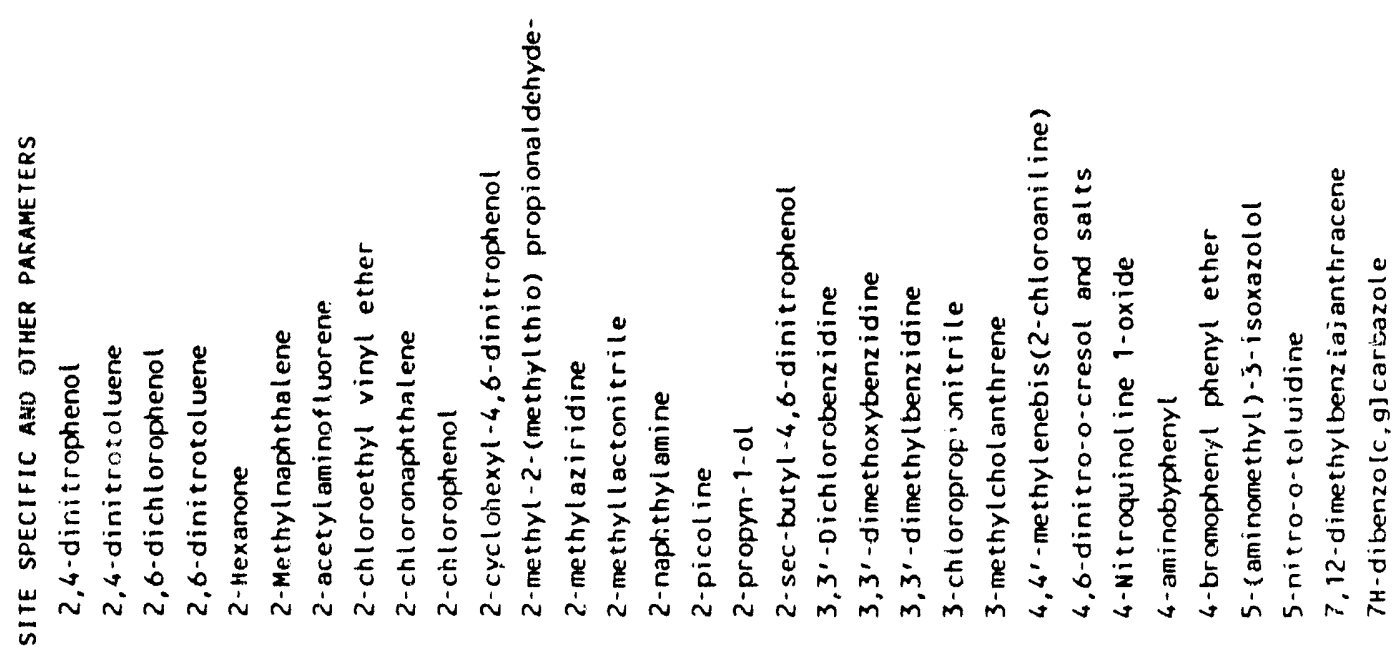




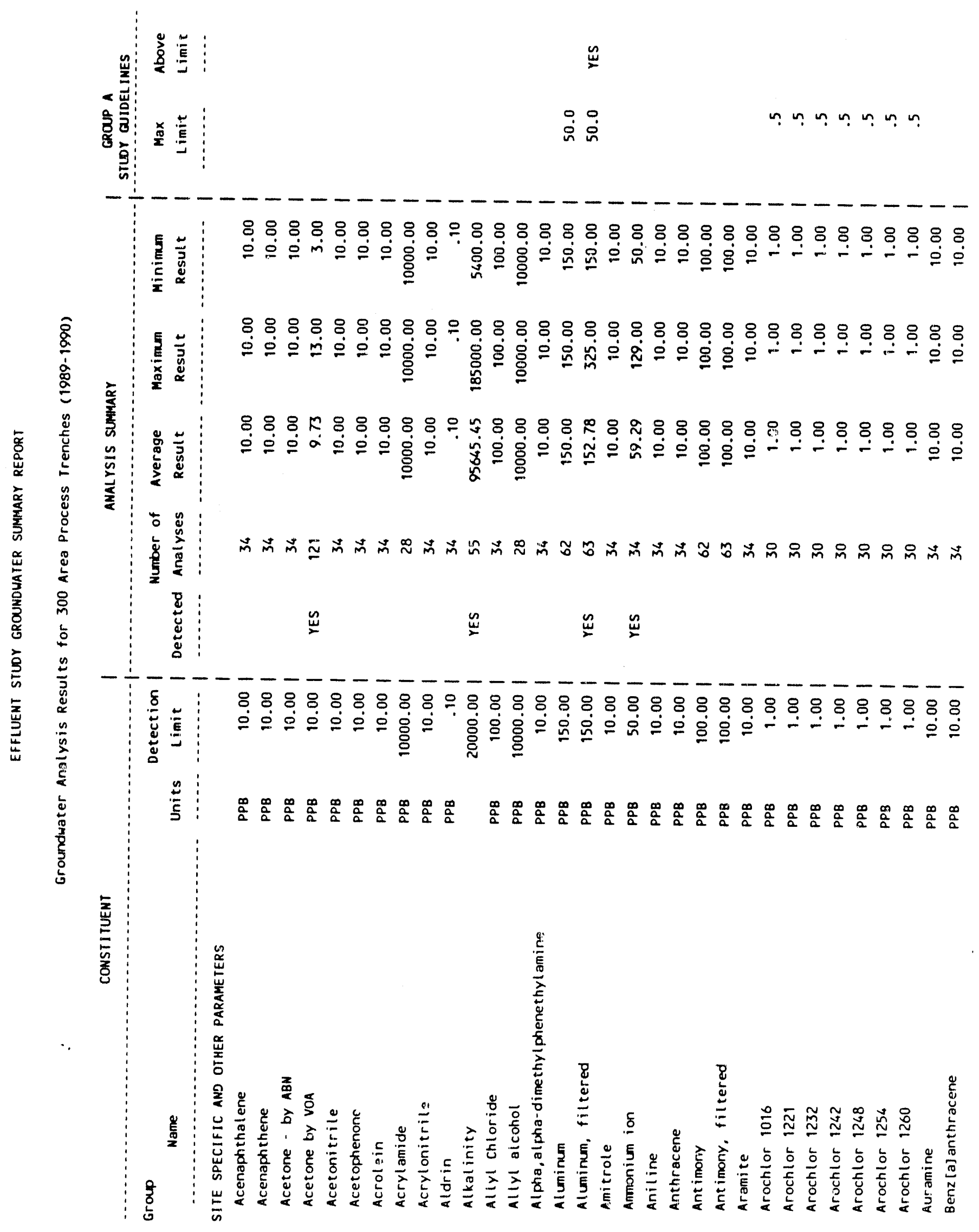


WHC -EP-0366

䀂:

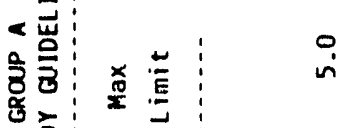

$\dot{\square} \stackrel{\dot{0}}{\dot{0}}$

点:

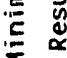

88888888888888888888888888888888

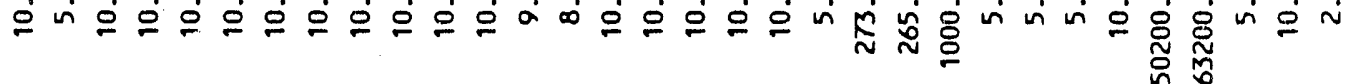

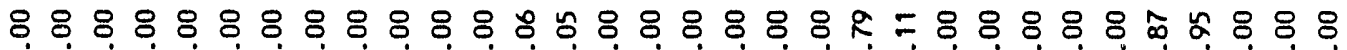

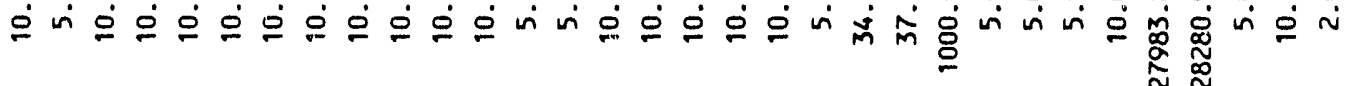

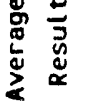

屯

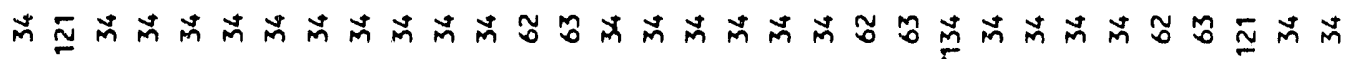

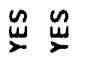
$\underset{\sim}{\stackrel{w}{x}}$

峞㥕

ڤั

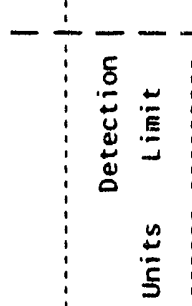

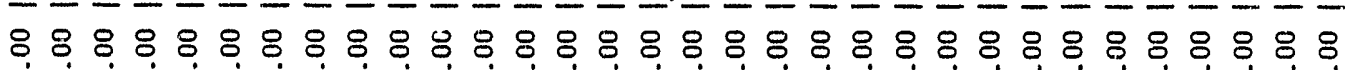

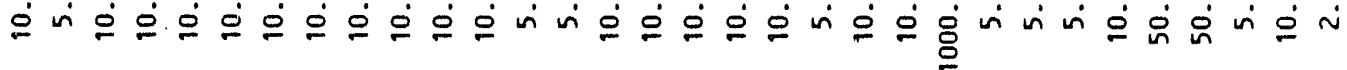

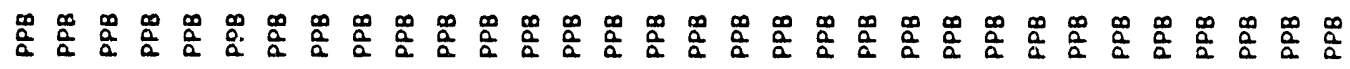

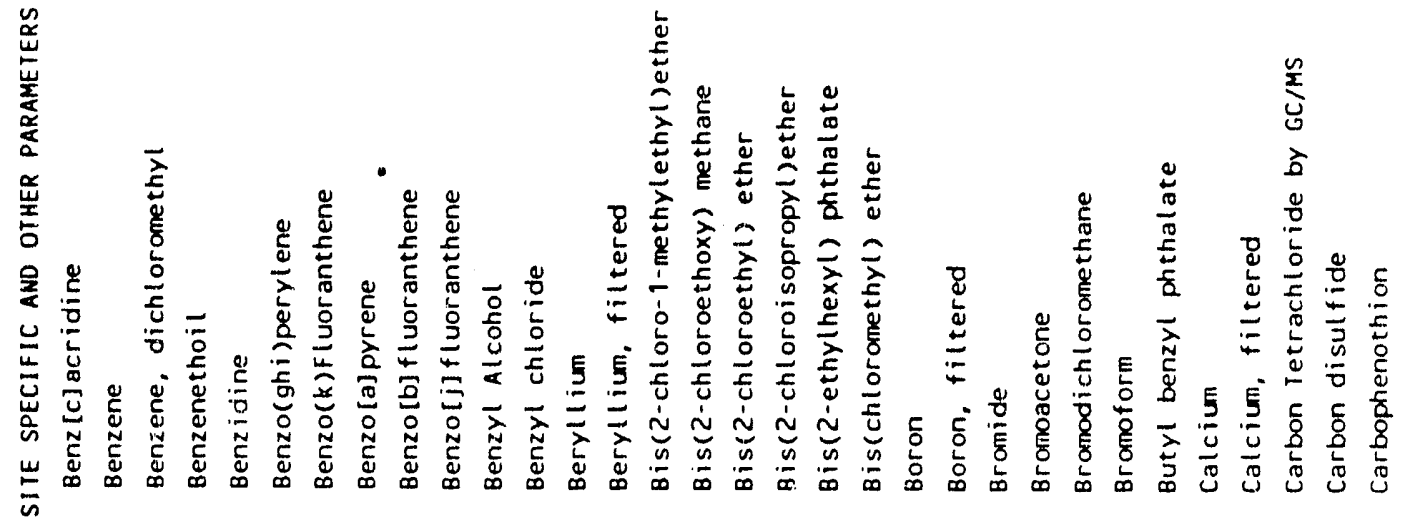


WHC-EP-0366

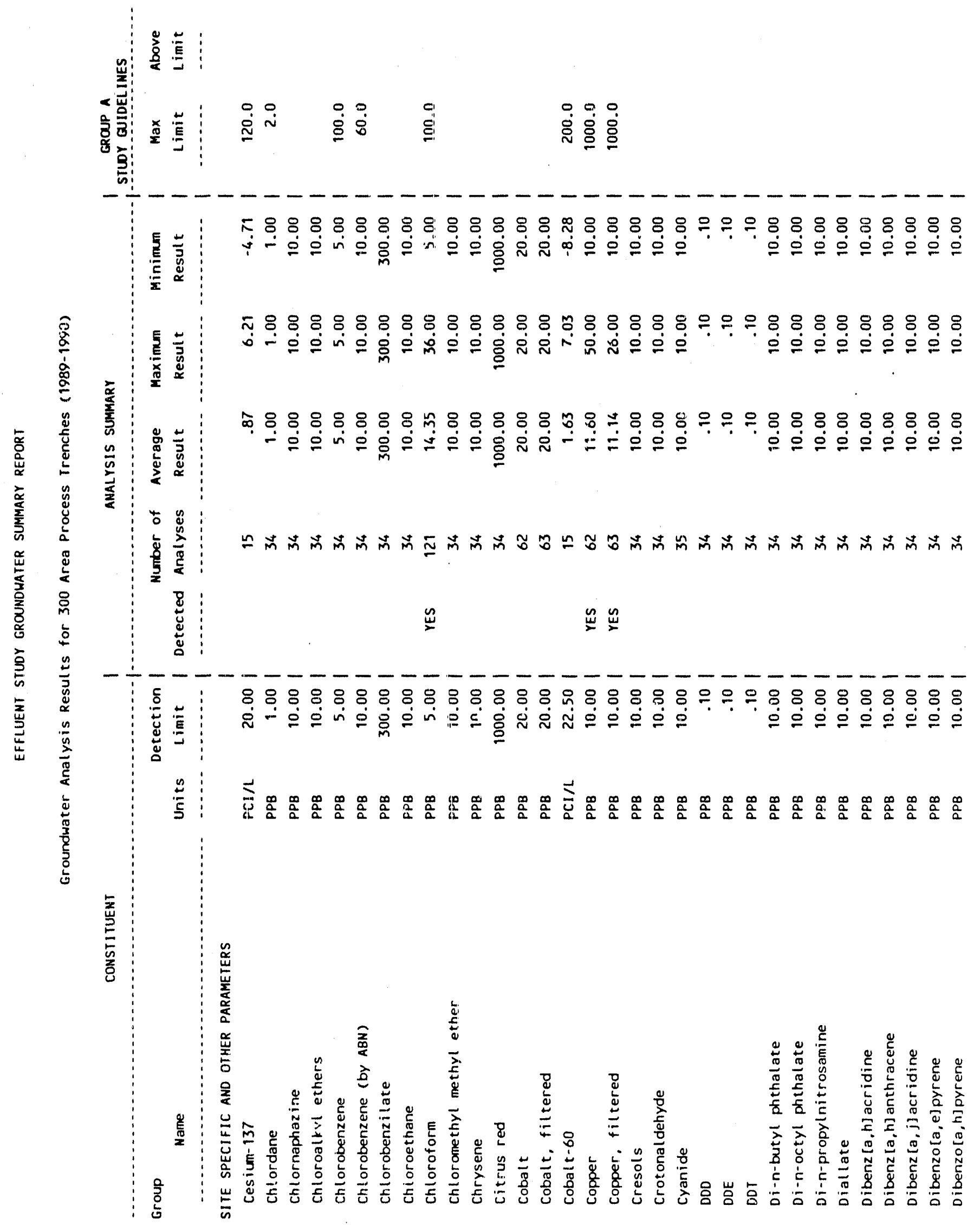


WHC - EP- 0366

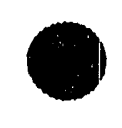

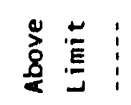

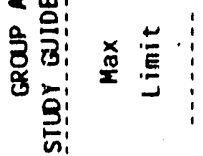

$\stackrel{0}{\dot{0}}$

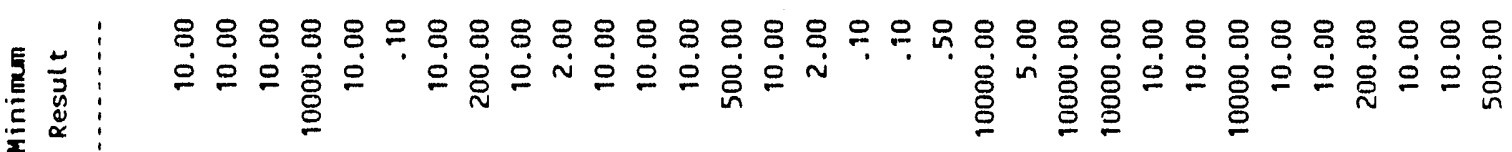

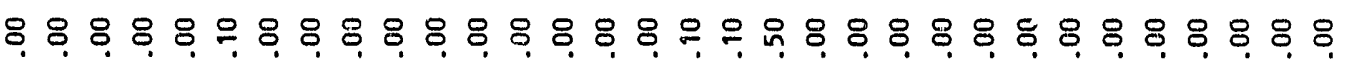

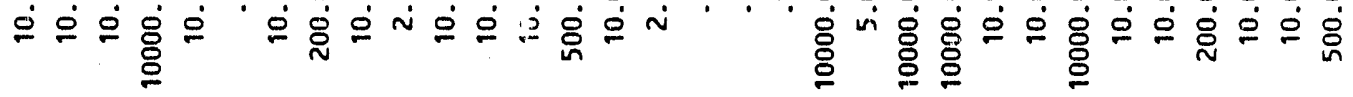

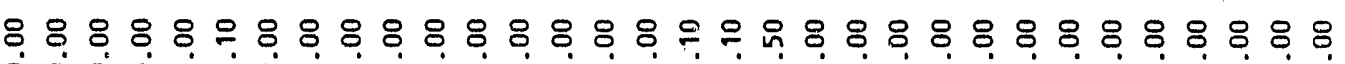

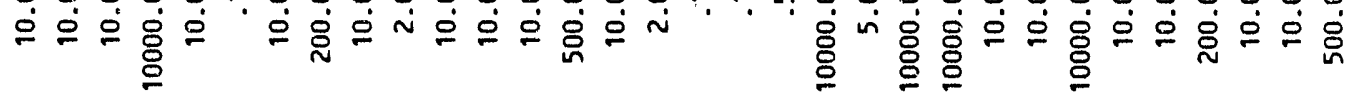

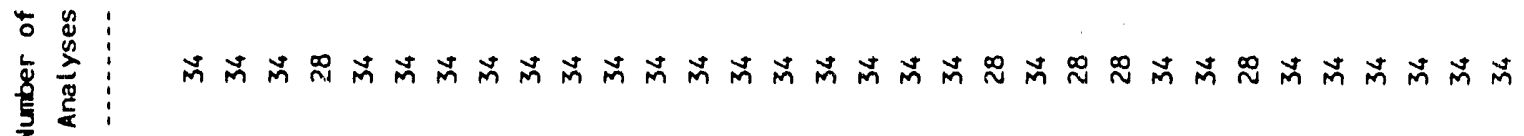

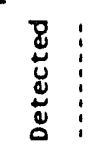

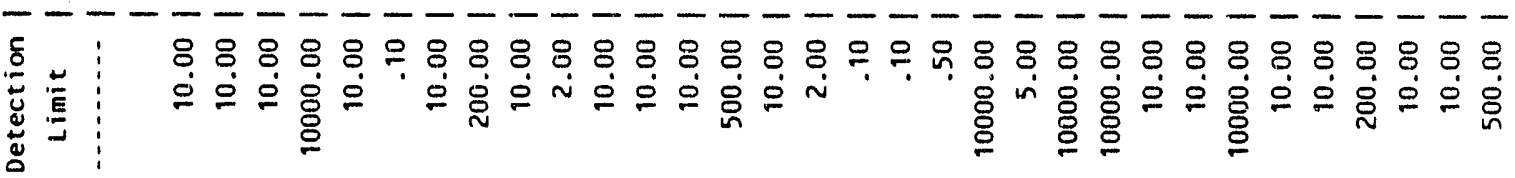

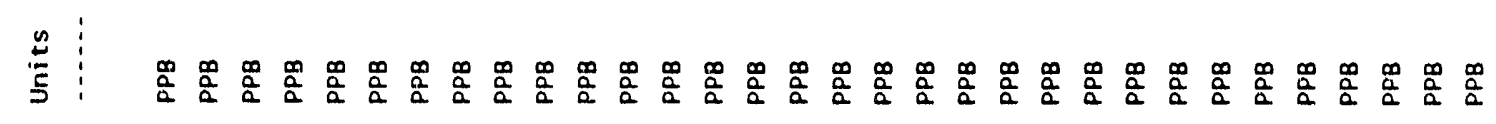
5

岸

亳 
WHC-EP-0366

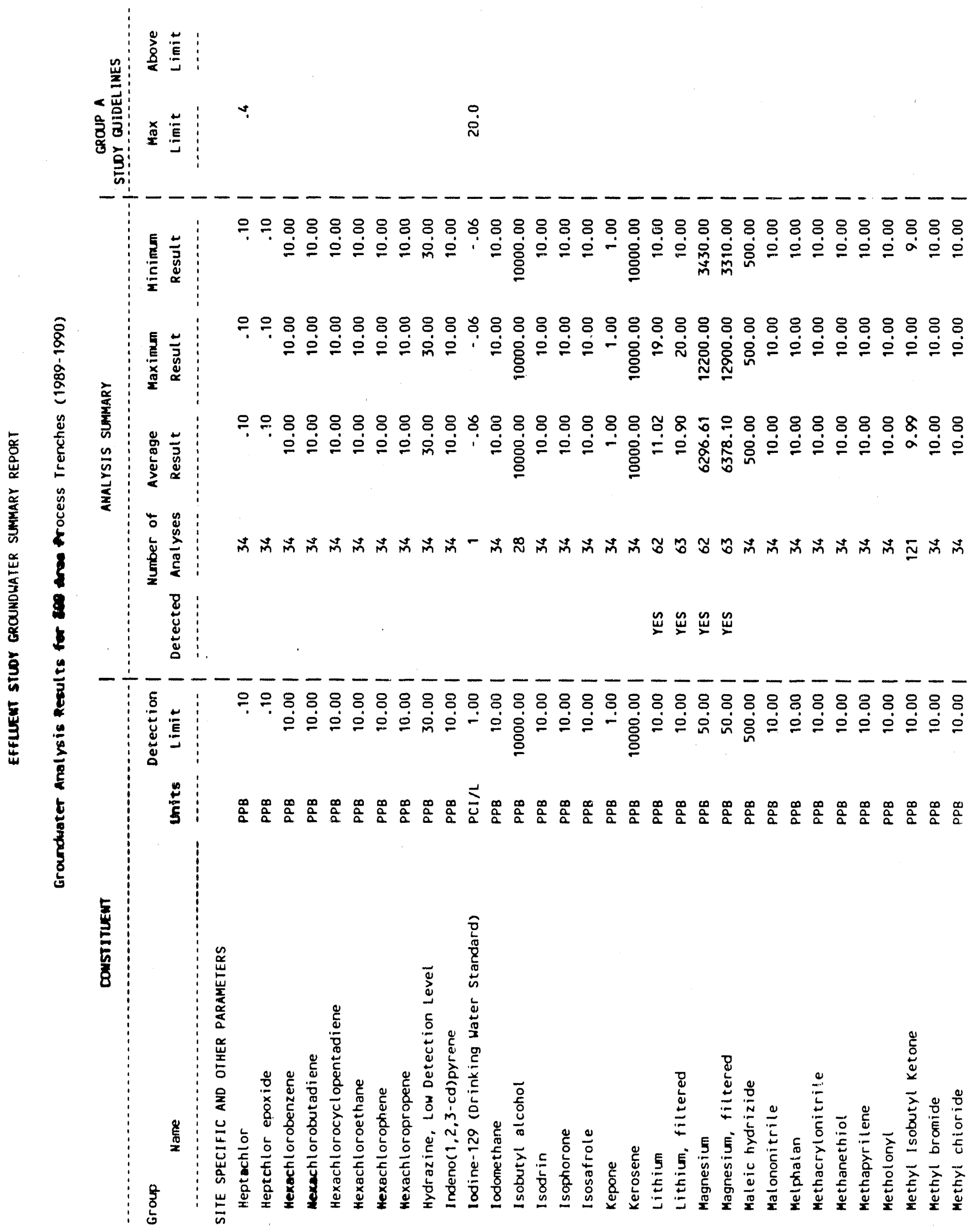




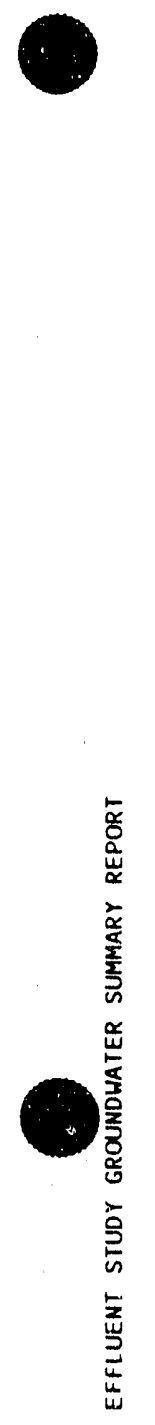

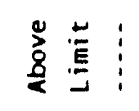

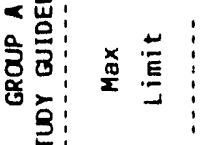

$\stackrel{\circ}{\stackrel{m}{m}}$

$-1$

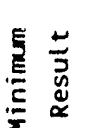

88898888898888388888888988888888

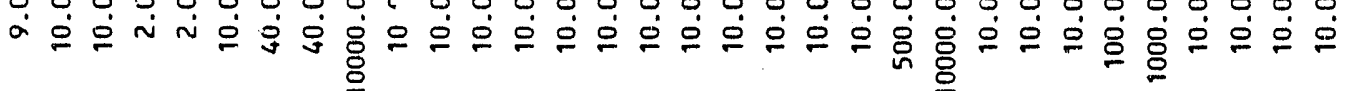

要

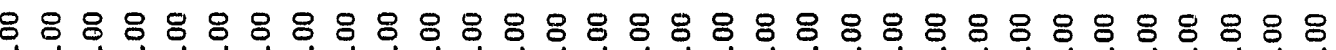

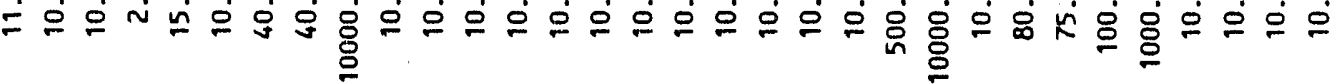

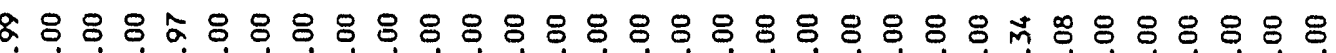

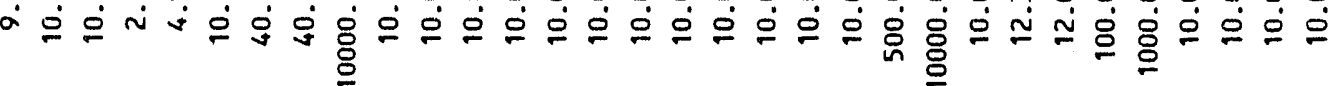

焉蓠

它

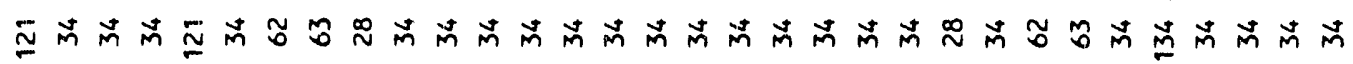

:

:

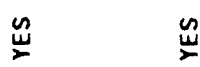

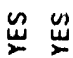

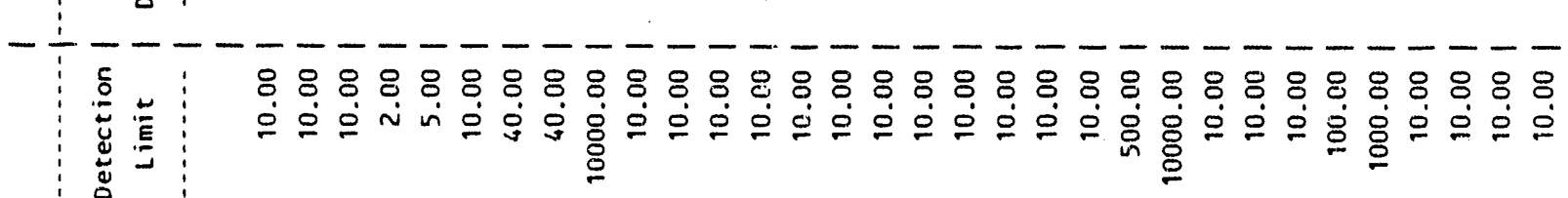

$\stackrel{n}{5}$

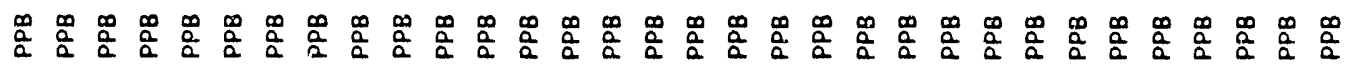

s

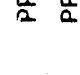




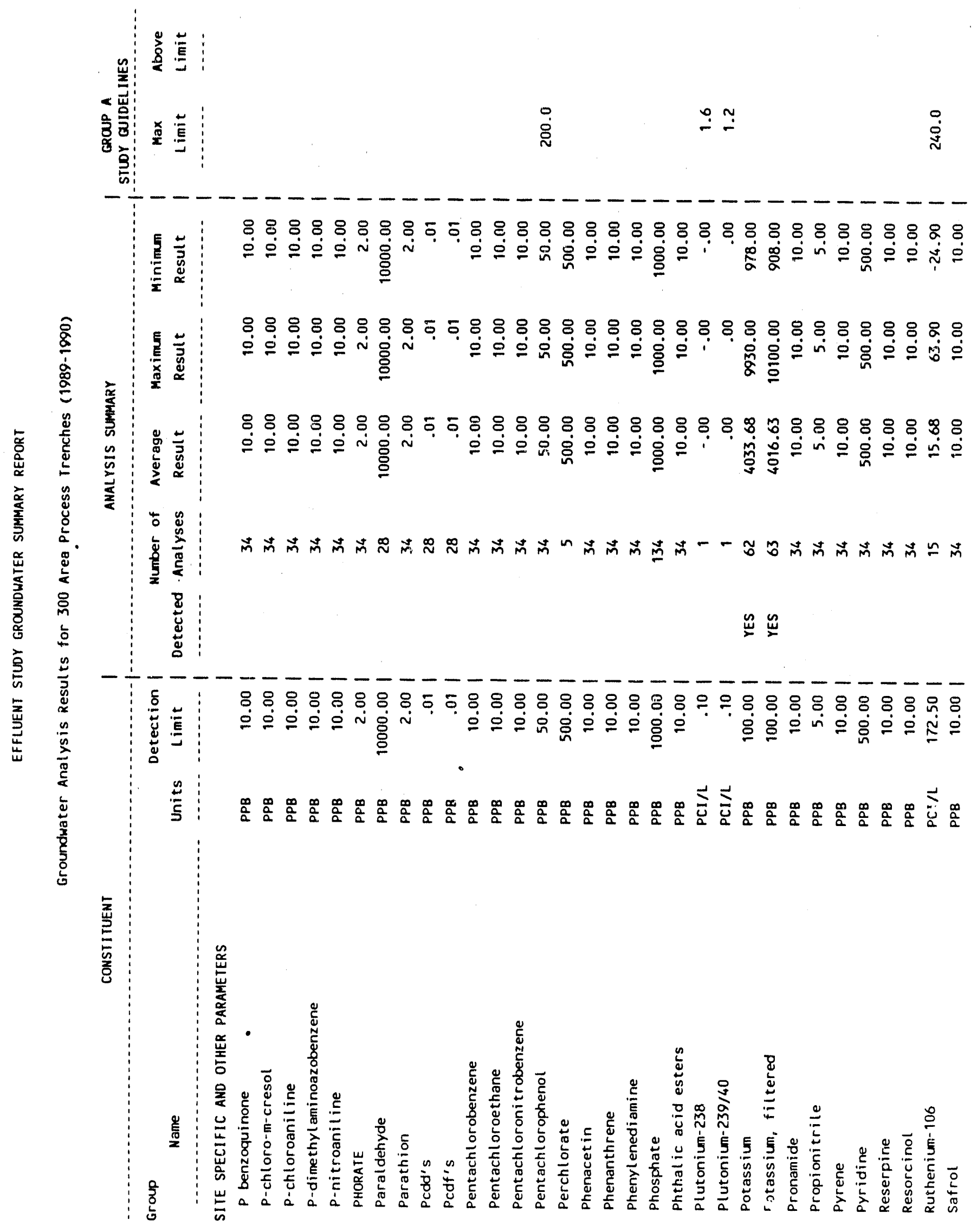


WHC-EP-.0366

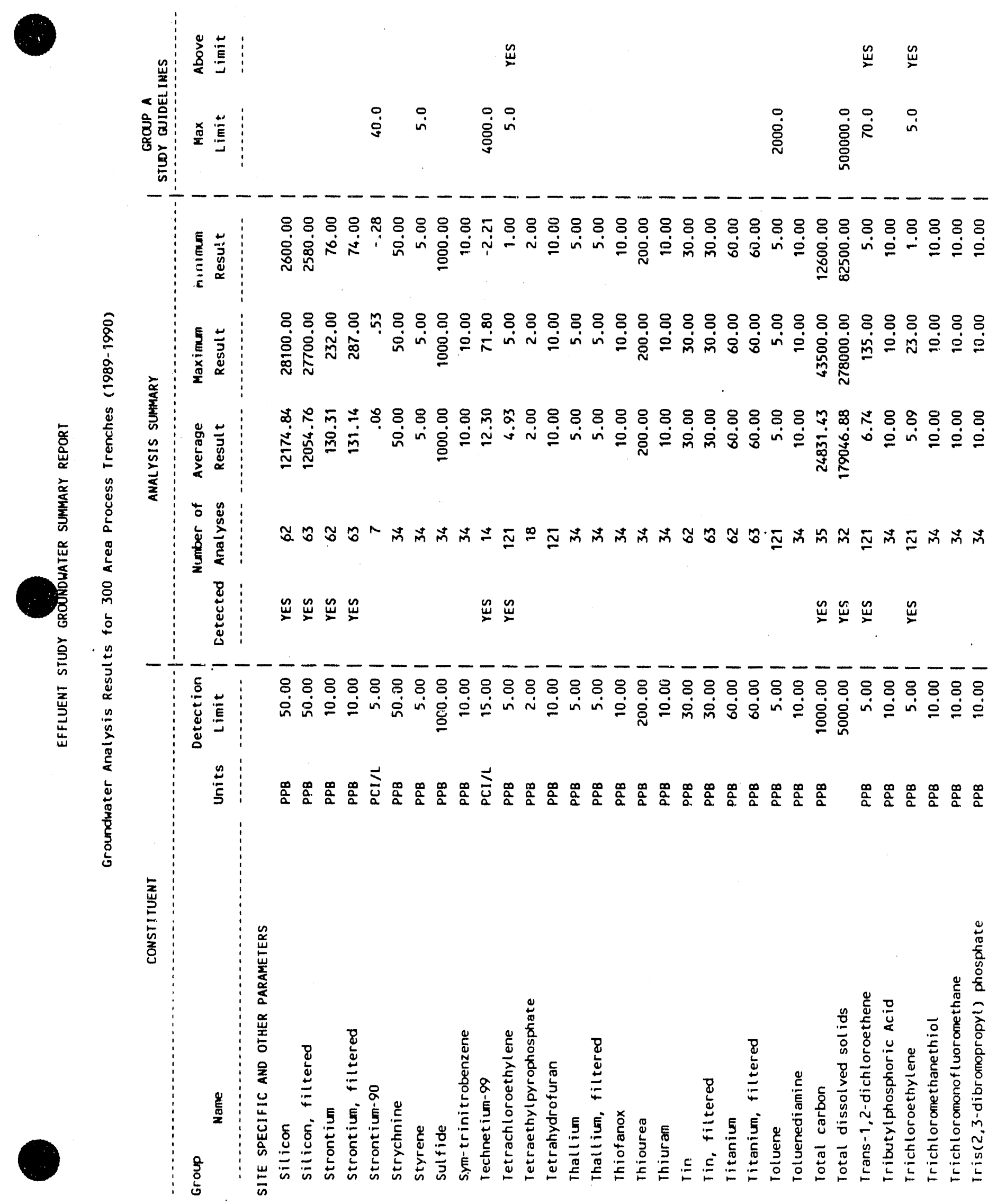


WHC - EP-0366

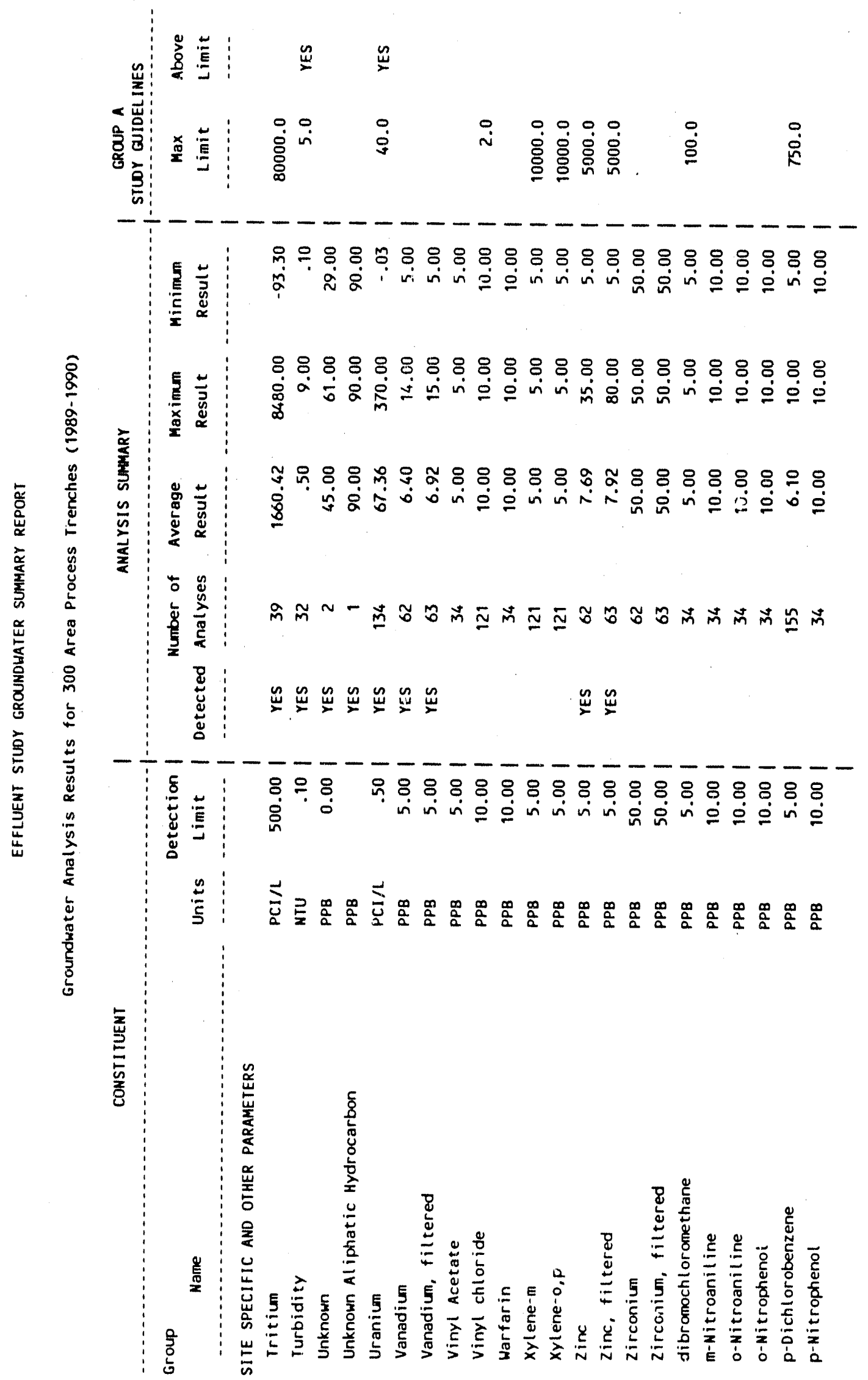


WHC-EP-0366

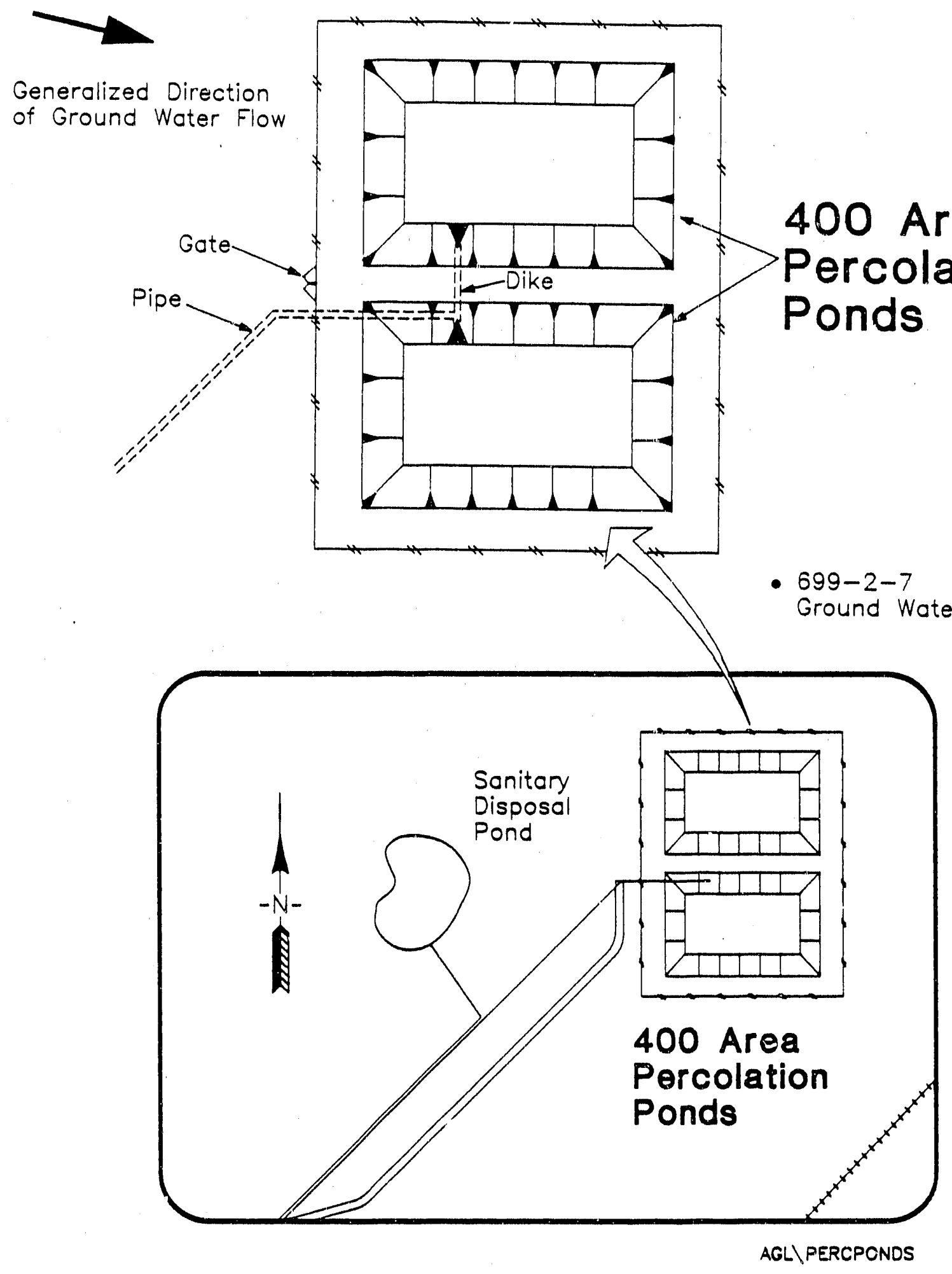

Well Location and Site Map for 400 Area Ponds 


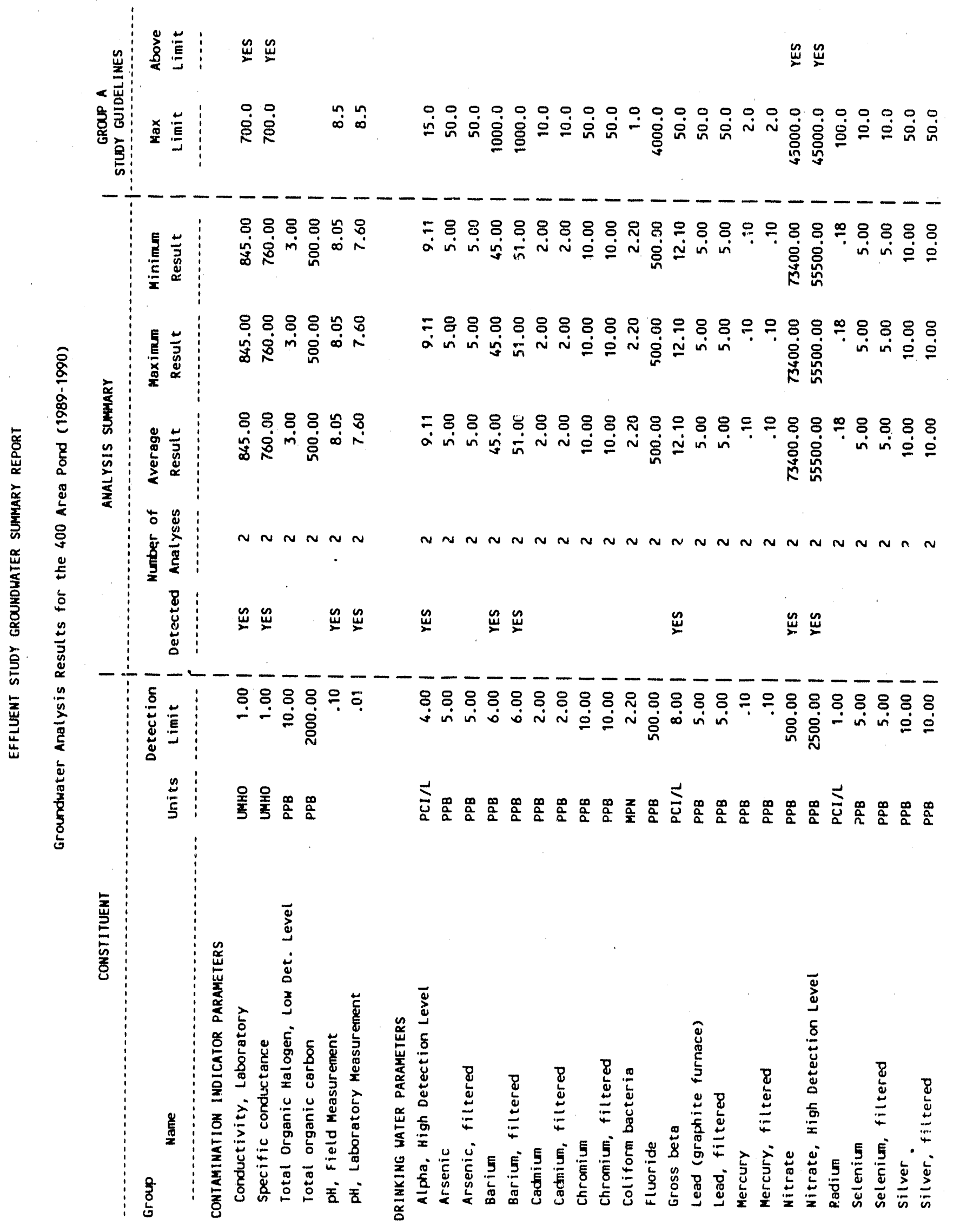


WHC - EP- 0366

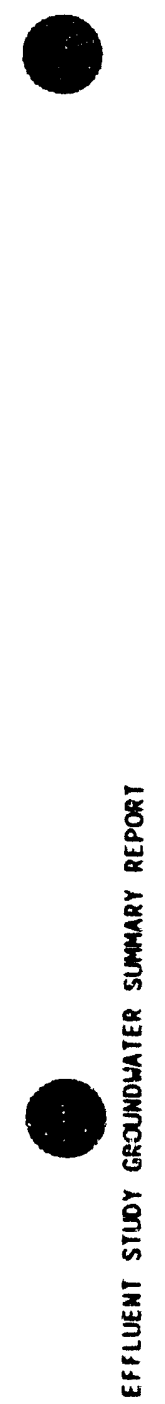

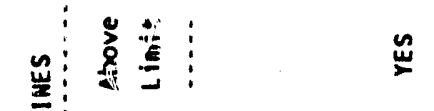

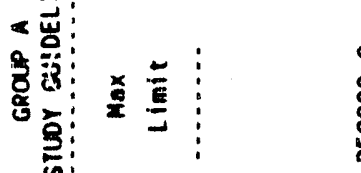

員总总

悹

$\stackrel{\circ}{\circ} \stackrel{0}{\circ}$

? $\quad \stackrel{0}{\dot{n}}$ i

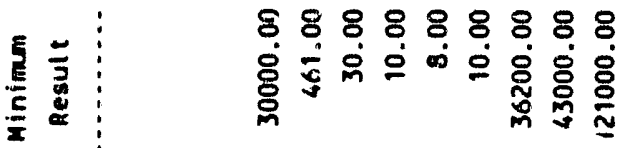

8. 8 8 8 8 8888888888888

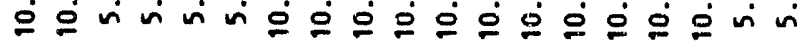

\% 8.88888

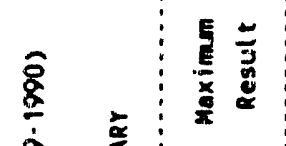

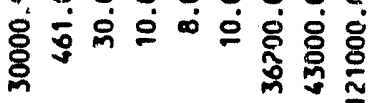

8888888088888888888

888888888

官

¿ 888888888888888888

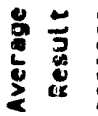

은 in

5
$\frac{8}{2}$
5
$\frac{8}{2}$

$\sim \sim N \sim \sim \sim N \sim N$

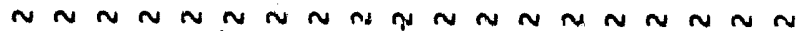

:

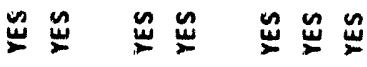

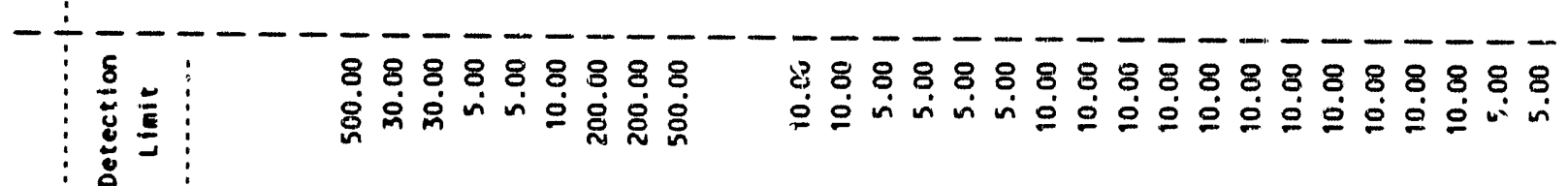

$\frac{5}{5}$

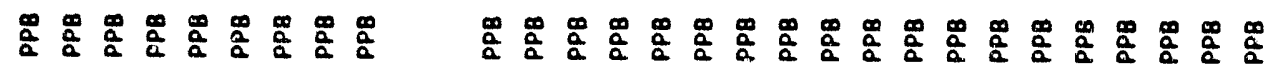

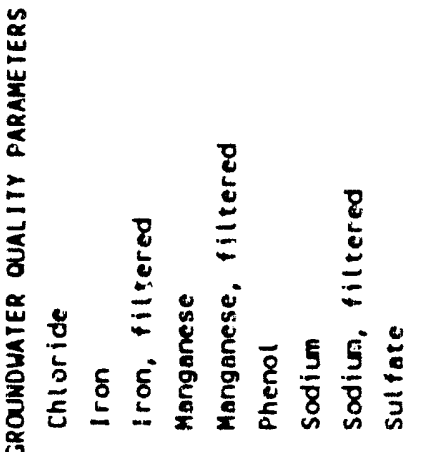

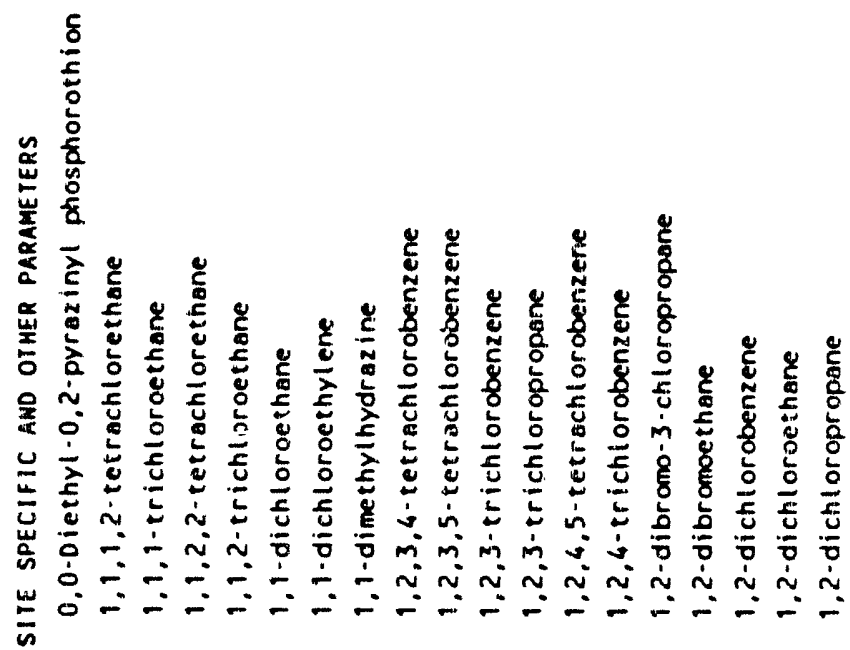


WHC - EP-0366
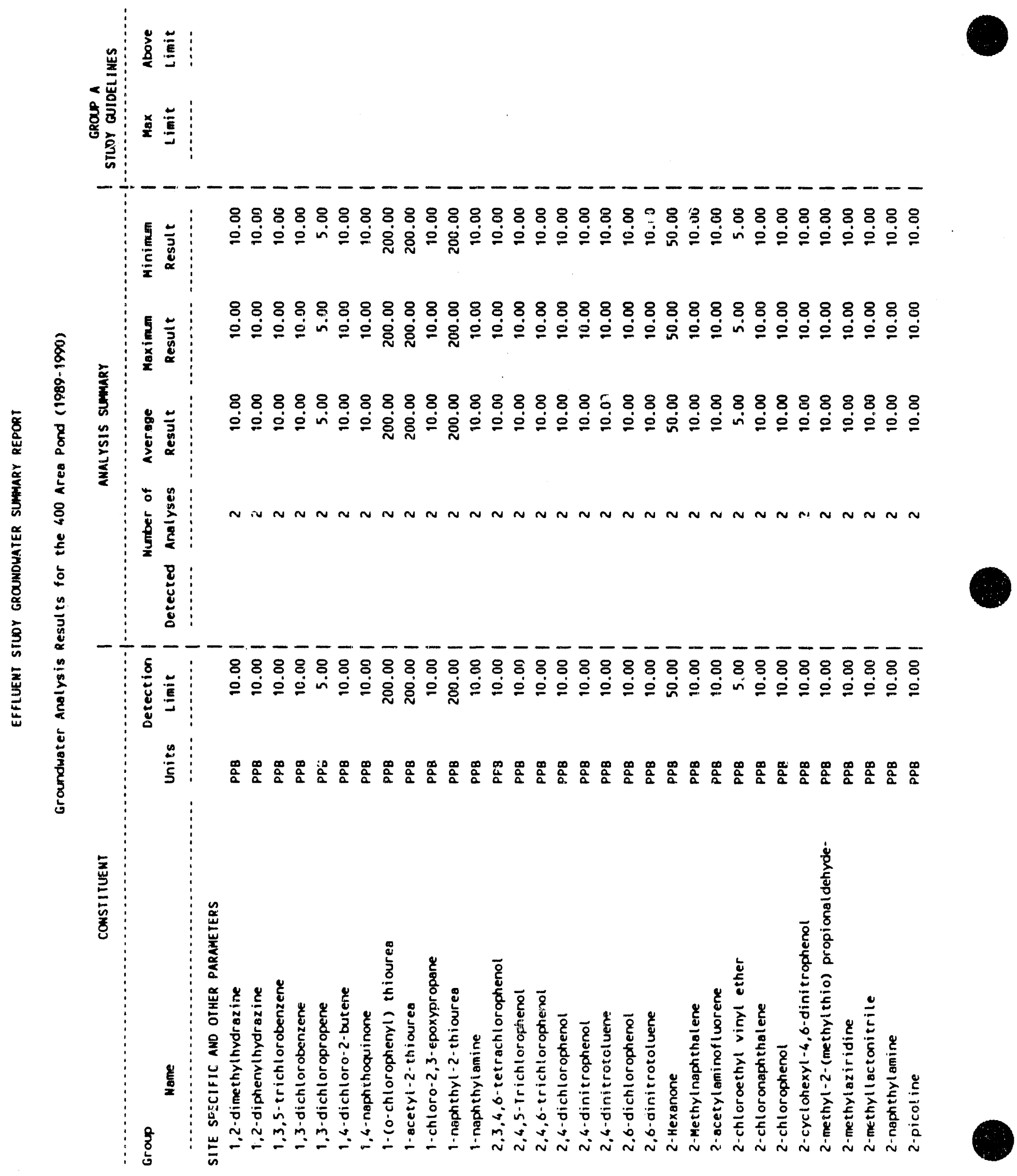
WHC -EP-0366

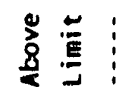

客:

i̊ i⿱ 口̊

焉

88888888888888888888888888888888

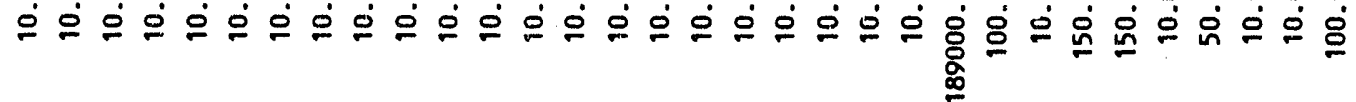

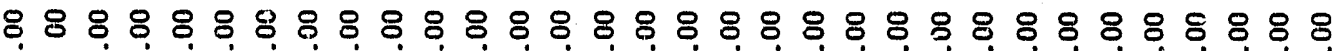

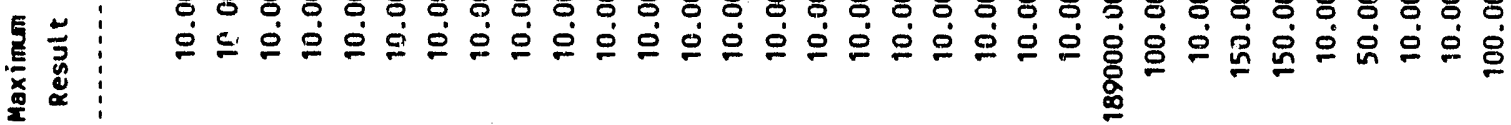

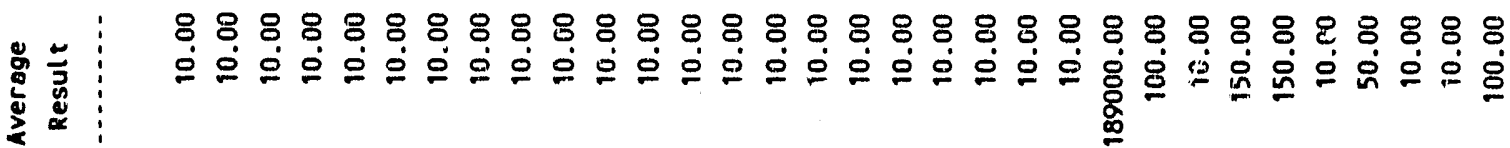
$\div$ : 要这

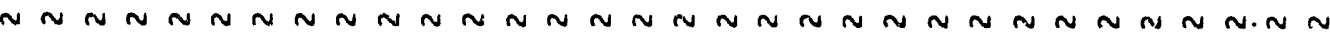
: $\stackrel{\mathscr{2}}{\longleftarrow}$

ร 8888888888888888888888888888888

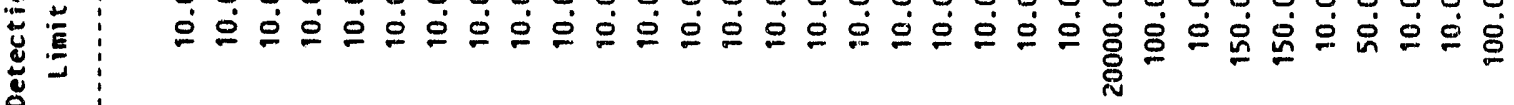

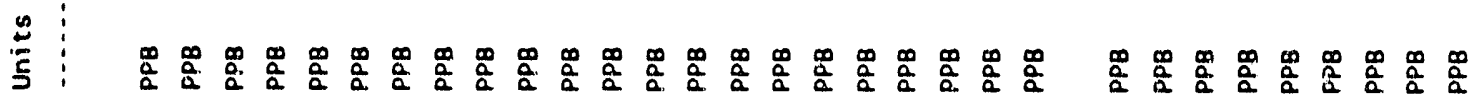

$$
\text { 氙 }
$$
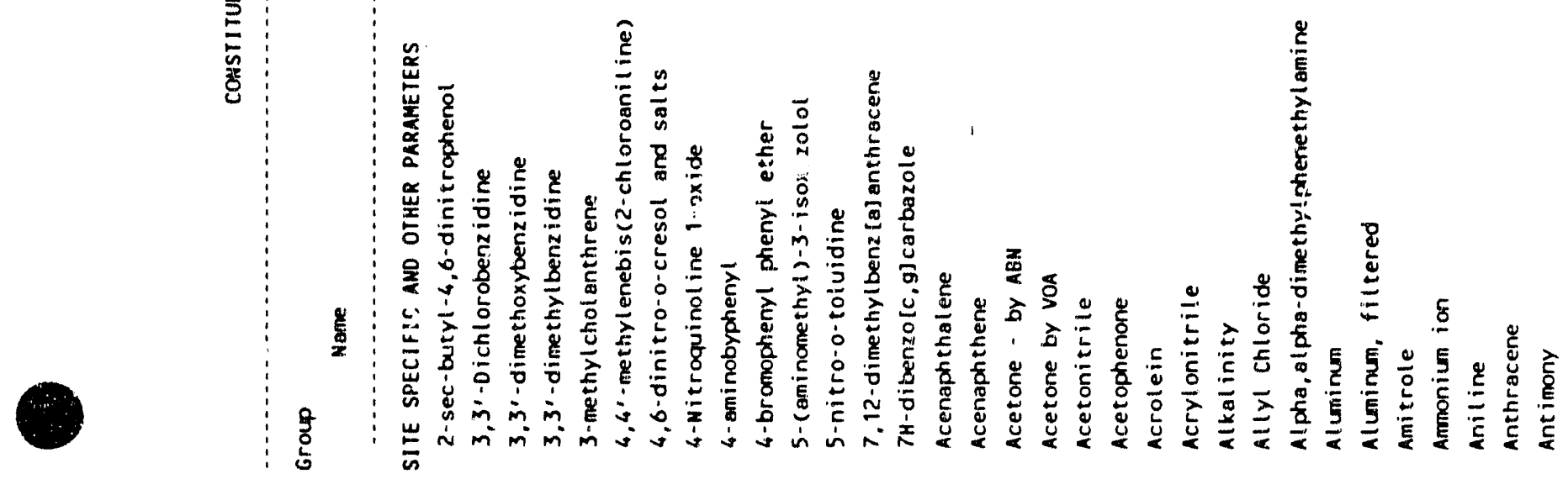


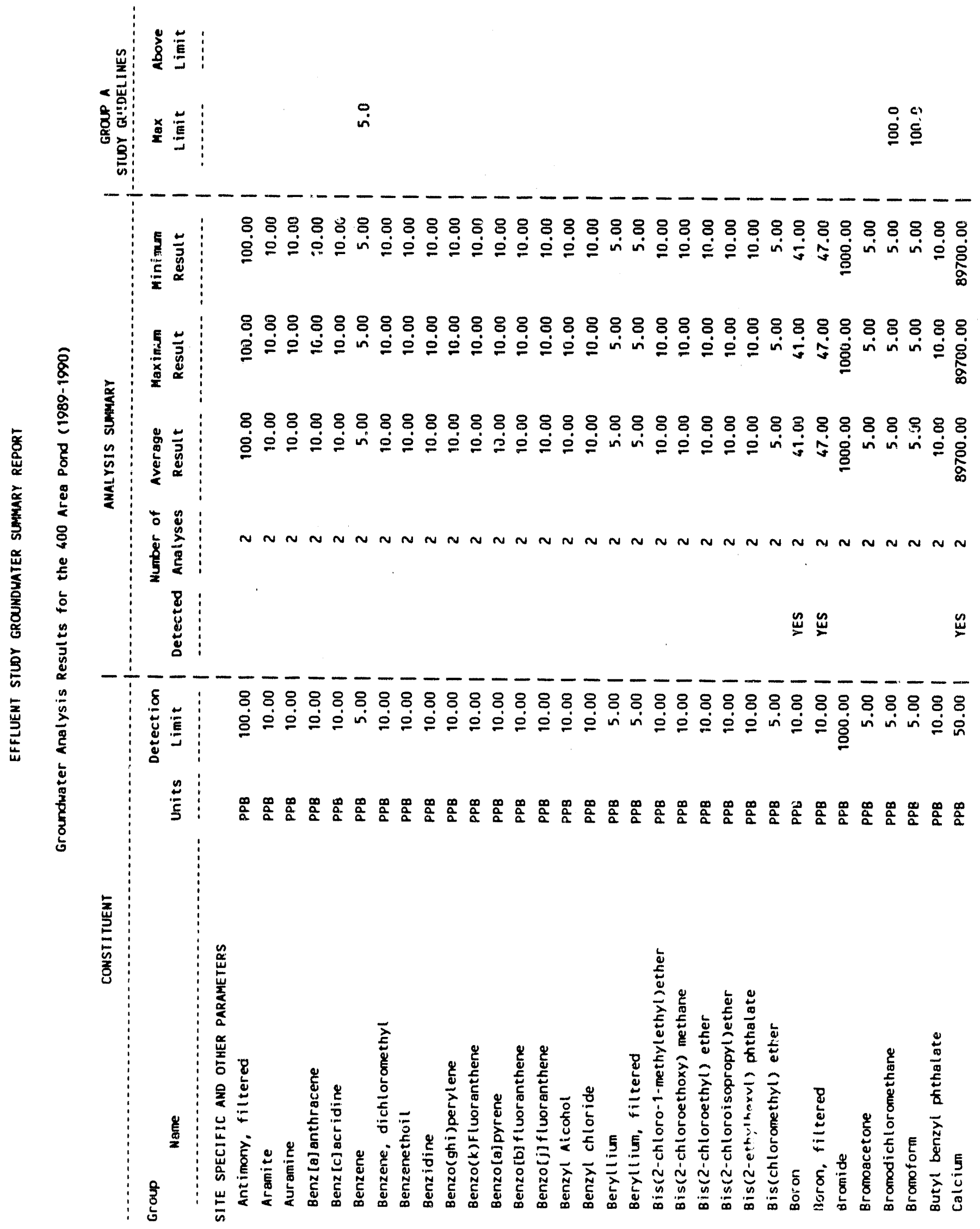


WHC -EP-0366

资:

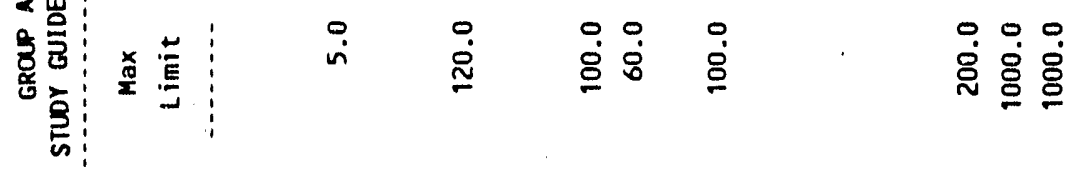

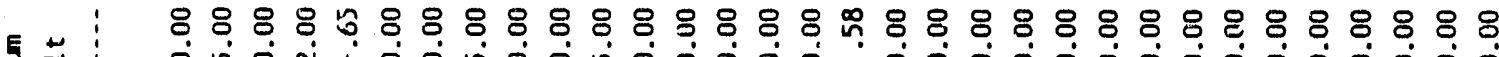

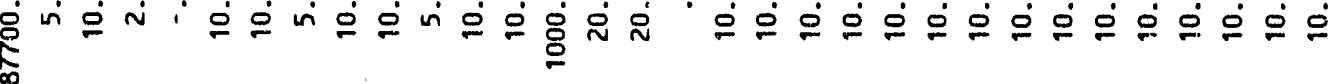

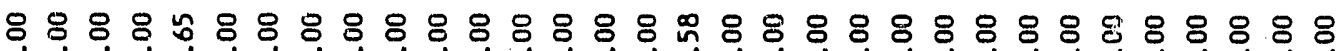
通

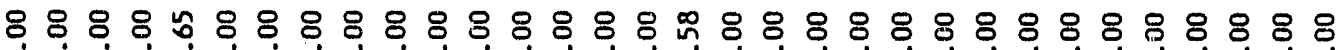
通

岁苛 $\infty$

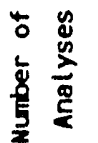

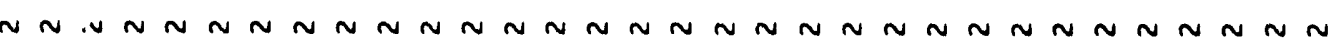

马

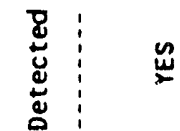

-

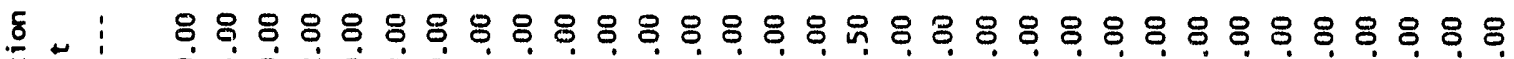

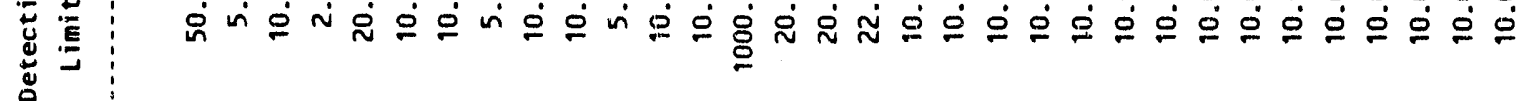

$\stackrel{0}{5} \quad$ D

$$
\text { 晃 }
$$

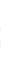

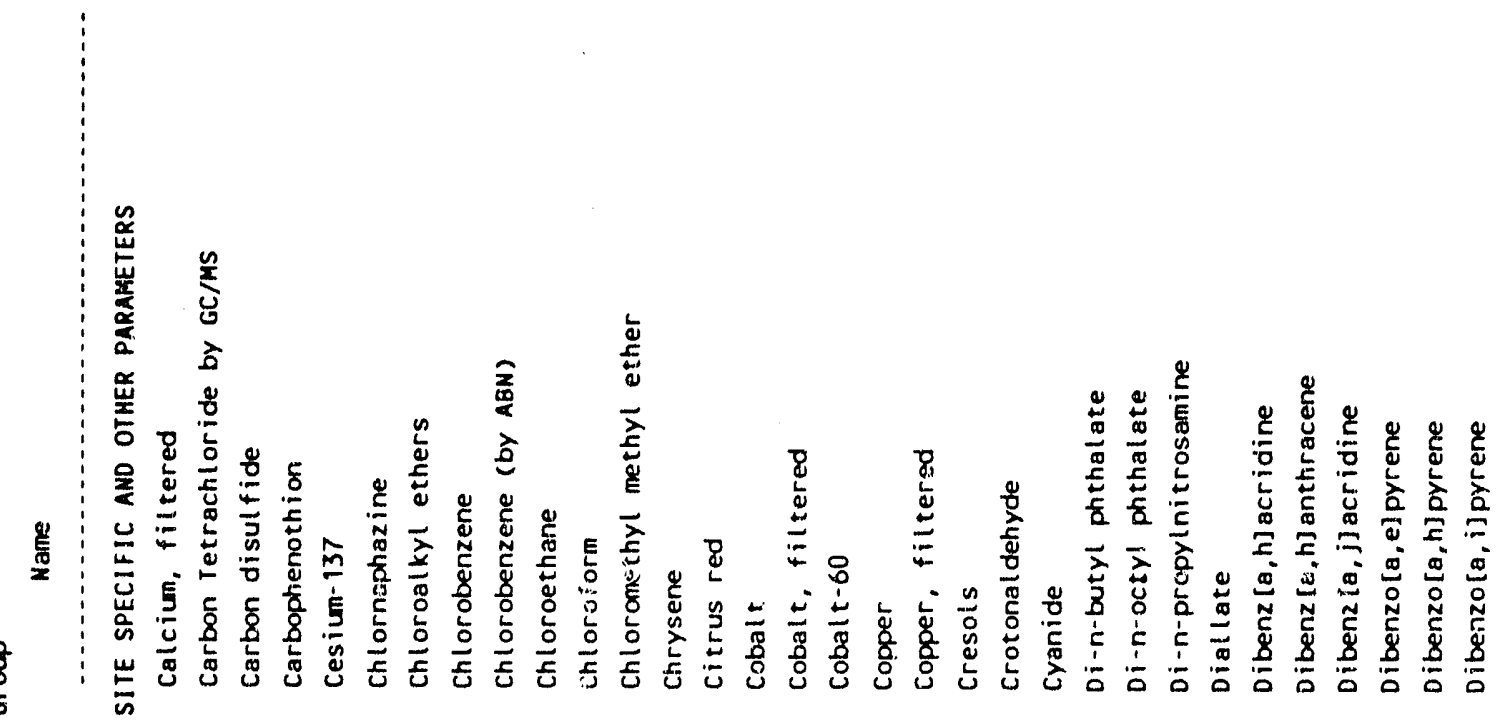



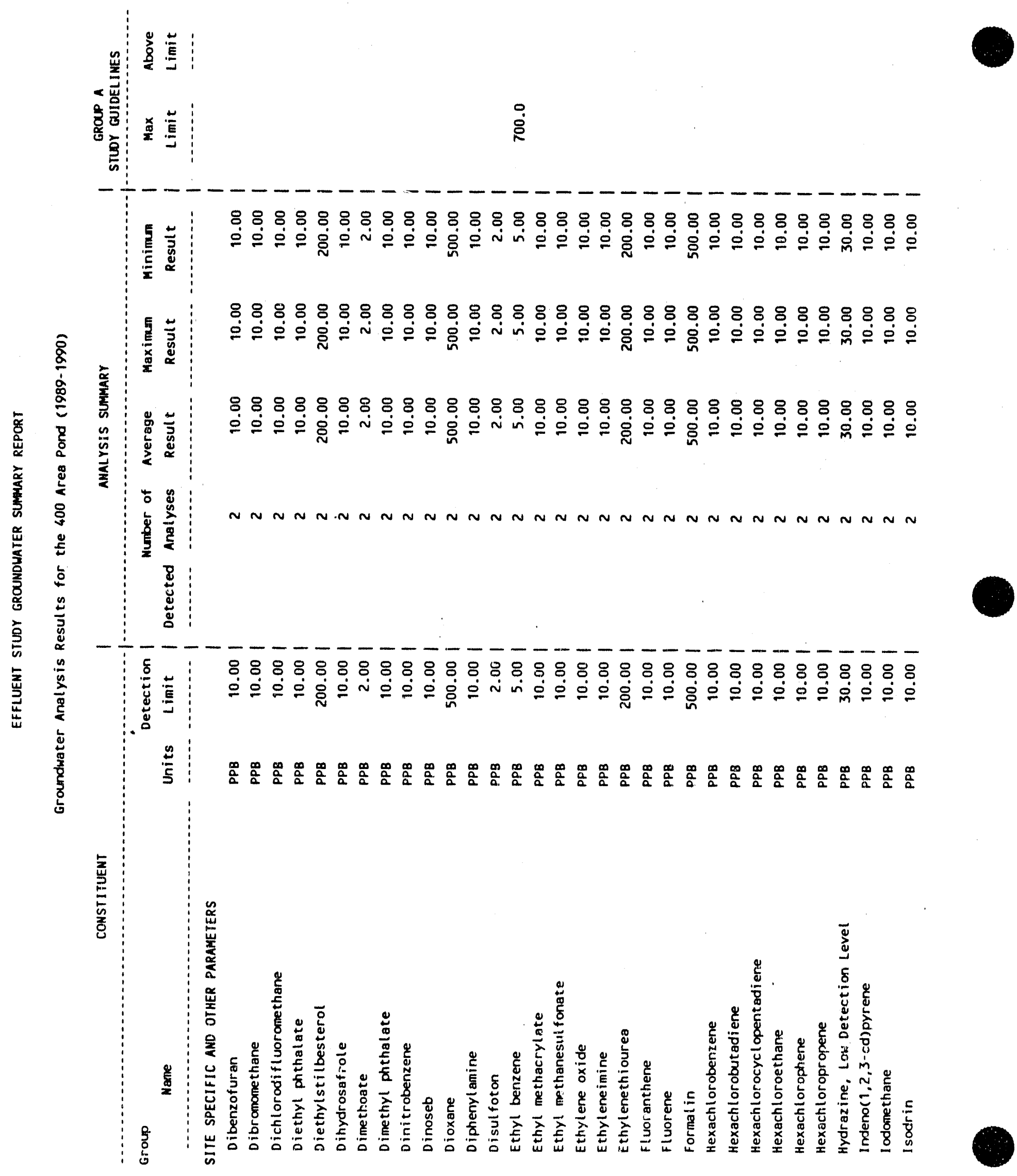


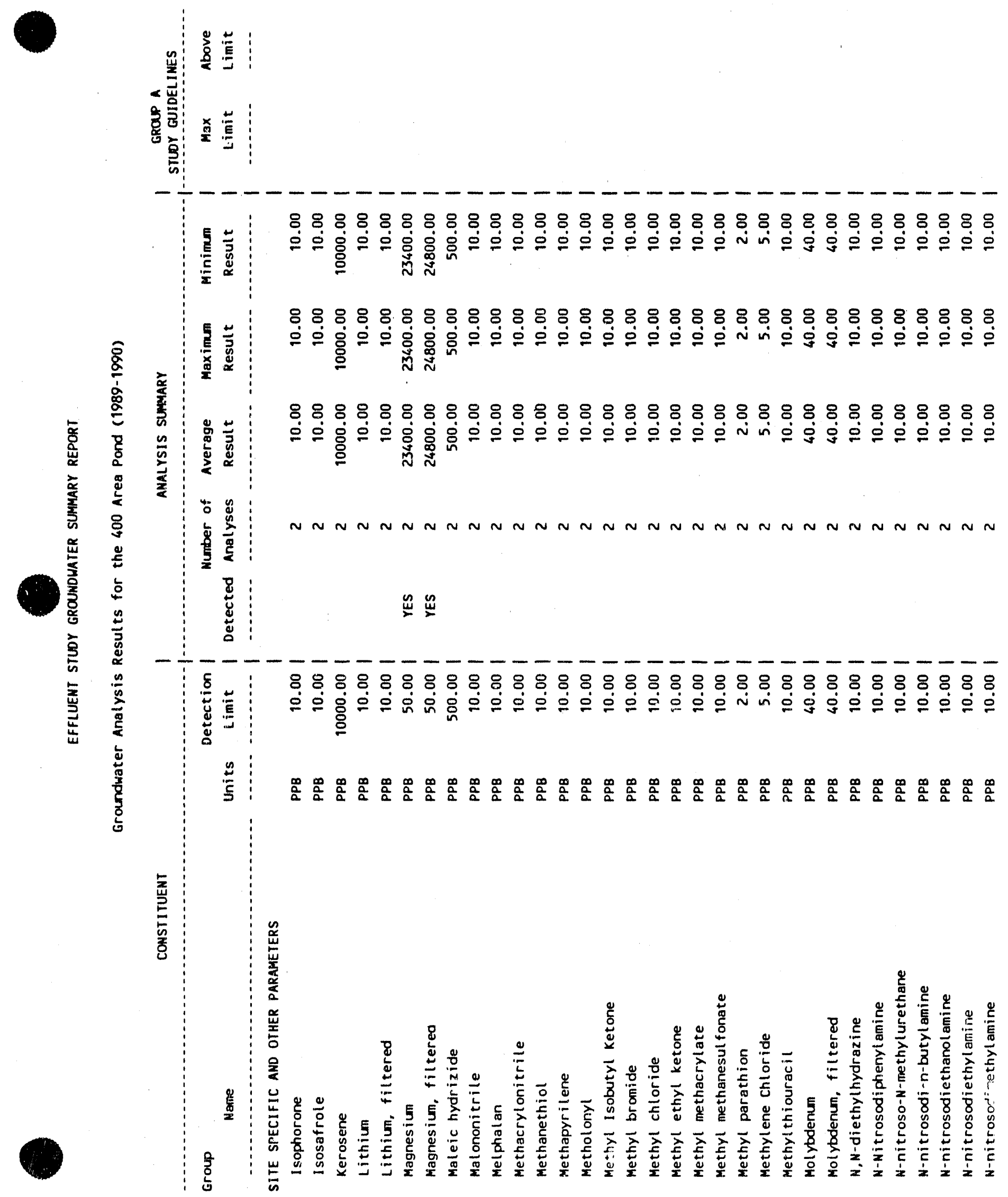




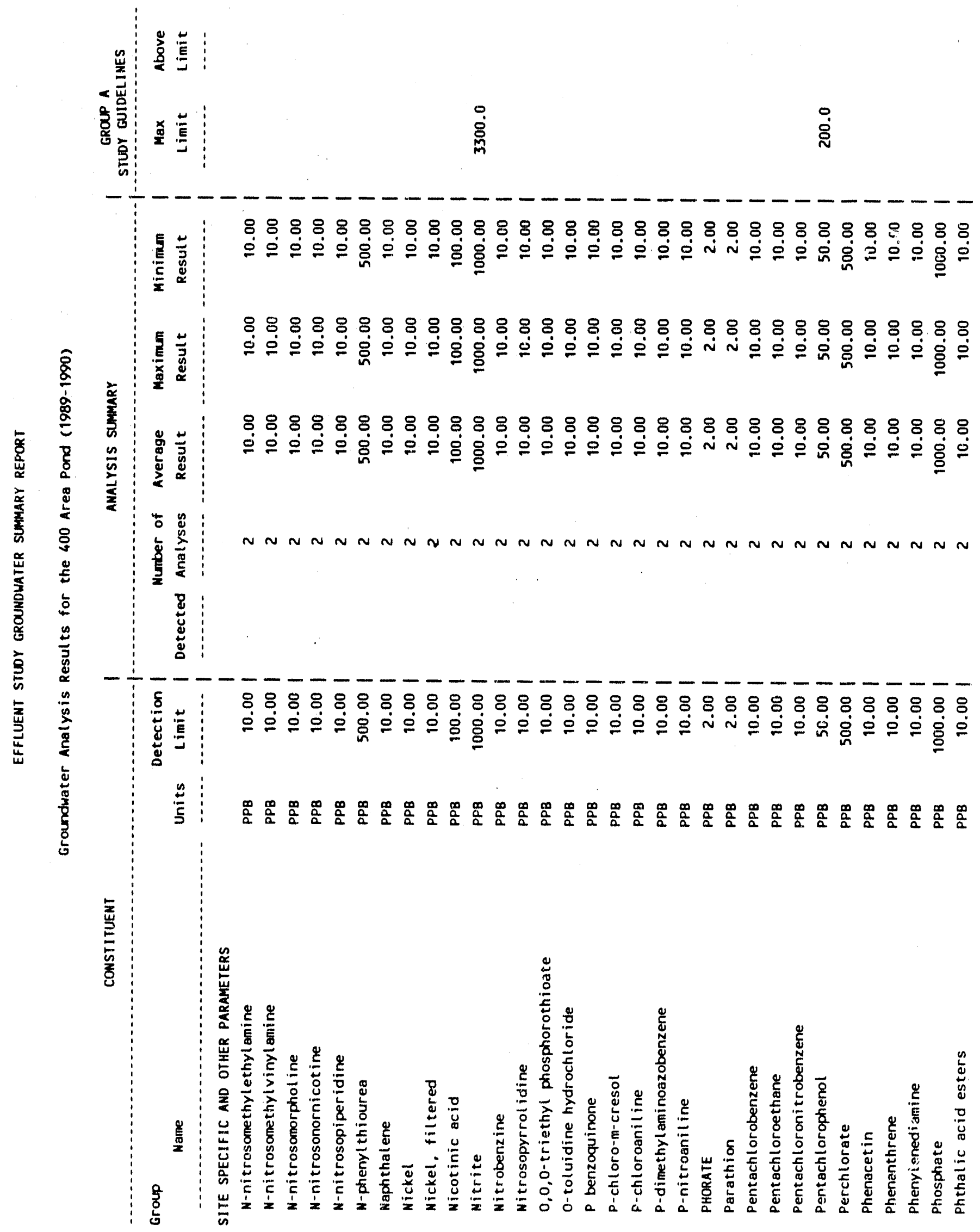


WHC -EP-0366

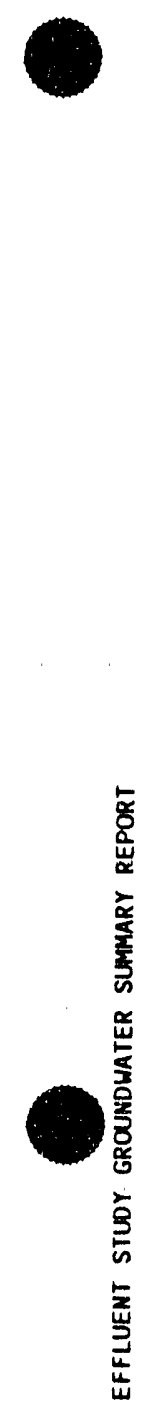

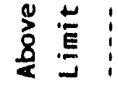

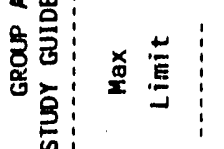

$\dot{\dot{x}} \quad \dot{i} \quad \dot{m}$

苂

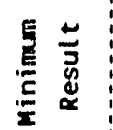

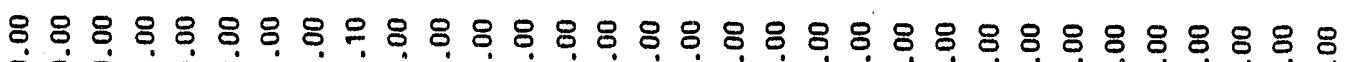

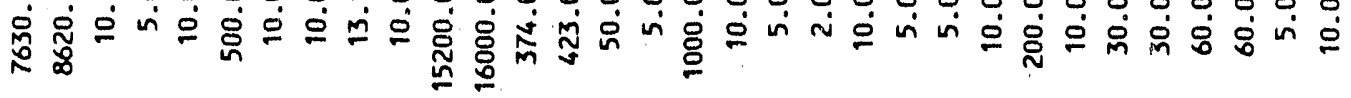

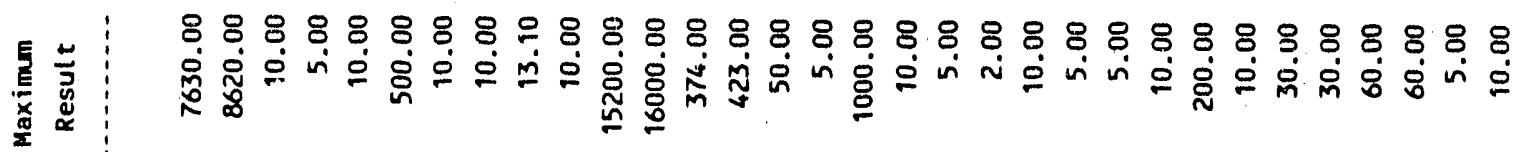

888880880908088888888888088888888

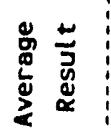

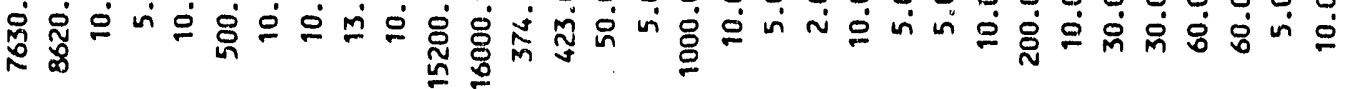

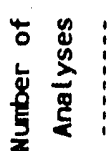

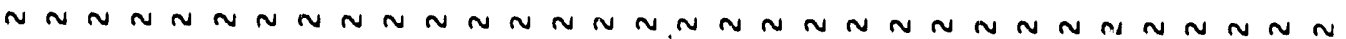

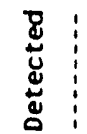

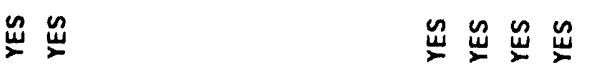

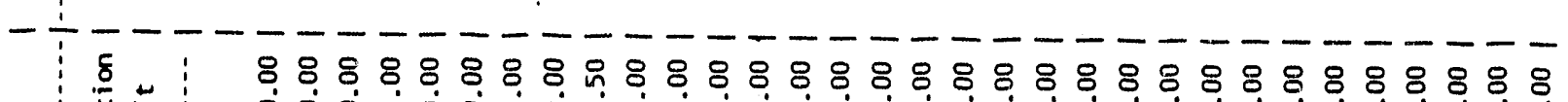

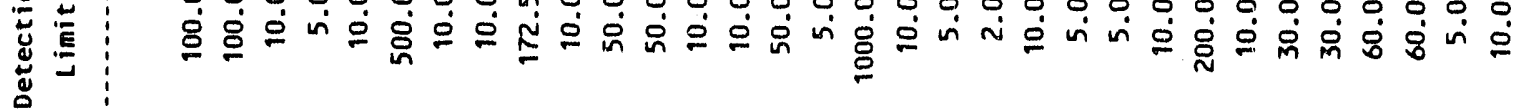

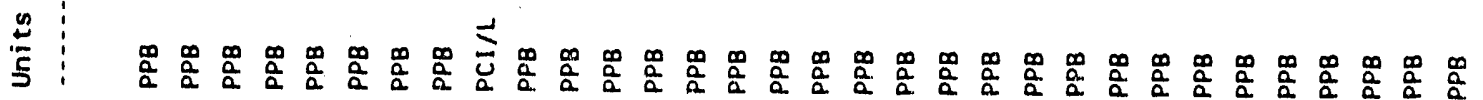

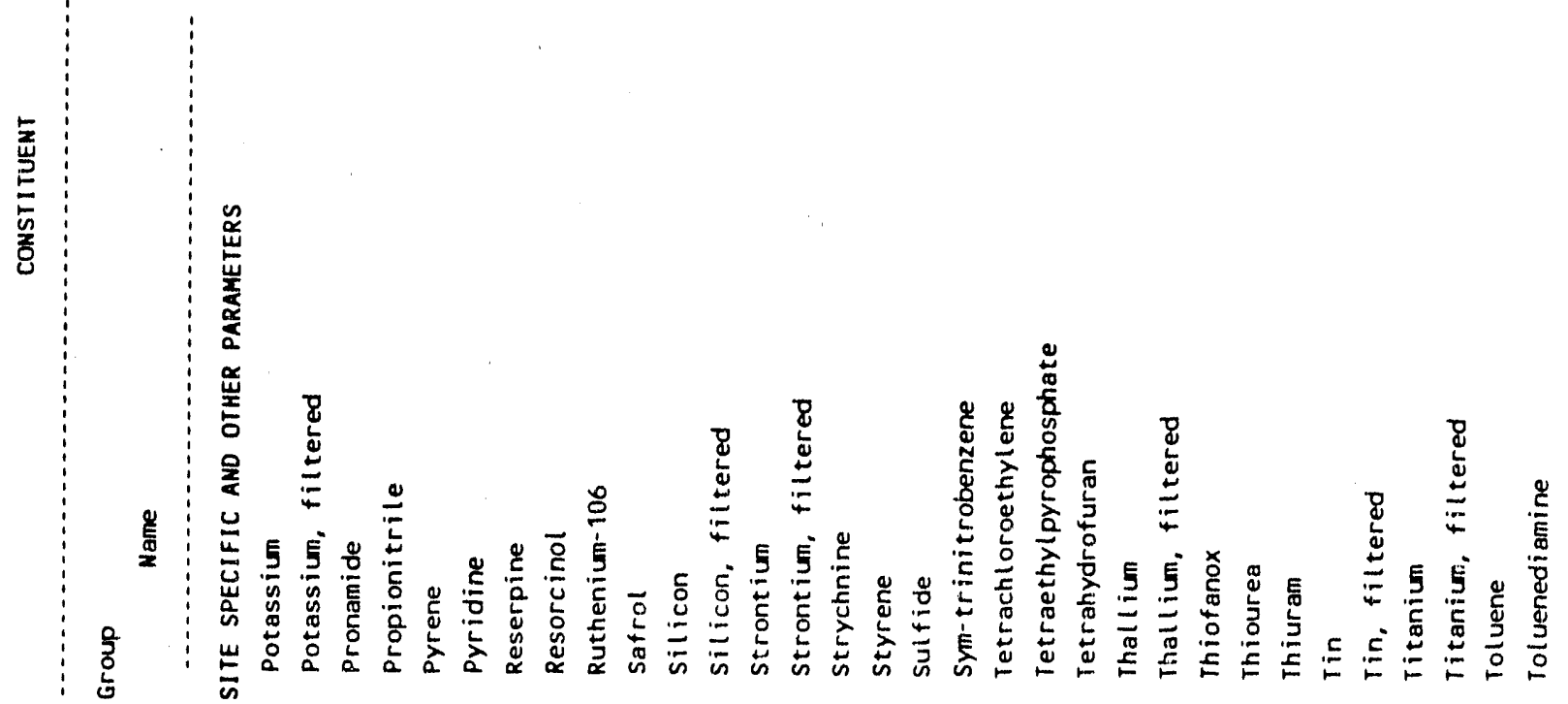


WHC-EP-0366

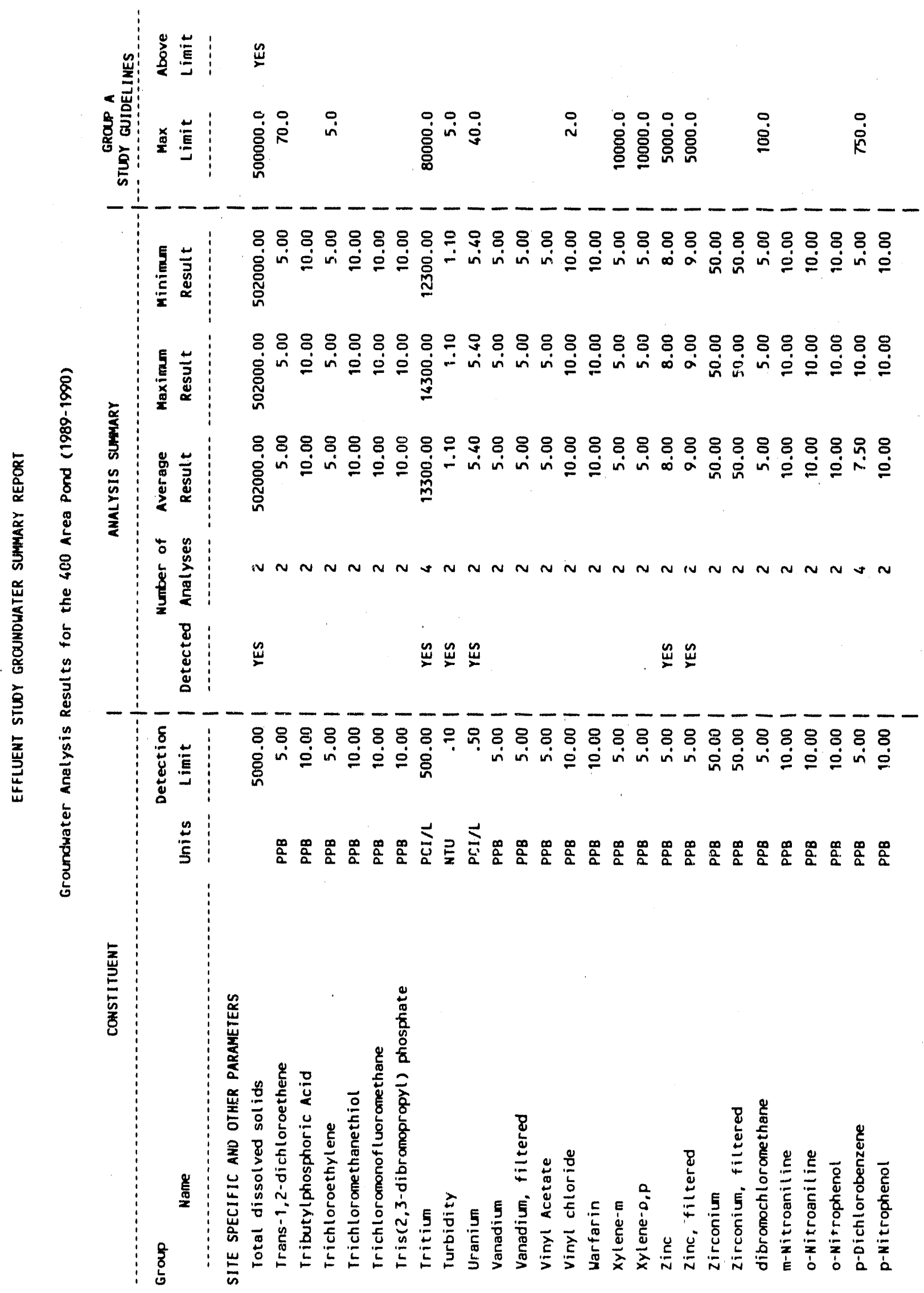


WHC -EP-0366

APPENDIX B

DATA VALIDATION SUMMARY SHEETS 


\section{APPENDIX B}

\section{RCRA DATA VALIDATION}

Unlike the U.S. Environmental Protection Agency (EPA) contract laboratory program (CLP), RCRA data validation procedures are not defined or required by the EPA. Reporting formats are much more flexible and varied due to the broad scope of RCRA analyses available. This data validation process implements three levels of evaluation based on type and origin of the sample as well as final disposition of the analytical results. The requirements to be reviewed during the validation process are defined below for Levels $A, B$, and $C$.

Level $A$ is the minimum requirement for all RCRA data. The primary application is for data used in waste designation/disposal.

- Review requirements:

- requested versus reported analyses

- analysis holding times

Level B provides a more in-depth review for programs whose data are compiled for use in later reports.

- Review requirements in addition to those isted for Level A:

- matrix spike/matrix spike duplicate analysis

- surrogate recoveries

- duplicate analysis

- analytical blank analysis

Level C requires data be reported in sample delivery group (SDG) data packages and is applicable to CERCLA analytical work conducted in non-CLP laboratories, as well as CLP special analytical services (SAS).

- Review requirements (where applicable) in addition to those above:

- initial and continuing instrument calibrations

- GC/MS tune criteria

- internal standards

- laboratory control samples (LCS)

- interference check sample (ICS)

- any other QC checks performed or required by the method of analys is

Review requirements through Level $B$ validation are described below.

This RCRA validation is heavily based on SW-846 methodology and QC requirements. Because of the wide scope of analyses contained in SW-846 and the limited requirements and guidelines that exist regarding the evaluation of RCRA data, this proceess represents a conservative approach to qualification of the data. Using professional judgement, the reviewer may at any time qualify data as estimated ( $J$ or $U J$ ) or unusable ( $R$ ) provided that the action is clearly documented. 


\section{B.1 REQUESTED VERSUS REPORTED ANALYSES (A, B, or C)}

The objective is to ensure that all analyses requested by the customer were reported by the laboratory.

Criteria - All requested analyses shall be reported or accounted for.

Evaluation - Review the Sample Analysis Request (SAR) or Chain of Custory (COC) and verify that all analyses requested were reported by the laboratory or accounted for in the case of any anomalies. A copy of the SAR or COC shall be attached to the validation documentation.

Action - Contact the laboratory and/or the field sampling team in the event of any unreported analyses and document the cause of the discrepancy. Make any necessary arrangements to resample or run the analyses on existing samples as required by the customer.

\section{B.2 HOLDING TIMES (A, B)}

The objective is to determine the validity of results based on the holding time of the sample from its date of collection to the date of analysis or sample preparation, as appropriate.

Criteria - Holding time requirements shall be applied to all sample matrices. Holding time requirements for analyses must be clearly documented.

Evaluation - Holding times for each analysis are established by comparing the sampling date on the chain-of-custody form with dates of analysis and/or extraction found in the analytical data.

Action - If holding time requirements are not met, qualify all positive results as estimated (J) and nondetects as estimated (UJ), and document that holding times were exceeded. Professional judgement must be used in the case of grossly exceeded holding times. The expected bias would be low and the reviewer may determine that nondetect data are unusable $(R)$.

\section{B.3 SURROGATE RECOVERY $(B, C)$}

Laboratory performance on individual samples is established by spiking activity. Prior to purging or extraction, samples, blanks, MS/MSD's, and standards may be spiked with surrogate compounds not expected to be detected in environmental media. Surrogate analyses provide information on laboratory accuracy as well as sample matrix effects. This is applicabie only to organic analyses.

Criteria - Sample and blank surrogate recoveries must be within the QC limits as required by the specific method in question. If no recovery limits are specified in the method, control limits of 80 to $120 \%$ shall be applied. 
Evaluation - Review the surrogate recovery data and verify that a 11 results are within the specified control limits.

Action - Document all surrogate compounds, recoveries, and control limits that do not meet $Q C$ specifications. If surrogate recoveries are out of the specified QC limits but $>10 \%$, qualify all positive results as estimated (J) and nondetects as estimated (UJ) and document any bias observed. If recoveries are $<10 \%$, nondetects shall be qual ified as unusatle (R).

\section{B.4 MATRIX SPIKE/MATRIX SPIKE DUPLICATE $(B, C)$}

MS/MSD data are an indication of the precision and accuracy of the analytical method on various matrices. The data are generated for long term evaluation and cannot be used alone to determine the precision and accuracy of individual samples.

Criteria - A matrix spike or MS/MSD must be analyzed with every analytical batch or every 20 samples, whichever is more frequent. Recoveries (\%R) and relative percent differences (RPD) between MS and MSD recoveries must be within the method specified control limits. Control limits of $75-125 \% R$, and \pm 20 RPD shall be applied in the case where none are specified.

Evaluation - Review the MS or MS/MSD results and verify that QC criteria are met. Observe any trends in bias that may be present for recoveries or RPD's which are outside the QC limits.

Action - If recoveries or RPD's are out of the specified control limits, qualify all associated sample data as estimated ( $J$ for positive results, and UJ for nondetects). For Inorganics analyses with recoveries $<30 \%$, nondetects shall be qualified as unusable (R). Document the \%R or RPD and the control limits applied for all compounds outside those limits, and indicate any bias observed in the results.

\section{B.5 DUPLiCATE ANALYSES $(B, C)$}

Duplicate analyses provide an indication of laboratory precision based on each sample matrix.

Criteria - Duplicate analysis must be performed with every analytical batch or every 20 samples, whichever is more frequent. This requirement may be satisfied with the analysis of one MS/MSD sample. Method specified control limits shall be applied to sample results where they exist, otherwise the 1 imit shall be $+20 \%$. If both sample and duplicate results are below the method detection limit (MDL) or sample quantitation limit (SQL), then no control limit applies.

Evaluation - Review the duplicate analysis results and verify that all data fall within the control limits where applicable. 
Action - If duplicate analysis result.s are outside the specified control limits, qualify all associated positive sample results as estimated ( $J)$, and nondetects as estimated (UJ). Document the RPD and limits for those compounds out of control.

\section{B.6 ANALYTICAL BLANKS $(B, C)$}

Assessment of analytical blank analysis results provide information on the existence and magnitude of any laboratory contamination problems.

Criteria - A minimum of one analytical blank must be analyzed for every batch or every 20 samples. No contaminants should be detected in the bianks.

Evaluation - Review the analysis results for evidence of blank contamination.

Action - In the case of unsuitable blank results, no positive sample results should be reported unless the concentration of the coinpound in the sample exceeds $5 x$ the amount in any biank. If contaminants are detected above the MOL or SQL in any blank associated with the sample, the following guidelines are used to qualify all associated data. No aciion is taken for compounds detected in an associated blank but not in the sample.

- Qualify other positive sample results $<5 x$ the amount detected in any blank as not detected (U).

- A special case exists for positive sample results reported $\angle M O L$ or SQL. If the $5 x$ criteria dictate qualification (U), then the result reported is raised to the MOL or SQL as in the example below.

\section{5x Criteria}

$\begin{array}{ll}\text { Blank result } & 7 \\ \text { MDL or SQL } & 5 \\ \text { Sample result } & 4 \\ \text { Qualified result } & 5 U\end{array}$

This is the only case where a reported laboratory value is changed. Sample results must not be corrected by subtracting any blank value.

- The 5x criteria shall also be applied to tentatively identified compounds (TIC) and unknown compounds which are found in both the sample and associated blank(s) for GC/MS analyses.

\section{B.7 VALIDATION COVERSHEETS}

The following pages are examples of RCRA data validation coversheets. Data validation coversheets are used to summarize specific areas checked and to note deficiencies requiring attention, as well as overall laboratory per- 
formance and any discernable trends in the quality of the data. Supplementary information is contained in attachment pages to the zoversheets. Each coversheet summarizes the validation results for a single sampling trip to a single well.

The following qualifiers are applied to the data as a result of the RCRA validation process and override any previous flags reported by the laboratory. If any other qualifiers are necessary to describe the quality of the data, they must be clearly documented.

The material was analyzed for, but was not detected. The associated value is the MDL or SQL.

UJ The material was analyzed for, but was not detected. The MDL or SQL is an estimated quantity.

J

The associated value is an estimated quantity.

R

The data are unusable. 
OFFICE OF SAMPLE MANAGEMENT RCRA DATA VALIDATION - LEVEL B

Date $8 / 12 / 90$

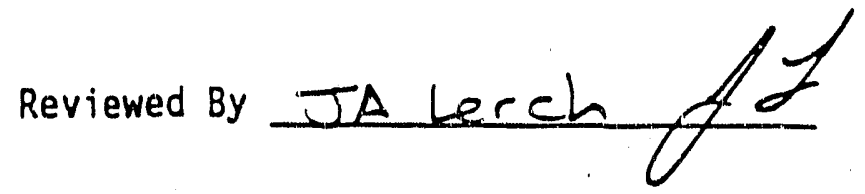

DATA ASSESSMENT SUMMARY

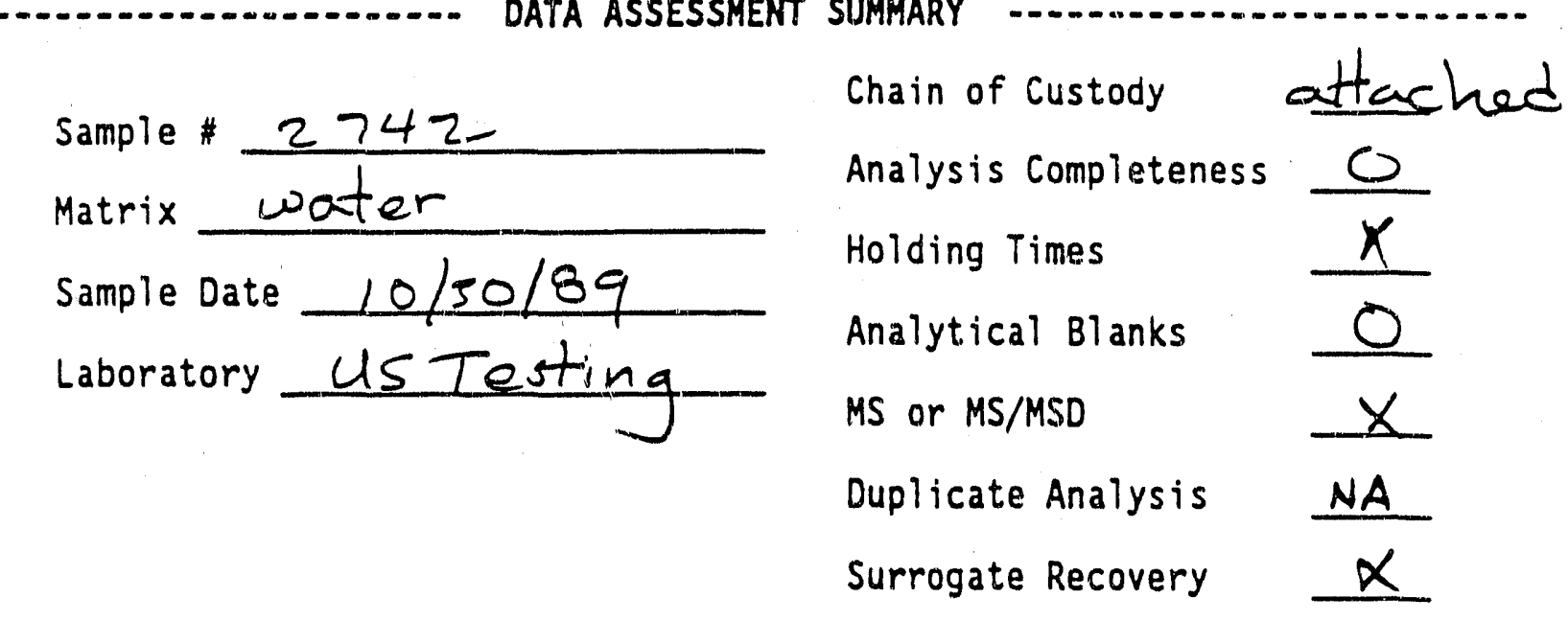

0 -data had no problems; $X$-data qualified due to minor problems

$M$-data qualified due to major problems/some data may be unusable

Overall Assessment: Well $\# 2-E 25-35$

No major problems-all results acceptable with attached qualification

- herbicides (737) estimated (UJ) because of low surrogate recovery, high MSD POR, mised HT

- hydrazine ( $\mathrm{H}_{62}$ ) estimated (UJ) due to low MS,MSD Z.R

- DAI qualified as estimated (UJ) due to miseed HT 
Westinghouse

WHC-EP-0366

OFFICE OF SAMPLE MANAGEMENT RCRA DATA VALIDATION - LEVEL B

Date $8 / 12 / 90$

Reviewed By JA Leech

DATA ASSESSMENT SUMMARY

Sample \#2670

Chain of custody attached

Matrix water

Sample Date $10 / 30 / 89$

Analys is Completeness

Holding Times

Laboratory us Testing

Analytical Blanks

MS or MS/MSD

Duplicate Analysis

NA

Surrogate Recovery

0 -data had no problems; $X$-data qualified due to minor problems

$M$-data qualified due to major problems/some data may be unusable

Overall Assessment: well Zz-W19-20

No major problems - all results acceptable with attached qualification

- DAI qualified as estimated (UJ) due to missed HT

- Herbicides (7JT) estimated (UJ) due to high MS 7.R

- hydrazine (HGC) estimated (UJ) due to low MS, MSD ?.R

RCRA B - Cover

B-7

n now on 
WHC-EP-0366

Westinghouse

Hanford Company

OFFICE OF SAMPLE MANAGEMENT RCRA DATA VALIDATION - LEVEL B

Date $8 / 12 / 90$

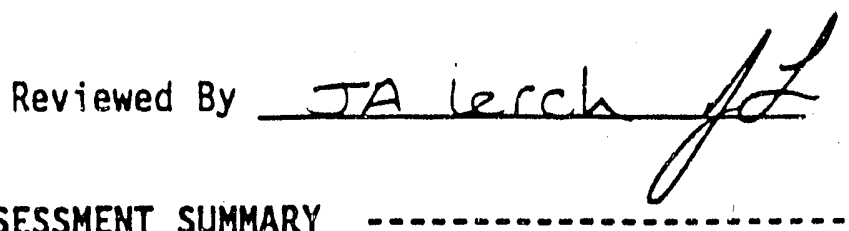

DATA ASSESSMENT SUMMARY

Sample \#2663

Chain of Custody attached

Matrix water

Analysis Completeness

Holding Times

Sample Date $10 / 27 / 89$

Analytical Blanks

Laboratory US Testing

MS or MS/MSD

Duplicate Analysis NA

Surrogate Recovery $\mathrm{O}$

0 -data had no problems; $X$-data qualified due to minor problems

$M$-data qualified due to major problems/some data may be unusable

Overall Assessment: Well $\# 2-\omega_{19-23}$

No major problems -all results acceptable with attached qualification

- hydrazine (HOZ) estimated (UJ) due to low MS, MSD IO R

- herbicides (732) estimated due to rig MS

RCRA B - Cover

B-8

r nos on 
Q. Westinghouse

WHC-EP-0366

OFFICE OF SAMPLE MANAGEMENT RCRA DATA VALIDATION - LEVEL B

Date $8 / 12 / 90$

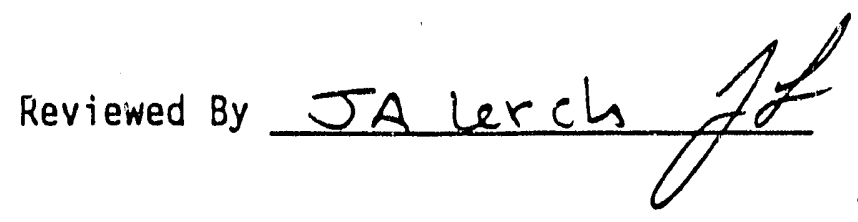

DATA ASSESSMENT SUMMARY

Sample \#-2405 well z-EZ5-28

Matrix water

Sample Date $10 / 27 / 90$

Chain of custody attached Analysis Completeness

Laboratory UsTesting

Holding Times

Analytical Blanks

MS or MS/MSD

Duplicate Analysis

Surrogate Recovery

$x$

0

$x$

NA

0 -data had no problems; $X$-data qualified due to minor problems

$M$-data qualified due to major problems/some data may be unusable

overall Assessment: No major problems -all results

acceptable w/attached qualification

- TuI (763) qualified due to exceeded HT

- Herbicides (232) quad ified (US) due to high MS ZOR

- Hydrazine (HC2) qualified (US) due to low MS, MSD ToR

RCRA B - Cover

B-9

$x \operatorname{mon}$ 
OFFICE OF SAMPLE MANAGEMENT RCRA DATA VALIDATION - LEVEL B

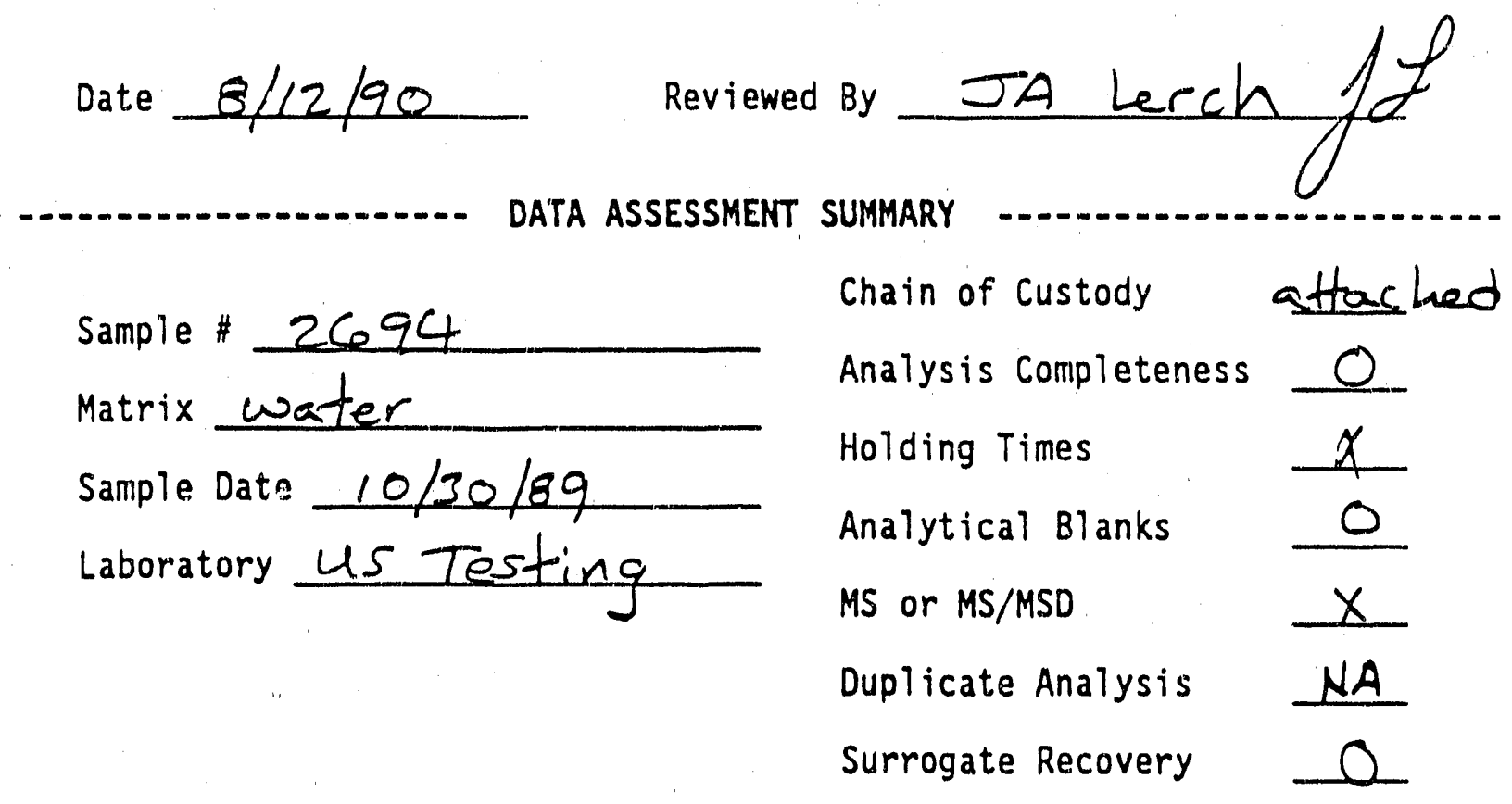

0 -data had no problems; $X$-data qualified due to minor problems

$M$-data qualified due to major problems/some data may be unusable

Overall Assessment: Well \# 2-W19-24

No major problems, all results acceptable with

atteched qualification

- DAI (763) estimated (UV) due to exceeded HT

- hydcazine (HE.z) estimated (WO) due to low MS, MSD T.R

- harbicidor(737) estimated (UJ) due to hiqh MS ?.R

RCRA B - Cover 
Q7. Whanghouse

WHC-EP-0366

Hanford Company

OFFICE OF SAMPLE MANAGEMENT RCRA DATA VALIDATION - LEVEL B

Date $8 / 12 / 90$

Reviewed By

DATA ASSESSMENT SUMMARY

Sample \#2730

Matrix water

Sample Date $10 / 31 / 90$

Laboratory us Testing
Chain of Custody attached

Analysis Completeness

Holding Times

Analytical Blanks

MS or MS/MSD

Duplicate Analysis

Surrogate Recovery

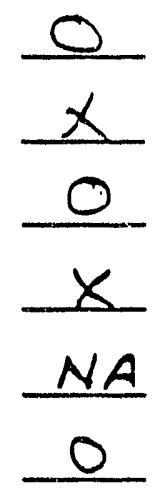

0 -data had no problems; $x$-data qualified due to minor problems

$M$-data qualified due to major problems/some data may be unusable

Overall Assessment: well \# 2-E25-32

No major problems - all results acceptable with attached qualification

- DAI (263) estimated due to exceeded HT

- Herbicides (73i) estimated (us) due to high MS T.R

RCRA B - Cover

$\bar{B}-i \mathrm{i}$

has on 
QV Westinghouse

WHC-EP-0366

Hanford Company

OFFICE OF SAMPLE MANAGEMENT RCRA DATA VALIDATION - LEVEL B

Date $08 / 11 / 90$

Reviewed By

DATA ASSESSMENT SUMMARY

Sample \#2231

Matrix water

Sample Date $10 / 31 / 8 q$

Laboratory Us Testing
Chain of Custody attached

Analysis Completeness

Holding Times

Analytical Blanks

MS or MS/MSD

Duplicate Analysis

Surrogate Recovery

0 -data had no problems; $X$-data qualified due to minor problems

$M$-data qualified due to major problems/some data may be unusable

Overall Assessment: Well \# 2-E18-1

No major problems - all results acceptable with attached qualification

- DAI (263) estimated (u) du to exceeded HI

- herbicides (237) estimated (W0) due to high MS ZOR

RCRA B - Cover

$\mathrm{P}-12$ 
We Westinghouse

WHC-EP-0366 Hanford Company

OFFICE OF SAMPLE MANAGEMENT RCRA DATA VALIDATION - LEVEL B

Date $08 / 12 / 90$

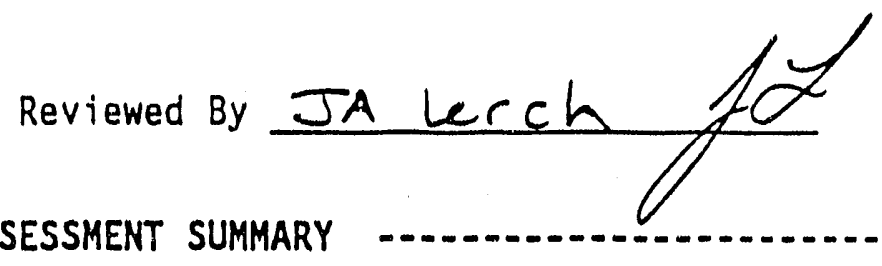

DATA ASSESSMENT SUMMARY

Sample \# 2666

Matrix water

Sample Date $10 / 27 / 89$

Laboratory
Chain of Custody

Analys is Completeness

Holding Times

Analytical Blanks

MS or MS/MSD

Duplicate Analysis

Surrogate Recovery attached

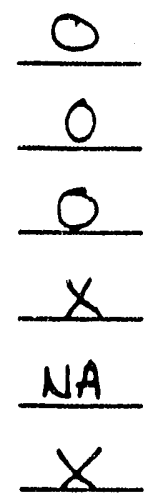

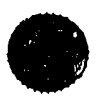

0 -data had no problems; $X$-data qualified due to minor problems

$M$-data qualified due to major problems/some data may be unusable

Overall Assessment: Well \#2-w19-26

No major problems -all results acceptable

with attached qualification

- Dioxins (752) estimated (w) due to low surrogate $2 R$

- hydrazine (HGZ) estimated (UT) due to low MS, MSD TaR

- Pest (229) MS, MSD 7.R high is ample results all $\angle D L$, acceptable wont qualification

RCRA B - Cover

B-13

sa moo nate 
OFFICE OF SAMPLE MANAGEMENT RCRA DATA VALIDATION - LEVEL B

Date $8 / 14 / 90$

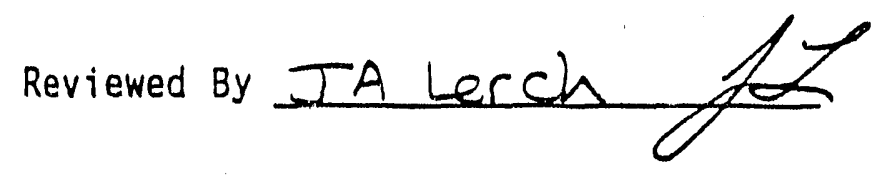

DATA ASSESSMENT SUMMARY

Sample \#2807 Well 2-w19-19

Chain of Custody

attached

Matrix water

Analysis Completeness

Halding Times

Sample Date $-10-31-89$

Laboratory US Testing

Analytical Blanks

MS or MS/MSD

Duplicate Analysis

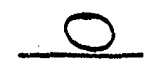

Surrogate Recovery

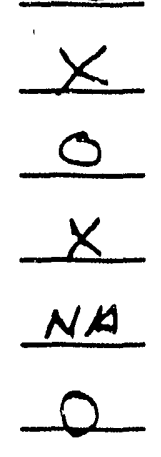

0 -data had no problems; $X$-data qualified due to minor problems

M -data qualified due to major problems/some data may be unusable

overa11 Assessment: No major problems-ail results ace eptable with qualification - DAI (2603) estimated due to exceeded HT - herbicides (237) estimated (UJ) due to high MS 2.R 
(w)

Westinghouse

WHC-EP-0367

Hanford Company

OFFICE OF SAMPLE MANAGEMENT RCRA DATA VALIDATION - LEVEL B

Date $8 / 12 / 90$

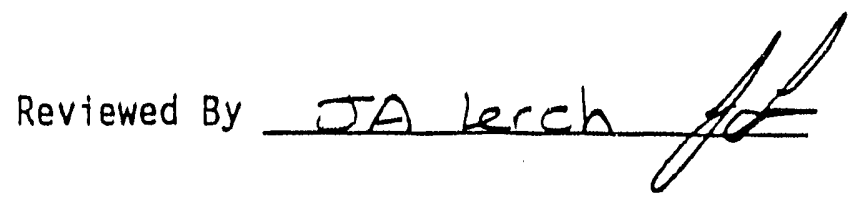

DATA ASSESSMENT SUMMARY

Sample \#2741

Matrix water

Sample Date $10 / 31 / 90$

Laboratory uses Testing
Chain of Custody

Analys is Completeness

Holding Times

Analytical Blanks MS or MS/MSD

Duplicate Analysis

Surrogate Recovery attached

$\frac{\frac{0}{x}}{\frac{0}{x}} \frac{N A}{0}$

0 -data had no problems; $X$-data qualified due to minor problems $M$-data qualified due to major problems/some data may be unusable

overall Assessment: Well 2 Z $2-625-34$

No major problems - all results acceptable with attached qualification

- DAI (765) estimated $(U T)$ du to exceeded HT

- herbicider(237) estimated (UJ) due to high MS $2 R$

RCRA B - Cover

B-15 
WHC-EP-0366

APPENDIX C

WELL LISTING AND SAMPLING DATES

C-1 


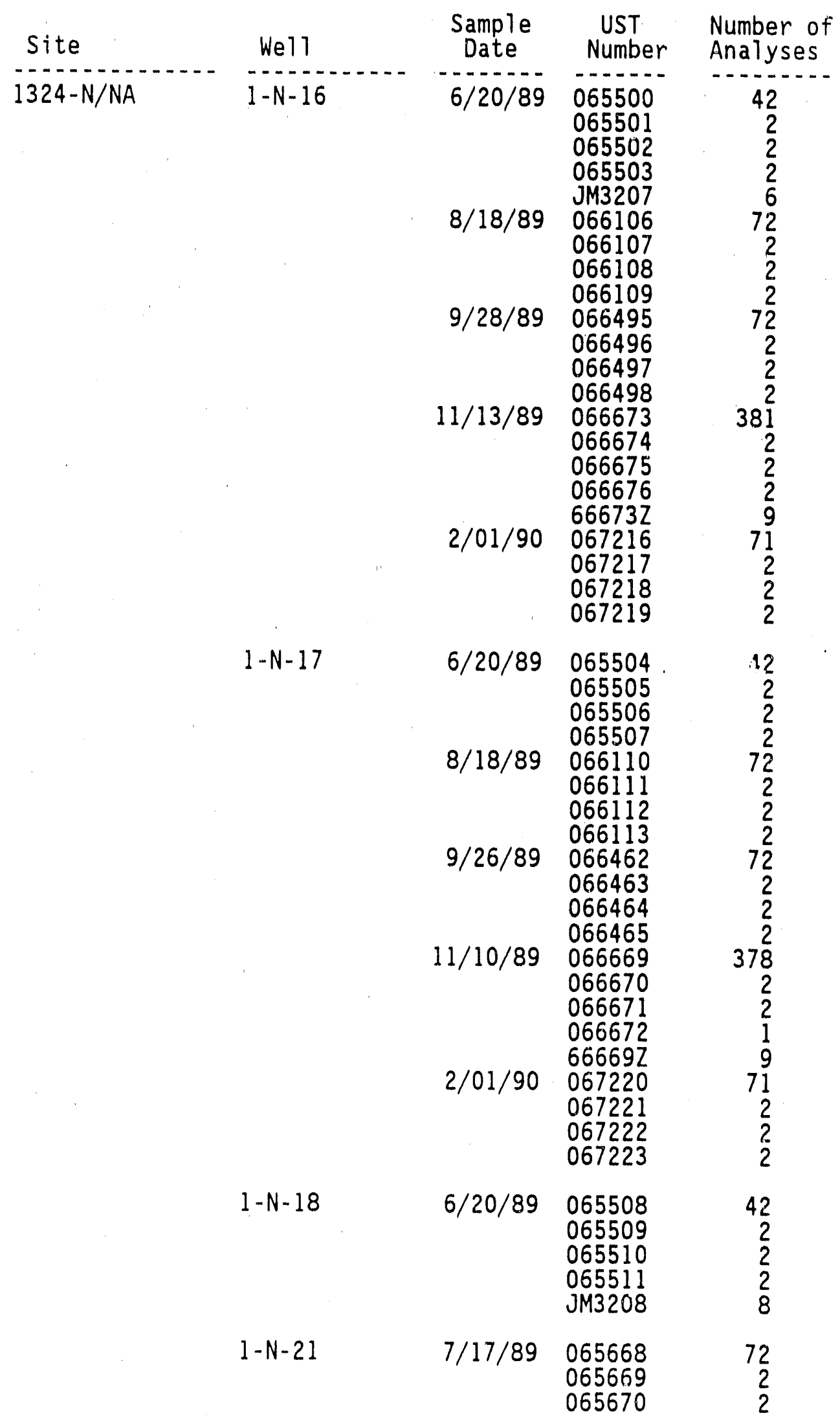


WHC -EP-0366

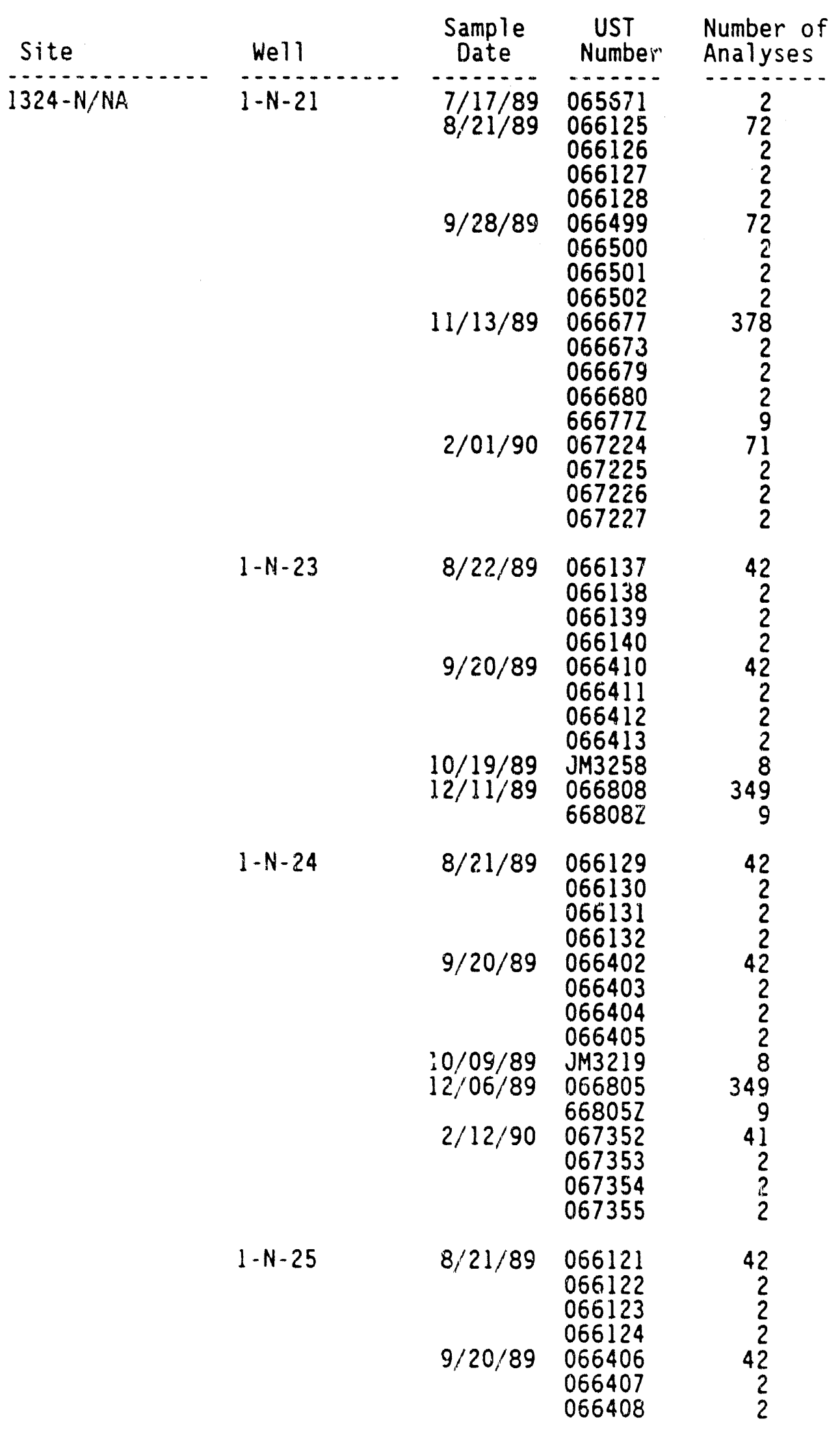




\begin{tabular}{|c|c|c|c|c|}
\hline Site & Well & $\begin{array}{c}\text { Sample } \\
\text { Date }\end{array}$ & $\begin{array}{l}\text { UST } \\
\text { Number }\end{array}$ & $\begin{array}{l}\text { Number of } \\
\text { Analyses }\end{array}$ \\
\hline \multirow[t]{4}{*}{$1324-N / N A$} & $1-N-25$ & $\begin{array}{r}9 / 20 / 89 \\
10 / 09 / 89 \\
12 / 06 / 89\end{array}$ & $\begin{array}{l}066409 \\
\text { JM3220 } \\
066806 \\
668062\end{array}$ & $\begin{array}{r}2 \\
8 \\
349 \\
9\end{array}$ \\
\hline & $1-N-26$ & $\begin{array}{l}8 / 22 / 89 \\
9 / 18 / 89 \\
12 / 12 / 89 \\
2 / 12 / 90\end{array}$ & $\begin{array}{l}066141 \\
066142 \\
066143 \\
066144 \\
066361 \\
066362 \\
066363 \\
066364 \\
066810 \\
668102 \\
067356 \\
067357 \\
067358 \\
067359\end{array}$ & $\begin{array}{r}42 \\
2 \\
2 \\
2 \\
42 \\
2 \\
2 \\
2 \\
349 \\
9 \\
41 \\
2 \\
2 \\
2\end{array}$ \\
\hline & $1-N-47$ & $\begin{array}{c}8 / 21 / 89 \\
9 / 18 / 89 \\
12 / 11 / 89 \\
2 / 08 / 90\end{array}$ & $\begin{array}{l}066133 \\
066134 \\
066135 \\
066136 \\
066365 \\
066366 \\
066367 \\
066368 \\
066809 \\
668092 \\
067332 \\
067333 \\
067334 \\
067335\end{array}$ & $\begin{array}{r}42 \\
2 \\
2 \\
2 \\
42 \\
2 \\
2 \\
2 \\
349 \\
9 \\
71 \\
2 \\
2 \\
2\end{array}$ \\
\hline & $I-N-54$ & $\begin{array}{l}6 / 21 / 89 \\
8 / 15 / 89 \\
10 / 02 / 89 \\
11 / 01 / 89 \\
2 / 01 / 90\end{array}$ & $\begin{array}{l}065524 \\
065525 \\
065526 \\
065527 \\
066063 \\
066064 \\
066065 \\
066066 \\
066530 \\
066531 \\
066532 \\
066533 \\
066630 \\
666302 \\
067232 \\
067233 \\
067234 \\
067235\end{array}$ & $\begin{array}{r}72 \\
2 \\
2 \\
2 \\
72 \\
2 \\
2 \\
2 \\
72 \\
2 \\
2 \\
2 \\
378 \\
9 \\
71 \\
2 \\
2 \\
2\end{array}$ \\
\hline
\end{tabular}




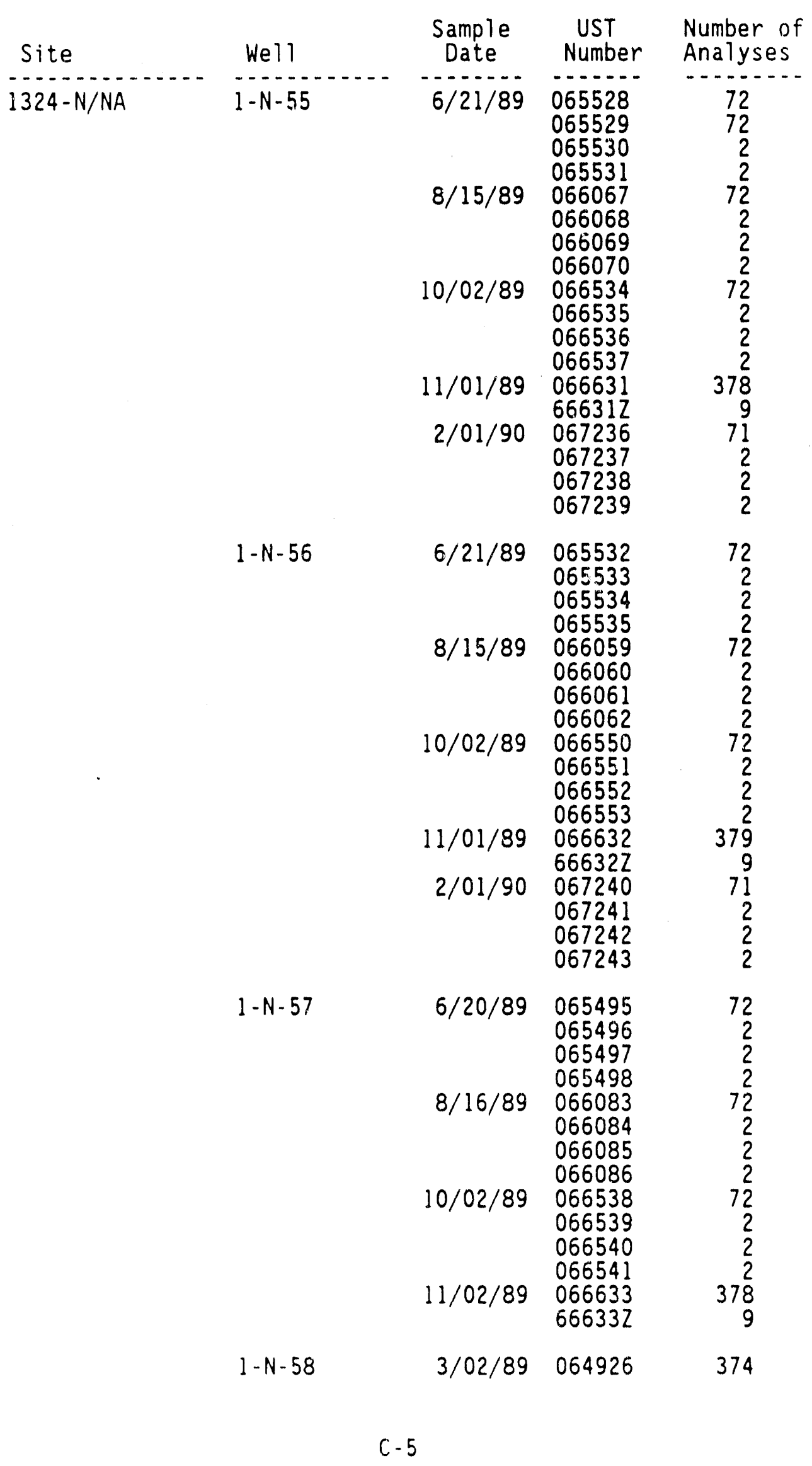




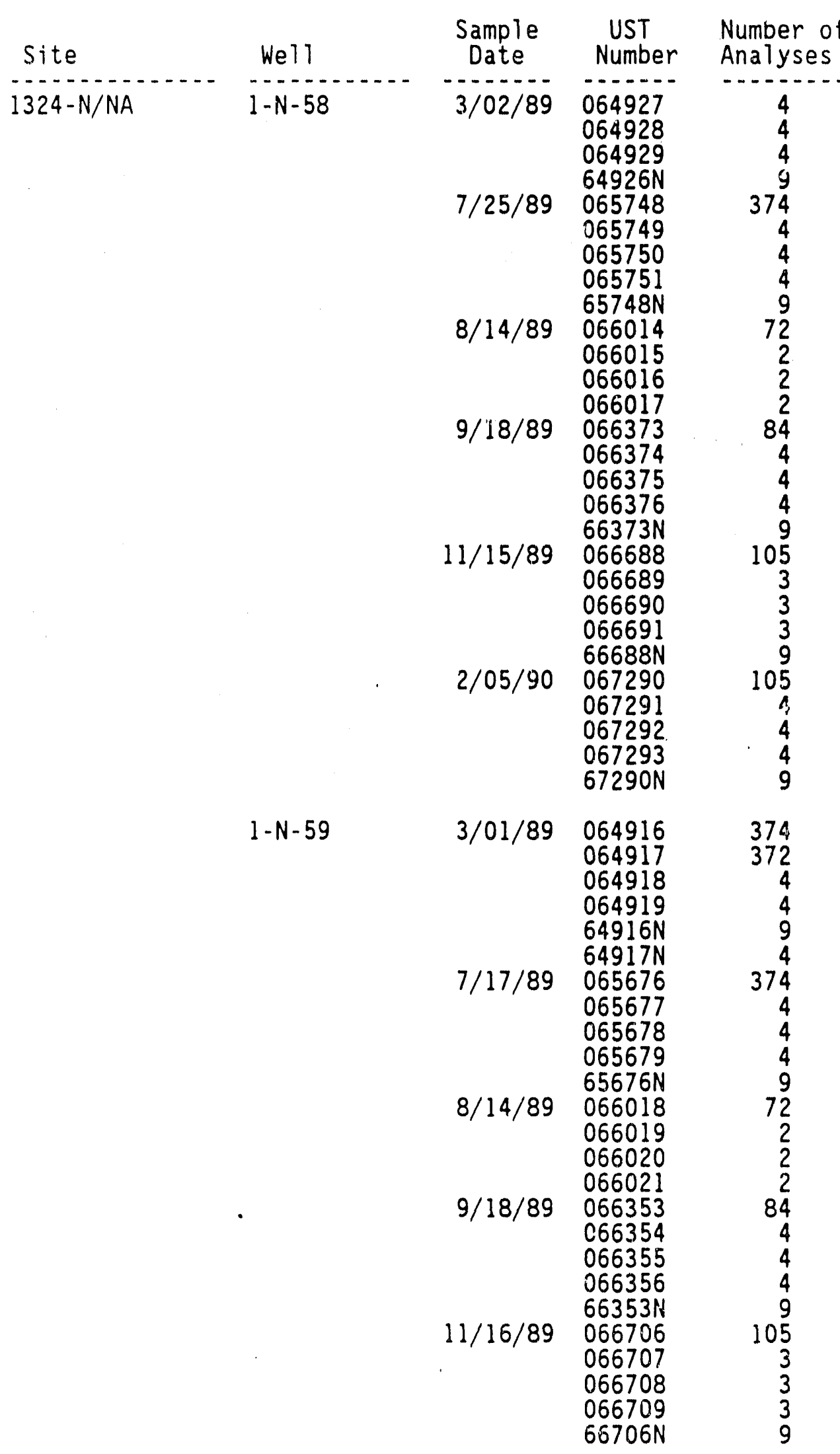


WHC-EP-0366

\begin{tabular}{|c|c|c|c|c|}
\hline Site & Well & $\begin{array}{c}\text { Sample } \\
\text { Date }\end{array}$ & $\begin{array}{l}\text { UST } \\
\text { Number }\end{array}$ & $\begin{array}{l}\text { Number of } \\
\text { Analyses }\end{array}$ \\
\hline \multirow[t]{3}{*}{$1324-N / N A$} & $1-N-59$ & $2 / 02 / 90$ & $\begin{array}{l}067244 \\
067245 \\
067246 \\
067247 \\
67244 N \\
67245 N\end{array}$ & $\begin{array}{r}106 \\
82 \\
4 \\
4 \\
9 \\
9\end{array}$ \\
\hline & $1-N-60$ & $\begin{array}{l}8 / 14 / 89 \\
9 / 18 / 89\end{array}$ & $\begin{array}{l}064930 \\
064931 \\
064932 \\
064933 \\
64930 N \\
065853 \\
065854 \\
065855 \\
065856 \\
65853 N \\
65854 N \\
066022 \\
066023 \\
066024 \\
066025 \\
066357 \\
066358 \\
066359 \\
066360 \\
66357 N \\
066710 \\
066711 \\
066712 \\
066713 \\
66710 N \\
067286 \\
067287 \\
067288 \\
067289 \\
67286 N\end{array}$ & $\begin{array}{r}374 \\
4 \\
4 \\
4 \\
9 \\
374 \\
374 \\
4 \\
4 \\
9 \\
9 \\
72 \\
2 \\
2 \\
2 \\
84 \\
4 \\
4 \\
4 \\
9 \\
105 \\
89 \\
3 \\
3 \\
9 \\
106 \\
4 \\
4 \\
4 \\
9\end{array}$ \\
\hline & $1-N-61$ & $\begin{array}{l}8 / 14 / 89 \\
9 / 19 / 89\end{array}$ & $\begin{array}{l}064934 \\
064935 \\
064936 \\
064937 \\
64934 N \\
065795 \\
065796 \\
065797 \\
065798 \\
65795 N \\
056028 \\
066029 \\
066030 \\
066031 \\
066381 \\
066382\end{array}$ & $\begin{array}{r}374 \\
4 \\
4 \\
4 \\
9 \\
9 \\
374 \\
4 \\
4 \\
4 \\
9 \\
9 \\
72 \\
2 \\
2 \\
2 \\
84 \\
82\end{array}$ \\
\hline
\end{tabular}




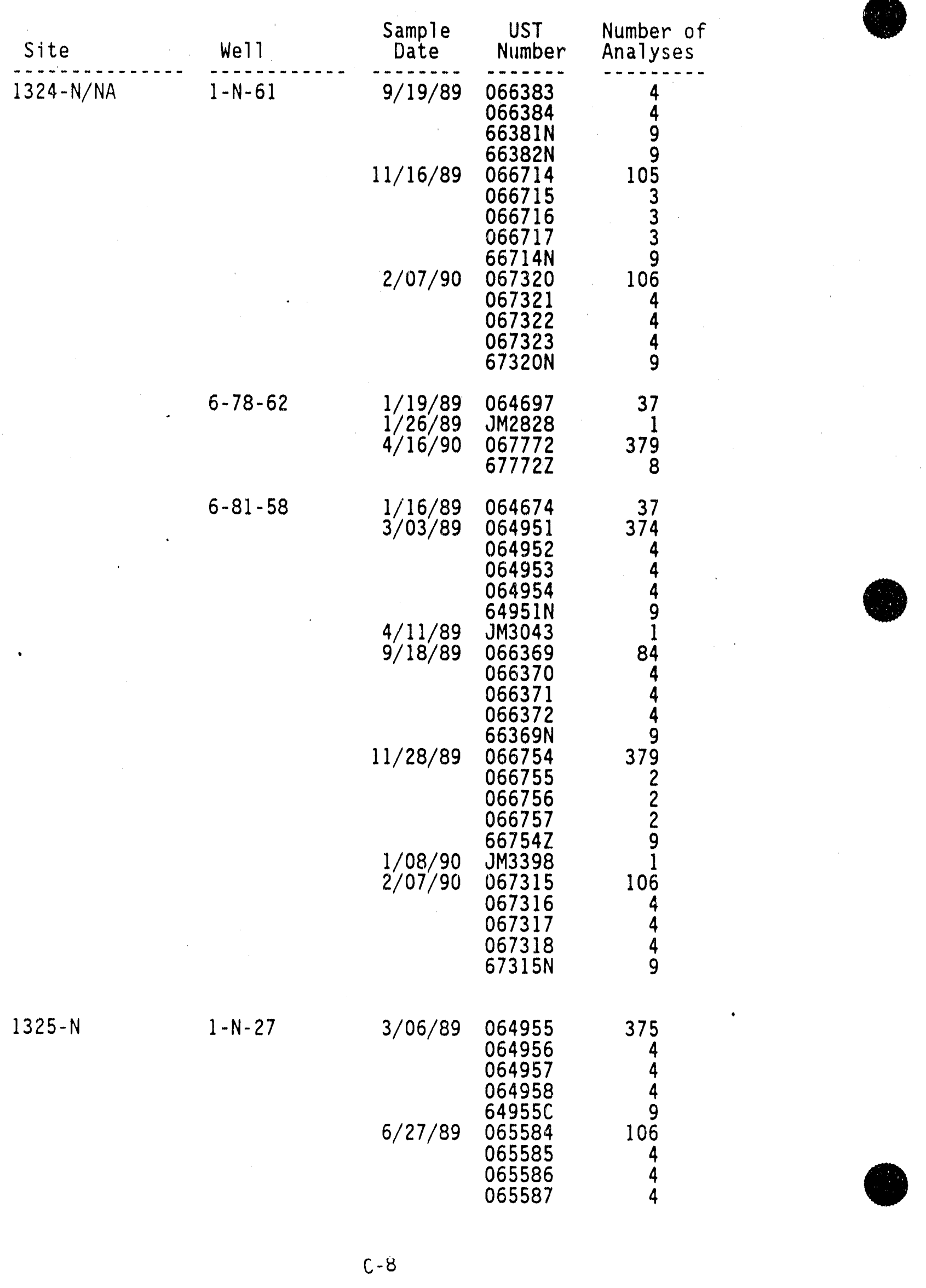




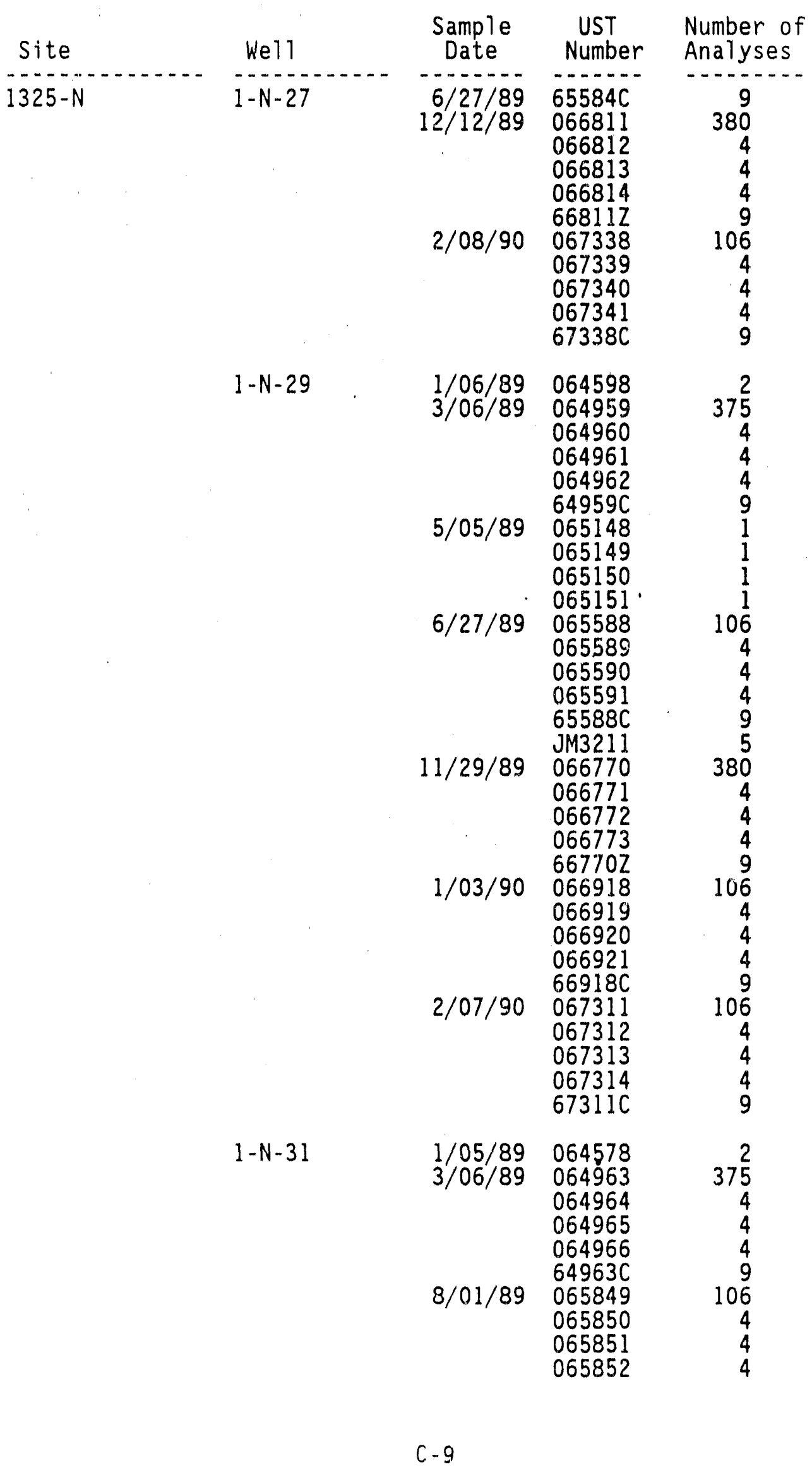


WHC-EP-0366

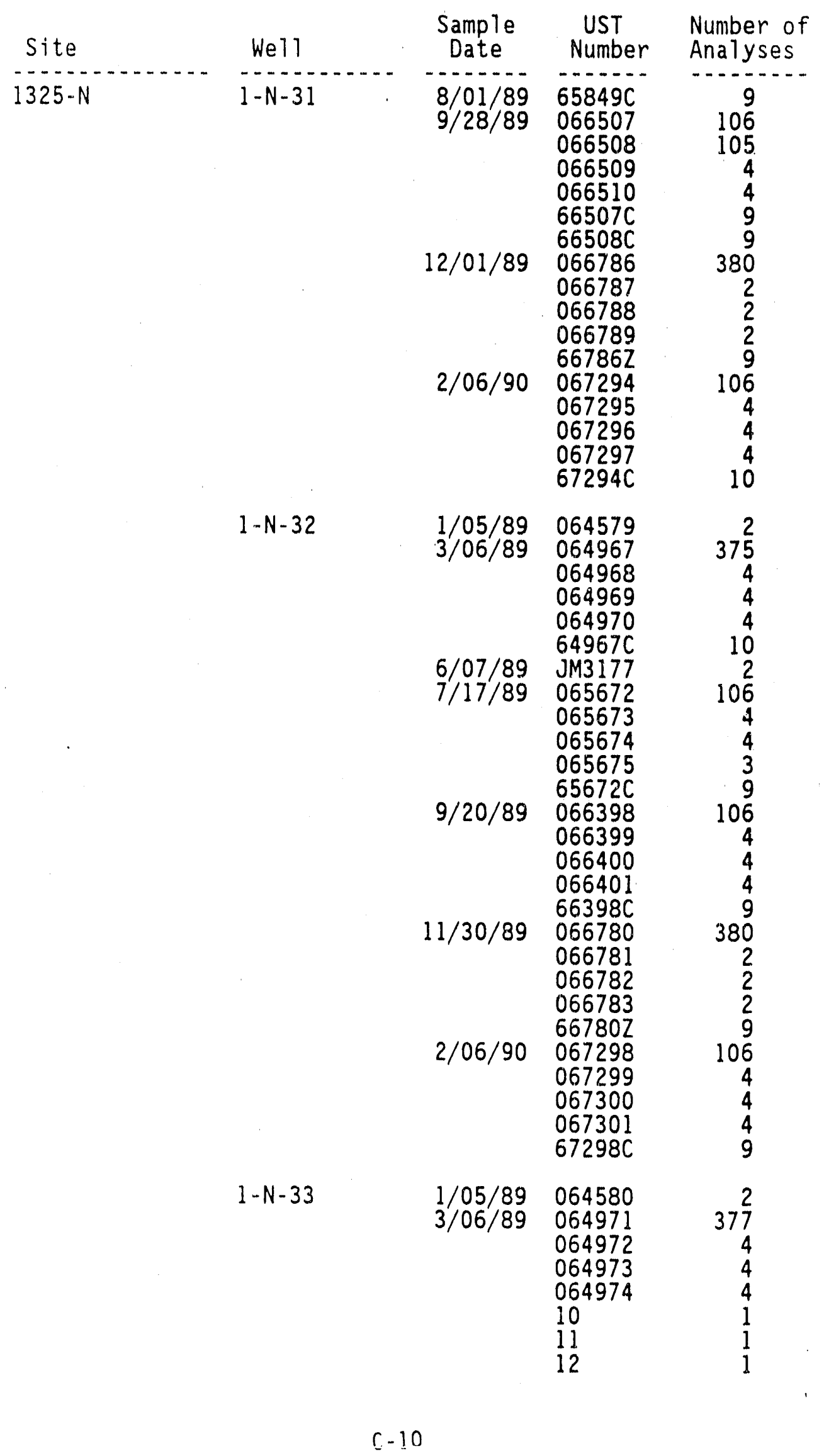


WHC-EP-0366

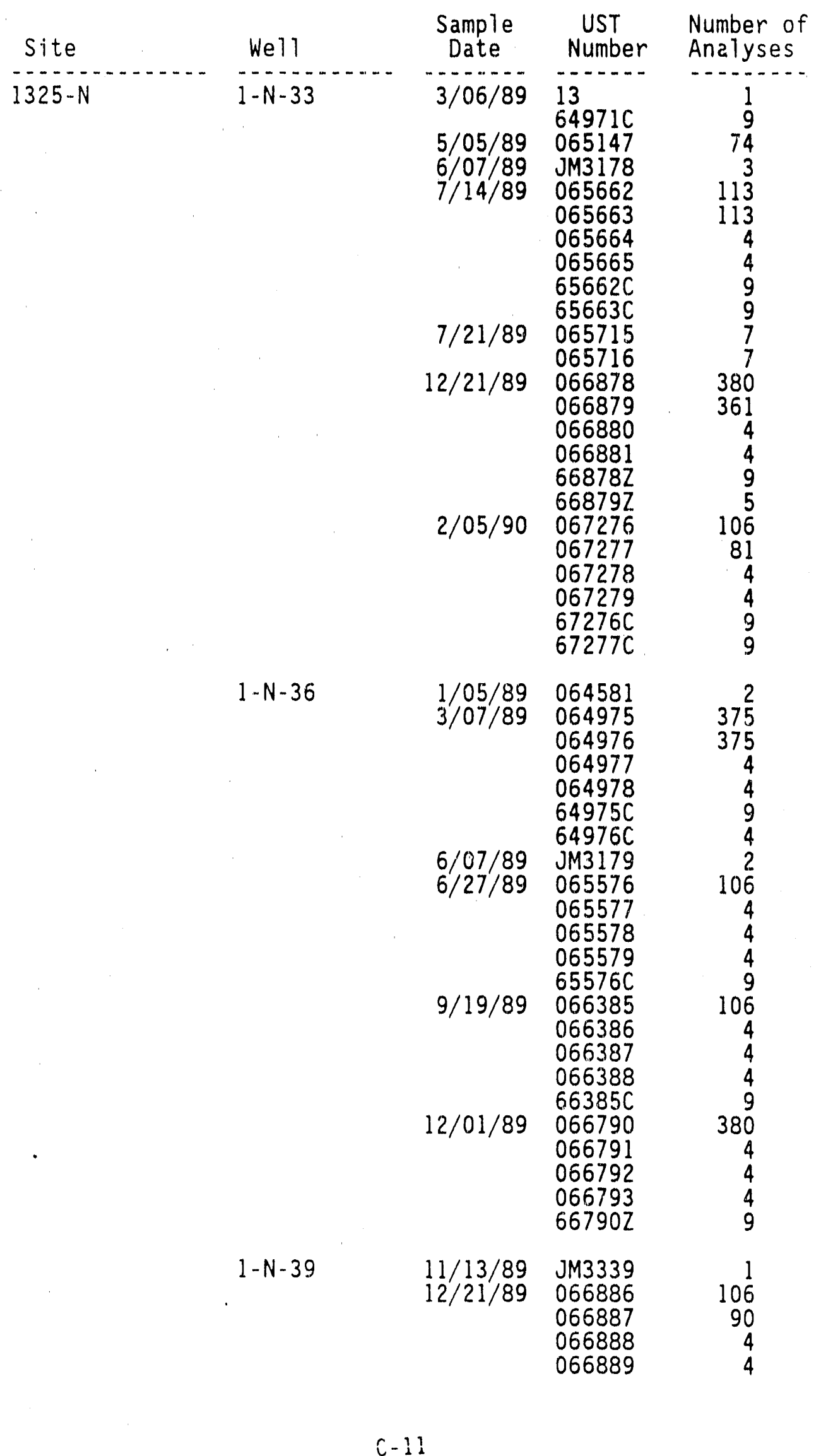




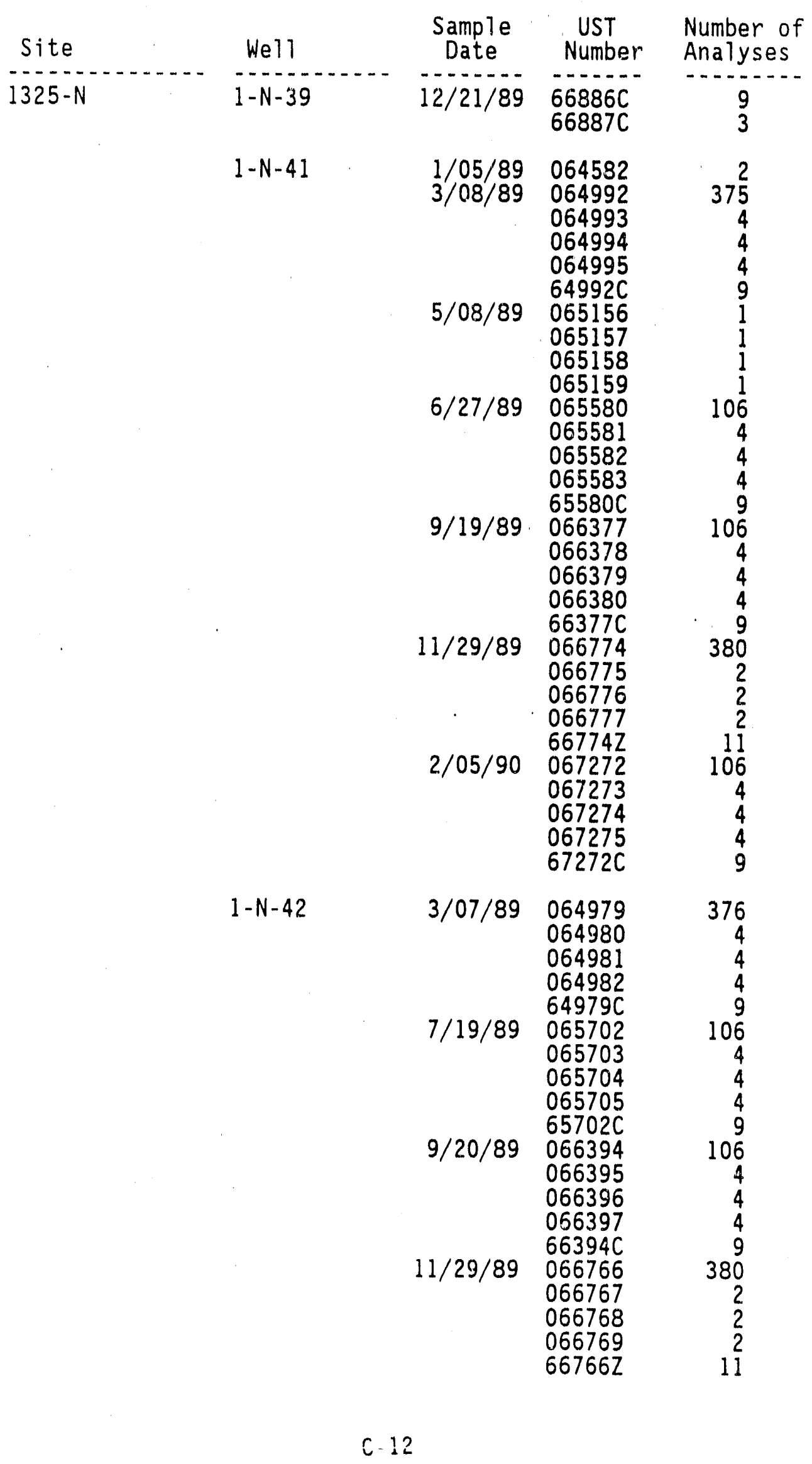


WHC-EP-0366

\begin{tabular}{|c|c|c|c|c|}
\hline Site & We11 & $\begin{array}{c}\text { Sample } \\
\text { Date }\end{array}$ & $\begin{array}{l}\text { UST } \\
\text { Number }\end{array}$ & $\begin{array}{l}\text { Number of } \\
\text { Analyses }\end{array}$ \\
\hline $1325-\mathrm{N}$ & $1-N-42$ & $2 / 07 / 90$ & $\begin{array}{l}067324 \\
067325 \\
067326 \\
067327 \\
67324 \mathrm{C}\end{array}$ & $\begin{array}{r}106 \\
4 \\
4 \\
4 \\
9\end{array}$ \\
\hline & $1-N-52$ & $\begin{array}{r}9 / 20 / 89 \\
12 / 01 / 89\end{array}$ & $\begin{array}{l}064988 \\
064989 \\
064990 \\
064991 \\
64988 C \\
065152 \\
065153 \\
065154 \\
065155 \\
065592 \\
065593 \\
065594 \\
065595 \\
65592 C \\
J M 3212 \\
066414 \\
066415 \\
066416 \\
066417 \\
66414 C \\
066794 \\
066795 \\
066796 \\
066797 \\
667942 \\
067268 \\
067269 \\
067270 \\
067271 \\
67268 C\end{array}$ & $\begin{array}{r}375 \\
4 \\
4 \\
4 \\
9 \\
1 \\
1 \\
1 \\
1 \\
106 \\
4 \\
4 \\
4 \\
9 \\
2 \\
106 \\
4 \\
4 \\
4 \\
9 \\
380 \\
4 \\
4 \\
4 \\
9 \\
106 \\
4 \\
4 \\
4 \\
9\end{array}$ \\
\hline & $1-N-70$ & $\begin{array}{l}5 / 08 / 89 \\
7 / 18 / 89\end{array}$ & $\begin{array}{l}064583 \\
064996 \\
064997 \\
064998 \\
064999 \\
64996 C \\
065160 \\
065161 \\
065162 \\
065163 \\
065694 \\
065695 \\
065696 \\
065697 \\
65694 C \\
J M 3213 \\
066525\end{array}$ & $\begin{array}{r}2 \\
375 \\
4 \\
4 \\
4 \\
9 \\
1 \\
1 \\
1 \\
1 \\
106 \\
4 \\
4 \\
4 \\
9 \\
1 \\
106\end{array}$ \\
\hline
\end{tabular}




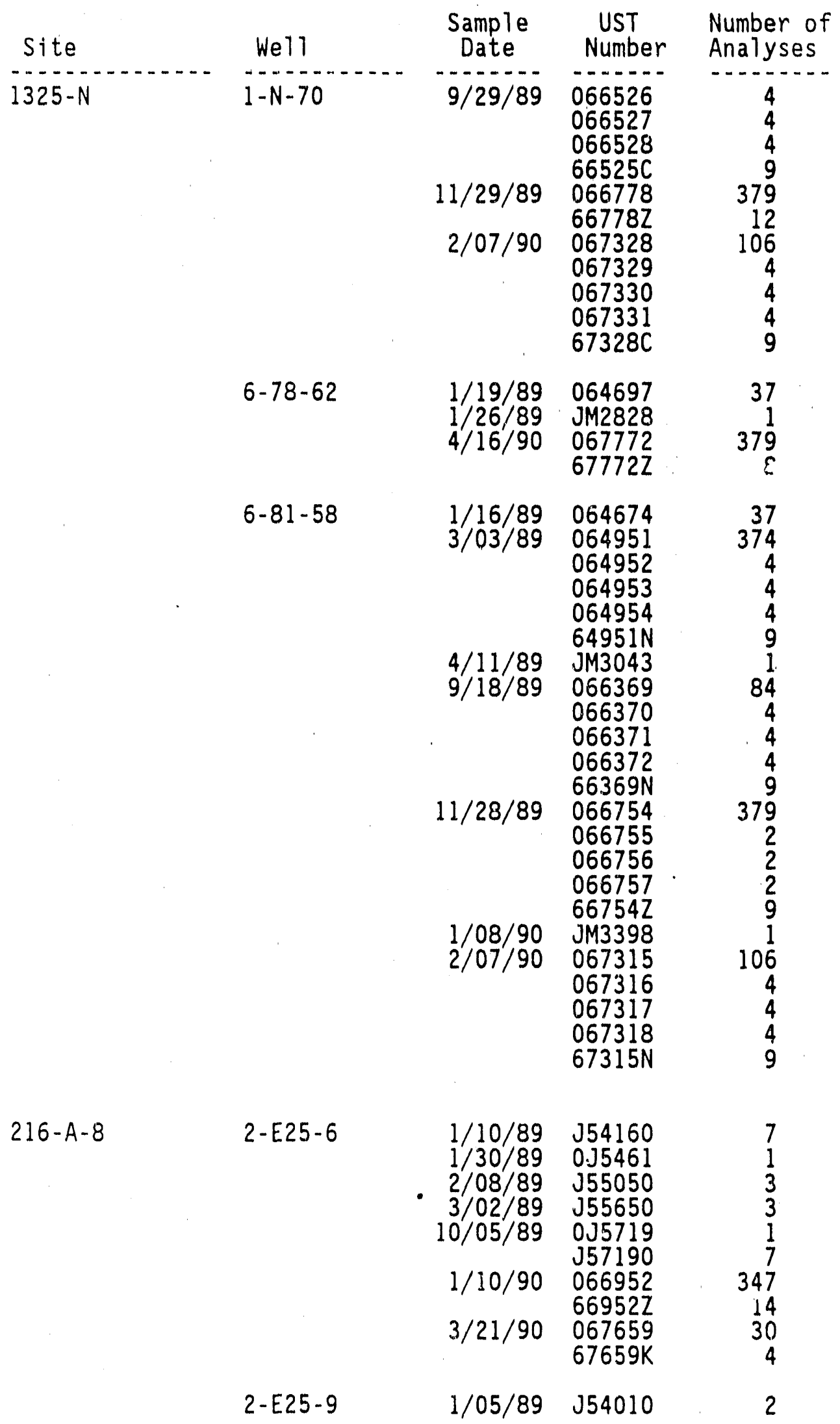


WHC-EP-0366

\begin{tabular}{lrrrr} 
Site & We11 & $\begin{array}{c}\text { Sample } \\
\text { Date }\end{array}$ & $\begin{array}{c}\text { UST } \\
\text { Number }\end{array}$ & $\begin{array}{c}\text { Number of } \\
\text { Analyses }\end{array}$ \\
\hdashline $216-A-8$ & $2-E 25-9$ & $1 / 10 / 89$ & $j 54170$ & 8 \\
& $1 / 30 / 89$ & $0 j 5462$ & 1 \\
$2 / 08 / 89$ & $J 55060$ & 2 \\
$3 / 02 / 89$ & $j 55660$ & 2 \\
$10 / 05 / 89$ & $0 j 5720$ & 1 \\
& & $J 57200$ & 8 \\
& $1 / 10 / 90$ & 066953 & 347 \\
& & 669532 & 14 \\
& $3 / 21 / 90$ & 067656 & 30
\end{tabular}

$216-A-29$

$2-E 18-1$

2/15/89

064792

064793

064794

064795

064796

$64792 \mathrm{M}$

$64796 \mathrm{P}$

$5 / 26 / 89 \quad 065300$

065301

065302

065303

065308

$65300 \mathrm{M}$

$\begin{array}{ll}8 / 08 / 89 & 065956 \\ & 065957\end{array}$

065958

065959

065960

$65956 \mathrm{M}$

$10 / 31 / 89 \quad 066624$

666242

$11 / 29 / 89066762$

066763

066764

066765

83

4

21

$2 / 28 / 89 \quad 064907$

064908

064909

064910

064911

64907B

$7 / 12 / 89 \quad 065621$

065622

065623

065624

065625

65622B

$8 / 11 / 89065996$

065997

065998

065999

81
2
2
2
26
3
113
81
2
2
2
3
138
2
2
2


WHC-EP-0366

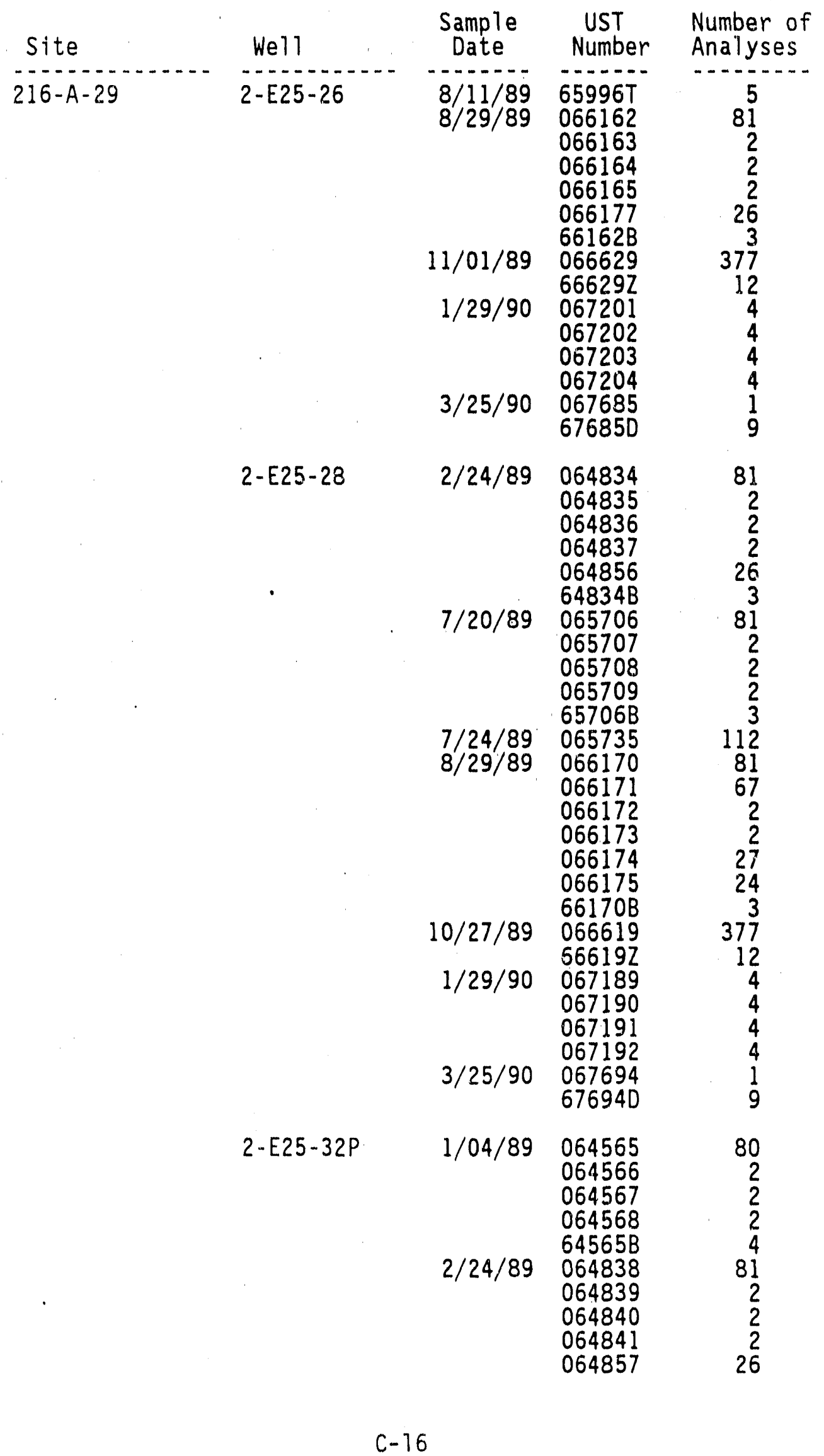


WHC -EP-0366

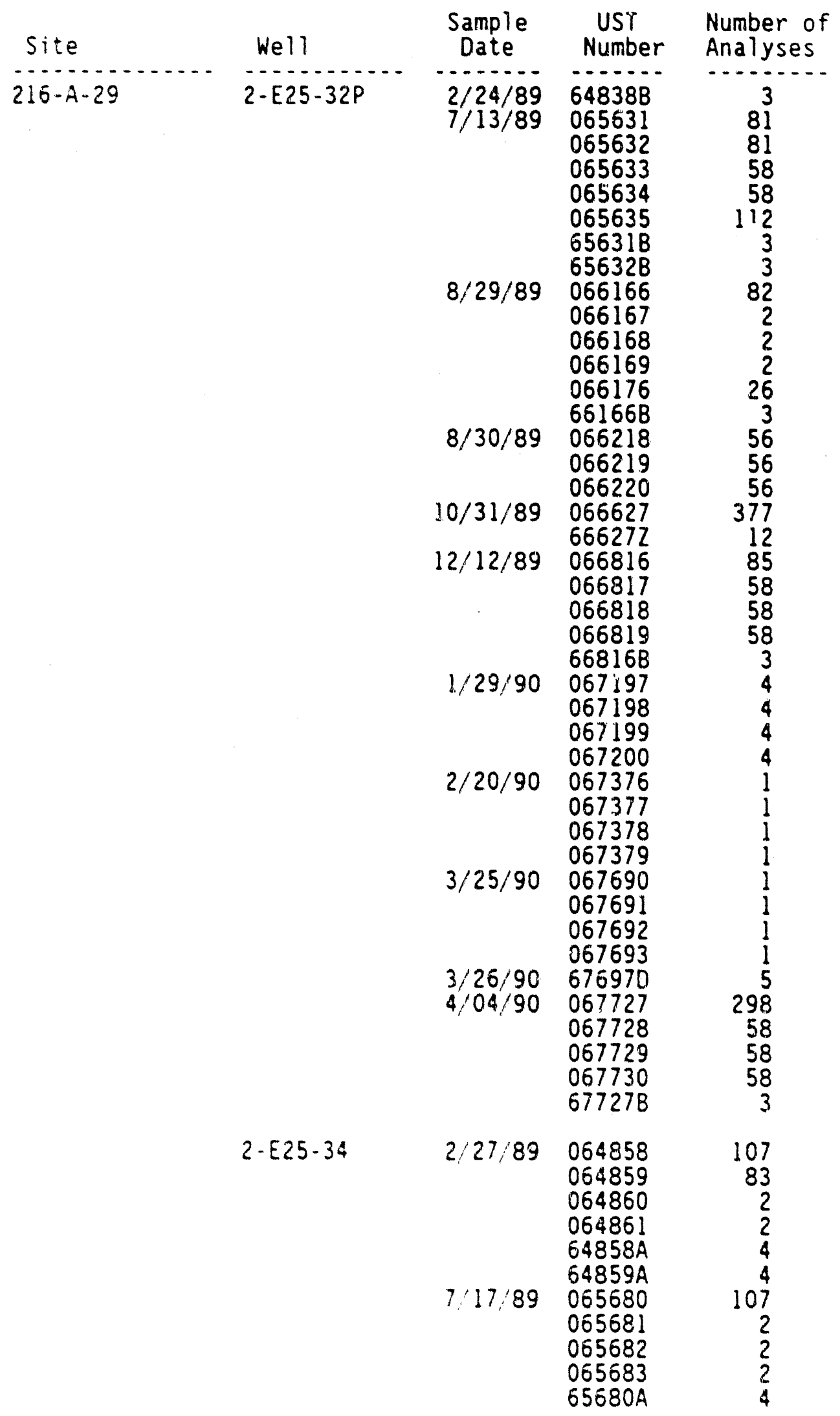




\begin{tabular}{|c|c|c|c|c|}
\hline Site & Well & $\begin{array}{l}\text { Sample } \\
\text { Date }\end{array}$ & $\begin{array}{l}\text { UST } \\
\text { Number }\end{array}$ & $\begin{array}{l}\text { Number of } \\
\text { Analyses }\end{array}$ \\
\hline \multirow[t]{2}{*}{$216-A-29$} & $2-E 25-34$ & $\begin{array}{r}8 / 30 / 89 \\
10 / 31 / 89 \\
1 / 29 / 90\end{array}$ & $\begin{array}{l}066198 \\
066199 \\
066200 \\
066201 \\
66198 A \\
066626 \\
666262 \\
067181 \\
067182 \\
067183 \\
067184\end{array}$ & $\begin{array}{r}107 \\
2 \\
2 \\
2 \\
4 \\
377 \\
12 \\
4 \\
4 \\
4 \\
4\end{array}$ \\
\hline & $2-E 25-35$ & $\begin{array}{l}2 / 27 / 89 \\
7 / 17 / 89 \\
8 / 30 / 89 \\
10 / 30 / 89 \\
1 / 29 / 90\end{array}$ & $\begin{array}{l}054864 \\
064865 \\
064866 \\
064867 \\
54864 A \\
065684 \\
065685 \\
065686 \\
065687 \\
65684 A \\
65685 A \\
066202 \\
066203 \\
066204 \\
066205 \\
66202 A \\
066622 \\
666222 \\
067185 \\
067186 \\
067187 \\
067188\end{array}$ & $\begin{array}{r}107 \\
2 \\
2 \\
2 \\
4 \\
107 \\
105 \\
2 \\
2 \\
4 \\
4 \\
107 \\
2 \\
2 \\
2 \\
4 \\
377 \\
12 \\
4 \\
4 \\
4 \\
4\end{array}$ \\
\hline \multirow[t]{2}{*}{$216-A-30$} & $2-E 16-2$ & $\begin{array}{c}1 / 10 / 89 \\
2 / 10 / 89 \\
3 / 02 / 89 \\
10 / 19 / 89 \\
12 / 14 / 89 \\
3 / 22 / 90\end{array}$ & $\begin{array}{l}0 J 5418 \\
J 54180 \\
0 J 5516 \\
J 55160 \\
0 J 5567 \\
J 55670 \\
J 57420 \\
066838 \\
668382 \\
067666 \\
67666 \mathrm{~K}\end{array}$ & $\begin{array}{r}1 \\
7 \\
1 \\
6 \\
1 \\
6 \\
7 \\
347 \\
12 \\
30 \\
4\end{array}$ \\
\hline & $2-E 25-11$ & $\begin{array}{l}1 / 09 / 89 \\
2 / 10 / 89 \\
3 / 06 / 89\end{array}$ & $\begin{array}{l}\text { OJ } 5410 \\
\text { J54 } 100 \\
0 J 5515 \\
\text { J55 } 5150 \\
0 J 5575 \\
\text { J55750 }\end{array}$ & $\begin{array}{l}1 \\
7 \\
1 \\
6 \\
1 \\
6\end{array}$ \\
\hline & & & & \\
\hline
\end{tabular}


WHC-EP-0366

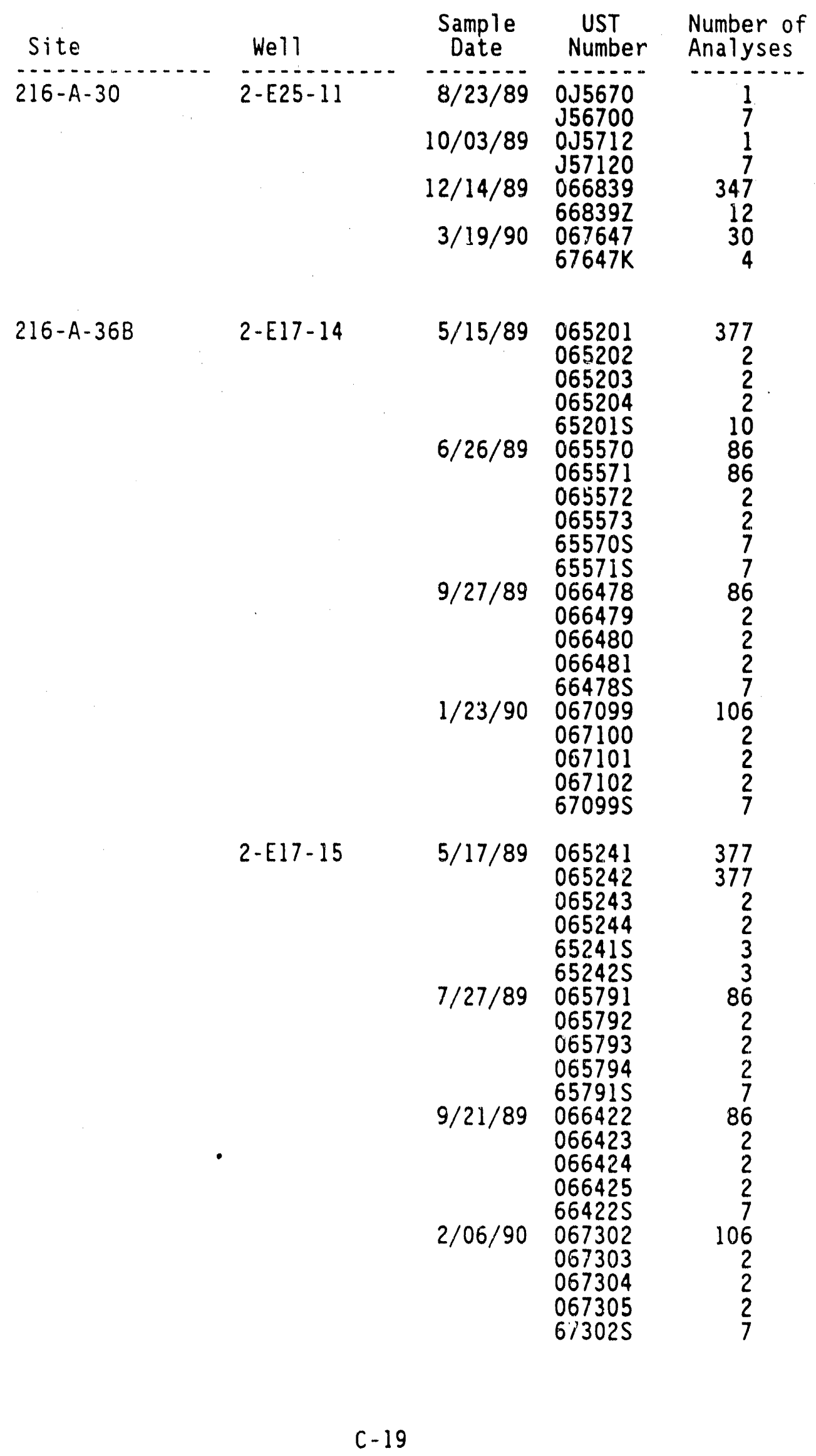




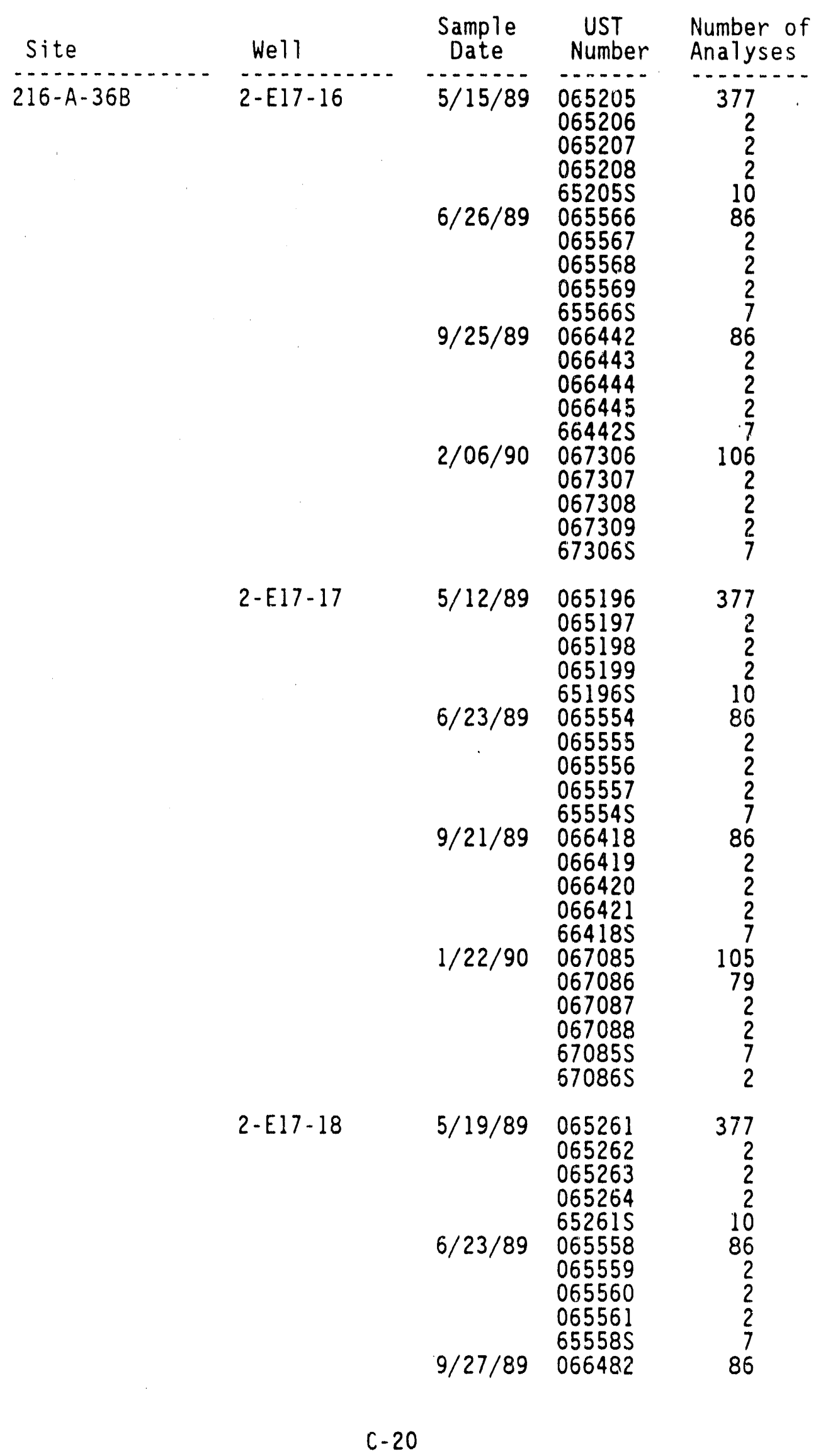


WHC-EP-0366

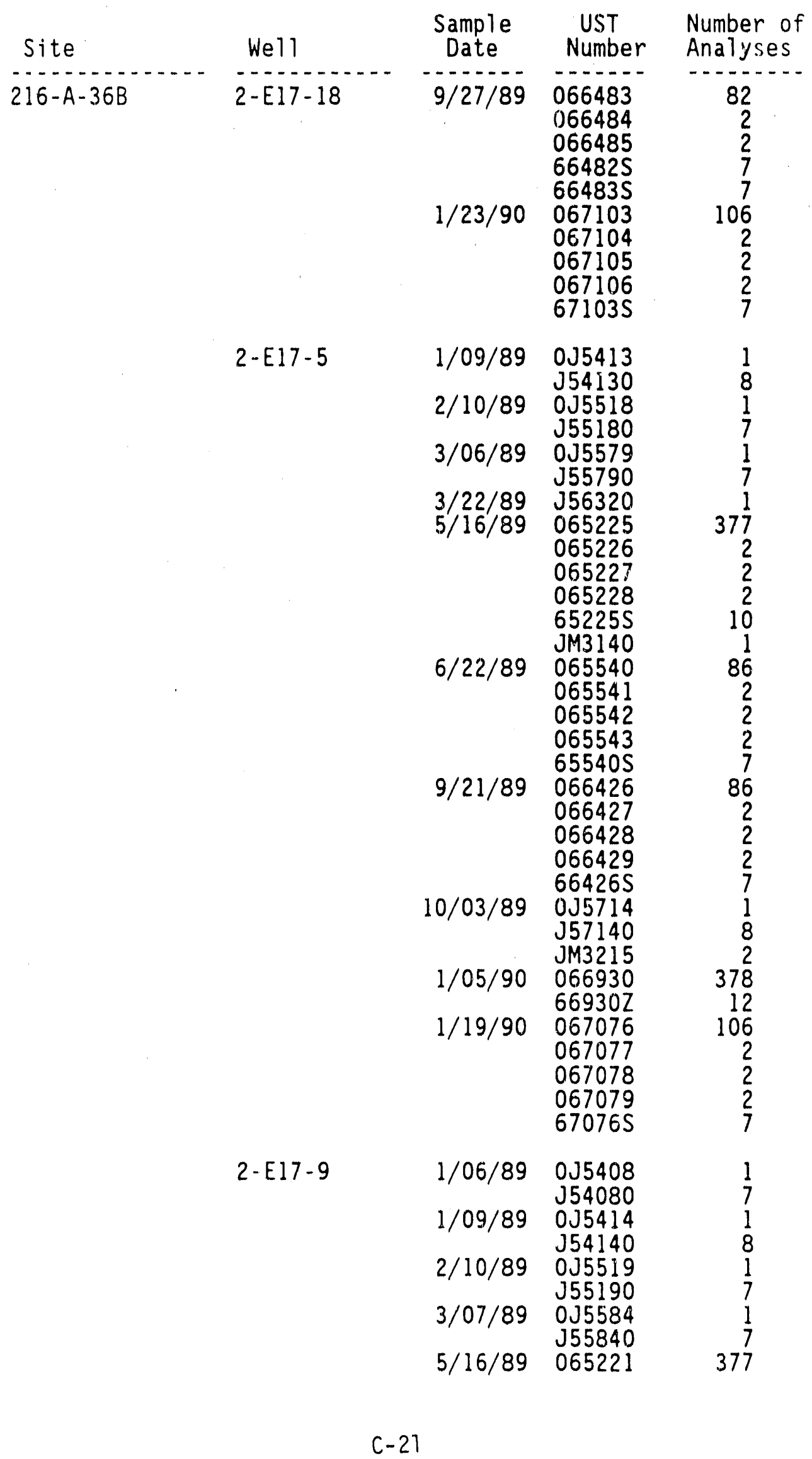




\begin{tabular}{|c|c|c|c|c|}
\hline Site & Well & $\begin{array}{c}\text { Sample } \\
\text { Date }\end{array}$ & $\begin{array}{l}\text { UST } \\
\text { Number }\end{array}$ & $\begin{array}{l}\text { Number of } \\
\text { Analyses }\end{array}$ \\
\hline $216-A-36 B$ & $2-E 17-9$ & $\begin{array}{l}5 / 16 / 89 \\
6 / 22 / 89 \\
9 / 28 / 89 \\
10 / 03 / 89 \\
1 / 05 / 90 \\
1 / 22 / 90\end{array}$ & $\begin{array}{l}065222 \\
065223 \\
065224 \\
65221 S \\
065544 \\
065545 \\
065546 \\
065547 \\
655445 \\
066503 \\
066504 \\
066505 \\
066506 \\
665035 \\
0 J 5715 \\
J 57150 \\
066931 \\
66931 Z \\
067081 \\
067082 \\
067083 \\
067084 \\
67081 S\end{array}$ & $\begin{array}{r}2 \\
2 \\
2 \\
10 \\
86 \\
2 \\
2 \\
2 \\
7 \\
86 \\
2 \\
2 \\
2 \\
7 \\
1 \\
8 \\
378 \\
12 \\
106 \\
2 \\
2 \\
2 \\
7\end{array}$ \\
\hline $216-A-37-1$ & $2-E 25-17$ & $\begin{array}{c}1 / 10 / 89 \\
2 / 10 / 89 \\
3 / 02 / 89 \\
10 / 06 / 89 \\
1 / 04 / 90 \\
3 / 19 / 90\end{array}$ & $\begin{array}{l}0 J 5420 \\
J 54200 \\
0 J 5517 \\
J 55170 \\
0 J 5568 \\
J 55680 \\
0 J 5725 \\
J 57250 \\
066929 \\
669292 \\
067648 \\
67648 \mathrm{~K}\end{array}$ & $\begin{array}{r}1 \\
7 \\
1 \\
3 \\
1 \\
3 \\
1 \\
7 \\
346 \\
14 \\
30 \\
7\end{array}$ \\
\hline & $2-E 25-18$ & $\begin{array}{l}2 / 01 / 89 \\
2 / 28 / 89 \\
6 / 23 / 89 \\
8 / 30 / 89\end{array}$ & $\begin{array}{l}0 J 5483 \\
J 54830 \\
064883 \\
064884 \\
064885 \\
064886 \\
648838 \\
065550 \\
065551 \\
065552 \\
065553 \\
65550 B \\
066206 \\
066207 \\
066208 \\
066209\end{array}$ & $\begin{array}{r}1 \\
7 \\
81 \\
2 \\
2 \\
2 \\
3 \\
81 \\
2 \\
2 \\
2 \\
3 \\
81 \\
2 \\
2 \\
2\end{array}$ \\
\hline
\end{tabular}


WHC-EP-0366

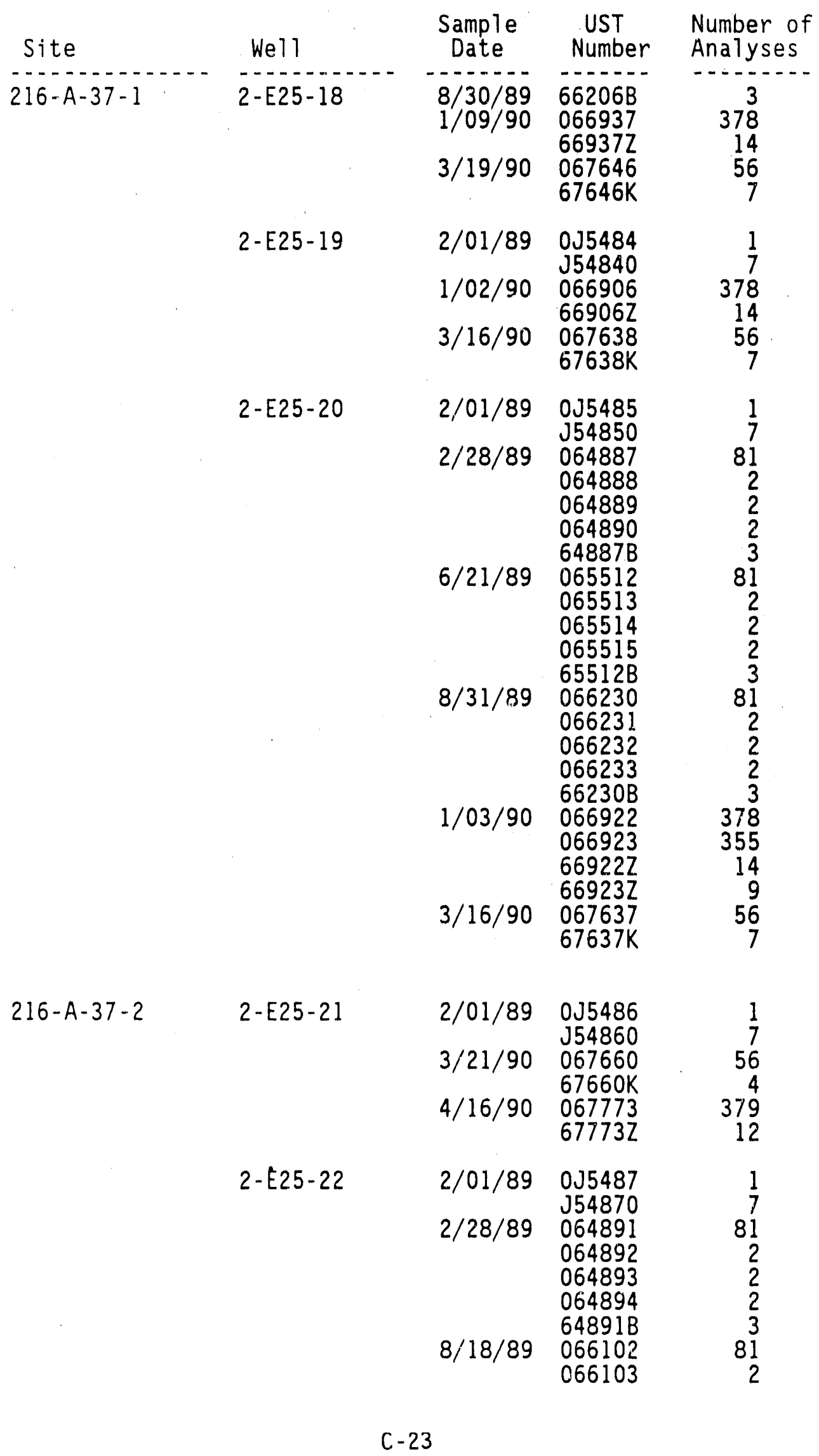




\begin{tabular}{|c|c|c|c|c|}
\hline Site & We11 & $\begin{array}{c}\text { Sample } \\
\text { Date }\end{array}$ & $\begin{array}{l}\text { UST } \\
\text { Number }\end{array}$ & $\begin{array}{l}\text { Number of } \\
\text { Analyses }\end{array}$ \\
\hline $216-A-37-2$ & $2-E 25-22$ & $\begin{array}{l}8 / 18 / 89 \\
8 / 30 / 89 \\
12 / 14 / 89 \\
3 / 21 / 90\end{array}$ & $\begin{array}{l}066104 \\
066105 \\
66102 B \\
066190 \\
066191 \\
066192 \\
066193 \\
66190 \mathrm{~B} \\
066840 \\
668402 \\
067657 \\
67657 \mathrm{~K}\end{array}$ & $\begin{array}{r}2 \\
2 \\
3 \\
81 \\
2 \\
2 \\
2 \\
3 \\
378 \\
12 \\
56 \\
4\end{array}$ \\
\hline & $2-E 25-23$ & $\begin{array}{l}2 / 01 / 89 \\
1 / 29 / 90 \\
3 / 21 / 90\end{array}$ & $\begin{array}{l}0 J 5488 \\
J 54880 \\
067193 \\
671932 \\
067661 \\
67661 \mathrm{~K}\end{array}$ & $\begin{array}{r}1 \\
7 \\
378 \\
11 \\
56 \\
4\end{array}$ \\
\hline & 2-E25-24 & $\begin{array}{l}2 / 01 / 89 \\
2 / 28 / 89 \\
6 / 21 / 89 \\
8 / 30 / 89 \\
12 / 20 / 89 \\
3 / 21 / 90\end{array}$ & $\begin{array}{l}0 J 5489 \\
J 54890 \\
064899 \\
064900 \\
064901 \\
064902 \\
64899 \mathrm{~B} \\
065516 \\
065517 \\
065518 \\
065519 \\
65516 \mathrm{~B} \\
066210 \\
066211 \\
066212 \\
066213 \\
66210 \mathrm{~B} \\
066865 \\
668652 \\
067658 \\
67658 \mathrm{~K}\end{array}$ & $\begin{array}{r}1 \\
7 \\
81 \\
2 \\
2 \\
2 \\
3 \\
3 \\
81 \\
2 \\
2 \\
2 \\
3 \\
81 \\
2 \\
2 \\
2 \\
3 \\
378 \\
12 \\
56 \\
4\end{array}$ \\
\hline $\begin{array}{c}216-A-45 \\
.\end{array}$ & $2-E 17-12$ & $\begin{array}{l}1 / 09 / 89 \\
1 / 26 / 89 \\
2 / 09 / 89 \\
3 / 06 / 89 \\
3 / 16 / 90 \\
4 / 17 / 90\end{array}$ & $\begin{array}{l}0 J 5411 \\
J 54110 \\
J 54470 \\
0 J 5511 \\
J 55110 \\
0 J 5577 \\
J 55770 \\
067628 \\
67628 \mathrm{~K} \\
067780 \\
677802\end{array}$ & $\begin{array}{r}1 \\
13 \\
1 \\
1 \\
13 \\
1 \\
13 \\
56 \\
7 \\
379 \\
12\end{array}$ \\
\hline
\end{tabular}


WHC - EP-0366

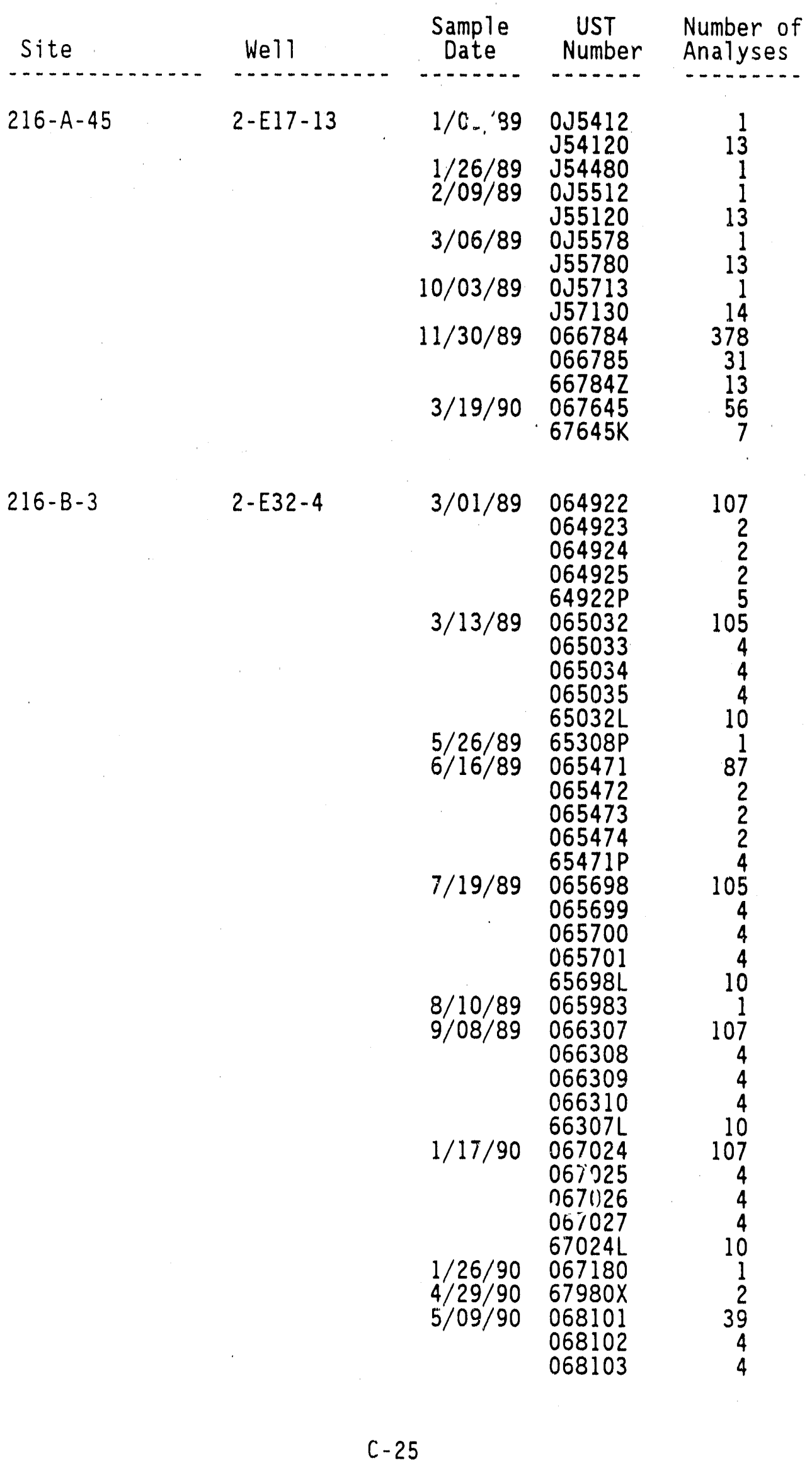




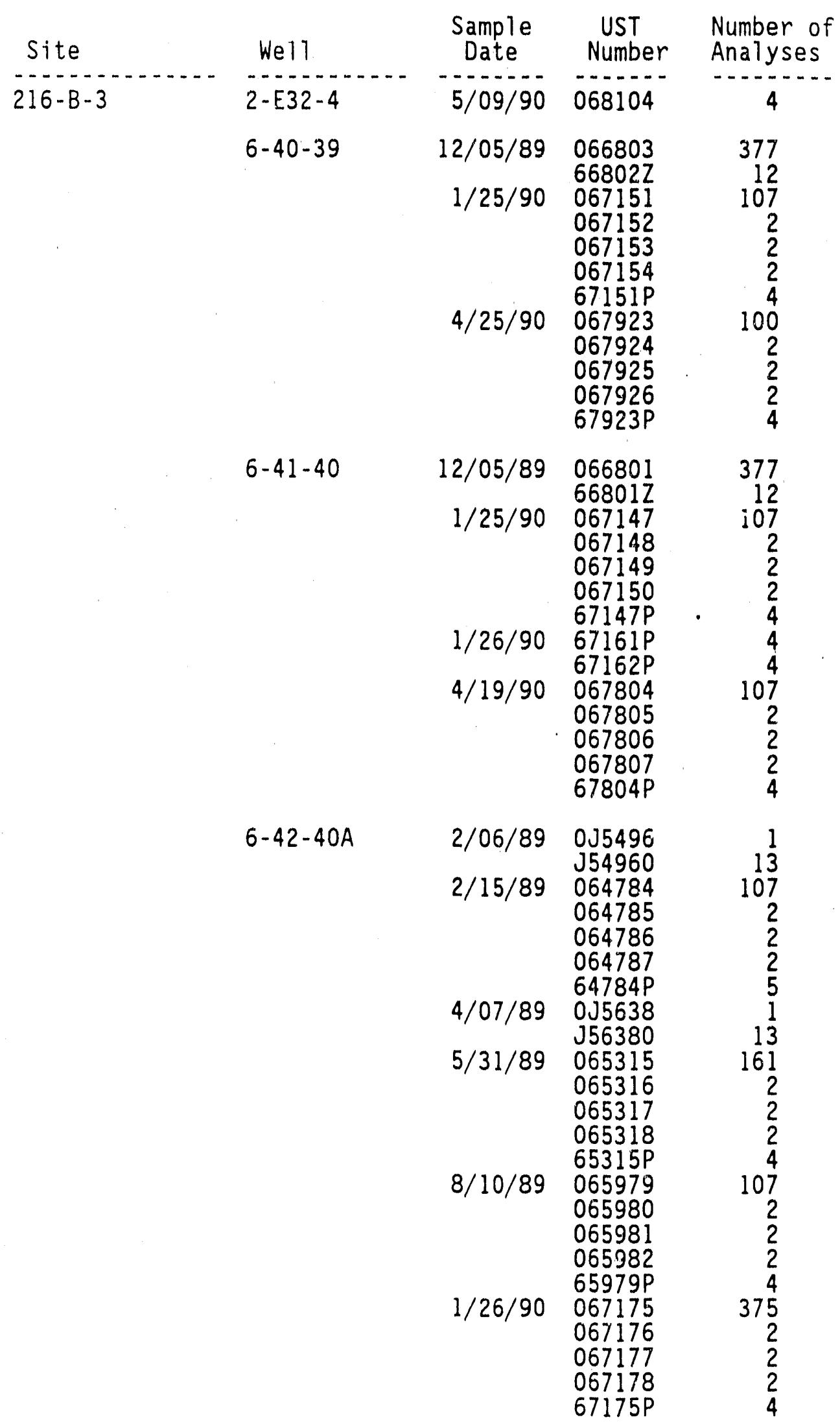


WHC -EP-0366

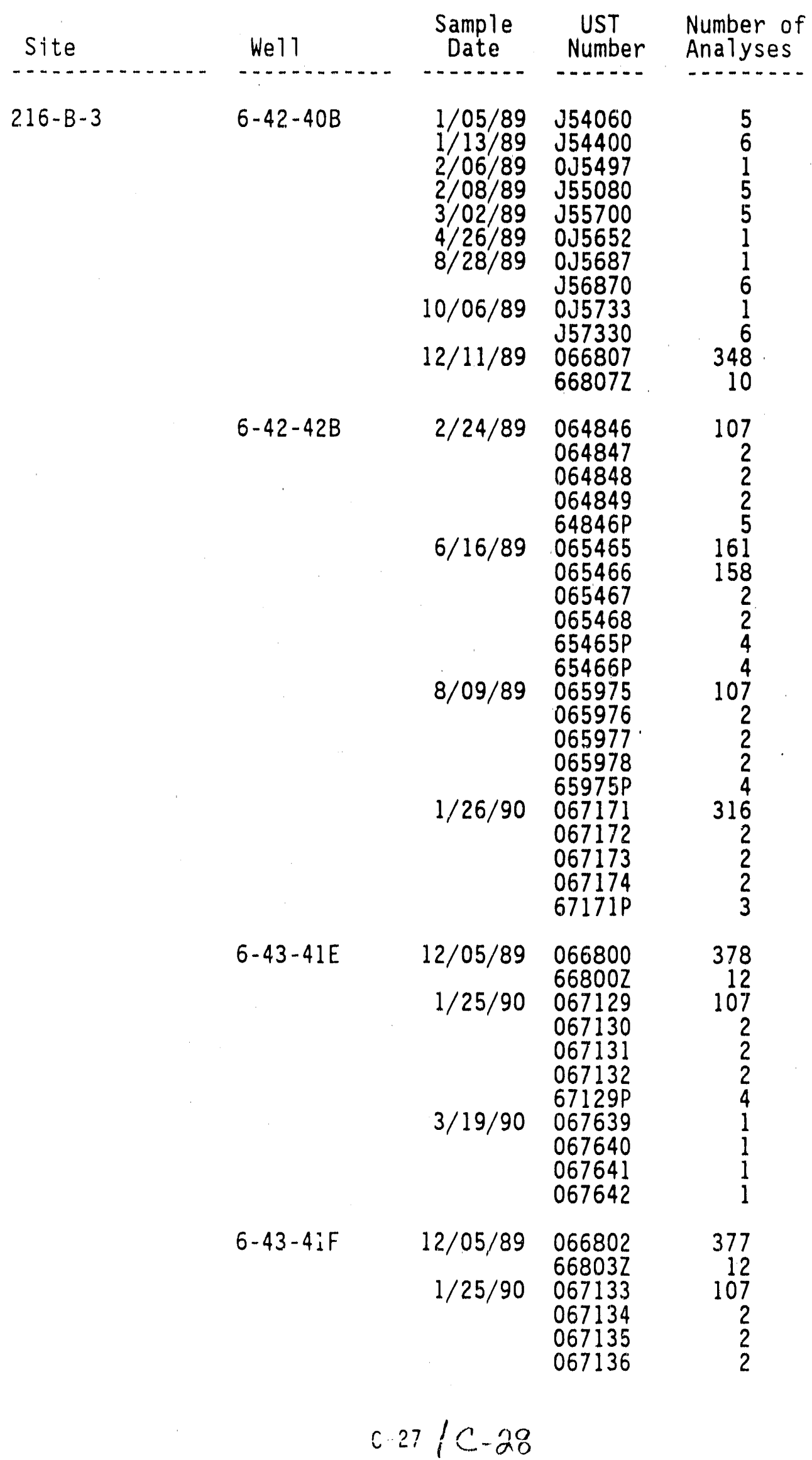




\begin{tabular}{|c|c|c|c|c|}
\hline Site & Well & $\begin{array}{c}\text { Sample } \\
\text { Dálo }\end{array}$ & $\begin{array}{l}\text { UST } \\
\text { Number }\end{array}$ & $\begin{array}{l}\text { Number of } \\
\text { Analyses }\end{array}$ \\
\hline $216-B-3$ & $6-43-45$ & $1 / 26 / 90$ & $\begin{array}{l}067157 \\
067158 \\
067159 \\
067160 \\
67157 \mathrm{P} \\
067792 \\
067793 \\
067794 \\
067795 \\
67792 \mathrm{P}\end{array}$ & $\begin{array}{r}107 \\
2 \\
2 \\
2 \\
4 \\
107 \\
2 \\
2 \\
2 \\
4\end{array}$ \\
\hline & $6-44-42$ & $\begin{array}{l}2 / 17 / 89 \\
6 / 15 / 89 \\
8 / 08 / 89 \\
1 / 31 / 90 \\
4 / 26 / 90\end{array}$ & $\begin{array}{l}064815 \\
064816 \\
064817 \\
064818 \\
64815 P \\
065443 \\
065444 \\
065445 \\
065446 \\
65443 P \\
065961 \\
065962 \\
065963 \\
065964 \\
65961 P \\
067208 \\
067209 \\
067210 \\
067211 \\
67208 P \\
67955 x\end{array}$ & $\begin{array}{r}107 \\
2 \\
2 \\
2 \\
4 \\
161 \\
2 \\
2 \\
2 \\
4 \\
107 \\
2 \\
2 \\
2 \\
4 \\
107 \\
2 \\
2 \\
2 \\
4 \\
2\end{array}$ \\
\hline - & $6-44-43 B$ & $\begin{array}{l}12 / 20 / 89 \\
1 / 26 / 90 \\
4 / 18 / 90\end{array}$ & $\begin{array}{l}066866 \\
066867 \\
668662 \\
668672 \\
067161 \\
067162 \\
067163 \\
067164 \\
067788 \\
067789 \\
067790 \\
067791 \\
67788 P \\
67956 x\end{array}$ & $\begin{array}{r}377 \\
356 \\
13 \\
13 \\
107 \\
80 \\
2 \\
2 \\
107 \\
2 \\
2 \\
2 \\
4 \\
2\end{array}$ \\
\hline $216-B-55$ & $2-E 28-12$ & $\begin{array}{r}1 / 10 / 89 \\
2 / 07 / 89 \\
3 / 06 / 89 \\
8 / 23 / 89 \\
10 / 05 / 89\end{array}$ & $\begin{array}{l}J 54190 \\
J 55000 \\
J 55760 \\
J 56710 \\
J 57180\end{array}$ & $\begin{array}{l}5 \\
5 \\
5 \\
5 \\
5\end{array}$ \\
\hline
\end{tabular}




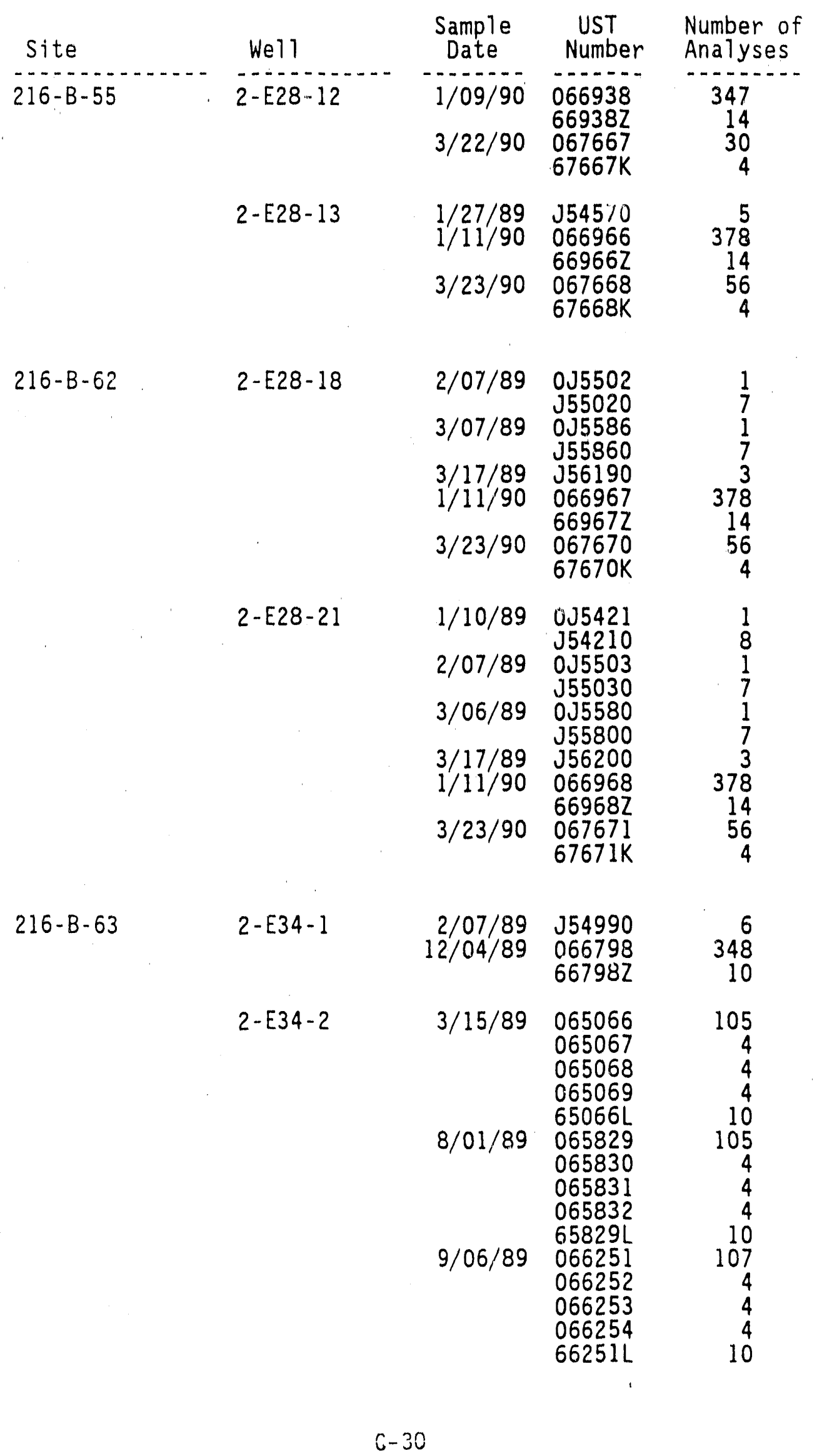




\begin{tabular}{|c|c|c|c|c|}
\hline Site & Well & $\begin{array}{c}\text { Sample } \\
\text { Date }\end{array}$ & $\begin{array}{l}\text { UST } \\
\text { Number }\end{array}$ & $\begin{array}{l}\text { Number of } \\
\text { Analyses }\end{array}$ \\
\hline \multirow[t]{2}{*}{$216-B-63$} & \multirow[t]{2}{*}{$2-E 34-2$} & $11 / 27 / 89$ & \multirow{2}{*}{$\begin{array}{l}066742 \\
667422 \\
067544 \\
067545 \\
067546 \\
067547 \\
675441 \\
676840\end{array}$} & \multirow{2}{*}{$\begin{array}{r}379 \\
10 \\
107 \\
4 \\
4 \\
4 \\
10 \\
2\end{array}$} \\
\hline & & $3 / 07 / 90$ & & \\
\hline \multirow[t]{3}{*}{$216-3-26$} & \multirow[t]{3}{*}{$2-W 27-1$} & $2 / 01 / 89$ & \multirow{3}{*}{$\begin{array}{l}0 J 5490 \\
J 54900 \\
066958 \\
669582 \\
067764 \\
067765 \\
67764 K \\
67765 K\end{array}$} & \multirow{3}{*}{$\begin{array}{r}1 \\
13 \\
378 \\
12 \\
56 \\
33 \\
4 \\
4\end{array}$} \\
\hline & & $1 / 11 / 90$ & & \\
\hline & & $4 / 12 / 90$ & & \\
\hline \multirow[t]{7}{*}{$216-U-14$} & $2-W 19-1$ & $1 / 02 / 90$ & $\begin{array}{l}066908 \\
669082\end{array}$ & $\begin{array}{r}347 \\
10\end{array}$ \\
\hline & \multirow[t]{3}{*}{$2-W 19-21$} & $1 / 04 / 89$ & \multirow{3}{*}{$\begin{array}{l}0 J 5394 \\
J 53940 \\
066634 \\
666342 \\
067739 \\
67739 \mathrm{~K}\end{array}$} & \multirow{3}{*}{$\begin{array}{r}1 \\
13 \\
378 \\
12 \\
-56 \\
5\end{array}$} \\
\hline & & $11 / 02 / 89$ & & \\
\hline & & $4 / 04 / 90$ & & \\
\hline & \multirow[t]{3}{*}{$2-W 19-27$} & $2 / 01 / 89$ & \multirow{3}{*}{$\begin{array}{l}0 J 5491 \\
J 54910 \\
066635 \\
666352 \\
067758 \\
67758 \mathrm{~K}\end{array}$} & \multirow{3}{*}{$\begin{array}{r}1 \\
13 \\
378 \\
12 \\
56 \\
5\end{array}$} \\
\hline & & $11 / 02 / 89$ & & \\
\hline & & $4 / 06 / 90$ & & \\
\hline \multirow[t]{7}{*}{$216-U-17$} & \multirow[t]{6}{*}{$2-W 19-19$} & $1 / 12 / 89$ & \multirow{6}{*}{$\begin{array}{l}0 J 5430 \\
J 54300 \\
J 54740 \\
0 J 5549 \\
J 55490 \\
0 J 5592 \\
J 55920 \\
0 J 5727 \\
J 57270 \\
066623 \\
666232 \\
067736 \\
67736 K\end{array}$} & \multirow{6}{*}{$\begin{array}{r}1 \\
6 \\
6 \\
1 \\
5 \\
1 \\
5 \\
1 \\
12 \\
378 \\
12 \\
56 \\
4\end{array}$} \\
\hline & & $\begin{array}{l}1 / 31 / 89 \\
2 / 22 / 89\end{array}$ & & \\
\hline & & $3 / 08 / 89$ & & \\
\hline & & $10 / 06 / 89$ & & \\
\hline & & $10 / 31 / 89$ & & \\
\hline & & $4 / 04 / 90$ & & \\
\hline & $2-W 19-20$ & $1 / 11 / 89$ & $\begin{array}{l}0 J 5426 \\
J 54260\end{array}$ & $\begin{array}{l}1 \\
6\end{array}$ \\
\hline
\end{tabular}


WHC -EP-0366

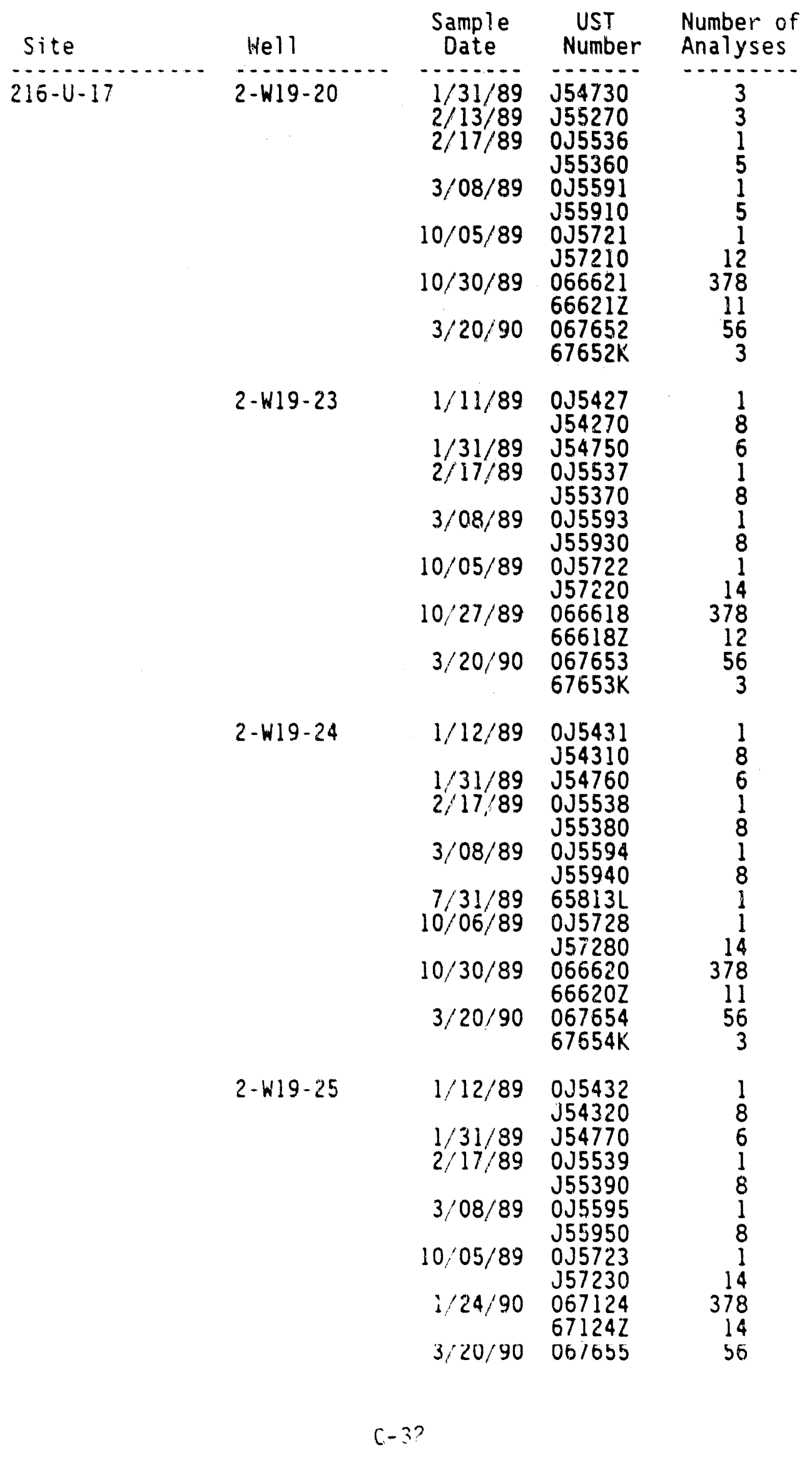




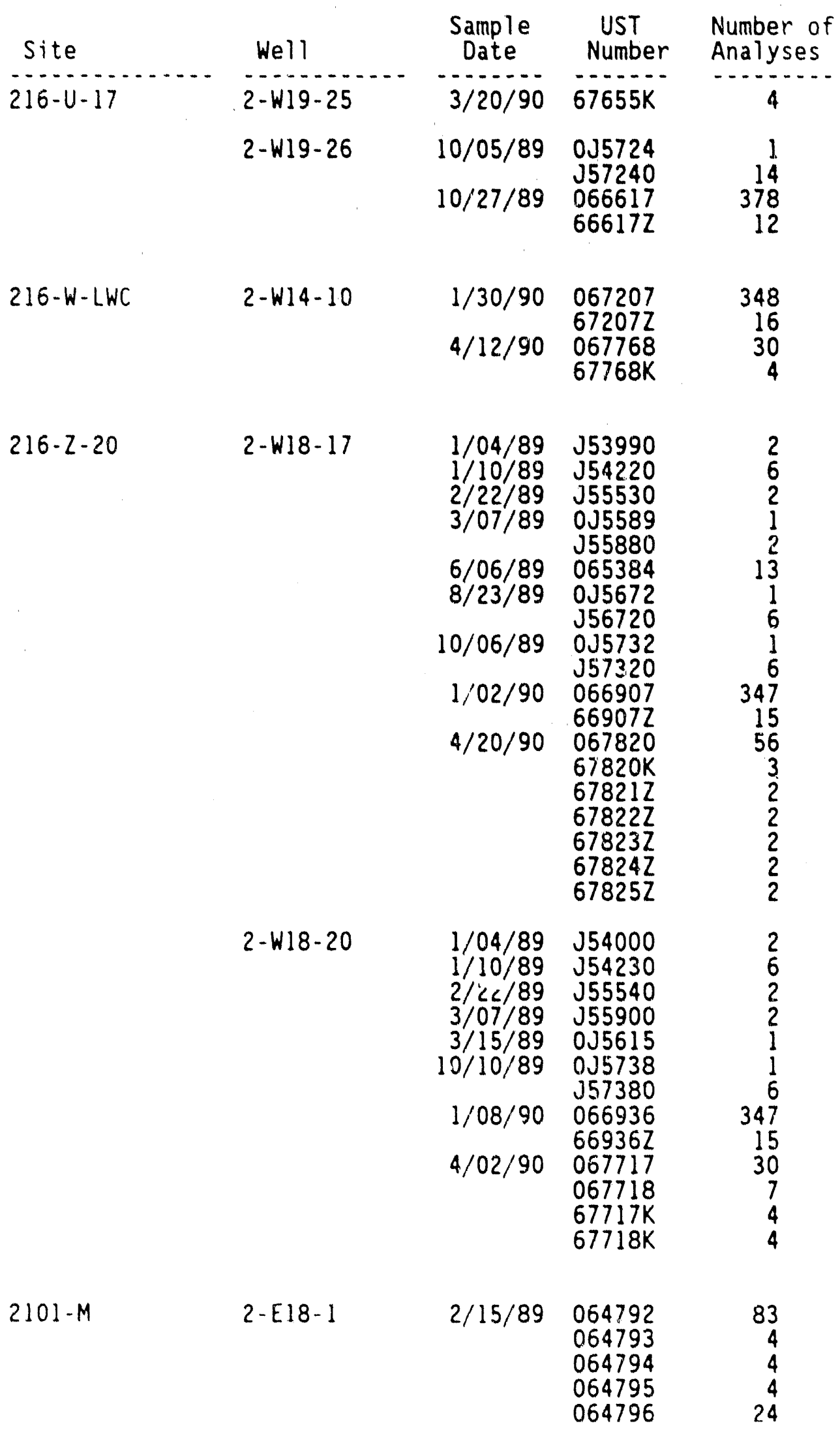


WHC - EP-0356

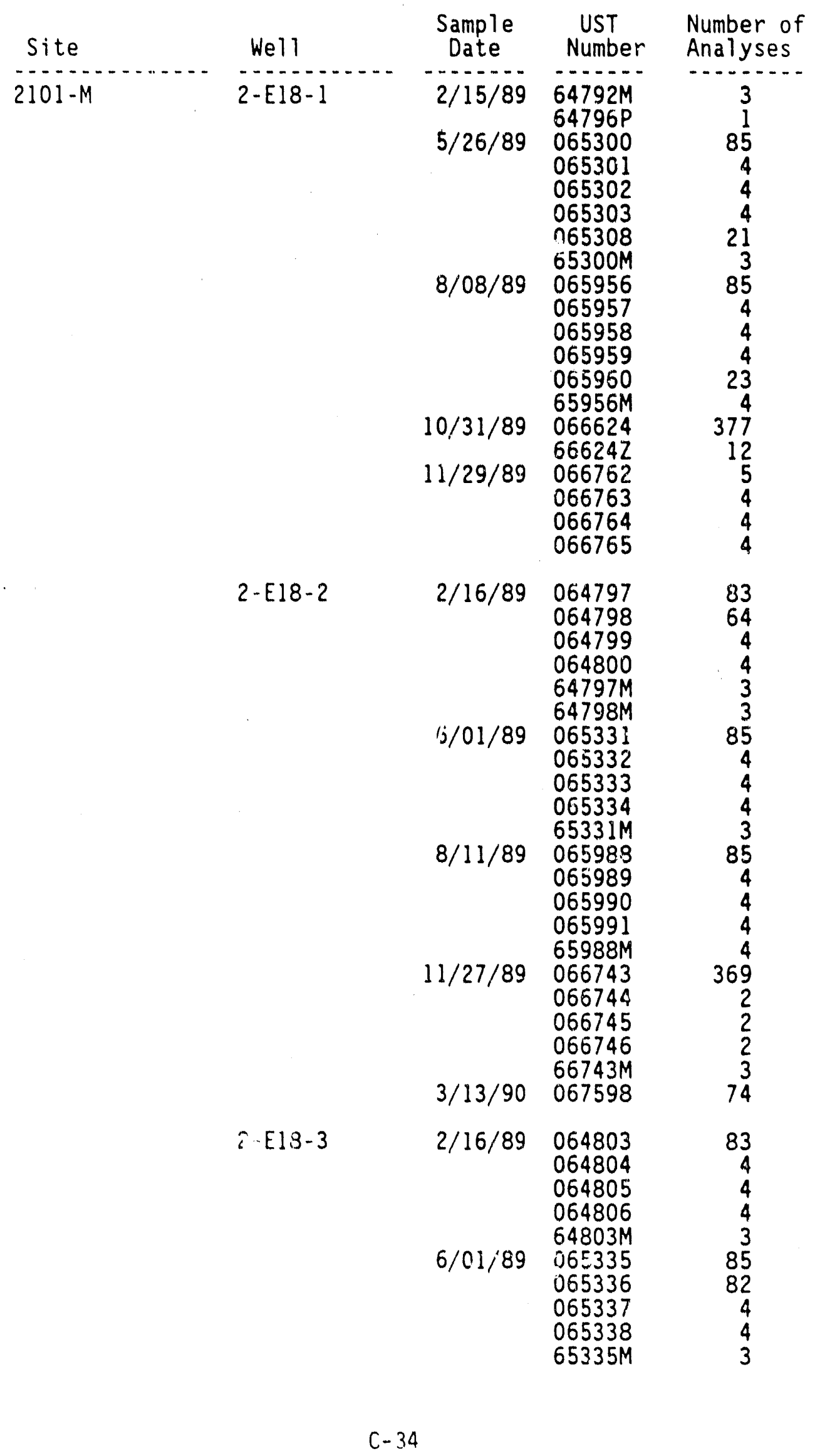




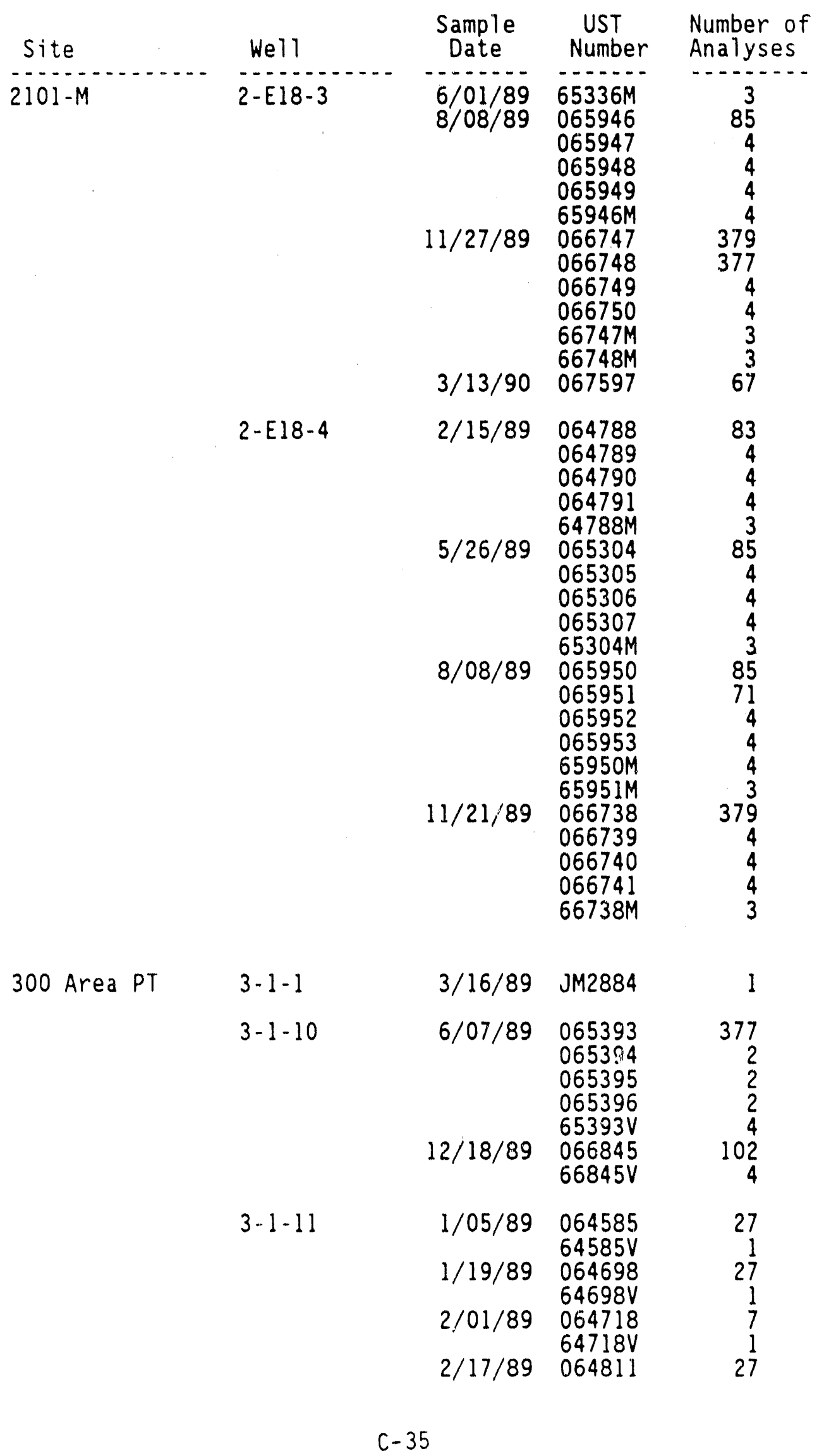


WHC-EP-0366

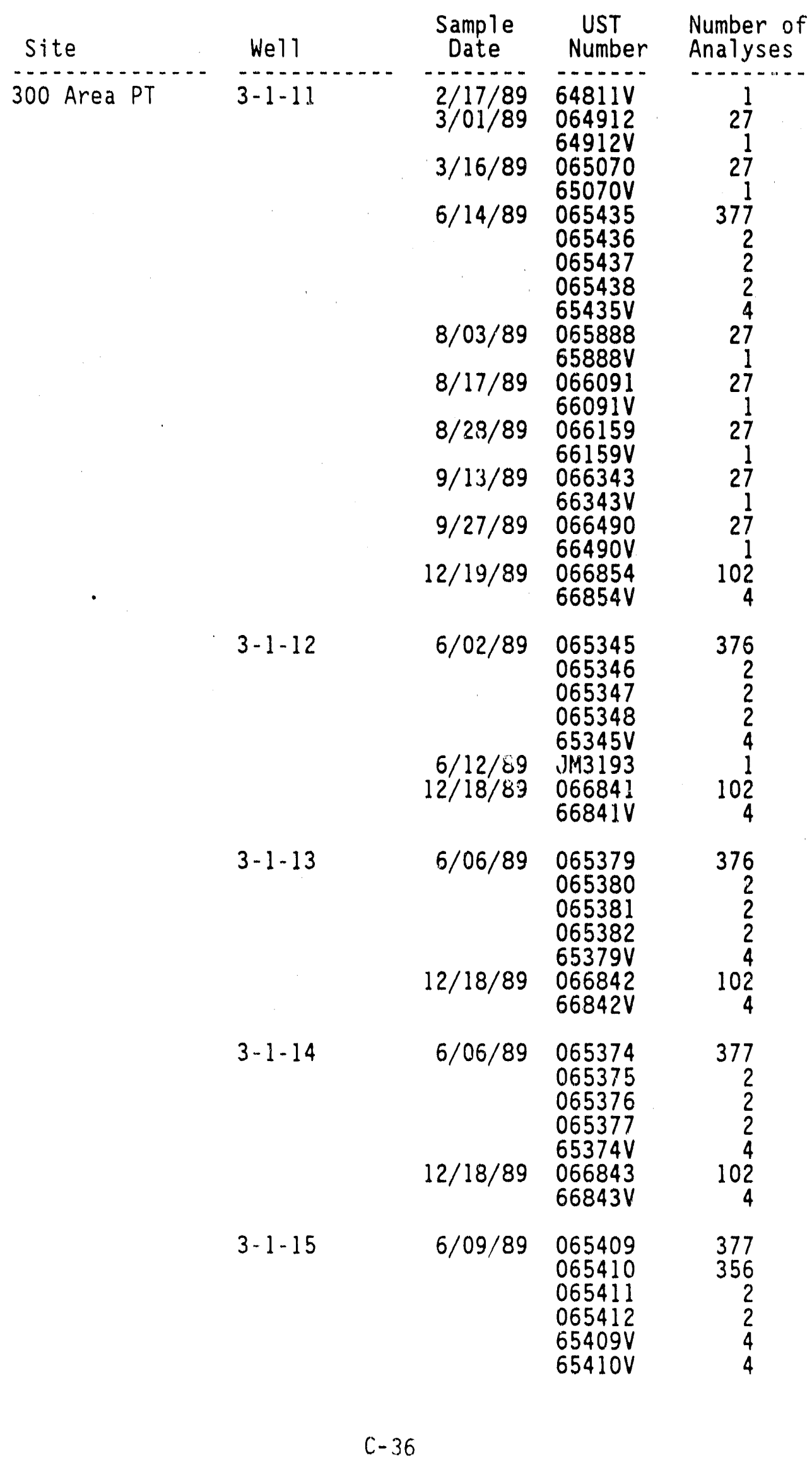




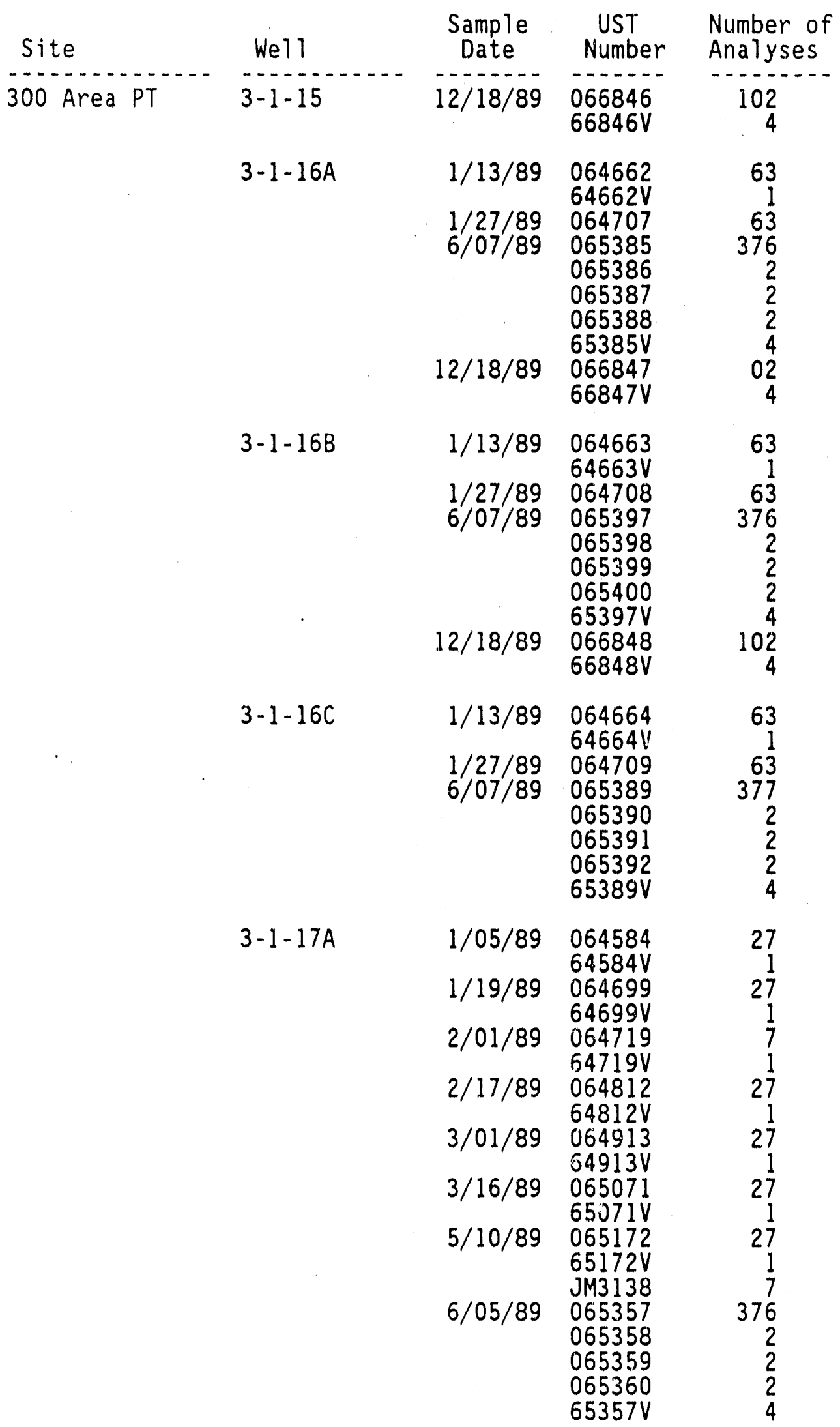


WHC-EP-0366

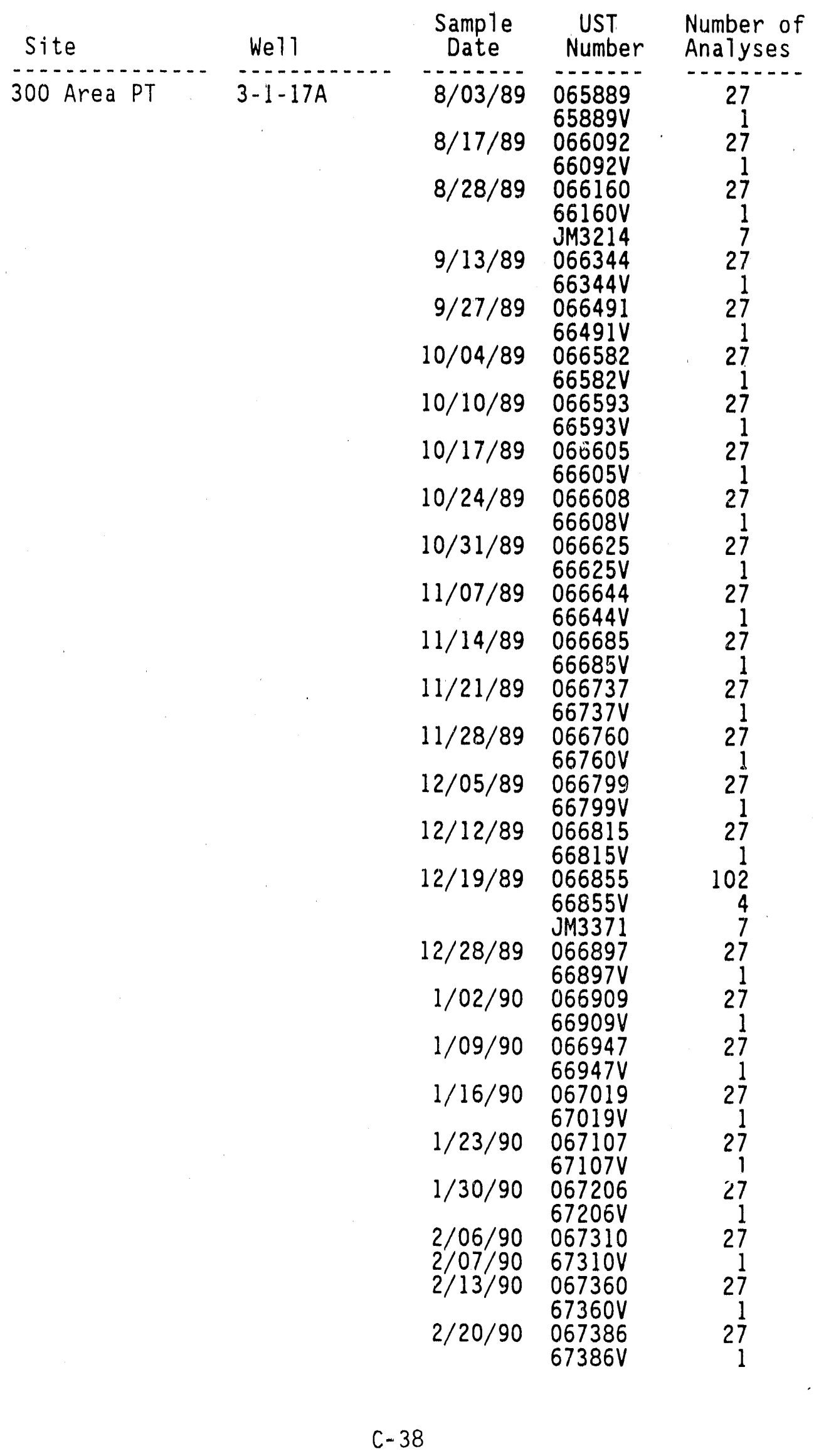




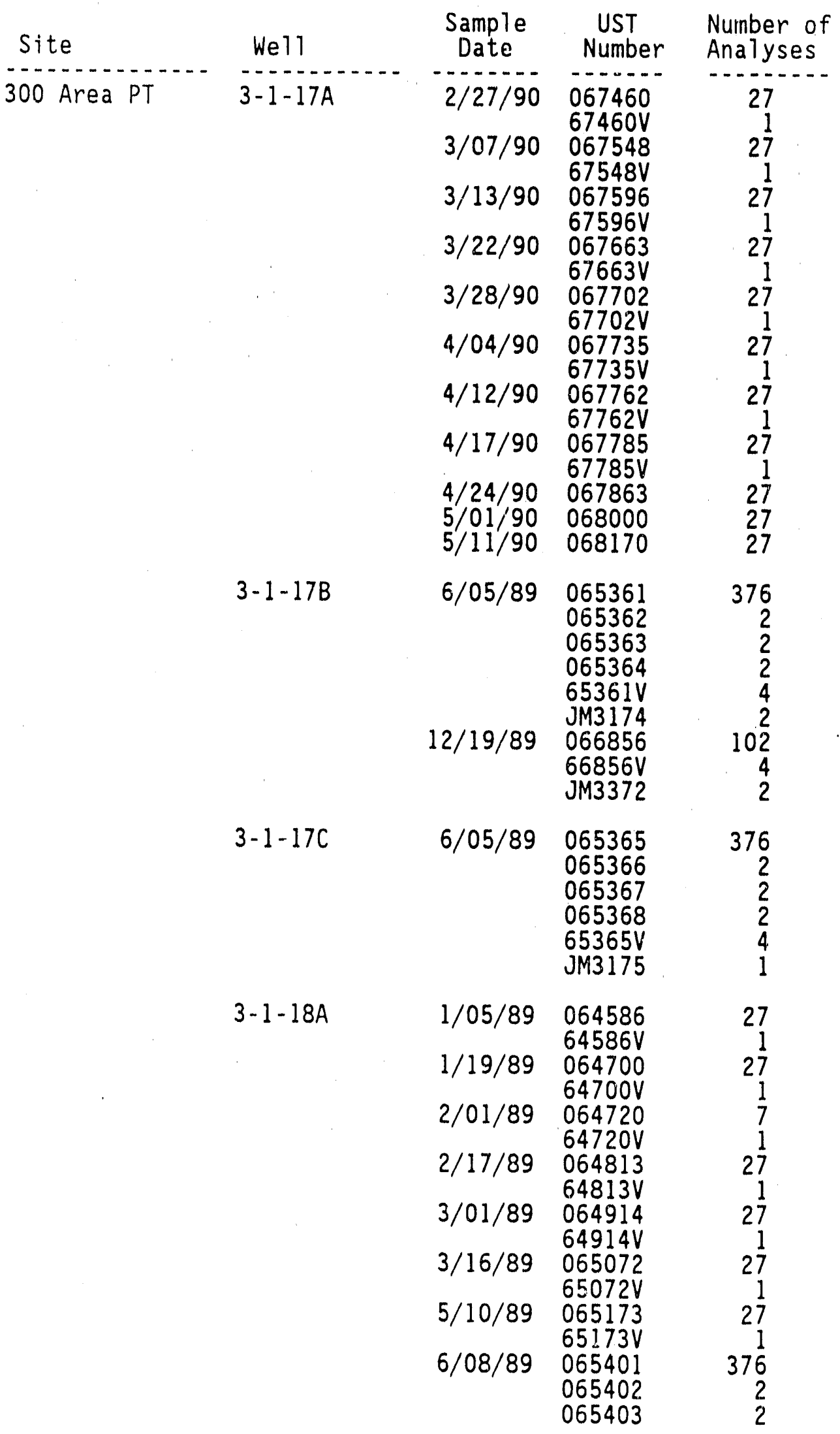




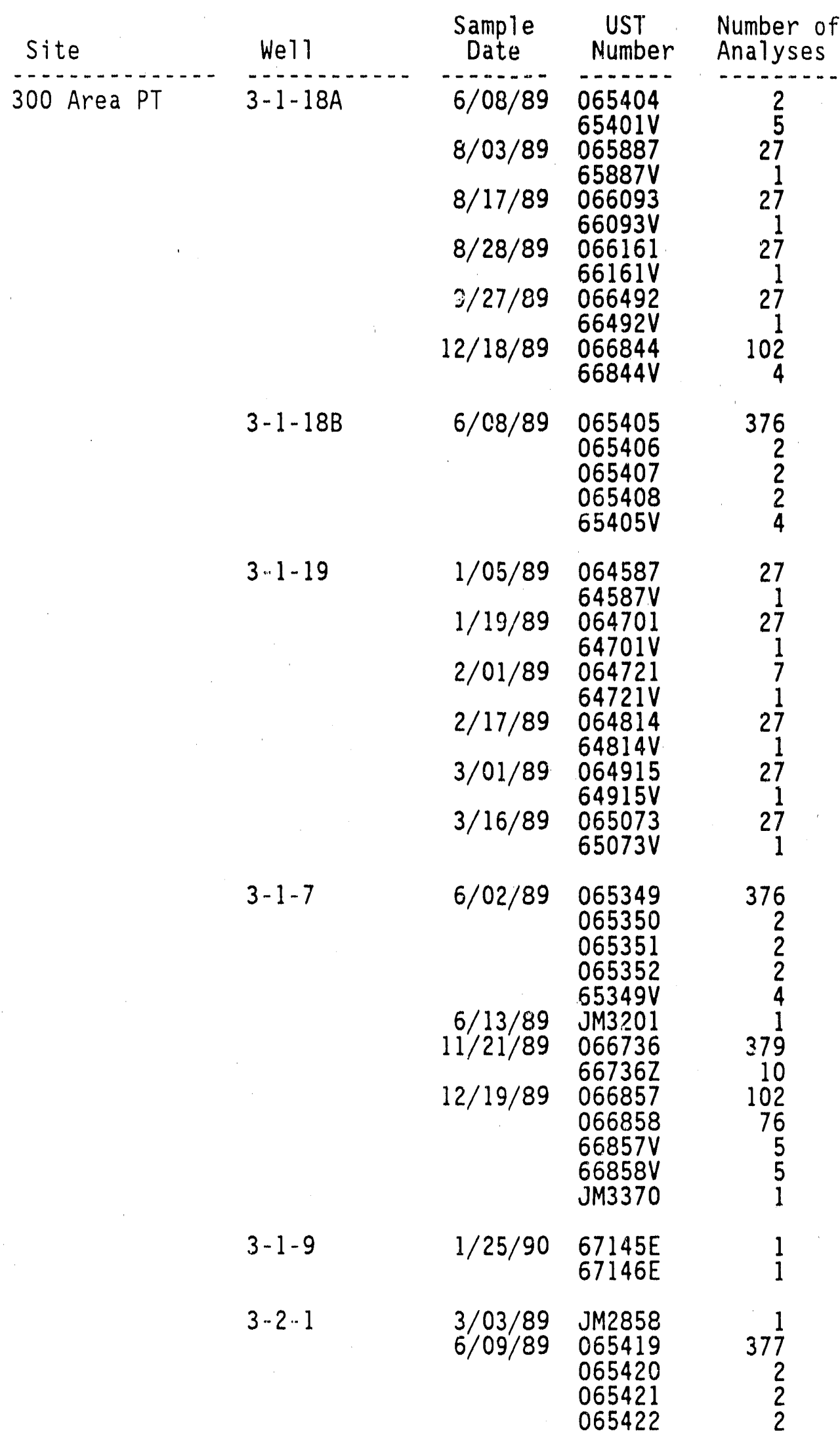




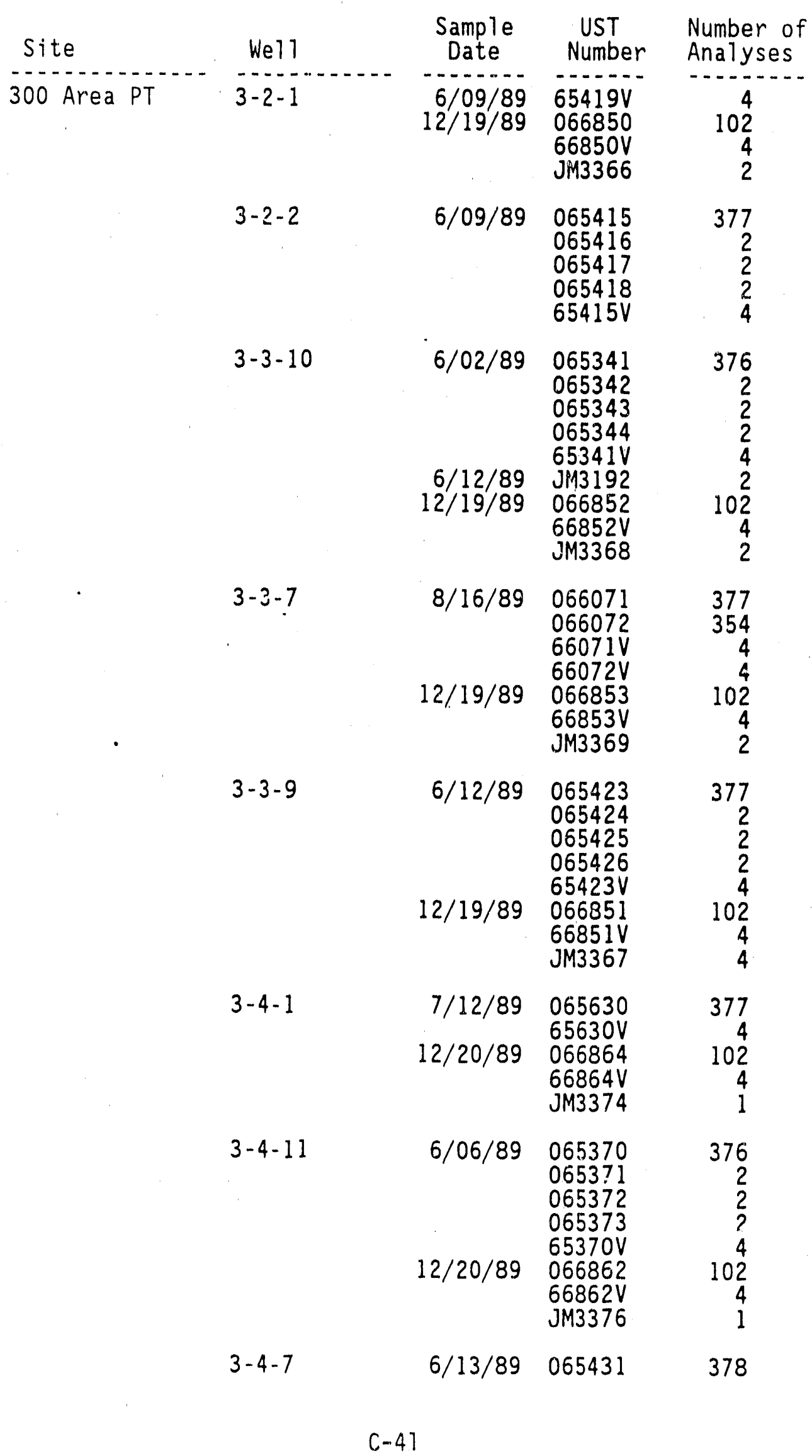


WHC -EP-0366

\begin{tabular}{|c|c|c|c|c|}
\hline Site & We11 & $\begin{array}{c}\text { Sample } \\
\text { Date }\end{array}$ & $\begin{array}{l}\text { UST } \\
\text { Number }\end{array}$ & $\begin{array}{l}\text { Number of } \\
\text { Analyses }\end{array}$ \\
\hline \multirow[t]{6}{*}{300 Area PT } & $3-4-7$ & $6 / 13 / 89$ & $\begin{array}{l}065432 \\
065433 \\
065434 \\
65431 V \\
066861 \\
66861 V \\
J M 3375\end{array}$ & $\begin{array}{r}2 \\
2 \\
2 \\
4 \\
102 \\
4 \\
2\end{array}$ \\
\hline & $3-8-1$ & $\begin{array}{r}12 / 20 / 89 \\
4 / 12 / 90\end{array}$ & $\begin{array}{l}065353 \\
065354 \\
065355 \\
065356 \\
65353 V \\
J M 3173 \\
066863 \\
66863 V \\
J M 3373 \\
067763 \\
677632\end{array}$ & $\begin{array}{r}376 \\
2 \\
2 \\
2 \\
4 \\
3 \\
3 \\
102 \\
4 \\
3 \\
379 \\
8\end{array}$ \\
\hline & $3-8-2$ & $6 / 13 / 89$ & $\begin{array}{l}065427 \\
065428 \\
065429 \\
065430 \\
65427 \mathrm{~V}\end{array}$ & $\begin{array}{r}377 \\
2 \\
2 \\
2 \\
4\end{array}$ \\
\hline & $3-8-3$ & $6 / 14 / 89$ & $\begin{array}{l}065439 \\
065440 \\
065441 \\
065442 \\
65439 V \\
J M 3205\end{array}$ & $\begin{array}{r}377 \\
2 \\
2 \\
2 \\
4 \\
3\end{array}$ \\
\hline & $6-S 19-E 13$ & $\begin{array}{r}4 / 11 / 89 \\
10 / 24 / 89 \\
4 / 13 / 90\end{array}$ & $\begin{array}{l}\text { JM3046 } \\
\text { JM3311 } \\
067771 \\
677712\end{array}$ & $\begin{array}{r}6 \\
6 \\
379 \\
8\end{array}$ \\
\hline & $6-S 30-E 15 A$ & $\begin{array}{r}1 / 20 / 89 \\
4 / 11 / 89 \\
10 / 23 / 89 \\
2 / 21 / 90\end{array}$ & $\begin{array}{l}J M 2825 \\
J M 3048 \\
J M 3293 \\
067389 \\
673890\end{array}$ & $\begin{array}{r}1 \\
8 \\
8 \\
35 \\
10\end{array}$ \\
\hline 400 Area Pond & $6-2-7$ & $\begin{array}{l}1 / 30 / 89 \\
4 / 13 / 90\end{array}$ & $\begin{array}{l}\text { JM2832 } \\
067770 \\
677702\end{array}$ & $\begin{array}{r}2 \\
376 \\
8\end{array}$ \\
\hline
\end{tabular}


WHC-EP-0366

\section{APPENDIX D}

INORGANIC SAMPLE REANALYSIS DATA 
WHC-EP-0366

\begin{tabular}{|c|c|c|c|c|c|c|c|c|}
\hline \multirow[b]{2}{*}{ Well } & \multirow[b]{2}{*}{ Date } & \multirow[b]{2}{*}{ UST \# } & \multirow[b]{2}{*}{ Element } & \multicolumn{3}{|c|}{ UST } & \multicolumn{2}{|c|}{ PNL } \\
\hline & & & & & Result & Error & Result & Error \\
\hline n & $\ldots$ & $\ldots$ & $\cdots$ & $\cdots$ & $\ldots$ & a.......... & $\ldots$ & $\ldots \ldots \ldots$ \\
\hline \multirow[t]{20}{*}{$2-E 25-17$} & $1 / 04 / 90$ & 066929 & $\mathrm{Ag}^{\star}$ & $<$ & 10.00 & 2.39 & .07 & .01 \\
\hline & & & $A l$ * & & 1180.00 & 149.00 & 362.00 & 36.20 \\
\hline & & & As* & & 56.00 & 10.10 & 9.23 & .92 \\
\hline & & & $B a^{\star}$ & & 343.00 & 35.30 & 350.00 & 35.00 \\
\hline & & & $\mathrm{Cd}^{\star}$ & & 211.00 & 21.70 & 51.00 & 5.10 \\
\hline & & & Co* & & 30.00 & 4.15 & 21.10 & 2.11 \\
\hline & & & $\mathrm{C} \Gamma^{\star}$ & & 321.00 & 34.60 & 28.50 & 2.85 \\
\hline & & & $C s^{\star}$ & & & & .81 & .08 \\
\hline & & & $\mathrm{Cu}^{\star}$ & & 73.00 & 9.84 & 73.00 & 7.30 \\
\hline & & & $\mathrm{Fe}^{\star}$ & & 2000.00 & 60000.00 & & \\
\hline & & & $M n^{\star}$ & & 6240.00 & & & \\
\hline & & & Mo* & $<$ & 40.00 & 4.83 & 7.67 & .77 \\
\hline & & & $\mathrm{Ni}$ & & 183.00 & 18.80 & 32.80 & 3.28 \\
\hline & & & $\mathrm{Pb}^{\star}$ & & & & 43.70 & 4.37 \\
\hline & & & $R b^{*}$ & & & & 1.59 & .16 \\
\hline & & & $s b^{\star}$ & $<$ & 30.00 & 5.80 & .33 & .03 \\
\hline & & & $S r^{\star}$ & & 292.00 & 35.70 & 287.00 & 28.70 \\
\hline & & & $V^{\star}$ & & 656.00 & 73.80 & 111.00 & 11.10 \\
\hline & & & $2 n^{*}$ & & 42.00 & 8.02 & 84.80 & 8.48 \\
\hline & & 669292 & $U^{\star}$ & & 12.49 & 5.36 & 11.80 & 1.18 \\
\hline \multirow{23}{*}{$2 \cdot E 25-20$} & $1 / 03 / 90$ & 066922 & $\mathrm{Ag}$ & $<$ & 10.00 & 2.39 & .02 & .00 \\
\hline & & & $A g^{*}$ & $<$ & 10.00 & 2.39 & .01 & .00 \\
\hline & & & Al & $<$ & 150.00 & 20.10 & 2.82 & .28 \\
\hline & & & $A \mid \star$ & $<$ & 150.00 & 20.10 & 3.85 & .39 \\
\hline & & & As & & 7.00 & 2.81 & 3.85 & .39 \\
\hline & & & $\mathrm{As}^{\star}$ & & 7.00 & 2.81 & 3.41 & .34 \\
\hline & & & $\mathrm{Ba}$ & & 59.00 & 6.68 & 63.60 & 6.36 \\
\hline & & & $B a^{\star}$ & & 56.00 & 6.39 & 63.60 & 6.36 \\
\hline & & & cd & $<$ & 2.00 & 1.38 & .20 & .02 \\
\hline & & & $C d^{*}$ & $<$ & 2.00 & 1.38 & .44 & .04 \\
\hline & & & Co & $<$ & 20.00 & 3.35 & .06 & .01 \\
\hline & & & Co* & $<$ & 20.00 & 3.35 & .08 & .01 \\
\hline & & & $\mathrm{Cr}$ & $<$ & 10.00 & 5.13 & 1.68 & .17 \\
\hline & & & $\mathrm{Cr}^{\star}$ & $<$ & 10.00 & 5.13 & 1.83 & .18 \\
\hline & & & Cs & & & & .01 & .00 \\
\hline & 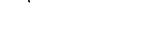 & & $\mathrm{Cs}^{\star}$ & & & & .17 & .02 \\
\hline & & & $\mathrm{Cu}$ & $<$ & 10.00 & 2.89 & .40 & .04 \\
\hline & & & Cu* & $<$ & 10.00 & 2.89 & 2.91 & .29 \\
\hline & & & $\mathrm{Fe}$ & $<$ & 30.00 & 9.50 & 37.00 & 3.70 \\
\hline & & & $\mathrm{Fe}^{\star}$ & & 84.00 & 13.00 & 72.00 & 7.20 \\
\hline & & & $M n$ & $<$ & 5.00 & 1.67 & 2.15 & .22 \\
\hline & & & $M n^{*}$ & $<$ & 5.00 & 1.67 & 3.59 & .36 \\
\hline & & & Mo & $<$ & 40.00 & 4.83 & 16.20 & 1.62 \\
\hline
\end{tabular}

\footnotetext{
*indicates unfiltered analyses, results in ppb.
} 
WHC-EP-0366

\begin{tabular}{|c|c|c|c|c|c|c|c|c|}
\hline \multirow[b]{2}{*}{ Well } & \multirow[b]{2}{*}{ Date } & \multirow[b]{2}{*}{ UST \# } & \multirow[b]{2}{*}{ Element } & \multicolumn{3}{|c|}{ UST } & \multicolumn{2}{|c|}{ PNL } \\
\hline & & & & & Result & Error & Resul t & Error \\
\hline n. & n........ & $\ldots \ldots$ & $\ldots \ldots$ & $\cdot-$ & n......... & $\ldots$ & n........... & .............. \\
\hline \multirow[t]{46}{*}{$2-E 25-20$} & $1 / 03 / 90$ & 066922 & $\mathrm{Ni}$ & $<$ & 10.00 & 3.15 & 1.01 & .10 \\
\hline & & & $\mathrm{Ni \hbar}$ & $<$ & 10.00 & 3.15 & .88 & .09 \\
\hline & & & $\mathrm{Pb}$ & $<$ & 5.00 & 2.55 & .10 & .01 \\
\hline & & & $\mathrm{Pb}^{*}$ & & & & .56 & .06 \\
\hline & & & $\mathbf{R b}$ & & & & 1.65 & .17 \\
\hline & & & $R b^{\star}$ & & & & 1.62 & .16 \\
\hline & & & $s b$ & $<$ & 30.00 & 5.80 & .29 & .03 \\
\hline & & & $s b^{*}$ & $<$ & 30.00 & 5.80 & .26 & .03 \\
\hline & & & $\mathrm{sr}$ & & 321.00 & 39.20 & 341.00 & 34.10 \\
\hline & & & $S r^{*}$ & & 301.00 & 36.80 & 322.00 & 32.20 \\
\hline & & & $v$ & & 33.00 & 5.60 & 39.60 & 3.96 \\
\hline & & & $V^{\star}$ & & 32.00 & 5.50 & 36.00 & 3.60 \\
\hline & & & $\mathrm{Zn}$ & & 6.00 & 2.69 & 5.40 & .54 \\
\hline & & & $2 n^{\star}$ & & 11.00 & 3.39 & 10.80 & 1.08 \\
\hline & & 066923 & Ag & $<$ & 10.00 & 2.39 & & \\
\hline & & & $A g^{*}$ & $<$ & 10.00 & 2.39 & .04 & .00 \\
\hline & & & Al & $<$ & 150.00 & 20.10 & & \\
\hline & & & $A l *$ & $<$ & 150.00 & 20.10 & 6.00 & .60 \\
\hline & & & As & & 6.00 & 2.67 & & \\
\hline & & & As ${ }^{\star}$ & & 7.00 & 2.81 & 3.45 & .35 \\
\hline & & & $\mathrm{Ba}$ & & 57.00 & 6.48 & & \\
\hline & & & Ba* & & 56.00 & 6.39 & 61.80 & 6.18 \\
\hline & . & & $\mathrm{cd}$ & $<$ & 2.00 & 1.38 & & \\
\hline & & & $\cot ^{\star}$ & $<$ & 2.00 & 1.38 & .52 & .05 \\
\hline & & & Co & $<$ & 20.00 & 3.35 & & \\
\hline & & & $\varepsilon_{0}{ }^{*}$ & $<$ & 20.00 & 3.35 & .07 & .01 \\
\hline & & & $\mathrm{Cr}$ & $<$ & 10.00 & 5.13 & & \\
\hline & & & $\mathrm{Cr}{ }^{\star}$ & $<$ & 10.00 & 5.13 & 1.54 & .15 \\
\hline & & & $C s^{*}$ & & & & .23 & .02 \\
\hline & & & $\mathrm{Cu}$ & $<$ & 10.00 & 2.89 & & \\
\hline & & & $\mathrm{Cu}^{*}$ & $<$ & 10.00 & 2.89 & 2.47. & .25 \\
\hline & & & $\mathrm{Fe}$ & $<$ & 30.00 & 9.50 & & \\
\hline & & & $\mathrm{Fe}^{\star}$ & & 86.00 & 13.10 & 41.50 & 4.15 \\
\hline & & & $M r ı$ & $<$ & 5.00 & 1.67 & & \\
\hline & & & $M n^{\star}$ & $<$ & 5.00 & 1.67 & 2.19 & .22 \\
\hline & & & Mo & $<$ & 40.00 & 4.83 & & \\
\hline & & & Mo* & $<$ & 40.00 & 4.83 & 16.00 & 1.60 \\
\hline & & & $\mathrm{Ni}$ & $<$ & 10.00 & 3.15 & & \\
\hline & & & $N i *$ & $<$ & 10.00 & 3.15 & .67 & .07 \\
\hline & & & $\mathrm{Pb}$ & $<$ & 5.00 & 2.55 & & \\
\hline & & & $\mathrm{Pb}^{*}$ & & & & .56 & .06 \\
\hline & & & $\mathrm{Rb}^{\star}$ & & & & 1.69 & .17 \\
\hline & & & sb & $<$ & 30.00 & 5.80 & & \\
\hline & & & $\mathrm{Sb}^{\star}$ & $<$ & 30.00 & 5.80 & .30 & .03 \\
\hline & & & Sr & & 313.00 & 38.20 & & \\
\hline & & & $S r^{\star}$ & & 302.00 & 36.90 & 323.00 & 32.30 \\
\hline
\end{tabular}


WHC-EP-0366

$5 / 29 / 80$

Analys is Comparison

\begin{tabular}{|c|c|c|c|c|c|c|c|c|}
\hline \multirow[b]{2}{*}{ Hell } & \multirow[b]{2}{*}{ Date } & \multirow[b]{2}{*}{ UST * } & \multirow[b]{2}{*}{ Element } & \multicolumn{3}{|c|}{ UST } & \multicolumn{2}{|c|}{ PNL } \\
\hline & & & & & Result & Error & Result & Error \\
\hline$\cdots \ldots \ldots \ldots$ & $\cdots \cdots$ & $\cdots \cdots$ & $\cdots \ldots$ & $\cdots$ & $\ldots \ldots$ & $\cdots+\cdots$ & $\cdots \cdots$ & $\cdots$ \\
\hline \multirow[t]{7}{*}{$2-E 25-20$} & $1 / 03 / 90$ & 066923 & $v$ & & 34.00 & 5.70 & & \\
\hline & & & $v^{*}$ & & 35.00 & 5.79 & 36.60 & 3.66 \\
\hline & & & $2 n$ & & 10.00 & 3.25 & & \\
\hline & & & $2 n *$ & & 11.00 & 3.39 & 11.80 & 1.18 \\
\hline & & 669222 & $u$ & & & & 2.00 & .20 \\
\hline & & & $u *$ & & 2.76 & .89 & 2.20 & .22 \\
\hline & & $66923 \mathrm{c}$ & $u *$ & & 2.03 & .67 & 2.10 & .21 \\
\hline \multirow[t]{38}{*}{$2-E 25-22$} & $12 / 14 / 89$ & 066840 & Ag & $<$ & 10.00 & 2.39 & .01 & .00 \\
\hline & & & $\mathrm{Ag}^{*}$ & $<$ & 10.00 & 2.39 & .02 & .00 \\
\hline & & & Al & $<$ & 150.00 & 20.10 & 5.33 & .53 \\
\hline & & & $A l^{*}$ & $<$ & 950.00 & 20.10 & 8.10 & .81 \\
\hline & & & As & & 8.00 & 2.95 & 4.21 & .42 \\
\hline & & & As* & & 8.00 & 2.95 & 4.71 & .47 \\
\hline & & & $\mathrm{Ba}$ & & 13.00 & 2.64 & 14.80 & 1.48 \\
\hline & & & $B a^{*}$ & & 13.00 & 2.64 & 16.80 & 1.48 \\
\hline & & & $\operatorname{cod}$ & $<$ & 2.00 & 1.38 & .05 & .00 \\
\hline & & & $\cos$ & $<$ & 2.00 & 1.38 & .04 & .00 \\
\hline & & & Co & $<$ & 20.00 & 3.35 & .03 & .00 \\
\hline & & & co* & $<$ & 20.00 & 3.35 & .07 & .01 \\
\hline & & & $\mathrm{Cr}$ & $<$ & 10.00 & 5.13 & 1.74 & .17 \\
\hline & & & cr* & $<$ & 10.00 & 5.13 & 1.54 & .15 \\
\hline & & & Cs & & & & .19 & .02 \\
\hline & & & Cs* & & & & .23 & .02 \\
\hline & & & $\mathrm{Cu}$ & $<$ & 10.00 & 2.89 & .72 & .07 \\
\hline & & & $\mathrm{Cu}^{*}$ & $<$ & 10.00 & 2.99 & 1.20 & .12 \\
\hline & & & $\mathrm{Fe}$ & $<$ & 30.00 & 9.50 & 18.00 & 1.80 \\
\hline & & & $\mathrm{Fe}^{\mathrm{N}}$ & & 59.00 & 11.20 & 41.50 & 4.15 \\
\hline & & & Mn & $<$ & 5.00 & 1.67 & 1.01 & .10 \\
\hline & & & $M n^{*}$ & $<$ & 5.00 & 1.67 & 2.19 & .22 \\
\hline & & & Mo & $<$ & 40.00 & 4.83 & 4.21 & .42 \\
\hline & & & Mo* & $<$ & 40.00 & 4.83 & 4.55 & .46 \\
\hline & & & $\mathrm{Ni}$ & $<$ & 10.00 & 3.15 & 1.02 & .10 \\
\hline & & & $\mathrm{Ni*}$ & $<$ & 10.00 & 3.15 & .67 & .07 \\
\hline & & & $\mathrm{Pb}$ & $<$ & 5.00 & 2.55 & .08 & .01 \\
\hline & & & Po* & & & & .23 & .02 \\
\hline & & & $R b$ & & & & .76 & .08 \\
\hline & & & $R b^{*}$ & & & & .87 & .09 \\
\hline & & & sb & $<$ & 30.00 & 5.80 & .25 & .03 \\
\hline & & & sb* & $<$ & 30.00 & 5.80 & .22 & .07 \\
\hline & & & $\mathrm{Sr}$ & & 106.00 & 13.20 & 110.00 & 11.00 \\
\hline & & & sit & & 106.00 & 13.20 & 116.00 & 11.60 \\
\hline & & & $v$ & & 42.00 & 6.42 & 37.80 & 3.78 \\
\hline & & & $v=$ & & 62.00 & 6.49 & 41.70 & 6.17 \\
\hline & & & $2 n$ & $<$ & 5.00 & 2.55 & 3.73 & .37 \\
\hline & & & $2 n^{*}$ & & 7.00 & 2.83 & 8.56 & .86 \\
\hline
\end{tabular}

*indicates unfiltered analyses, results in $\mathrm{ppb}$. 
WHC-EP-0366

$5 / 29 / 90$

Anolys is Comparison

\begin{tabular}{|c|c|c|c|c|c|c|c|c|}
\hline \multirow[b]{2}{*}{ Well } & \multirow[b]{2}{*}{ Date } & \multirow[b]{2}{*}{ UST * } & \multirow[b]{2}{*}{ Element } & \multicolumn{3}{|c|}{ UST } & \multicolumn{2}{|c|}{ PNL } \\
\hline & & & & & Result & Error & Result & Error \\
\hline$\ldots \ldots \ldots$ & $\cdots \ldots$ & $\ldots \ldots$ & $\ldots \ldots$ & $\cdots$ & $\cdots \ldots$ & $\cdots \cdots$ & $\ldots \ldots \ldots$ & $\cdots \ldots$ \\
\hline \multirow[t]{2}{*}{$2-E 25 \cdot 22$} & $12 / 14 / 89$ & 068402 & $u$ & & & & 1.36 & .14 \\
\hline & & & $u *$ & & 1.19 & .44 & 1.31 & .13 \\
\hline \multirow[t]{37}{*}{$2-E 28 \cdot 13$} & $1 / 11 / 90$ & 066966 & Ag & $<$ & 10.00 & 2.39 & .02 & .00 \\
\hline & & & $A g^{*}$ & $<$ & 10.00 & 2.39 & & \\
\hline & & & Al & $<$ & 150.00 & 20.10 & 2.92 & .29 \\
\hline & & & $A 1 *$ & $<$ & 150.00 & 20.10 & & \\
\hline & & & As & $<$ & 5.00 & 2.53 & 1.47 & .15 \\
\hline & & & As* & $<$ & 5.00 & 2.53 & & \\
\hline & & & Ba & & 76.00 & 8.34 & 79.20 & 7.92 \\
\hline & & & $B a^{*}$ & & 82.00 & 8.93 & & \\
\hline & & & $\mathrm{cd}$ & $<$ & 2.00 & 1.38 & .04 & .00 \\
\hline & & & $\cot$ & $<$ & 2.00 & 1.38 & & \\
\hline & & & Co & $<$ & $20 . c 0$ & 3.35 & .06 & .01 \\
\hline & & & $\mathrm{CO}^{*}$ & $s$ & 20.00 & 3.35 & & \\
\hline & & & $\mathrm{Cr}$ & $<$ & 10.00 & 5.13 & $2 . \pi$ & .28 \\
\hline & & & $\mathrm{Cr}$ & $<$ & 10.00 & 5.13 & & \\
\hline & & & cs & & & & .24 & .02 \\
\hline & & . & $\mathrm{Cu}$ & $<$ & 10.00 & 2.89 & .38 & .04 \\
\hline & & & $\mathrm{Cu}$ & $<$ & 10.00 & 2.89 & & \\
\hline & & & Fe & $<$ & 30.00 & 9.50 & 23.40 & 2.34 \\
\hline & & & $\mathrm{Fe}^{*}$ & & 88.00 & 13.30 & & \\
\hline & & & $M n$ & $<$ & 5.00 & 1.67 & 1.50 & .15 \\
\hline & & & $M n^{*}$ & $<$ & 5.00 & 1.67 & & \\
\hline & & & Mo & $<$ & 40.00 & 4. 33 & 5.63 & .56 \\
\hline & & & Mo* & $<$ & 40.00 & 4.83 & & \\
\hline & & & $\mathrm{Ni}$ & $<$ & 10.00 & 3.15 & 1.51 & .15 \\
\hline & & & $\mathrm{Ni*}$ & $<$ & 10.00 & 3.15 & & \\
\hline & & & $\mathrm{Pb}$ & $<$ & 5.00 & 2.55 & .37 & .04 \\
\hline & & & $R D$ & & & & 2.51 & .25 \\
\hline & & & Sb & $<$ & 30.00 & 5.80 & .12 & .01 \\
\hline & & & sb* & $<$ & 30.00 & 5.80 & & \\
\hline & & & Sr & & 228.00 & 28.00 & 245.00 & 24.50 \\
\hline & & & $S r^{*}$ & & 234.00 & 28.70 & & \\
\hline & & & $v$ & & 19.00 & 4.31 & 17.20 & 1.72 \\
\hline & & & $v^{*}$ & & 17.00 & 4.14 & & \\
\hline & & & $2 n$ & & 10.00 & 3.25 & 8.26 & .83 \\
\hline & & & $2 n^{*}$ & & 11.00 & 3.39 & & \\
\hline & & 669652 & u & & & & 3.67 & .35 \\
\hline & & & $u^{*}$ & & 3.00 & .96 & & \\
\hline
\end{tabular}

\begin{tabular}{|c|c|c|c|c|c|c|c|c|}
\hline \multirow[t]{5}{*}{$2-E 2.8-18$} & \multirow{5}{*}{$1 / 11 / 90$} & \multirow{5}{*}{066967} & $A y$ & $<$ & 10.00 & 2.30 & \multirow{3}{*}{.06} & \multirow[b]{2}{*}{.01} \\
\hline & & & $A^{*}$ & $<$ & 10.00 & 2.38 & & \\
\hline & & & Al & $c$ & 150.00 & 20.10 & & \\
\hline & & & $A 1 *$ & $<$ & 150.00 & 20.10 & 5.68 & .57 \\
\hline & & & As & c & 5.00 & 2.53 & & \\
\hline
\end{tabular}

*inuicajes uníitiered anaiyses, resules in ppD. 
WHC-EP-0366

$5 / 29 / 90$

Analys is Comparison

\begin{tabular}{|c|c|c|c|c|c|c|c|c|}
\hline \multirow{2}{*}{ Well } & \multirow[b]{2}{*}{ Date } & \multirow[b]{2}{*}{ UST \# } & \multirow[b]{2}{*}{ Element } & \multicolumn{3}{|c|}{ UST } & \multicolumn{2}{|c|}{ PNL } \\
\hline & & & & & esult & Error & Result & Error \\
\hline$\cdots \ldots$ & $\cdots \ldots$ & $\cdots \ldots$ & $\ldots \ldots$ & $\cdots$ & (n...... & $\ldots$ & $\cdots \ldots$ & $\ldots$ \\
\hline \multirow[t]{27}{*}{$2 \cdot E 28 \cdot 18$} & $1 / 11 / 90$ & 066967 & As* & $<$ & 5.00 & 2.53 & 2.60 & .26 \\
\hline & & & Ba & & 24.00 & 3.46 & & \\
\hline & & & Ba* & & 28.00 & 3.80 & 26.30 & 2.63 \\
\hline & & & $\mathrm{cd}$ & $<$ & 2.00 & 1.38 & & \\
\hline & & & $\cot$ & $<$ & 2.00 & 1.38 & .11 & .01 \\
\hline & & & Co & $<$ & 20.00 & 3.35 & & \\
\hline & & & Co* & $<$ & 20.00 & 3.35 & .14 & .01 \\
\hline & & & $\mathrm{Cr}$ & s & 10.00 & 5.13 & & \\
\hline & & & $\mathrm{Cr}^{\star}$ & $<$ & 10.00 & 5.13 & 4.80 & .48 \\
\hline & & & $6 s^{\star}$ & & & & .05 & .00 \\
\hline & & & $\mathrm{Cu}$ & $<$ & 10.00 & 2.89 & & \\
\hline & & & Cu* & $<$ & 10.00 & 2.89 & .50 & .05 \\
\hline & & & Fe & $<$ & 30.00 & 9.50 & & \\
\hline & & & $\mathrm{Fe}^{\prime \prime}$ & & 308.00 & 33.60 & 210.00 & 21.00 \\
\hline & & & Mn & $<$ & 5.00 & 1.67 & & \\
\hline & & & $M n^{*}$ & $<$ & 5.00 & 1.67 & 3.20 & .32 \\
\hline & & & Mo & $<$ & 40.00 & 4.83 & & \\
\hline & & & Mo* & $<$ & 40.00 & 4.83 & 10.00 & 1.00 \\
\hline & & & $\mathrm{Ni}$ & $<$ & 10.00 & 3.15 & & \\
\hline & & & $N i *$ & $<$ & 10.00 & 3.15 & 1.05 & .11 \\
\hline & & & $\mathrm{Pb}$ & $<$ & 5.00 & 2.55 & & \\
\hline & & & $\mathrm{Pb}^{*}$ & & & & 1.10 & .11 \\
\hline & & & $R b^{*}$ & & & & 1.35 & .14 \\
\hline & & & $\mathrm{sb}$ & $<$ & 30.00 & 5.80 & & \\
\hline & & & $\mathrm{Sb}^{\star}$ & $<$ & 30.00 & 5.80 & .24 & .02 \\
\hline & & & sr & & 222.00 & 27.20 & & \\
\hline & & & $S r^{*}$ & & 236.00 & 28.90 & 252.00 & 25.20 \\
\hline \multirow[t]{5}{*}{ ' } & & & $v$ & & 18.00 & 4.22 & & \\
\hline & & & $v^{*}$ & & 18.00 & 4.22 & 18.50 & 1.85 \\
\hline & & & $2 n$ & & 30.00 & 6.20 & & \\
\hline & & & $2 n *$ & & 33.00 & 6.66 & 25.50 & 2.55 \\
\hline & & 669672 & $u^{*}$ & & 24.46 & 6.80 & 41.00 & 4.10 \\
\hline \multirow[t]{13}{*}{$2-E 28-21$} & $1 / 11 / 90$ & 066968 & $\mathrm{Ag}$ & $<$ & 10.00 & 2.39 & .02 & .00 \\
\hline & & & $\therefore 2^{*}$ & $<$ & 10.00 & 2.39 & & \\
\hline & & & Al & $<$ & 150.00 & 20.10 & 2.50 & .25 \\
\hline & & & $A 1 *$ & $<$ & 150.00 & 20.10 & & \\
\hline & & & As & $<$ & 5.00 & 2.53 & 1.63 & .16 \\
\hline & & & As" & $<$ & 5.00 & 2.53 & & \\
\hline & & & $\mathrm{Bd}$ & & 40.00 & 6.87 & 40.80 & 4.08 \\
\hline & & & $B a^{*}$ & & 40.00 & 4.87 & & \\
\hline & & & $C d$ & $<$ & 2.00 & 1.38 & .06 & .01 \\
\hline & & & $\cot$ & $<$ & 2.00 & 1.38 & & \\
\hline & & & Co & $<$ & 20.00 & 3.35 & .06 & .01 \\
\hline & & & Cot & $<$ & 20.00 & 3.35 & & \\
\hline & & & $\mathrm{Cr}$ & $<$ & 10.00 & 5.13 & 4.20 & .42 \\
\hline
\end{tabular}

*indicates unfiitered anaiyses, resuits in ppo. 
WHC-EP-0366

\begin{tabular}{|c|c|c|c|c|c|c|c|c|}
\hline \multirow[b]{2}{*}{ Well } & \multirow[b]{2}{*}{ Date } & \multirow[b]{2}{*}{ UST \# } & \multirow[b]{2}{*}{ Element } & \multicolumn{3}{|c|}{ UST } & \multicolumn{2}{|c|}{ PNL } \\
\hline & & & & & esult & Error & Result & Error \\
\hline 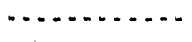 & $\cdots \ldots$ & $\ldots \ldots$ & $\cdots \ldots$ & $\cdots$ & $\ldots . .$. & ........... & $\ldots$ & $\ldots$ \\
\hline \multirow[t]{24}{*}{$2-E 28-21$} & $1 / 11 / 90$ & 066968 & $\mathrm{Cr}^{*}$ & $<$ & 10.00 & 5.13 & & \\
\hline & & & cs & & & & .01 & .00 \\
\hline & & & $\mathrm{Cu}$ & $<$ & 10.00 & 2.89 & .36 & .04 \\
\hline & & & $\mathrm{Cu}^{*}$ & $<$ & 10.00 & 2.89 & & \\
\hline & & & $\mathrm{Fe}$ & $<$ & 30.00 & 9.50 & 25.00 & 2.50 \\
\hline & & & $\mathrm{Fe}^{*}$ & & 223.00 & 25.40 & & \\
\hline & & & $M n$ & $<$ & 5.00 & 1.67 & 1.65 & .17 \\
\hline & & & $M n^{*}$ & & 6.00 & 1.72 & & \\
\hline & & & Mo & $<$ & 40.00 & 4.83 & 11.70 & 1.17 \\
\hline & & & Mo* & $<$ & 40.00 & 4.83 & & \\
\hline & & & $\mathrm{Ni}$ & $<$ & 10.00 & 3.15 & .80 & .08 \\
\hline & & & $\mathrm{Ni*}$ & $<$ & 10.00 & 3.15 & & \\
\hline & & & $\mathrm{Pb}$ & $<$ & 5.00 & 2.55 & .27 & .03 \\
\hline & & & $R \mathbf{B}$ & & & & 1.19 & .11 \\
\hline & & & sb & $<$ & 30.00 & 5.80 & .12 & .09 \\
\hline & & & $S_{b *}$ & $<$ & 30.00 & 5.80 & & \\
\hline & & & $\mathrm{Sr}$ & & 231.00 & 28.30 & 230.00 & 23.00 \\
\hline & & & $s r^{*}$ & & 226.00 & 27.70 & & \\
\hline & & & $v$ & & 11.00 & 3.66 & 14.60 & 1.46 \\
\hline & & & $v * \cdot$ & & 16.00 & 4.05 & & \\
\hline & & & $2 n$ & $<$ & 5.00 & 2.55 & 6.70 & .67 \\
\hline & & & $2 n *$ & & 6.00 & 2.69 & & \\
\hline & & 669682 & $u$ & & & & 34.30 & 3.43 \\
\hline & & . & $\mathrm{u}^{\star}$ & & 27.32 & 7.55 & & \\
\hline \multirow[t]{21}{*}{ 2-W10-14 } & $1 / 10 / 90$ & 066954 & $A g$ & $<$ & 10.00 & 2.39 & .02 & .00 \\
\hline & & & $A Q^{*}$ & $<$ & 10.00 & 2.39 & .02 & .00 \\
\hline & & & Al & $<$ & 150.00 & 20.10 & 2.75 & .28 \\
\hline & & & $A l *$ & $<$ & 150.00 & 20.10 & 6.10 & .61 \\
\hline & & & As & $<$ & 5.00 & 2.53 & .85 & .08 \\
\hline & & & As* & $<$ & 5.00 & 2.53 & .76 & .08 \\
\hline & & & $\mathrm{Ba}$ & & 68.00 & 7.55 & 67.00 & 6.70 \\
\hline & & & $B a *$ & & 70.00 & 7.75 & 66.60 & 6.66 \\
\hline & & & $\operatorname{cd}$ & $<$ & 2.00 & 1.38 & .02 & .00 \\
\hline & & & Cd* & $<$ & 2.00 & 1.38 & .05 & .00 \\
\hline & & & Co & $<$ & 20.00 & 3.35 & .16 & .02 \\
\hline & & & Co* & $<$ & 20.00 & 3.35 & .31 & .03 \\
\hline & & & $\mathrm{Cr}$ & $<$ & 10.00 & 5.13 & 5.46 & .55 \\
\hline & & & $\mathrm{Cr}$ & & 91.00 & 11.50 & 22.00 & 2.20 \\
\hline & & & Es & & & & .21 & .02 \\
\hline & & & Cs* & & & & .22 & .02 \\
\hline & & & $\mathrm{Cu}$ & $<$ & 10.00 & 2.89 & .59 & .06 \\
\hline & & & $\mathrm{Cu}^{\star}$ & $<$ & 10.00 & 2.89 & 1.04 & .10 \\
\hline & & & $\mathrm{Fe}$ & & 37.00 & 9.85 & 34.80 & 3.48 \\
\hline & & & $\mathrm{Fe}^{*}$ & & 392.00 & 41.90 & 75.10 & 7.51 \\
\hline & & & Mn & 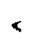 & 5.00 & 1.67 & 2.94 & .29 \\
\hline
\end{tabular}

\footnotetext{
*indicates unfiltered analyses, results in ppb.
} 
WHC-EP-0366

$5 / 29 / 90$

Analysis Comparison

\begin{tabular}{|c|c|c|c|c|c|c|c|c|}
\hline \multirow[b]{2}{*}{ Well } & \multirow[b]{2}{*}{ Date } & \multirow[b]{2}{*}{ UST \# } & \multirow[b]{2}{*}{ Element } & \multicolumn{3}{|c|}{ UST } & \multicolumn{2}{|c|}{ PNL } \\
\hline & & & & & Result & Error & Result & Error \\
\hline$\ldots \ldots \ldots \ldots$ & $\ldots \ldots$ & $\ldots \ldots$ & $\ldots \ldots$ & & $\ldots \ldots$ & $\ldots \ldots$ & $\ldots$ & $\ldots \ldots \ldots$ \\
\hline \multirow[t]{19}{*}{$2 \cdot-W 10-14$} & $1 / 10 / 90$. & 066954 & $M n^{\star}$ & & 9.00 & 1.88 & 4.33 & .43 \\
\hline & & & Mo & $<$ & 40.00 & 4.83 & 2.07 & .21 \\
\hline & & & Mo* & $<$ & 40.00 & 4.83 & 2.30 & .23 \\
\hline & & & $\mathrm{Ni}$ & $<$ & 10.00 & 3.15 & 4.96 & .50 \\
\hline & & & $\mathrm{Ni*}$ & & 44.00 & 5.48 & 12.60 & 1.26 \\
\hline & & & $\mathrm{Pb}$ & $<$ & 5.00 & 2.55 & .05 & .00 \\
\hline & & & $\mathrm{Pb}^{*}$ & & & & .12 & .01 \\
\hline & & & $\mathrm{Rb}$ & & & & 3.29 & .33 \\
\hline & & & $R b^{*}$ & & & & 3.01 & .30 \\
\hline & & & Sb & $<$ & 30.00 & 5.80 & .10 & .01 \\
\hline & & & sb* & $<$ & 30.00 & 5.80 & .10 & .01 \\
\hline & & & $\mathrm{sr}$ & & 179.00 & 22.00 & 185.00 & 18.50 \\
\hline & & & Sr* & & 185.00 & 22.80 & 185.00 & 18.50 \\
\hline & & & $v$ & & 35.00 & 5.79 & 33.40 & 3.34 \\
\hline & & & $V^{\star}$ & & 35.00 & 5.79 & 30.40 & 3.04 \\
\hline & & & $2 n$ & & 7.00 & 2.83 & 8.42 & .84 \\
\hline & & & $2 n^{\star}$ & & 7.00 & 2.83 & 8.68 & .87 \\
\hline & & $66954 \mathrm{~L}$ & $U$ & & & & .84 & .08 \\
\hline & & & $U^{*}$ & & .51 & .24 & .86 & .09 \\
\hline \multirow[t]{20}{*}{$2 \cdot W 18-17$} & $1 / 02 / 90$ & 066907 & $\mathrm{Ag}^{*}$ & $<$ & 10.00 & 2.39 & .03 & .00 \\
\hline & & & $A l^{*}$ & & 589.00 & 75.20 & 266.00 & 26.60 \\
\hline & & & As* & $<$ & 5.00 & 2.53 & .99 & .10 \\
\hline & & & $\mathrm{Ba}^{\star}$ & & 32.00 & 4.15 & 35.70 & 3.57 \\
\hline & & & $\mathrm{Cd}^{*}$ & & 16.00 & 2.28 & 4.81 & .48 \\
\hline & & & $\mathrm{CO}^{*}$ & $<$ & 20.00 & 3.35 & 1.97 & .20 \\
\hline & & & $\mathrm{Cr}^{*}$ & & 85.00 & 11.00 & 64.20 & 6.42 \\
\hline & & & Cs* & & & & .06 & .01 \\
\hline & & & $\mathrm{Cu}^{*}$ & & 11.00 & 2.98 & 11.20 & 1.12 \\
\hline & & & $\mathrm{Fe}^{\star}$ & & 49900.00 & 5060.00 & & \\
\hline & & & $M n^{*}$ & & 604.00 & 61.20 & 437.00 & 43.70 \\
\hline & & & Mo* & $<$ & 40.00 & 4.83 & .29 & .03 \\
\hline & & & $\mathrm{Ni}$ & & 41.00 & 5.23 & 33.30 & 3.33 \\
\hline & & & $\mathrm{Pb}^{\star}$ & & & & 6.79 & .68 \\
\hline & & & $\mathrm{Rb}^{*}$ & & & & 2.99 & .30 \\
\hline & & & sb* & $<$ & 30.00 & 5.80 & .07 & .01 \\
\hline & & & $\mathrm{Sr}^{*}$ & & 77.00 & 9.72 & 79.10 & 7.91 \\
\hline & & & $V^{\star}$ & & 41.00 & 6.39 & 28.40 & 2.84 \\
\hline & & & $2 n^{*}$ & & 27.00 & 5.75 & 29.80 & 2.98 \\
\hline & & 669072 & $y^{\star}$ & & .62 & .26 & .95 & .10 \\
\hline \multirow[t]{5}{*}{ 2-W27-1 } & $1 / 11 / 90$ & 066958 & $\mathrm{Ag}$ & $<$ & 10.00 & 2.39 & & \\
\hline & & & $A g^{*}$ & $<$ & 10.00 & 2.39 & .03 & .00 \\
\hline & & & Al & $<$ & 150.00 & 20.10 & & \\
\hline & & & $A I^{*}$ & $<$ & 150.00 & 20.10 & 28.30 & 2.83 \\
\hline & & & As & $<$ & 5.00 & 2.53 & & \\
\hline
\end{tabular}

*indicates unfiltered analyses, results in ppp. 
WHC-EP-0366

\begin{tabular}{|c|c|c|c|c|c|c|c|c|}
\hline \multirow[b]{2}{*}{ Well } & \multirow[b]{2}{*}{ Date } & \multirow[b]{2}{*}{ UST \# } & \multirow[b]{2}{*}{ Element } & \multicolumn{3}{|c|}{ UST } & \multicolumn{2}{|c|}{ PNL } \\
\hline & & & & & Result & Error & Result & Error \\
\hline 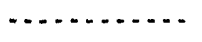 & ........... & $\ldots \ldots$ & $\ldots . . . .$. & - & .............. & $\ldots$ & $\ldots \ldots$ & $\ldots \ldots$ \\
\hline \multirow[t]{32}{*}{$2-w 27-1$} & $1 / 11 / 90$ & 005058 & As & $<$ & 5.00 & 2.53 & 1.30 & .13 \\
\hline & & & Ba & & 40.00 & 4.87 & & \\
\hline & & & $3 a^{*}$ & & 40.00 & 4.87 & 39.20 & 3.92 \\
\hline & & & $\mathrm{cd}$ & $<$ & 2.00 & 1.38 & & \\
\hline & & & $c d *$ & $<$ & 2.00 & 1.38 & .13 & .01 \\
\hline & & & Co & $<$ & 20.00 & 3.35 & & \\
\hline & & & $\mathrm{CO}^{*}$ & $<$ & 20.00 & 3.35 & .10 & .01 \\
\hline & & & $\mathrm{Cr}$ & & 12.00 & 5.23 & & \\
\hline & & & $C r^{\star}$ & & 13.00 & 5.27 & 16.20 & 1.62 \\
\hline & & & Cs* & & & & .04 & .00 \\
\hline & & & $\mathrm{Cu}$ & $<$ & 10.00 & 2.89 & & \\
\hline & & & $\mathrm{Cu}^{*}$ & $<$ & 10.00 & 2.89 & .54 & .05 \\
\hline & & & $\mathrm{Fe}$ & $<$ & 30.00 & 9.50 & & \\
\hline & & & $\mathrm{Fe}^{*}$ & & 177.00 & 21.10 & 172.00 & 17.20 \\
\hline & & & Mn & $<$ & 5.00 & 1.67 & & \\
\hline & & & $M n^{*}$ & $<$ & 5.00 & 1.67 & 4.00 & .40 \\
\hline & & & Mo & $<$ & 40.00 & 4.83 & & \\
\hline & & & Mo* & $<$ & 40.00 & 4.83 & 5.60 & .56 \\
\hline & & & $\mathrm{Ni}$ & $<$ & 10.00 & 3.15 & & \\
\hline & & & $\mathrm{Ni*}$ & $<$ & 10.00 & 3.15 & 1.56 & .16 \\
\hline & & & $\mathrm{Pb}$ & $<$ & 5.00 & 2.55 & & \\
\hline & & & $\mathrm{Pb}^{*}$ & & & & .53 & .05 \\
\hline & & & $R^{*} *$ & & & & 1.16 & .12 \\
\hline & & & Sb & $<$ & 30.00 & 5.80 & & \\
\hline & & & $\mathrm{Sb}^{*}$ & $<$ & 30.00 & 5.80 & .08 & .01 \\
\hline & & & $\mathrm{sr}$ & & 263.00 & 32.20 & & \\
\hline & & & $\mathrm{Sr}^{\star}$ & & 264.00 & 32.30 & 271.00 & 27.10 \\
\hline & & & $v$ & & 18.00 & 4.22 & & \\
\hline & & & $v^{*}$ & & 19.00 & 4.31 & 18.80 & 1.88 \\
\hline & & & $2 n$ & & 27.00 & 5.75 & & \\
\hline & & & $2 n^{*}$ & & 31.00 & 6.35 & 28.00 & 2.80 \\
\hline & & 669582 & $u^{*}$ & & 7.94 & 2.33 & 13.80 & 1.38 \\
\hline \multirow[t]{13}{*}{$6-44-438$} & $12 / 20 / 89$ & 0668667 & $\mathrm{Ag}$ & $<$ & 10.00 & 2.39 & & \\
\hline & & & $A g^{*}$ & $<$ & 10.00 & 2.39 & .0 .3 & .00 \\
\hline & & & Al & $<$ & 150.00 & 20.10 & & \\
\hline & & & $A l \star$ & & 168.00 & 22.40 & 196.00 & 19.60 \\
\hline & & & As & & 6.00 & 2.67 & & \\
\hline & & & $A S^{\star}$ & & 7.00 & 2.81 & 3.63 & .36 \\
\hline & & & $\mathrm{Ba}$ & & 52.00 & 6.00 & & \\
\hline & & & $B a^{\star}$ & & 47.00 & 5.53 & 53.70 & 5.37 \\
\hline & & & $\mathrm{cd}$ & $<$ & 2.00 & 1.38 & & \\
\hline & & & $\mathrm{Cd}^{\star}$ & $<$ & 2.00 & 1.38 & .07 & .01 \\
\hline & & & Co & $<$ & 20.00 & 3.35 & & \\
\hline & & & $\mathrm{CO}^{*}$ & $<$ & 20.00 & 3.35 & .40 & .04 \\
\hline & & & $\mathrm{Cr}$ & & 15.00 & 5.38 & & \\
\hline
\end{tabular}

*indicates unfiltered analyses, results in ppb. 
WHC-EP-0366

$5 / 29 / 90$

Analys is Comparison

\begin{tabular}{|c|c|c|c|c|c|c|c|}
\hline \multirow[b]{2}{*}{ Well } & \multirow[b]{2}{*}{ Date } & \multirow[b]{2}{*}{ UST \# } & \multirow[b]{2}{*}{ Element } & \multicolumn{2}{|c|}{ UST } & \multicolumn{2}{|c|}{ PNL } \\
\hline & & & & Result & Error & Result & \multirow[t]{2}{*}{ Error } \\
\hline & .... & .......... & ......... & -............. & . & …........ & \\
\hline \multirow{25}{*}{$6-44-43 B$} & $12 / 20 / 89$ & 066867 & $\mathrm{Cr}{ }^{*}$ & 30.00 & 6.30 & 8.00 & .80 \\
\hline & & & $\mathrm{Cs}^{\star}$ & & & .03 & .00 \\
\hline & & & Cu & 10.00 & 2.89 & & \\
\hline & & & $\mathrm{Cu}^{\star}$ & 10.00 & 2.89 & 1.09 & .11 \\
\hline & & & $\mathrm{Fe}$ & 318.00 & 34.60 & & \\
\hline & & & $\mathrm{Fe}^{*}$ & 562.00 & 58.90 & 207.00 & 20.70 \\
\hline & & & $M n$ & 7.00 & 1.77 & & \\
\hline & & & $M n^{\star}$ & 19.00 & 2.01 & 8.90 & .89 \\
\hline & & & Mo & 40.00 & 483 & & \\
\hline & & & Mo* & 40.00 & 4.83 & 8.69 & .87 \\
\hline & & & $\mathrm{Ni}$ & 10.00 & 3.15 & & \\
\hline & & & $\mathrm{Ni*}$ & 16.00 & 3.43 & 4.80 & .48 \\
\hline & & & $\mathrm{Pb}$ & 5.00 & 2.55 & & \\
\hline & & & $\mathrm{Pb}$ & & & .41 & .04 \\
\hline & & & $\mathrm{Rb}^{*}$ & & & 7.23 & .72 \\
\hline & & & Sb & 30.00 & 5.80 & & \\
\hline & & & Sb* & 30.00 & 5.80 & .15 & .02 \\
\hline & . & & $\mathrm{sr}$ & 182.00 & 22.40 & & \\
\hline & & & $S r^{*}$ & 168.00 & 20.70 & 190.00 & 19.00 \\
\hline & & & v & 36.00 & 5.69 & & \\
\hline & & & $v^{*}$ & 35.00 & 5.79 & 33.50 & 3.35 \\
\hline & & & $2 n$ & 25.00 & 5.45 & & \\
\hline & & & $2 n^{\star}$ & 27.00 & 5.75 & 28.40 & 2.84 \\
\hline & & 668662 & $u^{\star}$ & 2.16 & .69 & & \\
\hline & & 668672 & $u *$ & 2.70 & .85 & 3.08 & .31 \\
\hline
\end{tabular}

*indicates unfiltered analyses, results in ppb. 


\begin{tabular}{|c|c|c|c|c|}
\hline Site & Well & $\begin{array}{c}\text { Sample } \\
\text { Date }\end{array}$ & $\begin{array}{l}\text { UST } \\
\text { Number }\end{array}$ & $\begin{array}{l}\text { Number of } \\
\text { Analyses }\end{array}$ \\
\hline $216-B-3$ & $6-43-41 F$ & $\begin{array}{l}1 / 25 / 90 \\
4 / 18 / 90\end{array}$ & $\begin{array}{l}67133 P \\
067796 \\
067797 \\
067798 \\
067799 \\
67796 P\end{array}$ & $\begin{array}{r}4 \\
107 \\
2 \\
2 \\
2 \\
4\end{array}$ \\
\hline , & $6-43-42 J$ & $\begin{array}{l}2 / 24 / 89 \\
6 / 15 / 89 \\
8 / 09 / 89 \\
1 / 31 / 90\end{array}$ & $\begin{array}{l}064842 \\
064843 \\
064844 \\
064845 \\
64842 \mathrm{P} \\
065447 \\
065448 \\
065449 \\
065450 \\
65447 \mathrm{P} \\
065965 \\
065966 \\
065967 \\
065968 \\
65965 \mathrm{P} \\
65966 \mathrm{P} \\
067212 \\
067213 \\
067214 \\
067215 \\
67212 \mathrm{P}\end{array}$ & $\begin{array}{r}107 \\
2 \\
2 \\
2 \\
2 \\
5 \\
161 \\
2 \\
2 \\
2 \\
4 \\
107 \\
103 \\
2 \\
2 \\
4 \\
4 \\
55 \\
2 \\
2 \\
2 \\
4\end{array}$ \\
\hline & $6-43-43$ & $\begin{array}{l}2 / 24 / 89 \\
6 / 15 / 89 \\
8 / 09 / 89 \\
1 / 26 / 90 \\
4 / 26 / 90\end{array}$ & $\begin{array}{l}064850 \\
064851 \\
064852 \\
064853 \\
64850 P \\
64851 P \\
065451 \\
065452 \\
065453 \\
065454 \\
65451 P \\
065971 \\
065972 \\
065973 \\
065974 \\
65971 P \\
067167 \\
067168 \\
067169 \\
067170 \\
67167 P \\
67954 x\end{array}$ & $\begin{array}{r}107 \\
90 \\
2 \\
2 \\
4 \\
3 \\
3 \\
161 \\
2 \\
2 \\
2 \\
4 \\
107 \\
2 \\
2 \\
2 \\
4 \\
375 \\
2 \\
2 \\
2 \\
4 \\
2\end{array}$ \\
\hline & $6-43-45$ & $12 / 06 / 89$ & $\begin{array}{l}066804 \\
66804 Z\end{array}$ & $\begin{array}{r}377 \\
12\end{array}$ \\
\hline
\end{tabular}



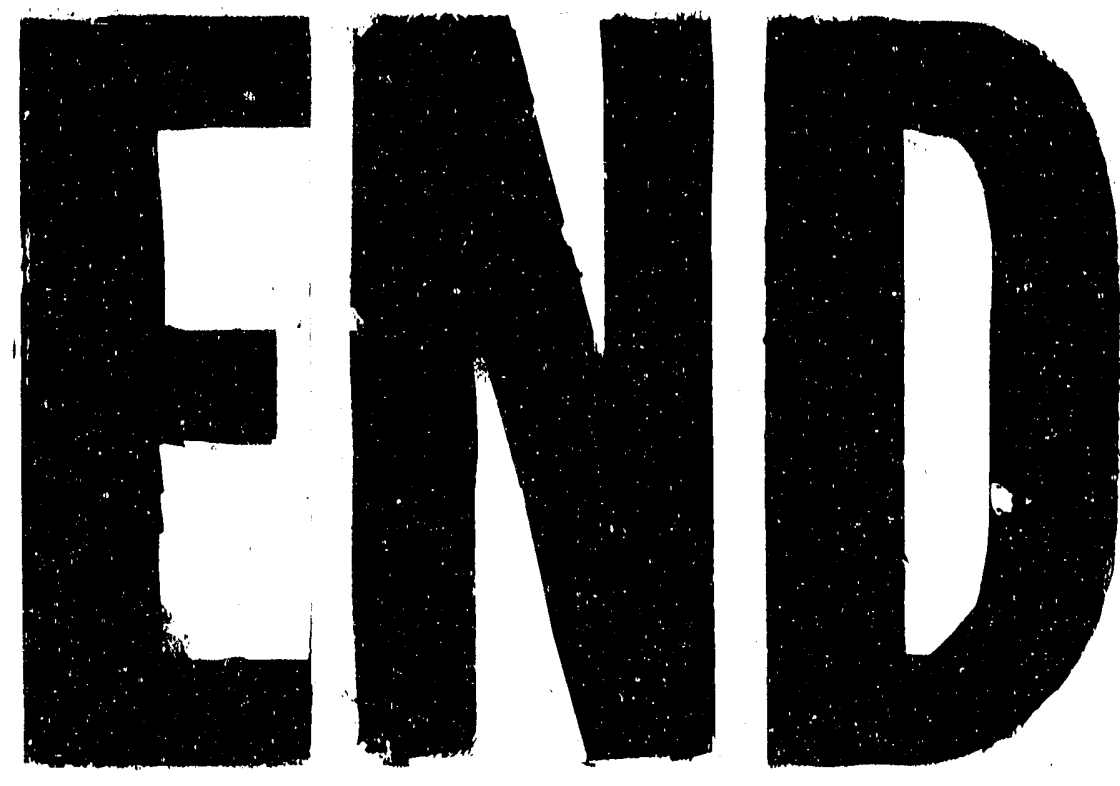

4
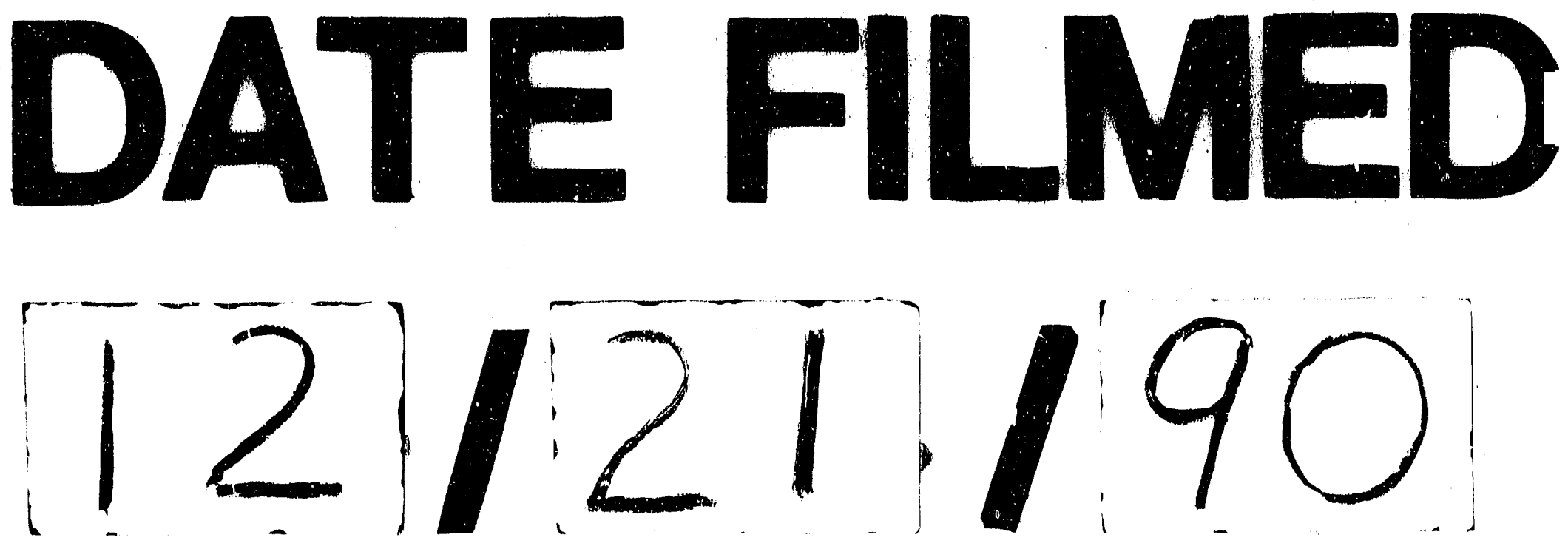
i 